FACUltad de Ciencias Astronómicas y Geofísicas

Universidad NACIONAL DE LA Plata

Tesis presentada para optar al grado de Doctora en Astronomía

\title{
ESTUDIO MULTIESPECTRAL DE REGIONES DE FORMACIÓN ESTELAR
}

María Mercedes Vazzano 

Foto de portada: La Nebulosa de la Laguna (también conocida como Messier 8 o NGC 6523) es una nube gigante de gas y polvo interestelar ubicada en la constelación de Sagitario, a unos 1200 parsec de distancia. Esta imagen tomada por el telescopio Hubble muestra solo una pequeña parte de esta turbulenta región de formación estelar. 
Dedicado a Pipo, Gonzalo y Emilia, las verdaderas estrellas de mi cielo. 
'La superficie de la Tierra es la orilla del océano cósmico.

Desde ella hemos aprendido la mayor parte de lo que sabemos.

Recientemente nos hemos adentrado un poco en el mar, vadeando

lo suficiente para mojamos los dedos de los pies, o como máximo

para que el agua nos llegara al tobillo.

El agua parece que nos invita a continuar. El océano nos llama.

Hay una parte de nuestro ser conocedora de que nosotros venimos de allí.'

Carl Sagan

'Multiplicar los muelles no disminuye el mar'

Emily Dickinson 
Mi interés por la Astronomía apareció casi de un día para el otro, cuando me dí cuenta que era la perfecta combinación entre ciencia dura (siempre sentí interés por la física y la química) y un 'no se que' que le otorgaba cierta mística. Casi trece años después de ese día he descubierto que la Astronomía es mucho más de lo que pensaba. La dimensión y belleza de la ciencia que elegí supera mis espectativas por mucho. La mayor revelación fue comprender que en el universo había mucho más que estrellas, y que era posible 'ver' lo invisible. Mi destino de radioastrónoma se había definido.

Deseo profundamente que este trabajo ayude a develar alguno de los miles misterios que nos presenta el universo, y que aquellos que lo lean seapan apreciar la dedicación y la pasión que conlleva. Aquí dejo un pedazo de mi vida.

\section{María Mercedes Vazzano}

Agosto de 2018 


\section{Agradecimientos}

En primer lugar quisiera agradecer a la Doctora Cristina Cappa, que es, como ella misma una vez se definió, mi 'madre científica', y a quien realmente siento de ese modo porque ha sido no solamente quien me ha enseñado y orientado en lo que respecta a los concimientos científicos, sino que ha sabido como acompañarme durante estos años en un sentido mucho más amplio. Gracias Cristina, no sólo por haber cumplido con tu tarea como directora, sino también por la gran persona que sos.

A mis codirectores: a Guillermo Bosch, a Javier Vasquez por haberme acompañado en los primeros años del doctorado y a Nicolás Duronea por haberse animado a terminar la tarea.

A todas las personas que han colaborado de algun modo en esta tesis: Mónica Rubio, Verónica Firpo y Laura Suad.

A la Facultad de Ciencias Astronómicas y Geofísicas de La Plata por ser la institución responsable de mi formación académica de grado y posgrado y a todos sus docentes por compartir sus conocimientos conmigo.

Al Instituto Argentino de Radioastronomía por brindarme un lugar de trabajo y a todo su personal.

Al CONICET por haberme otorgado la beca gracias a la cual pude llevar a cabo esta tesis.

A todos mis amigos por haber sido ese cable a tierra que se necesita para seguir adelante, y especialmente a Lucía Rizo con quién además comparto la pasión por mirar hacia el cielo.

A mis padres, quienes siempre me han acompañado en todas mis elecciones y me han enseñado el valor del esfuerzo.

Y finalmente a las tres personas más importantes en mi vida: a Pipo, mi amor y mi mejor amigo, y a Gonzalo y Emilia. Todo en la vida es más facil y más hermoso junto a ustedes. LOS AMO. 


\section{Resumen}

Gran parte del Universo en el que vivimos resulta invisible a nuestros ojos. Si fuéramos capaces de ver toda la radiación electromagnética descubriríamos mucho más que estrellas: enormes regiones de gas y polvo esculpiendo hermosas formas en el cielo. Es en estas regiones invisibles a nuestros ojos en donde ocurre la formación de nuevas estrellas.

La formación de estrellas en la galaxia es un proceso dinámico. Las estrellas masivas evolucionan rápidamente modificando el medio en el que se encuentran inmersas, calentando, comprimiendo y enriqueciendo químicamente el gas que las rodea y desencadenando la formación de nuevas generaciones de estrellas.

Estas regiones 'invisibles' se pueden estudiar a partir de la emisión desde el infrarrojo cercano hasta longitudes de onda de radio, originada en el gas y el polvo interestelar.

En esta tesis se estudia un conjunto de regiones con diferentes características pero que tienen algo en común: todas ellas son sitios potenciales de formación de estrellas.

El estudio llevado a cabo en esta tesis es de carácter observacional. Para estudiar las distintas regiones de formación estelar se analizaron datos de observaciones submilimétricas e infrarrojas. Se cuenta con datos propios de líneas moleculares observados con el telescopio submilimétrico APEX y con observaciones en el infrarrojo cercano realizadas con el instrumento Flamingos2 de Gemini Sur. Estos han sido complementados con datos públicos tanto de líneas moleculares (MALT90) como de imágenes en un amplio rango de longitudes de onda del infrarrojo (Spitzer-IRAC, Spitzer-MIPS, Herschel). Se completó el estudio analizando la presencia de objetos estelares jóvenes detectados en el infrarrojo y clasificados a partir de datos de catálogos (IRAC, WISE, 2MASS).

Las regiones estudiadas en esta tesis son la región Hir Gum 31, la nube infrarroja oscura SDC 341.232-0.268, y el complejo de burbujas infrarrojas S108-S109-S110-S111.

En el Capítulo 1 se describen las características de medio interestelar y los efectos que las estrellas masivas pueden tener sobre el mismo. En la Capítulo 2 se detallan los distintos procedimientos utilizados para calcular parámetros de las regiones estudiadas. En el Capítulo 3 se hace una breve descripción de los conceptos básicos de instrumentación radioastronómica y de las bases de datos utilizadas. En los Capítulos 4, 5, 6 y 7 se analiza el gas molecular en base a las observaciones de ${ }^{12} \mathrm{CO},{ }^{13} \mathrm{CO}, \mathrm{C}^{18} \mathrm{O}$ y trazadores moleculares de alta densidad que, junto con los datos infrarrojos y el análisis de la presencia de fuentes puntuales, permiten proponer posibles escenarios para cada región. 


\section{Índice general}

$\begin{array}{ll}\text { Objetivos } & 1\end{array}$

I Fundamentos teóricos 3

1. El medio interestelar

1.1. Componentes de medio interestelar . . . . . . . . . . . . . . 5

1.2. Moléculas en el medio interestelar . . . . . . . . . . . . . . . . . 9

1.3. Efecto de las estrellas masivas en el medio interestelar . . . . . . . . . . . . 11

1.3.1. Regiones Hir . . . . . . . . . . . . . . . . . . . . . . . 11

1.3.2. Regiones de fotodisociación . . . . . . . . . . . . . 15

1.3.3. Burbujas interestelares . . . . . . . . . . . . . . . 16

1.3.4. Burbujas infrarrojas de polvo . . . . . . . . . . . . . . . . . 17

1.3.5. Remanentes de supernova . . . . . . . . . . . . . . . . . . 19

1.4. Formación estelar . . . . . . . . . . . . . . . . . . . . . 19

1.4.1. Formación de estrellas de baja masa . . . . . . . . . . . . . . . . . . . . 19

1.4.2. Formación de estrellas de gran masa . . . . . . . . . . . . . . 21

1.4.3. Formación estelar inducida . . . . . . . . . . . . . . . 22

1.4.4. Identificación de YSOs a partir de observaciones infrarrojas . . . . . . . 25

1.4.5. Identificación de YSOs a partir de catálogos infrarrojos . . . . . . . . . . . . . . . . . 25

1.4.6. Jets y flujos moleculares . . . . . . . . . . . . . . . . . . 29

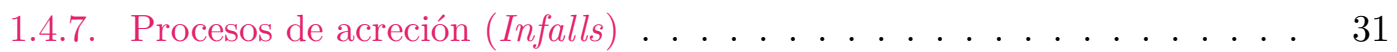

1.4.8. Extended Green Objects . . . . . . . . . . . . . . . . . . . . . . . . . . . . 32

1.4.9. Máseres interestelares . . . . . . . . . . . . . . . . . . . . . . . . 33

1.4.10. Nubes infrarrojas oscuras (IRDCs) . . . . . . . . . . . . . 33

2. Cómo obtener información del MIE $\quad 37$

2.1. Líneas espectrales moleculares en la banda de radio . . . . . . . . . . . . . . . . 37

2.2. Procesos físicos en las nubes moleculares . . . . . . . . . . . . . . . . . . . 37

2.2.1. Transiciones moleculares . . . . . . . . . . . . . . . . . 37

2.2.2. Transiciones rotacionales. . . . . . . . . . . . . . . . 39

2.2.3. Parámetros físicos de las nubes moleculares . . . . . . . . . . . . . . . . . . . . 42

2.2.4. Moléculas trazadoras de alta densidad . . . . . . . . . . . . . . . . . 47

2.3. Emisión térmica del polvo interestelar . . . . . . . . . . . . 50

2.4. Emisión en el continuo de radio . . . . . . . . . . . . . . . . 52

2.5. Ajuste de distribuciones espectrales de energía . . . . . . . . . . . . 53 
II Observaciones $\quad 55$

$\begin{array}{lr}\text { 3. Observaciones } & \mathbf{5 7}\end{array}$

3.1. Radiotelescopios ........................ . 57

3.1.1. Conceptos de instrumentación radioastronómica . . . . . . . . . . 57

3.2. Telescopios utilizados . . . . . . . . . . . . . . . . . . . 61

3.2.1. Telescopio NANTEN . . . . . . . . . . . . . . . . . 61

3.2.2. Telescopio APEX . . . . . . . . . . . . . . . 61

3.2.3. Telescopio Gemini . . . . . . . . . . . . . . . . . 61

3.3. Bases de datos . . . . . . . . . . . . . . . . . . . . . . 62

3.3.1. Spitzer ...................... 62

3.3.2. Herschel ..................... 63

3.3.3. ATLASGAL ................ . . . 63

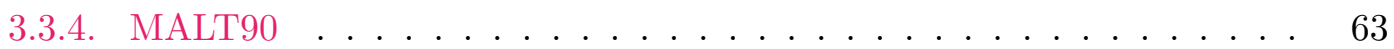

3.3.5. SUMSS .................... . . . . 64

3.4. Catálogos de fuentes puntuales infrarrojas . . . . . . . . . . . . . . . . . . . 64

3.5. Reducción de datos y confeccción de cubos de datos . . . . . . . . . . . . . 64

3.6. Regiones estudiadas en esta tesis . . . . . . . . . . . . . . . . . . 65

$\begin{array}{lll}\text { III Resultados } & 67\end{array}$

4. La región Hiı Gum $31 \quad 69$

4.1. Antecedentes de la región . . . . . . . . . . . . . . . . . . . . . . 69

4.2. Observaciones y bases de datos . . . . . . . . . . . . . . . . . . . . . 74

4.3. IRAS 10351-5816 . . . . . . . . . . . . . . . . . . . 76

4.4. IRAS $10349-5824$. . . . . . . . . . . . . . . . . . . . . . 79

4.5. 2MASSJ10365763-5844052 . . . . . . . . . . . . . . . . . 85

4.6. Conclusiones ......................... . . . . 92

5. Espectroscopía Flamingos 2 hacia IRAS 10349-5824 95

5.1. Observaciones ........................ 95

5.2. Resultados. . . . . . . . . . . . . . . . . . . . . 96

5.3. Modelos de objetos $\mathrm{HH}$. . . . . . . . . . . . . . . . . . . . . . 99

5.4. Cocientes de líneas . . . . . . . . . . . . . . . . . . . . 102

5.5. Conclusiones ........................... 104

6. IRDC SDC 341.232-0.268 107

6.1. Antecedentes de la región . . . . . . . . . . . . . . . . . . 107

6.2. Datos utilizados . . . . . . . . . . . . . . . . . . . 108

6.2.1. Observaciones moleculares . . . . . . . . . . . . . 108

6.2.2. Datos de Herschel . . . . . . . . . . . . . . . . . . 109

6.3. Gas molecular: distribución y cinemática . . . . . . . . . . . . . . . . 109

6.4. Parámetros derivados de la emisión de CO . . . . . . . . . . . . . . . . . 112

6.5. Análisis de datos de MALT90 . . . . . . . . . . . . . . . . . . . . . . 115

6.6. Distribución del polvo . . . . . . . . . . . . . . . . . . . . . 117

6.7. Análisis y discusión . . . . . . . . . . . . . . . . . . . . . . . 120

6.7.1. Evidencias de infalls y outflows . . . . . . . . . . . . . . . . . . . . . . . . . . . . . . . . . . . . . . . . .

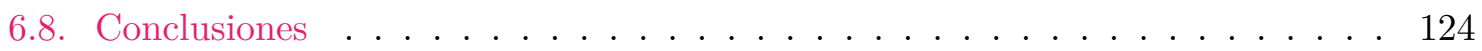


7. Complejo S108-S109-S110-S111 127

7.1. Antecedentes de la región . . . . . . . . . . . . . . . . . . 127

7.2. Observaciones y base de datos . . . . . . . . . . . . . . . . 131

7.3. Análisis de imágenes en el infrarrojo y el continuo de radio . . . . . . . . . . 133

7.4. Análisis de datos de líneas moleculares . . . . . . . . . . . . . . . . . 136

7.4.1. Distribución global del gas molecular . . . . . . . . . . . . . 136

7.4.2. El filamento en la zona de interacción de las burbujas . . . . . . . . 138

7.4.3. Burbuja tripolar . . . . . . . . . . . . . . . . . . . . 138

7.4.4. Identificación de grumos densos . . . . . . . . . . . . . . . . . . 142

7.4.5. IRDCs . . . . . . . . . . . . . . . . . . . . . . 149

7.4.6. Regiones observadas con MALT90 . . . . . . . . . . . . . 150

7.5. Parámetros del gas molecular . . . . . . . . . . . . . . . . . 154

7.5.1. Calculados a partir de CO . . . . . . . . . . . . . . 154

7.5.2. Calculados a partir de trazadores de alta densidad . . . . . . . . . 160

7.6. Parámetros calculados de emisión en continuo de radio y del polvo . . . . . . 160

7.7. Cúmulos y formación estelar en el complejo . . . . . . . . . . . . . . . . 161

7.7.1. Cúmulos infrarrojos . . . . . . . . . . . . . . . . . . 162

7.7.2. Búsqueda de candidatos a YSO . . . . . . . . . . . . . . . . 162

7.7.3. Clasificación de fuentes Spitzer . . . . . . . . . . . . . . . 163

7.7.4. Clasificación de fuentes WISE . . . . . . . . . . . . . . . 166

7.7.5. Clasificación de fuentes 2MASS . . . . . . . . . . . . . . 167

7.7.6. Clasificación de fuentes MSX . . . . . . . . . . . . . . . . 167

7.7.7. Correlación de fuentes IR y gas molecular . . . . . . . . . . . . . . . 167

7.8. Discusión . . . . . . . . . . . . . . . . . . . . . 172

7.8.1. Escenario propuesto . . . . . . . . . . . . . . . . 172

7.8.2. Estrellas excitatrices y edad de las regiones Hiı . . . . . . . . . . . . 173

7.8.3. ¿Formación estelar inducida? . . . . . . . . . . . . . . . . . 174

7.9. Conclusiones . . . . . . . . . . . . . . 176

8. Conclusiones generales $\quad 185$

$\begin{array}{ll}\text { Apéndice } & 187\end{array}$

$\begin{array}{ll}\text { Lista de figuras } & 191\end{array}$

$\begin{array}{ll}\text { Lista de tablas } & 195\end{array}$ 
ÍNDICE GENERAL 


\section{Objetivos}

\section{Objetivo específico}

El objetivo específico del presente trabajo es estudiar una muestra de regiones de formación estelar a fin de analizar las características del gas y polvo asociados, investigar la presencia de flujos bipolares y evaluar el estado evolutivo de los objetos estelares jóvenes embebidos. Para investigar la distribución del gas molecular en los grumos densos se utilizaron observaciones de líneas moleculares en el rango milimétrico, mientras que observaciones de continuo en el lejano infrarrojo permitieron analizar el polvo frío asociado. El estudio incluye el análisis de imágenes en otras bandas del espectro electromagnético, como el continuo de radio en la banda centimétrica y el infrarrojo cercano y mediano, y el análisis del contenido estelar de las regiones a partir de relevamientos disponibles en el infrarrojo cercano. Para llevar a cabo este trabajo se han seleccionado algunas regiones de formación estelar que carecen de estudios previos detallados y que están ubicadas preferentemente en la vecindad de regiones Hiı y burbujas, lo cual permite además investigar si los procesos de formación estelar se han visto favorecidos por el efecto expansivo de estas estructuras sobre el medio interestelar circundante.

\section{Objetivo general}

Se espera que estos estudios contribuyan a elucidar aspectos de los procesos de formación estelar que aún hoy se debaten. En particular, la observación y determinación de las características de núcleos densos y su interpretación son fundamentales para poner límites tanto en las teorías de formación de estrellas como en simulaciones de formación y evolución de nubes moleculares. Por otro lado, dado que se consideran objetos estelares jóvenes precursores de estrellas de gran masa y de baja masa, estos estudios pueden contribuir a elucidar las diferencias en la formación estelar entre estrellas de ambos grupos. Además, dado que algunos de los objetos analizados están ubicados en la vecindad de regiones HiI, estos estudios permiten arrojar luz en el debate acerca de la factibilidad de estos procesos en las regiones de interés. Estudios de este tipo se verán facilitados con la instalación en los próximos años del radiotelescopio LLAMA (Long Latin American Millimeter Array) en la Puna Salteña y con las ventajas de los telescopios Gemini. Así este tema de investigación tendrá un impacto importante y podrá insertarse en los desarrollos instrumentales de las facilidades observacionales en los rangos óptico y cercano infrarrojo más importante a la que nuestro país pertenece (Gemini) y en el rango milimétrico con el radiotelescopio LLAMA. 


\section{Parte I}

\section{Fundamentos teóricos}





\section{Capítulo 1}

\section{El medio interestelar}

\subsection{Componentes de medio interestelar}

El medio interestelar (MIE) de nuestra galaxia se encuentra formado por una fase gaseosa y una fase sólida. El gas consiste en un $70 \%$ de Hidrógeno (en sus tres fases, molecular, neutra y ionizada), $28 \%$ de Helio y $2 \%$ de elementos pesados. Por otra parte, la fase sólida está compuesta por el polvo interestelar. En la Tabla 1.1 se muestran las principales características de las diferentes fases de gas que componen el MIE.

\begin{tabular}{lcccc}
\hline \hline Fase & & $n$ & $\mathrm{~T}_{K}$ & $\mathrm{M}$ \\
& & {$\left[\mathrm{cm}^{-3}\right]$} & {$[\mathrm{K}]$} & {$\left[\mathrm{M}_{\odot}\right]$} \\
\hline Atómica & frío & $\sim 1-20$ & $\sim 100$ & $1.5 \times 10^{9}$ \\
& tibio & $\sim 0.25$ & $\sim 8000$ & $1.5 \times 10^{9}$ \\
\hline Molecular & & $\geq 1000$ & $\leq 30$ & $10^{9}$ \\
\hline Ionizada & regiones HII & $1-10^{4}$ & $10^{4}$ & $5 \times 10^{7}$ \\
& difuso & $\sim 0.03$ & $\sim 8000$ & $10^{9}$ \\
& caliente & $\sim 6 \times 10^{-3}$ & $\sim 5 \times 10^{5}$ & $10^{8}$ \\
\hline Masa total & & & & $5-6 \times 10^{9}$ \\
\hline \hline
\end{tabular}

Tabla 1.1: Características de las fases gaseosas del MIE de nuestra galaxia tomadas de Lequeux (2005). Se listan la densidad ambiental, la temperatura cinética y la masa total involucrada.

Hidrógeno neutro Aproximadamente la mitad de la masa de la galaxia está formada de Hidrógeno atómico neutro (HI). Las regiones frías atómicas tienen temperaturas de entre 50 y $150 \mathrm{~K}$ y se encuentran concentradas mayormente sobre el plano galáctico. Sus tamaños típicos son de unos $5 \mathrm{pc}$, con densidades de $1-20 \mathrm{~cm}^{-3}$ y masas de $50-500 \mathrm{M}_{\odot}$. Se detectan principalmete por medio de la línea de $21 \mathrm{~cm}$ del HI, aunque también se pueden identificar observando líneas en absorción de Ca II, Na I, etc. sobre el fondo estelar. La Figura 1.1 muestra la distribución global de HI en la Vía Láctea, donde se hace evidente su concentración sobre el plano galáctico.

Gas molecular El gas molecular de la galaxia se encuentra en las nubes moleculares. Estas están compuestas fundamentalmente por Hidrógeno molecular $\left(\mathrm{H}_{2}\right)$, con trazas de otras moléculas como $\mathrm{CO}, \mathrm{NH}_{3}, \mathrm{H}_{2} \mathrm{O}$, etc. Sin embargo el $\mathrm{H}_{2}$ carece de transiciones fácilmente excitables en las condiciones reinantes en las nubes moleculares, por lo que éste se detecta 


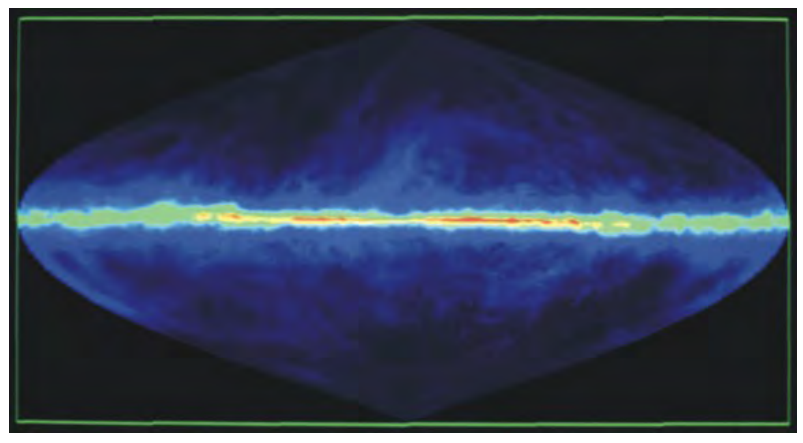

Figura 1.1: Distribución de la emisión de Hidrógeno atómico neutro en la Via Láctea. El color rojo indica direcciones de alta densidad de Hidrógeno mientras que el azul y negro muestran áreas con poco Hidrógeno. La figura se centra en el centro galáctico y la longitud galáctica aumenta hacia la izquierda. Los datos provienen de mediciones de la línea de Hidrógeno de $21 \mathrm{~cm}$ obtenidas de varios relevamientos.

\begin{tabular}{lccc}
\hline \hline & Nube oscura & Grumo & Núcleo \\
\hline Masa $\left(\mathrm{M}_{\odot}\right)$ & $10^{3}-10^{4}$ & $5-500$ & $0.5-5$ \\
Tamaño $(\mathrm{pc})$ & $2-15$ & $0.3-3$ & $0.03-2$ \\
Densidad media $\left(\mathrm{cm}^{-3}\right)$ & $50-500$ & $10^{3}-10^{4}$ & $10^{4}-10^{5}$ \\
Extensión en velocidad $\left(\mathrm{km} \mathrm{s}^{-1}\right)$ & $2-5$ & $0.3-3$ & $0.1-0.3$ \\
Temparatura del gas $(\mathrm{K})$ & $\sim 10$ & $10-20$ & $8-12$ \\
Ejemplos & Taurus, Oph, Musca & $\mathrm{B} 213, \mathrm{~L} 1709$ & $\mathrm{~L} 1544, \mathrm{~L} 1498, \mathrm{~B} 68$ \\
\hline \hline
\end{tabular}

Tabla 1.2: Propiedades de nubes oscuras, grumos y núcleos. Tomado de Bergin \& Tafalla (2007).

principalmente a partir de la emisión de transiciones rotacionales del $\mathrm{CO}$, que es la molécula más abundante en las nubes moleculares luego del $\mathrm{H}_{2}$, y de otras moléculas de menor abundancia.

Las nubes moleculares poseen temperaturas de entre 10 y $30 \mathrm{~K}$, con dimensiones típicas de unos 5 pc, densidades de entre $10^{3}$ y $10^{5} \mathrm{~cm}^{-3}$ y masas de unas miles de $\mathrm{M}_{\odot}$. Tienen un aspecto grumoso, son altamente estructuradas y con grandes variaciones en densidad: cuando se las observa con resolución angular suficiente evidencian una estructura jerárquica, con grumos y núcleos en pequeña escala (Blitz \& Williams, 1999) (ver Figura 1.2). En la Tabla 1.2 se muestran las propiedades físicas de nubes oscuras, grumos y núcleos.

Las nubes moleculares son los sitios adecuados para la formación de nuevas estrellas. Las observaciones indican que cuanto más jóvenes son las estrellas, más denso es el material que tienen asociado, lo que sugiere que las estrellas se forman por condensación de material interestelar debido a su propia gravedad. Los núcleos densos serían un lugar adecuado para la formación estelar. En las nubes moleculares se encuentran indicadores de formación estelar reciente, como fuentes infrarrojas, máseres de $\mathrm{H}_{2} \mathrm{O}$ y regiones HiI compactas.

Según sus densidades, las nubes moleculares se clasifican en nubes interestelares difusas, nubes oscuras y nubes moleculares gigantes. En la Tabla 1.3 se muestran las caracteríaticas de cada una de ellas.

También las moléculas se encuentran, por ejemplo, en cáscaras circunestelares, regiones donde actúan frentes de choques y regiones de fotodisociación.

Gas ionizado El gas ionizado de la galaxia se encuentra en tres medios diferentes: regiones HiI o nebulosas gaseosas, medio ionizado difuso (Warm Ionized Medium, WIM), y medio 


\begin{tabular}{lcccccc}
\hline \hline Tipo de nube molecular & $\mathrm{A}_{V}$ & $\mathrm{n}_{t o t}\left(\mathrm{~cm}^{-3}\right)$ & $\mathrm{L}(\mathrm{pc})$ & $\mathrm{T}_{K}(\mathrm{~K})$ & $\mathrm{M}\left(\mathrm{M}_{\odot}\right)$ & Ejemplos \\
\hline Difusas & 1 & 500 & 3 & 50 & 50 & $\zeta$ Ophiuchi \\
\hline Gigantes & 2 & 100 & 50 & 15 & $10^{5}$ & Orion \\
\hline Oscuras & & & & & & \\
$\quad$ Complejos & 5 & 500 & 10 & 10 & $10^{4}$ & Taurus-Auriga \\
$\quad$ Individuales & 10 & $10^{3}$ & 2 & 10 & 30 & B1 \\
\hline Núcleos densos/Glóbulos de Bok & 10 & $10^{4}$ & 0.1 & 10 & 10 & TMC-1/B335 \\
\hline \hline
\end{tabular}

Tabla 1.3: Propiedades físicas de las nubes moleculares. La primer columna indica el tipo de nube, mientras que valores típicos de absorción visual $\mathrm{A}_{V}$, densidad volumétrica $\mathrm{n}_{t o t}$, medida total $\mathrm{L}$, temperatura cinética $\mathrm{T}_{K}$ y masa $\mathrm{M}$ se indican en las columnas siguientes. Tabla obtenida de Stahler \& Palla (2004).
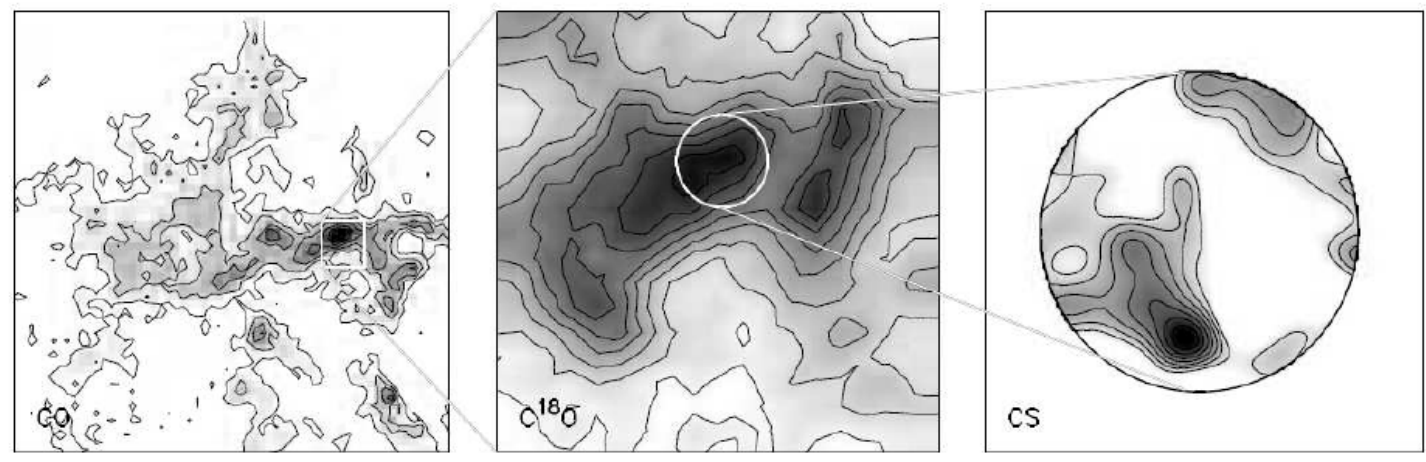

Figura 1.2: Estructura jerárquica de una nube molecular. Los tres paneles muestran una nube, un grumo y un núcleo. La nube se observa fácilmente en la emisión de ${ }^{12} \mathrm{CO}$, que a pesar de ser ópticamente gruesa, describe fielmente la ubicación de la nube (panel izquierdo). La estructura interna (panel central) se observa en más alta resolución a través de una línea ópticamente delgada como la de $\mathrm{C}^{18} \mathrm{O}$. Con un trazador de más alta densidad como $\mathrm{CS}$ se puede apreciar el núcleo (panel derecho). Figura extraida de Blitz \& Williams (1999).

ionizado caliente (Hot Ionized Medium, HIM).

Las regiones HII son regiones alrededor de estrellas con gran flujo de fotones UV (con energías mayores a $13.6 \mathrm{eV}$ ), capaces de ionizar átomos como $\mathrm{H}$, He y O. Se encuentran confinadas al plano galáctico dentro de nubes neutras y son regiones creadas y mantenidas por los fotones ultravioletas estelares. Se detectan a partir de la emisión libre-libre en el continuo de radio y de las líneas de recombinación en radio y óptico. Sus densidades electrónicas típicas varían entre 10 y $10^{5} \mathrm{~cm}^{-3}$, comparables en muchos casos a las de las nubes moleculares (excepto las regiones HiI compactas y ultracompactas cuyas densidades son del orden de $10^{6}$ y $10^{7} \mathrm{~cm}^{-3}$, respectivamente), sus tamaños varían desde $1 \mathrm{pc}$ hasta algunas decenas de pc y sus temperaturas entre 5000 y $10000 \mathrm{~K}$.

El gas ionizado difuso podría provenir del gas que escapa de las regiones Hiı o de la ionización de estrellas aisladas. Esta componente tiene mucha más masa que las regiones HII, con temperaturas del orden de $10^{3}-10^{4} \mathrm{~K}$ y densidades de $0.02-0.05 \mathrm{~cm}^{-3}$.

Finalmente, el gas ionizado caliente se originaría en los remanentes de supernova y las burbujas interestelares que luego se disipan y mezclan con el MIE. Posee temperaturas características de $10^{5}-10^{7} \mathrm{~K}$ (coronales) y densidades muy bajas, del orden de $10^{-4} \mathrm{~cm}^{-3}$. 
Polvo interestelar El polvo interestelar está formado por granos con un tamaño característico de entre 0.01 y $1 \mu \mathrm{m}$, del orden de la longitud de onda de la luz visible, lo que permitió su descubrimiento a partir de los efectos de extinción que éste producía en la luz de las estrellas. Se llama extinción a la atenuación de la radiación que atraviesa una nube debido a la suma de dos fenómenos: la absorción y la dispersión de fotones por los granos de polvo. Otros efectos que produce el polvo sobre la luz estelar son el enrojecimiento y la polarización.

El valor establecido de la absorción en la Galaxia es de aproximadamente una magnitud por cada kpc de distancia. La absorción es aproximadamente proporcional a la inversa de la longitud de onda en la región visible del espectro, con un máximo en el UV, a $2200 \AA$. Hay una buena correlación entre la densidad columnar de gas molecular y la extinción en el visible producida por el polvo interestelar, $A_{v}$,

$$
\left[\frac{N_{H_{2}}}{\mathrm{~cm}^{-2}}\right] \sim 10^{21}\left[\frac{A_{v}}{\mathrm{mag}}\right]
$$

Para explicar la polarización interestelar es necesario suponer que los granos de polvo son alargados y que están alineados parcialmente. Esto último se explica a partir de la rotación de los granos, que tiende a producirse alrededor de un eje en la dirección del campo magnético local.

La masa total de polvo de la galaxia es del orden de $\sim 5 \times 10^{7} \mathrm{M}_{\odot}$, lo que representa tan solo un $1 \%$ de la masa de gas. Sin embargo su presencia es fundamental durante el proceso de formación de algunas moléculas, entre ellas $\mathrm{H}_{2}$, ya que los granos de polvo funcionan como catalizadores. Una vez formadas las moléculas, el polvo cumple un importante rol protegiéndolas de la radiación ultravioleta. Algunos átomos y moléculas pueden depositarse sobre la superfice de los granos de polvo al colisionar con éstos formando un manto helado en donde ocurren gran número de reacciones químicas. Estas nuevas moléculas pueden inyectarse posteriormente al MIE a partir de la evaporación de los mantos.

Los granos de polvo se forman principalmente en las envolturas de estrellas frías, desde donde son barridos hacia el medio interestelar por la presión de radiación.

Se encuentran dos tipos de polvo interestelar que poseen núcleos de materiales refractarios: los silicatos $([\mathrm{O}] /[\mathrm{C}]>1)$ compuestos de $\mathrm{Si}, \mathrm{O}, \mathrm{Mg}$, Fe y Al, y los ricos en Carbono $([\mathrm{O}] /[\mathrm{C}]$ $<1$ ) compuestos principalmente por Carbono. Ambos poseen mantos de hielo de materiales volátiles condensados en su superficie. Los mantos, que se forman en las nubes moleculares, consisten en un manto interno compuesto mayormente de $\mathrm{H}_{2}$, además de $\mathrm{NH}_{3} \mathrm{y} \mathrm{CH}_{4}$, y por un manto externo compuesto de moléculas como $\mathrm{O}_{2}, \mathrm{C}_{2}, \mathrm{~N}_{2}$ y $\mathrm{CO}$, que son acretados del MIE.

Un tipo particular de partículas de polvo interestelar son los Hidrocarburos Aromáticos Policíclicos (Polycyclic Aromatic Hydrocarbons, PAHs). Estas son las moléculas más grandes presentes en el MIE, con tamaños de entre 4 y 10 Å, cuya unidad básica es el anillo de benceno y pueden llegar a contener hasta 80 átomos de Carbono. En estas macromoléculas los átomos se acomodan en estructuras con forma de panal de abeja y en láminas rígidas ligadas por fuerzas débiles (ver Figura 1.3). Los PAHs presentan emisión intensa de origen vibracional en diferentes bandas del infrarrojo cercano (NIR) centradas por ejemplo en 3.3, 6.2, 7.7, 8.6 y $11.3 \mu \mathrm{m}$.

Los PAHs se detectan fácilmente en regiones de fotodisociación (Photodissociation Regions, PDRs), donde son excitados e ionizados por fotones de las estrellas masivas con energías en el rango $4.5 \leq h \nu \leq 13.6 \mathrm{eV}$. Las PDRs, presentes en los bordes de regiones Hil y nubes moleculares, determinan la interfase entre el gas ionizado y el gas molecular (ver Sección 1.3.2). 

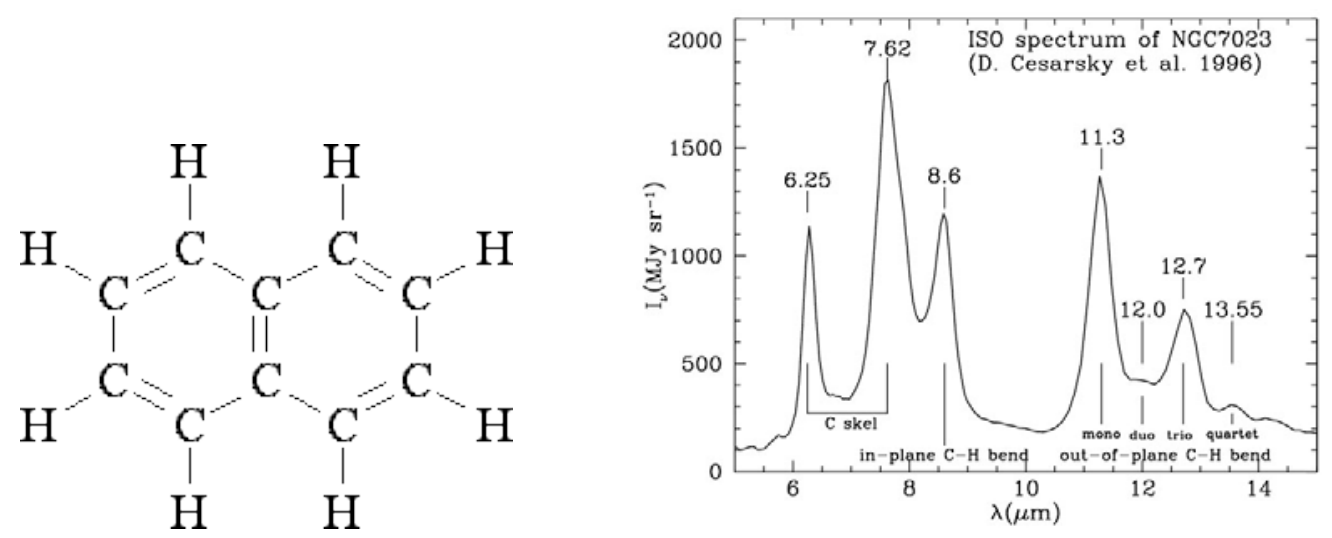

Figura 1.3: Izquierda: Estructura básica de una cadena de PAHs. Derecha: Emisión de PAHs en el espectro de 5 a $15 \mu \mathrm{m}$ de la nebulosa de reflexión NGC 7023 (Cesarsky et al., 1996).

Rayos cósmicos Toda la Galaxia es atravesada por partículas relativistas, sobre todo electrones y protones de alta energía. La aceleración de los electrones en el campo magnético galáctico produce la radiación sincrotrón galáctica de fondo, concentrada en el plano galáctico, con un máximo en la dirección del centro galáctico.

Campo magnético El campo magnético galáctico es $\mathrm{B} \sim 2-3 \times 10^{-6} \mathrm{G}$. Su intensidad depende de la densidad del medio interestelar, $\mathrm{B} \propto \mathrm{n}^{\alpha}$, con un índice $\alpha=\frac{1}{3}-\frac{2}{3}$, aunque hay variaciones locales importantes. Su dirección es en general hacia $1 \sim 90^{\circ}$, es decir, en dirección de la rotación galáctica.

\subsection{Moléculas en el medio interestelar}

Debemos a la radioastronomía nuestro conocimiento de la existencia de gran parte de las moléculas en el medio interestelar. Hoy conocemos cerca de 200 especies moleculares y se sabe de la existencia de muchas más que fueron detectadas pero aún no están identificadas. La mayor parte de estas especies son observadas a altas frecuencias, del orden del $\mathrm{GHz}$, que corresponden a longitudes de onda milimétricas y submilimétricas.

La mayor parte de las especies identificadas contienen átomos de Carbono, como puede verse en la Figura 1.4, que lista las 143 moléculas interestelares identificadas hasta el año 2007. En la Figura 1.5 se muestra un espectro observado hacia la nube molecular Sgr B2(N) en el que se observa la gran cantidad de moléculas presentes en dicha nube.

En el medio interestelar hay varias maneras de formar moléculas y muchas maneras de destruirlas. El hecho de que las especies moleculares efectivamente se encuentran en el espacio significa que el proceso de formación es generalmente más eficaz que el de la destrucción. Esto se debe también a la presencia de polvo en las nubes interestelares, que actúa protegiendo las moléculas de todo tipo de radiación capaz de destruirlas, especialmente la ultravioleta.

La mayoría de las moléculas interestelares son asimétricas, o por lo menos lo son las que se han detectado. Estas moléculas son capaces de girar de varias maneras dependiendo de su momento de inercia. Cada molécula posee varios estados de energía y las transiciones pueden ocurrir entre ellos, permitiendo la emisión de energía en una longitud de onda específica que será única para esa molécula. Algunas especies moleculares tienen cientos de posibles estados de energía, dependiendo de su estructura, lo que permite que puedan emitir en muchas frecuencias distintas. Los espectros contienen múltiples líneas en un amplio rango de longitudes 


\begin{tabular}{|c|c|c|c|c|c|}
\hline $\begin{array}{l}\text { Chemical } \\
\text { Fonmula }\end{array}$ & Nane & $\begin{array}{l}\text { Chemical } \\
\text { Formuta }\end{array}$ & Name & $\begin{array}{l}\text { Chemical } \\
\text { Fommila }\end{array}$ & Name \\
\hline $\mathrm{AlCl}$ & Aluminum chloride & $\mathrm{CH}_{3} \mathrm{CHO}$ & Acetaldehyde & HCS ${ }^{+}$ & Thioformy! radjeal lon \\
\hline AlF & Aluminum flunride & $\mathrm{CH}_{3} \mathrm{CN}$ & Methyl cyanide & HD & Deuterated hydrogen \\
\hline AINC & Alumimum iskxyanide & $\mathrm{CH}_{4} \mathrm{COOH}$ & Acelic acid & $\mathrm{HF}$ & Hydrogen fluoride \\
\hline $\mathrm{C}_{2}$ & Diatomic cartion & $\mathrm{CH}_{3} \mathrm{NC}$ & Methyl jwocyanide & HNC & Hydrogen isocyanide \\
\hline $\mathrm{C}_{2} \mathrm{H}$ & Ethyny I radical & $\mathrm{CH}_{3} \mathrm{NH}_{2}$ & Methylanine & HNCCC & Ethinylisocyanide \\
\hline $\mathrm{C}_{2} \mathrm{H}_{2}$ & Acetylene & $\mathrm{CH}_{3} \mathrm{OH}$ & methanol (wood alcohol) & $\mathrm{HNCO}$ & tsocyantis acid \\
\hline $\mathrm{C}_{2} \mathrm{H}_{4}$ & Ethylene & $\mathrm{CH}_{3} \mathrm{SH}$ & methyl nereaptan & HNCS & Fsothiocyamic acad \\
\hline $\mathrm{O}_{2} \mathrm{H}_{3} \mathrm{OCH}_{3}$ & Ethyl methyl ether & $\mathrm{CH}_{4}$ & Methane & HNO & Nitrosyl radical \\
\hline $\mathrm{C}_{2} \mathrm{O}$ & Carbon suboxide & $\mathrm{CN}$ & Cyanogen & $\mathrm{HOCH}_{2} \mathrm{CH}_{2} \mathrm{OH}$ & Ethylene glycot \\
\hline $\mathrm{C}_{2} \mathrm{~S}$ & & co & Carton monoxide & $\mathrm{HOCH}_{2} \mathrm{CHO}$ & Gilycoaldehyde \\
\hline $\mathrm{C}_{3}$ & Tricarbon radical & $\mathrm{CO}^{\prime}$ & Cirhon monoxide inn & $\mathrm{HOCO}^{+}$ & Protonated carkon disxide \\
\hline $\operatorname{cg} \mathrm{N}$ & Cyamoethynyl nudical & $\mathrm{CO}_{2}^{\prime}$ & Curtoon dioxide & $\mathrm{KCl}$ & Potassium ctilonid: \\
\hline $\mathrm{C}, \mathrm{O}$ & Thearbon onoxide & $\mathrm{CP}$ & Phosphonus cartiule & $\mathrm{H}-\mathrm{C}, \mathrm{H}$ & Propyny fidync \\
\hline Cys & & cs & Curbon monosultide & $\mathrm{MgCN}$ & Magnesium yanide \\
\hline$c_{4}$ & Fout sarbon radical & $\mathrm{FeO}^{t}$ & Iron oxide & $\mathrm{MgNC}$ & Magnesium isocyanide: \\
\hline $\mathrm{C}_{4} \mathrm{H}$ & Buadiynyl radical & $\mathrm{H}_{2}$ & Molecular hydrogen & $\mathrm{N}_{2}^{+}$ & Dinitrogen ion \\
\hline$c_{5}$ & & $\mathrm{H}_{2} \mathrm{C}$. & Hexapentacnylidene & $\mathrm{N}_{2} \mathrm{H}$ & Diazenylium \\
\hline $\mathrm{C}_{5} \mathrm{~N}$ & Cyanobutadiyny! & $\mathrm{H}_{2} \mathrm{CCC}$ & Propadienylidene & $\mathrm{N}_{2} \mathrm{O}$ & Nitrou oxide \\
\hline CsO & & $\mathrm{H}_{2} \mathrm{CCCC}$ & Butatrienylidene & $\mathrm{NaCl}$ & Sodium clileride \\
\hline $\mathrm{C}_{5} \mathrm{~S}$ & & $\mathrm{H}_{2} \mathrm{CCO}$ & Ketene & $\mathrm{NaCN}$ & Sodium cyanide \\
\hline 1. $\mathrm{C}_{5} \mathrm{HI}$ & Pentynylidyne radical & $\mathrm{H}_{2} \mathrm{CN}$ & Methylene amidogen & NH & Nitrogen hydride: \\
\hline $\mathrm{C}_{6} \mathrm{H}$ & Hexatriynyl radical & $\mathrm{H}_{2} \mathrm{CO}$ & Formaldehyde & $\mathrm{NH}_{z}$ & Aminyl radical \\
\hline $\mathrm{C}_{6} \mathrm{H}_{6}^{\mathrm{i}}$ & Benzene & $\mathrm{H}_{2} \mathrm{COH}^{+}$ & Protonated formaldeliyde & $\mathrm{NH}_{2} \mathrm{CHO}$ & Formanude \\
\hline $\mathrm{C}, \mathrm{H}$ & & $\mathrm{H}_{2} \mathrm{CS}$ & Thioformaldehyde & $\mathrm{NH}_{2} \mathrm{CN}$ & Cyanamide \\
\hline $\mathrm{C}_{8} \mathrm{H}$ & Octatetranyl radical & $\mathrm{H}_{2} \mathrm{D}^{\prime}$ & & $\mathrm{NH}_{3}$ & Ammonia \\
\hline $\mathrm{C}_{2} \mathrm{H}_{4} \mathrm{O}$ & Ellyylene oxide & $\mathrm{H}_{2} \mathrm{O}$ & Water & No & Nilric oxide \\
\hline $\mathrm{C}, \mathrm{CH}$ & Cyclic propynhidyne & $\mathrm{H}_{2} \mathrm{~S}$ & Hydrogen sulfide & NS & Nitrogen sulfide \\
\hline $\mathrm{C}_{\mathrm{C}} \mathrm{H}_{2}$ & Cyclopropynylidene & $\mathrm{H}_{j}^{i}$ & Protonated hydrogen & OCS & Carbonyl sulfide \\
\hline $\mathrm{CH}$ & Methyladine & $\mathrm{H}_{3} \mathrm{O}^{\prime}$ & Profonated water & $\mathrm{OH}$ & Hydroxyl \\
\hline $\mathrm{CH}^{+}$ & Methyladyne & $B C_{11} N$ & Cyanotetracetylenc & PN & Phosphorus mitride \\
\hline $\mathrm{CH}_{2}$ & Methylene & $\mathrm{HCSN}_{3}$ & Cyanoacetylene & $S_{2}$ & Deatomic sulfur \\
\hline $\mathrm{CH}_{2} \mathrm{CHCHO}$ & Propenal & $\mathrm{HC}_{3} \mathrm{NH}^{+}$ & Protonaled $\mathrm{HC}_{3} \mathrm{~N}$ & SH & Sultur hydride \\
\hline $\mathrm{CH}_{2} \mathrm{CHCN}$ & Vinyl cyanide & $\mathrm{HC}_{4} \mathrm{~N}$ & & $\mathrm{SiC}$ & Silicon carbide \\
\hline $\mathrm{CH}_{2} \mathrm{CHOH}$ & Vinyl alcohol & $\mathrm{HC}_{5} \mathrm{~N}$ & Cyanodiacetylene & $\mathrm{SiC}_{2}$ & Silicon dicarbide \\
\hline $\mathrm{CH}_{2} \mathrm{CN}$ & Cyanomethyl radical & $\mathrm{HC}_{\mathrm{n}} \mathrm{N}$ & & $\mathrm{SiC}_{5}$ & Silicon tricarbide \\
\hline $\mathrm{CH}_{2} \mathrm{D}^{+}$ & & $\mathrm{HC}_{7} \mathrm{~N}$ & Cyanotriacelylene & $\mathrm{SiC}_{4}$ & \\
\hline $\mathrm{CH}_{2} \mathrm{NH}$ & Methanimine & $\mathrm{HC}_{9} \mathrm{~N}$ & Cyamo-sctatetra-yne & $\mathrm{SiCN}$ & Silicon cyanide \\
\hline$\left(\mathrm{CH}_{2} \mathrm{OH}\right)_{2} \mathrm{CO}$ & & НССCHO & Propynal & Sil & Silicon hydride \\
\hline $\mathrm{CH}_{5}$ & Mettyl ratical & $\mathrm{HCCN}$ & & $\mathrm{SiH}_{2}^{i}$ & Silylene \\
\hline$\left(\mathrm{CH}_{3}\right)_{2} \mathrm{CO}$ & Acctone & HCCNC & Isocyanoacetylene & $\mathrm{SitH}_{4}$ & Silane \\
\hline$\left(\mathrm{CH}_{3} h_{2} \mathrm{O}\right.$ & Dimethyl ether & $\mathrm{HCl}$ & Hydrogen chloride & SiN & Silicon nitride \\
\hline $\mathrm{CH}_{3} \mathrm{C}_{3} \mathrm{~N}$ & Methyl cyanoacetylene & $\mathrm{HCN}$ & Hydrogen cyanide & SiNC & Silicon isocyanide \\
\hline $\mathrm{CH}_{3} \mathrm{C}_{4} \mathrm{H}$ & Methyl diacetylene & $\mathrm{HCNH}^{+}$ & Protonated hydrogen cyanide & SiO & Silicon monoxide \\
\hline $\mathrm{CH}_{3} \mathrm{C}_{5} \mathrm{~N}$ & 2.4.Hexadiynenitrile & $\mathrm{HCO}$ & Fomyl radical & Sis & Silicon sulfide \\
\hline $\mathrm{CH}, \mathrm{CCH}$ & Methyl acetylene & $\mathrm{HCO}_{4}$ & Formyl radical jon & so & Sulfur monoxide \\
\hline $\mathrm{CH}_{3} \mathrm{CH}_{2} \mathrm{CHO}$ & Propanat & $\mathrm{HCO}^{\circ}$ & Fonnyl radical ion & $\mathrm{SO}^{+}$ & Sulfur oxide ion \\
\hline $\mathrm{CH}_{3} \mathrm{CH}_{2} \mathrm{CN}$ & Ethyl cyanide & $\mathrm{HCOOCH}_{3}$ & Methyl formate & $\mathrm{SO}_{2}$ & Sulfur dioxide \\
\hline $\mathrm{CH}_{3} \mathrm{CH}_{2} \mathrm{OH}$ & Elhamol (ethyl alcohol) & $\mathrm{HCOOH}$ & Formic acid & & \\
\hline
\end{tabular}

Figura 1.4: Moléculas interestelares, tabla tomada de Verschuur (2007).

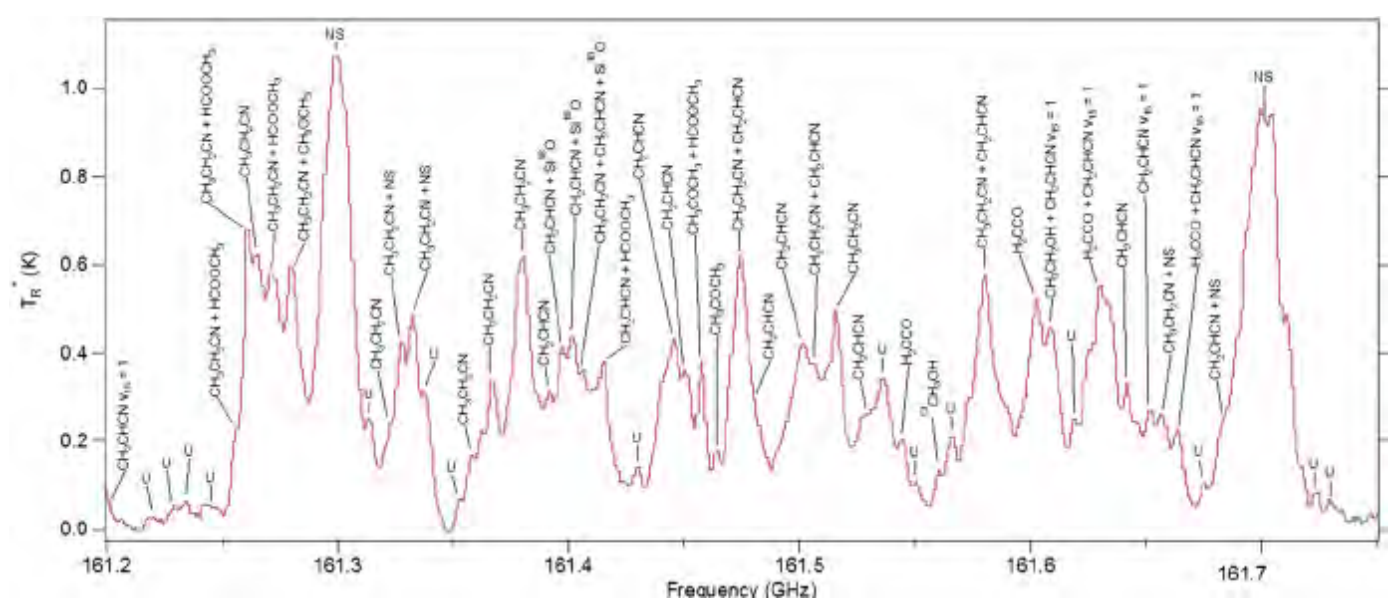

Figura 1.5: Espectro observado hacia la nube molecular Sgr B2(N) a $161.5 \mathrm{GHz}$, utilizando el telescopio Arizona Radio Observatory (ARO). Se indican las líneas conocidas, mientras que las no identificadas se marcan con 'U'. Tomada de Ziurys et al. (2015). 
de onda, que van desde radio hasta infrarrojo o ultravioleta, correspondiendo a regiones del MIE con distintas condiciones físicas.

La molécula más abundante en el medio interestelar es la de Hidrógeno molecular $\left(\mathrm{H}_{2}\right)$, que se cree representa más del $50 \%$ de la masa molecular de la galaxia. La molécula de $\mathrm{H}_{2}$ tiene transiciones vibracionales y electrónicas (por ejemplo en $2.16 \mu \mathrm{m}$ y $1090 \AA$ ), pero sólo se producen en la fase atómica fría $(\sim 100 \mathrm{~K})$ o en regiones donde actúan frentes de choques (por ejemplo en los outflows). Sin embargo, dado que la molécula de $\mathrm{H}_{2}$ está formada por dos átomos de Hidrógeno idénticos, el centro de carga del $\mathrm{H}_{2}$ coincide con su centro de masa, por lo que no posee momento dipolar eléctrico permanente $y$, por lo tanto, no tiene transiciones rotacionales permitidas que conduzcan a la emisión de líneas espectrales en la banda de radio en el medio frío $(\lesssim 20 \mathrm{~K})$.

Por lo general, la presencia de Hidrógeno molecular en el medio frío se infiere a partir de la distribución de Monóxido de Carbono (CO), que es la segunda molécula más abundante y se cree que se forma sólo en las nubes que contienen una gran cantidad de Hidrógeno molecular, lo que la convierte en un excelente trazador de $\mathrm{H}_{2}$.

Las moléculas de $\mathrm{H}_{2}$ y $\mathrm{CO}$ son las principales componentes de las nubes moleculares gigantes, grandes regiones del medio interestelar (típicamente entre 50 y 80 pc de diámetro y con varios millones de masas solares) plagadas de todo tipo de moléculas. Estas regiones son los principales sitios en donde ocurre la formación de nuevas estrellas. La evidencia de formación estelar en estas regiones viene dada por la presencias de fuentes infrarrojas brillantes, máseres y outflows.

\subsection{Efecto de las estrellas masivas en el medio interestelar}

Las estrellas masivas son muy pocas si se las compara con la cantidad de estrellas de baja masa (Salpeter, 1955), sin embargo los efectos que éstas producen pueden modificar drásticamente su entorno y juegan un rol fundamental en la evolución física y química del medio que las rodea.

La estrellas masivas interactúan con el medio interestelar circundante de dos maneras: a través de fotones ultravioletas (UV) y a través de vientos estelares que transfieren materia, momento y energía de las estrellas al medio circundante. Los fotones UV ionizan el gas atómico y disocian moléculas en los alrededores de las estrellas, formando regiones Hir. Estas regiones al expandirse por diferencia de presión con su entorno, ya que están más calientes que el mismo, generan ondas de choque que en ocasiones pueden originar inestabilidades gravitacionales y desencadenar la formación de nuevas estrellas. Por su parte los vientos estelares empujan el material circundante formando regiones de baja densidad y alta temperatura llamadas burbujas interestelares (BI, ver Sección 1.3.3). Por medio de los vientos estelares, una buena porción de la masa inicial de las estrellas es volcada al MIE, contribuyendo a su enriquecimiento químico. Estas estructuras suelen aparecer rodeadas por cáscaras densas de gas neutro proveniente del mismo medio que ha sido barrido. Las estrellas masivas terminan su vida en una violenta explosión de supernova (SN) que crea una fuerte onda de choque que barre material del medio previamente modificado por la estrella y lo enriquece químicamente.

\subsection{1. $\quad$ Regiones HII}

Las regiones Hiı son regiones ionizadas alrededor de estrellas o cúmulos de estrellas que emiten una alta tasa de fotones del continuo de Lyman, originadas dentro de la nube molecular en la que se formó su estrella o cúmulo estelar excitatriz. Estas regiones son visibles en líneas de recombinación, particularmente de $\mathrm{H}$ y He, en el óptico, infrarrojo y en radio, ya que los 

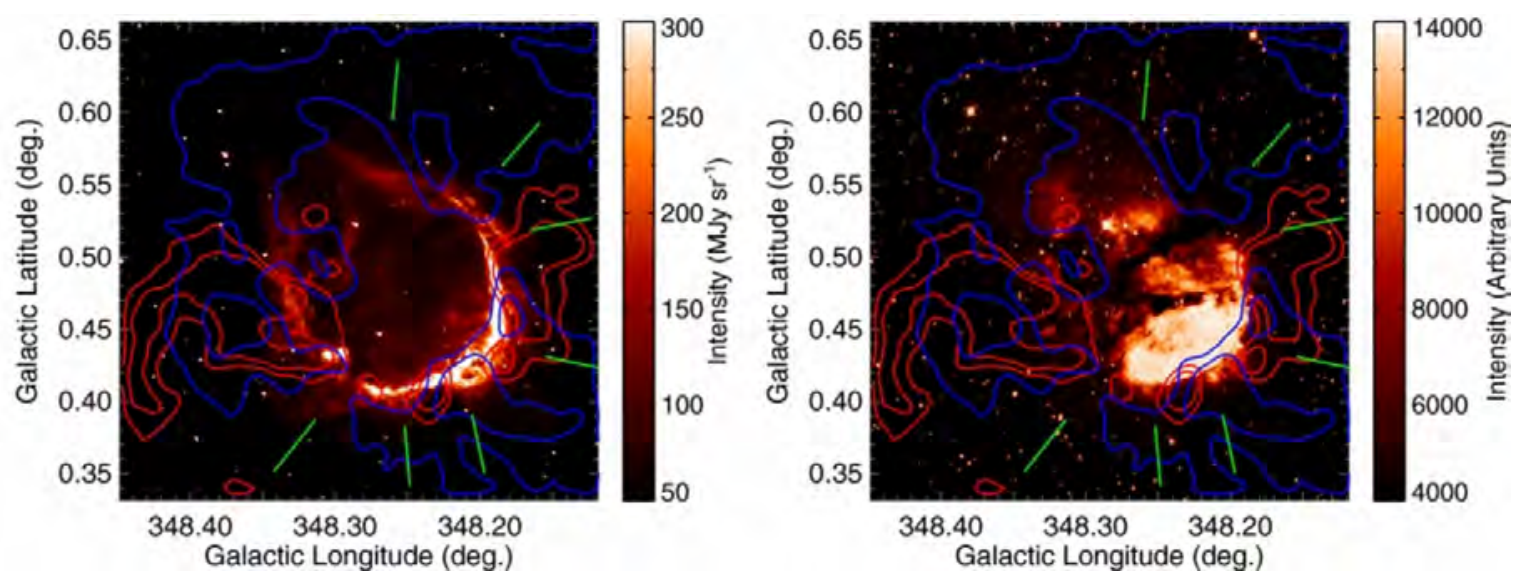

Figura 1.6: Región HiI RCW 120. Se muestra la emisión a $8 \mu \mathrm{m}$ que indica la posición de la PDR (izquierda) y la emisión óptica de $\mathrm{H} \alpha$ (derecha). Los contornos rojos muestran la emisión de ${ }^{13} \mathrm{CO}$ a $-6.0 \mathrm{~km} \mathrm{~s}^{-1}$ y los azules a $-8.9 \mathrm{~km} \mathrm{~s}^{-1}$. Imagen tomadad de Anderson et al. (2015).

átomos ionizados pueden recombinarse con electrones libres dentro de la región, cayendo en forma de cascada hasta el nivel fundamental, y emitiendo fotones de baja energía.

Inicialmente las regiones HiI son pequeñas y densas, y son llamadas regiones HiI hipercompactas y ultra-compactas (UCHII, Wood \& Churchwell, 1989). Si el flujo de fotones ultravioleta de la estrella masiva es suficientemente alto, la región HiI evoluciona a una región His clásica.

Durante la fase de expansión, una cáscara de gas neutro rodea la región Hiı (e.g. Spitzer 1978). La presencia de esta cáscara neutra puede observarse a partir de la emisión de la línea de $21 \mathrm{~cm}$ del Hidrógeno neutro (HI) (e.g. Deharveng et al., 2003) y de líneas de CO. El estudio de líneas moleculares permite la identificación de gas molecular siguiendo los bordes externos de las regiones HII, e indicando la presencia de regiones de fotodisociación (PDRs).

Las regiones Hiı resultan de particular interés para el estudio de la formación estelar, ya que las cáscaras neutras que las rodean, al ser perturbadas por la expansión de la región, presentan las condiciones adecuadas para ser sitos de formación estelar.

La Figura 1.6 muestra la región Hir RCW 120. Allí se ve cómo el gas molecular delinea el borde de la región HiI y coincide con la PDR que muestra la interfaz entre el gas neutro y el gas ionizado, mientras que la emisión en el óptico se encuentra en el interior.

\section{Evolución de regiones HII}

El tamaño de una región HiI está determinado por el flujo de fotones UV de la estrella excitatriz y por la densidad del medio en que la misma está inmersa, y viene dado por el radio de Strömgren (se considera una región formada solamente por Hidrógeno) (Strömgren, 1939)

$$
R_{S_{0}}=\sqrt[3]{\frac{3 N_{L y}}{4 \pi n_{i} n_{e} \alpha_{B}}}
$$

donde $N_{L y}$ es el número total de fotones emitidos por la estrella con $E \geq 13.6 \mathrm{eV}, n_{i}$ y $n_{e}$ son las densidades volumétricas de iones y electrones (iguales a $n_{0}$, densidad ambiental volumétrica del $\mathrm{HI}$ ), y $\alpha_{B}$ es el coeficiente de recombinación a todos los niveles del átomo de $\mathrm{H}$, salvo al fundamental, y está dado por $\alpha_{B}=2 \times 10^{-12} T_{e}^{-3 / 4} \mathrm{~cm}^{3} \mathrm{seg}^{-1}$. El tamaño de 
Tabla 1.4: Para cada tipo espectral se listan la temperatura efectiva, y número de fotones ionizantes para el $\mathrm{H}$ y el He obtenidos a partir de modelos de atmósfera para estrellas OB de secuencia principal. $\mathrm{R}_{S}(\mathrm{n} \sim 10)$ y $\mathrm{R}_{S}(\mathrm{n} \sim 50)$ son los radios de Strömgren para un medio compuesto sólo por Hidrógeno, con una densidad de 10 y 50 partículas por $\mathrm{cm}^{-3}$, respectivamente.

\begin{tabular}{lccccc}
\hline Tipo especrtral & $\begin{array}{c}\mathrm{T}_{\text {eff }} \\
{\left[\times 10^{3} \mathrm{~K}\right]}\end{array}$ & $\begin{array}{c}\log \mathrm{Q}\left(\mathrm{H}^{0}\right) \\
{\left[\mathrm{s}^{-1}\right]}\end{array}$ & $\begin{array}{c}\log \mathrm{Q}\left(\mathrm{He}^{0}\right) \\
{\left[\mathrm{s}^{-1}\right]}\end{array}$ & $\begin{array}{c}\mathrm{R}_{S_{0}}\left(\mathrm{n} \sim 10 \mathrm{~cm}^{-3}\right) \\
{[\mathrm{pc}]}\end{array}$ & $\begin{array}{c}\mathrm{R}_{S_{0}}\left(\mathrm{n} \sim 50 \mathrm{~cm}^{-3}\right) \\
{[\mathrm{pc}]}\end{array}$ \\
\hline O3V & 50.0 & 49.5 & 48.8 & 21 & 7.0 \\
O4V & 45.7 & 49.4 & 48.7 & 19 & 6.6 \\
O5V & 42.6 & 49.2 & 48.5 & 17 & 5.6 \\
O7V & 40.0 & 49.0 & 48.2 & 14 & 4.8 \\
O7.5V & 37.2 & 48.7 & 47.6 & 11 & 3.8 \\
O8V & 34.6 & 48.5 & 46.6 & 10 & 3.3 \\
O9V & 32.3 & 47.9 & 45.7 & 6.0 & 2.1 \\
B0V & 30.2 & 47.4 & 44.8 & 4.5 & 1.5 \\
B0.5V & 28.1 & 47.0 & 44.5 & 3.1 & 1.0 \\
B1V & 26.3 & 46.5 & 46.1 & 2.0 & 0.7 \\
B1.5V & 25.0 & 46.1 & 43.4 & 1.4 & 0.5 \\
\hline \hline
\end{tabular}

estas regiones puede ir desde unos pocos hasta decenas de parsecs, según el tipo espectral de la estrella excitatriz y la densidad ambiental. En la Tabla 1.4 se listan los posibles tipos espectrales de estrellas excitatrices junto con su temperatura efectiva, el número de fotones estelares que pueden ionizar el Hidrógeno, $\mathrm{Q}\left(\mathrm{H}^{0}\right)$, y el helio, $\mathrm{Q}\left(\mathrm{He}^{0}\right)$, y el radio de Strömgren de la región Hiı que son capaces de generar para dos densidades ambientales. Tanto $\mathrm{Q}\left(\mathrm{H}^{0}\right)$ como $\mathrm{Q}\left(\mathrm{He}^{0}\right)$ se pueden determinar a partir de modelos de atmósferas estelares (e. g. Smith et al., 2002), conociendo el tipo espectral, del cual es fuertemente dependiente.

Esto vale solamente si se considera que la región His es estática. Sin embargo, éstas necesitan un cierto tiempo desde su nacimiento para alcanzar el radio de Strömgren (primera fase de expansión $R<R_{S_{0}}$ ). Durante este tiempo sólo una pequeña fracción de los fotones UV emitidos por la estrella excitatriz es utilizada para mantener la ionización de la nebulosa. Los fotones que alcanzan el borde la región Hir sin ser absorbidos junto con la diferencia de presión entre gas ionizado y neutro hacen avanzar el frente de ionización a través del gas neutro con una cierta velocidad.

Mientras que el radio de la región Hir $R$, es menor que $R_{S_{0}}$, la velocidad del frente de ionización es mayor que la velocidad del sonido en el gas ionizado, pero a medida que $R$ se acerca a $R_{S_{0}}$ la velocidad del frente de ionización se hace menor que la velocidad del sonido en el medio ionizado, pero mayor que la velocidad del sonido en el medio neutro, originándose entonces un frente de choque delante del frente de ionización que avanza comprimiendo el gas neutro. En ese momento la región His tiene un tamaño de $0.95 R_{S_{0}}$ y el frente de choque avanza empujado por la diferencia de presión del gas ionizado respecto al gas neutro.

La velocidad de expansión es aproximadamente igual a la velocidad del sonido en el gas ionizado, supersónica con respecto al gas neutro. Entonces es el gas ionizado el que empuja una onda de choque que comprime el gas neutro que lo rodea.

Se puede desarrollar un modelo simple que describa cómo la región HiI va evolucionando cuando $R>R_{S_{0}}$ (Dyson \& Williams, 1997). Para esto se supone que la presión detrás de la onda de choque $P_{S}$ (ram pressure) es igual a la presión en el gas ionizado $P_{i}$.

$$
P_{s}=n_{0} m_{H} V_{S}^{2}=P_{i}=2 n_{i} m_{H} k T_{e}=n_{i} m_{H} C_{I I}^{2}
$$


donde $C_{I I}$ es la velocidad del sonido en el gas ionizado, $n_{0}$ es la densidad ambiental volumétrica original, $n_{i}$ es la densidad el gas ionizado, $m_{H}$ es la masa del átomo de Hidrógeno, $\mathrm{T}_{e}$ la temperatura electrónica y $V_{S}$ es $\left(\frac{d R}{d t}\right)$. Entonces se tiene que

$$
\left(\frac{d R}{d t}\right)^{2}=\frac{n_{i}}{n_{0}} C_{I I}^{2}
$$

Por otro lado, se puede despejar de la ecuación 1.2

$$
R^{3} n_{i}^{2}=\frac{3 N_{L y}}{4 \pi \alpha_{B}}
$$

y evaluar el radio y la densidad electrónica al inicio y al final de la expansión. Como el lado derecho de la ecuación es una constante, las expresiones para $R^{3} n_{i}^{2}$ al inicio y al final de la expansión se pueden igualar, de donde se obtiene

$$
\frac{n_{i}}{n_{0}}=\left(\frac{R_{S_{0}}}{R}\right)^{3 / 2}
$$

Reemplazando en 1.4

$$
\frac{d R}{d t}=\left(\frac{R_{S_{0}}}{R}\right)^{3 / 4} C_{I I}
$$

Integrando ambos lados de esta ecuación y despejando $\frac{R}{R_{S_{0}}}$ se obtiene la relación entre el radio de Strömgren original $R_{S_{0}}$ y el radio $\mathrm{R}$ durante esta segunda fase de expansión $\left(R>R_{S_{0}}\right)$.

$$
\frac{R}{R_{S_{0}}}=\left(1+\frac{7 C_{I I} t}{4 R_{S_{0}}}\right)^{4 / 7}
$$

donde $t$ es la edad de la región HiI. La realidad difiere muchas veces de este modelo simple debido a que generalmente el medio que rodea las regiones HiI es inhomogéneo, entre otras causas.

La expansión de la región cesa cuando se alcanza el equilibrio de presiones

$$
n_{0} k T_{0}=2 n_{f} k T_{e}
$$

donde $\mathrm{T}_{0}$ y $n_{0}$ son la temperatura y densidad volumétrica correspondientes al gas neutro, y $\mathrm{T}_{e} \mathrm{y} n_{f}$ las correspondientes al gas ionizado $\left(n_{f}\right.$ es la densidad electrónica al término de esta fase de expansión).

En la Figura 1.7 se muestra de modo esquemático la evolución de una región HiI hasta alcanzar el equilibrio de presiones.

A partir de aquí y considerando temperaturas $\mathrm{T}_{0}=100 \mathrm{~K}$ y $\mathrm{T}_{e}=10^{4} \mathrm{~K}$, se obtiene

$$
n_{f}=\frac{1}{200} n_{0}
$$

La ecuación 1.5 puede aplicarse a las condiciones en el inicio y en el final de la segunda fase de expansión, y las ecuaciones que surgen de evaluar esta ecuación antes y después de la expansión tendrán sus lados izquierdos iguales, y podrán igualarse obteniendo así

$$
R_{f}^{3} n_{f}^{2}=R_{S_{0}}^{3} n_{0}^{2}
$$

donde $R_{f}$ es el radio al término de la segunda fase de expansión. Considerando la ecuación 1.9 se puede obtener la expresión para $R_{f}$ en función del radio de Strömgren original. 


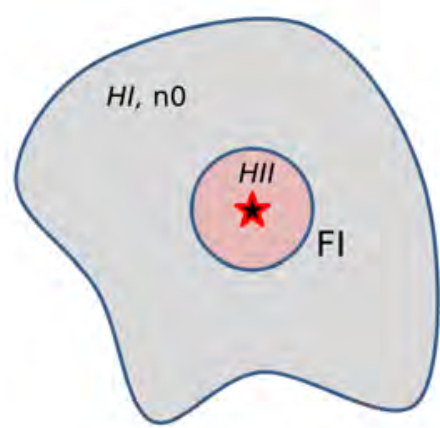

Formación

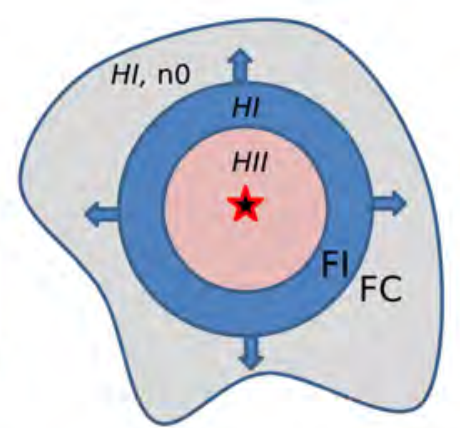

Expansión

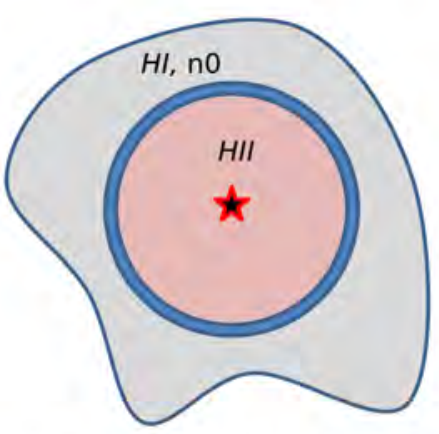

Equilibrio de presiones

Figura 1.7: Esquema de la estructura de una región His expandiéndose en un medio compuesto por Hidrógeno neutro. Se indican el frente de ionización (FI) y el frente de choque (FC).

$$
R_{f}=\left(\frac{2 T_{e}}{T_{0}}\right)^{2 / 3} R_{S_{0}}=34 R_{S_{0}}
$$

La masa ionizada final $M_{i, f}$ dentro de $R_{f}$, referida a la masa inicial $M_{i, 0}$ es

$$
\frac{M_{i, f}}{M_{i, 0}}=\left(\frac{R_{f}}{R_{S_{0}}}\right)^{3} \frac{n_{f}}{n_{0}}=\frac{2 T_{e}}{T_{0}}=200
$$

La densidad final es mucho menor que la inicial, lo que significa que el gas se desplaza desde su ubicación inicial durante la expansión de la región ionizada.

A partir de la ecuación 1.8 se puede obtener una expresión para el tiempo de expansión de la región Hir. Despejando $t$ de esta ecuación se obtiene

$$
t=\left[\left(\frac{R}{R_{S_{0}}}\right)^{7 / 4}-1\right] \frac{4 R_{S_{0}}}{7 C_{I I}}
$$

que indica el tiempo que la región tardaría en terminar de expandirse al equilibrarse la presión del gas ionizado y neutro. Calculando este valor se obtiene que los tiempos de expansión de las regiones Hir son mayores que los tiempos de vida de las estrellas excitatrices, por lo tanto la estrella evoluciona y deja de producir fotones antes de que la región Hit alcance el estado final.

\subsubsection{Regiones de fotodisociación}

Las regiones de fotodisociación (PDR), también conocidas como 'regiones dominadas por fotones', están presentes en todo el MIE y son los sitios donde el campo de radiación UV es lo suficientemente fuerte para disociar moléculas. Estas regiones están formadas predominantemente por gas neutro y polvo iluminado por radiación ultravioleta, la cual determina la física y la química el gas. Las PDRs se hallan en el volumen comprendido entre las nubes moleculares densas y las regiones difusas de gas ionizado y atómico, y son las capas de transición entre el gas ionizado, irradiado por los campos de radiación UV, y el gas neutro frío protegido de la radiación (Tielens \& Hollenbach, 1985). En las PDRs fotones con energías en el rango $4.5 \leq h \nu \leq 13.6 \mathrm{eV}$ excitan e ionizan los PAHs, que delinean la posición de la región de fotodisociación que se identifica en 5.8 y $8.0 \mu \mathrm{m}$ (entre otras longitudes de onda). Estos fotones suelen surgir de las estrellas masivas creadoras de regiones Hir, y en el contexto 


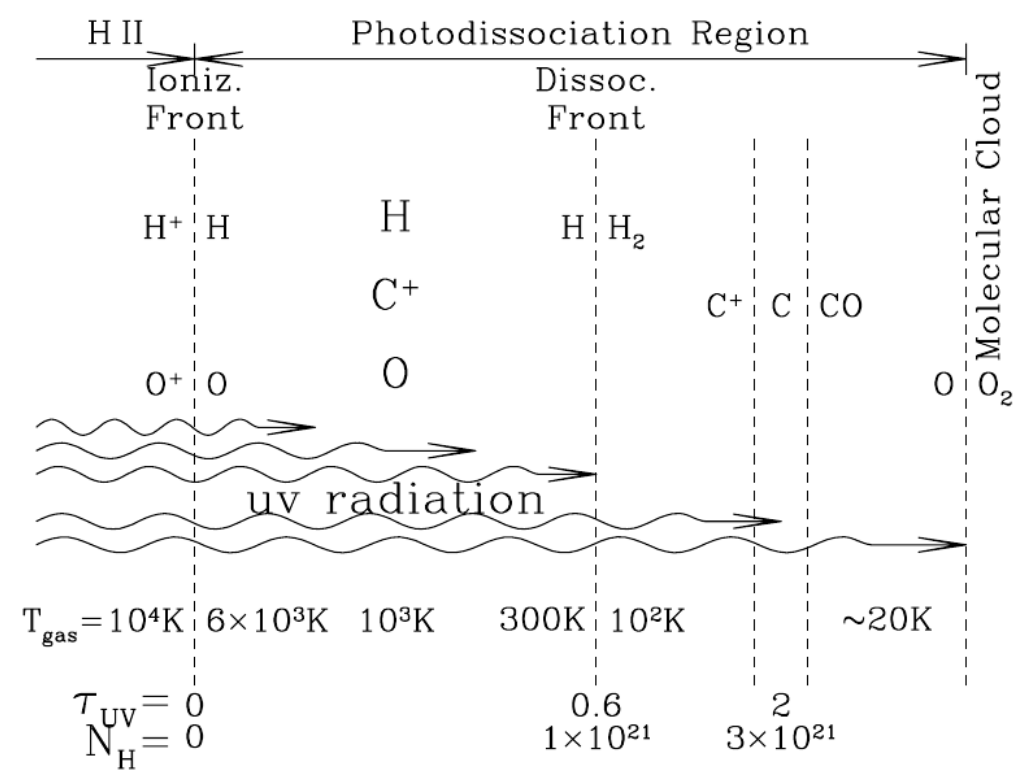

Figura 1.8: Esquema de la estructura de una región de fotodisociación. Imagen tomada de Draine (2010).

extragaláctico de núcleos activos de galaxias que producen emisión intensa ultravioleta y en rayos $\mathrm{X}$.

Las PDRs se encuentran en diferentes regiones, desde las galaxias con formación estelar a las nebulosas de reflexión y discos protoplanetarios. A menudo son adyacentes a regiones HII. Las PDRs dominan la emisión en el FIR e MIR, siendo también emisoras de líneas en longitudes de onda milimétricas, submilimétricas, etc. En ellas ocurre el intercambio de energía entre las estrellas masivas y el medio interestelar que las rodea. La comprensión de los procesos químicos y físicos que suceden en estas regiones es necesaria para el estudio de la evolución de las nubes moleculares, de las galaxias, de los discos protoplanetarios e incluso de la falta de masa en algunas galaxias.

Cuando una región His se expande en una nube molecular aparece un frente de disociación asociado con la destrucción de las moléculas, y se produce una estratificación en el medio (ver Figura 1.8), en la cual el Hidrógeno aparece primero ionizado $\left(\mathrm{H}^{+}\right)$, luego se recombina en $\mathrm{HI}$ y finalmente forma la molécula de $\mathrm{H}_{2}$. De manera similar, el Carbono se recombina con los electrones partiendo del $\mathrm{C}^{++}$en la región His para formar $\mathrm{C}^{+}$en regiones más externas de la PDRs, hasta formar $\mathrm{C}$ y más profundo en la nube, $\mathrm{CO}$. El Oxígeno se encuentra en forma atómica, con pequeñas trazas de $\mathrm{O}_{2}$ y $\mathrm{H}_{2} \mathrm{O}$. Los fotones UV de la estrella excitatriz logran penetrar hasta cierta profundidad en la nube molecular ya que para densidades grandes las moléculas de $\mathrm{H}_{2}$ y $\mathrm{CO}$ se autoapantallan, protegiendo las regiones más internas de la acción destructiva de la radiación. Este mecanismo es fundamental para garantizar la supervivencia de las nubes moleculares, que de otra manera serían rápidamente disociadas por el campo de radiación presente en el MIE.

\subsubsection{Burbujas interestelares}

La interacción entre el viento estelar y el MIE circundante modifica la dinámica de ese medio. La colisión del viento con el MIE da lugar a la generación de una burbuja interestelar (BI). Una BI es una cavidad de baja densidad y alta temperatura, rodeada por una envoltura 


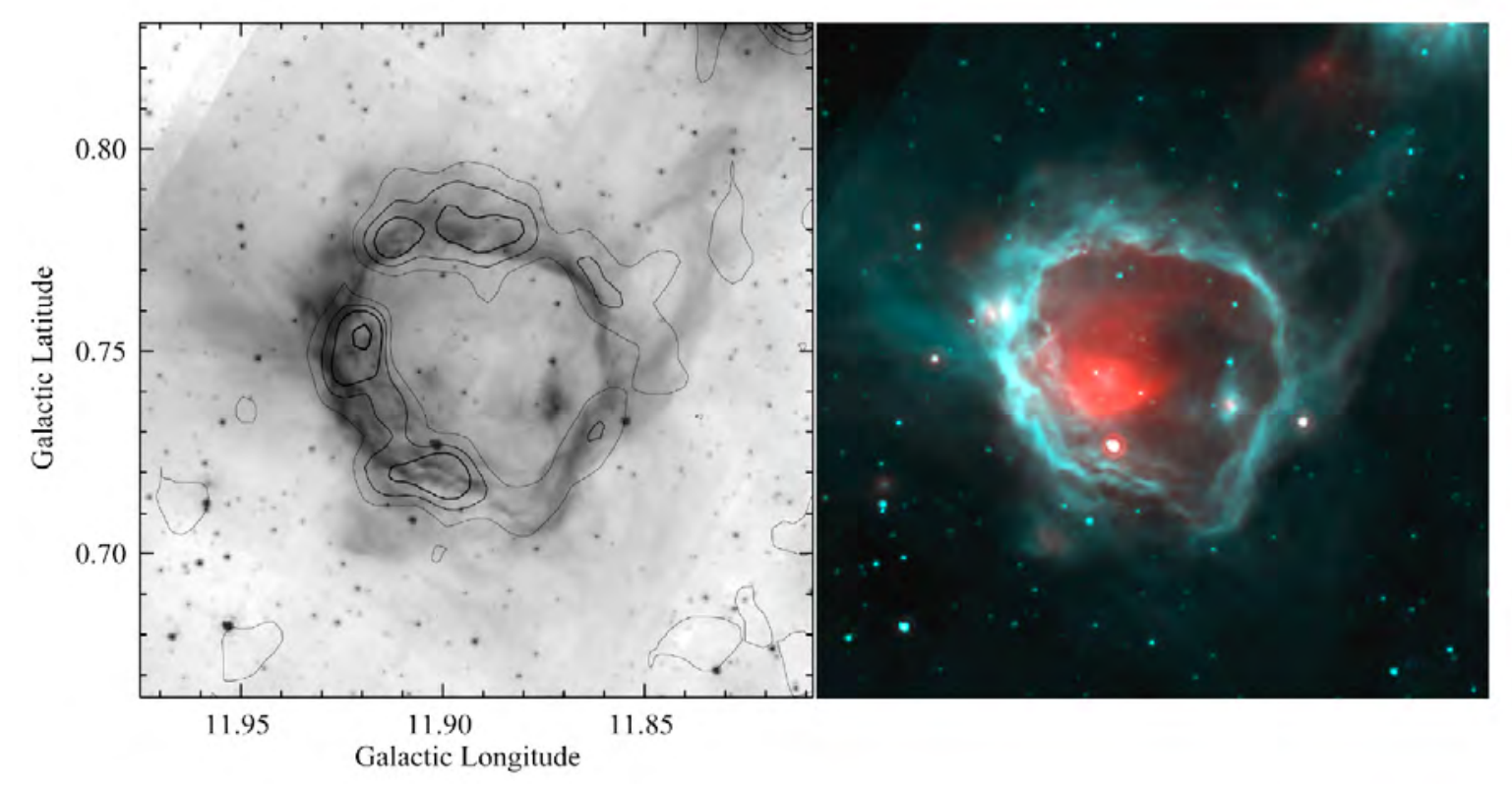

Figura 1.9: Burbuja infrarroja de polvo N 49. A la izquierda se muestra la emisión en $8 \mu \mathrm{m}$, que indica la presencia de una PDR, junto con los contornos correspondientes a la emisión en $870 \mu \mathrm{m}$ proveniente del polvo frío, asociado con material barrido. A la derecha se muestra en turquesa la emisión en $8 \mu \mathrm{m}$ y en rojo en $24 \mu \mathrm{m}$. Imagen tomada de Deharveng et al. (2010).

o cáscara formada por gas estelar desacelerado y gas interestelar barrido (Weaver et al., 1977; Freyer et al., 2003, 2006). Las formas que pueden adquirir este tipo de estructuras, idealmente esféricas, dependen fuertemente de las características del viento estelar, del MIE en el que evolucionan y de la propia estrella.

Particularmente, las estrellas tempranas modifican drásticamente su entorno debido a las altas tasas de pérdida de masa y altas velocidades terminales de los vientos que en ellas se generan. Las estrellas masivas arrojan al MIE durante su vida $\left(\sim 10^{6}-10^{7}\right.$ años $)$ una cantidad de energía similar a la entregada en un evento de supernova $\left(\sim 10^{51} \mathrm{erg}\right)$.

Se han encontrado numerosas burbujas interestelares asociadas a estrellas masivas en diferentes estados evolutivos, en particular O, Of y Wolf-Rayet. Éstas se manifiestan en distintos rangos espectrales: en el óptico se las reconoce generalmente por su aspecto anular, al igual que en el continuo de radio de naturaleza térmica; en el infrarrojo evidencian la presencia de polvo interestelar y PAHs rodeando la región ionizada, mostrando muchas veces la componente molecular que encierra todo el conjunto.

\subsubsection{Burbujas infrarrojas de polvo}

Las burbujas infrarrojas no son generalmente visibles en longitudes de onda del óptico, pero sí en el infrarrojo (ver Figura 1.9). El Observatorio Espacial Infrarrojo (ISO, Kessler et al., 1996) y el satélite Midcourse Space Experiment (MSX) fueron los primeros en revelar la existencia de estos objetos. Sin embargo fueros las observaciones realizadas con la cámara IRAC a bordo del satélite Spitzer, con la que se llevó a cabo el relevamiento GLIMPSE (Galactic Legacy Infrared Midplane Survey Extraordinaire), con una resolución espacial 10 veces superior y 100 veces mejor sensibilidad que MSX, las que han permitido la detección de muchas más burbujas y la buena determinación de su estructura morfológica. El satélite Spitzer observó el plano galáctico en longitudes de onda entre 3.6 y $70 \mu \mathrm{m}$, y detectó un gran 
número de estructuras con forma de burbujas. Estas estructuras poseen cáscaras externas brillantes en $8 \mu \mathrm{m}$ y, generalmente, un interior brillante en $24 \mu \mathrm{m}$. La emisión en $8 \mu \mathrm{m}$ ha permitido catalogar unas $600 \mathrm{BI}$ en una región que se extiende 130 grados en el plano galáctico, con un ancho de 2.2 grados (Churchwell et al., 2006, 2007). Estos autores tabularon las propiedades morfológicas de las burbujas, tales como el diámetro angular, el grosor de la cáscara y la excentricidad. Churchwell et al. (2006) demostraron que las burbujas infrarrojas están distribuidas en la galaxia del mismo modo que las estrellas O y B, que más del $25 \%$ coincide con regiones Hiı y que $\sim 13 \%$ encierran cúmulos estelares conocidos.

Sin embargo, en un trabajo más reciente, en base a una muestra de 100 objetos, Deharveng et al. (2010) encontraron que el $86 \%$ de las burbujas contienen gas ionizado detectado por medio de emisión en continuo de radio a $20 \mathrm{~cm}$, por lo que la mayoría de las burbujas observadas a $8 \mu \mathrm{m}$ encierran regiones HiI. También determinaron que el $98 \%$ de las burbujas muestran emisión a $24 \mu \mathrm{m}$ en su interior dado que contienen polvo caliente. La emisión de PAHs a $8 \mu \mathrm{m}$ se observa en la dirección de las regiones de fotodisociación que rodean el gas ionizado.

El número de burbujas infrarrojas se extendió a 5106 con el Milky Way Project ${ }^{(1)}$ utili- $^{-}$ zando los satélites Spitzer y WISE (Simpson et al., 2012).

El estudio de las burbujas infrarrojas proporciona información sobre los vientos estelares, los fotones UV y el tipo de estrellas necesarios para producirlas, la estructura y las propiedades físicas del ambiente en el que se están expandiendo, y así presentan una nueva y poderosa herramienta para estudiar la interacción de estrellas jóvenes, masivas y calientes con su entorno. Las estrellas jóvenes modifican el medio interestelar al calentar el polvo, ionizar el gas y disociar las moléculas, y las burbujas pueden ser producidas por una combinación de vientos estelares y presión debida a la ionización y al calentamiento por radiación estelar UV. Los tamaños y morfologías de las burbujas están determinados por la estructura de densidad del medio, la luminosidad de las estrellas centrales responsables de producir la burbuja, la luminosidad del viento estelar y el movimiento de las estrellas centrales en relación con su entorno.

Las burbujas infrarrojas, que evolucionan en medios más densos que las llamadas burbujas interestelares, marcan los lugares donde la radiación de las estrellas masivas recién formadas está impactando sobre el material de la nube circundante, por lo que indican sitios de formación estelar activa, ya que, por lo general, las estrellas masivas no viven lo suficiente como para alejarse demasiado de las nubes donde se formaron.

Las estrellas masivas ionizan el gas (a partir de fotones con energías superiores a 13.6 $\mathrm{eV}$ ), aumentan la temperatura del polvo interestelar que las rodea, y suelen tener vientos estelares. El gas ionizado, a mayor temperatura, se expande, y junto con los vientos estelares, que contribuyen también a la expansión, comprimen el gas atómico y molecular que circunda a la burbuja, favoreciendo la formación de cáscaras de gas molecular denso y de polvo a bajas temperaturas asociado a este gas frío y denso. Tienen así un interior ionizado y caliente (detectable en emisión a $24 \mu \mathrm{m}$ y continuo de radio térmico) y un exterior frío y denso, en cuya interfase se forma una región de fotodisociación. Los fotones con energías menores a $13.6 \mathrm{eV}$ excitan los PAHs que están mezclados con el gas y polvo interestelares emitiendo radiación detectable en 5.8 y $8 \mu \mathrm{m}$.

La formación de estrellas podría ser desencadenada en las cáscaras de gas molecular o cuando el frente de choque delantero sobrepasa y comprime un núcleo molecular preexistente, resultando en ambos casos en un colapso gravitacional del núcleo. Las burbujas que exhiben estos mecanismos de formación estelar proporcionan un camino crucial para comprender mejor cómo funciona la formación estelar.

(1) https://blog.milkywayproject.org/ 


\subsubsection{Remanentes de supernova}

Por completitud se mencionan los remanentes de supernova, ya que contribuyen a perturbar el MIE aunque no tienen relación con los estudios realizados para esta tesis doctoral.

Existen dos tipos de explosiones de supernovas. Las supernovas tipo Ia ocurren en sistemas binarios de estrellas de baja masa cuando una de las componentes (una enana blanca) acreta material de su compañera (generalmente una gigante roja) dando lugar a una explosión termonuclear. Las supernovas tipo II, Ib y Ic ocurren por el colapso nuclear de una estrella masiva $\left(\mathrm{M}>8 \mathrm{M}_{\odot}\right)$ en el final de su vida. Este colapso ocurre cuando la estrella ha usado todo su combustible y el desbalance entre la presión gravitatoria y la presión de radiación lleva a una implosión del núcleo hacia una estrella de neutrones o un agujero negro. La envoltura de la estrella rebota contra el núcleo y es expulsada al espacio a velocidades de varios miles de kilómetros por segundo.

En cualquier caso los efectos que estas explosiones, que liberan energías de unos $\sim 10^{51}$ erg, tienen sobre el MIE son drásticos. El material estelar expulsado a altas velocidades crea ondas de choque que modifican su entorno física y químicamente y lo enriquecen con elementos pesados. Así, se llama remanente de supernova (RSN) al conjunto del material estelar eyectado, el gas ambiente chocado y barrido, y el objeto compacto que queda en algunos casos (estrella de neutrones o agujero negro).

Un análisis general de estos remanentes de supernova puede verse en Dubner \& Giacani (2015).

\subsection{Formación estelar}

Las estrellas se forman en las nubes moleculares gigantes, a partir de la fragmentación y colapso de las mismas atravesando las siguientes etapas:

1- Fragmentación de la nube molecular gigante y formación de grumos o filamentos moleculares densos (con densidad y temperatura características $n \sim 10^{5} \mathrm{~cm}^{-3}$ y T $\sim 10-15 \mathrm{~K}$, respectivamente, tamaños de $\sim 0.1 \mathrm{pc}$ con masas de unas pocas hasta varias decenas de masas solares).

2- Colapso gravitacional de los grumos en núcleos protoestelares.

3- Acreción de material en los núcleos protoestelares.

4- Disipación de la nube originaria, debido a la acción combinada del viento estelar, radiación UV, eyecciones de materia en forma de outflows y eventualmente eventos de supernova de las estrellas masivas que evolucionan rápidamente.

\subsubsection{Formación de estrellas de baja masa}

La formación de estrellas de baja masa $(<8 \mathrm{M} \odot)$ está caracterizada por cuatro etapas, que definen cuatro clases de objetos estelares jóvenes (Young Stellar Objects, YSOs). Esta clasificación en clases se basa en el índice espectral de sus distribuciones espectrales de energía (Spectral Energy Distribution, SED).

Un trabajo pionero de Lada (1987) cuantificaba la juventud estelar de las fuentes IR considerando el índice espectral infrarrojo $\alpha_{I R}$

$$
\alpha_{I R}=\frac{d \log \lambda F_{\lambda}}{d \log \lambda}
$$

Las fuentes IR con $\alpha_{I R}>0$ eran clasificadas como Clase I, objetos generalmente asociados a grumos densos. Las fuentes IR con $-1.5<\alpha_{I R}<0$ eran estrellas menos inmersas en la nube madre y se clasifican como Clase II. Las fuentes IR con $\alpha_{I R}<-1.5$ correspondían a 


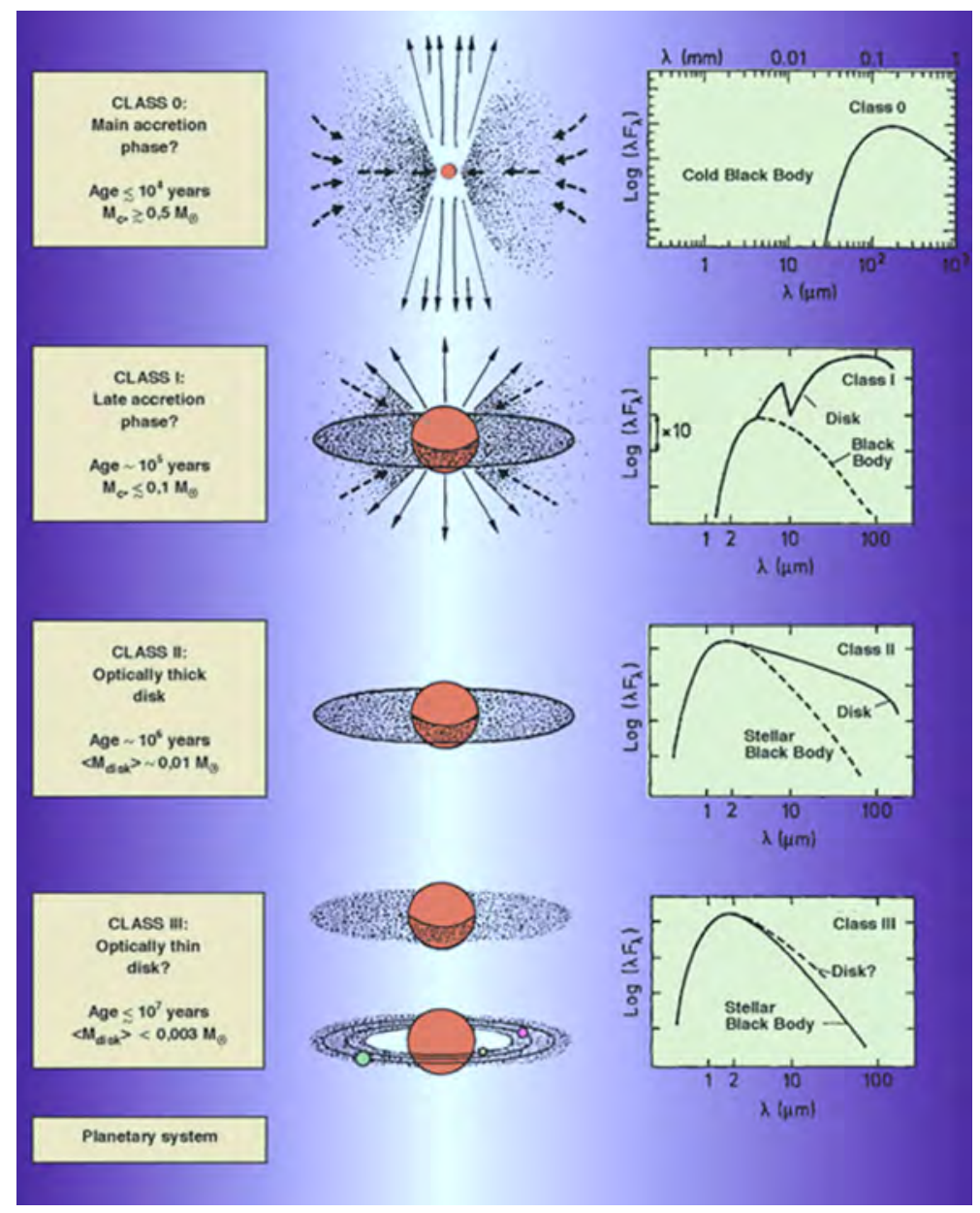

Figura 1.10: Clasificación de YSOs según su exceso infrarrojo para estrellas de baja masa. Los paneles de la derecha muestran el rango de longitud de onda en que los objetos son detectables (Fuente, 2001). 
las Clase III. Por último las fuentes Clase 0 son aquellas tan profundamente inmersas en la envoltura de polvo que sólo pueden detectarse en longitudes de onda del infrarrojo lejano y milimétricas, y que en este trabajo pionero no están incluidas pues las observaciones eran en el rango de $\sim 2-25 \mu \mathrm{m}$. Estas etapas se ilustran en la Figura 1.10 y corresponden a fuentes IR con las siguientes características:

- Los YSOs más jóvenes son aquellos que se hallan embebidos en un núcleo compuesto por gas molecular denso $\left(10^{4} \mathrm{~cm}^{-3}\right)$ y frío $(\mathrm{T}<10 \mathrm{~K})$ y por polvo interestelar, y se pueden detectar solamente en longitudes de onda milimétricas y submilimétricas, a $\lambda>40-100 \mu \mathrm{m}$. Los objetos en esta fase evolutiva, de unos $10^{4-5}$ años de duración, se conocen como objetos de Clase 0. Su comportamiento espectral se muestra en el panel superior de la Figura 1.10.

- La siguiente etapa está dominada por la caída de material desde el grumo molecular parental hacia el objeto central con la eventual aparición de flujos de material (outflows) desde el objeto hacia los alrededores. Cuando parte de la envoltura es disipada por efecto de los outflows, el objeto se vuelve detectable en el cercano infrarrojo (ver segundo panel de la Figura 1.10) y es llamado de Clase I.

- En aproximadamente $10^{6}$ años la envoltura se disipa, la estrella se hace visible ópticamente como una estrella TTauri clásica (CTTS) y pasa a ser de Clase II (Lada \& Kylafis, 1991). Se puede hablar entonces de una estrella de pre-secuencia principal.

- La estrella termina de dispersar el material circundante por efecto de los vientos estelares y evoluciona hacia un objeto de Clase III, que puede identificarse como una T Tauri de líneas débiles (WTTS). En esta etapa se podría comenzar a formar un sistema planetario a partir del remanente del disco circunestelar.

\subsubsection{Formación de estrellas de gran masa}

Las estrellas masivas son poco frecuentes en comparación con las estrellas de baja masa. Si se considera la Función Inicial de Masas (Initial Mass Function, IMF) de Salpeter (1955) por cada 10 estrellas que se forman con masas entre 1-2 M $\odot$ se forma sólo una con masa $\mathrm{M}>$ $8 \mathrm{M} \odot$. Sin embargo, las estrellas masivas juegan un papel importante en la determinación de las propiedades morfológicas, dinámicas y químicas de las galaxias que las albergan, a través de fuertes vientos estelares, una intensa radiación UV y explosiones de supernova, y suponen la principal fuente de inyección de energía y turbulencia en el medio interestelar.

A pesar de su gran importancia, sus primeras etapas evolutivas aún no se conocen en detalle, y no hay evidencia observacional que muestre una evolución tan definida como en el caso de las estrellas de baja masa. Los modelos teóricos (e. g., Larson, 1969; Shu et al., 1987) que permiten explicar de forma acertada la formación de estrellas de baja masa, presentan problemas cuando se aplican a las estrellas más masivas.

El principal problema teórico radica en el hecho que las estrellas masivas empiezan a producir reacciones termonucleares en su interior cuando aún están acretando material del disco y de la envolvente. Si la estrella masiva en formación alcanza una determinada luminosidad (equivalente a la de una estrella de tipo espectral B), será capaz de producir un número de fotones con energías $h \nu>13.6 \mathrm{eV}$, suficiente como para ionizar el gas que la rodea y la presión de radiación impedirá que la estrella siga acretando material. En el caso que esto ocurriera la estrella no sería capaz de seguir aumentando su masa y no sería posible la existencia de las estrellas de tipo O. 
Actualmente hay dos modelos teóricos diferentes que tratan de solucionar éste y otros problemas y así poder explicar la formación de las estrellas masivas:

El primer modelo, conocido como colapso monolítico (Yorke \& Sonnhalter, 2002), intenta adaptar el mecanismo de formación de estrellas de baja masa para resolver el problema de la presión de radiación, modificando en los modelos la simetría y las características en el modo como es acretado el material. De esta manera es un mecanismo amplificado del proceso que forma las estrellas de baja masa. Algunos trabajos teóricos revelan que la radiación de la protoestrella masiva podría escapar a través de la cavidad creada por un jet y un outflow sin obstaculizar la acreción a través del disco (Wolfire \& Cassinelli, 1987; Tan \& McKee, 2002; Yorke \& Sonnhalter, 2002). Sin embargo, otros autores advierten que esta versión ampliada de la formación de estrellas de baja masa puede ser factible sólo hasta estrellas B tempranas (ver Zinnecker \& Yorke, 2007).

El segundo método, acreción competitiva o coalescencia, se basa en el hecho que las estrellas masivas se encuentran en cúmulos numerosos (e. g., Bonnell et al., 1998; Bonnell, 2007; Stahler et al., 2000), y que éstas se pueden formar, por ejemplo, a partir de la fusión de estrellas de menor masa. Una estrella aislada en formación acreta la mayor parte del gas de la nube parental, lo que determina su masa final. Sin embargo, en un cúmulo, las estrellas deben 'competir' para poder acretar material de la nube y así poder ganar masa. La capacidad de una protoestella de acretar material depende de su masa y de la distribución de las protoestrellas cercanas, y así una estrella masiva puede acretar más masa que una estrella de baja masa, y llegar a ser aún más masiva. Bajo los supuestos de este escenario, las estrellas más masivas que se encuentran en los cúmulos deben ubicarse en el centro del cúmulo, donde el potencial gravitacional es mayor, y hay más gas disponible para que la protoestrella aumente su masa. El escenario de coalescencia, que requiere altas densidades protoestelares $\left(>10^{6}-10^{8} \mathrm{pc}^{-3}\right)$, sugiere que la fusión de dos o más estrellas de baja masa puede formar una estrella más masiva. Aunque estas densidades no suelen encontrarse en los cúmulos protoestelares, este modelo explicaría naturalmente por qué se encuentran estrellas masivas en cúmulos.

Estos procesos se relacionan con los núcleos moleculares calientes (hot cores) y con los núcleos preestelares, detectados en el infrarrojo lejano. Los núcleos moleculares calientes alcanzan valores de densidad, temperatura y masa de $n \sim 10^{7-9} \mathrm{~cm}^{-3}, T \sim 300 \mathrm{~K}, M \sim$ $100-1000 M_{\odot}$, respectivamente. En estos sitios se dan las condiciones apropiadas para la formación de estrellas de gran masa. Los núcleos preestelares son pequeñas nubes que están sufriendo una contracción cuasi-estática y aún no poseen estrellas formándose en su interior. Éstos están asociados a los estadíos más tempranos del proceso de formación de estrellas de gran masa. Como este estadío dura $\sim 10^{4}$ años, esta fase es difícil de detectar.

\subsubsection{Formación estelar inducida}

El impacto de una onda de choque sobre una nube molecular, la colisión entre sí de nubes moleculares, el avance de un frente de ionización sobre una nube molecular o la fragmentación que pueda tener lugar en una estructura densa en expansión actúan como disparadores de formación estelar. La formación de estrellas que pudiera ocurrir debido a alguno de estos efectos se conoce como formación estelar inducida. Las regiones HII, las burbujas infrarrojas y los remanentes de supernova son considerados agentes disparadores de formación estelar, aún cuando no se han encontrado casos concretos de formación estelar inducida por remanentes de supernova.

La formación estelar requiere para su desarrollo, una masa de gas densa y autogravitante, así como también un proceso de compresión que le dé origen. Para que se forme una estrella de baja masa basta con el efecto de la turbulencia interna de una nube molecular, pero para 
formar estrellas masivas, que requieren núcleos de mayor masa, la probabilidad de formación espontánea es menor, por lo tanto la formación de estrellas de gran masa también se asocia a los procesos de formación estelar inducida.

Para inducir la formación estelar es necesaria una fuente de energía que pueda cambiar el estado de una nube molecular de estable a inestable y provocar el colapso gravitacional. Los vientos estelares, regiones Hiı y explosiones de supernova generadas por estrellas de gran masa, barren y comprimen el gas molecular circundante. Así, en las envolturas moleculares asociadas pueden desarrollarse las condiciones necesarias para la formación estelar inducida o formación estelar secuencial.

Una vez producido el primer evento de formación estelar masiva, la formación estelar de objetos de gran masa es un proceso que se realimenta, siempre que haya suficiente material disponible para seguir formando estrellas.

Respecto de las regiones HiI se han sugerido tres mecanismos principales de formación estelar inducida en sus alrededores: collect and collapse, radiative driven implosion, y la colisión de nubes moleculares. Estos mecanismos se describen brevemente a continuación.

\section{Collect and collapse}

Propone el colapso y fragmentación de las envolturas moleculares circundantes a regiones Hir como resultado de la acción de frentes de choque generados en la expansión de estas regiones, que luego darían lugar a la formación de nuevas estrellas.

Elmegreen \& Lada (1977) analizaron la estabilidad gravitacional de las cáscaras de material neutro acumuladas entre los frentes de choque y de ionización originados en la interacción de los vientos estelares y la radiación UV de estrellas de gran masa con el gas neutro.

Las cáscaras neutras en expansión alrededor de regiones His y burbujas IR se alejan del centro de expansión, empujadas por la presión de gas ionizado. El frente de choque comprime el material circundante modificando drásticamente sus condiciones ambientales, cambiando su estado dinámico y aumentando su temperatura, densidad y presión, originando una cáscara densa. Este material comprimido que se encuentra entre el frente de choque y el frente de ionización, con el tiempo, se fragmenta y se vuelve dinámicamente inestable. Si la acumulación de material no es frenada por las inestabilidades, una gran cantidad de material se acopia en grumos moleculares y en los núcleos más densos en los que se fragmentan los grumos. De esta manera, se generan fragmentos altamente densos que permiten la formación de estrellas de gran masa o cúmulos de estrellas (e. g. Vazzano et al., 2014).

La formación estelar ocurrirá en los grumos densos de nubes moleculares en los que los efectos de la gravedad son mayores que los de la turbulencia, campos magnéticos y presión térmica (teorema del virial).

Varios trabajos (e. g. Whitworth et al., 1994) establecen que los fragmentos formados son de gran masa, por lo que este mecanismo favorece la formación de estrellas o cúmulos de estrellas masivas. Desde el punto de vista observacional, Deharveng et al. (2005) sugieren que la mera presencia de una cáscara de material molecular denso y de fragmentos distribuidos regularmente a lo largo del frente de ionización de una región Hiı son un fuerte indicio de que el mecanismo de collect and collapse está actuando.

Numerosos estudios muestran que este mecanismo es efectivo, identificándose numerosos YSOs en las cáscaras densas de regiones Hit y burbujas infrarrojas, aunque es posible que no todos los YSOs identificados se hayan originado en este proceso. Dale et al. (2015) proponen que algunos de los YSOs se formarían de todos modos aunque no existiera un mecanismo que disparara la formación de estrellas.

El panel izquierdo de la Figura 1.11 muestra la región His RCW 79, en $8 \mu$ m y en $\mathrm{H} \alpha$, donde estaría actuando el mecanismo collect and collapse. 

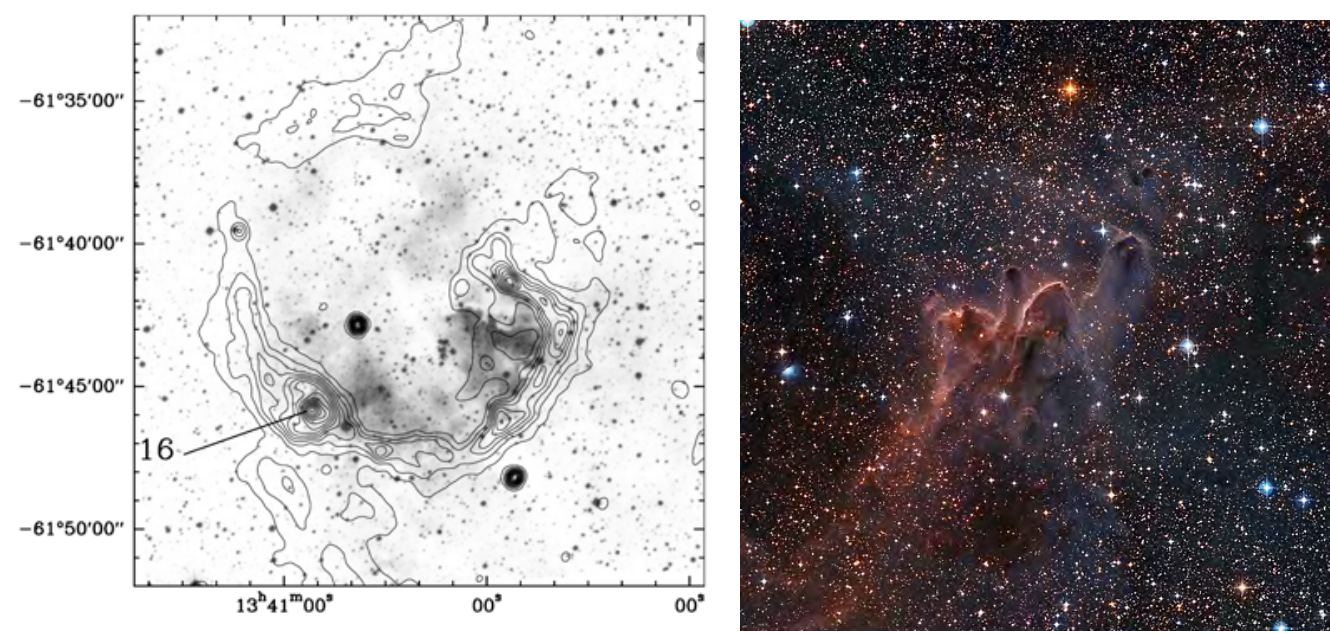

Figura 1.11: Izquierda: Región HiI RCW 79. Contornos de banda A ( $8 \mu \mathrm{m})$ de MSX superpuesto a la imagen de $\mathrm{H} \alpha$ de SuperCOSMOS. Las observaciones sugieren que el mecanismos collect and collapse estaría operando. Los contornos muestran la posición de la PDR. Imagen tomada de Deharveng et al. (2005). Derecha: Glóbulos cometarios en Vela-Puppis. Estas estructuras compuestas de gas y polvo tienen un tamaño de 0.3 pc y se encuentran a unos 400 pc de distancia. La radiación UV de las estrellas cercanas modela los glóbulos e ioniza sus bordes brillantes.

\section{Radiative driven implosion (RDI)}

Ocurre cuando un grumo denso preexistente colapsa por efecto de una alta presión que rodea a la nube (Sandford et al., 1982; Lefloch \& Lazareff, 1994). Estas altas presiones podrían ser originadas por regiones Hiı jóvenes que al comenzar su expansión interactúan con nubes moleculares circundantes dando origen a bordes brillantes y a pequeños glóbulos cometarios (Bertoldi, 1989; Bertoldi \& McKee, 1990).

Debido a la compresión inicial y dependiendo de la cantidad de fotones UV, el glóbulo colapsa rápidamente y comienzan a formarse estrellas en su núcleo. Posteriormente, le sigue una etapa de expansión, causada por la inyección de energía y turbulencia por parte de las estrellas recién formadas. Finalmente el glóbulo alcanza un nuevo equilibrio, adquiriendo una forma cometaria y bordes brillantes (Bright Rimmed Clouds, BRC) debidos al material ionizado (Sugitani et al., 1991).

Las BRC son nubes que han sido comprimidas por un frente externo de choque o ionización que concentra el gas neutro en glóbulos compactos. La capa límite entre el gas neutro y el gas ionizado se denomina bright rim (borde brillante), pero los grumos suelen clasificarse también como speck globules (glóbulos con forma de punto) o glóbulos cometarios dependiendo de su apariencia. Los glóbulos pequeños con bordes brillantes se consideran sitios potenciales de formación estelar y han sido estudiados por varios autores.

En el panel derecho de la Figura 1.11 se muestran los glóbulos cometarios presentes en la constelación de Vela-Puppis, un posible ejemplo del mecanismo RDI.

\section{Colisión de nubes moleculares}

La colisión entre nubes moleculares puede actuar como disparador de formación estelar, ya que al colisionar el gas que las compone sufre una compresión generando una capa densa en la interfase entre las nubes en la que pueden desencadenarse procesos de formación estelar. Este mecanismo es importante ya que se cree que tales colisiones son frecuentes en las galaxias con disco como la Vía Láctea (Tasker \& Tan, 2009). 
0

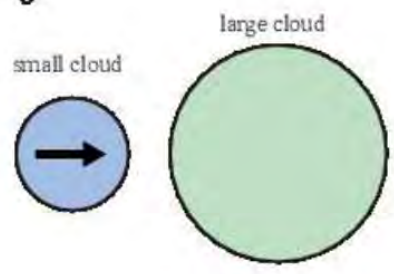

1

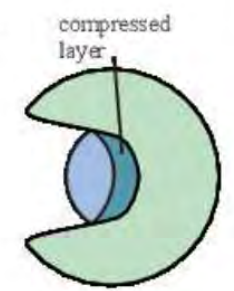

2

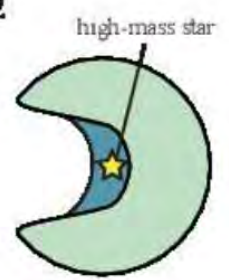

3

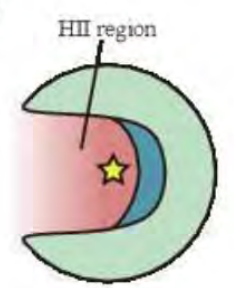

Figura 1.12: Esquema que muestra la formación estelar inducida por colisión de nubes moleculares. La fase 0 corresponde al estadío previo a la colisión. Las fases 1 a 3 muestran la secuencia temporal desde la colisión hasta la formación de una estrella. Tomada de Fukui et al. (2017).

Simulaciones hidrodinámicas (e.g. Habe \& Ohta, 1992; Anathpindika, 2010) han mostrado que la colisión entre nubes puede inducir la formación de grumos densos en la interfaz comprimida por el impacto. Dichos grumos son propensos a sufrir inestabilidades gravitacionales que conducen a la formación de estrellas.

Observaciones moleculares han demostrado que en muchos casos la formación de estrellas de tipo $\mathrm{O}$ en el disco de la Vía Láctea se desencadena por colisiones supersónicas entre dos nubes a una velocidad de 10-30 $\mathrm{km} \mathrm{s}^{-1}$ (e. g. Furukawa et al., 2009; Ohama et al., 2010). Simulaciones numéricas muestran que las colisiones entre las nubes son bastante frecuentes, con tiempos de $\sim 10 \mathrm{Myr}$, similares a la escala de tiempo evolutiva de las nubes moleculares gigantes. De este modo, es posible considerar la colisión de nubes como uno de los modos de formación de estrellas de gran masa. En la Figura 1.12 se ve un esquema que muestra la colisión entre dos nubes.

\subsubsection{Identificación de YSOs a partir de observaciones infrarrojas}

La observación directa de YSOs en nubes moleculares es muy difícil debido a que éstos se encuentran en zonas oscurecidas por el polvo y de altas densidades, por lo que la única forma de investigar qué está sucediendo en estas regiones es a partir de su emisión infrarroja y observaciones en longitudes de onda milimétricas y en radio.

El exceso de energía en la banda infrarroja de una estrella en formación no surge del enrojecimiento debido a granos de polvo distantes, sino de emisión de polvo ubicado relativamente cerca de la superficie de la estrella. Es decir, el fenómeno es circunestelar, en lugar de interestelar.

Una vez que la distribución espectral de energía de una fuente se establece a partir de observaciones, se puede utilizar la absorción visual estimada a través de la nube, junto con la curva de extinción, para calcular la extinción a otras longitudes de onda. De esta manera se puede reconstruir la distribución de la energía de la estrella y de su material circunestelar.

\subsubsection{Identificación de YSOs a partir de catálogos infrarrojos}

Es de gran interés en el estudio de la formación estelar poder detectar fuentes que sean posibles objetos en formación (YSOs). Esto se puede llevar a cabo a partir del análisis de fuentes puntuales identificadas en catálogos infrarrojos.

A continuación se detallan los criterios para la selección de YSOs a partir de diversos catálogos que se utilizarán en esta tesis. 


\section{Criterios de selección y análisis de las fuentes 2MASS}

El catálogo Two Micron All Sky Survey (2MASS) (Cutri et al., 2003), cuenta con observaciones en las bandas J, H y Ks centradas en $1.25,1.65$ y $2.2 \mu \mathrm{m}$, respectivamente. Las herramientas más usadas con las fuentes del catálogo 2MASS para la distinción entre fuentes con exceso IR candidatas a YSOs y estrellas gigantes o de secuencia principal, son los diagramas color-color (CC) y color-magnitud (CM) (ver Figura 1.13). El primero de estos dos gráficos se basa en la comparación de índices de color $I_{\mathrm{ij}}$ en diferentes bandas, donde

$$
I_{\mathrm{ij}}=m_{\mathrm{i}}-m_{\mathrm{j}}=M_{\mathrm{i}}-M_{\mathrm{j}}=-2.5 \log \left(\frac{S_{\mathrm{i}}}{S_{\mathrm{j}}}\right)
$$

donde $m_{\mathrm{i}}$ y $m_{\mathrm{j}}$ son las magnitudes en las bandas $i$ y $j$, pudiendo ser estas J, H o Ks; y $M_{\mathrm{i}} \mathrm{y}$ $M_{\mathrm{j}}$, y $S_{\mathrm{i}}$ y $S_{\mathrm{j}}$ son las magnitudes absolutas y los flujos en esas bandas. El diagrama CM, en cambio, relaciona un valor de $I_{\mathrm{ij}}$ con la magnitud en alguna de esas bandas.

Se pueden tomar las magnitudes absolutas para las estrellas de la ZAMS en la banda Ks de Hanson et al. (1997), y sus correspondientes valores para el diagrama CC de Koornneef (1983).

Se establecieron reglas de selección según la posición de las fuentes en el diagrama colorcolor (CC) a partir del trabajo de Comerón et al. (2005). En este trabajo se determinó el parámetro $\mathrm{q}=(\mathrm{J}-\mathrm{H})-1.83 \times(\mathrm{H}-\mathrm{Ks})$, cuyo rango de valores distingue básicamente tres tipos de fuentes con naturaleza distinta: objetos de secuencia principal $(-0.15<\mathrm{q}<0.10)$, generalmente de baja masa, estrellas gigantes de la rama asintótica $(q>0.10)$ y objetos con exceso IR $(q<-0.15)$, siendo los últimos los que se consideran candidatos a YSOs. Este criterio no es concluyente, ya que otros tipos de objetos, como las estrellas evolucionadas de tipo espectral M presentan exceso infrarrojo. Una manera de sortear este inconveniente es buscar una correlación con otras bases de datos de fuentes puntuales IR, como por ejemplo, las de los satélites MSX o IRAS.

Diagramas similares pueden realizarse utilizando datos de VVV (Vista Variables in the Via Lactea, Minniti et al., 2010), un relevamiento del bulge de la galaxia realizado utilizando VISTA, un telescopio de 4.1 metros que posee una cámara infrarroja (e.g. Cappa et al., 2017).

\section{Criterios de selección para las fuentes MSX}

Para la selección de fuentes infrarrojas detectadas por el satélite MSX se considera la emisión en las bandas A, C, D y E que corresponden a longitudes de onda de 8.3, 12, 14, y 21 $\mu \mathrm{m}$, respectivamente. La resolución angular del relevamiento es de $\sim 6^{\prime \prime}$. Mayor información sobre este relevamiento puede encontrarse en Egan et al. (1999).

La clasificación se lleva a cabo considerando los criterios definidos por Lumsden et al. (2002) a partir de la ubicación de las fuentes en el diagrama $F_{21} / F_{8}$ vs $F_{14} / F_{12}$, donde $F_{8}$, $\mathrm{F}_{12}, \mathrm{~F}_{14}$ y $\mathrm{F}_{21}$ son los flujos medidos a 8.3, 12, 14, y $21 \mu \mathrm{m}$, respectivamente. Las fuentes detectadas por MSX pueden clasificarse como objetos estelares jóvenes masivos (Massive Young Stellar Objects, MYSOs) o como regiones HII compactas. Los MYSOs poseen cocientes de flujos $\mathrm{F}_{21} / \mathrm{F}_{8}>2$ y $\mathrm{F}_{14} / \mathrm{F}_{12}>1$, mientras que para las regiones HII compactas los cocientes son $\mathrm{F}_{21} / \mathrm{F}_{8}>2$ y $\mathrm{F}_{14} / \mathrm{F}_{12}<1$. Aquellas fuentes con $\mathrm{F}_{21} / \mathrm{F}_{8}<2$ son consideradas estrellas evolucionadas.

\section{Criterios de selección para las fuentes Spitzer}

Considerando que Spitzer-IRAC tiene observaciones en las bandas a 3.6, 4.5, 5.8 y $8 \mu \mathrm{m}$, se clasifican como YSOs a las fuentes que cumplen

$$
[4.5]-[5.8]>0.35
$$




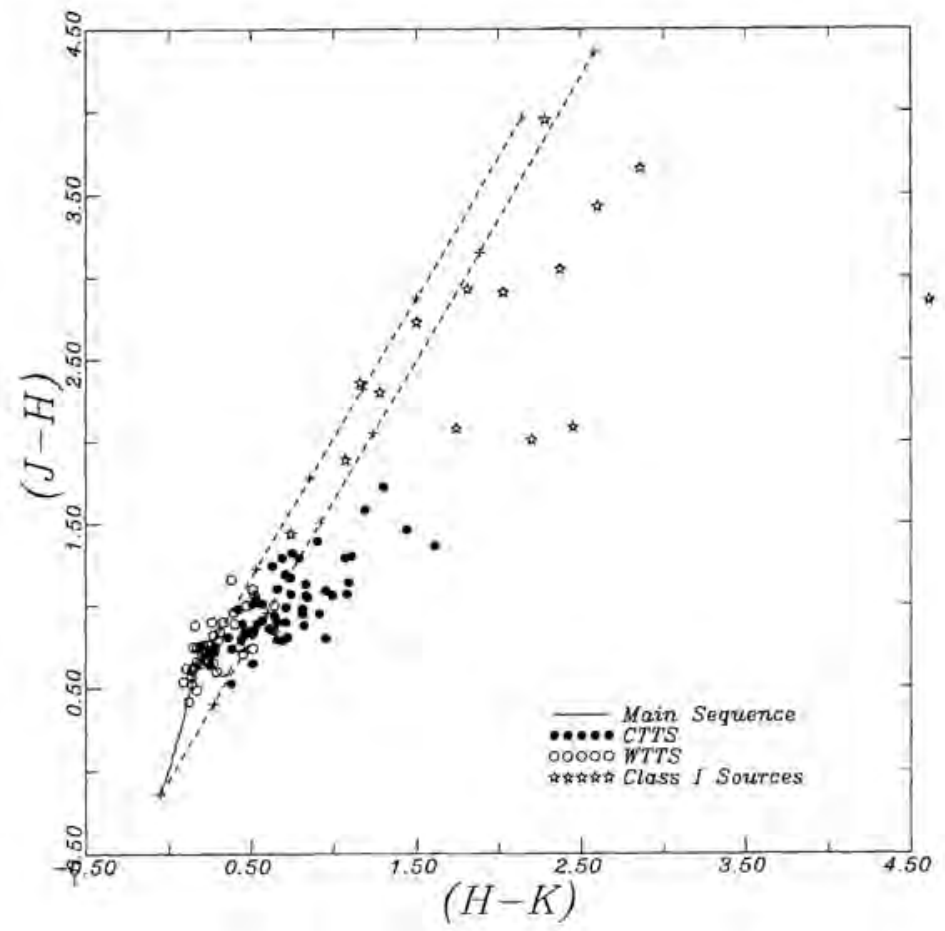

Figura 1.13: Diagrama color-color JHK para YSOs de baja masa en el complejo de nubes oscuras Taurus-Auriga. La línea contínua indica la posición de la secuencia principal no enrojecida y con líneas discontinuas se muestran las líneas de enrojecimiento. Estas dos líneas forman la banda de enrojecimiento para las fotósferas normales estelares. Los objetos con colores que caen fuera y a la derecha de esta banda son fuentes con exceso de emisión infrarroja intrínseca. Los puntos llenos y vacíos indican la posición de estrellas T-Tauri clásicas (CTTS) y de líneas débiles (WTTS), respectivamente, mientras que las estrellas muestran las posiciones de fuentes clase I. Las fuentes de clase I ocupan una región del diagrama caracterizada por valores de extinción muy grandes, mientras que las WTTS (clase II) están ubicadas en la región correspondiente a estrellas sin enrojecimiento o solo ligeramente enrojecidas. Las WTTS se caracterizan por tener valores de extinción más modestos que las de Clase I. Además, la mayoría de las fuentes de Clase I y CTTS muestran fuerte exceso infrarrojo. Tomado de Lada \& Adams, 1992. 


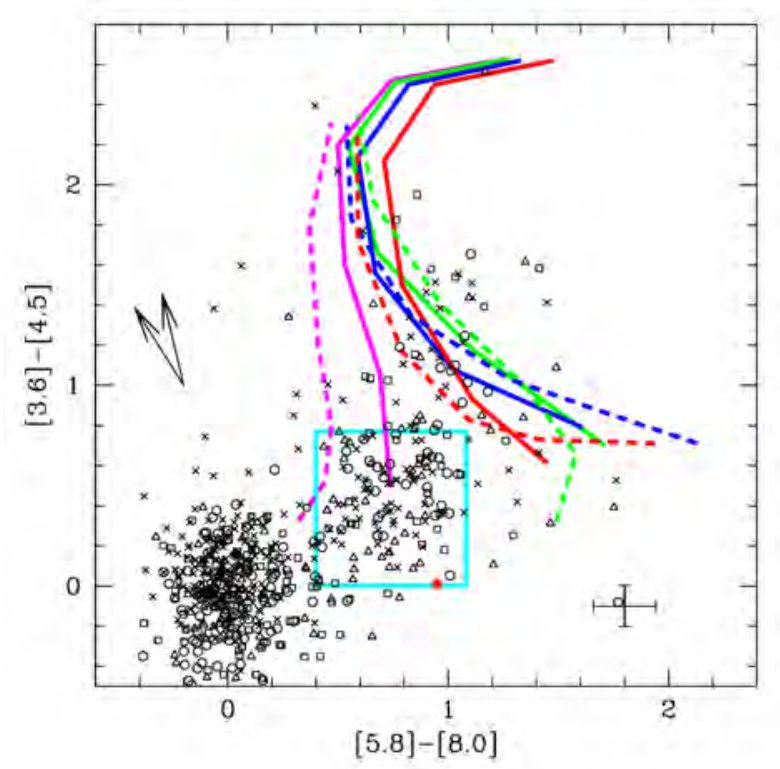

Figura 1.14: Diagrama color-color para fuentes Spitzer. Las líneas de colores muestran modelos de fuentes con envolturas (objetos clase I) para diferentes valores de luminosidades de la fuente central (magenta, verde, azul y rojo para $0.1,1,10$ y $100 \mathrm{~L} \odot$, respectivamente). El cuadrado celeste delinea el dominio aproximado de las fuentes de clase II. Se muestran barras de error representativas, que incluyen incertidumbres fotométricas promedio y una incertidumbre estimada del $10 \%$ en la calibración de flujo absoluto. El grupo de fuentes cerca de $(0,0)$ corresponde a clase III. Tomado de Allen et al. (2004).

Las fuentes que cumplan con esta condición se clasifican como clase I o clase II según su posición en el diagrama color-color (CC) [5.8]-[8] vs [3.6]-[4.5]. Este diagrama puede usarse para ayudar a distinguir entre las estrellas jóvenes con solo emisión de disco y las protoestrellas con envoltura circunestelar. Según el criterio determinado empíricamente por Allen et al. (2004) se consideran clase II a aquellas fuentes que caen en la región determinada por los rangos de colores $0<[3.6]-[4.5]<0.8$ y $0.4<[5.8]-[8.0]<1.1$ (ver recuadro celeste en Figura 1.14). $\mathrm{El}$ exceso infrarrojo de estas fuentes se debería a la presencia de discos de acreción en torno a objetos estelares. Las fuentes arriba y a la derecha de esta región son considerados Clase I y su exceso infrarrojo se debería a la presencia de envolturas circunestelares de polvo en torno a las estrellas (Allen et al., 2004).

\section{Criterios de selección para las fuentes WISE}

En cuanto a las fuentes pertenecientes al relevamiento Wide-field Infrared Survey Explorer (WISE), cuyas bandas están centradas en 3.4, 4.6, 12, y $22 \mu \mathrm{m}$, se utiliza para clasificarlas el criterio de Koenig et al. (2012).

La clasificación de las fuentes WISE se lleva a cabo a través del diagrama [3.4]-[4.6] vs [4.6]-[12]. Después de remover la contaminación proveniente de objetos de fondo como galaxias enrojecidas en [4.6]-[12], AGNs y emisión de PAHs, se procede clasificando como clase I a aquellas fuentes que cumplan con

$$
[3.4]-[4.6]>1.0 \quad[4.6]-[12.0]>2.0
$$

Las fuentes restantes quedan clasificadas como clase II. 


\section{Criterios de selección para las fuentes IRAS}

La base de datos del satélite IRAS posee observaciones a 12, 25, 60 y $100 \mu \mathrm{m}$. El bajo poder resolvente de los datos de IRAS (entre $6^{\prime}$ y $2^{\prime}$ para $12,25,60$ y $100 \mu \mathrm{m}$ ) en comparación con el del resto de los datos de fuentes infrarrojas sólo permite identificar las regiones de formación estelar. Es común encontrar varios YSOs utilizando los otros catálogos dentro del área ocupada por una fuente IRAS (ver por ejemplo Vazzano et al., 2014).

Al relevar no sólo el mediano sino también parte del lejano infrarrojo, la información sirve para detectar regiones de formación estelar que aún poseen grandes cantidades de material molecular y polvo en sus alrededores.

Siguiendo el criterio de Junkes et al. (1992) se clasifican como regiones de formación estelar ${ }^{(2)}$ aquellas fuentes que cumplen con:

- $\mathrm{S}_{100}>20 \mathrm{Jy}$

- $1.2<\mathrm{S}_{100} / \mathrm{S}_{60}<6$

- $\mathrm{S}_{60} / \mathrm{S}_{25}>1$

- $\mathrm{q}_{100}+\mathrm{q}_{60}>4$

donde $\mathrm{S}_{25} \mathrm{~S}_{60}$ y $\mathrm{S}_{100}$ son los flujos a 25, 60 y $100 \mu \mathrm{m}$, respectivamente, y q100 y $\mathrm{q}_{60}$ son las calidades fotométricas de los flujos en 100 y $60 \mu \mathrm{m}$. El rango de calidades fotométricas va de 1 a 3 , correspondiendo $\mathrm{q}=1$ a flujo detectado; $\mathrm{q}=2$ a flujo de calidad moderada y $\mathrm{q}=3$ a flujo de alta calidad.

\subsubsection{Jets y flujos moleculares}

\section{Objetos Herbig-Haro}

La observación de los fenómenos que ocurren en las zonas más densas de las nubes moleculares es difícil debido a la extinción producida por el polvo. Sin embargo existen excepciones. Los objetos Herbig-Haro (HH) se observan generalmente como regiones nebulares visibles en el óptico que se encuentran proyectadas sobre nubes moleculares en donde tienen lugar los procesos de formación estelar.

Los objetos Herbig-Haro (HH) fueron descubiertos alrededor de 1950 por G. Herbig y G. Haro (Herbig, 1951; Haro, 1952). Ellos independientemente notaron en la Nube Molecular de Orion dos zonas nebulares brillantes en $\mathrm{H} \alpha$ cerca de la región NGC 1999 que contiene un gran número de estrellas T Tauri. Además encontraron que estos objetos emitían en continuo y en líneas prohibidas en el óptico como S[II], N[II], Fe[II], O[I], O[II] e incluso O[III]. La presencia de estas líneas indicaba que era poco probable que el medio fuera una nube o grumo, ya que se trata de transiciones originadas en átomos desexcitados colisionalmente con bajas densidades. Por otro lado la presencia de ciertas especies neutras descartaba que se tratara de una región HiI. Finalmente se encontró que los objetos HH provenían del impacto de vientos estelares a alta velocidad (jets) originados en objetos en formación contra una nube molecular (Reipurth \& Bally, 2001).

El panel izquierdo de la Figura 1.15 muestra el outflow de HH 47 con sus objetos HH asociados.

\section{Jets ópticos}

$\mathrm{Al}$ igual que los objetos $\mathrm{HH}$ los jets son objetos que se observan en el rango óptico del espectro. Se trata de chorros de gas de muy alta velocidad altamente colimados que

\footnotetext{
${ }^{(2)}$ Junkes et al. (1992) las clasifica como YSOs, pero en la actualidad su pobre poder resolvente sólo permite identificarloas como regiones de formación estelar.
} 

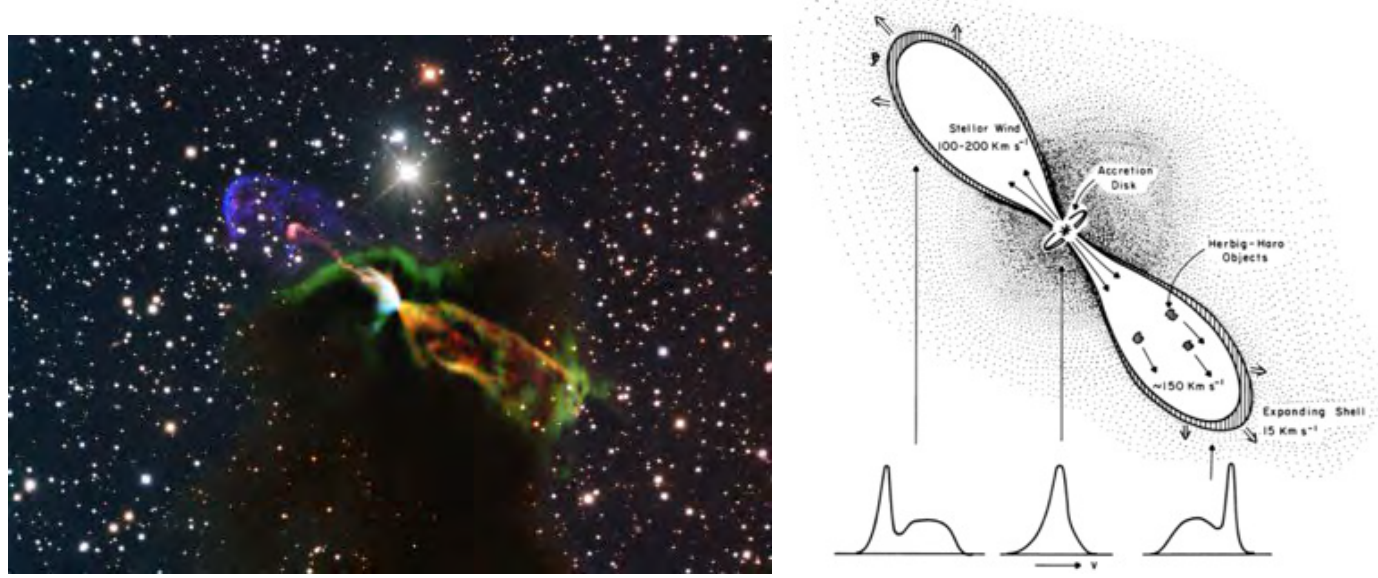

Figura 1.15: Izquierda: Imagen del outflow molecular HH 47 ejectado de una protoestrella. La imagen combina observaciones de radio adquiridas con Atacama Large Millimeter/submillimeter Array (ALMA) en naranja y verde, con observaciones en el visible del New Technology Telescop (NTT) de ESO en rosa y morado. Derecha: Esquema de un outflow molecular indicando el perfil de CO esperado para diferentes posiciones a lo largo de la fuente. Los objetos HH no están necesariamente ubicados dentro de la región molecular en que se forman, ya que las altas velocidades pueden expulsarlos hacia el medio circundante (imagen tomada de Snell et al., 1980).

son emitidos por las protoestrellas y pueden viajar a través de la nube molecular en la que se encuentran inmersos. La emisión óptica se produce cuando los jets colisionan con el material circundante. Si el gas impactante tiene una densidad suficientemente alta también puede generarse emisión máser. Presentan una estructura de 'nudos' (knots) espaciados cuasiregularmente lo que permite deducir que la eyección de material por parte de la fuente central no se realiza de manera continua, sino que son eventos esporádicos.

Su interacción con el medio ambiente ocurre a través de la excitación de átomos e iones, cuyas líneas de enfriamiento muestran la presencia del jet. Dichas líneas de enfriamiento se extienden en un amplio rango de frecuencias desde el UV hasta el IR lejano. En particular, la línea rotovibracional del Hidrógeno molecular a $2.12 \mu \mathrm{m}(\mathrm{v}=1-0 \mathrm{~S}(1))$ se usa para identificar nudos de emisión molecular, mientras que otras líneas rotovibracionales de $\mathrm{H}_{2}$ en el rango del IR cercano $(1-5 \mu \mathrm{m})$ son útiles tanto para sondear el gas molecular a altas temperaturas como para inferir los mecanismos de excitación principales (Giannini et al., 2013).

\section{Flujos moleculares (outflows)}

Los flujos moleculares son grandes áreas turbulentas de gas de una nube molecular empujados por los jets de la fuente protoestelar. Se detectan fácilmente aún cuando están inmersos en el gas molecular, razón por la cual se encuentran cientos de flujos moleculares y pocos jets ópticos.

Se trata de movimientos hipersónicos de gas con velocidades de 10 a $100 \mathrm{~km} \mathrm{~s}^{-1}$ asociados a YSOs. La masa de estos flujos va desde 0.1 hasta $100 \mathrm{M}_{\odot}$, con energías cinéticas de entre $10^{43}$ y $10^{47}$ erg. El tiempo de vida de estos fenómenos es corto (menor que $10^{5}$ años) y se requieren vientos estelares masivos para producirlo (tasas de pérdida de masa de entre $10^{-7} \mathrm{y}$ $10^{-3} \mathrm{M}_{\odot} \mathrm{yr}^{-1}$ ). Aproximadamente el $75 \%$ de los outflows conocidos muestran una estructura bipolar sugiriendo que los vientos son colimados.

El panel derecho de la Figura 1.15 muestra el esquema de un outflow molecular junto con el perfil de la línea ${ }^{12} \mathrm{CO}$ esperado para cada posición a lo largo del outflow. Hacia la fuente 

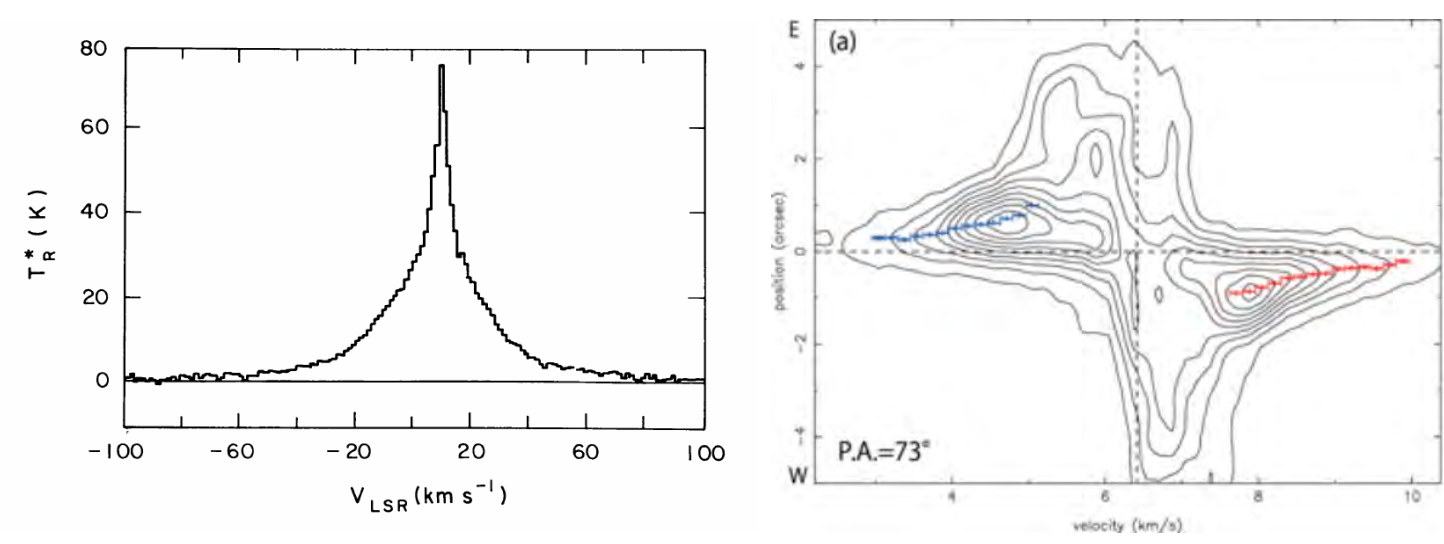

Figura 1.16: Izquierda: Espectro de ${ }^{12} \mathrm{CO}(2-1)$ observado en la posición de un objeto, en Orión, que presenta un flujo molecular de alta velocidad. La emisión de las alas de alta velocidad se extiende hasta velocidades de $\pm 90 \mathrm{~km} \mathrm{~s}^{-1}$ con respecto a la nube en que se encuentra el objeto. Imagen tomada de Snell (1987). Derecha: Diagrama posición-velocidad de la emisión de $\mathrm{C}^{18} \mathrm{O}(2-1)$ hacia la fuente TMC-1A. La línea de puntos vertical indica la velocidad sistémica y la horizontal la posición de la fuente. Imagen tomada de Aso et al. (2015).

central se observa un solo máximo mientras que hacia los lóbulos se observa una configuración de doble pico. El pico secundario cambia de lado debido a que movimiento del gas cambia de dirección. En algunos casos el material se dirige en la línea de la visual y los lóbulos son difíciles de identificar. Los lóbulos pueden terminar bruscamente si se acaba la nube molecular.

En el espectro observado en dirección a la fuente, que posee un único máximo, suelen observarse las llamadas 'alas de alta velocidad', y se deben a la emisión de gas con velocidades típicas de decenas de $\mathrm{km} \mathrm{s}^{-1}$, pero que pueden llegar incluso a centenares de $\mathrm{km} \mathrm{s}^{-1}$. Estos ensanchamientos son mucho mayores que los que se podrían producir por turbulencia del gas. Se denomina 'ala roja' a la emisión de alta velocidad correspondiente a gas que se aleja del observador y 'ala azul' a la que corresponde a gas que se mueve acercándose al observador (ver panel izquierdo de la Figura 1.16). Las velocidades observadas en los espectros corresponden únicamente a la componente radial de la velocidad, por lo que se trata de un límite inferior.

Otra forma de identificar observacionalmente a los outflows es a través de un diagrama posición-velocidad tal como el que se muestra en el panel derecho de la Figura 1.16. Estos diagramas permiten analizar cómo varía la emisión en una de las coordenadas respecto de la velocidad, dejando la otra coordenada fija. De esta forma es posible identificar la emisión de gas a altas velocidades respecto de la velocidad sistémica. En el caso de los outflows se suelen observar diagramas con simetría central, donde la emisión a uno y otro lado de la velocidad de la fuente central corresponde a cada uno de los lóbulos.

\subsubsection{Procesos de acreción (Infalls)}

La auto-absorción de líneas moleculares es una característica común en regiones de formación estelar. Se trata de una depresión en la emisión de una línea molecular que coincide con la velocidad central de la nube, producida por el gas más externo perteneciente a la envoltura que rodea la fuente, el cual absorbe la radiación proveniente de las regiones más densas del interior. La auto-absorción sería evidencia de la presencia de gradientes de densidad en el gas en caída hacia el YSO (infalls). 


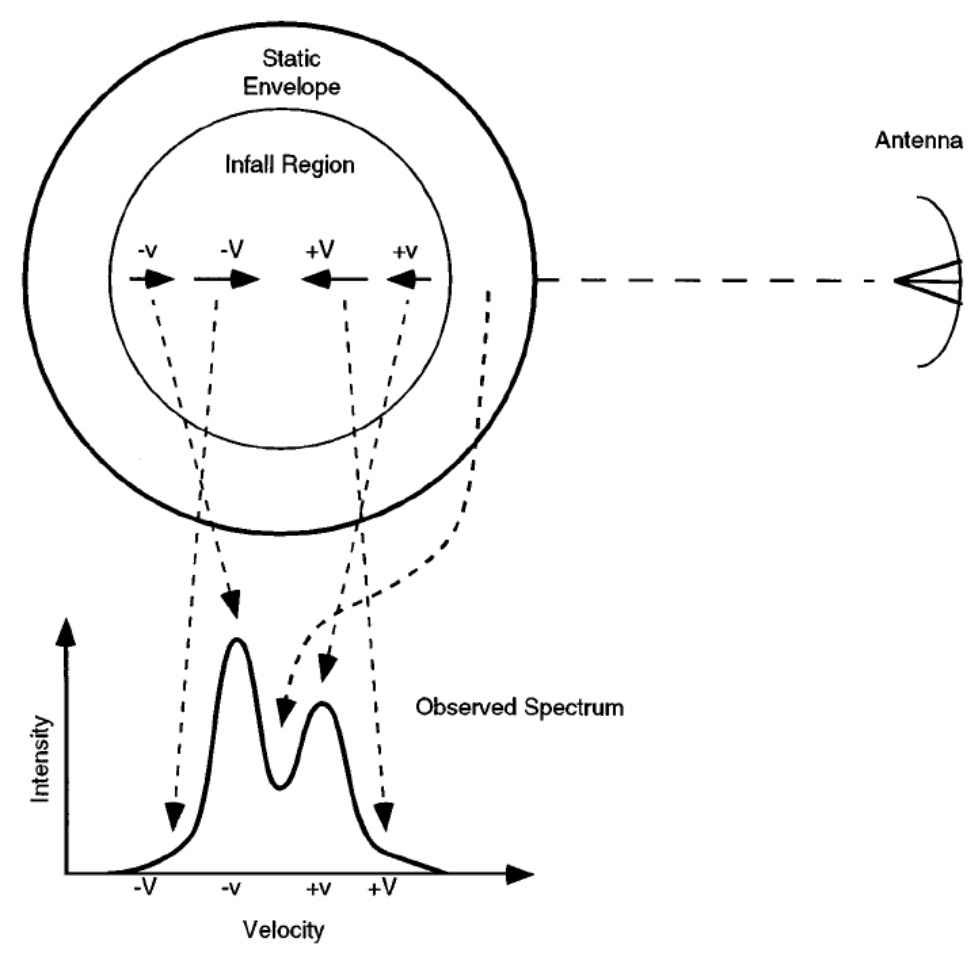

Figura 1.17: Esquema de la formación de líneas moleculares en una envoltura en caída hacia un objeto central. Las alas pueden formarse si el gas en caída cercano al objeto central cae con mayor velocidad que el gas más externo. La envoltura, que no se encuentra en caída, produce el dip de absorción central, mientras que el máximo hacia el azul proviene de la parte más alejada de la nube y el máximo que se encuentra hacia el rojo es emitido por la parte frontal de la nube molecular. Imagen tomada de Evans (1999).

Otra característica espectral interesante en los espectro hacia la región central es la componente roja menos intensa que la componente azul (ver Figura 1.17). Si el medio es opaco, los fotones que se detectan son emitidos principalmente de la superficie de estas componentes. Los fotones de la componente roja que llegan al observador provienen de las regiones más externas al grumo central y por lo tanto menos calientes, mientras que los fotones de la componente azul que llegan al observador provienen de las regiones más internas al grumo central y por lo tanto más calientes. Esta característica espectral es una prueba de la presencia de gas en caída hacia el objeto central, posiblemente debido a la acreción de material del entorno por parte del YSO (Evans, 1999).

\subsubsection{Extended Green Objects}

El estudio del plano central de la Vía Láctea realizado por el telescopio Spitzer reveló una característica inusual en algunas regiones masivas de formación estelar: se encontraron fuentes extendidas que se detectaban en la banda de $4.5 \mu \mathrm{m}$. A estos objetos se los llamó Extended Green Objects (EGOs) y su nombre se debe a que habitualmente la banda de $4.5 \mu \mathrm{m}$ se muestra en color verde en las imágenes de IRAC compuestas de tres colores (Cyganowski et al., 2008).

Los EGOs se hallan comúnmente asociados con objetos estelares masivos jóvenes (MYSOs) en regiones de formación estelar, a objetos $\mathrm{HH}$ y, en algunos casos, a outflows. La emisión extendida de $4.5 \mu \mathrm{m}$ es de particular interés como un diagnóstico de la presencia de MYSOs 
que complementa a los de los métodos tradicionales de identificación de candidatos MYSO. La banda de $4.5 \mu \mathrm{m}$ contiene las líneas de $\mathrm{H}_{2}(\mathrm{v}=0-0, \mathrm{~S}(9,10,11)$ y cabezas de banda de $\mathrm{CO}(\mathrm{v}=1-0)$, que pueden ser excitadas por choques tales como los que se esperan cuando flujos bipolares moleculares o jets asociados a protoestrellas en formación interactúan con el MIE.

\subsubsection{Máseres interestelares}

En 1955 Gordon, Zeiger y Townes descubrieron un método para enfocar un haz de $\mathrm{NH}_{3}$ excitado en una cavidad resonante (máser de laboratorio). En 1965 el fenómeno es encontrado en el espacio interestelar, donde no hay cavidades o espejos para que se produzca la amplificación.

El aumento de la intensidad de la radiación cuando pasa por una zona densa de material molecular es del orden de $10^{10}$ veces. Este escenario ocurre habitualmente en las vecindades perturbadas de estrellas jóvenes, aunque también pueden detectarse en cometas, atmósferas de estrellas gigantes, remanentes de supernovas y núcleos de galaxias externas.

A esta emisión amplificada se la llama MASER, (Microwave Amplification by Stimulated Emission of Radiation) y se produce debido a la inversión de la población de los niveles de las moléculas, es decir, los niveles superiores están más poblados que los inferiores. Para que esto suceda debe existir algún mecanismo de bombeo del nivel inferior al superior.

La emisión máser es uno de los principales indicadores de formación estelar masiva. Los máseres son relativamente comunes y debido a que tienen transiciones a longitudes de onda centimétricas, no se ven afectados por la alta extinción presente en los sitios de formación estelar. Además, la emisión máser es compacta e intensa, lo que significa que puede estudiarse con una resolución angular muy alta utilizando técnicas de VLBI. Por estas razones los máseres sirven para estudiar la cinemática del gas en el que surgen (e. g. Torrelles et al., 2005) y las condiciones físicas de las regiones donde se encuentran (e. g. Cragg et al., 2001). En el MIE se han detectado máseres de distintas moléculas, tales como $\mathrm{OH}, \mathrm{H}_{2} \mathrm{O}, \mathrm{CH}_{3} \mathrm{OH}$, $\mathrm{SiO}, \mathrm{H}_{2} \mathrm{CO}, \mathrm{NH}_{3}$.

El análisis de cientos de outflows ha demostrado que por lo menos el $50 \%$ presenta emisión en $1.35 \mathrm{~cm}(22.2 \mathrm{GHz})$ que corresponde a un máser de $\mathrm{H}_{2} \mathrm{O}$. Los spots de máser están localizados cerca de la protoestrella.

\subsubsection{Nubes infrarrojas oscuras (IRDCs)}

Las nubes oscuras infrarrojas (Infrared Dark Clouds, IRDCs) son sitios de reciente formación estelar dentro de las nubes moleculares. Fueron descubiertas en la década del 90 a partir de los satélites infrarrojos ISO (Perault et al., 1996) y MSX (Egan et al., 1998) como siluetas oscuras sobre un fondo brillante en el infrarrojo medio. Se trata de regiones frías ( $\mathrm{T}$ $<25 \mathrm{~K})$ y densas $\left(>10^{5} \mathrm{~cm}^{-3}\right)$ con tamaños entre 1 y 3 pc y masas entre $10^{2}$ y $10^{4} \mathrm{M}_{\odot}$ (Rathborne et al., 2006, 2007) que son invisibles en longitudes de onda correspondientes al infrarrojo cercano y medio, y se detectan en el infrarrojo lejano. La Figura 1.18 muestra la diferencia en la emisión en el IR medio (Spitzer) y lejano (Herschel) en una IRDC. Estas regiones se corresponden con las regiones más densas e inmersas de las nubes moleculares.

Simon et al. (2006) identificaron 10931 candidatos a IRDCs basándose en datos en $8 \mu \mathrm{m}$ de MSX observados en el plano galáctico en $l=0-360^{\circ}$ y $|b| \leq 5^{\circ}$. Se encontraron mayormente localizadas en el primer y cuarto cuadrante de la galaxia y cerca del plano galáctico (Jackson et al., 2008). 


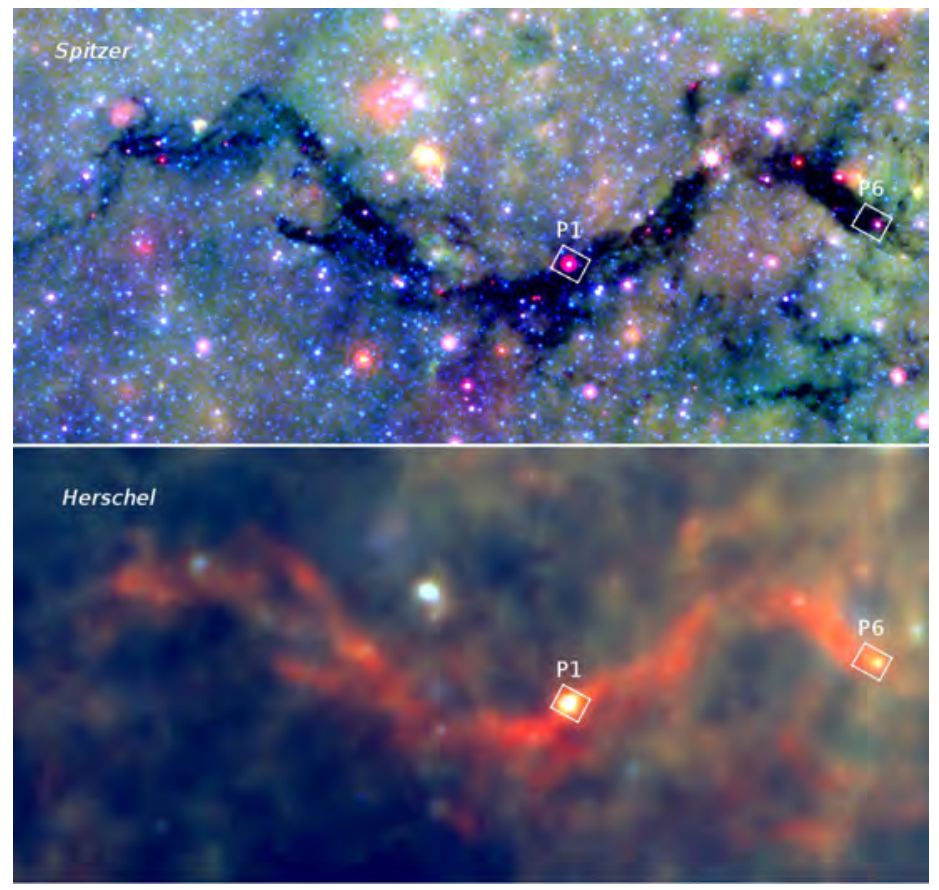

Figura 1.18: Imágenes de la nebulosa de la Serpiente tomadas por los telescopios espaciales Spitzer (arriba) y Herschel (abajo). En las longitudes de onda del infrarrojo medio el material de la nube bloquea la luz de las estrellas más distantes, mientras que en las longitudes de onda del infrarrojo lejano la nebulosa brilla debido a la emisión de polvo frío.

Se cree las que IRDC representan la etapa más temprana en la formación de estrellas masivas y son, por tanto, de gran importancia para la comprensión del proceso de formación de estrellas en su conjunto (Rathborne et al., 2007; Chambers et al., 2009). La formación de estrellas en las IRDCs puede ser inferida por la presencia de regiones HiI ultra compactas (Battersby et al., 2010), hot cores (Rathborne et al., 2008), fuentes embebidas detectadas en 24 $\mu \mathrm{m}$ indicativas de la presencia de polvo tibio (Chambers et al., 2009), outflows moleculares (Beuther \& Sridharan, 2007; Sanhueza et al., 2010), y emisión máser (Wang et al., 2006; Chambers et al., 2009).

Las IRDCs alojan a numerosos candidatos a núcleos preestelares, que corresponden a la primera fase de formación estelar. Debido a las características de las IRDCs y a la gran cantidad de estadíos evolutivos que albergan, se cree que estos son sitios de formación de las estrellas masivas y cúmulos de estrellas.

Con el objetivo de caracterizar los diferentes estados evolutivos de estos núcleos encontrados en las IRDCs, Chambers et al. (2009) propusieron una secuencia evolutiva en donde un grumo 'inactivo' evoluciona a un grumo 'intermedio', 'activo' y 'rojo'. Este esquema se basa en los colores de Spitzer-IRAC y la presencia o ausencia de fuentes puntuales detectadas por Spitzer-MIPS a $24 \mu \mathrm{m}$.

Un grumo es llamado 'inactivo' si no posee emisión en las bandas de Spitzer (o sea es oscuro en el infrarrojo cercano y medio), 'intermedio' si contiene emisión extendida en $4.5 \mu \mathrm{m}$ (EGOs) o emisión en $24 \mu \mathrm{m}$ pero no en ambas longitudes de onda, 'activo' si está asociado a un EGO y a una fuente embebida detectada en $24 \mu \mathrm{m}$, y 'rojo' si está vinculado a emisión en $8 \mu \mathrm{m}$, que se asocia a la presencia de regiones HiI.

Los grumos inactivos son los mejores candidatos a encontrarse en la fase preestelar, y los grumos inactivos masivos son los lugares en donde es más probable que transcurran las 
etapas más tempranas en la evolución de las estrellas de gran masa.

Las IRDCs se observan en continuo milimétrico (por ejemplo en las bandas observadas por Herschel en el lejano IR) y revelan la emisión de polvo frío asociado. 


\section{Capítulo 2}

\section{Cómo obtener información del medio interestelar}

\subsection{Líneas espectrales moleculares en la banda de radio}

Las diferentes especies químicas que se hallan en el universo emiten o absorben radiación a diferentes longitudes de onda originando líneas espectrales, siguiendo las leyes de la mecánica cuántica. En la región de radio del espectro electromagnético se originan transiciones de baja energía de las moléculas presentes en el universo. Estas líneas suelen observarse en emisión pero también pueden observarse en absorción sobre un fondo de continuo.

Numerosas líneas originadas en moléculas se encuentran presentes en los rangos milimétrico y submilimétrico del espectro electromagnético. En particular, las líneas rotacionales de los distintos isótopos del Monóxido de Carbono (CO) son el principal trazador del contenido de Hidrógeno molecular, principal componente de las nubes moleculares, al estar íntimamente asociado al contenido de $\mathrm{H}_{2}$. El $\mathrm{H}_{2}$ se forma sobre los granos de polvo que funcionan como catalizadores, mientras que el $\mathrm{CO}$ se forma fácilmente en la fase gaseosa. También se observan líneas de otras moléculas como el $\mathrm{NH}_{3}, \mathrm{OH}, \mathrm{HCN}$, etc, que trazan diferentes propiedades físicas y químicas de las regiones más densas del medio interestelar.

A continuación, se hará una breve descripción de los espectros moleculares, en particular de CO, y de la obtención de las propiedades de las nubes moleculares a través de ellos.

\subsection{Procesos físicos en las nubes moleculares}

\subsubsection{Transiciones moleculares}

Las transiciones moleculares pueden dividirse en tres grupos, según cual sea su origen. Las transiciones electrónicas se produccen entre los diferentes niveles de energía electrónicos de una molécula y poseen energías de unos pocos eV que corresponden a longitudes de onda del visual y del UV; las transiciones vibracionales son causadas por las oscilaciones de las posiciones relativas de los núcleos con respecto a sus posiciones de equilibrio y poseen energías típicas son 0.1-0.01 eV correspondientes a la región infrarroja del espectro; las transiciones rotacionales se originan en la rotación de los núcleos y poseen energías típicas de $10^{-3} \mathrm{eV}$, correspondientes al rango de longitudes de onda milimétricas principalmente. La energía total está dada por la suma de la energía correspondiente a cada tipo de transiciones:

$$
E_{t o t}=E_{e l}+E_{v i b}+E_{r o t}
$$




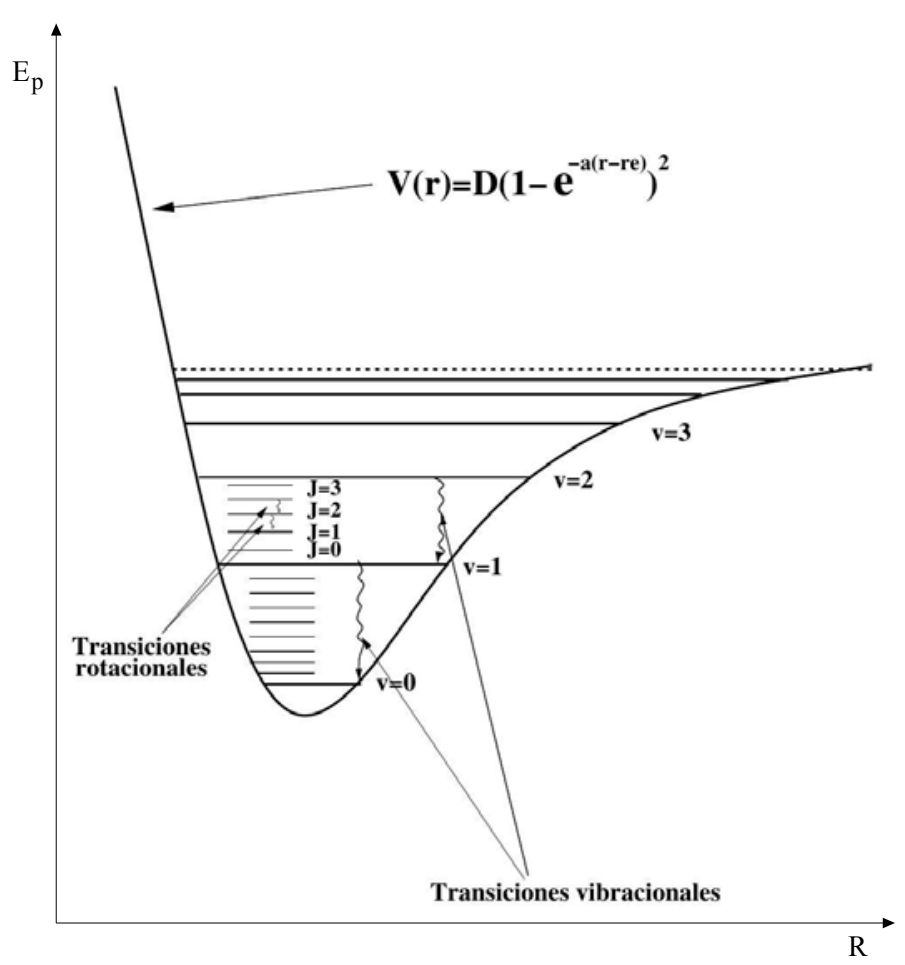

Figura 2.1: Representación esquemática de los niveles vibracionales y rotacionales en una molécula diatómica en un nivel electrónico.

Tabla 2.1: Valores típicos de energía, frecuencia, longitud de onda y temperatura para diferentes tipos de transiciones moleculares.

\begin{tabular}{lccccc}
\hline \hline & $\begin{array}{c}\text { Energía } \\
{[\mathrm{eV}]}\end{array}$ & $\begin{array}{c}\nu \\
{[\mathrm{GHz}]}\end{array}$ & $\lambda$ & $\begin{array}{c}\mathrm{T}_{K} \\
{[\mathrm{~K}]}\end{array}$ & Rango del espectro \\
\hline Electrónica & 7.5 & $1.8 \times 10^{6}$ & $1700 \AA$ & 90000 & $\mathrm{UV}$ \\
Vibracional & 0.06 & $1.3 \times 10^{4}$ & $23 \mu \mathrm{m}$ & 600 & $\mathrm{IR}$ \\
Rotacional & $10^{-4}$ & $9.8 \times 10^{1}$ & $3.1 \mathrm{~mm}$ & 5 & radio $(\mathrm{mm})$ \\
\hline \hline
\end{tabular}

Para calcular la energía típica que se requiere para cada tipo de transición se considera un tamaño molecular típico $a \simeq 1 \AA$, una masa molecular típica $M$, y la masa del electrón $m$. Para las transiciones electrónicas la energía se calcula como $E_{e l} \simeq \frac{\hbar}{m a^{2}}$, de lo cual resulta que para los valores típicos indicados la energía es $E_{e l} \simeq 1.2 \times 10^{-11} \mathrm{erg}=7.5 \mathrm{eV}$, que corresponde a una frecuencia de la transición $\nu_{e l}=E_{e l} / h=1.8 \times 10^{6} \mathrm{GHz}$. Para las transiciones vibracionales la energía es $E_{v i b} \simeq\left(\frac{m}{M}\right)^{1 / 2} E_{e l}$, de lo que resulta $E_{v i b} \simeq 8.9 \times 10^{-14} \mathrm{erg}=0.06 \mathrm{eV}$, que corresponde a una frecuencia de la transición $\nu_{v i b}=E_{v i b} / h=1.3 \times 10^{4} \mathrm{GHz}$. Finalmente para las transiciones rotacionales la energía se calcula como Erot $\simeq\left(\frac{m}{M}\right) E_{e l}$, por lo tanto $E_{\text {rot }} \simeq$ $6.5 \times 10^{-16} \mathrm{erg}=4 \times 10^{-4} \mathrm{eV}$, que corresponde a una frecuencia de la transición $\nu_{\text {rot }}=E_{\text {rot }} / h$ $=98 \mathrm{GHz}$. La energía, frecuencia, longitud de onda, temperatura cinética y rango del espectro electromagnético que corresponde a cada uno de los tipos de transiciones están indicadas en la Tabla 2.1. La Figura 2.1 muestra esquemáticamente los niveles vibracionales y rotacionales en una molécula diatómica, que es una representación de la energía potencial versus la distancia entre los átomos que componen dicha molécula.

La energía disponible en una nube molecular para excitar una transición mediante coli- 
siones es del orden de $k T_{K}$, donde $\mathrm{T}_{K}$ es la temperatura cinética de la nube. Dado que las temperaturas cinéticas típicas de nubes moleculares son de orden de $\mathrm{T}_{K} \simeq 10 \mathrm{~K}$, las únicas transiciones moleculares que tendrán lugar serán las rotacionales.

La molécula de $\mathrm{H}_{2}$ es la componente fundamental de las nubes moleculares. Sin embargo esta molécula no es observable directamente debido a que no posee momento dipolar eléctrico permanente, por lo que no puede emitir radiación en transiciones puramente rotacionales. Sólo en regiones de elevada temperatura, como zonas afectadas por frentes de choque, la molécula de $\mathrm{H}_{2}$ puede emitir radiación en transiciones vibracionales, o en el medio atómico neutro, donde emite en líneas del UV. Sin embargo las condiciones en que estas líneas aparecen no son representativas de las nubes moleculares.

Es por ello que el estudio de la distribución general de gas molecular en la galaxia se realiza a través de isótopos de la molécula de $\mathrm{CO}$, que presenta transiciones fácilmente detectables, y que es la segunda molécula más abundante luego del $\mathrm{H}_{2}$.

\subsubsection{Transiciones rotacionales}

Se analizarán en particular las transiciones rotacionales dado que en esta tesis se utilizarán datos de estas transiciones. Se seguirá en este punto el texto de Estalella \& Anglada Pons (2008).

Para una molécula diatómica, la energía rotacional es

$$
E_{r}=\frac{1}{2} I \omega^{2}=\frac{L^{2}}{2 I}
$$

donde $\mathrm{L}$ es el momento angular e I el momento de inercia, que está dado por $I=\frac{m_{1} m_{2}}{m_{1}+m_{2}} r_{0}$, donde $m_{1}$ y $m_{2}$ son las masas de los átomos y $r_{0}$ es la distancia entre los núcleos. Resolviendo la ecuación de Schrödinger para una molécula diatómica se obtiene que el momento angular para un cierto nivel $J$ responde a

$$
L_{J}=\sqrt{J(J+1)} \hbar
$$

donde $J=1,2,3, \ldots$ es el número cuántico rotacional. Por lo tanto, la energía correspondiente a un cierto nivel rotacional $J$ será

$$
E_{J}=\frac{\hbar^{2} J(J+1)}{2 I}
$$

Cuando se produce una transición de un cierto nivel rotacional $J$ a uno de menor energía $J-1$, se emite un fotón cuya frecuencia corresponde a

$$
\nu_{J, J-1}=\frac{E_{J}-E_{J-1}}{h}=\frac{h}{4 \pi^{2} I} J
$$

Definiendo para cada molécula la constante rotacional

$$
B_{0}=\frac{h}{8 \pi^{2} I}
$$

la energía del nivel $J$ queda determinada por

$$
E_{J}=h B_{0} J(J+1)
$$

Por lo tanto la frecuencia de la transición $J \rightarrow(J-1)$ quedará determinada por

$$
\nu_{J, J-1}=2 B_{0} J
$$


Para las diferentes especies isotópicas de una misma molécula las frecuencias de una misma transición son ligeramente diferentes, puesto que los momentos de inercia son también ligeramente distintos debido principalmente a la diferencia en la masa reducida (ya que se puede considerar que la distancia entre los núcleos, determinada fundamentalmente por las fuerzas Coulombianas, no varía en primera aproximación). Se observa además que la frecuencia de una transición $J \rightarrow J-1$ es proporcional a $J$, y por lo tanto, para moléculas diatómicas, los niveles serán equidistantes en frecuencia.

La Tabla 2.2 muestra las frecuencias de las líneas moleculares que se usan en esta tesis.

\section{Densidades críticas}

Las líneas moleculares se originan a través de colisiones con la molécula más abundante $\left(\mathrm{H}_{2}\right)$. En regiones donde escasean estos fotones y si la densidad del medio es alta las transiciones colisionales dominan sobre las radiativas y la población de los niveles está gobernada por las colisiones, entonces la temperatura de excitación de la transición $\mathrm{T}_{e x c}$, definida como aquella que domina las transiciones entre niveles energéticos, coincide con la temperatura cinética $\mathrm{T}_{K}$. En estos casos se dice que hay equilibrio termodinámico local (LTE) o que la región está termalizada.

Para que una cierta transición rotacional se produzca por colisiones con $\mathrm{H}_{2}$ la densidad de la nube molecular debe ser mayor que una cierta densidad crítica $n_{\text {crit }}$, de modo tal que la transición esté termalizada. La densidad crítica está dada por la expresión

$$
n_{\text {crit }}=\frac{A_{u l}}{\gamma_{u l}}
$$

donde $A_{u l}$ es el coeficiente de emisión espontánea, con $u$ y $l$ niveles superior e inferior de la transición, respectivamente, y $\gamma_{u l}$ es el coeficiente de desexcitación colisional, que está dado por

$$
\gamma_{u l}=\int \sigma_{u l}(v) v f(v) \mathrm{d} v
$$

donde $\sigma_{u l}(v)$ es la sección eficaz de colisión correspondiente a la velocidad $v$ y $f(v)$ es la distribución de velocidad de las partículas colisionantes. El coeficiente de desexcitación colisional suele expresarse, con suficiente aproximación, por

$$
\gamma_{u l}=\sigma_{u l}\langle v\rangle=\sigma_{u l} \sqrt{\frac{8 k \mathrm{~T}_{k}}{\pi m_{H_{2}}}}
$$

No todas las moléculas se excitan colisionalmente a la misma densidad crítica. Mientras que a algunas les basta con bajas densidades de $\mathrm{H}_{2}$ para hacerse detectables a través de líneas espectrales (tales como el ${ }^{12} \mathrm{CO}$ ), otras necesitan altas densidades para termalizarse (e.g. $\mathrm{HCO}^{+}$y $\mathrm{CS}$ ). A estas últimas las llamamos trazadores de alta densidad, ya que su presencia indica regiones de alta densidad ambiental de $\mathrm{H}_{2}$.

La Tabla 2.2 lista las densidades críticas de las moléculas utilizadas en esta tesis, así como el uso principal de cada línea. En los valores de densidades críticas mostrados en esta tabla se puede ver que, para una misma molécula, cuanto mayor es el nivel superior de la transición, mayor es la densidad del gas que muestra. Esto se puede apreciar en los valores de densidad crítica tabulados para ${ }^{12} \mathrm{CO}(1-0),{ }^{12} \mathrm{CO}(2-1)$ y ${ }^{12} \mathrm{CO}(3-2)$, o para $\mathrm{HCO}^{+}(1-0)$ y $\mathrm{HCO}^{+}(4-3)$. $\mathrm{El}{ }^{12} \mathrm{CO}$ es trazador de regiones de baja densidad, sin embargo sus isótopos muestran regiones más densas cuanto más escasos son. De este modo el ${ }^{13} \mathrm{CO}$ indica la presencia de regiones un poco más densas que el ${ }^{12} \mathrm{CO}$, y el $\mathrm{C}^{18} \mathrm{O}$ sin llegar a ser una trazador de alta densidad como otras moléculas mostradas en la tabla, tiene una distribución más compacta e indica la presencia de grumos considerablemente densos. 
Tabla 2.2: Frecuencia de las transiciones moleculares utilizadas en esta tesis y densidades críticas. Los valores indicados con ${ }^{*}$ fueron obtenidos de http://th.nao.ac.jp/MEMBER/ tomisaka/research_resources/critical_density.html y corresponden a un medio con una temperatura de $10 \mathrm{~K}$, y el resto, obtenidas de Sanhueza et al. (2012), corresponden a un medio con $\mathrm{T}=20 \mathrm{~K}$.

\begin{tabular}{|c|c|c|c|}
\hline Molécula & $\begin{array}{c}\text { Frecuencia } \\
\text { [GHz] }\end{array}$ & $\begin{array}{c}n_{\text {crit }} \\
{\left[\mathrm{cm}^{-3}\right]}\end{array}$ & Trazador \\
\hline $\mathrm{HCO}^{+}(4-3)$ & 356.734288 & $9 \times 10^{6} *$ & Gas denso y outflows \\
\hline${ }^{12} \mathrm{CO}(3-2)$ & 345.795989 & $7 \times 10^{4} *$ & Gas de baja densidad \\
\hline${ }^{13} \mathrm{CO}(3-2)$ & 330.587965 & - & \\
\hline $\mathrm{C}^{18} \mathrm{O}(3-2)$ & 329.330552 & - & \\
\hline${ }^{12} \mathrm{CO}(2-1)$ & 230.538000 & $2 \times 10^{4 *}$ & Gas de baja densidad \\
\hline${ }^{13} \mathrm{CO}(2-1)$ & 220.398684 & - & \\
\hline $\mathrm{C}^{18} \mathrm{O}(2-1)$ & 219.560354 & - & \\
\hline${ }^{12} \mathrm{CO}(1-0)$ & 115.271201 & $4 \times 10^{3} *$ & Gas de baja densidad \\
\hline${ }^{13} \mathrm{CO}(1-0)$ & 110.201354 & - & \\
\hline $\mathrm{C}^{18} \mathrm{O}(1-0)$ & 109.782173 & - & \\
\hline $\mathrm{CS}(2-1)$ & 97.980953 & $6 \times 10^{5} *$ & Gas denso \\
\hline $\mathrm{N}_{2} \mathrm{H}^{+}(1-0)$ & 93.173480 & $3 \times 10^{5}$ & Gas inactivo \\
\hline $\mathrm{HC}_{3} \mathrm{~N}(10-9)$ & 90.978989 & $5 \times 10^{5}$ & Núcleos calientes, gas denso \\
\hline $\mathrm{HNC}(1-0)$ & 90.663572 & $3 \times 10^{5}$ & Gas denso \\
\hline $\mathrm{HCO}^{+}(1-0)$ & 89.188526 & $2 \times 10^{5}$ & Gas denso y outflows \\
\hline $\mathrm{HCN}(1-0)$ & 88.631847 & $3 \times 10^{6}$ & Gas denso e infalls \\
\hline $\mathrm{C}_{2} \mathrm{H}(1-0)$ & 87.316925 & $2 \times 10^{5}$ & Etapas tempranas de formación \\
\hline $\mathrm{HN}^{13} \mathrm{C}(1-0)$ & 87.090859 & $3 \times 10^{5}$ & Densidad columnar \\
\hline $\mathrm{H}^{13} \mathrm{CO}^{+}$ & 86.754330 & $2 \times 10^{5}$ & Gas denso \\
\hline $\mathrm{CS}(1-0)$ & 48.990954 & $1 \times 10^{5 *}$ & Gas denso \\
\hline
\end{tabular}




\subsubsection{Parámetros físicos de las nubes moleculares}

Dado que la base de datos a utilizar está constituída básicamente por observaciones de líneas de $\mathrm{CO}$, se detallará la metodología para la obtención de los parámetros físicos del gas molecular en base a las líneas de diferentes isótopos de esta molécula.

Para obtener los parámetros del gas en LTE es necesario tener observaciones de líneas de dos isótopos de CO, uno que sea ópticamente delgado (es decir que la profundidad óptica de la línea sea $\tau \ll 1)$ y uno que sea ópticamente grueso $(\tau>1)$. La profundidad óptica es una medida adimensional que sirve para dar una idea de qué tan transparente es un medio para un longitud de onda determinada.

Habitualmente las líneas de ${ }^{12} \mathrm{CO}$ son ópticamente gruesas debido a la gran abundancia de este isótopo, mientras que las de ${ }^{13} \mathrm{CO}$ son generalmente ópticamente delgadas. Sin embargo, en ocasiones en que la densidad ambiental es muy alta puede resultar que las líneas de ${ }^{13} \mathrm{CO}$ resulten ópticamente gruesas, por lo que pueden utilizarse como líneas ópticamente delgadas las correspondientes a isótopos menos abundantes tales como el $\mathrm{C}^{18} \mathrm{O}$.

\section{Temperatura de excitación}

Para poder derivar la temperatura de excitación de las nubes moleculares a partir de observaciones de Monóxido de Carbono, se debe observar una transición ópticamente gruesa. Se considera la ecuación de transporte

$$
\frac{d I_{\nu}}{d \tau_{\nu}}=-I_{\nu}+S_{\nu}(T)
$$

En esta expresión $I_{\nu}$ es la intensidad específica y $S_{\nu}=\frac{j_{\nu}}{\kappa_{\nu}}$ es la función fuente, que bajo condiciones de equilibrio termodinámico local estará bien representada por una función de Plank para un cuerpo negro

$$
B_{\nu}(T)=\frac{2 k \nu^{3}}{c^{2}} \frac{1}{e^{k \nu / k T}-1}
$$

Integrando la ecuación desde la región observada hasta el observador, se encuentra que su solución es

$$
I_{\nu}(l)=I_{\nu}(0) e^{-\tau(l)}+\frac{j_{\nu}}{\kappa_{\nu}}\left(1-e^{-\tau(l, \nu)}\right)
$$

donde $l$ es la longitud de la nube en la línea de la visual, $j_{\nu}$ y $\kappa_{\nu}$ son los coeficientes de emisión y absorción, respectivamente, y $\tau$ es la profundidad óptica, definida como la inversa del camino libre medio de un fotón de frecuencia $\nu$, y expresada a través de

$$
\tau(l, \nu)=\int_{l} \kappa_{\nu} \mathrm{d} l
$$

Corrigiendo por la emisión de continuo de fondo $I_{\nu}(0)$ y definiendo $\Delta I_{\nu}=I_{\nu}(l)-I_{\nu}(0)$ se obtiene la expresión

$$
\Delta I_{\nu}=\left(\frac{j_{\nu}}{k_{\nu}}-I_{\nu}(0)\right)\left(1-e^{-\tau(l, \nu)}\right)
$$

Suponiendo que la intensidad incidente sobre la nube molecular y la recibida por el instrumento corresponden a dos cuerpos negros con temperaturas $\mathrm{T}_{\text {rad }}$ y $\mathrm{T}_{b}$, respectivamente (donde $\mathrm{T}_{r a d}$ es la temperatura del fondo de radiación y $\mathrm{T}_{b}$ es la temperatura de brillo del objeto observado), entonces $I_{\nu}(0)$ y $\Delta I_{\nu}$ pueden escribirse como: 


$$
\begin{gathered}
I_{\nu}=B_{\nu}\left(T_{r a d}\right)=\frac{2 h \nu^{3}}{c^{2}} \frac{1}{e^{\frac{T^{*}}{T_{\text {rad }}}}-1} \\
\Delta I_{\nu}=B_{\nu}\left(T_{b}\right)=\frac{2 h \nu^{3}}{c^{2}} \frac{1}{e^{\frac{T^{*}}{T_{b}}}-1}
\end{gathered}
$$

donde $T^{*}=\frac{h \nu}{k}$. Reemplazando 2.16 y 2.17 en 2.15 resulta

$$
B_{\nu}\left(T_{b}\right)=\left[B_{\nu}\left(T_{e x c}\right)-B_{\nu}\left(T_{r a d}\right)\right]\left(1-e^{-\tau(\nu)}\right)
$$

donde $\mathrm{T}_{\text {exc }}$ es la temperatura de excitación de la nube, que es aquella que domina las transiciones entre niveles energéticos ya que determina la población de los niveles rotacionales por la ecuación de Boltzmann. A partir de aquí se puede obtener

$$
T_{b}(\nu)=T^{*}\left(\frac{1}{e^{\frac{T^{*}}{T_{e x c}}}-1}-\frac{1}{e^{\frac{T^{*}}{T_{\text {rad }}}}-1}\right)\left(1-e^{-\tau(\nu)}\right)
$$

Si la línea observada es ópticamente gruesa $(\tau \gg 1)$, se puede aproximar la expresión anterior por

$$
T_{b}=T^{*}\left(\frac{1}{e^{\frac{T^{*}}{T_{e x c}}}-1}-\frac{1}{e^{\frac{T^{*}}{T_{\text {rad }}}}-1}\right)
$$

Por lo tanto, conociendo la temperatura de brillo $\mathrm{T}_{b}$ de una línea con $\tau \gg 1 \mathrm{y}$ adoptando un valor para la temperatura de fondo $\left(\mathrm{T}_{\text {rad }}=2.7 \mathrm{~K}\right)$, se puede hallar la temperatura de excitación de la línea simplemente despejando de 2.20, ya que $\mathrm{T}_{\text {exc }}$ es la única incógnita de la ecuación. Entonces se puede escribir

$$
T_{e x c}=\frac{T^{*}}{\ln \left[\frac{1}{\frac{T_{b}}{T_{*}}+\left(e^{\frac{T^{*}}{T_{r a d}}}-1\right)^{-1}}+1\right]}
$$

\section{Profundidad óptica}

Una vez que se conoce la temperatura de excitación y asumiendo que es la misma para todas las especies isotópicas de una molécula se pueden considerar las observaciones de la línea ópticamente delgada y aplicar la ecuación 2.19. La expresión de $\tau$ obtenida a partir de esta ecuación es

$$
\tau_{0}=-\ln \left[1-\frac{T_{b}}{T^{*}}\left(\frac{1}{e^{\frac{T^{*}}{T_{e x c}}-1}}-\frac{1}{e^{\frac{T^{*}}{T_{\text {rad }}}-1}}\right)^{-1}\right]
$$

donde $\tau_{0}$ es la profundidad óptica en el centro de la línea y $T^{*}=\frac{h \nu}{k}$.

A partir de la definición del coeficiente de absorción e integrando a lo largo de la línea de la visual se obtiene

$$
\tau_{0}=\frac{c^{3}}{8 \pi \nu_{J, J-1}^{3} \Delta \mathrm{v}} A_{J, J-1} N_{J}\left(e^{\frac{h \nu_{J, J-1}}{k T_{e x c}}}-1\right)
$$

donde $A_{J, J-1}$ es el coeficiente de emisión espontánea, $N_{J}$ es la densidad columnar de moléculas en el estado $J$, y $\Delta \mathrm{v}$ es el ancho en velocidad de la línea. 
En algunos casos resulta útil calcular el cociente de profundidades ópticas de dos especias isotópicas

$$
\frac{\tau_{0(1)}}{\tau_{0(2)}}=\frac{N_{J(1)} \Delta \mathrm{v}_{(2)} \nu_{(2)}^{2}}{N_{J(2)} \Delta \mathrm{v}_{(1)} \nu_{(1)}^{2}}
$$

donde los subíndices 1 y 2 indican que se trata de la densidad columnar, ancho en velocidad y frecuencia de dos especies isotópicas distintas.

Considerando las especies isotópicas ${ }^{12} \mathrm{CO}$ y ${ }^{13} \mathrm{CO}$ del Monóxido de Carbono y el cociente de sus profundidades ópticas resulta

$$
\frac{\tau_{0}^{13}}{\tau_{0}^{12}}=\frac{N_{J}^{13} \Delta \mathrm{v}^{12} \nu_{12}^{2}}{N_{J}^{12} \Delta \mathrm{v}^{13} \nu_{13}^{2}}
$$

Teniendo en cuenta las abundancias isotópicas terrestres resulta $\frac{N^{13}}{N^{12}} \simeq \frac{X\left({ }^{13} C O\right)}{X\left({ }^{12} C O\right)}=\frac{1}{70}$ (Langer \& Penzias, 1990; Wilson \& Rood, 1994), entonces $\tau_{0}^{12} \gg \tau_{0}^{13}$. Finalmente, determinando $\tau_{0}^{13}$ de $2.22, \tau_{0}^{12}$ se puede obtener como

$$
\tau_{0}^{12}=\tau_{0}^{13} \frac{70 \Delta \mathrm{v}^{13}}{\Delta \mathrm{v}^{12}}\left(\frac{\nu^{13}}{\nu^{12}}\right)^{2}
$$

Para obtener la ecuación anterior se debe considerar que una de las líneas es ópticamente delgada. Sin embargo no siempre es posible estar seguro de que esta aproximación sea válida.

En ese caso se pueden obtener valores de las profundidades ópticas de las líneas a partir del cociente de intensidades integradas por medio de la siguiente ecuación

$$
\frac{\int T_{m b, 1} d \mathrm{v}}{\int T_{m b, 2} d \mathrm{v}}=\frac{1-\exp \left(-\tau_{1}\right)}{1-\exp \left(-\tau_{2}\right)}
$$

donde $\tau_{2}=\tau_{1} / A$, siendo $A$ la abundancia de la molécula 1 respecto de la 2 .

\section{Densidad columnar}

Una vez que se conoce la profundidad óptica y la temperatura de excitación de la nube, se puede obtener la densidad columnar utilizando la especie ópticamente delgada.

Dado que interesa la densidad columnar total de una especie isotópica $N$, se debe tener en cuenta la población en todos los niveles cuánticos $J$. Para obtener este valor se supone que todos los niveles están poblados según la ley de Boltzmann para una misma temperatura de excitación, o sea que el sistema se encuentra en LTE. Así la relación entre un nivel $j$ cualquiera y el nivel $J$ considerado estará dada por

$$
\frac{n_{j}}{n_{J}}=\frac{g_{j}}{g_{J}} e^{-\frac{E_{j}-E_{J}}{k T_{e x c}}}
$$

donde $E_{J}=h B_{0} J(J+1)$ es la energía correspondiente a un dado nivel $J$ y $g_{J}=2 J+1 \mathrm{su}$ peso estadístico.

La densidad volumétrica de moléculas tomando en cuenta todos los niveles será

$$
n=\sum_{j=0}^{\infty} n_{j}=\frac{n_{J}}{g_{J}} e^{\frac{E_{J}}{k T_{e x c}}} \sum_{j=0}^{\infty} g_{j} e^{\frac{-E_{j}}{k T_{e x c}}}=\frac{n_{J}}{2 J+1} e^{\frac{h B_{0} J(J+1)}{k T_{e x c}}} Q
$$

donde $\mathrm{Q}$ es la función de partición 
Tabla 2.3: Abundancias moleculares respecto al $\mathrm{H}_{2}$.

\begin{tabular}{lcc}
\hline \hline Molécula & Abundancia & Referencia \\
\hline${ }^{13} \mathrm{CO}$ & $1.3 \times 10^{-6}$ & Wilson \& Rood (1994) \\
$\mathrm{C}^{18} \mathrm{O}$ & $1.7 \times 10^{-7}$ & Sanhueza et al. (2010) \\
$\mathrm{C}_{2} \mathrm{H}$ & $5.0 \times 10^{-8}$ & Ohishi et al. (1992) \\
$\mathrm{HCO}^{+}$ & $8.0 \times 10^{-9}$ & Ohishi et al. (1992) \\
$\mathrm{HNC}$ & $6.0 \times 10^{-9}$ & Ohishi et al. (1992) \\
$\mathrm{HCN}$ & $4.0 \times 10^{-9}$ & Ohishi et al. (1992) \\
$\mathrm{N}_{2} \mathrm{H}^{+}$ & $5.0 \times 10^{-10}$ & Ohishi et al. (1992) \\
$\mathrm{HC}_{3} \mathrm{~N}$ & $1.8 \times 10^{-11}$ & Ohishi et al. (1992) \\
\hline \hline
\end{tabular}

$$
Q=\sum_{j=0}^{\infty}(2 j+1) e^{\frac{-h B_{0} j(j+1)}{k T_{e x c}}}
$$

Reemplazando la sumatoria por una integral en la función de partición y considerando que el sistema está termalizado se puede aproximar $Q \approx \frac{k T_{e x c}}{h B_{0}}$ y reemplazar en la expresión de $n$.

Por otro lado, se acepta que la relación entre las densidades columnares es la misma que para las densidades volumétricas, lo que permite escribir la densidad columnar de una molécula en el estado $J\left(N_{J}\right)$ en función de la densidad columnar total considerando todos los estados $(N)$

$$
N_{J}=(2 J+1) \frac{h B_{0}}{k T_{\text {exc }}} N e^{\frac{-h B_{0} J(J+1)}{k T_{e x c}}}
$$

Una vez obtenido $N_{J}$ se lo puede reemplazar en la expresión para $\tau_{0}$, dada por la ecuación 2.23

$$
\tau_{0}=\frac{(2 J+1) h c^{3} A_{J, J-1}}{16 J \pi k \nu_{J, J-1}^{2} \Delta \mathrm{v} T_{e x c}} N\left(e^{\frac{h \nu_{J, J-1}}{k T_{e x c}}}-1\right) e^{\frac{-(J+1) h \nu_{J, J-1}}{2 k T_{e x c}}}
$$

donde se utilizó que $\nu_{J, J-1}=2 B_{0} J$.

Despejando $N$ de la ecuación anterior se obtiene

$$
N=\tau_{0} \frac{16 J \pi k \nu_{J, J-1}^{2} \Delta \mathrm{v} T_{e x c}}{(2 J+1) h c^{3} A_{J, J-1}}\left[\frac{e^{(J+1) h \nu / 2 k T_{e x c}}}{e^{h \nu / k T_{e x c}}-1}\right]
$$

Si se considera que las densidades columnares de las distintas especies isotópicas guardan relación con las abundancias isotópicas de los átomos constituyentes del monóxido de carbono que se hallan presentes en la Tierra, se tiene que

$$
\left[\frac{N\left({ }^{12} \mathrm{CO}\right)}{N\left({ }^{13} \mathrm{CO}\right)}\right] \sim 70, \quad\left[\frac{N\left({ }^{12} \mathrm{CO}\right)}{N\left(\mathrm{C}^{18} \mathrm{O}\right)}\right] \sim 500, \quad\left[\frac{N\left({ }^{12} \mathrm{CO}\right)}{N\left({ }^{13} \mathrm{C}^{18} \mathrm{O}\right)}\right] \sim 70 \times 500
$$

Una vez obtenida la densidad columnar de la molécula se puede hallar la correspondiente a $\mathrm{H}_{2}$ conociendo la abundancia relativa entre las mismas. En la Tabla 2.3 se muestran las abundancias relativas de algunas de las moléculas más comunes respecto de la de $\mathrm{H}_{2}$.

En el procedimiento descripto son necesarias observaciones de dos especies moleculares, una ópticamente gruesa y otra ópticamente delgada, para determinar un valor para la densidad columnar. Sin embargo, es posible hacer una estimación de la densidad columnar de $\mathrm{H}_{2}$ cuando se tienen datos de la línea de ${ }^{12} \mathrm{CO}$ únicamente a través del siguiente producto 


$$
N_{H_{2}}=X W_{C O}
$$

donde $W_{C O}$ es la intensidad integrada de ${ }^{12} \mathrm{CO}$

$$
W_{C O}=\int_{v_{1}}^{v_{2}} \mathrm{~T}_{m b} \mathrm{~d} v
$$

con $v_{1}$ y $v_{2}$ correspondientes al rango de velocidad en que se integra la emisión.

El parámetro $X$ es un factor empírico aproximadamente constante para nubes moleculares galácticas y toma el valor $X=1.9 \times 10^{20} \mathrm{~cm}^{-2}\left(\mathrm{~K} \mathrm{~km} \mathrm{~s}^{-1}\right)^{-1}$ (Strong \& Mattox 1996; Bolatto et al. 2013). Dado que fue obtenido para la línea de la transición $J=1-0$, cuando se utilizan las líneas $\mathrm{J}=2-1$ y $\mathrm{J}=3-2$, debe dividirse por 0.8 y 0.7 , respectivamente, a fin de tener en cuenta la diferente población molecular (Azimlu et al., 2009; Oka et al., 2012).

\section{Masa molecular}

Una vez que se conoce la densidad columnar de Hidrógeno molecular $\left(N_{H_{2}}\right)$ se puede obtener la masa de $\mathrm{H}_{2}$ de la nube molecular, siempre que se conozca la distancia y se pueda medir el ángulo sólido que subtiende la nube de $\mathrm{CO}, \Omega_{C O}$. Entonces,

$$
M_{H_{2}}=m_{H_{2}} N_{H_{2}} \Omega_{C O} d^{2}
$$

donde $m_{H_{2}}$ es igual a $\mu m_{H}$. En esta expresión $m_{H}$ es la masa del átomo de Hidrógeno $\left(=1.67 \times 10^{-24} g\right)$ y $\mu$ es el peso molecular medio, $\mu=2.8$. Este último valor incluye una abundancia de $25 \%$ de Helio (por masa) (Yamaguchi et al. 1999, Miettinen 2012).

A los fines prácticos del cálculo, $M_{H_{2}}$ se obtiene de un valor promedio de $N_{H_{2}}$ sobre la región molecular, y $\Omega_{C O}$ como el ángulo sólido de la estructura de $\mathrm{CO}$ sobre el plano del cielo (que se puede calcular a partir de la cantidad de pixeles ocupados por la fuente y el tamaño angular de los mismos), de esta forma se obtiene

$$
\left[\frac{M_{H_{2}}}{M_{\odot}}\right]=C\left[\frac{N_{H_{2}}}{c m^{-2}}\right]\left[\frac{\Omega_{C O}}{s t r}\right]\left[\frac{d^{2}}{k p c}\right]
$$

donde $C=5.17 \times 10^{-25}$.

\section{Densidad ambiental}

Conociendo la masa total de la región y el tamaño de la misma y considerando que la nube posee una geometría esférica, se puede obtener la densidad ambiental a partir de la siguiente ecuación

$$
n_{H_{2}}=\frac{M_{H_{2}}}{\mu m_{H} \frac{4}{3} \pi r^{3}}
$$

donde $M_{H_{2}}$ es la masa calculada a partir de la ecuación 2.38 y $r$ es el radio medio de la región o radio efectivo ${ }^{(1)}$.

\section{Masa del virial}

La masa del virial se obtiene a partir de la siguiente expresión (MacLaren et al., 1988).

$$
\left[\frac{M_{V I R}}{M_{\odot}}\right]=k\left[\frac{r}{p c}\right]\left[\frac{\Delta \mathrm{v}^{2}}{k m s^{-1}}\right]
$$

\footnotetext{
${ }^{(1)}$ El radio efectivo de una región se define como el radio de un círculo cuya área sea igual al área de la región considerada $\mathrm{r}=\sqrt{\text { Area } / \pi}$
} 
donde $r$ y $\Delta \mathrm{v}$ son el radio de la región y el ancho a potencia mitad del perfil de una línea ópticamente delgada y $k$ es un coeficiente que depende de la distribución de densidad asumida, siendo $k=210$ para una densidad constante, $k=190$ para $n \propto 1 / r$, y $k=126$ para $n \propto 1 / r^{2}$.

La masa del virial es proporcional al ancho de la línea $(\Delta \mathrm{v})$ y por lo tanto a la energía cinética de las partículas de la nube. Calcular la masa de virial es útil para determinar la estabilidad gravitacional de una nube o grumo, y es un parámetro importante a la hora de evaluar si hay formación estelar.

- Si $M_{V I R} / M_{H_{2}}<1$ la nube no tiene suficiente energía cinética como para frenar el colapso gravitacional.

- Si $M_{V I R} / M_{H_{2}}>1$ la energía cinética de la nube es mayor que la gravitacional y por lo tanto no puede colapsar.

\section{Cálculo de errores}

La principal fuente de incerteza en el cálculo de los parámetros de las nubes moleculares es el error en la distancia. A lo largo de esta tesis se calculan las distancias a partir del modelo de rotación circular galáctica, y sus errores surgen de considerar una dispersión en velocidad de $6 \mathrm{~km} \mathrm{~s}^{-1}$, que es la dispersion en velocidades del HI en el MIE (Kellermann \& Verschuur, 1988).

En aquellos parámetros que dependen de modo lineal con la distancia (e. g. tamaño lineal de las fuentes, masa del virial) los errores son porcentualmente iguales al error en distancia; en aquellos que dependen de la distancia al cuadrado (e.g. masa) son el doble que el error en distancia; y para aquellos que dependen de la distancia al cubo (e.g. densidad ambiental) son el triple. Estos errores son una cota mínima, ya que existen otras fuentes de error tales como los errores en calibración, en las mediciones de los parámetros observacionales y en los ajustes realizados.

Los errores en calibración de los datos observados con el telescopio APEX $\left(\mathrm{T}_{m b}\right)$ son de aproximadamente un $10 \%$. Este error afecta a todos los parámetros determinados a partir de los datos moleculares. Sin embargo, como suele hacerse en la literatura, el error en calibración no será considerado y solamente los parámetros que dependan de la distancia serán presentados con errores.

\subsubsection{Moléculas trazadoras de alta densidad}

Ya se ha mencionado la existencia de ciertas moléculas que son trazadoras de alta densidad, es decir, que sólo son capaces de excitarse colisionalmente y emitir en regiones muy densas del medio. A continuación se describen brevemete los procesos de formación y destrucción de algunas de estas moléculas (Miettinen, 2014).

\section{$\mathrm{HCO}^{+}$y $^{13} \mathrm{CO}^{+}$}

En nubes moleculares densas, $\mathrm{HCO}^{+}$se forma principalmente a través de la reacción $\mathrm{H}_{3}^{+}$ $+\mathrm{CO} \rightarrow \mathrm{HCO}^{+}+\mathrm{H}_{2}$ (e.g. Herbst \& Klemperer, 1973). La abundancia de esta molécula puede aumentar en regiones en donde se generen choques, debido a, por ejemplo, la presencia de flujos provenientes de YSOs inmersos en el gas denso. Cuando los choques calientan el gas y producen radiación UV a través de emisión Ly- $\alpha$, los mantos helados de los granos de polvo se evaporan y la abundancia de $\mathrm{HCO}^{+}$aumenta debido a la evaporación de $\mathrm{CO}$ y $\mathrm{H}_{2} \mathrm{O}$. Esta última molécula puede formar $\mathrm{HCO}^{+}$a partir de la reacción $\mathrm{C}^{+}+\mathrm{H}_{2} \mathrm{O} \rightarrow \mathrm{HCO}^{+}+$ 


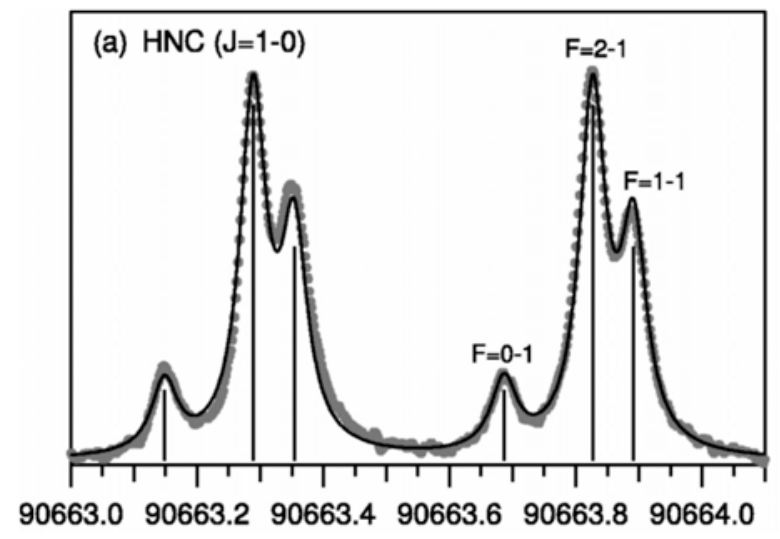

Figura 2.2: Estructura hiperfina de la molécula HNC(1-0). Imagen tomada de Bechtel et al. (2006)

$\mathrm{H}$ (Rawlings et al., 2000, 2004). La destrucción del $\mathrm{HCO}^{+}$es causada principalmente por la recombinación con electrones, $\mathrm{HCO}^{+}+\mathrm{e}^{-} \rightarrow \mathrm{CO}+\mathrm{H}$.

$\mathrm{HCO}^{+}$suele mostrar signos de infalls y alas de outflows (Rawlings et al., 2004; Fuller et al., 2005). Los outflows de YSOs inmersos en gas denso originan choques que aumentan la abundancia de $\mathrm{HCO}^{+}$, transformando a esta molécula en una herramienta útil para estudiar este tipo de fenómenos.

\section{$\mathrm{HCN}, \mathrm{HNC}$ y $\mathrm{HN}^{13} \mathrm{C}$}

Los modelos químicos sugieren que la molécula HCN y su isómero geométrico metaestable $\mathrm{HNC}$, son formados principalmente a través de la reacción $\mathrm{HCNH}^{+}+\mathrm{e}^{-} \rightarrow \mathrm{HCN}+\mathrm{H}$ o $\mathrm{HNC}$ $+\mathrm{H}$ (e.g. Herbst, 1978). El cociente $\mathrm{HNC} / \mathrm{HCN}$ predicho es en este caso 0.9, cercano a la unidad. La formación de $\mathrm{HNC}$ puede también tener lugar a partir de las reacciones $\mathrm{H}_{2} \mathrm{CN}^{+}$ $+\mathrm{e}^{-}$ó $\mathrm{H}_{2} \mathrm{NC}^{+}+\mathrm{e}^{-} \rightarrow \mathrm{HNC}+\mathrm{H}$ (Pearson \& Schaefer, 1974; Allen et al., 1980). Como resultado de esta reacción adicional, el cociente $\mathrm{HNC} / \mathrm{HCN}$ podría superar la unidad.

Otros procesos que forman $\mathrm{HCN}$ y $\mathrm{HNC}$ son las reacciones neutro-neutro $\mathrm{CH}_{2}+\mathrm{N} \rightarrow$ $\mathrm{HCN}+\mathrm{H}$ y $\mathrm{NH}_{2}+\mathrm{C} \rightarrow \mathrm{HNC}+\mathrm{H}$ (Herbst et al., 2000).

En nubes densas y PDRs, HCN puede ser fotodisociado en CN, ya sea directamente o a través de fotodisociación inducida por rayos cósmicos (Boger \& Sternberg, 2005). Otro proceso de destrucción de $\mathrm{HCN}$ en nubes densas es $\mathrm{HCN}+\mathrm{H}^{+} \rightarrow \mathrm{HCN}^{+}+\mathrm{H}$. y $\mathrm{HCN}+$ $\mathrm{HCO}^{+} \rightarrow \mathrm{H}_{2} \mathrm{CN}^{+}+\mathrm{CO}$ (Boger \& Sternberg, 2005). La molécula de HNC puede ser destruida a través de reacciones con átomos de Hidrógeno y Oxígeno, $\mathrm{HNC}+\mathrm{H} \rightarrow \mathrm{HCN}+\mathrm{H}$ y $\mathrm{HNC}$ $+\mathrm{O} \rightarrow \mathrm{NH}+\mathrm{CO}$ (Schilke et al., 1992; Talbi et al., 1996). HNC también puede convertirse en $\mathrm{HCN}$ a partir de la reacción $\mathrm{HNC}+\mathrm{H}^{+} \rightarrow \mathrm{HCN}+\mathrm{H}^{+}$(Herbst et al., 2000).

$\mathrm{HNC}$ es especialmente preponderante en el gas frío y se usa para trazar gas denso en nubes moleculares.

La molécula HNC en su transición (1-0), centrada en un frecuencia $\nu=90.663564 \mathrm{GHz}$, posee estructura hiperfina que consta de tres componentes (ver Figura 2.2). La estructura hiperfina de una molécula está dada por la interacción del spin del núcleo I (que es la resultante del momento de spin de los protones y neutrones) y el momento angular de los electrones J, y origina que una línea proveniente de una dada transición se divida en más de una componente. 


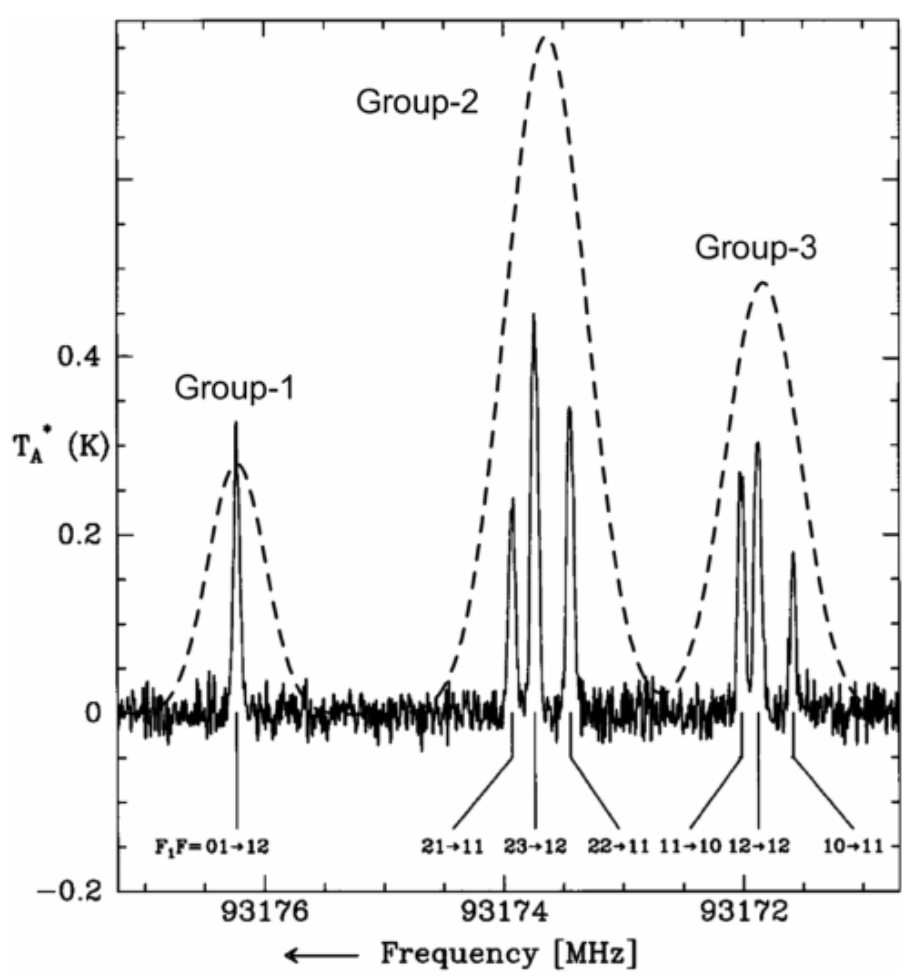

Figura 2.3: Estructura hiperfina de $\mathrm{N}_{2} \mathrm{H}^{+}$. Imagen tomada de Purcell et al. (2009).

\section{$\mathbf{C}_{2} \mathbf{H}$}

Se cree que el origen de la molécula $\mathrm{C}_{2} \mathrm{H}$ en las regiones de fotodisociación del medio interestelar, se encuentra en la fotodisociación del acetileno $\left(\mathrm{C}_{2} \mathrm{H}^{+}\right): \mathrm{C}_{2} \mathrm{H}^{+}+h \nu \rightarrow \mathrm{C}_{2} \mathrm{H}$ $+\mathrm{H}$ (e.g. Fuente et al., 1993). La reacción neutro-neutro $\mathrm{CH}_{2}+\mathrm{C} \rightarrow \mathrm{C}_{2} \mathrm{H}+\mathrm{H}$ puede también producir $\mathrm{C}_{2} \mathrm{H}$ en donde el átomo de Carbono precursor es formado a partir de la fotodisociación del CO (e.g. Turner et al., 2000). La recombinación disociativa de los iones $\mathrm{CH}^{+}$(Rimmer et al., 2012), $\mathrm{C}_{2} \mathrm{H}_{2}^{+}$and $\mathrm{C}_{2} \mathrm{H}_{3}^{+}$también produce moléculas de etileno en las nubes moleculares densas ( $\mathrm{Mul} \&$ McGowan, 1980). $\mathrm{C}_{2} \mathrm{H}$ es también destruida a través de las reacciones $\mathrm{C}_{2} \mathrm{H}+\mathrm{O} \rightarrow \mathrm{CO}+\mathrm{CH}$ y $\mathrm{C}_{2} \mathrm{H}+\mathrm{C}^{+} \rightarrow \mathrm{C}_{3}^{+}+\mathrm{H}$ (Watt et al., 1988).

\section{$\mathbf{N}_{2} \mathbf{H}^{+}$}

$\mathrm{N}_{2} \mathrm{H}^{+}$es una molécula más resistente a la congelación sobre los granos que las especies que contienen Carbono, por lo que permanece en la fase gaseosa a mayores densidades que otras moléculas. Esto se debe a la alta volatilidad de su molécula precursora $\mathrm{N}_{2}$ que permite varios mecanismos de desorción para mantener una abundancia significativa de $\mathrm{N}_{2}$, y por lo tanto de $\mathrm{N}_{2} \mathrm{H}^{+}$, en la fase gaseosa a altas densidades (Bergin et al., 2001). Esta molécula posee siete componentes hiperfinas, seis de ellas en dos grupos de tres líneas cada uno más una componente aislada (ver Figura 2.3).

La estructura hiperfina de $\mathrm{N}_{2} \mathrm{H}^{+}$también permite una determinación de la profundidad óptica en la línea, por lo que puede realizarse una comprobación interna de la delgadez de la línea aislada $\mathrm{N}_{2} \mathrm{H}^{+}$(101-012). Los anchos de línea $\mathrm{N}_{2} \mathrm{H}^{+}$y los componentes hiperfinos lo convierten en una sonda ideal de la velocidad sistémica hacia los objetos estelares jóvenes. 


\section{Determinación de parámetros a través de $\mathrm{N}_{2} \mathbf{H}^{+}$.}

Utilizando las componentes hiperfinas de $\mathrm{N}_{2} \mathrm{H}^{+}$puede calcularse la profundidad óptica y densidad columnar de grumos moleculares densos. Asumiendo que los anchos de todas las componentes hiperfinas son iguales, las intensidades integradas de los tres grupos deben cumplir la relación 1:5:3 bajo condiciones ópticamente delgadas. Por lo tanto la profundidad óptica puede ser derivada a partir del cociente de las intensidades integradas a partir de la siguiente ecuación:

$$
\frac{\int T_{m b, 1} d \mathrm{v}}{\int T_{m b, 2} d \mathrm{v}}=\frac{1-\exp \left(-\tau_{1}\right)}{1-\exp \left(-\tau_{2}\right)}=\frac{1-\exp \left(-\tau_{1}\right)}{1-\exp \left(-a \tau_{1}\right)}
$$

donde $\tau_{1}$ y $\tau_{2}$ son las profundidades ópticas para dos de los tres grupos y a es el cociente esperado de $\tau_{2} / \tau_{1}$ bajo condiciones ópticamente delgadas.

Caselli et al. (1995) reportaron una excitación anómala en las componentes $\mathrm{F}_{1}, \mathrm{~F}=1,0 \rightarrow 1,1$ y $1,2 \rightarrow 1,2$, pertenecientes al grupo 3 . Por esta razón para determinar profundidades ópticas se utilizan cocientes de intensidades de los grupos 1 y 2 .

A partir de la expresión de $\mathrm{T}_{m b}$ dada por Rohlfs \& Wilson (2004), la temperatura de excitación y la densidad columnar para $\mathrm{N}_{2} \mathrm{H}^{+}$pueden ser calculadas usando las siguientes expresiones de Chen et al. (2013):

$$
\begin{gathered}
T_{e x c}=4.47 / \ln \left[1+\left(\frac{T_{m b}}{4.47(1-\exp (-\tau))}+0.236\right)^{-1}\right] \\
N\left(N_{2} H^{+}\right)=\frac{3 k W}{8 \pi^{3} \nu S \mu^{2}}\left(\frac{T_{e x c}}{T_{e x c}-T_{b g}}\right)\left(\frac{\tau}{1-\exp (-\tau)}\right) Q\left(T_{e x c}\right) \exp \left(E_{u} / k T_{e x c}\right)
\end{gathered}
$$

En esta última expresión $k$ es la constante de Boltzmann, $W$ es la intensidad integrada de la línea observada, $\nu$ es la frecuencia de la transición, y $S \mu^{2}$ es el producto entre el valor intrínseco de la fuerza de línea $S$ (o factor de Hönl-London) en $\mathrm{erg}^{\mathrm{cm}} \mathrm{cm}^{3} \mathrm{D}^{-2}$ y el cuadrado del dipolo eléctrico $\mu$ en $\mathrm{D}^{2(2)}$. $\mathrm{T}_{\text {exc }} \mathrm{y}_{b g}$ son la temperatura de excitación y la temperatura de fondo cósmico de radiación, respectivamente. $E_{u} / k$ es la energía del nivel superior de la transición en Kelvin, $Q\left(T_{\text {exc }}\right)$ es la función de partición a la temperatura $\mathrm{T}_{\text {exc }} \mathrm{y} \tau$ es la profundidad óptica.

\subsection{Emisión térmica del polvo interestelar}

El proceso más importante de emisión en el continuo infrarrojo en las regiones de formación estelar y en el MIE en general, es la radiación térmica de los granos de polvo. Las pequeñas partículas de polvo que existen en las nubes moleculares absorben de manera muy eficiente la radiación de longitud de onda más corta que su tamaño y, cuando hay equilibrio entre calentamiento y enfriamiento, la reemiten como emisión en el continuo, principalmente a longitudes de onda en el infrarrojo. El espectro resultante es muy parecido al de un cuerpo negro caracterizado por una cierta temperatura $T_{d}$ (temperatura del polvo). Así, el flujo observado a una frecuencia $\nu$, vendrá dado por:

$$
S_{\nu}=B_{\nu}\left(\mathrm{T}_{d}\right)\left(1-e^{-\tau_{\nu}}\right) \Omega_{S}
$$

donde $B_{\nu}\left(\mathrm{T}_{d}\right)$ es la función planckiana a una temperatura $\mathrm{T}_{d}, \Omega_{S}$ es el ángulo sólido que subtiende a la fuente y $\tau_{\nu}$ es la profundidad óptica. Esta última está dada por

\footnotetext{
${ }^{(2)}$ El símbolo D corresponde al Debye, una unidad de momento dipolar eléctrico y equivale a $10^{-18}$ statC $\mathrm{cm}$.
} 


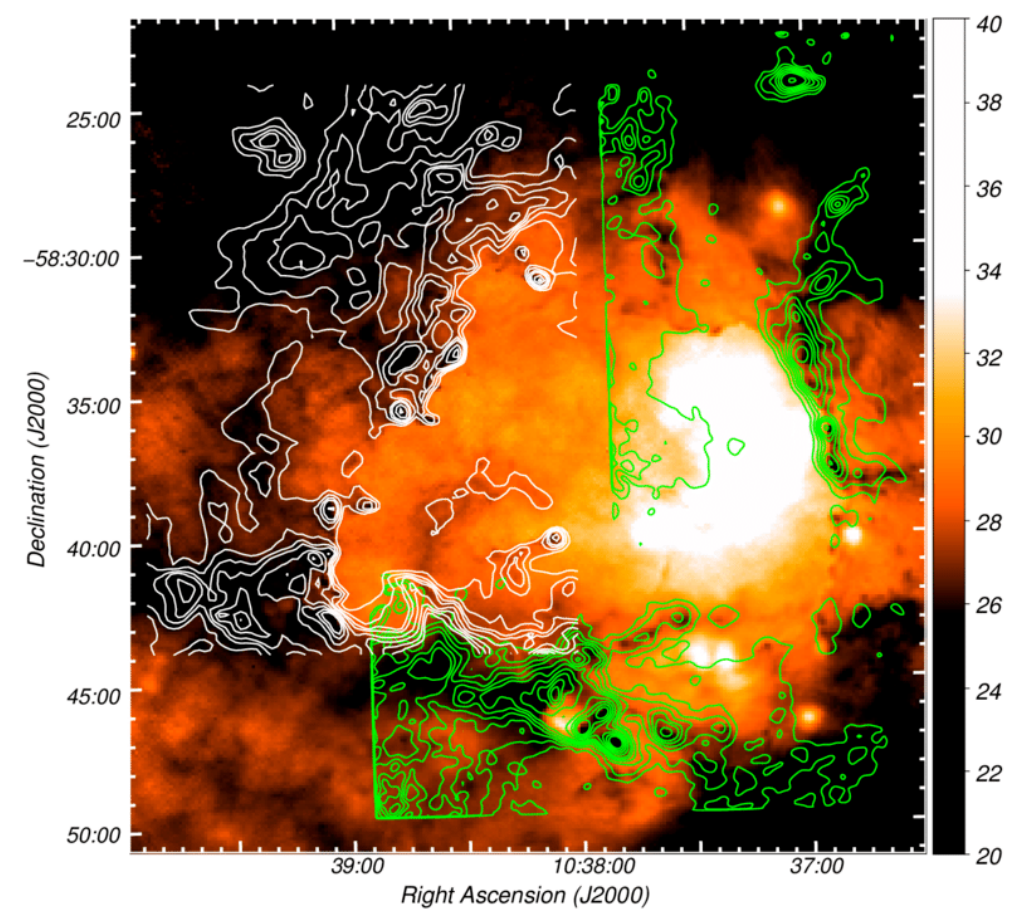

Figura 2.4: Mapa de temperatura de polvo de la región Hir Gum 31. Los contornos verdes muestran la emisión de continuo IR a $870 \mu \mathrm{m}$ y los blancos a $250 \mu \mathrm{m}$. Imagen tomada de Duronea et al. (2015)

$$
\tau_{\nu}=\kappa_{\nu} \int \rho \mathrm{d} l
$$

donde $\kappa_{\nu}$ es el coeficiente de absorción por unidad de densidad de masa y la integral es la densidad columnar de polvo. Se ha encontrado que $\kappa_{\nu} \propto \nu^{\beta}$, donde $\beta$ es el índice de emisividad del polvo, cuyos valores se determinaron observacionalmente entre $\sim 1$ y $\sim 3$ (Preibisch et al., 2012). Los valores que puede tomar $\kappa_{\nu}$ dependen también del tipo de polvo y fueron calculados por Ossenkopf \& Henning (1994).

A longitudes de onda submilimétricas o más largas la emisión es, en general, ópticamente delgada, por lo que se puede aproximar $\left(1-e^{-\tau_{\nu}}\right) \sim \tau_{\nu}$. Además, se puede usar la aproximación de Rayleigh-Jeans y expresar la función planckiana como $B_{\nu}(T)=\left(2 k \nu^{2} / c^{2}\right) T_{d}$, por lo que el flujo será

$$
S_{\nu}=\frac{2 k \nu^{2}}{c^{2}} \mathrm{~T}_{d} \tau_{\nu} \Omega_{S}
$$

Reemplazando $\tau_{\nu}$ por 2.45 y $\Omega_{S}$ por $A / D^{2}$, donde $A$ es el área y $D$ la distancia a la fuente

$$
S_{\nu}=\frac{2 k \nu^{2}}{c^{2}} \mathrm{~T}_{d} \frac{A}{D^{2}} \kappa_{\nu} \int \rho \mathrm{d} l=\frac{2 k \nu^{2}}{c^{2}} \mathrm{~T}_{d} \frac{M_{d}}{D^{2}} \kappa_{\nu}
$$

donde $\mathrm{M}_{d}$ es la masa de polvo. De aquí es posible despejar una expresión que, conociendo la tempetarura $\mathrm{T}_{d}$, permite calcular la masa del polvo (Hildebrand, 1983)

$$
M_{d}=\frac{S_{\nu} D^{2}}{\kappa_{\nu} B_{\nu}\left(T_{d}\right)}
$$


Tabla 2.4: Factores de conversión de los modelos para calcular la masa de gas ionizado y densidad electrónica de una región HıI

\begin{tabular}{lcc}
\hline \hline & $u_{1}$ & $u_{2}$ \\
\hline Modelo I & 0.775 & 1.291 \\
Modelo II & 0.857 & 1.167 \\
Modelo III & 0.911 & 3.106 \\
\hline \hline
\end{tabular}

Si se desea obtener la masa total (polvo+gas) hay que multiplicarla por el valor del cociente gas/polvo, que fue estimado por Draine et al. (2007) para la Vía Láctea a partir de la diferencia entre las abundancias interestelares totales (que se consideran equivalentes a las abundancias solares) y las abundancias observadas en la fase gaseosa en nubes interestelares difusas. Este cociente es una de las mayores fuentes de incerteza en el cálculo de las masas totales a partir de la emisión IR.

A partir de imágenes infrarrojas es posible construir mapas de temperatura de polvo, como la inversa de un mapa de cociente de imágenes a dos frecuencias distintas, $\mathrm{T}_{d}=f(\mathrm{~T})^{-1}$ (Preibisch et al., 2012; Ohlendorf et al., 2013), donde

$$
f(T)=\frac{S_{\nu_{1}}}{S_{\nu_{2}}}=\frac{B_{\nu}\left(\nu_{1}, T\right)}{B_{\nu}\left(\nu_{2}, T\right)}=\left(\frac{\nu_{1}}{\nu_{2}}\right)^{\beta}
$$

En la Figura 2.4 se muestra un mapa de temperatura del polvo en la región HiI Gum 31 (Duronea et al., 2015), obtenido a partir del cociente de las imágenes de Herschel a 70 y 160 $\mu \mathrm{m}$. Allí se puede apreciar cómo la temperatura es mayor en el interior de la región HiI y menor en las regiones en las que coincide con la emisión más intensa en el lejano IR.

\subsection{Emisión en el continuo de radio}

Las regiones HiI son ionizadas por el campo radiativo de las estrellas cercanas, que emiten radiación originada por procesos libre-libre que se detecta en el continuo de radio. Esta emisión permite calcular algunos parámetros físicos de las regiones HiI, tales como la densidad electrónica $\left(n_{e}\right)$ y la masa de gas ionizado $\left(\mathrm{M}_{i o n}\right)$. Esta emisión libre-libre se detecta a frecuencias mayores que $1 \mathrm{GHz}$, donde la región HiI es ópticamente delgada.

Para la determinación de estos parámetros es necesario usar un modelo geométrico para la región Hiı. Mezger \& Henderson (1967) propusieron tres modelos sencillos:

Modelo I: Densidad electrónica constante dentro de una esfera de diámetro aparente $\theta_{\text {esf }}$ y 0 fuera de ésta.

Modelo II: Densidad electrónica constante dentro de un cilindro de diámetro aparente $\theta_{c i l}$ cuyo eje de simetría es paralelo al eje de la visual y con largo igual al diámetro, y 0 fuera de éste.

Modelo III: Distribución exponencial decreciente con densidad máxima $\mathrm{N}_{0}$ y ancho a potencia mitad $\theta_{G}$.

Con estos modelos pueden obtenerse las siguientes expresiones para la densidad electrónica 


$$
\left(\frac{N_{e}}{c m^{-3}}\right)=u_{1} a^{1 / 2} 6.351 \times 10^{2}\left(\frac{T_{e}}{10^{4} \mathrm{~K}}\right)^{0.175}\left(\frac{\nu}{\mathrm{GHz}}\right)^{0.05}\left(\frac{S_{\nu}}{\mathrm{Jy}}\right)^{0.5}\left(\frac{d}{\mathrm{Kpc}}\right)^{-0.5}\left(\frac{\theta_{G}}{\prime}\right)^{-1.5}
$$

y para la masa de hidrógeno ionizado

$$
\left(\frac{M}{M_{\odot}}\right)=u_{2} a^{1 / 2} 0.3864\left(\frac{T_{e}}{10^{4} \mathrm{~K}}\right)^{0.175}\left(\frac{\nu}{\mathrm{GHz}}\right)^{0.05}\left(\frac{S_{\nu}}{\mathrm{Jy}}\right)^{0.5}\left(\frac{d}{\mathrm{Kpc}}\right)^{2.5}\left(\frac{\theta_{G}}{\prime}\right)^{1.5}
$$

donde $T_{e}$ es la temperatura electrónica, $\nu$ la frecuencia observada, $S_{\nu}$ el flujo observado a la frecuencia $\nu, d$ es la distancia y $\theta_{G}$ es el ancho a potencia mitad. $a$ es un factor que toma valores cercanos a la unidad y mide la desviación entre el valor de la profundidad óptica para la emisión libre-libre real y su aproximación. Estos valores dependen de la temperatura electrónica y de la frecuencia y se encuentran listados en las Tabla 6 del trabajo de Mezger \& Henderson (1967). $u_{1} y u_{2}$ dependen en particular del modelo elegido para la distribución de densidad de la región HII. Los valores de $u_{1}$ y $u_{2}$ para los modelos I, II y III se listan en la Tabla 2.4 (Mezger \& Henderson, 1967).

La Ecuación 2.51 considera solamente la masa originada en la ionización de HI. Este valor debe multiplicarse por 1.27 para considerar la contribución del He ionizado (25\% de He por masa, $10 \%$ por número de partículas de $\mathrm{He})$.

\subsection{Ajuste de distribuciones espectrales de energía}

La herramienta desarrollada por Robitaille et al. (2007) consiste en la comparación de una distribución espectral de energía (SED) observada con un conjunto de SEDs teóricas, generadas mediante el código de Whitney et al. (2003b). El procedimiento empleado selecciona el mejor modelo para una dada distribución espectral de energía en base a un criterio de minimización de residuos $\left(\chi^{2}\right)$, aplicado en una grilla de modelos de objetos estelares jóvenes presentada por Robitaille et al. (2006). Esta grilla consiste de 20000 modelos con simetría azimutal de transporte radiativo (Whitney et al., 2003a) de objetos estelares jóvenes. Cada uno de ellos están calculados, a su vez, para 10 ángulos de visión diferentes, lo que hace un total de 200000 modelos de SEDs, en el rango de longitudes de onda de 0.36-1000 $\mu \mathrm{m}$.

La grilla de modelos para objetos estelares jóvenes cubre un amplio rango de etapas evolutivas y masas: desde las protoestrellas embebidas hasta los objetos de clase III con discos tenues, ópticamente finos, y masas estelares entre $0.1 \mathrm{M}_{\odot}$ y $50 \mathrm{M}_{\odot}$.

Además, permite barrer un amplio rango de modelos físicamente aceptables en un espacio multiparamétrico de 16 variables. Los parámetros físicos y geométricos de cada protoestrella derivados de este análisis resultan confiables y permiten caracterizar o restringir los valores de los parámetros físicos y geométricos, no sólo de la estrella central sino también de las envolventes y de los discos que acompañan la formación de la nueva estrella.

Los parámetros del modelo de transporte radiativo pueden dividirse en tres grupos:

- Parámetros de la fuente central: masa estelar, radio y temperatura.

- Parámetros de la envolvente: tasa de acreción de la envolvente, radio externo de la envolvente, densidad dentro de la cavidad, ángulo de apertura de la cavidad (medido desde el eje de rotación hacia el radio externo de la envolvente). 
- Parámetros del disco: masa del disco, radios interno y externo del disco, tasa de acreción de masa del disco, escala de altura del disco, exponente de la densidad radial del disco, y exponente de la escala de altura del disco.

El decimoquinto parámetro es la densidad ambiente que rodea a la estrella joven.

Estos parámetros afectan la forma de la SED de manera diferente, dependiendo de la fase evolutiva y el rango de longitudes de onda de la fuente. Recíprocamente, la forma de una dada SED puede ser utilizada para restringir los valores posibles de un dado parámetro. Durante la etapa de la envolvente en colapso (objetos de clase I), los parámetros que mejor pueden determinarse mediante el modelado de las respectivas SEDs son los relacionados con la envolvente, es decir, la tasa acreción de masa de la envolvente y su masa, mientras que para los parámetros relacionados con el disco, sólo pueden obtenerse cotas menos precisas o rangos más grandes para los posibles valores. De manera análoga, durante la etapa siguiente (objetos de clase II), donde predomina la contribución del disco, los parámetros mejor determinados son los que se relacionan con el mismo.

La ventaja del modelado de SEDs a partir de una grilla con respecto a un único mejor modelo, consiste en que es posible determinar cuáles de los parámetros pueden ser acotados con precisión y cuáles no. Por ejemplo, si para cierto parámetro se obtiene un rango grande de modelos, entonces, ese parámetro no estará bien determinado.

Este método presenta algunas limitaciones que pueden provenir de: (1) los YSOs son objetos tridimensionales complejos con estructuras de densidad no muy bien axisimétricas, por lo que los modelos son incorrectos en comparación con las distribuciones de densidad real, pero aún se puede esperar que sean lo suficientemente similares a la realidad para proporcionar información interesante sobre esos objetos; (2) suele haber mezcla de fuentes y muchos objetos aparecen como un único YSO, pero a menudo son dos o más objetos. Si este es el caso, algunas de las propiedades generales, como la luminosidad o la masa de polvo, pueden estar lo suficientemente cerca de los valores reales, pero para otros parámetros más detallados los valores pueden no ser representativos; (3) incluso en el caso de un YSO aislado la variabilidad es una complicación adicional, ya que los datos con los que se suelen construir las SEDs pertenecen a diferentes relevamientos que se han realizado en diferentes años y pueden producir discontinuidades en las SEDs de una longitud de onda a otra (ver Robitaille, 2017). 


\section{Parte II}

\section{Observaciones}





\section{Capítulo 3}

\section{Observaciones}

\subsection{Radiotelescopios}

\subsubsection{Conceptos de instrumentación radioastronómica}

Las observaciones en longitudes de onda de radio se llevan a cabo utilizando radiotelescopios, cuya antena actúa como colectora de ondas de radio del modo análogo a como lo hacen las lentes o los espejos de los telescopios ópticos. Las señales pasan luego por un proceso de predetección donde se transforman en señales eléctricas en un receptor que las amplifica, filtra e integra. Finalmente son traducidas en señales legibles a un analizador digital.

\section{Camino de la señal}

Filtrado y amplificación

La radiación es recogida por la superficie de la antena y enviada hacia el subreflector localizado en el foco de la parábola. Desde allí se refleja hacia un punto de la parábola en donde se encuentran los receptores. Allí se selecciona mediante filtros la frecuencia deseada, y luego de su paso por un amplificador de bajo ruido se envía la señal resultante hacia un convertidor de frecuencia intermedia.

\section{Disminución de frecuencia}

Las señales de alta frecuencia, como las que se reciben en un radiotelescopio, se atenúan muy rápidamente al transmitirlas por un cable. Para evitar las grandes inestabilidades que sufriría el sistema receptor por trabajar con altas frecuencias y lograr una pérdida mínima es conveniente bajar la frecuencia de las señales.

Para esto se utiliza un conversor de frecuencia intermedia, que no es más que un mezclador que combina la señal original recibida por la antena con otra señal artificial y monocromática proporcionada por un oscilador local. Esta mezcla tiene como resultado la obtención de dos señales: una cuya frecuencia es la suma de la original más la del oscilador local y otra cuya frecuencia es la resta (en valor absoluto) de las mismas. Esta última señal, llamada de frecuencia intermedia, es la que se utiliza para ser propagada con las mínimas pérdidas, ya que proporciona exactamente la misma información que la señal inicial y es más fácil de procesar.

\section{Análisis final de la señal}

La señal obtenida con la nueva frecuencia intermedia es amplificada nuevamente y enviada a un medidor de potencias o a un espectrómetro para su análisis.

El medidor de potencia cuantifica la energía recibida por unidad de tiempo en el intervalo de frecuencias determinado por los filtros. Es el tipo de sistema de medida que se utiliza 


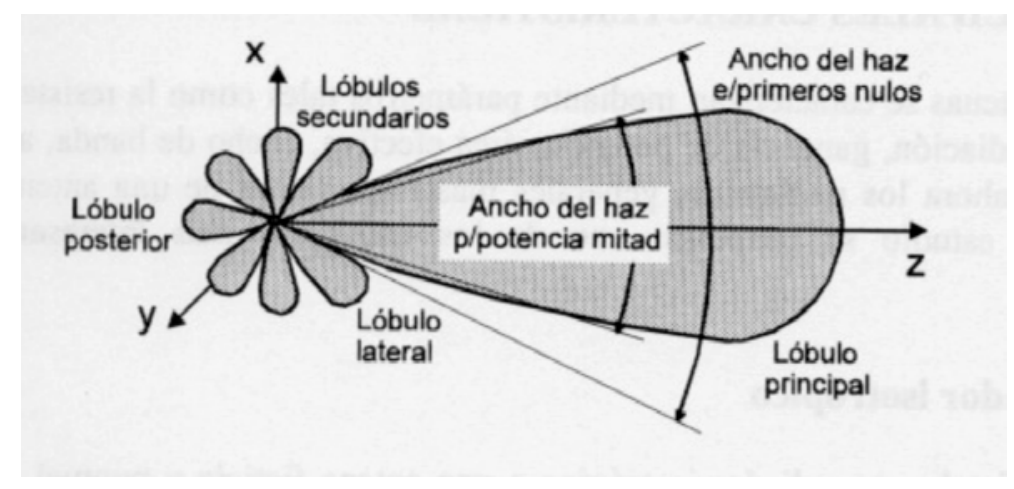

Figura 3.1: Patrón estándar de una antena.

para medir radiación continua. Cuanto mayor sea el ancho de banda en que se observa, más cantidad de energía se recibe y, por tanto, mejor será la relación señal a ruido. Si se amplía demasiado el ancho de banda (más de $1 \mathrm{GHz}$ ), puede que las variaciones de emisión de la fuente dentro de la banda observada comiencen a ser importantes.

El espectrómetro puede analizar cómo varía la emisión en función de la frecuencia dentro del intervalo de frecuencias que estemos observando. Este tipo de equipos es útil para observar líneas de emisión, que sólo emiten en una zona de frecuencias muy estrecha.

\section{Parámetros de las antenas}

\section{Diagrama de antena}

Cuando se observa con un radiotelescopio se apunta la antena hacia aquel punto del cielo que se desea observar. Sin embargo, debido a la difracción de la radiación que llega al borde de la antena, también se detecta radiación proveniente de otros puntos del cielo. A pesar de que, desafortunadamente, es imposible evitar la entrada de esta radiación no deseada, las antenas están diseñadas de modo que la respuesta de las mismas sea mayor en la dirección en que la antena es apuntada.

El diagrama de difracción de la radiación está formado por los lóbulos primario y secundarios, y es llamado diagrama de antena. Para representar la respuesta de la antena a la radiación se utiliza el llamado diagrama polar. En él se representa la cantidad de radiación recibida por la antena procedente de cada zona del cielo, cuando la antena apunta a un punto determinado.

La dirección de apuntamiento será aquella en la que la radiación es máxima, y se llama lóbulo principal al margen angular en torno a la dirección de máxima radiación (ver Figura 3.1). El ángulo entre los puntos del lóbulo principal en los que la intensidad recibida es la mitad del máximo se llama ancho a potencia mitad (Half-Power Beam-Width, HPBW), y se suele definir como el tamaño del haz de la antena. Este depende de la longitud de onda de observación y del tamaño de la antena, y se puede calcular como HPBW=1.22 $\frac{\lambda}{D}$. El tamaño del haz determina el poder resolvente del telescopio, y será más estrecho cuanto mayor sea el diámetro de la antena o menor sea la longitud de onda observada. Los lóbulos secundarios son los otros máximos relativos, de valor inferior al principal, y apuntan en una dirección distinta de la de apuntamieto. Las antenas se construyen de manera que la radiación que entra por estos lóbulos secundarios (spillover) sea mínima.

Directividad y eficiencia del haz

A partir del diagrama de antena pueden definirse otros parámetros. Para ello se establece previamente el ángulo sólido del haz, como la integral del diagrama de la antena a lo largo 
de todas las direcciones del cielo.

$$
\Omega_{a}=\iint_{4 \pi} P d \Omega
$$

Si en lugar de integrar en todo el cielo se integra en las direcciones ocupadas por el lóbulo principal, se tiene el ángulo sólido del lóbulo principal $\left(\Omega_{m}\right)$.

Se define la eficiencia del haz como $\eta=\frac{\Omega_{m}}{\Omega_{a}}$. Esta eficiencia varía entre 0 y 1 , y será mayor cuanto más concentrado esté el diagrama de la antena en el lóbulo principal.

Una antena tiene mayor directividad cuanto más estrecho es su lóbulo principal y más pequeños son sus lóbulos secundarios. Una antena ideal, totalmente directiva, sería aquella que sólo tuviese un lóbulo principal de ancho infinitesimal. En este caso detectaría el máximo de emisión cuando se apuntase a la fuente y cero cuando se desplazase cualquier distancia fuera de ella.

En función del ángulo sólido del haz, se define la directividad como $D=\frac{4 \pi}{\Omega_{a}}$.

Se llama eficiencia de apertura al cociente entre la área efectiva y el área física de la antena. Este valor varía entre 0 y 1, donde 1 es el valor que tendría una antena perfecta.

\section{Parámetros de la radiación}

\section{Densidad de flujo e intensidad}

Se define la densidad de flujo como la potencia total que se recibe de un cuerpo emisor, y se trata de una magnitud extensiva, mientras que la intensidad es la emisión por unidad de ángulo sólido y es una magnitud intensiva. Por lo tanto, la densidad de flujo será el resultado de integrar la intensidad en todo el ángulo sólido subtendido por el emisor. Si se tiene suficiente resolución angular como para ver al objeto emisor como una fuente extendida, se puede realizar un mapa de intensidades.

La intensidad emitida por un objeto es la potencia (W, energía por unidad de tiempo) emitida por unidad de área, frecuencia y ángulo sólido

$$
I_{\nu}=\frac{d \mathrm{~W}}{\cos (\theta) d \sigma d \nu d \Omega}
$$

La densidad de flujo es la integral de la intensidad a lo largo del ángulo sólido subtendido por la fuente

$$
S_{\nu}=\int I_{\nu} d \Omega
$$

Los subíndices $\nu$ se deben a que estos parámetros dependen de la frecuencia a la que se esté observado.

La intensidad recibida no depende de la distancia a la que esté el objeto pero sí la densidad de flujo, por lo que, esta última, no es una magnitud característica del objeto que emite, sino que se refiere a la energía que recibe el observador, y disminuye con el cuadrado de la distancia.

La unidad habitual de densidad de flujo en radioastronomía es el Jansky y se define como $1 \mathrm{Jy}=10^{-26} \mathrm{~W} \mathrm{~m}^{-2} \mathrm{~Hz}^{-1}=10^{-23} \mathrm{erg} \mathrm{s}^{-1} \mathrm{~cm}^{-2} \mathrm{~Hz}^{-1}$. Por consiguiente, la unidad básica de intensidad será el Jansky por esterorradián o por haz (beam).

Temperatura de brillo

En Radioastronomía se suele usar una escala equivalente para referirse a la intensidad de la emisión de un cuerpo. Esta escala equivalente es una escala de temperaturas. Se define así la temperatura de brillo del haz principal como la temperatura de un cuerpo negro que emite la misma intensidad que el objeto que se está estudiando dentro del haz principal. Esto permite expresar la intensidad en unidades de temperatura (K). Se puede usar esta escala 
de temperaturas para medir intensidades, porque la intensidad que un cuerpo negro emite a una determinada frecuencia, depende únicamente de su temperatura

$$
I_{\nu}=\frac{2 h \nu}{c} \frac{1}{e^{h \nu / k T}-1}
$$

La relación entre la intensidad y la temperatura es, en principio, compleja. Sin embargo, en el caso de las ondas de radio, en las que $h$ es mucho menor que $k T$, se puede hacer un desarrollo en serie de Taylor de la exponencial, que da como resultado

$$
I_{\nu}=\frac{2 h \nu^{2}}{c^{2}} k T
$$

Esta simplificación en el rango de ondas de radio se llama aproximación de Rayleigh-Jeans. Se puede ver que la intensidad emitida por el cuerpo negro es directamente proporcional a la temperatura, y, por esta razón, en la mayoría de las fórmulas en las que aparece la intensidad, ésta puede sustituirse simplemente por la temperatura de brillo.

Esta fórmula puede ilustrar la utilidad de usar la temperatura de brillo, en lugar de la intensidad en unidades de $\mathrm{Jy} \mathrm{sr}^{-1}$. Si el objeto que se está estudiando es aproximadamente un cuerpo negro, la temperatura de brillo es constante, independientemente de la frecuencia a la que se esté observando, pero la intensidad será muy diferente al cambiar de frecuencia. Si el objeto no es un cuerpo negro, la temperatura de brillo variará, pero siempre menos que la correspondiente intensidad. Por lo tanto, resulta más sencillo comparar las observaciones tomadas en diferentes frecuencias cuando se utiliza la escala de temperaturas de brillo.

\section{Temperatura de antena}

Cuando se realiza una observación con un radiotelescopio lo que se mide es una temperatura de antena. La temperatura de antena no solamente tiene la contribución de la fuente $\left(\mathrm{T}_{f}\right)$ sino también de la atmósfera $\left(\mathrm{T}_{a t m}\right)$ por la que la radiación de la fuente debe propagarse hasta llegar a la antena, y de del sistema receptor $\left(\mathrm{T}_{\text {rec }}\right)$, es decir, de la propia antena, cables, amplificadores, mezcladores, y cualquier otro equipo que se encuentre entre el lugar al que llega la radiación y el aparato final de medida. De modo que la temperatura de antena medida es

$$
T_{A}=T_{a t m}+T_{r e c}+T_{f} A_{a t m}
$$

$\mathrm{A}_{a t m}$ es un factor que representa la absorción de la radiacion proveniente de la fuente producida por la atmósfera terrestre. Su valor varía entre 0 (cuando la atmósfera absorbe toda la radiación que le llega) y 1 (cuando la atmósfera es totalmente transparente). Es por esta razón que los telescopios milimétricos y submilimétricos están ubicados a grandes alturas y en climas secos, en donde la cantidad de vapor de agua en la atmósfera es muy baja, ya que el vapor de agua absorbe la radiación en estos rangos de frecuencias.

Las contribuciones a la temperatura de antena que no provienen de la fuente pueden agruparse en un término que se denomina temperatura de sistema $\left(\mathrm{T}_{s i s}\right)$. Para obtener el valor de la temperatura de la fuente es necesario poder estimar el valor de la temperatura de sistema. Esto se hace realizando una observación fuera de la fuente pero en una posición cercana (de modo que la contribución de la atmósfera no cambie mucho) en la que se mide una temperatura $\mathrm{T}_{A}^{\prime}$ que no tiene la contribución de la fuente, y restándosela a $\mathrm{T}_{A}$. Entonces

$$
T_{A}-T_{A}^{\prime}=\left[T_{\text {sis }}+T_{f} A_{a t m}\right]-T_{\text {sis }}=T_{f} A_{a t m}
$$

Aunque la temperatura de sistema desaparece de la fórmula, su valor no es irrelevante en una observación, ya que la emisión del sistema se puede considerar como un ruido de fondo 
y determina directamente la calidad de la observación. Es por esta razón que el valor de $\mathrm{T}_{\text {sis }}$ debe minimizarse. Esto se hace manteniendo algunos de los elementos de los receptores refrigerados mediante helio o nitrógeno líquido, para disminuir su contribución a la temperatura de sistema. En cuanto a la contribución de la atmósfera a la temperatura de sistema, esta no depende de la temperatura ambiente sino de su opacidad, es decir, depende del tiempo meteorológico, aunque también puede atenuarse con técnicas in situ en tiempo real (e.g. chopper-wheel). En días despejados, la atmósfera es más transparente a las ondas de radio y, por tanto, la temperatura de sistema será menor.

\subsection{Telescopios utilizados}

En esta sección se hace una breve descripción de los telescopios utilizados para observar los datos de esta tesis.

\subsubsection{Telescopio NANTEN}

El radiotelescopio NANTEN ${ }^{(1)}$ es una colaboración entre institutos de investigación en Japón (Universidad de Nagoya y Osaka), Corea del Sur (Universidad Nacional de Seúl), Alemania (KOSMA, Universität zu Köln, Argelander-Institut Universität Bonn), Suiza (ETH Zürich), Universidad de Nueva Gales del Sur (UNSW) y Chile (Universidad de Chile).

El observatorio está ubicado a 4865 metros de altitud en Pampa la Bola, en el desierto de Atacama, Chile y se encuentra operativo desde mayo de 2006.

Equipado con un telescopio submilimétrico de 4 metros, NANTEN se utiliza para estudiar el cielo del sur en líneas espectrales moleculares y atómicas entre 110 y $880 \mathrm{GHz}$ (longitudes de onda de $2.6 \mathrm{~mm}$ a $350 \mu \mathrm{m}$ ) con una resolución angular entre $180^{\prime \prime}$ y $22^{\prime \prime}$.

Datos obtenidos con este telecopio se utilizan en el Capítulo 7.

\subsubsection{Telescopio APEX}

El radiotelescopio $\mathrm{APEX}^{(2)}$ (Atacama Pathfinder EXperiment), es una colaboración entre el Max Planck Institut für Radioastronomie, el Onsala Space Observatory (OSO) y el European Southern Observatory (ESO). Se encuentra ubicado a $5100 \mathrm{~m}$ de altura en el Llano de Chajnantor, en el desierto de Atacama al norte de Chile. Posee un diámetro de $12 \mathrm{~m}$ y cubre un rango espectral que va desde los 0.2 hasta los $1.3 \mathrm{~mm}$ (frecuencias entre 1500 y $210 \mathrm{GHz}$ ).

Todas las regiones estudiadas en esta tesis fueron observadas con este instrumento en diferentes fechas y utilizando diferentes modos de observación. Las observaciones fueron reducidas y analizadas utilizando el paquete CLASS (Continuum and Line Analysis Single-dish Software) del software GILDAS (Grenoble Image and Line Data Analysis Software).

\subsubsection{Telescopio Gemini}

El Observatorio Gemini está conformado por una cooperación internacional entre Estados Unidos, Canadá, Gran Bretaña, Brasil, Francia, Argentina, Australia, y Chile como país huésped. Consta de dos telescopios gemelos ópticos/infrarrojos de 8,1 m de diámetro ubicados en ambos hemisferios de la Tierra que en conjunto logran cubrir la totalidad del cielo durante todo el año.

Las observaciones presentes en esta tesis fueron tomadas con Gemini Sur, ubicado en el Cerro Pachón, a $80 \mathrm{~km}$ de La Serena, Chile, a una altura de $2700 \mathrm{~m}$.

\footnotetext{
${ }^{(1)}$ www.astro.uni-koeln.de/nanten2/

${ }^{(2)}$ www.apex-telescope.org/
} 


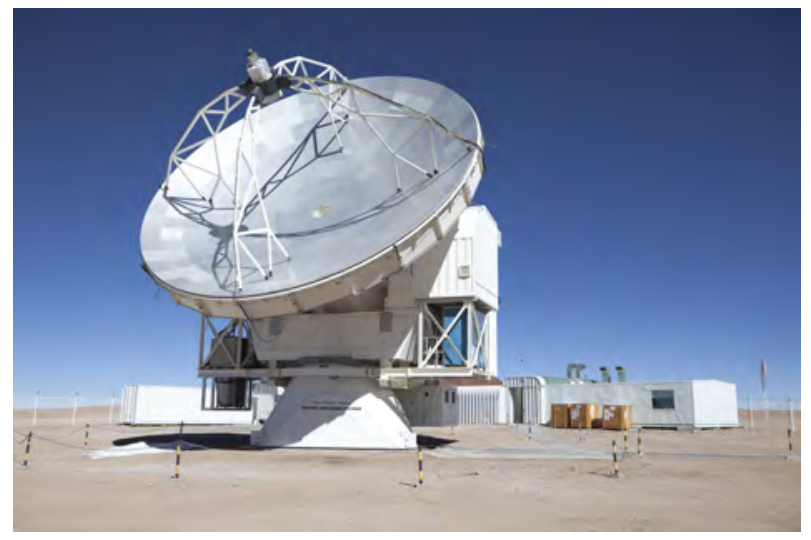

Figura 3.2: Telescopio APEX.

Se realizaron observaciones en el IR utilizando el instrumento Flamingos $2^{(3)}$ de Gemini Sur, haciendo uso de la red de alta resolución en la banda $\mathrm{K}$ combinada con una ranura de 4 pixeles de ancho, redundando en una resolución $\mathrm{R}=1500$.

Datos obtenidos con este telecopio se utilizan en el Capítulo 5.

\subsection{Bases de datos}

Las observaciones realizadas se complementaron con datos de relevamientos de acceso público a través de internet. Se utilizaron imágenes infrarojas y cubos de datos de observaciones de líneas moleclares. Los relevamientos utilizados se describen a continuación.

\subsubsection{Spitzer}

El telescopio espacial Spitzer (SST) es un observatorio espacial infrarrojo perteneciente a la NASA. El observatorio Spitzer consiste en un telescopio de 0.85 metros con tres instrumentos enfriados criogénicamente, capaces de tomar imágenes y espectros a longitudes de onda entre 3 a $180 \mu \mathrm{m}$.

Spitzer lleva a bordo los instrumentos IRAC (Infrared Array Camera) y MIPS (Multiband Infrared Photometer for Spitzer). Utilizando IRAC se llevó a cabo el relevamiento GLIM$\mathrm{PSE}^{(4)}$ (Galactic Legacy Infrared Mid-Plane Survey Extraordinaire, Benjamin et al., 2003). GLIMPSE es un relevamiento de la Via Láctea, que abarca los 360 grados en longitud y 2-4 grados de latitud. El telescopio fue apuntado a 111.000 posiciones diferentes en el cielo y las instantáneas fueron tomadas en cuatro longitudes de onda diferentes del infrarrojo $(3.6,4.5,5.8$, y $8 \mu \mathrm{m})$, creando un total de 444.000 imágenes. La resolución angular es de aproximadmente 1 !' 2 .

MIPSGAL $^{(5)}$ (Carey et al., 2005) es un relevamiento de 278 grados del plano galáctico llevado a cabo a través del fotómetro multibanda de infrarrojo (MIPS), instrumento a bordo del telescopio espacial Spitzer, con una resolución angular de $6^{\prime \prime}$. MIPSGAL complementa el relevamiento GLIMPSE (en 3-9 $\mu \mathrm{m}$ ) realizado utilizando el conjunto de cámaras infrarrojas (IRAC) de Spitzer. Los objetivos científicos principales de MIPSGAL son identificar y estudiar las estrellas masivas que se forman en el interior de la galaxia y estudiar la distribución del

\footnotetext{
${ }^{(3)}$ www.gemini.edu/sciops/instruments/flamingos2/

${ }^{(4)}$ www.astro.wisc.edu/sirtf/

${ }^{(5)}$ http://mipsgal.ipac.caltech.edu/a_mipsgal.html
} 
polvo interestelar de nuestra galaxia. El relevamiento MIPSGAL obtuvo alrededor de 400.000 imágenes a 24 y $70 \mu \mathrm{m}$.

La combinación de MIPSGAL y GLIMPSE es extremadamente sensible a los objetos protoestelares jóvenes.

\subsubsection{Herschel}

El Observatorio Espacial Herschel ${ }^{(6)}$ es una misión de la Agencia Espacial Europea. Con un espejo de un tamaño de $3.5 \mathrm{~m}$, es capaz de cubrir completamente el infrarrojo lejano y longitudes de onda submilimétricas. Posee tres instrumentos: Photodetector Array Camera and Spectrometer (PACS), Spectral and Photometric Imaging REceiver (SPIRE) y Heterodyne Instrument for the Far-Infrared (HIFI). PACS y SPIRE permiten observar en tres bandas diferentes cada uno dentro del infrarrojo lejano (de 60 a 210 y de 200 a $670 \mu \mathrm{m}$ respectivamente) y pueden funcionar como espectrómetros de baja resolución, mientras HIFI es un detector heterodino de un solo pixel que funciona como espectrómetro de muy alta resolución en siete bandas (de 157 a $625 \mu \mathrm{m}$ ).

Hi-GAL (Herschel infrared Galactic Plane Survey, Molinari et al., 2010) es un relevamiento del plano de la Vía Láctea en 5 bandas centradas en frecuencias de entre 60 y 600 $\mu \mathrm{m}$.

\subsubsection{ATLASGAL}

ATLASGAL (APEX Telescope Large Area Survey of the Galaxy, Schuller et al., 2009) consiste en un relevamiento del gas frío y denso que incluye regiones de formación estelar en la zona sur de la Vía Láctea y fue llevado a cabo usando el bolómetro LABOCA (LArge BOlometer Camera) del telescopio APEX. Los mapas de ATLASGAL cubren un área del cielo de 140 grados de largo y 3 de ancho.

Los datos de ATLASGAL complementan las observaciones realizadas con los satélites Planck y Herschel. La combinación de datos de Planck y APEX permitió detectar emisiones a lo largo de un área de cielo mayor y estimar la fracción de gas denso en la región interior de la galaxia. Los datos de ATLASGAL han sido usados también para crear un censo completo de nubes frías y masivas donde nuevas generaciones de estrellas se están formando.

\subsubsection{MALT90}

El relevamiento MALT90(7) (Millimetre Astronomy Legacy Team 90 GHz, Jackson et al., 2013, Foster et al., 2011, Foster et al., 2013) es un proyecto internacional cuyo objetivo es realizar mapas de líneas moleculares de más de 2000 grumos densos en el plano galáctico.

Utilizando la antena MOPRA se realizó un relevamiento simultáneo de 16 líneas moleculares con frecuencias cercanas a los $90 \mathrm{GHz}$, con una resolución angular de $40^{\prime \prime}$ y espectral de $0.1 \mathrm{~km} \mathrm{~s}^{-1}$, en dirección a grumos densos con emisión intensa en ATLASGAL. Las regiones observadas se encuentran en $+20^{\circ}>l>+3^{\circ}$ y $-3^{\circ}>l>-60^{\circ}$ y poseen un tamaño de $3^{\prime} \times$ $3^{\prime}$. Estas líneas moleculares permiten estudiar las condiciones físicas, el estado químico y el estado evolutivo de los grumos moleculares densos observados. Los grumos fueron seleccionados con el objetivo de abarcar toda la gama de estados evolutivos, dado que ATLASGAL contiene una mezcla heterogénea de núcleos cálidos y fríos que abarcan una amplia gama de masas.

\footnotetext{
${ }^{(6)}$ www.herschel.caltech.edu/

${ }^{(7)}$ http://malt90.bu.edu/
} 


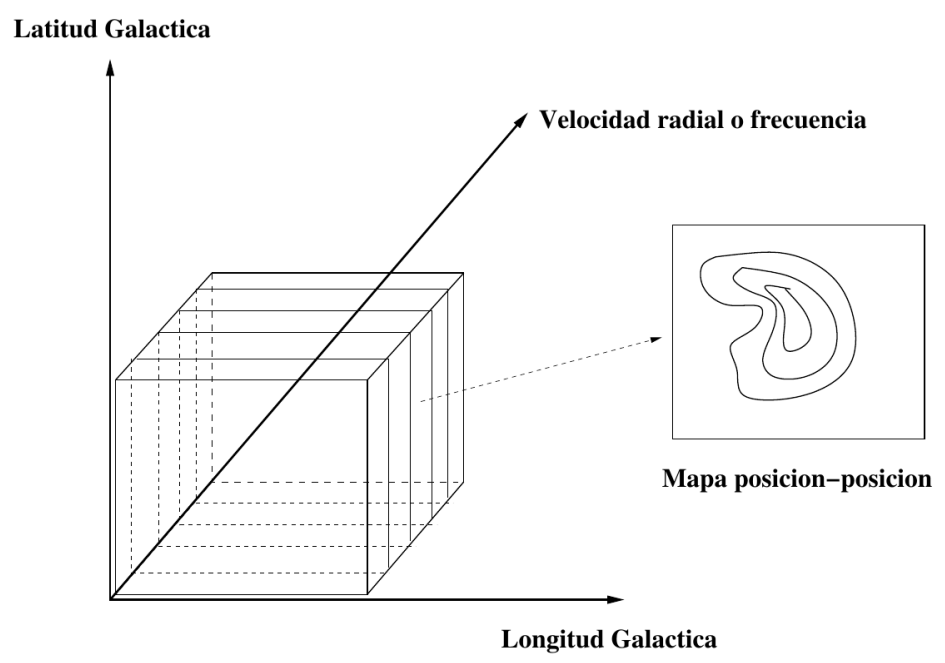

Figura 3.3: Esquema de un cubo de datos $(1, b, v)$ y de uno de los planos del mismo.

\subsubsection{SUMSS}

Sydney University Molonglo Sky Survey (SUMSS) es un relevamiento del cielo austral que se llevó a cabo con el Molonglo Observatory Synthesis Telescope (MOST) operando a $843 \mathrm{MHz}$ y abarca todo el cielo sur a $\delta>-30$ grados con $|b|>10^{\circ}$.

El relevamiento consta de 629 imágenes de $4.3 \times 4.3$ con una resolución de $45^{\prime \prime} \times 45^{\prime \prime} \times \operatorname{cosec}(\delta)$, y un brillo máximo de $6 \mathrm{mJy} /$ beam para declinaciones $\delta \leq 50^{\circ}$, y $10 \mathrm{mJy} /$ beam para $\delta>50^{\circ}$.

\subsection{Catálogos de fuentes puntuales infrarrojas}

En esta tesis se utilizaron los catálogos de fuentes puntuales infrarrojas 2MASS, MSX, Spitzer y WISE para identificar posibles objetos en formación. Una descripción de las características de estos catáologos fue dada en la Sección 1.4.5 del Capítulo 1.

\subsection{Reducción de datos y confeccción de cubos de datos}

Las observaciones fueron realizadas utilizando el Telescopio APEX en modo on source, en el que se observa sólo una posición en el cielo y se obtiene un único espectro en cada punto, y en modo On the Fly (OTF), en el que el telescopio realiza una observación de un área determinada del cielo. En ambos casos se requieren para realizar la observación las coordenadas de un punto de cielo sin emisión de la línea que se quiera observar (off). Este punto debe encontrase lo más cerca posible de la región a observar y se resta de las observaciones en cada punto. El modo OTF es útil para observación de líneas intensas en grandes áreas del cielo y permite construir cubos de datos que, a diferencia de lo que ocurre con las imágenes de continuo, proporcionan información de frecuecia o velocidad.

Un cubo de datos consiste en un arreglo de perfiles observados dispuestos de acuerdo a un conjunto de tres ejes coordenados. En un cubo de datos estándar, dos de los ejes corresponden a algún sistema de coordenadas astronómicas y el tercero a frecuencia o velocidad radial. En estas condiciones, el cubo es especificado como $(x, y, z)$ donde $x$ puede ser ascención reta $\alpha$ o latitud galáctica $l, y$ puede ser declinación $\delta$ o longitud galáctica $b$, y $z$ puede ser frecuencia 
$\nu$ o velocidad radial respecto del LSR $v$. En la Figura 3.3 se muestra un esquema de un cubo de datos $(l, b, v)$ y una imagen 'posición-posición' que puede extraerse de éste.

Los cubos también pueden rotarse de modo que sus ejes sean $(z, x, y)$ o $(z, y, x)$. Las imágenes extraídas de un cubo $(z, x, y)$ o $(z, y, x)$ son llamadas 'posición-velocidad', ya que la velocidad estará necesariamente en uno de sus ejes.

El proceso de reducción de los datos se llevó a cabo con el software CLASS, y consistió en los siguientes pasos:

- Identificación de los canales en que se encuentra la emisión.

- Ajuste de línea de base a cada espectro a partir de los canales en los que no hay emisión (se ajustaron polinomios de grado 0 y 1 ).

- Remuestreado de los espectros. Esto permite 'recortar' los espectros de modo de conservar solamente los canales en que se encuentra la emisión, centrar el espectro en un determinada velocidad y determinar el ancho de cada canal (siempre que sea mayor o igual a la resolución en velocidad observada).

- Armado de los cubos de datos a partir de los espectros.

- Unión de cubos de datos de regiones adyacentes observadas por separado para generar un solo cubo de mayor tamaño utilizando el paquete de procesamiento de datos MADR del Dominion Radio Astrophysical Observatory (DRAO) ${ }^{(8)}$.

En el caso de los espectros de ${ }^{13} \mathrm{CO}$ y $\mathrm{C}^{18} \mathrm{O}$, que al encontrarse muy cerca en frecuencia pueden observarse simultáneamente, fue necesario reducir el set de datos dos veces, modificando la velocidad y frecuencia de modo de generar un cubo para cada molécula.

\subsection{Regiones estudiadas en esta tesis}

En los siguientes capítulos se detallará el estudio realizado sobre una muestra de regiones de formación estelar, que permitirá testear los procesos de formación estelar en diferentes escenarios. Todas ellas han sido observadas utilizando el telescopio APEX, cuyos datos han sido reducidos utilizando el software CLASS y analizados usando CLASS, AIPS y ds9. Además para todas estas regiones se han utilizado datos disponibles de las bases de datos descriptas en la Sección 3.3.

En el Capítulo 4 se estudian tres regiones asociadas a YSOs que se encuentran sobre el borde la región Hiı Gum 31. En el Capítulo 5 se profundiza en el análisis de una de las regiones estudidas en el Capítulo 4 utilizando observaciones realizadas con el telescoipo Gemini. En el Capítulo 6 se estudia el gas molecular asociado a la IRDC SDC 341.232-0.268. En el Capítulo 7 se estudia el gas molecular asociado al complejo de burbujas infrarrojas S108-S109-S110S111. Finalmente en el Capítulo 8 se resumen los resultados obtenidos en los capítulos 4, 5, 6 y 7 , con la intención de arribar a una conclusión general que surge del trabajo realizado sobre las distintas regiones de formación estelar estudiadas.

${ }^{(8)}$ La unión de cubos fue llevado a cabo por la Dra. Laura Suad. 
Parte III

Resultados 



\section{Capítulo 4}

\section{La región Hiı Gum 31}

\subsection{Antecedentes de la región}

La región Hir Gum 31 está ubicada a $\sim 1^{\circ}$ al nor-oeste de la nebulosa de Carina, en $(l, b)$ $=(286.3,-0.2)$, a una distancia de $2.5 \pm 0.3 \mathrm{kpc}$ (Cappa et al., 2008) y tiene un diámetro de $\sim 15^{\prime}$ (10 pc a $\left.2.5 \mathrm{kpc}\right)$. Esta región está siendo excitada por estrellas OB, miembros del cúmulo abierto NGC 3324 .

El cúmulo estelar NGC 3324, que según determinaciones recientes se encontraría a una distancia de $2.5 \mathrm{kpc}$, está constituido principalmente por tres estrellas brillantes. Se trata de HD 92206, un sistema múltiple compuesto por las estrellas O6.5V HD 92206A y HD 92206B, y por la estrella O8.5V HD 92206C (Walborn, 1982). Además se encuentra la estrella supergigante A0 HD 92207, un objeto evolucionado muy luminoso y masivo con un fuerte viento estelar. La membresía de esta estrella al cúmulo no es completamente clara (Carraro et al., 2001).

Carraro et al. (2001) identificaron 25 candidatos a miembros de NGC 3324. Sin embargo, la función inicial de masa del campo de estrellas sugiere que debe haber 1500 estrellas de baja masa presentes $\left(0.1 \mathrm{M}_{\odot}<\mathrm{M}<2 \mathrm{M}_{\odot}\right)$, lo que implicaría que la mayoría de los miembros del cúmulo son aún desconocidos.

La Figura 4.1 muestra la región Gum 31 en óptico (ESO Wide Field Imager, WFI), mediano infrarrojo (Spitzer-GLIMPSE a $5.8 \mu \mathrm{m}$ y -MIPSGAL a $24 \mu \mathrm{m}$ ) y lejano infrarrojo (Herschel PACS a $70 \mu \mathrm{m}$ ). En la imagen de IRAC se distingue el cúmulo NGC 3324 cerca del centro de la región. Esta imagen y las de MIPS y PACS muestran una estructura en forma de arco que indica la presencia de polvo tibio, delineando la pared de la región HII. Además se pueden distinguir una serie de estructuras en forma de pilares que se extienden desde el borde hacia la parte interna de la región HII, sobre todo en el borde sur. La región de fotodisociación que delinea el borde de la región HII se observa claramente en la emisión a $5.8 \mu \mathrm{m}$ y se origina en la interacción de los fotones UV emitidos por el cúmulo con el gas molecular circundante. Como se mencionó en el capítulo 1, la emisión de los PAHs en 5.8 y $8 \mu \mathrm{m}$ delinea la PDR ubicada entre el gas ionizado y el gas molecular.

Tal como se muestra en la Figura 4.2 el gas molecular está concentrado en una estructura en forma de arco que se detecta a través de la emisión de ${ }^{12} \mathrm{CO}(1-0)$ en torno a la región HiI (Cappa et al., 2008). Sobre ésta se identifican zonas más densas que están determinadas por la presencia de emisión de $\mathrm{C}^{18} \mathrm{O}(1-0)$. Los datos de $\mathrm{CO}$ mostrados en esta figura tienen una resolución angular de 2!7 y corresponden al intervalo de velocidades $-27.2 \mathrm{a}-14.0 \mathrm{~km} \mathrm{~s}^{-1}$.

Numerosas fuentes infrarrojas encontradas en los catálogos de fuentes puntuales de los satélites IRAS (Infrared Astronomical Satellite) y MSX (Midcourse Space Experiment), y en el catálogo 2MASS ( Two Micron All Sky Survey), que aparecen proyectadas sobre la cáscara 

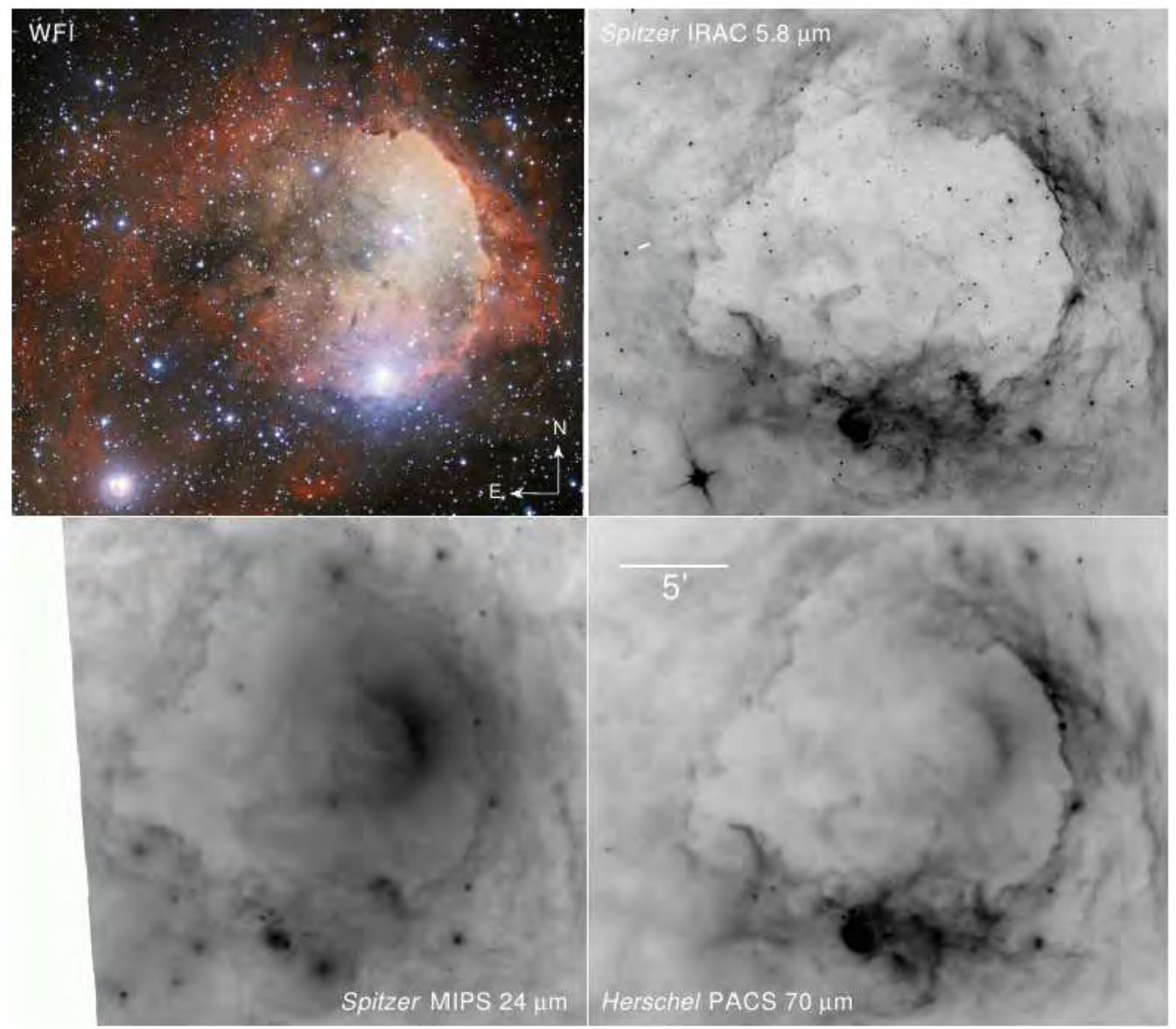

Figura 4.1: Región HiI Gum31. Arriba izquierda: Imagen óptica (ESO Wide Field Imager, WFI). Se muestra en azul la banda $V$, en amarillo la banda $R$, la emisión de O[III] $5007 \AA$ en verde y la emisión en $\mathrm{H} \alpha$ en rojo. Arriba derecha: Imagen de Spitzer-IRAC a $5.8 \mu \mathrm{m}$. Abajo izquierda: Imagen de Spitzer-MIPS a $24 \mu \mathrm{m}$. Abajo derecha: Imagen de Herschel-PACS a 70 $\mu \mathrm{m}$. Figura tomada de Ohlendorf et al. (2013). 


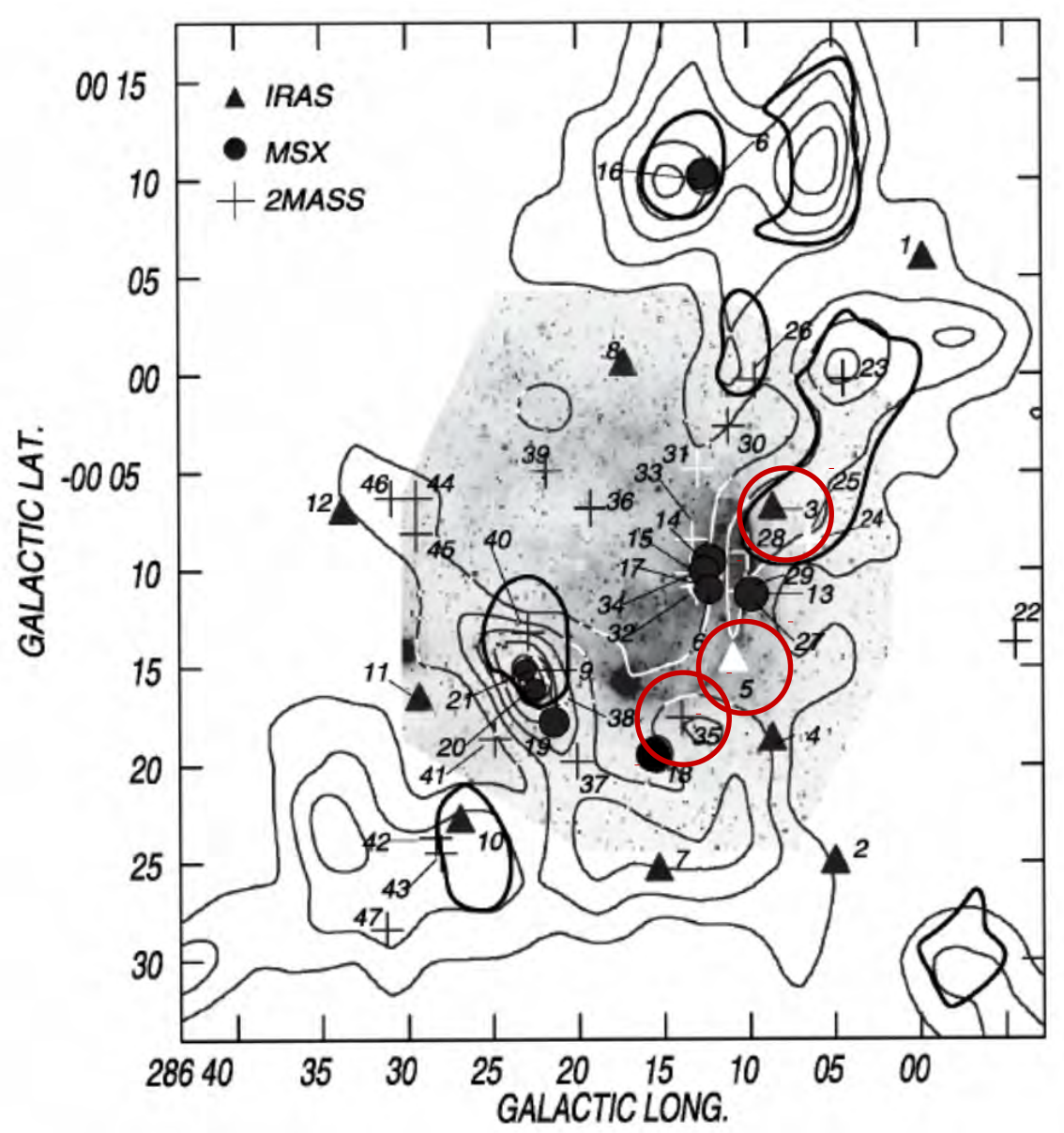

Figura 4.2: Fuentes puntuales de los catálogos IRAS (triángulos), MSX (puntos) y 2MASS (cruces) catalogadas como YSOs superpuestas a la imagen de SuperCOSMOS de Gum 31 y a los contornos de ${ }^{12} \mathrm{CO}$ (contornos finos) y $\mathrm{C}^{18} \mathrm{O}$ (contornos gruesos). Es evidente la cáscara molecular que bordea casi por completo la región Hiı. Los datos de ${ }^{12} \mathrm{CO}(1-0)$ y $\mathrm{C}^{18} \mathrm{O}(1-0)$ fueron obtenidos con el telescopio NANTEN con una resolución angular de 2.7 minutos de arco. Imagen tomada de Cappa et al. (2008). Las fuentes IRAS \#3 y \#5 y la fuentes 2MASS \#35 (encerradas en círculos rojos) son las estudiadas en esta tesis. Nótese que las coordenadas son longitud y latitud galácticas, mientras que en el resto del capítulo se utilizan coordenadas equatoriales RA, Dec.(J2000). 
densa que rodea a Gum 31 (ver Figura 4.2), han sido catalogadas como candidatas a objetos estelares jóvenes (YSOs) por Cappa et al. (2008) a partir de sus caracteríaticas fotométricas, evidenciando la presencia de regiones de formación estelar reciente. El medio interestelar en el cual los YSOs se encuentran inmersos, emite la mayor cantidad de radiación en las bandas del infrarrojo lejano y submilimétrica $(30 \mu \mathrm{m}-1 \mathrm{~mm})$ del espectro electromagnético, por lo que es fundamental un estudio de estas bandas del espectro.

Con el objetivo de estudiar las propiedades físicas del gas molecular y del polvo asociados a Gum 31 e investigar su correlación con la formación de estrellas, Duronea et al. (2015) llevaron a cabo un estudio del polvo frío en la región. Para esto utilizaron datos de continuo infrarrojo a $870 \mu \mathrm{m}$ tomados con el bolómetro Large APEX Bolometer Camera (LABOCA), que fueron complementados con datos de archivo en el infrarrojo lejano, continuo de radio y óptico (ver Figura 4.3). Estos autores determinaron que la distribución de la emisión IR coincide con la del gas molecular mostrado en la Figura 4.2 y sugieren que el polvo frío y el material molecular se han acumulado detrás del frente de ionización debido a la expansión de la nebulosa. La intensa emisión en $8 \mu \mathrm{m}$, continuo de radio y $870 \mu \mathrm{m}$ al oeste de las estrellas excitratrices indica la existencia de una PDR en la interfaz entre el gas ionizado y el gas molecular (ver Figura 4.3). Utilizando el algoritmo Gaussclumps identificaron 41 grumos en la imagen de $870 \mu \mathrm{m}$ con masas de gas y polvo de entre 70 y $2800 \mathrm{M}_{\odot}$, radios de entre 0.12 y 1.34 pc y temperaturas de polvo de entre 21 y $32 \mathrm{~K}$. Analizando la edad dinámica de la región Hit, el tiempo de fragmentación y el radio de la cáscara de polvo y gas molecular que la rodea, Duronea et al. (2015) determinaron que podría estar actuando el proceso collect and collapse. Este escenario se ve fortalecido por la presencia de candidatos a YSOs que se detectan proyectados sobre la emisión de $870 \mu \mathrm{m}$ adyacente al frente de ionización.

Dentro de la gran cantidad de fuentes catalogadas como candidatos a YSOs encontradas en la región se han seleccionado las fuentes IRAS 10351-5816, IRAS 10349-5824 y 2MASS J10365763-5844052 (indicadas en la Figura 4.2 con los números 3, 5 y 35, respectivamente), que se encuentran proyectadas sobre la envoltura densa que rodea a la región HII Gum 31. La elección de estas fuentes se debe a que son algunas de las fuentes infrarrojas identificadas sobre la cáscara que rodea a Gum 31 más cercanas a los bordes brillantes de la región, permitiendo así estudiar los posibles efectos de la expansión de la región HiI sobre el gas asociado a las fuentes. El objetivo es llevar a cabo un estudio del gas molecular, con resolución igual a $30^{\prime \prime}$ o mejor (los datos mostrados en la Figura 4.2 tienen una resolución angular de $2: 7$ ), en el que estas fuentes se encuentran inmersas a fin de analizar en detalle el entorno de las mismas e investigar la posible interacción de las fuentes con su medio circundante, y determinar parámetros del gas molecular que permitan inferir cuál es su estado evolutivo.

Una cuarta fuente, IRAS 10361-5830 (identificada con el número 9 en la Figura 4.2), localizada en el borde de Gum 31, sobre un grumo molecular detectado en $\mathrm{C}^{18} \mathrm{O}$ por Yonekura et al. (2005), y las fuentes asociadas MSX G286.3747-00.2630 y G286.3773-00.2563 fueron previamente estudiadas a partir de datos moleculares tomados con el telescopio APEX, imágenes infrarrojas de Spitzer GLIMPSE y MIPS, y de continuo de radio de SUMSS a $843 \mathrm{MHz}$, encontrándose una estructura en forma de cáscara en torno a las mismas. Se trata de una cáscara molecular con un radio medio de $0.32 \mathrm{pc}$ detectada en un intervalo de velocidades de $6.3 \mathrm{~km} \mathrm{~s}^{-1}$ a una velocidad de $-22.8 \mathrm{~km} \mathrm{~s}^{-1}$ y con una masa molecular de $40 \pm 8 \mathrm{M}_{\odot}$. La presencia de objetos estelares jóvenes proyectados en el interior de la cavidad y sobre la cáscara molecular indicaría que la formación estelar es activa en la región. La fuente MSX G286.3773-00.2563, candidata a estrella de tipo O tardía, pareciera ser la responsable de crear una región HII compacta dentro del grumo molecular (Vazzano et al., 2014).

A pesar de que en el trabajo de Cappa et al. (2008) se clasifican las fuentes IRAS como candidatos a objetos jóvenes, dada la baja resolución angular de IRAS, y tal como se mencionó 


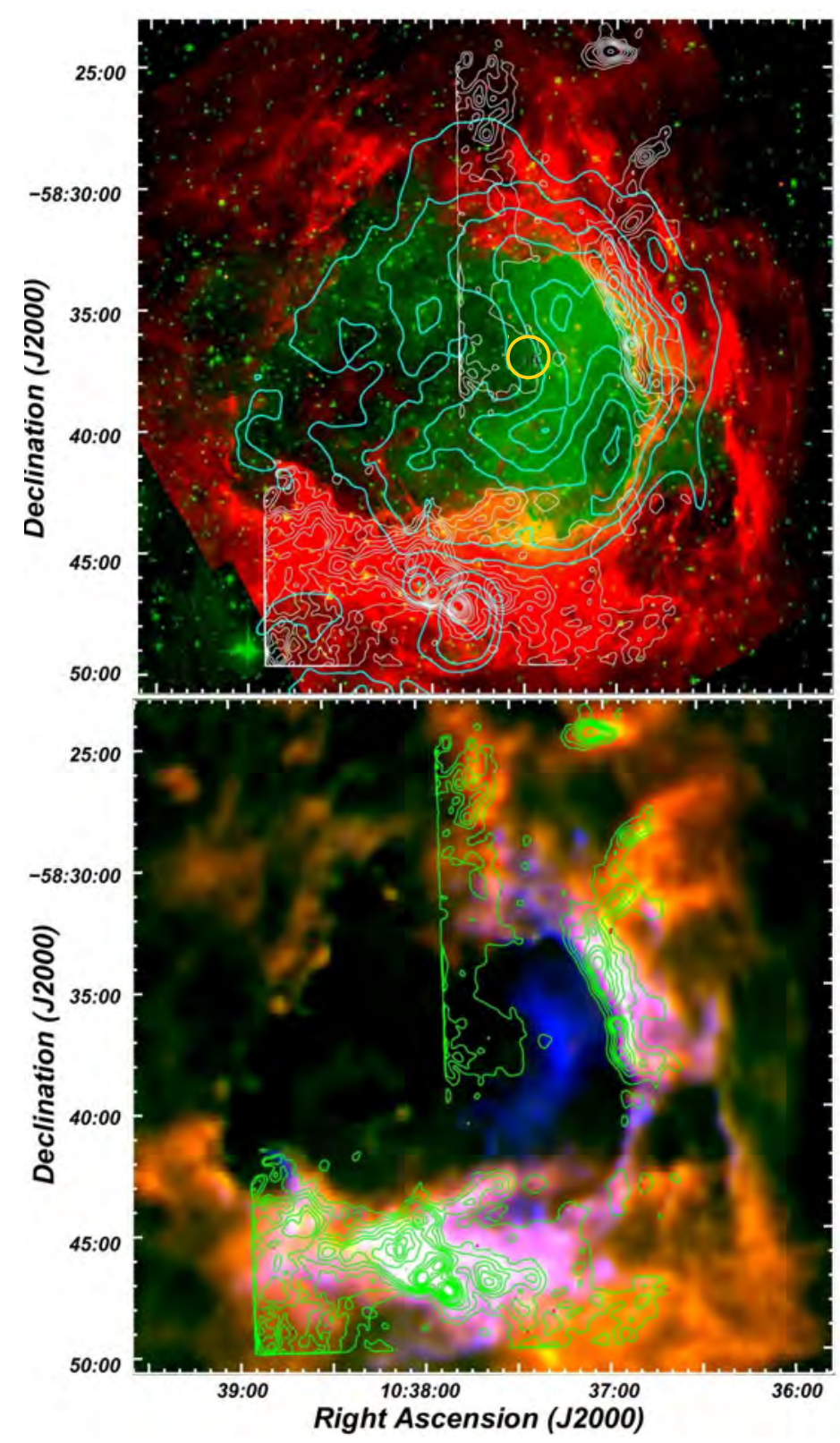

Figura 4.3: Arriba: Se muestra en rojo la emisión a $8 \mu \mathrm{m}$ de Spitzer-GLIMPSE y en verde la emisión óptica (DSSR2). Los contornos blancos corresponden a la emisión a $870 \mu \mathrm{m}$ y los cyan a la emisión de continuo de radio a $843 \mathrm{MHz}$. El círculo amarillo indica la posición del cúmulo NGC 3324. Abajo: Contornos de la emisión a $870 \mu \mathrm{m}$ superpuestos a las imágenes de Herschel a $70 \mu \mathrm{m}$ (azul), $250 \mu \mathrm{m}$ (verde) y $500 \mu \mathrm{m}$ (rojo). En ambas figuras los contornos de $870 \mu \mathrm{m}$ van desde $150 \mathrm{mJy}_{\text {beam }}{ }^{-1}(3 \sigma)$ hasta $650 \mathrm{mJy}$ beam $^{-1}$ en pasos de $100 \mathrm{mJy}$ beam $^{-1}$, y de $650 \mathrm{mJy}_{\text {beam }}{ }^{-1}$ en pasos de $200 \mathrm{mJy}^{\text {beam }}{ }^{-1}$. Figura tomada de Duronea et al. (2015). 


\begin{tabular}{lccccccc}
\hline \hline Nombre & $l$ & $b$ & \multicolumn{2}{c}{ Flujos[Jy] } & \multicolumn{3}{c}{$\mathrm{L}_{I R A S}\left[10^{3} \mathrm{~L}_{\odot}\right]$} \\
& & & $12 \mu \mathrm{m}$ & $25 \mu \mathrm{m}$ & $60 \mu \mathrm{m}$ & $100 \mu \mathrm{m}$ & \\
\hline IRAS 10351-5816 & $286^{\circ} 08 ! 58$ & $-0^{\circ} 06^{\prime} \cdot 96$ & 6.3 & 6.8 & 315 & 1480 & 18 \\
IRAS 10349-5824 & $286^{\circ} 10^{\prime} \cdot 92$ & $-0^{\circ} 14 ! 7$ & 5.9 & 9.0 & 163 & 1660 & 18 \\
\hline Nombre & $l$ & $b$ & $J[\mathrm{mag}]$ & $H[\mathrm{mag}]$ & $K_{s}[\mathrm{mag}]$ & $(J-H)$ & $(H-K)$ \\
\hline 2MASS J10365763-5844052 & $286^{\circ} 13 ! 92$ & $-0^{\circ} 17 ! 87$ & 12.22 & 11.882 & 11.51 & 0.338 & 0.372 \\
\hline \hline
\end{tabular}

Tabla 4.1: Candidatos a YSOs de los catálogos IRAS y 2MASS proyectados sobre la cáscara molecular que rodea a Gum 31, cuyo entorno molecular se analiza en esta tesis.

\begin{tabular}{lcc}
\hline \hline Fuente & Cubos de datos & Observaciones single point \\
\hline IRAS 10351-5816 & ${ }^{12} \mathrm{CO}(3-2), \mathrm{C}^{18} \mathrm{O}(3-2)$ & ${ }^{13} \mathrm{CO}(3-2), \mathrm{HCO}^{+}(4-3)$ \\
IRAS 10349-5824 & ${ }^{12} \mathrm{CO}(2-1), \mathrm{C}^{18} \mathrm{O}(2-1)$ & - \\
2MASS J10365763-5844052 & ${ }^{12} \mathrm{CO}(2-1),{ }^{13} \mathrm{CO}(2-1), \mathrm{C}^{18} \mathrm{O}(2-1)$ & - \\
\hline \hline
\end{tabular}

Tabla 4.2: Datos moleculares obtenidos usando el telescopio APEX.

en la Sección 1.4.5, se referirá a estas fuentes como a regiones de formación estelar y no como candidatos a YSOs.

El catálogo de fuentes puntuales IRAS permite identificar candidatos a regiones de formación estelar siguiendo el criterio de Junkes et al. (1992) (ver Sección 1.4.5), que establece relaciones entre los flujos obtenidos en 12, 25, 60 y $100 \mu \mathrm{m}$. En la Tabla 4.1, se listan los nombres de los candidatos seleccionadas, sus posiciones $(l, b)$, sus flujos a diferentes longitudes de onda del IR y sus luminosidades en el IR estimadas en base a

$$
L=2.96 \times 10^{-7}\left(\frac{d}{p c}\right)^{2} \Sigma\left[\Delta \nu_{\lambda}\left(\frac{F_{\lambda}}{J y}\right)\right]+5.3 \times 10^{-5}\left(\frac{F_{\lambda}}{J y}\right) \frac{d^{2}}{p c}\left(\frac{\lambda_{100}}{\mu m}\right)^{-1}
$$

donde $d$ es la distancia a la fuente, $\Delta \nu_{\lambda}$ y $F_{\lambda}$ son el ancho de banda efectivo y el flujo en la banda correspondiente a una dada longitud de onda $\lambda$, y la sumatoria se realiza sobre todas las bandas desde $12 \mu \mathrm{m}$ hasta $100 \mu \mathrm{m}$ (Yamaguchi et al., 1999). El valor de $F_{\lambda}$ en el segundo término se toma a $\lambda=100 \mu \mathrm{m}$ ya que este término representa una corrección bolométrica estimada por extrapolación de la longitud de onda más larga.

El catálogo de fuentes puntuales 2MASS provee detecciones en tres bandas del IR cercano: J, H y $K_{S}$, a $1.25 \mu \mathrm{m}, 1.65 \mu \mathrm{m}$ y $2.17 \mu \mathrm{m}$, respectivamente. Cappa et al. (2008) consideraron fuentes con $K_{S}<12$. Este criterio corresponde a estrellas con tipos espectrales más tempranos que B3 a una distancia de $3 \mathrm{kpc}$. Las fuentes con $q<-0.15$, donde $q=(J-H)-1.83 \times$ $\left(H-K_{S}\right)$, fueron clasificadas como objetos con exceso IR, candidatos a YSOs. Los datos de la fuente 2MASS J10365763-5844052 están también incluidos en la Tabla 4.1.

\subsection{Observaciones y bases de datos}

Para llevar a cabo el análisis del medio ambiente en que estas fuentes se encuentran inmersas se han observado, utilizando el telescopio APEX, tres regiones centradas en cada una de las fuentes (ver Figura 4.4). Las regiones analizadas en torno a las fuentes IRAS tienen un tamaño de $3^{\prime} \times 3^{\prime}$, y la que corresponde a la fuente 2 MASS de $4^{\prime} \times 4^{\prime}$. Las moléculas observadas en cada una de estas regiones y el modo de observación están detallados en la Tabla 4.2 y sus frecuencias en la Tabla 2.2. Los cubos de datos fueron observados utilizando la técnica On the Fly (OTF), mientras que las observaciones single point corresponden a la toma de espectros únicamente en dirección a la fuente.

Los datos fueron adquiridos con un espectrómetro de transformada rápida de Fourier, que consta de 4090 canales, con un ancho de banda total de $800 \mathrm{~km} \mathrm{~s}^{-1}$, lo que proporciona 


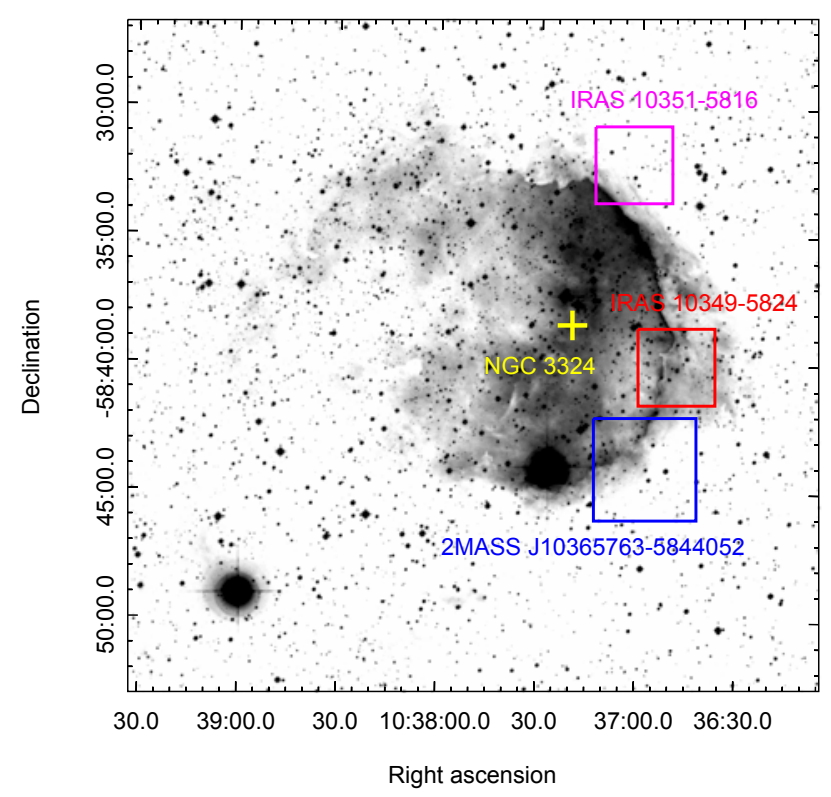

Figura 4.4: Imagen de SuperCOSMOS de Gum 31. Los cuadros indican las regiones observadas y la cruz la posición de cúmulo NGC 3324.

una resolución en velocidad de $0.2 \mathrm{~km} \mathrm{~s}^{-1}$. La posición libre de emisión de CO que se usó se encuentra en RA, Dec.(J2000) = 10:38:53.46, -59:18:48.6. La calibración se realizó utilizando el planeta Saturno, y las fuentes RAFGL5254 y RAFGL4211. El apuntamiento se comprobó dos veces durante las observaciones usando X-TrA, Venus y VY-CMa. La calibración de intensidad tiene una incertidumbre del $10 \%$.

Los espectros se redujeron utilizando el software CLASS (Continuum and Line Analysis Single-dish Software) de GILDAS (Grenoble Image and Line Data Analysis Software). Para el ajuste de línea de base se utilizaron polinomios de grado 1. El ruido cuadrático medio (rms) de los perfiles después de la resta y la calibración de la línea de base fue de $\sim 0.6 \mathrm{~K}$. Las intensidades de línea observadas se expresan como temperaturas de brillo del haz principal $\mathrm{T}_{m b}$, dividiendo la temperatura de antena $\mathrm{T}_{A}$ por la eficiencia de haz principal $\eta_{m b}$, igual a 0.72 para APEX-1 y 0.82 para APEX-2 (Vassilev et al., 2008). Para realizar el análisis de los datos moleculares se utilizaron los softwares AIPS (Astronomical Image Processing System) y CLASS.

Con el fin de completar la identificación de YSOs en las regiones a estudiar se ha llevado a cabo una búsqueda de fuentes en los catálogos Spitzer-GLIMPSE y WISE, estas últimas no disponibles al momento de realizar el trabajo Cappa et al. (2008). En los listados catalogados en Spitzer sólo se han encontrado hacia Gum 31 fuentes que poseen observaciones en dos bandas, por lo que no es posible realizar una clasificación de las mismas, y por lo tanto fueron excluidas del análisis de la región. Este nuevo análisis permitió encontrar un gran número de fuentes WISE, que fueron clasificadas como Clase I, II o III según su emisión en cada una de las bandas observadas en 3.4, 4.6, 12 y $22 \mu \mathrm{m}$, según el criterio de Koenig et al. (2012) (ver Sección 1.4.5 para detalles sobre el criterio).

A continuación se describirá el análisis realizado para cada una de las regiones observadas. 


\begin{tabular}{|c|c|c|c|c|c|c|c|c|}
\hline \multicolumn{9}{|c|}{ Región asociada a IRAS 10351-5816 } \\
\hline \multirow[t]{2}{*}{$\#$} & \multirow[t]{2}{*}{$\alpha$} & \multirow[t]{2}{*}{$\delta$} & \multirow[t]{2}{*}{ Nombre } & \multicolumn{4}{|c|}{ Magnitud } & \multirow{2}{*}{ Clase } \\
\hline & & & & $3.4 \mu \mathrm{m}$ & $4.6 \mu \mathrm{m}$ & $12 \mu \mathrm{m}$ & $22 \mu \mathrm{m}$ & \\
\hline 1 & $10: 37: 0.7984$ & $-58: 32: 41.020$ & J103700.79-583241.0 & 11.152 & 9.490 & 5.493 & 3.125 & I \\
\hline 2 & $10: 37: 2.3070$ & $-58: 32: 59.563$ & J103702.30-583259.5 & 10.560 & 10.126 & 5.732 & 3.990 & II \\
\hline 3 & $10: 37: 8.4814$ & $-58: 32: 17.477$ & J103708.48-583217.4 & 9.987 & 9.624 & 7.818 & 4.660 & II \\
\hline \multicolumn{9}{|c|}{ Región asociada a IRAS 10349-5824 } \\
\hline \multirow[t]{2}{*}{$\#$} & $\alpha$ & $\delta$ & Nombre & \multicolumn{4}{|c|}{ Magnitud } & Clase \\
\hline & & & & $3.4 \mu \mathrm{m}$ & $4.6 \mu \mathrm{m}$ & $12 \mu \mathrm{m}$ & $22 \mu \mathrm{m}$ & \\
\hline 4 & $10: 36: 48.9732$ & $-58: 40: 10.711$ & J103648.97-584010.7 & 9.451 & 8.496 & 4.441 & 1.154 & II \\
\hline 5 & $10: 36: 48.4064$ & $-58: 40: 0.7554$ & J103648.40-584000.7 & 10.087 & 9.292 & 4.549 & 1.404 & II \\
\hline
\end{tabular}

Tabla 4.3: Fuentes WISE catalogadas como YSOs detectadas en las regiones observadas en torno a las fuentes IRAS 10351-5816 y IRAS 10349-5824. No se han detectado fuentes WISE asociadas a la fuente 2MASS J10365763-5844052.

\subsection{IRAS 10351-5816}

El análisis de la distribución del gas molecular en la región de $3^{\prime} \times 3^{\prime}$ centrada en la posición de la fuente IRAS 10351-5816 revela un grumo denso detectado en ${ }^{12} \mathrm{CO}(3-2)$ y $\mathrm{C}^{18} \mathrm{O}(3-2)$ (indicado en azul en la Figura 4.5) a velocidades de alrededor de $-21 \mathrm{~km} \mathrm{~s}^{-1}$, referidas al LSR, cercano a la posición central de la fuente. Este coincide espacialmente con un grumo de polvo frío detectado en $870 \mu \mathrm{m}$ por Duronea et al. (2015) (indicado en amarillo en la Figura 4.5) . Este grumo, identificado con el número 32 en la Tabla 1 de Duronea et al. (2015), está centrado en RA, Dec. $(\mathrm{J} 2000)=(10: 37: 02,-58: 32: 31)$, tiene un radio de $0.3 \mathrm{pc}$, posee una masa total (gas + polvo) de $240 \mathrm{M}_{\odot}$ y una densidad volumétrica promedio de $27900 \mathrm{~cm}^{-3}$, valores obtenidos a partir de la emisión en el continuo IR en $870 \mu \mathrm{m}$ utilizando la ecuación 2.48, y una temperatura de polvo de $24 \mathrm{~K}$ obtenida del cociente de la emisión en 70 y 160 $\mu \mathrm{m}$.

En dirección al centro del mapa se detecta emisión de $\mathrm{HCO}^{+}(4-3)$ que coincide con el borde del grumo. Dado que la densidad crítica de $\mathrm{HCO}^{+}(4-3)$ es de $9 \times 10^{6} \mathrm{~cm}^{-3}$ (ver Tabla 2.2) esta emisión revela una región de alta densidad en el grumo. Como se puede ver en el panel derecho de la Figura 4.5 el máximo de esta emisión coincide en velocidad con el máximo de emisión del perfil de $\mathrm{C}^{18} \mathrm{O}$ en dirección al centro del mapa, lo que indica que se trata de emisión correspondiente al mismo grumo.

La búsqueda de fuentes YSOs en la región permitió identificar tres fuentes WISE, una de ellas clasificada como clase I y dos como clase II. Sus posiciones y magnitudes a distintas frecuencias se encuentran listados en la tabla 4.3.

La emisión en diferentes bandas del IR entre 8 y $870 \mu \mathrm{m}$ se muestra en la Figura 4.6. Como allí se puede ver, la emisión en $8 \mu$ m revela la presencia de la PDR, que delinea el borde de la región Hiı Gum 31, y la emisión en $24 \mu$ m muestra polvo tibio en el interior de la misma. El polvo tibio es también mostrado por la emisión a $70 \mu \mathrm{m}$. En los paneles de la Figura 4.6 correspondientes a frecuencias de 160, 350 y $500 \mu \mathrm{m}$ de Herschel se puede distinguir, cerca del centro de cada imagen, el grumo detectado en $870 \mu \mathrm{m}$ por Duronea et al. (2015). La parte más densa de este grumo coincide espacialmente con una fuente puntual detectada en $24 \mu \mathrm{m}$ y con la fuente WISE clasificada como Clase I (\# 1 en la Tabla 4.3), indicando que hay formación estelar.

Para estimar los parámetros físicos del grumo se realizaron ajustes gaussianos a los perfiles de ${ }^{12} \mathrm{CO}(3-2)$ y $\mathrm{C}^{18} \mathrm{O}(3-2)$ promediados en el grumo (ver figura 4.7). Los parámetros de las gaussianas ajustadas, que se muestran en la Tabla 4.4, son el área debajo de la curva (K 

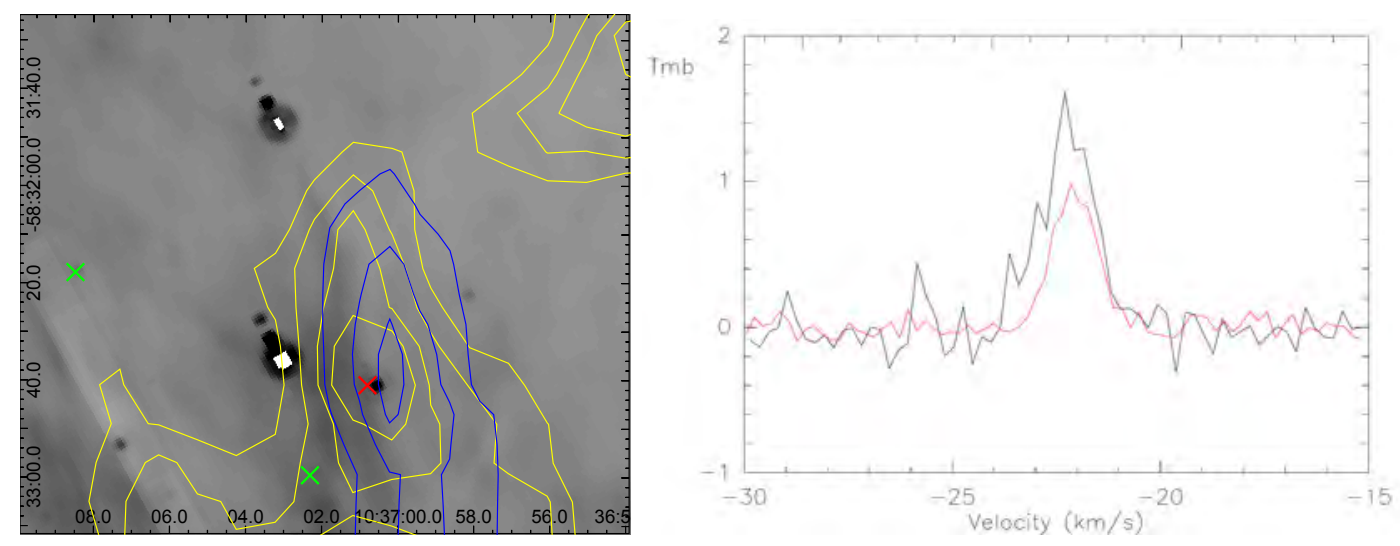

Figura 4.5: IRAS 10351-5816 Izquierda: En azul se muestran los contornos de $\mathrm{C}^{18} \mathrm{O}(3-2)$ $(1,1.5$ y $2 \mathrm{~K})$ correspondientes al gas denso con velocidades en el rango $-21.0 \mathrm{a}-22.3 \mathrm{~km} \mathrm{~s}^{-1}$, superpuestos a la imagen de $8 \mu \mathrm{m}$ de Spitzer-IRAC. El grumo de $\mathrm{C}^{18} \mathrm{O}(3-2)$ coincide espacialmente con un grumo detectado en $870 \mu \mathrm{m}$ (contornos amarillos, $0.3,0.4,0.5$ y $0.6 \mathrm{Jy} / \mathrm{beam}$ ). Las cruces indican las posiciones de las fuentes WISE clase I (rojo) y clase II (verde). Derecha: Espectros de $\mathrm{C}^{18} \mathrm{O}(3-2)$ (negro) y $\mathrm{HCO}^{+}$(4-3) (rojo) hacia la posición central de IRAS 10351-5816 (unidades de temperatura de brillo).
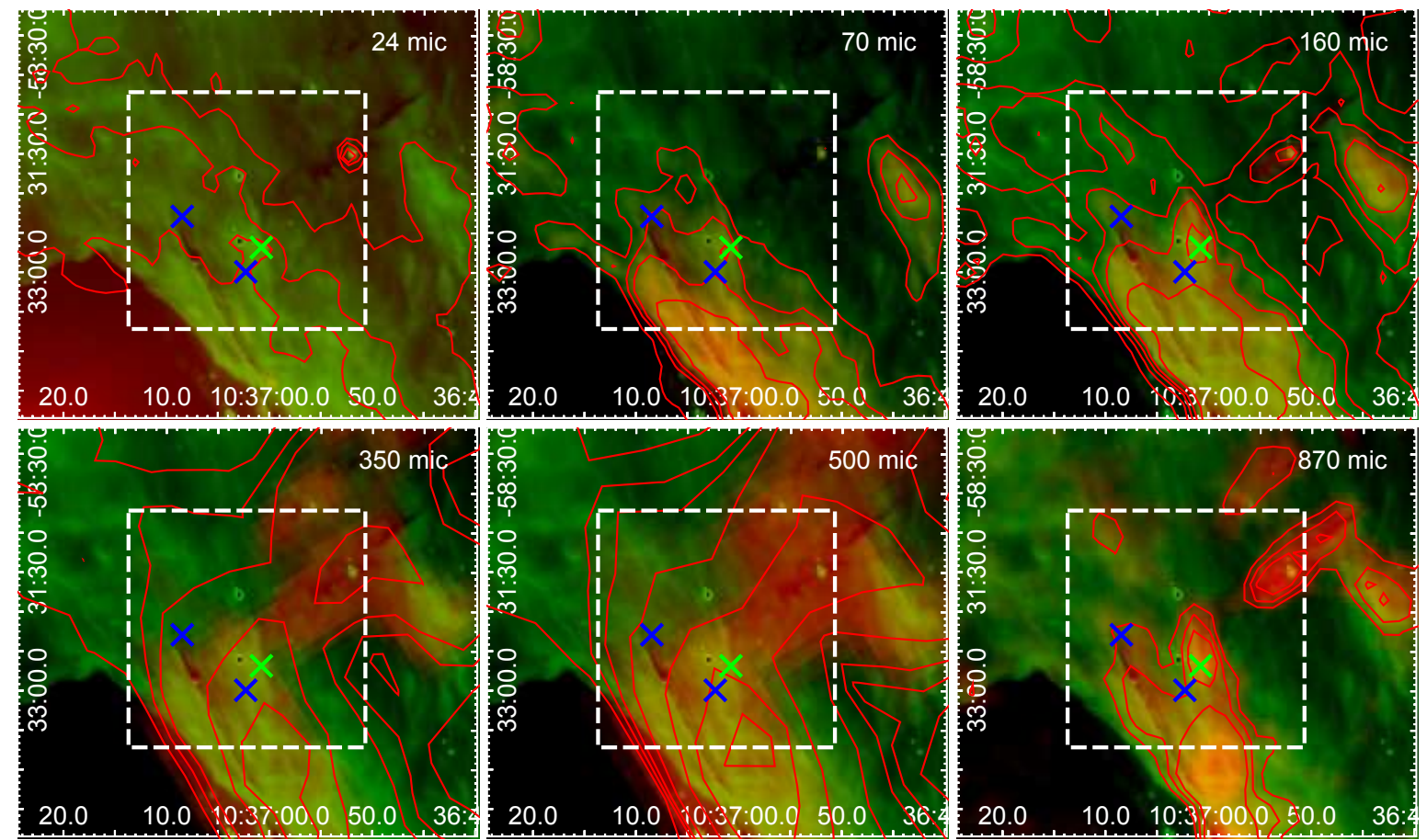

Figura 4.6: IRAS 10351-5816 Imagen de $8 \mu \mathrm{m}$ (verde) superpuesta a las de 24, 70, 160, 350,500 y $870 \mu \mathrm{m}$ (rojo y contornos). El cuadrado muestra la región observada con APEX. La cruces indican las posiciones de fuentes WISE clase I (verde) y clase II (azul). 


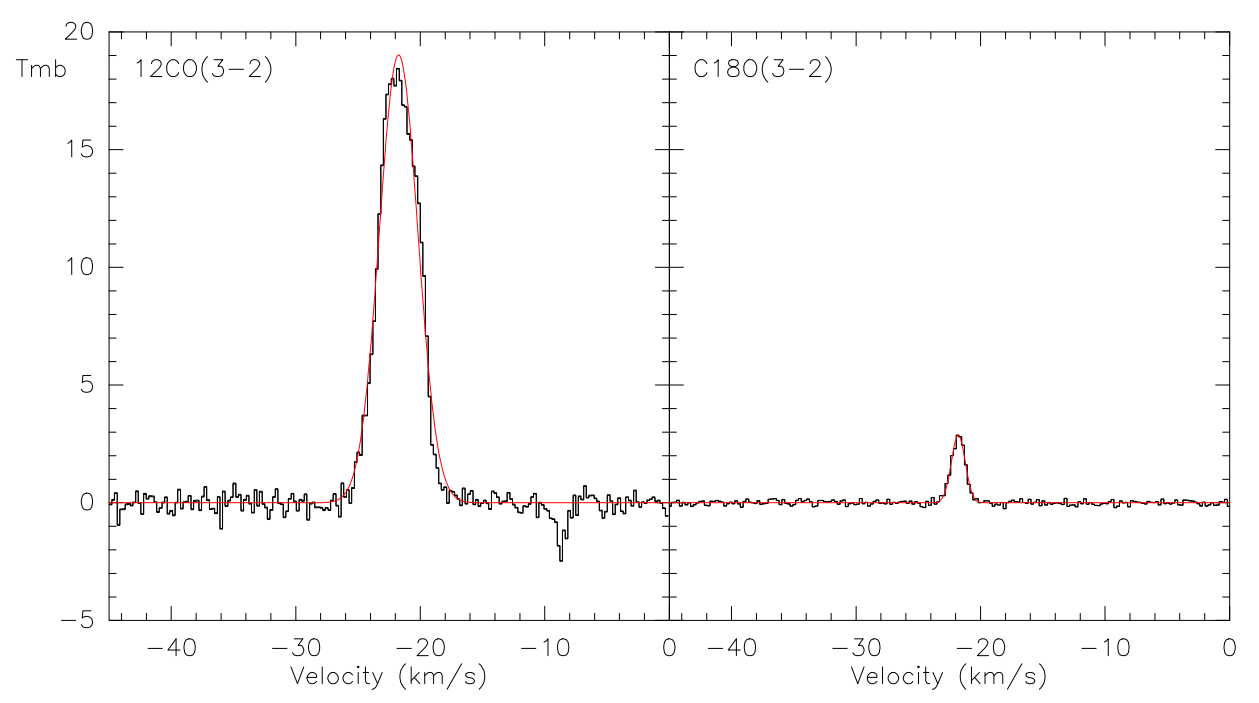

Figura 4.7: IRAS 10351-5816 Perfiles promedio de ${ }^{12} \mathrm{CO}$ y $\mathrm{C}^{18} \mathrm{O}$ en dirección al grumo identificado en esta región junto con sus ajustes gaussianos.

\begin{tabular}{ccccc}
\hline \hline & $\begin{array}{c}\text { Area } \\
{\left[\mathrm{K} \mathrm{km} \mathrm{s}^{-1}\right]}\end{array}$ & $\begin{array}{c}\text { Velocidad } \\
{\left[\mathrm{km} \mathrm{s}^{-1}\right]}\end{array}$ & $\begin{array}{c}\Delta \mathrm{v} \\
{\left[\mathrm{km} \mathrm{s}^{-1}\right]}\end{array}$ & $\begin{array}{c}\text { Temperatura máxima } \\
{[\mathrm{K}]}\end{array}$ \\
\hline${ }^{12} \mathrm{CO}(3-2)$ & 73.3 & -21.7 & 3.6 & 19 \\
$\mathrm{C}^{18} \mathrm{O}(3-2)$ & 4.0 & -21.8 & 1.3 & 3 \\
\hline \hline
\end{tabular}

Tabla 4.4: IRAS 10351-5816 Parámetros de los ajustes gaussianos utilizados para el cálculo de densidades y masas.

$\left.\mathrm{km} \mathrm{s}^{-1}\right)$, la velocidad a la que se encuentra el máximo $\left(\mathrm{km} \mathrm{s}^{-1}\right)$, el ancho a potencia mitad de la gaussiana $\left(\Delta \mathrm{v}\right.$, en $\left.\mathrm{km} \mathrm{s}^{-1}\right)$ y el valor máximo que alcanza $(\mathrm{K})$.

Asumiendo que la molécula de ${ }^{12} \mathrm{CO}(3-2)$ es ópticamente gruesa y la de $\mathrm{C}^{18} \mathrm{O}(3-2)$ es ópticamente delgada, y considerando los parámetros de los ajustes gaussianos realizados, se calcularon a partir de las ecuaciones 2.21 y 2.22 una temperatura de excitación promedio de $26 \mathrm{~K}$ y una profundidad óptica para el $\mathrm{C}^{18} \mathrm{O}(3-2)$ de 0.16 corroborando que la misma es ópticamente delgada. Esta temperatura calculada es similar a la temperatura de polvo determinada para el grumo por Duronea et al. (2015). Esta similitud está de acuerdo con el hecho de que las regiones de alta densidad, como la que se está estudiando, se encuentran térmicamente acopladas, o sea que el gas y el polvo están a la misma temperatura. A partir de una temperatura de excitación de $26 \mathrm{~K}$ y usando las ecuaciones 2.33, 2.38 y 2.39 se estimaron las densidades columnares, masas y densidades ambientales para el grumo en base al radio efectivo indicado. La principal fuente de error de estos parámetros es la incerteza en la distancia, que para Gum 31 es el $12 \%$. Por lo tanto el error en el tamaño lineal de la región será también del $12 \%(r \propto d)$, el error en la masa será de $24 \%\left(M \propto d^{2}\right)$ y el error en las densidades será del $36 \%\left(n \propto d^{3}\right)$. Estos parámetros se encuentran listados en la Tabla 4.5.

Una forma de determinar si el grumo es capaz de colapsar y dar lugar al nacimiento de nuevas estrellas es calcular la masa del virial y compararla con la masa estimada con algún otro método como puede ser la obtenida considerando equilibrio termodinámico local. Utilizando la ecuación 2.40 se obtuvieron valores de masas de virial entre 65 y $108 \mathrm{M}_{\odot}$ (considerando valores de $k=126$ - 210). Estos valores poseen al menos un $12 \%$ de incerteza debido al error en la distancia $\left(M_{V I R} \propto d\right)$. Además el perfil de densidad desconocido del grumo y la existencia de un soporte de campo magnético puede sobrestimar los valores de 


\begin{tabular}{|c|c|c|c|c|c|c|c|c|c|}
\hline \multicolumn{10}{|c|}{ IRAS 10351-5816 } \\
\hline & $\begin{array}{l}\mathrm{T}_{e x c} \\
{[\mathrm{~K}]}\end{array}$ & $\tau_{18}$ & $\tau_{12}$ & $\begin{array}{c}N\left(C^{18} \mathrm{O}\right) \\
10^{15}\left[\mathrm{~cm}^{-2}\right]\end{array}$ & $\begin{array}{c}N\left(\mathrm{H}_{2}\right) \\
\times 10^{22}\left[\mathrm{~cm}^{-2}\right]\end{array}$ & $\begin{array}{c}r \\
{[\mathrm{pc}]}\end{array}$ & $\begin{array}{c}M\left(H_{2}\right) \\
{\left[\mathrm{M}_{\odot}\right]}\end{array}$ & $\begin{array}{c}n_{H_{2}} \\
\times 10^{3}\left[\mathrm{~cm}^{-3}\right]\end{array}$ & $M_{V I R} / M\left(H_{2}\right)$ \\
\hline Grumo 1 & 26 & 0.16 & 4.7 & 1.77 & 1.04 & $0.30 \pm 0.04$ & $85 \pm 20$ & $10.7 \pm 3.8$ & $0.8-1.3$ \\
\hline \multicolumn{10}{|c|}{ IRAS 10349-5824 } \\
\hline & $\begin{array}{l}\mathrm{T}_{e x c} \\
{[\mathrm{~K}]}\end{array}$ & $\tau_{13}$ & $\tau_{12}$ & $\begin{array}{c}N\left({ }^{13} \mathrm{CO}\right) \\
10^{15}\left[\mathrm{~cm}^{-2}\right]\end{array}$ & $\begin{array}{c}N\left(H_{2}\right) \\
\times 10^{21}\left[\mathrm{~cm}^{-2}\right]\end{array}$ & $\begin{array}{c}r \\
{[\mathrm{pc}]}\end{array}$ & $\begin{array}{c}M\left(H_{2}\right) \\
{\left[\mathrm{M}_{\odot}\right]}\end{array}$ & $\begin{array}{c}n_{H_{2}} \\
\times 10^{3}\left[\mathrm{~cm}^{-3}\right]\end{array}$ & \\
\hline Grumo 1 & 21 & 0.27 & 19.2 & 4.15 & 2.42 & $0.28 \pm 0.04$ & $22 \pm 5$ & $3.5 \pm 1.3$ & $5.4-9(*)$ \\
\hline Grumo 2 & 22 & 0.37 & 23.5 & 5.15 & 3.96 & $0.29 \pm 0.04$ & $30 \pm 7$ & $4.2 \pm 1.5$ & $1.5-2.6$ \\
\hline Grumo 3 & 18 & 0.20 & 15.0 & 2.85 & 2.20 & $0.21 \pm 0.03$ & $9 \pm 2$ & $3.2 \pm 1.2$ & $14.8-24.7(*)$ \\
\hline \multicolumn{10}{|c|}{ 2MASS J10365763-5844052 } \\
\hline & $\begin{array}{l}\mathrm{T}_{e x c} \\
{[\mathrm{~K}]}\end{array}$ & $\tau_{18}$ & $\tau_{12}$ & $\begin{array}{c}N\left(C^{18} \mathrm{O}\right) \\
10^{15}\left[\mathrm{~cm}^{-2}\right]\end{array}$ & $\begin{array}{c}N\left(H_{2}\right) \\
\times 10^{21}\left[\mathrm{~cm}^{-2}\right] \\
\end{array}$ & $\begin{array}{c}r \\
{[\mathrm{pc}]}\end{array}$ & $\begin{array}{c}M\left(H_{2}\right) \\
{\left[\mathrm{M}_{\odot}\right]}\end{array}$ & $\begin{array}{c}n_{H_{2}} \\
\times 10^{3}\left[\mathrm{~cm}^{-3}\right] \\
\end{array}$ & \\
\hline Grumo 1 & 28 & 0.03 & 1.58 & 0.73 & 4.31 & $0.41 \pm 0.05$ & $65 \pm 15$ & $3.3 \pm 1.2$ & $2.3-4.0$ \\
\hline Grumo 2 & 26 & 0.10 & 4.02 & 1.40 & 8.24 & $0.48 \pm 0.06$ & $170 \pm 40$ & $5.3 \pm 1.9$ & $0.5-0.8$ \\
\hline
\end{tabular}

Tabla 4.5: Parámetros calculados para los grumos en las regiones de las fuentes IRAS 103515816, IRAS 10349-5824, y 2MASS J10365763-5844052 en base a datos de CO. Los cocientes $M_{V I R} / M\left(H_{2}\right)$ marcados con $\left(^{*}\right)$ fueron calculados utilizando masas de virial obtenidas a partir de ${ }^{13} \mathrm{CO}$, ya que no se detecta emisión de $\mathrm{C}^{18} \mathrm{O}$ en estos grumos.

\section{$M_{V I R}$ derivados (MacLaren et al., 1988)}

Los valores calculados de los cocientes $M_{V I R} / M\left(H_{2}\right)$ se muestran en la Tabla 4.5. Que el valor de la masa del virial sea menor que el de la masa en $\operatorname{LTE}\left(M_{V I R} / M\left(H_{2}\right)<1\right)$ significa que este grumo no tiene suficiente energía cinética como para frenar el colapso gravitacional, por lo que sería posible la formación de nuevas estrellas a partir de dicho grumo. En este grumo los valores del cociente $M_{V I R} / M\left(H_{2}\right)$ se encuentran por encima o por debajo de uno según qué modelo de perfil de densidad se considere, por lo que es posible que haya colapso. Sin embargo si se considera la estructura jerárquica de las nubes moleculares se puede suponer que los grumos aumentan su densidad hacia el interior. Si este fuera el caso se debe tomar un valor de $k=126$, lo que da como resultado $M_{V I R} / M\left(H_{2}\right)=0.8$ e indica que el grumo estaría colapsando, compatible con la identificación de YSOs clase I y II coincidentes con el grumo.

\subsection{IRAS 10349-5824}

La distribución espacial del gas molecular asociado a la fuente IRAS 10349-5824 revela la presencia de tres grumos proyectados sobre la región observada (a los que se hará referencia como Grumo 1, Grumo 2 y Grumo 3), en un rango de velocidades de entre -20 y $-25 \mathrm{~km} \mathrm{~s}^{-1}$, con una velocidad central de $-23 \mathrm{~km} \mathrm{~s}^{-1}$, que coincide con la asociada a Gum 31 (ver panel superior de Figura 4.8). Los tres grumos se detectan en ${ }^{12} \mathrm{CO}(2-1)$ y ${ }^{13} \mathrm{CO}(2-1)$, mientras que solamente el Grumo 2 se detecta en $\mathrm{C}^{18} \mathrm{O}(2-1)$. Claramente, el material molecular bordea la región HiI Gum 31. Se puede ver que estos tres grumos delimitan una región de baja emisión de gas molecular (indicada como cavidad en la Figura 4.8). La posición de esta región (RA, Dec.(J2000) $=10: 36: 48.5,-58: 40: 13)$ coincide con el máximo de la emisión extendida a 4.5 $\mu \mathrm{m}$ (ver panel inferior izquierdo de la Figura 4.8).

Dos de los grumos (Grumo 1 y Grumo 2) se encuentran en el borde del frente de ionización, delineando la región HII. Se puede ver además que el gas molecular coincide con la posición de la PDR, que queda evidenciada a través de la emisión en $8 \mu \mathrm{m}$ proveniente de los PAHs. La emisión de los PAHs indica la presencia de una interfase entre el gas ionizado perteneciente al interior de la región Hit y el gas molecular de la cáscara. En el panel inferior derecho de la Figura 4.8 se puede ver que la emisión en $8 \mu \mathrm{m}$ delinea perfectamente el borde de la región Gum 31, marcando la posición del frente de ionización y la PDR. 


\begin{tabular}{lc}
\hline \hline $\mathrm{M}_{\text {out }}$ & $9 \mathrm{M}_{\odot}$ \\
$p$ & $26.6 \mathrm{M}_{\odot} \mathrm{km} \mathrm{s}^{-1}$ \\
$\mathrm{E}$ & $6.6 \times 10^{45} \mathrm{erg}$ \\
$t$ & $1.3 \times 10^{5} \mathrm{yr}$ \\
$\dot{\mathrm{M}}$ & $7 \times 10^{-5} \mathrm{M}_{\odot} \mathrm{yr}^{-1}$ \\
$\mathrm{v}_{\max }$ & $7-9 \mathrm{~km} \mathrm{~s}^{-1}$ \\
\hline \hline
\end{tabular}

Tabla 4.6: Parámetros derivados para el outflow asociado a IRAS 10349-5824.

En el panel superior de la Figura 4.9 se muestra la emisión de ${ }^{12} \mathrm{CO}(2-1)$ a -20.5 y -25 $\mathrm{km} \mathrm{s}^{-1}$. Esta emisión, que indica la presencia de gas a velocidades mayores y menores que $-23 \mathrm{~km} \mathrm{~s}^{-1}$ (velocidad a la que se distinguen los tres grumos), sugiere la existencia de alas roja y azul de la emisión de ${ }^{12} \mathrm{CO}(2-1)$, lo que podría interpretarse como un flujo molecular bipolar cuyo eje es casi coincidente con el plano del cielo, y muestra lóbulos que se detectan a partir del gas molecular. El panel inferior de la Figura 4.9 muestra los perfiles en las direcciones correspondientes a los máximos de la emisión de cada lóbulo. Claramente estos espectros no son simétricos y no pueden ser ajustados por una única gaussiana ya que poseen alas ensanchadas, las cuales podrían ser evidencia de la acción del outflow de una estrella en formación sobre el gas molecular que lo rodea. Para ambos espectros se pudo realizar un ajuste gaussiano utilizando dos componentes: la más intensa, a velocidades cercanas a -23 $\mathrm{km} \mathrm{s}^{-1}$, indicaría la emisión correspondiente a la nube molecular, y la más débil indicaría la emisión proveniente de los lóbulos azul y rojo (más detalles de cómo se producen las alas en la Sección 1.4.6). Estos ajustes se muestran en la Figura 4.9 y sus parámetros en la Tabla 4.7 .

Los parámetros del outflow fueron claculados a partir de estos ajustes y usando las ecuaciones de Beuther et al. (2002),

$$
\begin{gathered}
\mathrm{M}_{\text {out }}=\left(\mathrm{N}_{b} \times \text { size }_{b}+\mathrm{N}_{r} \times \text { size }_{r}\right) \\
p=\left(\mathrm{M}_{b} \times v_{\text {max }_{b}}+\mathrm{M}_{r} \times v_{\text {max }_{r}}\right) m_{H_{2}} \\
\mathrm{E}=\frac{1}{2}\left(\mathrm{M}_{b} \times v_{\text {max }_{b}}^{2}+\mathrm{M}_{r} \times v_{\text {max }_{r}}^{2}\right) \\
\dot{\mathrm{M}}=\frac{\mathrm{M}_{\text {out }}}{t}
\end{gathered}
$$

donde $\mathrm{N}_{b}$ y $\mathrm{N}_{r}$ son las densidades columnares, size $b$ y size $r$ los tamaños, $\mathrm{M}_{b}$ y $\mathrm{M}_{r}$ las masas y $v_{m a z_{b}}$ y $v_{m a z_{r}}$ las velocidades de los lóbulos azul y rojo, respectivamente.

Estos parámetros se listan en la Tabla 4.6, en donde $\mathrm{M}_{\text {out }}$ es la masa, $p$ el momento lineal, E la energía, $t$ la edad, $\dot{M}$ la tasa de pérdida de masa y $v_{\max }$ la velocidad del outfow. Estos parámetros están de acuerdo con los mencionados en la Sección 1.4.6 y con los encontrados por Beuther et al. (2002).

La Figura 4.10 muestra la distribución del ${ }^{12} \mathrm{CO}(2-1)$ en la región observada en un rango de velocidades entre -20 y $-24.8 \mathrm{~km} \mathrm{~s}^{-1}$ cada $0.2 \mathrm{~km} \mathrm{~s}^{-1}$. Los contornos corresponden a 2, 5 , 10,15 y $20 \mathrm{~K}$. Entre -21.8 y $-24.8 \mathrm{~km} \mathrm{~s}^{-1}$ es evidente que el gas molecular delinea el borde de la región Hir, además de identificarse emisión perteneciente al ala roja, y entre -22 y -23 $\mathrm{km} \mathrm{s}^{-1}$ se pueden distinguir los tres grumos. La emisión proveniente del ala azul puede verse entre -20 y $-21.8 \mathrm{~km} \mathrm{~s}^{-1}$. 


\begin{tabular}{lcccc}
\hline \hline & $\begin{array}{c}\text { Area } \\
{\left[\mathrm{K} \mathrm{km} \mathrm{s}^{-1}\right]}\end{array}$ & $\begin{array}{c}\text { Velocidad } \\
{\left[\mathrm{km} \mathrm{s}^{-1}\right]}\end{array}$ & $\begin{array}{c}\Delta \mathrm{v} \\
{\left[\mathrm{km} \mathrm{s}^{-1}\right]}\end{array}$ & $\begin{array}{c}\text { Temperatura máxima } \\
{[\mathrm{K}]}\end{array}$ \\
\hline Lóbulo rojo & & & & 18.5 \\
\hline Componente principal & 39.3 & -22.3 & 2.0 & 2.8 \\
Componente secundaria & 3.9 & -20.5 & 1.3 & 19.7 \\
\hline Lóbulo azul & & & & 2.1 \\
\hline Componente principal & 39.8 & -23.0 & 1.9 & 1.7 \\
Componente secundaria & 3.8 & -24.6 & 1.7 & \\
\hline \hline
\end{tabular}

Tabla 4.7: IRAS 10349-5824: Parámetros de los ajustes gaussianos de los perfiles de ${ }^{12} \mathrm{CO}(2-1)$ en dirección a los lóbulos.

\begin{tabular}{lcccc}
\hline \hline & $\begin{array}{c}\text { Area } \\
{\left[\mathrm{K} \mathrm{km} \mathrm{s}^{-1}\right]}\end{array}$ & $\begin{array}{c}\text { Velocidad } \\
{\left[\mathrm{km} \mathrm{s}^{-1}\right]}\end{array}$ & $\begin{array}{c}\Delta \mathrm{v} \\
{\left[\mathrm{km} \mathrm{s}^{-1}\right]}\end{array}$ & $\begin{array}{c}\text { Temperatura máxima } \\
{[\mathrm{K}]}\end{array}$ \\
\hline Grumo 1 & & & & \\
\hline${ }^{12} \mathrm{CO}(2-1)$ & 37.0 & -23.1 & 2.2 & 16.1 \\
${ }^{13} \mathrm{CO}(2-1)$ & 7.7 & -23.2 & 1.8 & 3.9 \\
\hline Grumo 2 & & & & \\
\hline${ }^{12} \mathrm{CO}(2-1)$ & 37.1 & -23.1 & 2.0 & 17.3 \\
${ }^{13} \mathrm{CO}(2-1)$ & 9.0 & -23.3 & 1.5 & 5.5 \\
$\mathrm{C}^{18} \mathrm{O}(2-1)$ & 0.8 & -23.5 & 1.1 & 0.7 \\
\hline Grumo 3 & & & & \\
\hline${ }^{12} \mathrm{CO}(2-1)$ & 33.7 & -22.3 & 2.4 & 13.4 \\
${ }^{13} \mathrm{CO}(2-1)$ & 5.7 & -22.2 & 2.3 & 2.4 \\
\hline \hline
\end{tabular}

Tabla 4.8: IRAS 10349-5824 Parámetros de los ajustes gaussianos utilizados para el cálculo de densidades y masas.

La emisión extendida en $4.5 \mu \mathrm{m}$ (tal como la que se observa en la cavidad de gas molecular centrada en RA, Dec.(J2000) = 10:36:48.70, -58:40:11) es típica de los EGOs (Extended Green Objects) y sugiere la presencia de flujos bipolares ya que se origina en gas chocado (Cyganowski et al., 2008). Se observa que la distribución de esta emisión es elongada en sentido sudoeste-noreste, lo que sugiere la dirección en que se extenderían los flujos bipolares (ver panel inferior izquierdo de la Figura 4.8).

Esta dirección coincide con aquella en la que están alineados el lóbulo azul, la cavidad y el lóbulo rojo. Además se puede distinguir una fuente puntual a $24 \mu \mathrm{m}$ (ver Figura 4.11) en coincidencia con el máximo de emisión a $4.5 \mu \mathrm{m}$. Esta fuente puntual en $24 \mu \mathrm{m}$ indica la presencia de polvo tibio, lo que permite inferir que allí se encontraría una región de gas excitado posible consecuencia de la formación de una nueva estrella masiva.

Utilizando el criterio de Koenig et al. (2012) se identificaron 2 fuentes WISE clasificadas como clase II. Sus posiciones y flujos se encuentran listados en la Tabla 4.3. Una de ellas coincide con la fuente puntual detectada en $24 \mu \mathrm{m}$ (ver Figura 4.11) y con el máximo de la emisión en $4.5 \mu \mathrm{m}$ que indica la presencia de gas chocado. Además se encuentra rodeada por emisión en $8 \mu \mathrm{m}$ proveniente de los PAHs. Una pequeña cavidad coincidente con las fuentes WISE se evidencia también en el lejano IR (350, 500 y $870 \mu \mathrm{m}$, ver Figura 4.11). La imagen revela que las dos fuentes están alineadas en la dirección de elongación de la emisión en 4.5 $\mu \mathrm{m}$.

Para calcular los parámetros del gas molecular se ajustaron gaussianas a los perfiles promedio de cada grumo y se consideró la línea de ${ }^{12} \mathrm{CO}(2-1)$ como ópticamente gruesa y la de 

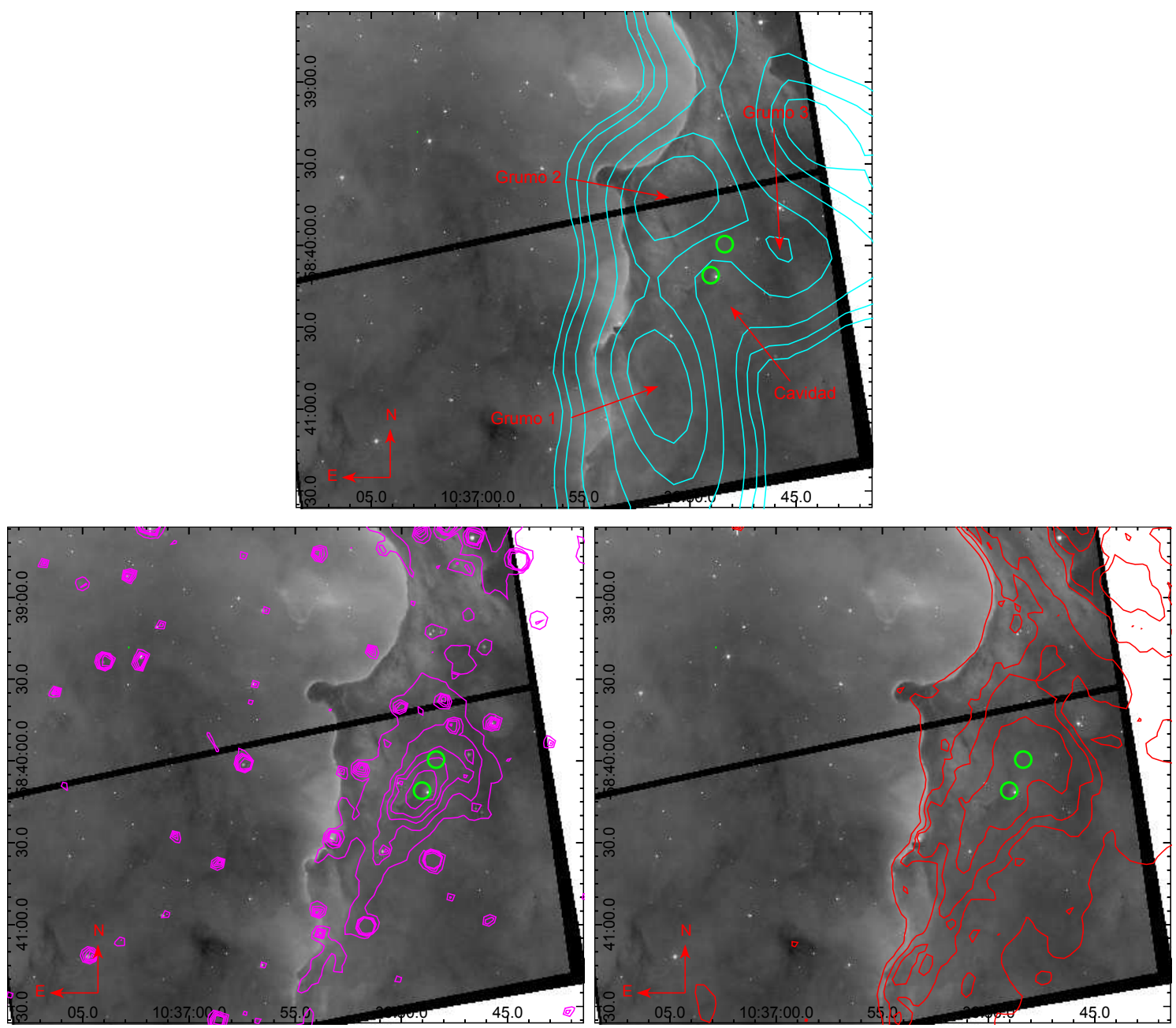

Figura 4.8: IRAS 10349-5824 Superposición de la imagen del Telescopio Espacial Hubble (Hubble Space Telescope, HST) de Gum 31 en la región de la fuente IRAS10349-5824 con los contornos correspondientes a: Arriba: emisión de ${ }^{12} \mathrm{CO}(2-1)$ a $-23 \mathrm{~km} \mathrm{~s}^{-1}(2,3,5,10,15 \mathrm{y}$ $20 \mathrm{~K})$. Los círculos verdes indican la posición y tamaño de las fuentes WISE detectadas en la región. Abajo izquierda: emisión en $4.5 \mu \mathrm{m}(4,6,8,10$, y $15 \mathrm{MJy} / \mathrm{sr})$. Abajo derecha: emisión en $8 \mu \mathrm{m}(50,75,100$ y $150 \mathrm{MJy} / \mathrm{sr})$. 

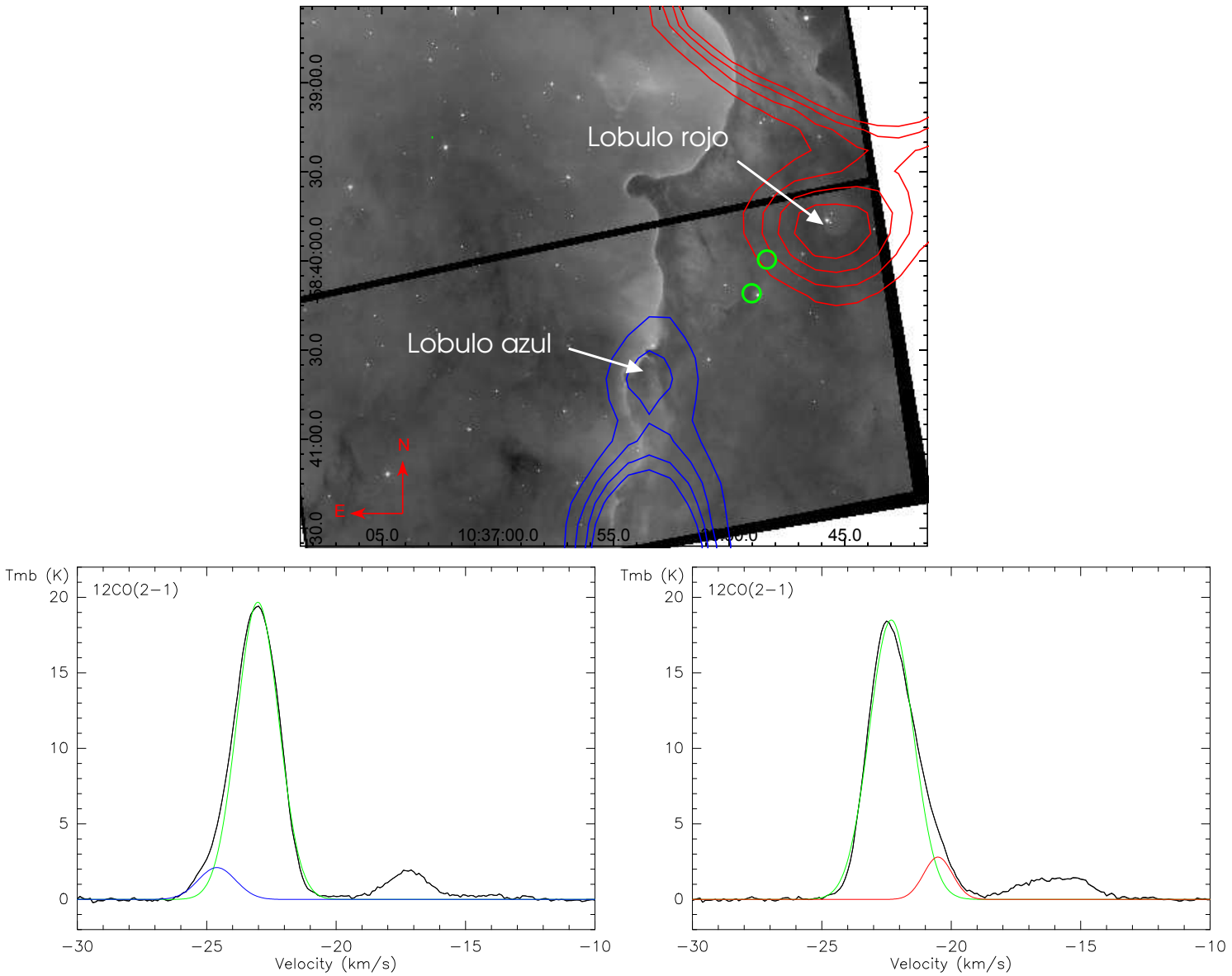

Figura 4.9: IRAS 10349-5824 En contornos se muestra la emisión de ${ }^{12} \mathrm{CO}(2-1)$ a -20.5 $\mathrm{km} \mathrm{s}^{-1}$ (contornos rojos, 1.5, 2.5, 3.5 y $4.5 \mathrm{~K}$ ) y a $-25 \mathrm{~km} \mathrm{~s}^{-1}$ (contornos azules, 1.5, 2.5, 3.5 y $4.5 \mathrm{~K}$ ) sobre la imagen de HST. Los círculos verdes indican la posición y tamaño de las fuentes WISE detectadas en la región. En los paneles inferiores se muestran los perfiles de ${ }^{12} \mathrm{CO}(2-1)$ en dirección a los máximos de emisión correspondientes a cada uno de los lóbulos. Se requiere de dos gaussianas para ajustar los perfiles. 


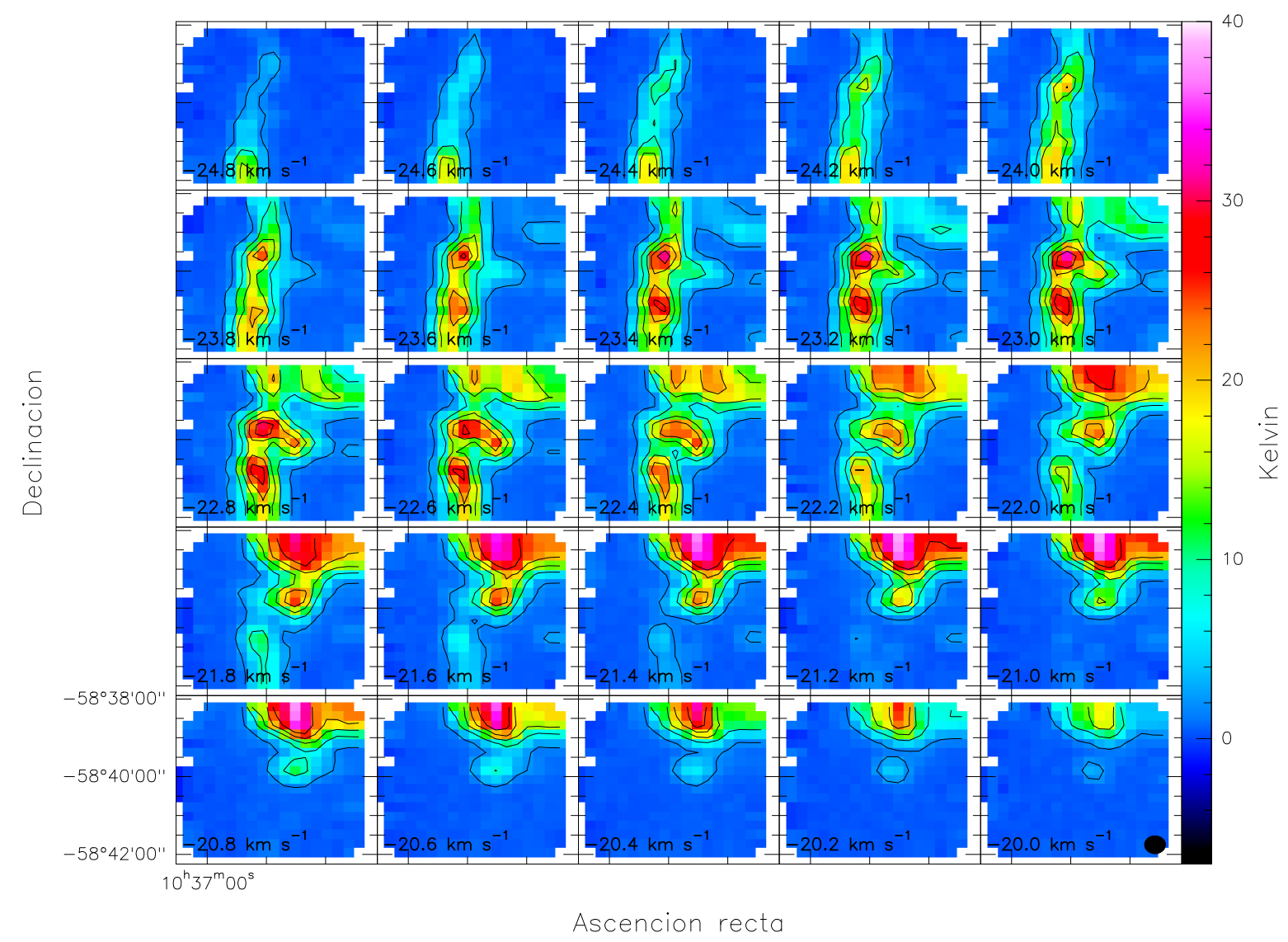

Figura 4.10: IRAS 10349-5824 Emisión de ${ }^{12} \mathrm{CO}$ en la región $3^{\prime} \times 3^{\prime}$ asociada a la fuente IRAS 10349-5824 en el rango de velocidades de $-20 \mathrm{a}-24.8 \mathrm{~km} \mathrm{~s}^{-1}$. Los contornos corresponden a 2, 8, 14, 20 y $26 \mathrm{~K}$. La posición de la fuente coincide con el centro del mapa. 
${ }^{13} \mathrm{CO}(2-1)$ como ópticamente delgada (para esta región no se usó $\mathrm{C}^{18} \mathrm{O}(2-1)$ para el cálculo de parámetros ya que sólo un grumo se ve en esta línea). Los perfiles promedio de cada grumo se muestran en la Figura 4.12 y los parámetros de sus ajustes en la Tabla 4.8. Para una temperatura de excitación de $20 \mathrm{~K}$ calculada a partir de la ecuación 2.21 se obtuvieron masas de 22, 30 у $9 \mathrm{M}_{\odot}$ para los grumos usando la ecuación 2.38. Además aplicando la ecuación 2.39 se estimaron densidades ambientales en el rango de $(3.2-4.2) \times 10^{3} \mathrm{~cm}^{-3}$. Los valores calculados de masas, densidades ambientales y radios (listados en la Tabla 4.5), junto con la distribución de la emisión en 24 y $8 \mu \mathrm{m}$ y la presencia de fuentes infrarrojas son compatibles con una región de formación estelar.

Para calcular las masas de virial es necesario contar con líneas ópticamente delgadas. Dado que las profundidades ópticas de ${ }^{13} \mathrm{CO}$ para los grumos definidos en esta región toman valores de entre 0.2 y 0.37 es posible utilizar este isótopo para calcular las masas de virial. Para los Grumos 1 y 3, que no se detectan en $\mathrm{C}^{18} \mathrm{O}(2-1)$, se utilizó ${ }^{13} \mathrm{CO}(2-1)$ y se obtuvieron masas de entre 119 y $198 \mathrm{M}_{\odot}$ para el Grumo 1 y de entre 134 y 223 para el Grumo 3 . Esto corresponde a cocientes $M_{V I R} / M\left(H_{2}\right)$ de 5.4 a 9 y de 14.8 a 24.7 , respectivamente. Para el Grumo 2, $M_{V I R}$ se calculó a partir de $\mathrm{C}^{18} \mathrm{O}(2-1)$. Se encontraron masa de virial de entre $47 \mathrm{y}$ $79 \mathrm{M}_{\odot}$, que corresponden a cocientes $M_{V I R} / M\left(H_{2}\right)$ de entre 1.5 y 2.6. Los errores en $M_{V I R}$ son de al menos $12 \%$ dado la incerteza en la distancia. Los valores de los cocientes mayores que uno indican que los grumos no estarían colapsando allí.

El escenario que se propone para esta región es el de un grumo que ya ha colapsado y comenzado a formar estrellas. Estas estrellas, que coincidirían con las fuentes IR y con la emisión a $24 \mu \mathrm{m}$, estarían calentando y perturbando el medio circundante y generando una pequeña cavidad que se detecta en el gas molecular y en la emisión del polvo en el lejano IR. Los Grumos 1, 2 y 3 formarían parte de los restos del grumo a partir del que se han empezado a formar las nuevas estrellas, lo que es consistente con el hecho de que sus masas sean pequeñas y no se encuentren candidatos a YSOs sobre ellos. Este escenario concuerda también con el hecho de que se observe emisión extendida a $4.5 \mu \mathrm{m}$, que indica la existencia de gas chocado. La forma elongada de esta emisión junto con la presencia de gas molecular detectado en las alas roja y azul indicaría un posible outflow, que es una característica típica de estrellas masivas en formación.

En el capítulo siguiente se complementará el análisis de esta región a partir de espectros observados en el IR cercano utilizando el telecopio Gemini, a fin de corroborar las hipótesis aquí enunciadas.

\subsection{MASS J10365763-5844052}

La Figura 4.13 muestra la emisión de ${ }^{12} \mathrm{CO}(2-1)$ en un rango de velocidades de -19.6 a $-26.8 \mathrm{~km} \mathrm{~s}^{-1}$, en intervalos de $0.3 \mathrm{~km} \mathrm{~s}^{-1}$. La emisión más intensa se encuentra entre $-22.6 \mathrm{y}$ -23.8. Esta emisión delinea el borde de la región Gum 31, a excepción de una cavidad cercana a la posición de la fuente 2MASS claramente visible en el rango de velocidades entre -22.9 y -24.1 .

Como se puede ver en las Figuras 4.14 y 4.15 la emisión de ${ }^{13} \mathrm{CO}(2-1)$ y $\mathrm{C}^{18} \mathrm{O}(2-1)$ revela dos grumos densos en un rango de velocidades entre -22 y $-24 \mathrm{~km} \mathrm{~s}^{-1}$, que coinciden con la velocidad en que se encuentra la emisión más intensa de ${ }^{12} \mathrm{CO}(2-1)$.

En la Figura 4.16 se muestra el promedio de la emisión de ${ }^{13} \mathrm{CO}(2-1)$ entre -25.9 y -21.1 $\mathrm{km} \mathrm{s}^{-1}$ en torno a la fuente 2MASS J10365763-5844052. La fuente se encuentra cerca del borde de la región HII que se ve delineado por la emisión en $8 \mu \mathrm{m}$ indicando la PDR. Como se mencionó antes, la emisión en $24 \mu \mathrm{m}$ muestra el polvo tibio en el interior de Gum 31. Además se detecta una fuente puntual muy intensa en $24 \mu \mathrm{m}$ en el centro del mapa que coincide con 

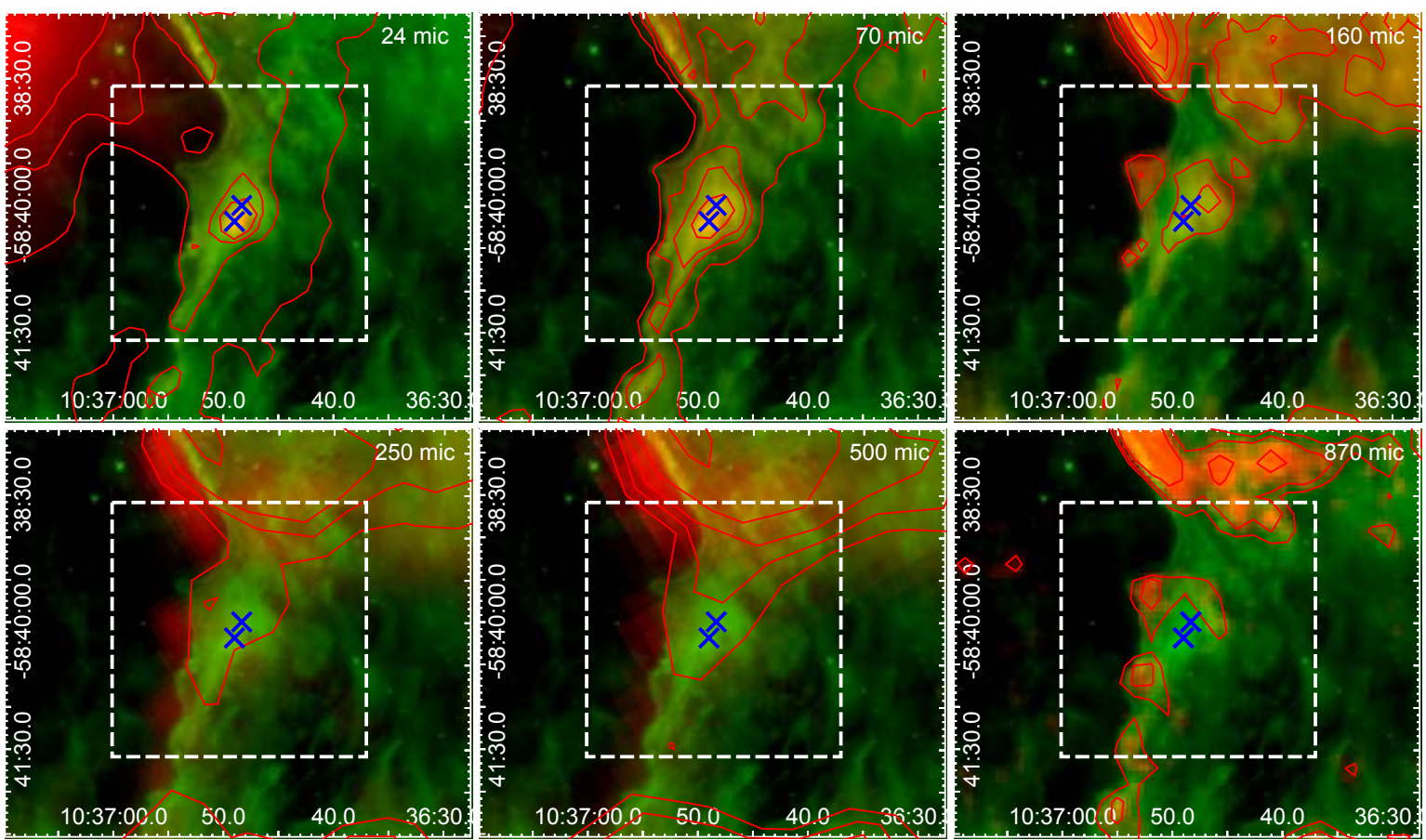

Figura 4.11: IRAS 10349-5824 Imagen de $8 \mu \mathrm{m}$ (verde) superpuesta a las de 24, 70, 160, 350, 500 y $870 \mu \mathrm{m}$ (rojo). El cuadrado muestra la región observada con APEX. La cruces indican las posiciones de fuentes WISE clase II.

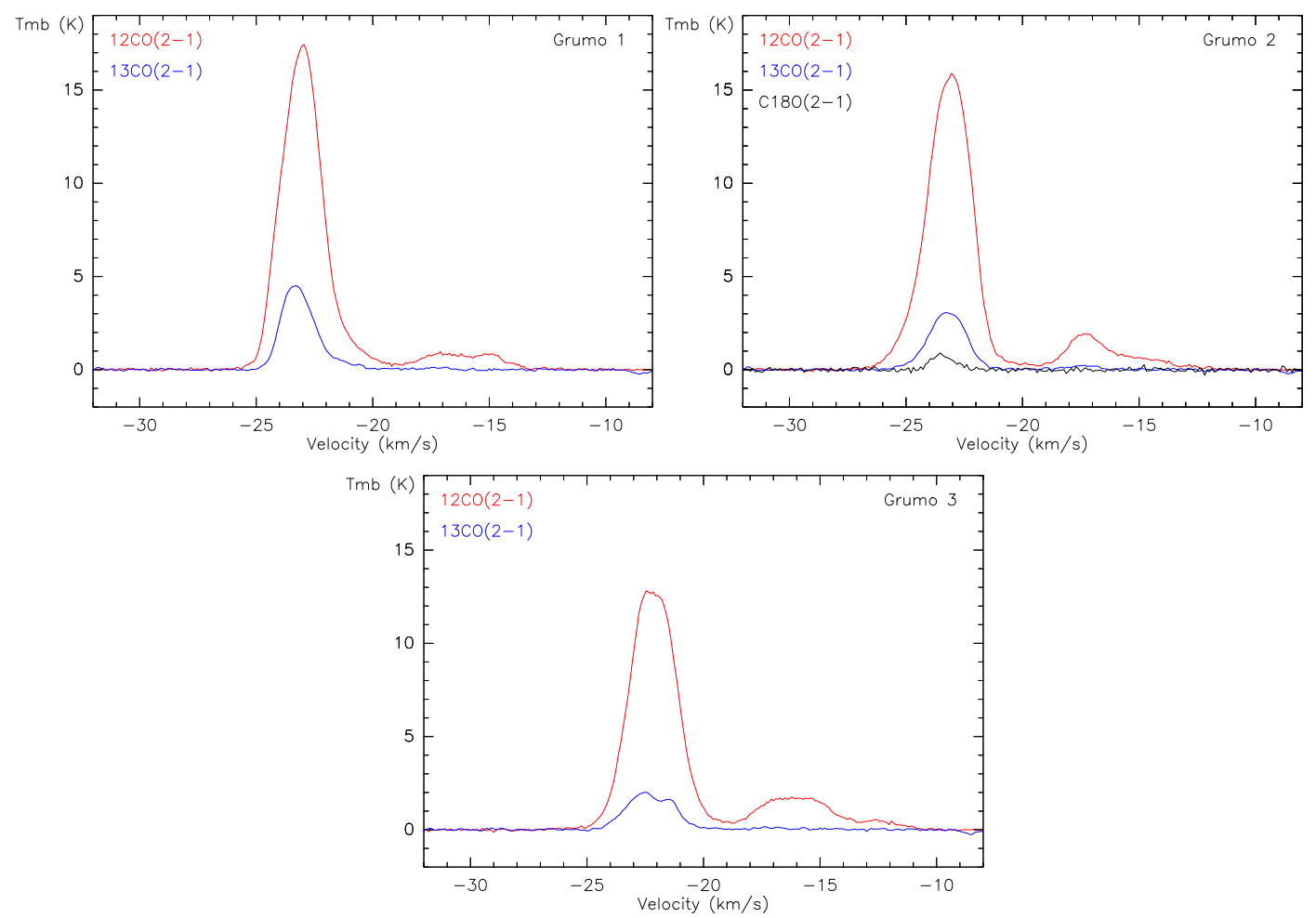

Figura 4.12: IRAS 10349-5824 Perfiles promedio para cada uno de los grumos. Se muestra en rojo la emisión de ${ }^{12} \mathrm{CO}(2-1)$, en azul la de ${ }^{13} \mathrm{CO}(2-1)$ y en negro la de $\mathrm{C}^{18} \mathrm{O}(2-1)$. Arriba izquierda: Grumo 1; Arriba derecha: Grumo 2; Abajo: Grumo 3. 
la posición de la fuente 2MASS J10365763-5844052. Ésta también presenta emisión en 4.5 y $8 \mu \mathrm{m}$. Como se ve en la Figura 4.17 estos grumos densos se detectan también en la emisión a 160, 350 y $500 \mu \mathrm{m}$ de Herschel y en $870 \mu \mathrm{m}$ LABOCA indicando la existencia de polvo frío.

La Figura 4.18 muestra los perfiles promedio correspondientes a cada uno de los grumos. En ambos la intensidad de la emisión de ${ }^{12} \mathrm{CO}(2-1)$ es similar, pero el Grumo 2 presenta emisión más intensa en ${ }^{13} \mathrm{CO}(2-1)$ y $\mathrm{C}^{18} \mathrm{O}(2-1)$ lo que indicaría que este grumo es más denso, tal como lo sugiere la emisión más intensa de polvo frío. Los ajustes gaussianos de estos perfiles se muestran en la Tabla 4.9.

A partir de estos datos no puede confirmarse si la pequeña cavidad de radio efectivo 1'.2 detectada en ${ }^{12} \mathrm{CO}(2-1)$ entre -24.1 y -23 se haya originado en la fuente 2MASS, en parte por su posición excéntrica y en parte porque no hay evidencia de la cavidad en 5.8 y en 8 $\mu \mathrm{m}$. Sin embargo la fuente en $24 \mu \mathrm{m}$ podría corresponder a una estrella excitatriz.

Asumiendo que la molécula de ${ }^{12} \mathrm{CO}(2-1)$ es ópticamente gruesa y la de $\mathrm{C}^{18} \mathrm{O}(2-1)$ es ópticamente delgada, y considerando los parámetros de los ajustes gaussianos realizados se calcularon a partir de las ecuaciones 2.21 y 2.22 temperaturas de excitación promedio de 28 y $26 \mathrm{~K}$ y profundidades ópticas para el $\mathrm{C}^{18} \mathrm{O}$ de 0.03 y 0.1 para los grumos 1 y 2 respectivamente, corroborando que la misma es ópticamente delgada. A partir de estas temperaturas de excitación y usando las ecuaciones 2.33, 2.38 y 2.39 se estimaron densidades columnares, masas y densidades ambientales para los grumos. Estos parámetros se encuentran listados en la Tabla 4.5 .

Para conocer el valor de la profundidad óptica de ${ }^{13} \mathrm{CO}$ y saber si se trata de una molécula ópticamente delgada o gruesa, se puede utilizar la ecuación 2.27. En este caso se obtuvieron valores de $\tau_{13}=0.4$ y 1.1 para los grumos 1 y 2 respectivamente, lo que significa que el ${ }^{13} \mathrm{CO}$ es ópticamente delgado sólo en el grumo menos denso.

Los valores de masa de virial obtenidos son de entre 150 y $251 \mathrm{M}_{\odot}$ para el Grumo 1 y de entre 84 y $140 \mathrm{M}_{\odot}$ para el Grumo 2 , lo que implica que para el Grumo $1 M_{V I R} / M\left(H_{2}\right)>1$ y para el Grumo $2 M_{V I R} / M\left(H_{2}\right)<1$, por lo que el primero podría frenar el colapso y el segundo podría estar contrayéndose.

No se han encontrado fuentes WISE en la región observada por APEX, ni asociadas a la fuente 2MASS ni a ninguno de los grumos. Sin embargo, como se puede ver en la Figura 4.17 se encuentra 1 fuente clase I y 3 fuentes clase II en los bordes de la región observada. A pesar de no tener observaciones de moléculas se pueden hacer algunas especulaciones respecto de lo que podría significar la presencia de estas fuentes IR analizando la emisión en distintas bandas del IR.

La fuente clase I (RA, Dec.(J2000)= 10:36:37.245, -58:42:44.21) coincide con emisión en el lejano IR $(\lambda \geq 160 \mu \mathrm{m})$ y se detecta claramente sobre un máximo en la emisión de 870 $\mu \mathrm{m}$. Ésta no ha sido clasificada como grumo por Duronea et al. (2015), posiblemente porque la emisión es débil. Sin embargo es posible que en esta posición se encuentre un grumo de gas molecular y la fuente IR podría ser una estrella muy joven formándose allí. Serían necesarias observaciones de gas molecular en esa región para determinar si efectivamente esto es así. Dos de las fuentes clase II, que son algo más evolucionadas que la de clase I, se encuentran asociadas a una fuente puntual detectada en 24 y $70 \mu \mathrm{m}$ (RA, Dec.(J2000) = 10:37:00.954, $-58: 46: 26.98)$, lo que indica la presencia de polvo tibio y fuentes excitatrices. Podría tratarse entonces de estrellas en formación algo más evolucionadas que la anterior. 


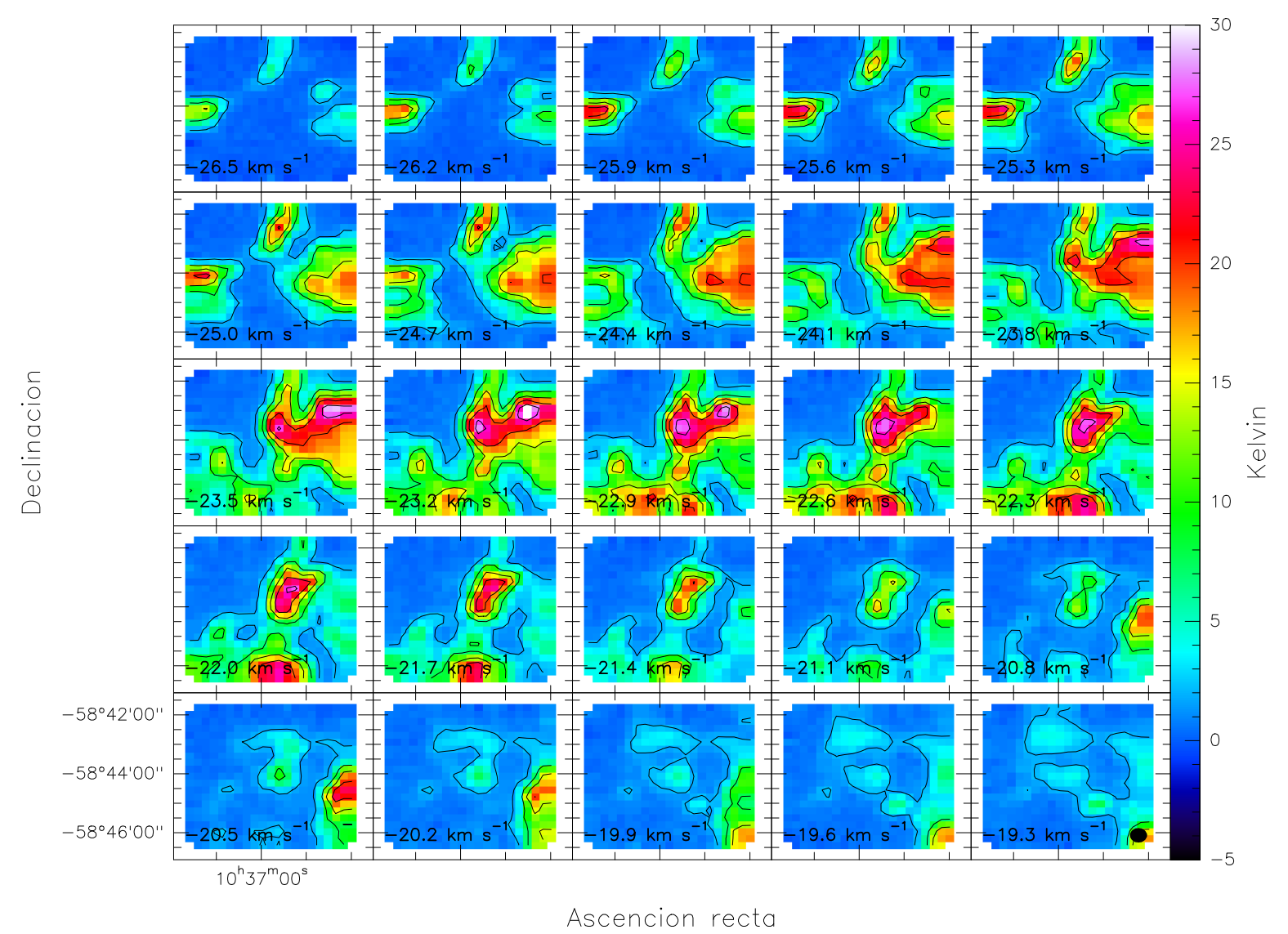

Figura 4.13: 2MASS J10365763-5844052 Emisión de ${ }^{12} \mathrm{CO}$ en la región $4^{\prime} \times 4^{\prime}$ asociada a la fuente 2MASS J10365763-5844052 en el rango de velocidades de $-19.3 \mathrm{a}-26.5 \mathrm{~km} \mathrm{~s}^{-1}$. Contornos: 2, 8, 14, 20 y $26 \mathrm{~K}$. La posición de la fuente 2MASS coincide con el centro del mapa.

\begin{tabular}{lcccc}
\hline \hline & $\begin{array}{c}\text { Area } \\
{\left[\mathrm{K} \mathrm{km} \mathrm{s}^{-1}\right]}\end{array}$ & $\begin{array}{c}\text { Velocidad } \\
{\left[\mathrm{km} \mathrm{s}^{-1}\right]}\end{array}$ & $\begin{array}{c}\Delta \mathrm{v} \\
{\left[\mathrm{km} \mathrm{s}^{-1}\right]}\end{array}$ & $\begin{array}{c}\text { Temperatura máxima } \\
{[\mathrm{K}]}\end{array}$ \\
\hline Grumo 1 & & & & \\
\hline${ }^{12} \mathrm{CO}(2-1)$ & 71.5 & -22.8 & 2.9 & 22.9 \\
${ }^{13} \mathrm{CO}(2-1)$ & 15.5 & -22.9 & 2.1 & 7.1 \\
$\mathrm{C}^{18} \mathrm{O}(2-1)$ & 1.4 & -23.0 & 1.7 & 0.8 \\
\hline Grumo 2 & & & & \\
\hline${ }^{12} \mathrm{CO}(2-1)$ & 55.1 & -23.5 & 2.4 & 21.3 \\
${ }^{13} \mathrm{CO}(2-1)$ & 18.0 & -23.5 & 1.6 & 10.7 \\
$\mathrm{C}^{18} \mathrm{O}(2-1)$ & 2.6 & -23.6 & 1.2 & 2.1 \\
\hline \hline
\end{tabular}

Tabla 4.9: 2MASS J10365763-5844052 Parámetros de los ajustes gaussianos utilizados para el cálculo de densidades y masas. 


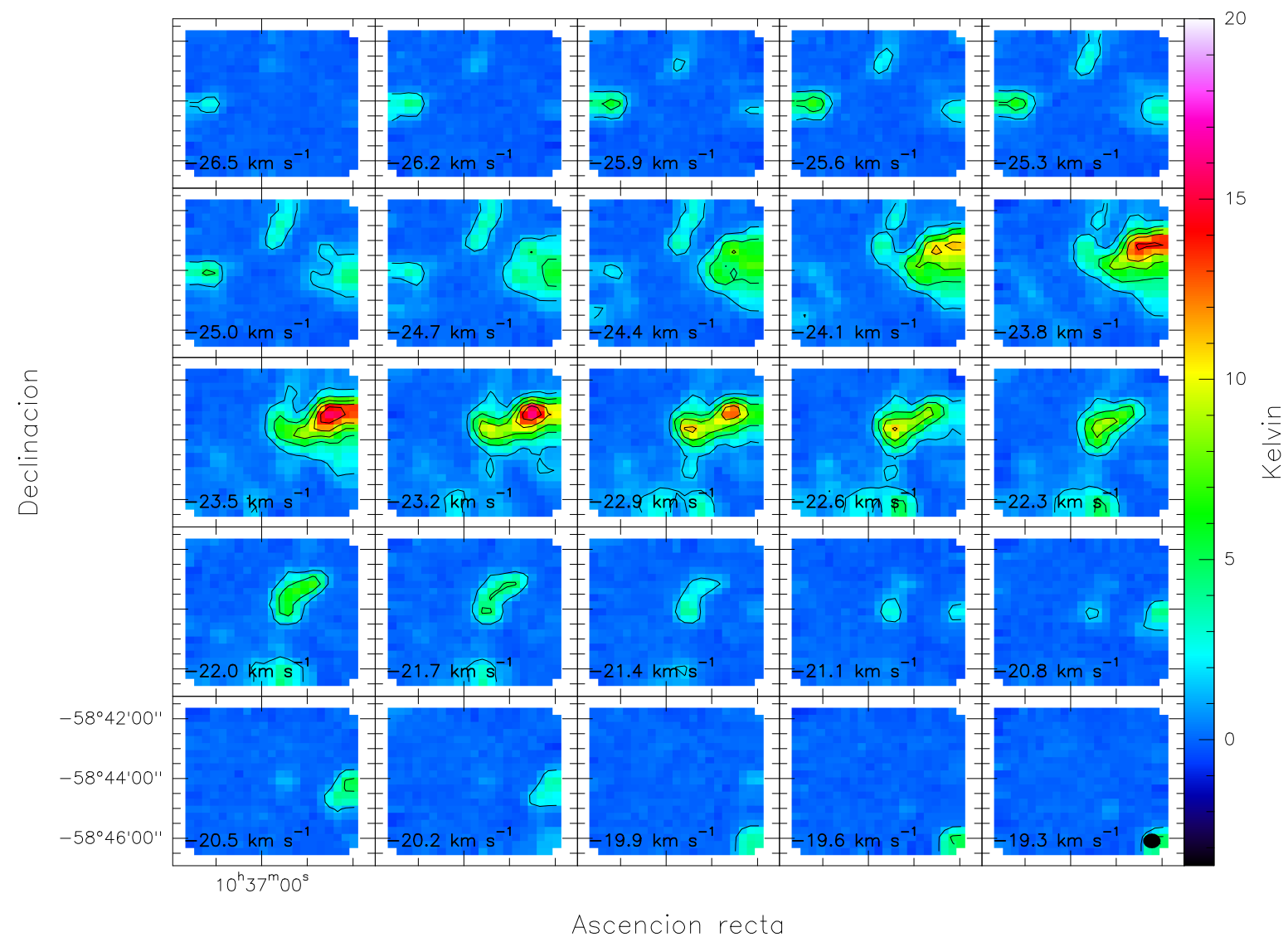

Figura 4.14: 2MASS J10365763-5844052 Emisión de ${ }^{13} \mathrm{CO}$ en la región $4^{\prime} \times 4^{\prime}$ asociada a la fuente 2MASS J10365763-5844052 en el rango de velocidades de $-19.3 \mathrm{a}-26.5 \mathrm{~km} \mathrm{~s}^{-1}$. Contornos: $1.5,4.5,7.5,10.5$ y $13.5 \mathrm{~K}$. La posición de la fuente 2MASS coincide con el centro del mapa. 


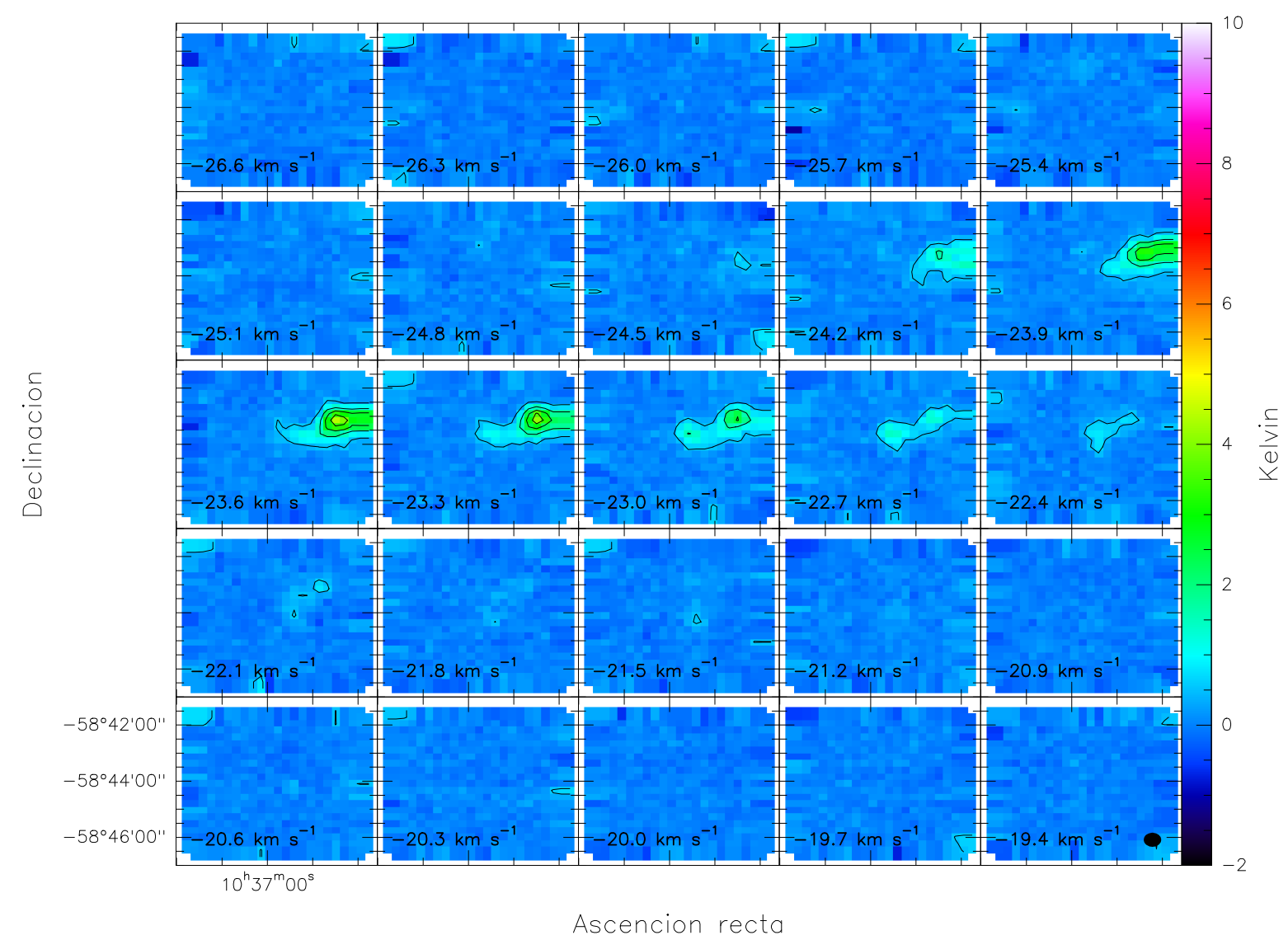

Figura 4.15: 2MASS J10365763-5844052 Emisión de $\mathrm{C}^{18} \mathrm{O}$ en la región $4^{\prime} \times 4^{\prime}$ asociada a la fuente 2MASS J10365763-5844052 en el rango de velocidades de -19.4 a $-26.6 \mathrm{~km} \mathrm{~s}^{-1}$. Contornos: $0.5,1,1.5$ y $2 \mathrm{~K}$. La posición de la fuente 2MASS coincide con el centro del mapa. 


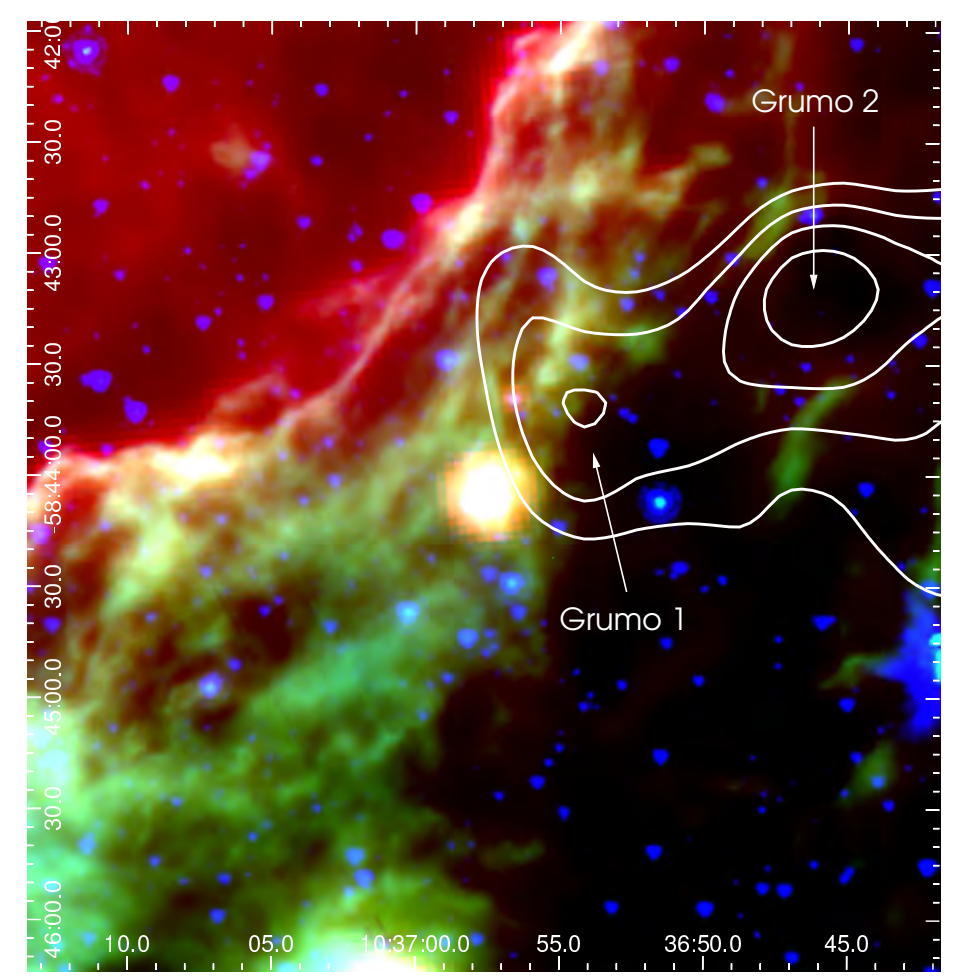

Figura 4.16: 2MASS J10365763-5844052 Región observada en torno a la fuente 2MASS J10365763-5844052 ubicada en el centro de la imagen. Se muestra la emisión de Spitzer-IRAC en 4.5 (azul) y $8 \mu \mathrm{m}$ (verde), Spitzer-MIPS en $24 \mu \mathrm{m}$ en rojo, y la emisión de ${ }^{13} \mathrm{CO}$ en contornos $(2,3,4$ y $5 \mathrm{~K})$ en el intervalo $-25.9 \mathrm{a}-21.1 \mathrm{~km} \mathrm{~s}^{-1}$.
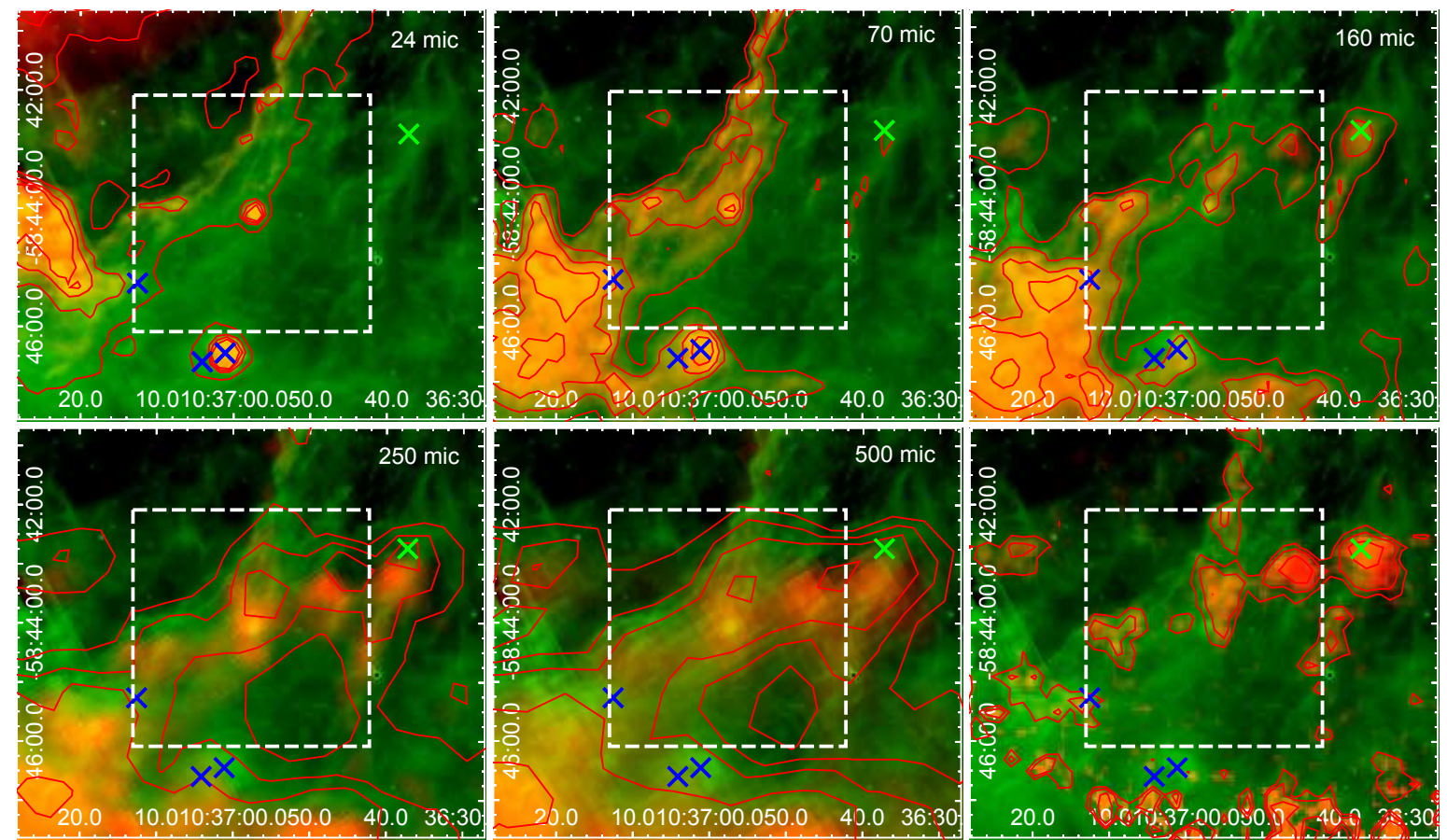

Figura 4.17: 2MASS J10365763-5844052 Imagen de $8 \mu \mathrm{m}$ (verde) superpuesta a las de 24, $70,160,350,500$ y $870 \mu \mathrm{m}$ (rojo). El cuadrado muestra la región observada con APEX. La cruces indican las posiciones de fuentes WISE clase I (verde) y clase II (azul). 

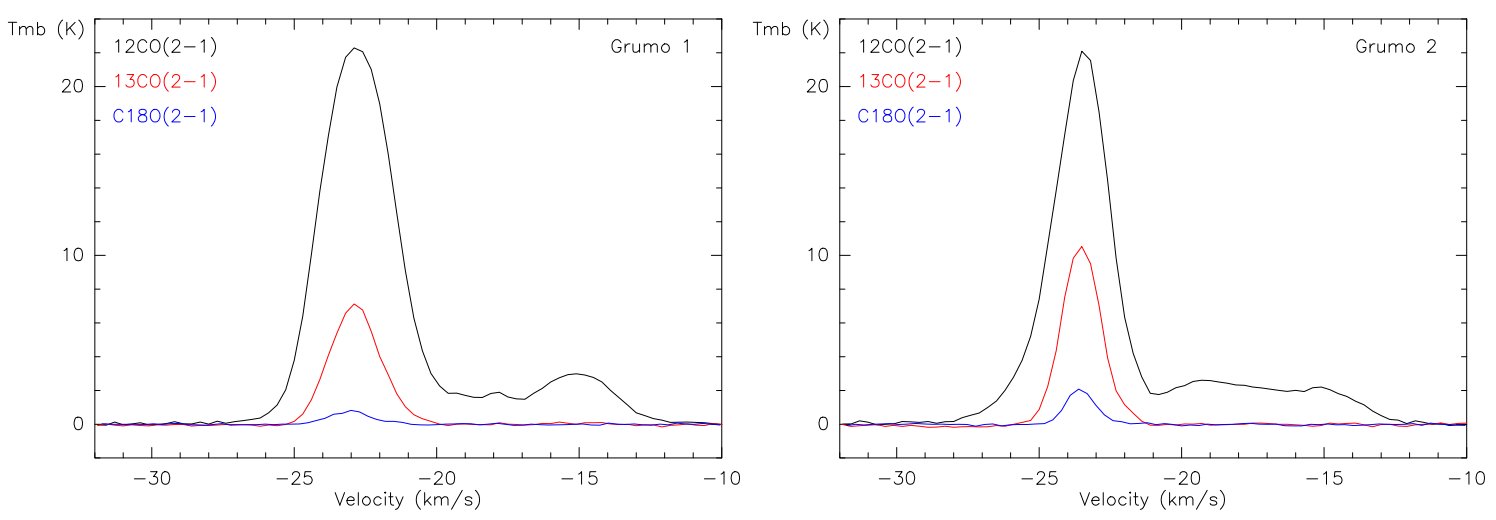

Figura 4.18: 2MASS J10365763-5844052 Perfiles promedio correspondientes al Grumo 1 (izquierda) y Grumo 2 (derecha). Se muestra en línea negra la emisión correspondiente a ${ }^{12} \mathrm{CO}(2-1)$, en rojo a ${ }^{13} \mathrm{CO}(2-1)$ y en verde a $\mathrm{C}^{18} \mathrm{O}(2-1)$.

\subsection{Conclusiones}

Según los parámetros calculados a partir de CO el Grumo asociado a la fuente IRAS 103515816 es el más denso de los estudiados en este capítulo y, además, es el único que coincide con un grumo de polvo identificado por Duronea et al. (2015). Se observa emisión de $\mathrm{HCO}^{+}(4-3)$ en dirección a este grumo lo que indica que, según los valores de densidades críticas mostrados en la Tabla 2.2, la densidad debe ser mayor de $9 \times 10^{6} \mathrm{~cm}^{-3}$. Posee una fuente WISE clasificada como candidato a YSO clase II y los valores de masas de virial calculados indican que podría estar colapsando. Es probable que se trate de un grumo denso con formación estelar.

Los tres grumos asociados a la fuente IRAS 10349-5824 se encuentran rodeando una cavidad que contiene fuentes WISE clasificadas como candidatos a YSOs clase II y en la que se observa emisión en $24 \mu \mathrm{m}$, lo que indica la presencia de fuentes excitatrices. Los valores de masas de virial calculados para los grumos indican que los mismos no estarían colapsando. Además se observa emisión extendida en $4.5 \mu \mathrm{m}$, que es típica de EGOs y sugiere la existencia de flujos bipolares indicados por la asimetría en los perfiles de ${ }^{12} \mathrm{CO}(2-1)$. El escenario propuesto para esta región es el de un grumo que ya ha colapsado y comenzado a formar estrellas, perturbando el medio circundante y formando una cavidad de $\mathrm{r} \sim 30^{\prime \prime}$. Los grumos detectados, que son de baja masa, serían los restos del grumo original que ha colapsado. Dada la evidencia de la posible presencia de flujos bipolares, y con el objetivo de detectar la línea rotovibracional de $\mathrm{H}_{2}$ en $2.16 \mu \mathrm{m}$ y líneas nebulares presentes en la misma banda IR, se llevaron a cabo observaciones en el IR utilizando el instrumento Flamingos 2 de Gemini Sur. El análisis de estos resultados se muestra en el Capítulo 5.

Finalmente en la región asociada a la fuente 2MASS J10365763-5844052 se encuentran dos grumos. El Grumo 2 es el de mayor masa y, según los valores calculados para la masa del virial, podría estar colapsando, aunque no se encuentran fuentes IR identificadas como candidatos a YSOs asociadas a los grumos. Se detectan, en cambio, fuentes IR en los bordes de la región observada, que están asociadas a emisión o bien de polvo tibio (24 y $70 \mu \mathrm{m})$ o de polvo frío (entre 160 y $870 \mu \mathrm{m}$ ).

Tal como se mencionó en la Sección 1.4.3 del Capítulo 1 las regiones Hir son consideradas agentes disparadores de formación estelar. Dada la evidencia mostrada en el presente Capítulo y la encontrada por Vazzano et al. (2014), se puede presumir que Gum 31 estaría desencadenando la formación de nuevas generaciones de estrellas en la cáscara densa que la rodea. El mecanismo que podría estar actuando es el collect and collapse, en el que la acción 
de frentes de choque generados en la expansión de una región HiI produce una cáscara densa en donde se dan las condiciones para el colapso y fragmentación de parte de su envoltura molecular. Es poco probable que en la región de Gum 31, donde están los objetos estudiados, se encuentre en marcha el proceso RDI, dada la ausencia de los típicos bordes brillantes y glóbulos cometarios que lo caracterizan. 


\section{Capítulo 5}

\section{Espectroscopía Flamingos 2 en la región de IRAS 10349-5824}

Como se mencionó en el capítulo anterior, usando catálogos de fuentes puntuales infrarrojas, Cappa et al. (2008) y Vazzano et al. (2014) identificaron varios candidatos a objetos estelares jóvenes (YSOs) asociados a la fuente IRAS 10349-5824, localizada en los bordes de la región HiI Gum 31. Estos YSOs están generalmente asociados a jets detectables en bandas ópticas e infrarrojas, emisión de $\mathrm{H}_{2}$ en el cercano IR, y objetos Herbig-Haro. En particular, la banda de $4.5 \mu \mathrm{m}$ de Spitzer-IRAC incluye, además de emisión $\operatorname{Br} \gamma$ y $\operatorname{Pf} \beta$, líneas rotovibracionales de $\mathrm{H}_{2}$ y $\mathrm{CO}$ típicas de gas chocado (Watson et al., 2008). Estas líneas de emisión molecular extendida son comunes en YSOs mientras que la emisión a $4.5 \mu \mathrm{m}$ es típica de los EGOs (Extended Green Objects), sugiriendo la presencia de outflows bipolares (Cyganowski et al., 2008) que son detectables a partir de la emisión de CO.

En este Capítulo se analiza la región asociada a IRAS 10349-5824, una zona de formación estelar joven ubicada en el borde de la región Hir Gum 31, utilizando observaciones de la línea $\mathrm{H}_{2}$ en banda $\mathrm{K}$ obtenida con Flamingos 2 en Gemini Sur y datos adicionales de Spitzer en $4.5,8$ y $24 \mu \mathrm{m}$ y de APEX en ${ }^{12} \mathrm{CO}(2-1)$, con el objetivo de corroborar la existencia de los flujos bipolares sugeridos en el Capítulo 4.

\subsection{Observaciones}

Para este estudio se combinaron las observaciones de ${ }^{12} \mathrm{CO}(2-1)$ y $\mathrm{C}^{18} \mathrm{O}(2-1)$ obtenidas con el telescopio APEX ya analizadas en el capítulo anterior que permiten determinar las características del medio ambiente en que las fuentes se encuentran, con observaciones infrarrojas obtenidas con Gemini Sur que permiten estudiar las características y estados evolutivos de las fuentes.

Con el objetivo de detectar la línea rotovibracional de $\mathrm{H}_{2}$ en $2.12 \mu \mathrm{m}$ y líneas nebulares presentes en la misma banda IR, se llevaron a cabo observaciones en el IR utilizando el instrumento Flamingos 2 de Gemini Sur. Se utilizó la red de alta resolución en la banda K combinada con una ranura de 4 pixeles de ancho, redundando en una resolución $\lambda / \Delta \lambda=$ 1500 .

La sustracción de las líneas de emisión de la atmósfera se realizó introduciendo desplazamientos espaciales a lo largo de la dirección de la ranura, de manera de optimizar el tiempo neto de observación de las fuentes de interés. La reducción de los datos se llevó a cabo utilizando las tareas específicas desarrolladas por el Observatorio Gemini, basadas en el código IRAF. Las mismas incluyen la determinación de la contribución de corriente térmica (darks), uniformidad de respuesta del detector (flats), calibración en longitud de onda, obtención 


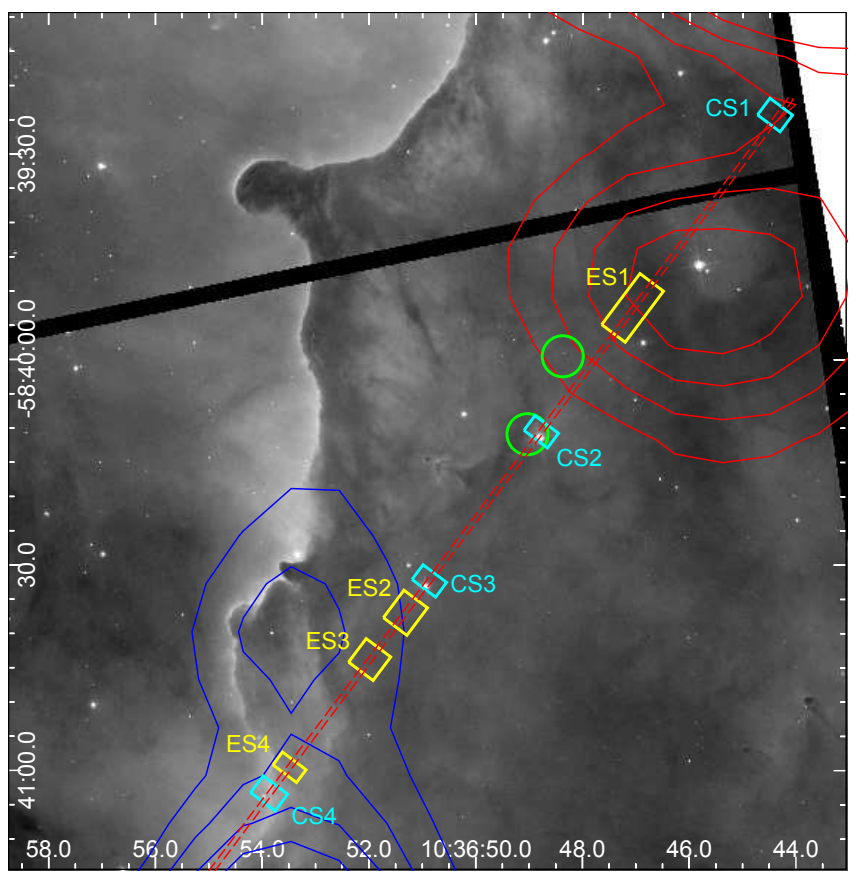

Figura 5.1: Se muestra la posición de la ranura observada con Gemini junto con las fuentes WISE catalogadas como candidatos a YSO (círculos verdes). Los contornos de ${ }^{12} \mathrm{CO}(2-1)$ son los mismos que se muestran en la Figura 4.9 del Capítulo 4. Las cajas amarillas y celestes indican las fuentes de continuo (CS) y las fuentes extendidas (ES) identificadas a partir de los datos de Gemini.

de espectros unidimensionales en regiones de interés (aperture extraction) y corrección por absorciones propias de la atmósfera terrestre (telluric absorptions) $)^{(1)}$.

Cabe aclarar que el proceso de extracción se realizó de manera artesanal, puesto que la falta de señal de continuo en algunas fuentes extendidas no permite identificar la traza del objeto en cuestión. Para ello se utiliza un objeto con emisión continua cercano a dicha fuente como guía y se realiza una extracción con una abertura desplazada de manera de incluir la señal de las líneas de emisión de interés.

Una segunda aclaración es que la estrella de referencia utilizada para la corrección por absorciones telúricas pertenece al conjunto de calibraciones obtenidas para la noche de observación por el Observatorio Gemini (Baseline Calibrations). Por lo tanto, si bien dicha calibración es suficiente para derivar cocientes relativos entre líneas de emisión detectadas, no permite obtener flujos absolutos de las líneas en cuestión. Esta estrategia de observación apunta a maximizar las chances de obtención de datos dentro del método de fila de observación propio del Observatorio, al reducir el tiempo total de la propuesta.

El estudio se completó con datos complementarios de Spitzer-IRAC en 4.5, 8 y $24 \mu \mathrm{m}$.

\subsection{Resultados}

En la Figura 4.8 del Capítulo 4 se mostraron los contornos correspondientes a la emisión de $4.5 \mu \mathrm{m}$ de Spitzer-IRAC superpuestos a la imagen óptica de la región obtenida con HST, junto con las posiciones de las fuentes WISE catalogadas como YSOs clasificados como clase II. Una de las fuentes (J103648.97-584010.7, de aquí en adelante WISE1) se encuentra proyectada

\footnotetext{
${ }^{(1)}$ La reducción de los datos de Gemini fue llevada a cabo por el Dr. Guillermo Bosch.
} 


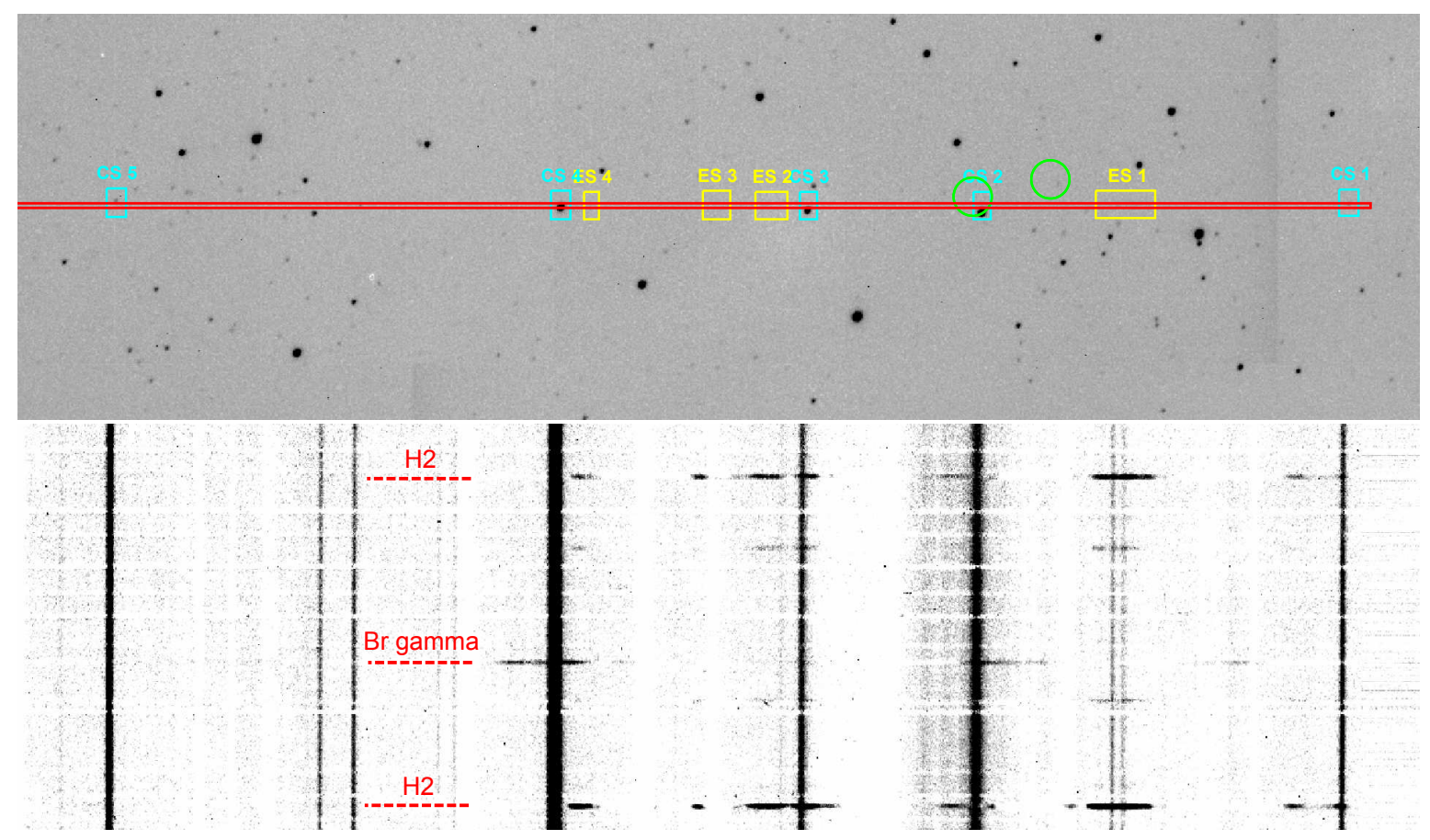

Figura 5.2: Imagen de doble panel mostrando la distribución de fuentes conspicuas a lo largo de la ranura. Las cajas celestes y amarillas identifican las fuentes de continuo (CS) y extendidas (ES) así definidas en función de la extensión espacial de sus rasgos espectrales.

sobre el máximo de la emisión extendida de $4.5 \mu \mathrm{m}$, que es típica de objetos estelares jóvenes (Cyganowski et al., 2009). Como también se mostró en la Figura 4.8 la emisión de ${ }^{12} \mathrm{CO}$ a una velocidad radial de $-23 \mathrm{~km} \mathrm{~s}^{-1}$ revela la presencia de 3 grumos en la región observada, que bordean la cavidad en la que WISE 1 está inmersa.

La Figura 5.1 muestra la posición de la ranura observada con Gemini junto con las posiciones de las fuentes WISE detectadas y los contornos de la emisión a $-20.5 \mathrm{~km} \mathrm{~s}^{-1}$ y -25 $\mathrm{km} \mathrm{s}^{-1}$ (los mismos mostrados en la Figura 4.9) que corresponden al gas molecular en las alas azul y roja a lo largo de las ranuras, lo que puede ser interpretado como outflows moleculares bipolares.

Las observaciones realizadas con Gemini permitieron identificar cinco fuentes de continuo y cuatro fuentes extendidas a lo largo de la ranura observada. Una de las fuentes de continuo no será estudiada en este capítulo ya que no se encuentra en la región de interés. En el panel superior de la Figura 5.2 se muestra la distribución de estas fuentes sobre la ranura sobre la imagen de adquisición en la banda H. Las cajas celestes y amarillas identifican las fuentes de continuo (CS) y fuentes extendidas (ES), respectivamente, definidas de acuerdo a sus características espectrales. El tamaño de las cajas amarillas define el área en donde se realizaron las extracciones para obtener los espectros unidimensionales, mientras que para las fuentes puntuales se realizó una extraccción convencional. Estas cajas se muestran también en la Figura 5.1 sobre la imagen de HST.

Tal como se muestra en la Figura 5.3, los espectros de las fuentes extendidas ES1, ES2, ES3 y ES4 presentan varias líneas de emisión de $\mathrm{H}_{2}$ y sólamente ES2 y ES4 exhiben emisión $\operatorname{Br} \gamma$. Como se puede ver en la Figura 5.1 las fuentes extendidas ES1 y ES3 coinciden con la emisión de CO identificada como los lóbulos rojo y azul, respectivamente, mientras que ES2 se encuentra en el borde del 'grumo azul' y ES4 en el borde de Gum 31. Esto podría explicar la presencia de la línea $\operatorname{Br} \gamma$ en las últimas, que revela la presencia de gas ionizado que podría 

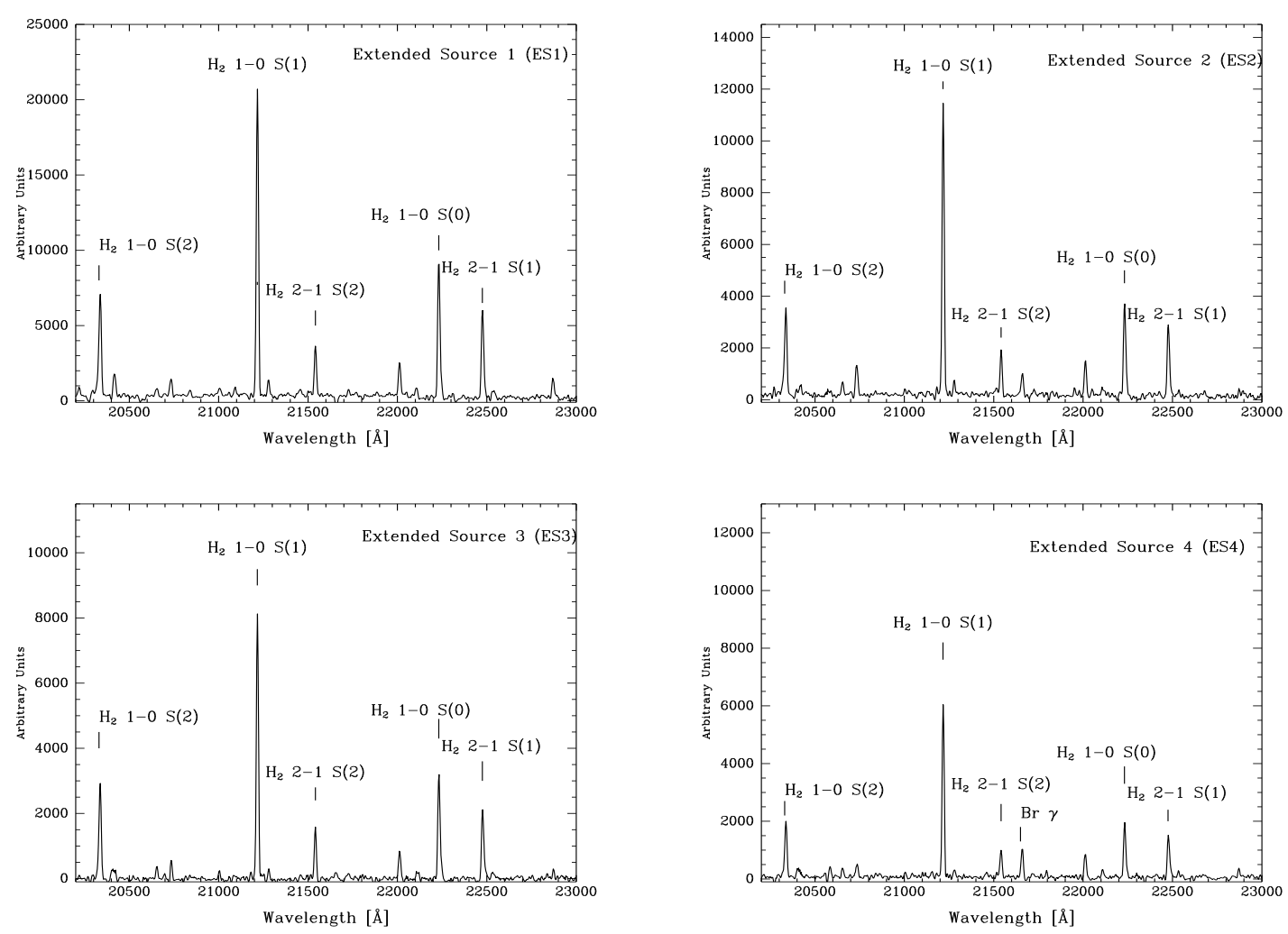

Figura 5.3: Espectro extraidos de las fuentes extendidas ES1, ES2, ES3 y ES4.
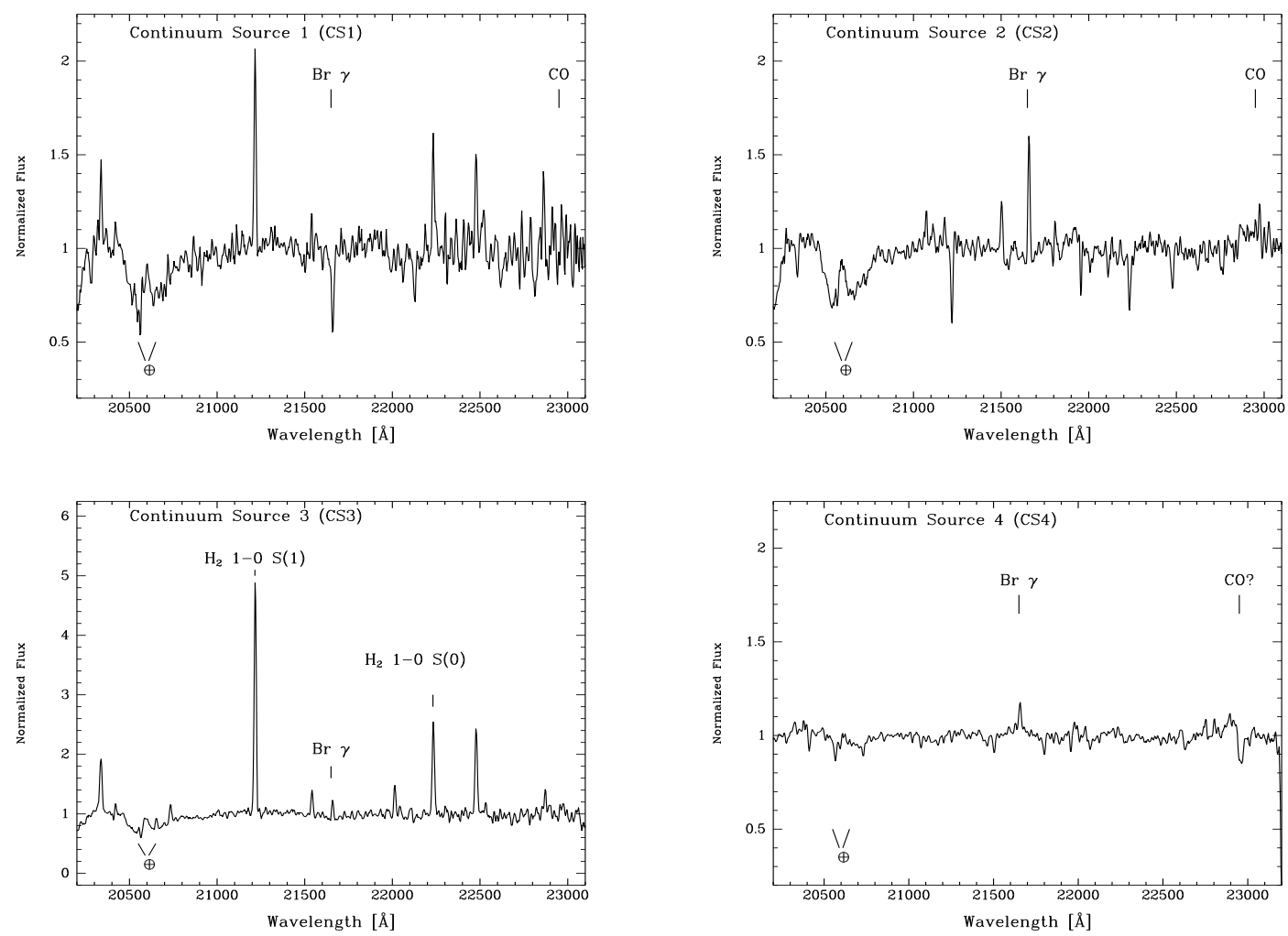

Figura 5.4: Espectro extraidos de las fuentes de continuo CS1, CS2, CS3 y CS4. 
originarse en la región HiI o ser parte de un invisible jet óptico. Se observa además que las intensidades de las líneas de $\mathrm{H}_{2}$ son mayores cuanto más alejada está la fuente del borde de la región Gum 31, siendo ES1 la que posee las líneas más intensas y ES4 las más débiles. Un análisis de la relación de las intensidades entre las líneas de $\mathrm{H}_{2}$ en una misma fuente se presentará en la Sección 5.4.

La Figura 5.4 muestra los espectros de las fuentes puntuales, en las que se puede apreciar un continuo sobre el que están montadas las líneas de emisión y/o absorción. La fuente CS1 muestra $\operatorname{Br} \gamma$ en absorción. Las líneas de Balmer en absorción corresponden a estrellas que no poseen envolturas, por lo que CS1 podría ser una estrella que no se encuentra relacionada con la región estudiada. Las líneas de $\mathrm{H}_{2}$ detectadas se encontrarían allí sólo por estar en la línea de la visual y no serían excitadas por CS1.

La fuente CS2, que coincide espacialmente con WISE 1, y la fuente CS3 presentan emisión $\operatorname{Br} \gamma$, lo que indica que tienen gas ionizado asociado. Estas fuentes se encuentran dentro de una cavidad que se detecta en el gas molecular y en la emisión del polvo en el lejano IR (ver Figura 4.8). CS3 muestra además líneas de emisión de $\mathrm{H}_{2}$, probablemente por estar en el borde de la cavidad, mientras que CS2 presenta líneas de $\mathrm{H}_{2}$ en absorción. Estas líneas de absorción podrían ser líneas espúreas que aparecen debido a la resta del cielo durante el proceso de reducción.

Finalmente, la fuente CS4, que, tal como se puede apreciar en la Figura 5.1, se encuentra en el borde de la región His, es una fuente intensa de continuo y muestra emisión $\operatorname{Br} \gamma$ pero no líneas de $\mathrm{H}_{2}$. Esto se debería a que se trata de una fuente que está fuera de las nube molecular estudiada. En todas las fuentes de continuo se puede apreciar absorción a una frecuencia de $\sim 20500 \AA$ que se debe a la absorción de nuestra atmósfera y emisión a $\sim 23000 \AA$ que corresponde a las cabezas de banda de CO.

Los resultados extraídos de los espectros mostrados en las Figuras 5.3 y 5.4 permiten proponer un escenario en el que las fuentes de continuo CS2 y CS3 se encuentran dentro de una cavidad en el gas molecular en la que hay presente gas ionizado evidenciado por la presencia de la línea $\operatorname{Br} \gamma$. Esta emisión está relacionada con el proceso de acreción, ya que se cree que la mayor parte de la emisión de Hidrógeno detectado por las líneas Br $\gamma$ identificada en los espectros de objetos estelares jóvenes proviene de las regiones de recombinación asociadas con la acreción magnetosférica del material del disco circunestelar en la estrella en formación (Najita et al., 1996; Beck et al., 2010). Las fuentes extendidas ES1, ES2 y ES3, que presentan varias líneas de emisión de $\mathrm{H}_{2}$ y se encuentran sobre la emisión de gas molecular podrían estar evidenciando la presencia de los lóbulos propuestos en el Capítulo 4.

\subsection{Modelos de objetos $\mathrm{HH}$}

Por lo descripto en el Capítulo 4 y en la Sección 5.2, la hipótesis propuesta para esta región es que la fuente WISE 1 posee outflows que están perturbando el medio. Una de las características de la interacción de estos con el gas circundante es la presencia de los llamados Objetos Herbig-Haro (ver Sección 1.4.6), que provienen del impacto de los jets originados en objetos en formación y se observan como regiones nebulares visibles en el óptico. Sin embargo en algunos casos en que las fuentes están muy inmersas en el gas y polvo es posible que no se encuentre emisión óptica. Los objetos HH pueden observarse también en longitudes de onda del IR cercano, por ejemplo en las bandas J, H y K (centradas en 1.235, 1.662 y $2.159 \mu \mathrm{m}$ ).

No se han detectado en la región asociada a IRAS 10349-5824 objetos HH en el óptico. Sin embargo no se puede descartar que estos objetos se encuentren por detrás de la emisión mostrada por la imagen de $\mathrm{H} \alpha$ de HST y parcialmente inmersos en el povo interestelar, por lo que se justifica hacer un estudio de las líneas en el IR. 


\begin{tabular}{cccccccc}
\hline \hline & $\lambda$ & Cont. UV & \multicolumn{2}{c}{ Choque no disociativo } & UV y & Formation \\
& $(\mu \mathrm{m})$ & & $\frac{n_{H}}{n}=3(-3)^{*}$ & $\frac{n_{H}}{n}=3(-2)^{*}$ & LTE & Rayos X & Pumping \\
\hline $1-0 \mathrm{~S}(2)$ & 2.033 & 0.44 & 0.29 & 0.30 & 0.36 & 0.43 & 0.53 \\
$2-1 \mathrm{~S}(3)$ & 2.073 & 0.19 & 0.018 & 0.037 & 0.074 & 0.63 & 0.65 \\
$1-0 \mathrm{~S}(1)$ & 2.121 & 1.0 & 1.0 & 1.0 & 1.0 & 1.0 & 1.0 \\
$2-1 \mathrm{~S}(2)$ & 2.154 & 0.16 & 0.013 & 0.026 & 0.029 & 0.18 & 0.17 \\
$3-2 \mathrm{~S}(3)$ & 2.201 & 0.086 & $\ll 0.01$ & $\ll 0.01$ & $\ll 0.01$ & $\ll 0.01$ & 0.26 \\
$1-0 \mathrm{~S}(0)$ & 2.223 & 0.45 & 0.22 & 0.22 & 0.21 & 0.18 & 0.16 \\
$2-1 \mathrm{~S}(1)$ & 2.247 & 0.53 & 0.046 & 0.090 & 0.080 & 0.43 & 0.43 \\
$3-2 \mathrm{~S}(2)$ & 2.286 & 0.070 & $\ll 0.01$ & $\ll 0.01$ & $\ll 0.01$ & $\ll 0.01$ & 0.069 \\
\hline \hline
\end{tabular}

Tabla 5.1: Parte de la Tabla 1 de Wolfire \& Konigl (1991) en que se listan las frecuencias y las intensidades de las líneas de $\mathrm{H}_{2}$ relativas a 1-0 S(1) para diferentes modelos de excitación de líneas.

$$
(*): a(b) \text { representa } a \times 10^{b} \text {. }
$$

Una herramienta que puede ser de utilidad para determinar el origen de las fuentes extendidas ES1, ES2, ES3 y ES4 es el análisis de los cocientes de intensidades de las diferentes líneas de $\mathrm{H}_{2}$. Wolfire \& Konigl (1991) analizaron los cocientes de intensidades de líneas de emisión de $\mathrm{H}_{2}$ para diferentes modelos de emisión de Hidrógeno molecular en objetos Herbig-Haro, que están asociados con las cabezas de los jets estelares.

Estos bowshocks se identifican con los choques que se producen cuando un outflow impacta en el medio interestelar, en las denominadas superficies de trabajo. Es posible discriminar dos tipos de superficies en la estructura del choque: el choque frontal (forward shock) y el choque trasero (reverse shock) (Hartigan, 1989). El primero corresponde al material que está siendo acelerado y que colisiona con el material circundante. En esta región frontal, que presenta forma de choque de proa, el efecto del choque provoca la excitación colisional del material molecular y por lo tanto un calentamiento del mismo. El segundo corresponde a la región que está por detrás del choque frontal, donde el material se desacelera. Esta región, también denominada 'disco de Mach', comprende una zona de enfriamiento, en donde se emiten las características líneas de recombinación del Hidrógeno y líneas prohibidas de metales.

Simulaciones numéricas de jets (Blondin, Konigl, \& Fryxell, 1989) han determinado que el material enfriado se acumula en una capa densa, dinámicamente inestable, entre los dos choques y que las inhomogeneidades de densidad que se desarrollan en esta capa pueden explicar el 'nudos' (knots) de emisión óptica detectados en estos objetos HH.

Los objetos $\mathrm{HH}$ también son fuente de emisión de líneas moleculares, especialmente $\mathrm{H}_{2}$ y $\mathrm{HCO}^{+}$. Líneas en el UV identificadas como emisión de $\mathrm{H}_{2}$ en la banda Lyman, líneas de transiciones vibracionales de $\mathrm{H}_{2}$ en el infrarrojo y emisión de ondas milimétricas en las líneas rotacionales de $\mathrm{HCO}^{+}$han sido detectadas en diversos objetos $\mathrm{HH}$. Además, existe evidencia de que el continuo UV detectado en varios objetos $\mathrm{HH}$ y previamente atribuido a la emisión de Hidrógeno atómico (e.g., Dopita, Binette, \& Schwartz, 1982; Brugel, Shull, \& Seab, 1982) puede surgir, al menos en parte, de la fotodisociación de $\mathrm{H}_{2}$.

Los niveles rotacionales en los estados vibracionales más bajos (principalmete $v=0,1,2$ ), son excitados por colisiones con $\mathrm{H}_{2}, \mathrm{H}$ y $e^{-}$. Los niveles decaen mediante transiciones de radiación cuadrupolar a estados vibracionales inferiores o a estados de rotación inferiores dentro del mismo nivel de vibración. Las transiciones con $\Delta v= \pm 1$ tienen frecuencias cercanas a $2 \mu \mathrm{m}$. Una transición particularmente intensa es $v=1, \mathrm{~J}=3 \rightarrow v=0, \mathrm{~J}=1$ o $1-0 \mathrm{~S}(1)(2.121$ $\mu \mathrm{m})$. Los estudios de cocientes de líneas suelen referirse a esta transición.

Wolfire \& Konigl (1991) investigaron los posibles mecanismos de excitación de las líneas 


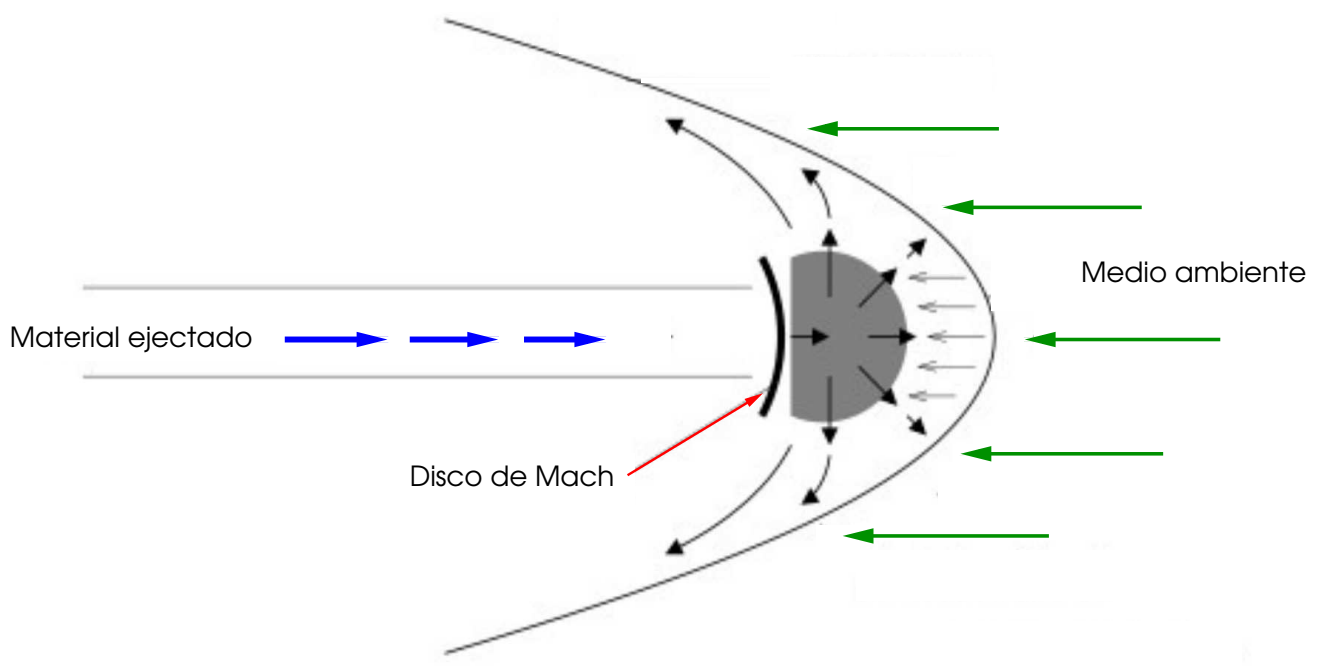

Figura 5.5: Esquema de la superficie de trabajo de un bowshock. En el marco de referencia del bowshck el material de jet (flechas azules) es desacelerado en el disco de Mach, y el material ambiental (flechas verdes) es desacelerado en el choque frontal. En el medio de éstos se forma una capa densa de material enfriado (región gris) desde la que el gas sale despedido e interactúa con el medio ambiente para formar la superficie de trabajo con forma de arco.

de emisión de $\mathrm{H}_{2}$ observadas en objetos $\mathrm{HH}$ y su relación con los diferentes modelos de choque para explicar la emisión óptica y UV en estas fuentes. En la Tabla 5.2 se muestra parte de la Tabla 1 del trabajo de Wolfire \& Konigl (1991), donde se listan los cocientes de intensidades de distintas líneas respecto a 1-0 S(1) (la más intensa). En la columna 3 se muestran los cocientes para una excitación producida por radiación de continuo UV, y en las columnas 4 a 8 para los modelos descriptos a continuación.

\section{I - Excitación por colisiones en un choque no disociativo}

El Hidrógeno molecular que entra en un choque no disociativo (o sea un choque lento al que las moléculas son capaces de sobrevivir) se excita de manera colisional en el gas caliente que queda detras del choque. La velocidad crítica para la disociación colisional depende del campo magnético y toma valores de entre $\sim 25$ y $50 \mathrm{~km} \mathrm{~s}^{-1}$.

Wolfire \& Konigl (1991) estimaron los cocientes de las líneas de $\mathrm{H}_{2}$ esperadas de un choque no disociativo mediante el cálculo de las poblaciones de los niveles excitados por colisiones a una densidad y una temperatura constantes. Adoptaron un valor para la velocidad del choque de $25 \mathrm{~km} \mathrm{~s}^{-1}$, un medio de una densidad $n_{0}=10^{3} \mathrm{~cm}^{-3}$, y un campo magnético pre-choque de $\mathrm{B}_{0}=30 \mu \mathrm{G}$ (Draine et al., 1983). Para la región de emisión de $\mathrm{H}_{2}$ usaron una densidad del gas de $n \sim 10^{3} \mathrm{~cm}^{-3}$, una abundancia de Hidrógeno atómico de $n_{H} / n \sim 3 \times 10^{-3}$, una abundancia de Hidrógeno molecular de $n_{H_{2}} / n \sim 0.5$, una abundancia electrónica de $n_{e} / n \sim$ $3 \times 10^{-7}$, una temperatura del gas $\mathrm{T} \sim 2000 \mathrm{~K}$ y una temperatura electrónica $\mathrm{T}_{e} \sim 3000 \mathrm{~K}$. Los valores calculados se muestran en la columna 4 de la Tabla 5.1.

Para las densidades apropiadas para los objetos HH, las tasas de colisión y las relaciones de intensidad entre líneas son sensibles a la abundancia de Hidrógeno atómico $n_{H} / n$. Esta dependencia se ilustra a través de los valores mostrados en la columna 5 de la Tabla 5.1 para los que se ha aumentado el valor de $n_{H} / n$ a $3 \times 10^{-2}$, manteniendo inalterados los otros parámetros del choque. 
Por comparación también se muestran los valores de los cocientes en LTE a T $\sim 2000 \mathrm{~K}$ (columna 6 de la Tabla 5.1).

Todos estos valores deben ser usados sólo como una guía ya que el gas post-choque en un choque real no se caracteriza por valores únicos de temperatura, densidad y abundancia química, sino más bien por un rango de estos parámetros.

\section{II - Excitación por radiación UV y Rayos X}

La excitación colisional de $\mathrm{H}_{2}$ en la capa densa en la cabeza de un jet radiativo también podría deberse al calentamiento por rayos X y radiación EUV (Extreme Ultraviolet) proveniente de un choque disociativo. La radiación ultravioleta (FUV,6-13.6 eV y EUV, 13.6-54.4 eV) y los Rayos X (>54.4 eV) tanto del bowshock como del choque producido por el jet pueden excitar las moléculas de $\mathrm{H}_{2}$.

En este caso, la molécula $\mathrm{H}_{2}$ se eleva a un estado de excitación electrónica a través de la absorción de fotones UV en las bandas de Lyman y Werner. La desintegración posterior conduce a la disociación de la molécula (en aproximadamente el $10 \%$ de todas las transiciones) o a la descomposición en niveles roto-vibracionales del estado fundamental. A partir de ahí, la molécula de $\mathrm{H}_{2}$ se desintegra a través de una cascada de transiciones roto-vibracionales (fluorescencia) (e. g., Black \& Dalgarno 1976, Black \& van Dishoeck 1987).

Los valores de los cocientes obtenidos por este modelo se muestran en la columna 7 de la Tabla 5.1.

\section{III - Formation Pumping (bombeo durante la formación)}

Las moléculas de Hidrógeno se forman en los granos de polvo a densidades columnares mayores que $4 \times 10^{21} \mathrm{~cm}^{-2}$ detrás de un choque disociativo. Una vez formada, la molécula es expulsada del grano en un estado roto-vibracional excitado. El decaimiento de los niveles excitados se produce de forma radiativa, colisional o mediante una combinación de ambos, según la densidad y la temperatura del gas. A bajas densidades, la desexcitación radiativa produce una cascada de emisión de líneas infrarrojas. A altas densidades, las desexcitaciones por colisión aplacan la emisión de la línea y la energía de excitación contribuye al calentamiento del gas.

Los valores de los cocientes obtenidos por este modelo se muestran en la colunma 8 de la Tabla 5.1.

Las relaciones de línea de formation pumping son muy similares a las encontradas para la excitación por rayos X y EUV. Estos procesos se pueden distinguir por medio de las transiciones puramente rotacionales o a través de las líneas producidas en niveles vibracionales más altos (datos con los que no se cuenta en esta tesis).

\subsection{Cocientes de líneas}

Se han detectado ocho líneas de $\mathrm{H}_{2}$ en la región a partir de las observaciones llevadas a cabo con Gemini en la banda K. Estas se encuentran listadas en la Tabla 5.2 junto con sus longitudes de onda. Con el fin de comparar estas observaciones con los modelos desarrollados por Wolfire \& Konigl (1991) se calcularon los cocientes de las líneas respecto a 1-0 S(1). Estos valores se muestran también en la Tabla 5.2 para cada una de las fuentes extendidas identificadas en la Sección 5.2. La comparación entre los cocientes de líneas listados en las Tablas 5.1 y 5.2 deben ser tomados con cautela ya que no es posibe determianar los parámetros sobre los lóbulos (velocidad del choque, densidad, temperatura) por lo que es probable que las condiciones en las que se emiten las líneas no sean idénticas a las consideradas en los modelos. 


\begin{tabular}{cccccc}
\hline \hline & $\begin{array}{c}\lambda \\
(\mu \mathrm{m})\end{array}$ & ES1 & ES2 & ES3 & ES4 \\
\hline 1-0 S $(2)$ & 2.033 & 0.35 & 0.40 & 0.35 & 0.39 \\
2-1 S $(3)$ & 2.073 & 0.12 & 0.07 & 0.14 & 0.08 \\
$1-0 \mathrm{~S}(1)$ & 2.121 & 1.0 & 1.0 & 1.0 & 1.0 \\
2-1 $\mathrm{S}(2)$ & 2.154 & 0.18 & 0.19 & 0.17 & 0.16 \\
3-2 $\mathrm{S}(3)$ & 2.201 & 0.17 & 0.12 & 0.13 & 0.12 \\
$1-0 \mathrm{~S}(0)$ & 2.223 & 0.34 & 0.44 & 0.36 & 0.48 \\
2-1 S $(1)$ & 2.247 & 0.28 & 0.29 & 0.28 & 0.31 \\
$3-2 \mathrm{~S}(2)$ & 2.286 & 0.05 & 0.03 & 0.00 & 0.07 \\
\hline \hline
\end{tabular}

Tabla 5.2: Cocientes calculados para las fuentes extendidas.

Un cociente de intensidades 2-1 S(1)/1-0 S(1) <0.1 generalmente implica excitación colisional (Shull \& Hollenbach, 1978). Como se muestra en la Tabla 5.2 para todas las fuentes extendidas este cociente toma valores cercanos a 0.3 , por lo que no resulta evidente que estén siendo excitadas colisionalmente. Sin embargo es posible que exista más de un proceso de exitacion de líneas en estas fuentes, por lo que no se puede descartar las excitación colisional.

En las Figuras 5.6 y 5.7 se comparan los cocientes calculados para las fuentes extendidas (puntos rojos con barras de error) con los distintos modelos (círculos de colores). Se puede ver que ninguna de las fuentes ajusta con exactitud a ninguno de los modelos. Esto puede deberse a que en realidad no existe un único proceso de excitación, sino una combinación de varios.

Para cuantificar cuál de los modelos ajusta mejor a las observaciones se estimó el apartamiento de cada modelo usando la siguiente ecuación

$$
\operatorname{Desvío~}_{j}=\sum_{j}\left(\frac{\operatorname{obs}_{j}-\bmod _{j}}{\bmod _{j}}\right)^{2}
$$

donde $\sum_{j}$ denota la suma sobre cada uno de los cocientes calculados. Esta suma se hizo para cada uno de los modelos (el subíndice $j$ representa cada modelo). Los resultados se muestran en la Tabla 5.3. Para todas las fuentes se encuentra que el menor desvío es respecto de los cocientes producidos por radiación de continuo UV, y en segundo lugar por formation pumping.

En particular en la fuente ES4 es muy evidente la coincidencia con los cocientes excitados por radiación de continuo UV. Tal como se puede ver en la Figura 5.1, esta fuente se encuentra en el borde de la región HiI Gum 31, por lo que es probable que esté siendo excitada por fotones provenientes de cúmulo NGC 3324. Después de ES4, la fuente ES2, que se encentra en el borde del lóbulo azul, es la que mejor concuerda con excitación por radiación UV.

Como se puede ver en la Figura 5.2, ES4 y ES2 tienen emisión Br $\gamma$ mientras que ES1 y ES3 no muestran esta línea. Es por esta razón que a pesar de que para ES1 y ES3 el desvío es menor para el modelo que considera excitación por emisión de continuo UV que para los modelo de choques, es probable que exista una mezcla de procesos excitando las fuentes. Cuando la opacidad es lo suficientemente grande, los fotones que emergen del frente de choque ionizan el gas no chocado produciendo lo que se conoce como precursor radiativo. En el caso de las fuentes embebidas, tal como ES1 y ES3, los fotones UV que estarían excitando las líneas podrían provenir de este mecanismo.

Por lo tanto es factible pensar que las líneas de $\mathrm{H}_{2}$ presentes en las fuentes extendidas ubicadas sobre los lóbulos esten siendo, en parte, excitadas por choques. Este hecho sería un 
Tabla 5.3: Desvío de los cocientes observados respecto a los modelos de Wolfire \& Konigl (1991).

\begin{tabular}{ccccccc}
\hline \hline Fuente & Cont. UV & \multicolumn{3}{c}{ Choque no disociativo } & UV y & Formation \\
& & $\frac{n_{H}}{n}=3(-3)^{*}$ & $\frac{n_{H}}{n}=3(-2)^{*}$ & LTE & Rayos X & Pumping \\
\hline ES1 & 1.51111 & 495.353 & 316.897 & 306.132 & 273.602 & 2.36661 \\
ES2 & 1.13069 & 348.005 & 171.632 & 163.927 & 127.99 & 4.64813 \\
ES3 & 1.63926 & 363.115 & 188.314 & 176.196 & 146.764 & 3.66515 \\
ES4 & 0.68114 & 331.181 & 192.375 & 187.338 & 160.639 & 5.21026 \\
\hline \hline
\end{tabular}

$(*): a(b)$ representa $a \times 10^{b}$.

indicio más de la posible presencia de un outflow. Dadas las caracteríasticas de la fuente CS2, en la que se observa emisión $\operatorname{Br} \gamma$ que indica la presencia de gas ionizado, y la coincidencia espacial de esta con WISE1, se propone que esta es la fuente responsable de generar la cavidad y excitar el Hidrógeno molecular.

\subsection{Conclusiones}

Los resultados sugieren que CS2 es una estrella masiva en sus etapas finales de formación. Esta hipótesis se basa en la coincidencia de CS2 con: (1) la fuente WISE 1, clasificada como YSO clase II, (2) emisión extendida a $4.5 \mu \mathrm{m}$, (3) una cavidad en el gas molecular, (4) emisión $\operatorname{Br} \gamma$.

La distribución del gas molecular sugiere que este ha sido disociado, mientras la emisión $\operatorname{Br} \gamma$ indica que parte de él fue posteriormente ionizado por CS2.

Las fuentes extendidas ES1, ES2 y ES3 detectadas en $\mathrm{H}_{2}$ a $2.12 \mu$ m muestran gas chocado posiblemente interactuando con outflows protoestelares, denotando la presencia de objetos $\mathrm{HH}$ en los extremos de los jets, mientras que ES4 podría tener otro origen ya que se encuentra en el borde de la región Hir Gum 31 y podría estar siendo excitada por fotones del cúmulo NGC 3324. Además ES2, que se encuentra cerca del borde de la cavidad de gas molecular, y ES4 que se localiza en el borde de Gum 31 poseen emisión de la línea Br $\gamma$, que revela la presencia de gas ionizado.

Esta hipótesis es apoyada por la detección de gas molecular en las alas roja y azul de CO. Otra evidencia de la existencia del sistema protostelar es la extensión hacia el sureste de la emisión a $4.5 \mu \mathrm{m}$.

El estudio de los cocientes de líneas de $\mathrm{H}_{2}$ y su comparación con diferentes modelos no permite asegurar que la excitación de las líneas presentes en las fuentes extendidas tengan un origen colisional. Sin embargo no se puede descartar que el origen de las mismas sea una combinacion de mecanismos, entre ellos alguno colisional.

Los resultados obtenidos concuerdan con el escenario propuesto en el Capítulo 4 para la región asociada a IRAS 10349-5824, en el que se propuso que un grumo ya ha colapsado y comenzado a formar una estrella, que estaría perturbando su entorno. 
ES1

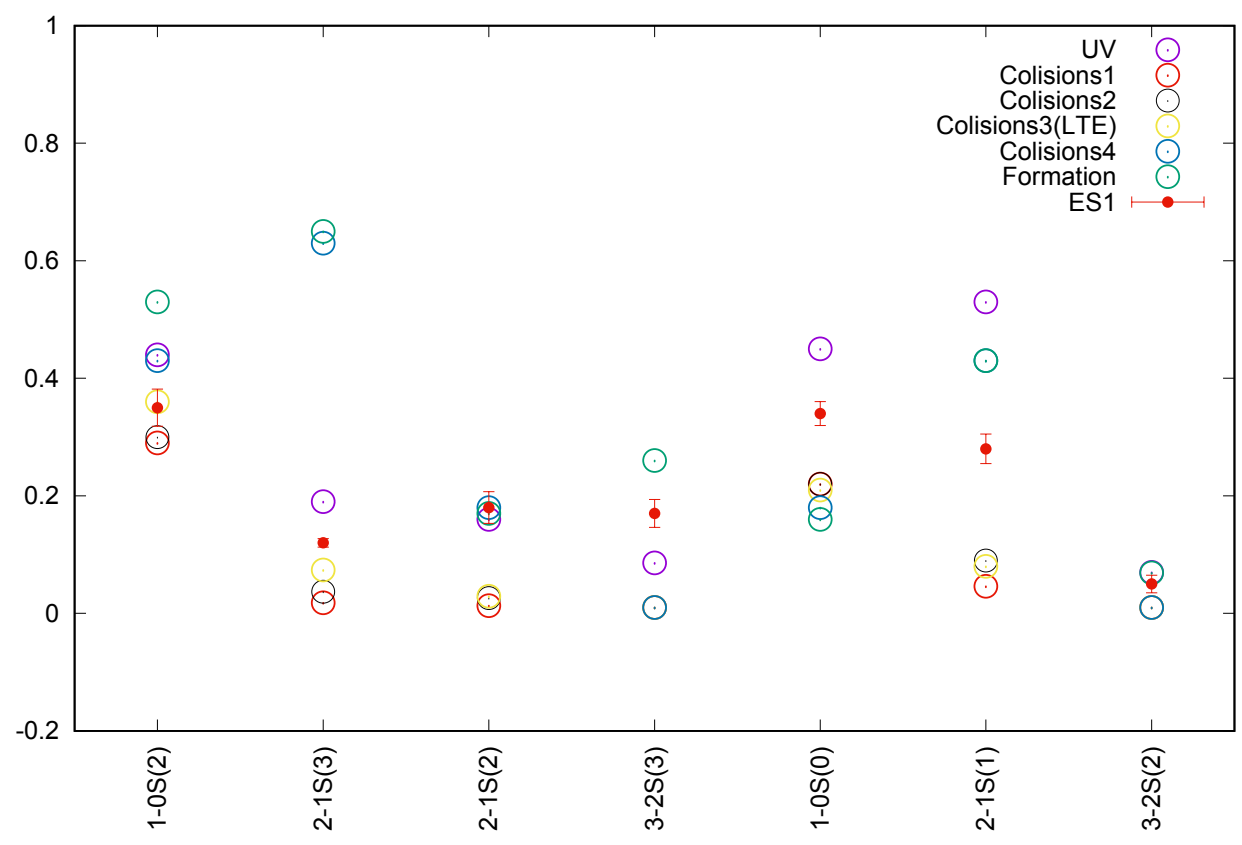

ES2

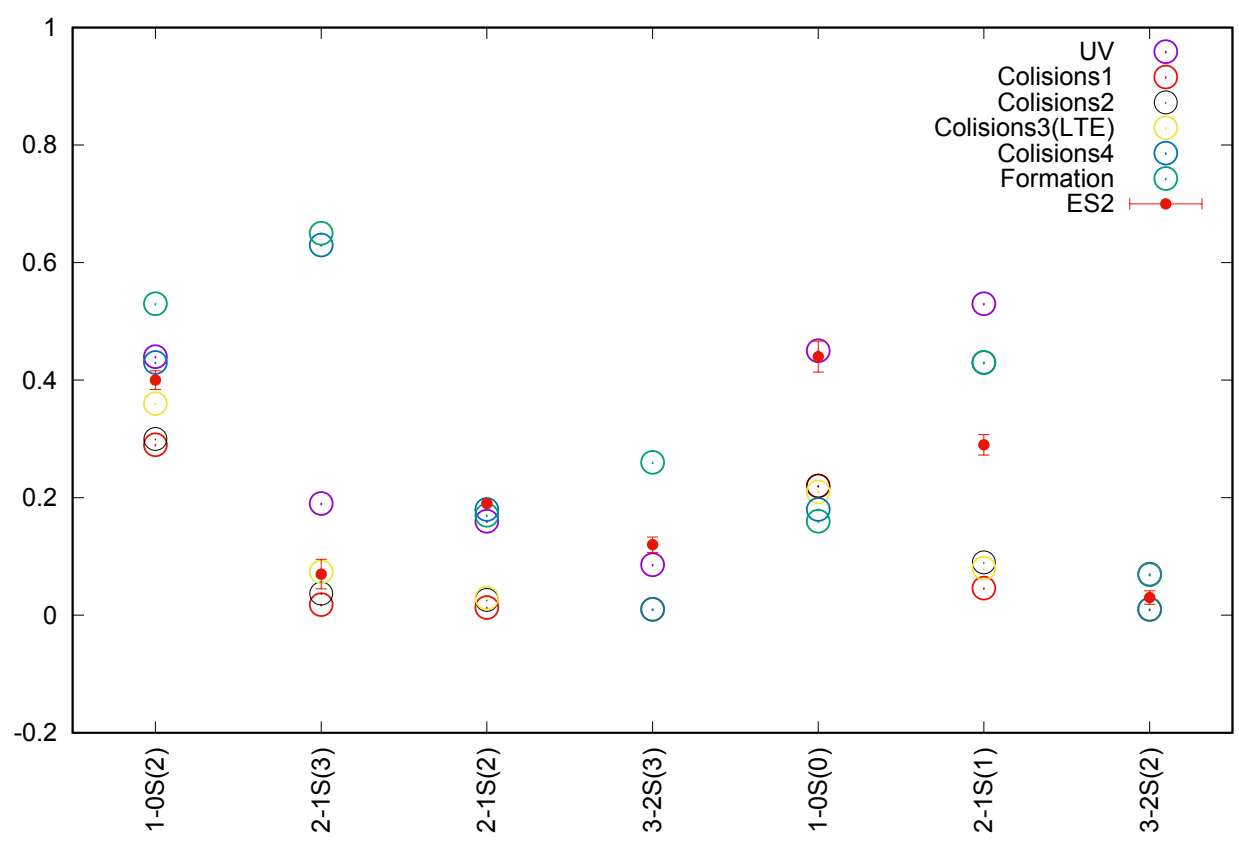

Figura 5.6: ES1 y ES2 Comparación de los cocientes calculados a partir de las observaciones con los modelos descriptos por Wolfire \& Konigl (1991). 
ES3

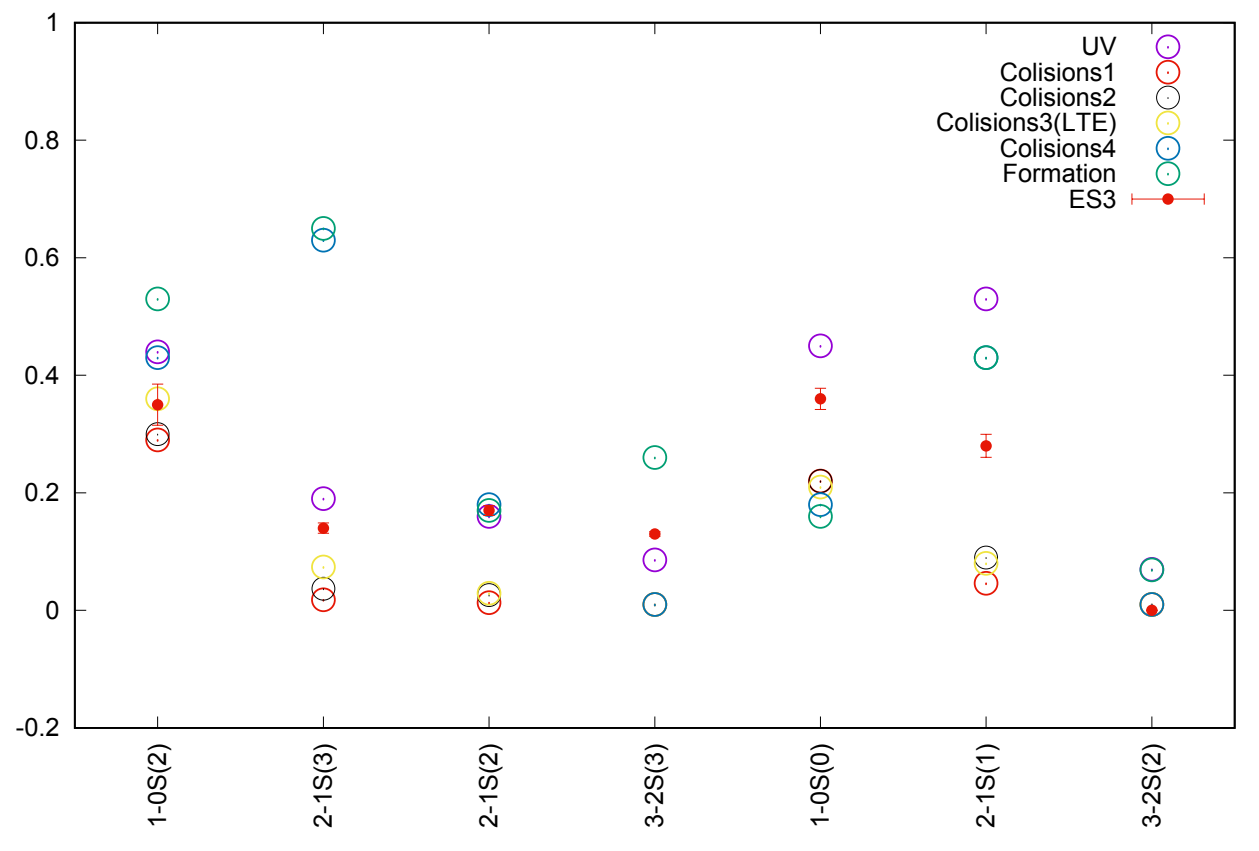

ES4

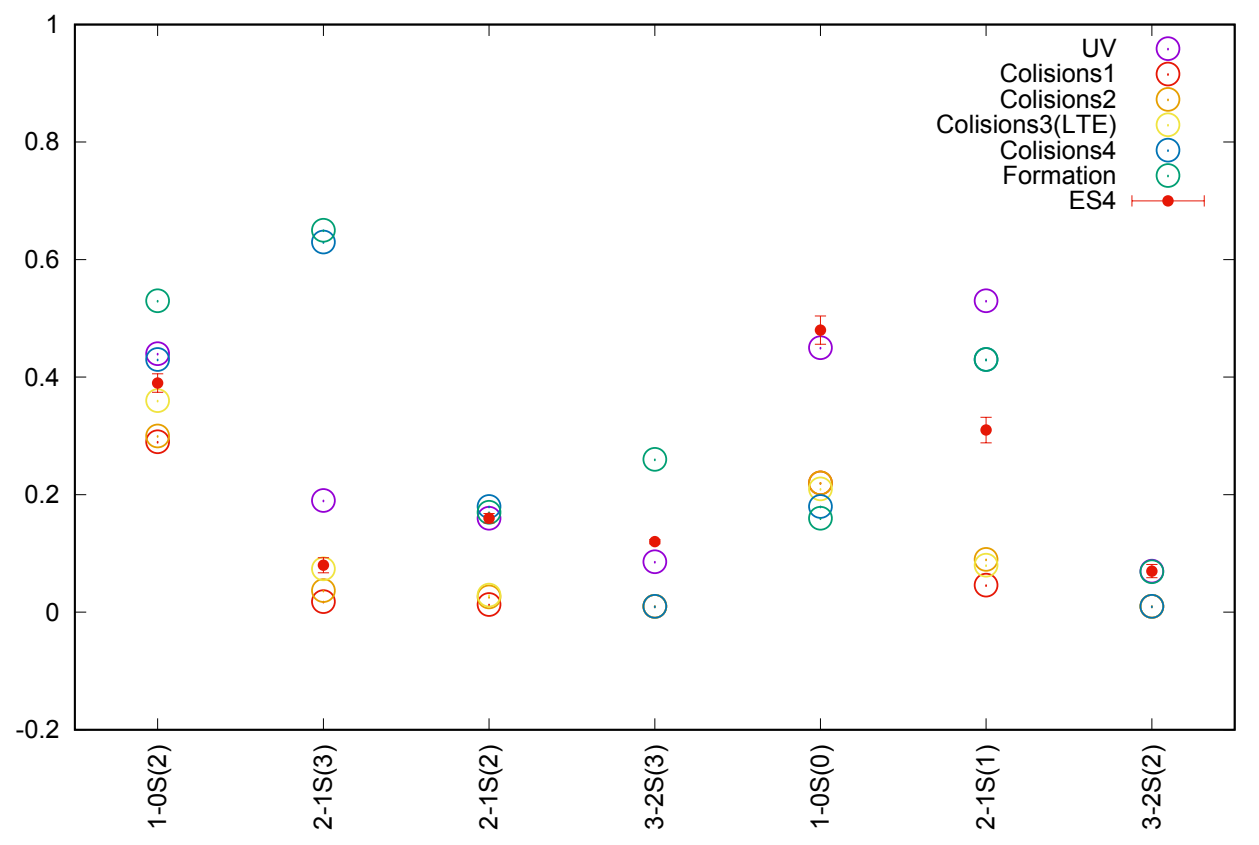

Figura 5.7: ES3 y ES4 Comparación de los cocientes calculados a partir de las observaciones con los modelos descriptos por Wolfire \& Konigl (1991). 


\section{Capítulo 6}

\section{IRDC SDC 341.232-0.268}

\subsection{Antecedentes de la región}

Usando imágenes de Spitzer-GLIMPSE y -MIPSGAL, Peretto \& Fuller (2009) catalogaron y caracterizaron $\sim 11000$ nubes oscuras infrarrojas (IRDCs) usando datos de Spitzer en 8 y $24 \mu \mathrm{m}$. La alta resolución angular de los datos de Spitzer permite estudiar detalladamente la estructura de estas regiones y su alta sensibilidad permite detectar protoestrellas y protocúmulos profundamente inmersos en el entorno gaseoso. En este catálogo las IRDCs fueron identificadas como estructuras conectadas con densidades columnares pico de Hidrógeno molecular mayores a $2 \times 10^{22} \mathrm{~cm}^{-2}$.

La IRDC SDC 341.232-0.268, ubicada en RA, Dec.(J2000) = (16:52:35.67, -44:28:21.8), tiene una intensidad de campo de radiación a $8 \mu \mathrm{m}$ de $\sim 88.3 \mathrm{MJy}$ ster $^{-1}$, mientras que la opacidad media en $8 \mu \mathrm{m}$ es de 0.5 y la opacidad máxima es 1.09 . Además, Peretto \& Fuller (2009) determinaron que la IRDC posee una subestructura compuesta por dos fragmentos, y en Spitzer-MIPS detectaron una fuente puntual a $24 \mu \mathrm{m}$ en el campo de la IRDC.

La Figura 6.1 muestra la superposición de las imágenes de Spitzer-GLIMPSE en $4.5 \mu \mathrm{m}$ y $8 \mu \mathrm{m}$, y la imagen de MIPSGAL en $24 \mu \mathrm{m}$, en la región de SDC 341.232-0.268. Los contornos verdes (80, 90 y $95 \mathrm{MJy} /$ ster) revelan ausencia de emisión en $8 \mu \mathrm{m}$ sobre la IRDC, en contraste con su entorno, una característica típica de estos objetos. En la región aparecen proyectados cuatro candidatos a EGO clasificados por Cyganowski et al. (2008): G341.22-0.26(b) (16:52:30.3, -44:28:40.0), G341.22-0.26(a) (16:52:32.2, -44:28:38.0), G341.23-0.27 (16:52:34.2, -44:28:36.0) y G341.24-0.27 (16:52:37.3, -44:28:09.0). La posición de estos candidatos a EGO, que de aquí en más llamaremos EGO 1, EGO 2, EGO 3 y EGO 4, respectivamente, se muestra con cruces en la Figura 6.1. EGO 4 está relacionado con la fuente compacta de ATLASGAL AGAL 341.219-00.259 (Contreras et al., 2013), tiene una densidad de flujo integrado y máximo de $\mathrm{S}_{\text {int }}=12.85 \pm 2.20$ Jy y $\mathrm{S}_{\max }=2.13 \pm 0.33$, respectivamente, y un radio efectivo de $\sim 37$ arcsecs.

La Figura 6.1 también revela que la emisión extendida a $4.5 \mu \mathrm{m}$ aparece proyectada sobre los candidatos a EGO. La emisión a esta longitud de onda incluye tanto las líneas $\mathrm{H}_{2}$ ( $\mathrm{v}=0-0$, $\mathrm{S}(9,10,11)$ ) como las cabezas de las bandas de $\mathrm{CO}(\mathrm{v}=1-0)$ (Cyganowski et al., 2008), que son excitadas por choques como los que se esperan cuando los flujos protoestelares afectan el medio interestelar (Cyganowski et al., 2008).

Como se muestra en la Tabla 6.1, se ha detectado emisión máser de metanol en $95 \mathrm{GHz}$ hacia EGO 3 y EGO 4, en un rango de velocidades de -43 a $-45 \mathrm{~km} \mathrm{~s}^{-1}$, a una distancia cinemática de 3.6 kpc (Chen et al., 2011). La detección de emisión máser es una clara indicación de que la IRDC es una región de formación estelar activa.

En este capítulo se analiza la contraparte molecular y el polvo asociados a SDC 341.232- 


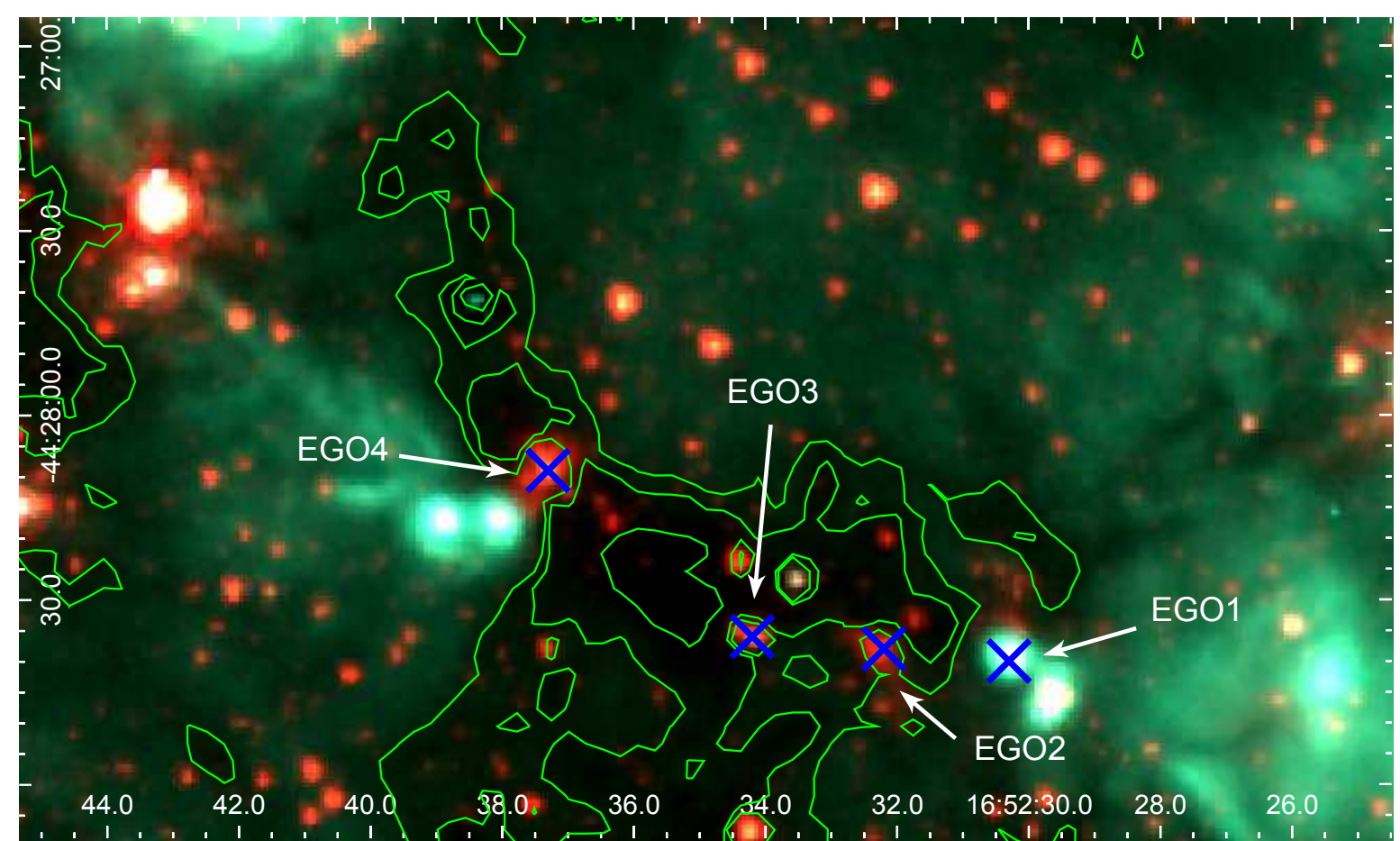

Figura 6.1: IRDC SDC 341.232-0.268. Se muestra la emisión a 4.5 (rojo) y $8.0 \mu \mathrm{m}$ (verde) de Spitzer-IRAC y a $24 \mu \mathrm{m}$ (azul) de Spitzer-MIPS. Los contornos corresponden a la emisión de $8.0 \mu \mathrm{m}$ a 80,90 y $95 \mathrm{mJy} / \mathrm{str}$ y delinean la IRDC. Las cruces indican las posiciones de los candidatos a EGO.

0.268, con el objetivo de caracterizar la región y determinar sus parámetros físicos, lo que permitirá una mejor comprensión de los procesos que allí ocurren. Para ello se ha llevado a cabo un análisis del gas molecular y polvo asociado a la región en donde EGO 1, EGO 2, EGO 3 y EGO 4 se encuentran inmersos. A tal fin se utilizaron datos de las líneas moleculares de ${ }^{12} \mathrm{CO}(2-1),{ }^{13} \mathrm{CO}(2-1)$ y $\mathrm{C}^{18} \mathrm{O}(2-1)$ obtenidas con el telescopio APEX, y datos de $\mathrm{HCO}^{+}(1-0)$, $\mathrm{HNC}(1-0)$ y $\mathrm{N}_{2} \mathrm{H}^{+}(1-0)$ del relevamiento MALT90, imágenes infrarrojas de Spitzer-IRAC y -MIPS, Herschel-PACS y -SPIRE, y del bolómetro LABOCA del telescopio APEX.

\subsection{Datos utilizados}

\subsubsection{Observaciones moleculares}

Los datos de ${ }^{12} \mathrm{CO}(2-1),{ }^{13} \mathrm{CO}(2-1)$ y $\mathrm{C}^{18} \mathrm{O}(2-1)$ fueron obtenidos con el receptor APEX1 (SHeFI; Vassilev et al. 2008) del telescopio APEX, utilizando el espectrómetro XFFTS2 (eXtended bandwidth Fast Fourier Transform Spectrometer2) con un ancho de banda de 2.5 $\mathrm{GHz}$ dividido en 32768 canales. Los principales parámetros de las transiciones moleculares (frecuencia en reposo $\nu_{0}$, tamaño del haz a potencia mitad HPBW, resolución en velocidad $\Delta \mathrm{v}_{\text {res }}$, y ruido rms de cada espectro obtenidos en el modo OTF) se encuentran listados en la Tabla 6.2. La posición libre de emisión de CO elegida fue RA, Dec.(J2000) $=(16: 36: 40.56$, $-42: 03: 40.6)$.

La corrección por la emisión atmosférica se llevó a cabo utilizando la técnica chopper-wheel (Kutner \& Ulich, 1981). La escala de temperatura de antena fue convertida a temperatura de brillo a través de $\mathrm{T}_{m b}=\mathrm{T}_{A} / \eta_{m b}$, donde $\eta_{m b}$ es la eficiencia de haz. Para el receptor SHeFI/APEX-1 se adoptó un valor de $\eta_{m b}=0.75$. Las condiciones ambientales durante la 
Tabla 6.1: Emisión máser de metanol a $95 \mathrm{GHz}$ hacia la IRDC. Se muestran los parámetros de los ajustes gaussianos (velocidad central $\mathrm{v}_{0}$, ancho de la gaussiana $\Delta \mathrm{v}$ y flujo pico $\mathrm{S}_{95}$ ) junto con la distancia y luminosidad estimada (Chen et al., 2011).

\begin{tabular}{cccccc}
\hline \hline & $\begin{array}{c}\mathrm{v}_{0} \\
\mathrm{~km} \mathrm{~s}^{-1}\end{array}$ & $\begin{array}{c}\Delta \mathrm{v} \\
\mathrm{km} \mathrm{s}^{-1}\end{array}$ & $\begin{array}{c}S_{95} \\
\mathrm{Jy}\end{array}$ & $\begin{array}{c}d_{\text {kin }} \\
\mathrm{kpc}\end{array}$ & $\begin{array}{c}L \\
L \odot\end{array}$ \\
\hline EGO 3 & $-44.58 \pm 0.03$ & $0.55 \pm 0.08$ & 5.14 & 3.6 & 7.0 \\
& $-44.13 \pm 0.12$ & $3.82 \pm 0.30$ & 3.50 & & \\
EGO 4 & $-44.90 \pm 0.29$ & $3.50 \pm 0.46$ & 3.4 & 3.5 & 10.4 \\
& $-43.78 \pm 0.02$ & $0.75 \pm 0.05$ & 13.82 & & \\
& $-43.02 \pm 0.04$ & $0.41 \pm 0.10$ & 4.23 & & \\
& $-42.33 \pm 0.06$ & $0.52 \pm 0.17$ & 2.79 & & \\
\hline \hline
\end{tabular}

Tabla 6.2: Parámetros observacionales de las líneas observadas.

\begin{tabular}{lcccc}
\hline \hline Línea & $\begin{array}{c}\nu_{0} \\
{[\mathrm{GHz}]}\end{array}$ & $\begin{array}{c}\mathrm{HPBW} \\
{[\operatorname{arcsec}]}\end{array}$ & $\begin{array}{c}\Delta \mathrm{v}_{\text {res }} \\
{\left[\mathrm{km} \mathrm{s}^{-1}\right]}\end{array}$ & $\begin{array}{c}r m s \\
{[\mathrm{~K}]}\end{array}$ \\
\hline${ }^{12} \mathrm{CO}(2-1)$ & 230.538 & 29.5 & 0.3 & 0.2 \\
${ }^{13} \mathrm{CO}(2-1)$ & 220.398 & 29.5 & 0.3 & 0.4 \\
$\mathrm{C}^{18} \mathrm{O}(2-1)$ & 219.560 & 30.2 & 0.1 & 0.4 \\
\hline \hline
\end{tabular}

observación fueron buenas, con valores de vapor de agua precipitable (PWV) entre 1.5 - 2.0 $\mathrm{mm}$. Los datos fueron reducidos utilizando el software CLASS90.

Además se han utilizado datos del relevamiento MALT90 (ver Sección 3.3.4). En particular se utilizaron cubos de datos de $\mathrm{HCO}^{+}(1-0), \mathrm{HNC}(1-0)$ y $\mathrm{N}_{2} \mathrm{H}^{+}(1-0)$ para detectar regiones de alta densidad hacia SDC 341.232-0.268. El análisis fue llevado a cabo utilizando el software CLASS90.

\subsubsection{Datos de Herschel}

Se utilizaron datos de archivo del relevamiento Hi-GAL (Herschel Infrared GALactic plane survey, Molinari et al., 2010). Los datos fueron tomados con el instrumento PACS (Poglitsch et al., 2010) a 70 y $160 \mu \mathrm{m}$, y con SPIRE a 250, 350 y $500 \mu \mathrm{m}$ (Griffin et al., 2010). Las resoluciones angulares de las distintas bandas fotométricas se extienden desde $8^{\prime \prime}$ hasta $35 ! 2$ para $70 \mu \mathrm{m}$ y $500 \mu \mathrm{m}$, respectivamente. Los datos fueron reducidos utilizando el software HIPE (Herschel interactive processing environment, Ott, 2010) con scripts de reducción del proceso estándar $^{(1)}$.

\subsection{Gas molecular: distribución y cinemática}

En esta sección se analiza la emisión de las líneas de $\mathrm{CO}$ que permiten trazar el gas molecular ligado a SDC 341.232-0.268 y estudiar su cinemática. Bronfman et al. (1996) detectaron emisión de CS(2-1) hacia la región HiI ultracompacta (UCHII) IRAS 16487-4423 $[$ RA, Dec. $(J 2000)=(16: 52: 21.39,-44: 28: 03.13)]$ localizada a una distancia angular de 2.5 de la IRDC, con una velocidad $\mathrm{v}_{L S R} \sim-43.4 \mathrm{~km} \mathrm{~s}^{-1}$. Como la emisión de esta molécula revela la presencia de regiones de gas de alta densidad $\left(n_{\text {crit }}=6 \times 10^{5} \mathrm{~cm}^{-3}\right.$, ver Tabla 2.2), ésta puede ayudar a determinar la velocidad sistémica de la IRDC.

\footnotetext{
${ }^{(1)}$ La reducción de los datos fue llevada a cabo por la Dra. Verónica Firpo
} 
Tabla 6.3: Posición, radio y velocidad de los Grumos A y B definidos a partir de la emisión de ${ }^{13} \mathrm{CO}(2-1)$.

\begin{tabular}{cccc}
\hline \hline & $\begin{array}{c}\text { Posición } \\
\text { RA, Dec.(J2000) }\end{array}$ & $\begin{array}{c}\text { Radio efectivo } \\
{\left[{ }^{\prime \prime}\right]}\end{array}$ & $\begin{array}{c}\text { Velocidad } \\
{\left[\mathrm{km} \mathrm{s}^{-1}\right]}\end{array}$ \\
\hline Grumo A & $16: 52: 37.06,-44: 28: 13.89$ & 37.6 & -44.0 \\
Grumo B & $16: 52: 29.72,-44: 28: 40.09$ & 45.6 & -43.5 \\
\hline \hline
\end{tabular}

Para visualizar la distribución espacial de la emisión molecular la Figura 6.2 muestra, en escala de colores, mapas de temperatura de brillo de ${ }^{13} \mathrm{CO}(2-1)$ desde $-47.6 \mathrm{a}-41.9 \mathrm{~km} \mathrm{~s}^{-1}$. La emisión más intensa de ${ }^{13} \mathrm{CO}(2-1)$ aparece en el rango de -44 a $-45.2 \mathrm{~km} \mathrm{~s}^{-1}$ y coincide espacialmente con la IRDC revelando la presencia de dos grumos. Esta coincidencia indicaría que el gas se encuentra asociado a ésta.

Con el objetivo de definir un tamaño y posición para estos grumos se hizo un promedio de la emisión de ${ }^{13} \mathrm{CO}(2-1)$ en el rango de velocidades $-47.0 \mathrm{a}-42.8 \mathrm{~km} \mathrm{~s}^{-1}$. El resultado de este promedio se muestra en la Figura 6.3. A partir de allí se definieron radios efectivos de 37!'6 y 45"6 para los Grumos A y B, respectivamente, y se definió la posición de los mismos como la que corresponde al máximo de emisión. El tamaño de los grumos se calculó considerando la emisión contenida dentro del contorno de $6.2 \mathrm{~K}$. Allí se midió la cantidad de pixeles y considerando el tamaño de los pixeles de la imagen se calculó un tamaño para cada grumo. El radio efectivo corresponde al que tendría un círculo con igual área que la medida para cada grumo. Los parámetros de los grumos se listan en la Tabla 6.3.

La Figura 6.4 muestra los perfiles de ${ }^{12} \mathrm{CO}(2-1),{ }^{13} \mathrm{CO}(2-1)$ y $\mathrm{C}^{18} \mathrm{O}(2-1)$ promediados en el área correspondiente a los Grumos A y B. El ruido $r m s$ del espectro promediado de ${ }^{12} \mathrm{CO}$ es $0.019 \mathrm{~K}$ para el Grumo A y $0.017 \mathrm{~K}$ para el Grumo B.

Hacia el Grumo A la línea de ${ }^{12} \mathrm{CO}(2-1)$ exhibe una estructura de múltiples componentes a diferentes velocidades, desde -70 hasta $-20 \mathrm{~km} \mathrm{~s}^{-1}$. Las componentes más intensas se encuentran entre -48 y $-35 \mathrm{~km} \mathrm{~s}^{-1}$, alcanzando sus máximos a velocidades de $-46.60 \mathrm{~km} \mathrm{~s}^{-1}$, $-42.15 \mathrm{~km} \mathrm{~s}^{-1} \mathrm{y}-36.80 \mathrm{~km} \mathrm{~s}^{-1}$. Las componentes fuera de este rango no serán consideradas parte de los grumos y serán analizadas en la Sección 6.7. Las espectros de ${ }^{13} \mathrm{CO}(2-1)$ y $\mathrm{C}^{18} \mathrm{O}(2-1)$ alcanzan sus máximos a velocidades de $-44.0 \mathrm{~km} \mathrm{~s}^{-1}$, en coincidencia con un mínimo en la emisión de ${ }^{12} \mathrm{CO}$.

Hacia el Grumo B, la emisión de ${ }^{12} \mathrm{CO}(2-1)$ también muestra una estructura de múltiples componentes entre -55 and $-25 \mathrm{~km} \mathrm{~s}^{-1}$, con el máximo a $-45.4 \mathrm{~km} \mathrm{~s}^{-1}$. Del mismo modo que para el Grumo A las componentes con velocidades menores que $-35 \mathrm{~km} \mathrm{~s}^{-1}$ no serán consideradas parte del grumo. Los perfiles de ${ }^{13} \mathrm{CO}(2-1)$ y $\mathrm{C}^{18} \mathrm{O}(2-1)$ tienen su máximo en $-43.5 \mathrm{~km} \mathrm{~s}^{-1}$.

Considerando que la emisión de $\mathrm{C}^{18} \mathrm{O}(2-1)$ es generalmemte ópticamente delgada y que la velocidad de su componente principal es similar a la velocidad de la línea de CS(2-1) de Bronfman et al. (1996), se adoptaron para los Grumos A y B las velocidades sistémicas $\mathrm{v}_{\text {sys }}$ de $-44.0 \mathrm{~km} \mathrm{~s}^{-1} \mathrm{y}-43.5 \mathrm{~km} \mathrm{~s}^{-1}$, respectivamente.

Los modelos de rotación circular galáctica predicen que el gas a velocidades de $-44 \mathrm{~km} \mathrm{~s}^{-1}$ está a una distancia cinemática de $3.6 \mathrm{kpc}$ (e. g. Brand \& Blitz, 1993) ${ }^{(2)}$. Asumiendo que la dispersión en velocidad del gas en el modelo de Brand \& Blitz (1993) es de 6 km s${ }^{-1}$, el

\footnotetext{
${ }^{(2)}$ El modelo de rotación circular galáctica proporciona dos posibles distancias para un dada velocidad en el primer y cuarto cuadrante, una cercana y una lejana (que en este caso son 3.6 y $12.5 \mathrm{kpc}$ ). En esta tesis todas las distancias calculadas con este método corresponden a la cercana, ya que las regiones se encuentran asociadas a burbujas infrarrojas, y estos objetos son difícilmente detectables a distancias mayores a $8 \mathrm{kpc}$ (Churchwell et al., 2006).
} 


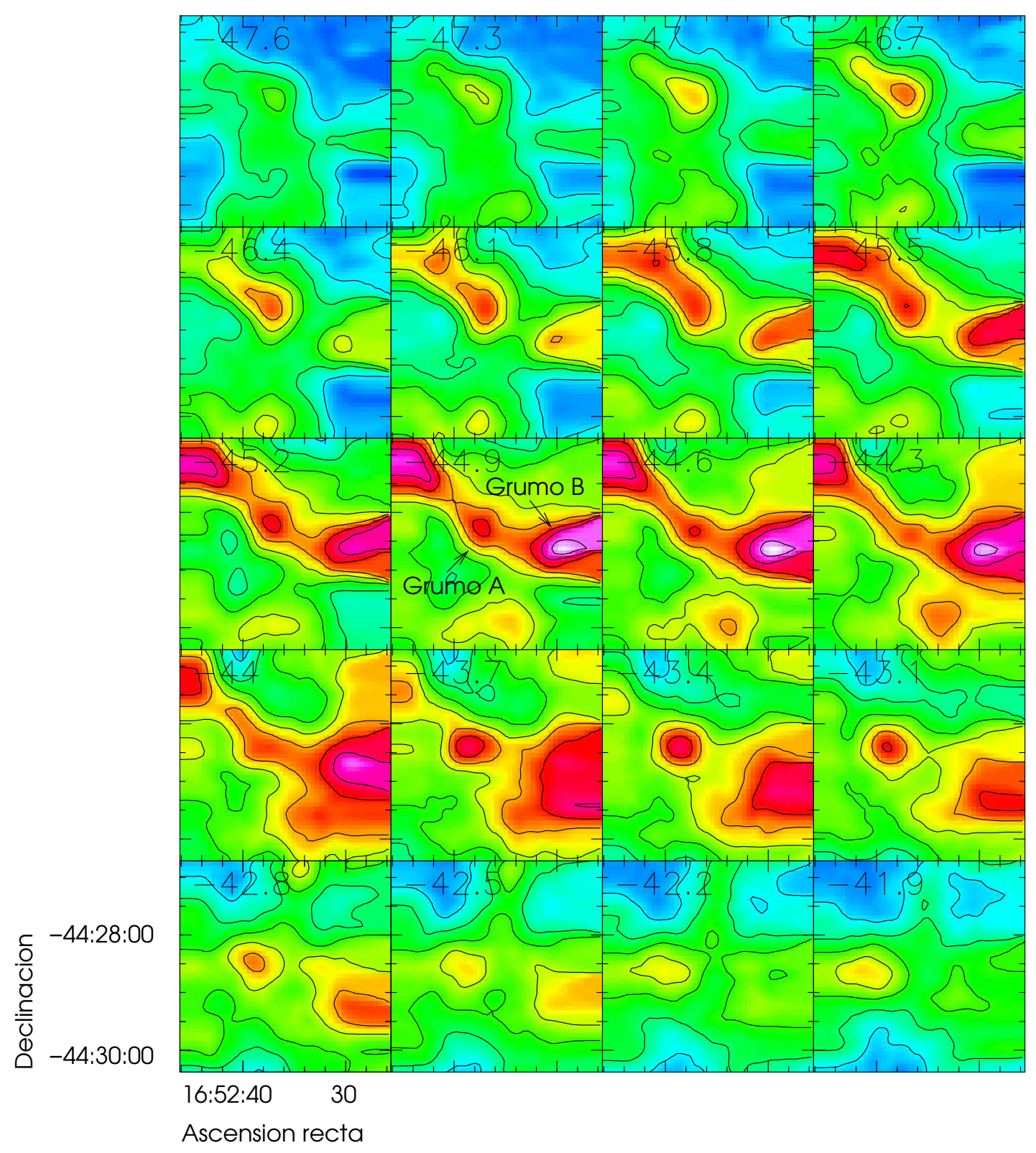

Figura 6.2: Mapas de temperatura de brillo de ${ }^{13} \mathrm{CO}(2-1)$ en el intervalo de velocidades desde $-47.6 \mathrm{a}-41.9 \mathrm{~km} \mathrm{~s}^{-1}$ en pasos de $0.3 \mathrm{~km} \mathrm{~s}^{-1}$. La emisión molecular asociada a la IRDC SDC 341.232-0.268 se ve claramente en los canales centrales (entre -45.2 y $-44.0 \mathrm{~km} \mathrm{~s}^{-1}$ ) aunque emisión correspondiente a los Grumos A y B se detecta entre -47.0 y $-42.8 \mathrm{~km} \mathrm{~s}^{-1}$. Los mapas cubren la zona comprendida entre RA(J2000)=16:52:26 a 16:52:46 y Dec(J200)= $-44: 26: 10$ y $-44: 29: 46$. 


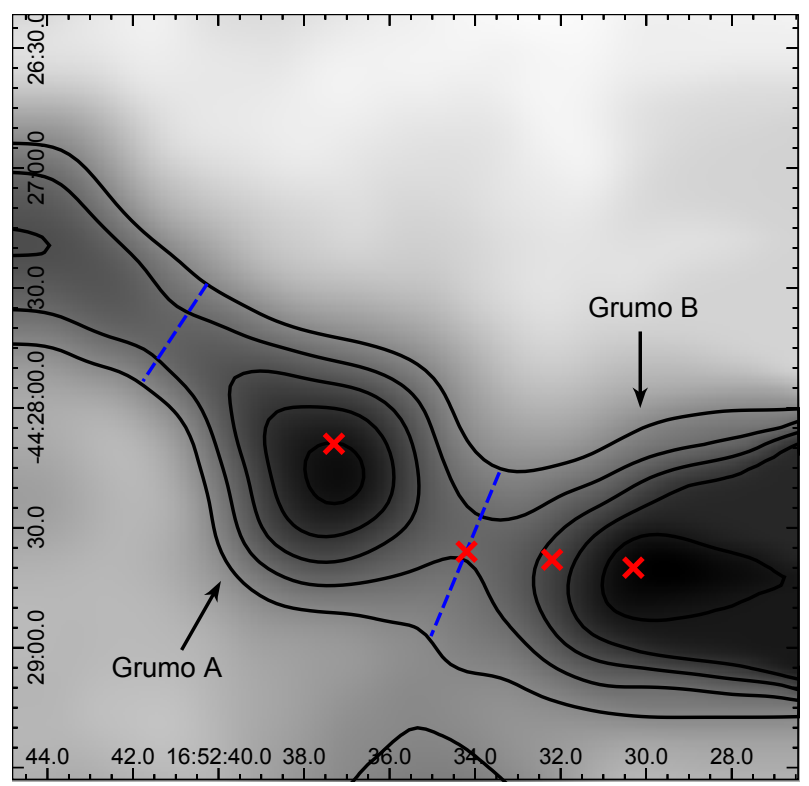

Figura 6.3: Emisión de ${ }^{13} \mathrm{CO}(2-1)$ promediada entre -47.0 a $-42.8 \mathrm{~km} \mathrm{~s}^{-1}$. Los contornos corresponden a $6.2,7.0,7.8,8.6$ y $9.4 \mathrm{~K}$. El contorno de $6.2 \mathrm{~K}$ y las líneas discontinuas de color azul delinean la región que corresponde a cada uno de los grumos. Las cruces rojas muestran la posición de los candidatos a EGO.

error en la distancia es de $0.4 \mathrm{kpc}(\sim 10 \%)$. Esta distancia coincide con la encontrada por Chen et al. (2011) para máseres, y con la distancia adoptada para la burbuja infrarroja S24 por Cappa et al. (2016), indicando que SDC 341.232-0.268 es parte del mismo complejo que S24.

\subsection{Parámetros derivados de la emisión de $\mathrm{CO}$}

Para estimar los parámetros del gas molecular se asumió que la nube se encuentra en equilibrio termodinámico local (LTE). Teniendo en cuenta que la distribución espacial de ${ }^{13} \mathrm{CO}(2-1)$ y $\mathrm{C}^{18} \mathrm{O}(2-1)$ coincide con la IRDC, se usaron estas líneas para estimar los principales parámetros del gas molecular asociado.

Se puede asegurar que $\mathrm{C}^{18} \mathrm{O}(2-1)$ es ópticamente delgado, pero de antemano no se puede decir nada sobre los valores que podría tomar $\tau_{13}$. Por lo tanto no es posible usar la ecuación 2.21 para calcular la temperatura de excitación, para la cual se supone que la línea es ópticamente gruesa. Es por esta razón que se determinaron los valores de $\tau_{18}$ y $\tau_{13}$ a partir de la ecuación 2.27, que en este caso toma la forma

$$
\frac{T_{m b}^{13}}{T_{m b}^{18}}=\frac{1-e^{-\tau^{13}}}{1-e^{-\tau^{13} / 7.6}}
$$

donde $\tau^{13}$ es la profundidad óptica de ${ }^{13} \mathrm{CO}(2-1)$ y $7.6=\left[{ }^{13} \mathrm{CO}\right] /\left[\mathrm{C}^{18} \mathrm{O}\right]$ (Sanhueza et al., $2010)$ es el cociente de abundancia de los isótopos. Las profundidades ópticas de ${ }^{13} \mathrm{CO}(2-1)$ obtenidas se muestran en la columna 2 de la Tabla 6.4. Para estimar la profundidad óptica de $\mathrm{C}^{18} \mathrm{O}(2-1)$ se usó la ecuación 2.24 que para el par de isótopos aquí considerados toma la forma 


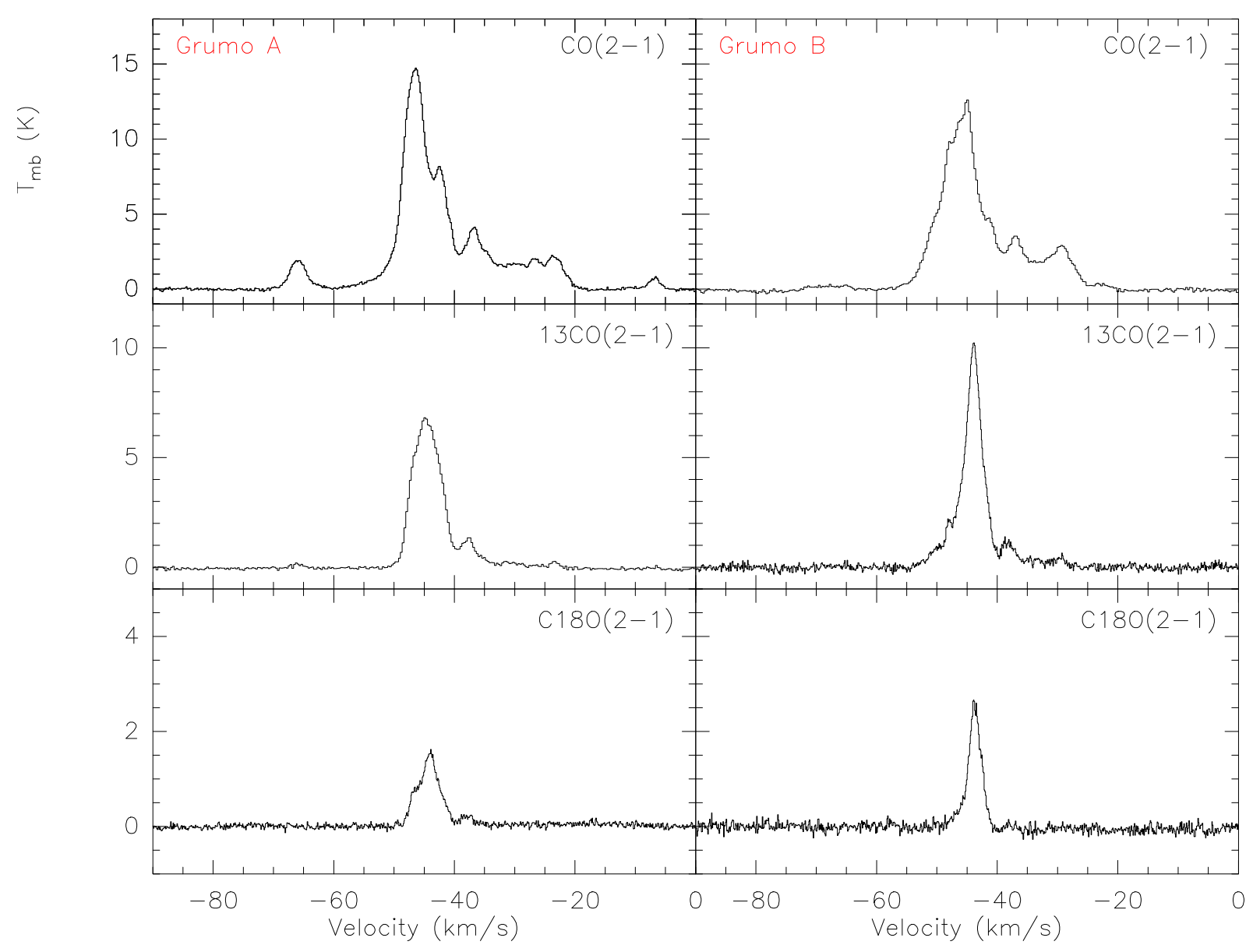

Figura 6.4: Espectros promedio de ${ }^{12} \mathrm{CO}(2-1),{ }^{13} \mathrm{CO}(2-1)$ and $\mathrm{C}^{18} \mathrm{O}(2-1)$ hacia el Grumo A (izquierda) y el Grumo B (derecha). 
Tabla 6.4: Parámetros de los grumos moleculares.

\begin{tabular}{ccccccccccc}
\hline \hline & $\tau_{13}$ & $\tau_{18}$ & $\begin{array}{c}\mathrm{T}_{\text {exc }} \\
\mathrm{K}\end{array}$ & $\begin{array}{c}N\left({ }^{13} \mathrm{CO}\right) \\
10^{16} \mathrm{~cm}^{-2}\end{array}$ & $\begin{array}{c}N\left(H_{2}\right) \\
10^{22} \mathrm{~cm}^{-2}\end{array}$ & $\begin{array}{c}r \\
\mathrm{pc}\end{array}$ & $\begin{array}{c}M\left(H_{2}\right) \\
M_{\odot}\end{array}$ & $\begin{array}{c}n_{H_{2}} \\
10^{4} \mathrm{~cm}^{-3}\end{array}$ & $\begin{array}{c}M_{\text {vir }} \\
M_{\odot}\end{array}$ & $\frac{M_{\text {vir }}}{M\left(H_{2}\right)}$ \\
\hline Grumo A & 3.4 & 0.6 & 11.5 & 14.20 & 7.1 & 0.7 & $2400 \pm 960$ & $2.6 \pm 1.3$ & $1000-1670$ & $0.41-0.69$ \\
Grumo B & 1.0 & 0.1 & 14.4 & 4.12 & 2.06 & 0.9 & $1200 \pm 480$ & $0.5 \pm 0.2$ & $750-1240$ & $0.62-1.0$ \\
\hline \hline
\end{tabular}

$$
\tau_{13}=\tau_{18} 7.6\left(\frac{\Delta \mathrm{v}_{18}}{\Delta \mathrm{v}_{13}}\right)\left(\frac{\nu^{18}}{\nu^{13}}\right)^{2}
$$

Para estos cálculos se consideraron para el Grumo $\mathrm{A}$ anchos a potencia mitad $(\Delta \mathrm{v})$ iguales a $3.8 \mathrm{~km} \mathrm{~s}^{-1}$ para el $\mathrm{C}^{18} \mathrm{O}$ y $5.2 \mathrm{~km} \mathrm{~s}^{-1}$ para el ${ }^{13} \mathrm{CO}(2-1)$, y para el Grumo B iguales a 1.9 y $3.9 \mathrm{~km} \mathrm{~s}^{-1}$. Los resultados se muestran en la Tabla 6.4.

De esta forma, una vez obtenido un valor para $\tau_{13}$, es posible calcular $\mathrm{T}_{\text {exc }}$ despejando directamente de la ecuación 2.19 sin necesidad de hacer la aproximación $\tau \gg 1$. Los resultados se muestran en la columna 4 de la Tabla 6.4.

La densidad columnar de ${ }^{13} \mathrm{CO}(2-1)$ se calculó a partir de la expresión de Rohlfs \& Wilson (2004)

$$
N\left({ }^{13} \mathrm{CO}\right)=2.4 \times 10^{14} \exp \left(\frac{T^{*}}{T_{\mathrm{exc}}}\right) \times \frac{T_{\text {exc }}}{1-e^{-T^{*} / T_{\text {exc }}}} \int \tau_{13} d \mathrm{v}
$$

en la que, para valores de la profundidad óptica como los obtenidos aquí para ${ }^{13} \mathrm{CO}(2-1)\left(\tau_{13}\right.$ $>1$ ), se usa la aproximación

$$
T_{\text {exc }} \int \tau_{13} d \mathrm{v} \simeq \times \frac{\tau^{13}}{1-\exp \left(-\tau^{13}\right)} \int T_{\mathrm{mb}}^{13} d \mathrm{v} .
$$

donde la integral se calcula como la temperatura de brillo promedio hacia el grumo multiplicada por $\Delta \mathrm{v}$.

Para estimar la densidad columnar de Hidrógeno molecular $N\left(H_{2}\right)$ (columna 6 en Tabla 6.4) se consideró una abundancia $\left[\mathrm{H}_{2}\right] /\left[{ }^{13} \mathrm{CO}\right]=7.7 \times 10^{5}$ (Wilson \& Rood, 1994). La masa molecular (columna 8 en Tabla 6.4) se calculó usando la ecuación 2.38. Los radios efectivos de los grumos calculados a partir de ${ }^{13} \mathrm{CO}$ y las densidades volumétricas $n_{H_{2}}$ calculadas a partir de la ecuación 2.39 se muestran en las columnas 7 y 9 de la Tabla 6.4.

La masa del virial puede determinarse a partir de la ecuación 2.40 y los resultados se hallan listados en la columna 10 de la Tabla 6.4. El cociente entre la masa del virial y la masa en LTE (columna 11 de la Table 6.4) toma valores mayores o menores que la unidad según qué perfil de densidad del grumo se considere, por lo que no se puede asegurar que los grumos estén colapsando pero tampoco se puede descartar la posibilidad.

Las incertezas en la masa molecular calculada bajo condiciones de LTE, $M\left(H_{2}\right)$, y en la masa del virial, $M_{V I R}$, se originan principalmente en la indeterminación en distancia (10\%), produciendo un $20 \%$ de error en $M\left(H_{2}\right)$ y un $10 \%$ en $M_{V I R}$. Las masas de virial no están libres de incertidumbres, debido a la existencia de un soporte de campo magnético que puede sobrestimar los valores derivados (MacLaren et al., 1988) y al perfil de densidad desconocido del grumo.

Bergin \& Tafalla (2007) utilizan el término grumo (clump) para referirse a fuentes con un tamaño de $\sim 0.3-3 \mathrm{pc}$, temperaturas de entre 10 y $20 \mathrm{~K}$, masas de $\sim 5-500 \mathrm{M}_{\odot}, \mathrm{y}$ densidades volumétricas de $\sim 10^{3}-10^{4} \mathrm{~cm}^{-3}$ (ver Tabla 1.2). Los valores encontrados para el gas analizado en esta sección coinciden con los definidos por estos autores excepto para 
Tabla 6.5: Parámetros de los ajustes de los perfiles extraidos de MALT90

\begin{tabular}{llcccc}
\hline \hline Grumo & Molécula & $\begin{array}{c}\text { Area } \\
{\left[\mathrm{K} \mathrm{km} \mathrm{s}^{-1}\right]}\end{array}$ & $\begin{array}{c}\text { Velocidad } \\
{\left[\mathrm{km} \mathrm{s}^{-1}\right]}\end{array}$ & $\begin{array}{c}\Delta \mathrm{v} \\
{\left[\mathrm{km} \mathrm{s}^{-1}\right]}\end{array}$ & $\begin{array}{c}\mathrm{T}_{\max } \\
{[\mathrm{K}]}\end{array}$ \\
\hline Grumo A & $\mathrm{HCO}+(1-0)$ & 3.55 & -46.41 & 3.45 & 0.97 \\
& $\mathrm{HNC}(1-0)$ & 3.48 & -45.27 & 3.16 & 1.04 \\
& $\mathrm{~N}_{2} \mathrm{H}^{+}(1-0)_{\text {grupo } 1}$ & 1.46 & -52.58 & 3.32 & 0.41 \\
& $\mathrm{~N}_{2} \mathrm{H}^{+}(1-0)_{\text {grupo } 2}$ & 6.47 & -44.05 & 3.24 & 1.87 \\
& $\mathrm{~N}_{2} \mathrm{H}^{+}(1-0)_{\text {grupo }}$ & 3.63 & -38.27 & 3.05 & 1.12 \\
\hline Grumo B & $\mathrm{HCO}^{+}(1-0)$ & 3.82 & -46.13 & 3.36 & 1.07 \\
& $\mathrm{HNC}(1-0)$ & 6.09 & -44.83 & 3.92 & 1.46 \\
& $\mathrm{~N}_{2} \mathrm{H}^{+}(1-0)_{\text {grupo } 1}$ & 1.91 & -52.41 & 2.58 & 0.69 \\
& $\mathrm{~N}_{2} \mathrm{H}^{+}(1-0)_{\text {grupo } 2}$ & 7.82 & -44.18 & 3.48 & 2.11 \\
& $\mathrm{~N}_{2} \mathrm{H}^{+}(1-0)_{\text {grupo }}$ & 3.79 & -38.29 & 2.66 & 1.34 \\
\hline \hline
\end{tabular}

la masa. Sin embargo se considera que el término grumo es correcto para referirse a estas estructuras.

\subsection{Análisis de datos de MALT90}

De los cubos de datos disponibles de MALT90 en esta región sólo las moléculas $\mathrm{HCO}^{+}(1-0)$, $\mathrm{HNC}(1-0)$ y $\mathrm{N}_{2} \mathrm{H}^{+}(1-0)$ muestran emisión. Estas moléculas tienen densidades ambientales críticas de $2 \times 10^{5}, 3 \times 10^{5}$ y $3 \times 10^{5} \mathrm{~cm}^{-3}$, respectivamente, lo que suponiendo LTE permite inferir un límite inferior para la densidad de la región. Estos valores son mayores que los valores de la densidad ambiental de $\mathrm{H}_{2}$ derivada en la sección anterior (ver Tabla 6.4), indicando que están mostrando regiones más densas. Los espectros de estas moléculas promediados en el área del grumo en cada uno de los grumos se muestran en la Figura 6.5 junto con sus ajustes gaussianos.

Tal como se dijo en la Sección 2.2.4, $\mathrm{N}_{2} \mathrm{H}^{+}(1-0)$ posee siete componentes hiperfinas, seis de ellas agrupadas en dos grupos de tres líneas cada uno, más una componente aislada. Esto justifica la detección de tres componentes en la emisión de $\mathrm{N}_{2} \mathrm{H}^{+}(1-0)$, mientras se detecta sólo una en las otras moléculas. En la Tabla 6.5 se listan los parámetros de los ajustes gaussianos realizados sobre las líneas detectadas por MALT90. Las velocidades de estas líneas coinciden con las velocidades sistémicas derivadas para los grumos a partir de CO (para el caso de $\mathrm{N}_{2} \mathrm{H}^{+}(1-0)$ se consideró la velocidad del grupo 2).

La Figura 6.6 muestra los mapas de $\mathrm{HCO}^{+}(1-0), \mathrm{HNC}(1-0)$ y $\mathrm{N}_{2} \mathrm{H}^{+}(1-0)$ integrados desde $-47.6 \mathrm{a}-44.0 \mathrm{~km} \mathrm{~s}^{-1},-46.7 \mathrm{a}-43.1 \mathrm{~km} \mathrm{~s}^{-1}, \mathrm{y}-47 \mathrm{a}-44.1 \mathrm{~km} \mathrm{~s}^{-1}$, respectivamente. Ambos grumos son detectados en las tres líneas moleculares indicando densidades medias de $10^{5} \mathrm{~cm}^{-3}$. La distribución de la emisión de $\mathrm{N}_{2} \mathrm{H}^{+}$es similar a la que se observa en el lejano IR (ver Figura 6.7). Las especies que contienen Carbono, como el CO, tienden a desaparecer de la fase gaseosa en los centros de alta densidad de los grumos por deposición en los granos de polvo, mientras que las que contienen nitrógeno, como $\mathrm{N}_{2} \mathrm{H}^{+}$sobreviven casi sin ser afectadas hasta densidades mucho más altas dado que son más resistentes a la congelación de los granos que las especies que contienen Carbono. Por lo tanto la aparición de un grumo depende fuertemente del trazador usado para mapearlo, y luce similar cuando es observado en $\mathrm{N}_{2} \mathrm{H}^{+}$o continuo de polvo.

Usando las ecuaciones 2.41, 2.42 y 2.43 se calcularon las profundidades ópticas, temperaturas de excitación y densidades columnares de los grumos a partir de los parámetros de los ajustes realizados sobre los perfiles de $\mathrm{N}_{2} \mathrm{H}^{+}$. Para estos cálculos se consideró que los valores 


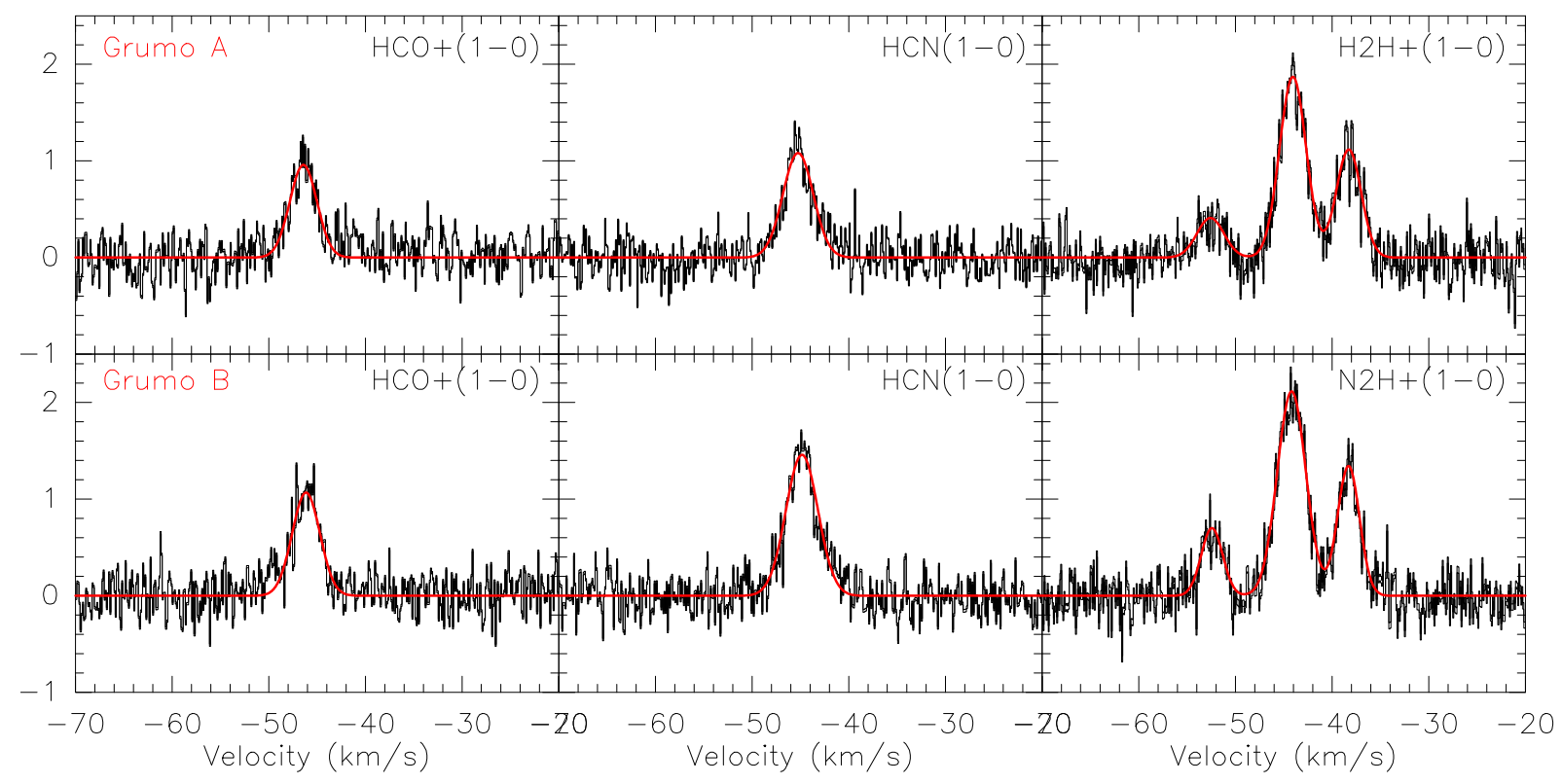

Figura 6.5: Perfiles de $\mathrm{HCO}^{+}(1-0), \mathrm{HNC}(1-0)$ y $\mathrm{N}_{2} \mathrm{H}^{+}(1-0)$ en dirección al Grumo A (arriba) y al Grumo B (abajo). En líneas rojas se muestra el ajuste en componentes gaussianas.

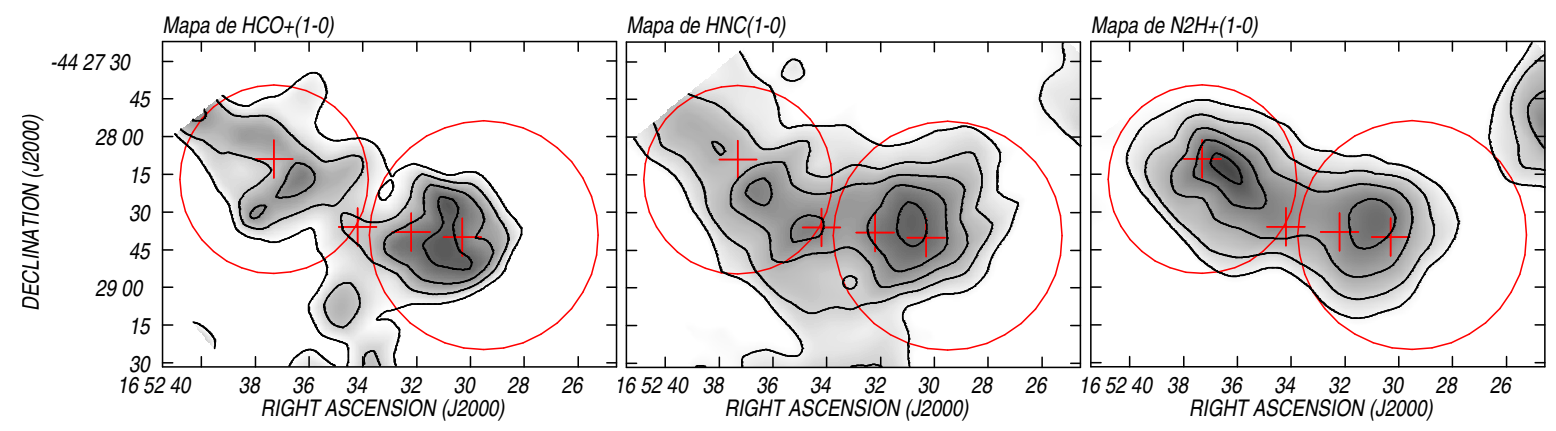

Figura 6.6: Mapas de $\mathrm{HCO}^{+}(1-0)$ (contornos: 0.6, 0.7, 0.8 and $0.9 \mathrm{~K}$ ), $\mathrm{HNC}(1-0)$ (contornos: $0.65,0.8,0.95,1.1$ y $1,25 \mathrm{~K})$ y $\mathrm{N}_{2} \mathrm{H}^{+}(1-0)$ (contornos: $0.65,0.8,1.0,1.2$ and $1.4 \mathrm{~K}$ ). Las cruces indican la posición de los candidatos a EGO y los círculos la posición y tamaño de los Grumos A y B tal como se definieron a partir de CO. 
de $\nu, S \mu^{2}$ y $E_{u} / k$ para el grupo 1 son $93176.2526 \mathrm{MHz}, 12.42 \mathrm{D}^{2}$ y $4.47 \mathrm{~K}$ (valores tomados del catálogo SPLATALOGUE $\left.{ }^{(3)}\right)$.

Para los Grumos A y B se derivaron valores de $\mathrm{T}_{e x c}=17.4$ y $12.5 \mathrm{~K}$, y $N\left(N_{2} H^{+}\right)=$ $8.7 \times 10^{13}$ y $8.1 \times 10^{13}$, respectivamente. Considerando que la abundancia en nubes moleculares oscuras es $\left[\mathrm{N}_{2} \mathrm{H}^{+}\right] /\left[\mathrm{H}_{2}\right]=5 \times 10^{-10}$ (Ohishi et al., 1992), se obtuvieron densidades columnares de $\mathrm{H}_{2}$ de $1.7 \times 10^{23}$ y $1.6 \times 10^{23} \mathrm{~cm}^{-2}$ para los Grumos A y B, respectivamente. Las densidades columnares son mayores que las calculadas a partir de CO. Esta diferencia podría deberse al hecho de que, como se explicó anteriormente, las especies nitrogenadas sobreviven casi sin ser afectadas hasta densidades mucho más altas que las portadoras de Carbono (Caselli et al., 1999; Tafalla, 2011).

Considerando radios efectivos $\mathrm{r}=0.53 \mathrm{pc}$ para los Grumos A y B (medidos a partir del mapa de $\mathrm{N}_{2} \mathrm{H}^{+}(1-0)$ ) se obtuvieron densidades volumétricas $n_{H_{2}} \sim 1.7 \times 10^{5} \mathrm{~cm}^{-3}$ para cada grumo, compatibles con lo esperado.

\subsection{Distribución del polvo}

En la Figura 6.7 se muestra la emisión en el infrarrojo lejano hacia SDC 341.232-0.268 en imágenes de Herschel-PACS, SPIRE, y LABOCA. Los contornos mostrados en los seis paneles de esta figura corresponden a 20 veces el rms de cada una de las imágenes, mientras que los círculos indican la posición y tamaño de los grumos definidos a partir de CO en la sección 6.3. En todos los casos la emisión en el infrarrojo resulta menos extendida que la del gas molecular. Los candidatos a EGO se ven resueltos en 70 y $160 \mu \mathrm{m}$, mientras que a longitudes de onda mayores solo se pueden distinguir dos grumos de polvo superpuestos a la emisión más extendida en el rango submilimétrico.

La detección de emisión a $24 \mu \mathrm{m}$ (ver Figura 6.1) y a $70 \mu \mathrm{m}$ asociada a EGO1, EGO 2 y EGO 3 indica la presencia de polvo tibio posiblemente relacionado con estos candidatos a EGO.

Los flujos en el lejano IR $(\lambda>160 \mu \mathrm{m})$ de estos dos grumos de polvo, que coinciden con el Grumo A y el GrumoB identificados a partir de la emisión del gas molecular, fueron obtenidos luego de llevar las imágenes a la resolución angular de la imagen a $500 \mu \mathrm{m}\left(36^{\prime \prime}\right)$ y están listados en la Tabla 6.6. Los flujos a 70, 160, 250, 350 y $500 \mu \mathrm{m}$ se calcularon con HIPE y el flujo a $870 \mu \mathrm{m}$ con AIPS, usando una apertura circular (37!'6 para Grumo A y 45".6 para Grumo B) y midiendo la fotometría del cielo en una región rectangular $\left(52^{\prime \prime} .5 \times 25^{\prime \prime} 8\right)$. Para cada apertura, se ha medido el brillo promedio de la superficie con una incertidumbre derivada de la desviación estándar del brillo de la superficie dentro de cada apertura y la incertidumbre de calibración del flujo.

A los valores calculados para los flujos se les ajustó una función Plankiana, lo que permitió hacer una estimación de la temperatura $\mathrm{T}_{d}{ }^{(4)}$. El mejor ajuste obtenido para cada grumo se muestra en la Figura 6.8 y corresponde a una temperatura de 13.6 y $13.5 \mathrm{~K}$, y a un valor del parámetro $\beta$ de 2.75 y 2.76 para los Grumos A y B, respectivamente.

Puede estimarse también la temperatura de color del polvo a partir de la relación de los flujos observados en dos bandas de Herschel $\left(S_{\nu}\right)$. La distribución de la temperatura de polvo $\left(\mathrm{T}_{d}\right)$ puede ser obtenida a partir del cociente entre la emisión en 160 y $250 \mu \mathrm{m}$. A pesar de que el cociente 70/160 tiene mejor resolución angular, el cociente 160/250 es más apropiado para esta región ya que es una zona fria.

Asumiendo que la emisión térmica del polvo observada es ópticamente delgada, el cociente entre los flujos observados está relacionado con la temperatura de color a partir de la siguiente

\footnotetext{
${ }^{(3)}$ www.splatalogue.net

${ }^{(4)} \mathrm{El}$ ajuste fue llevado a cabo por la Dra. Verónica Firpo
} 

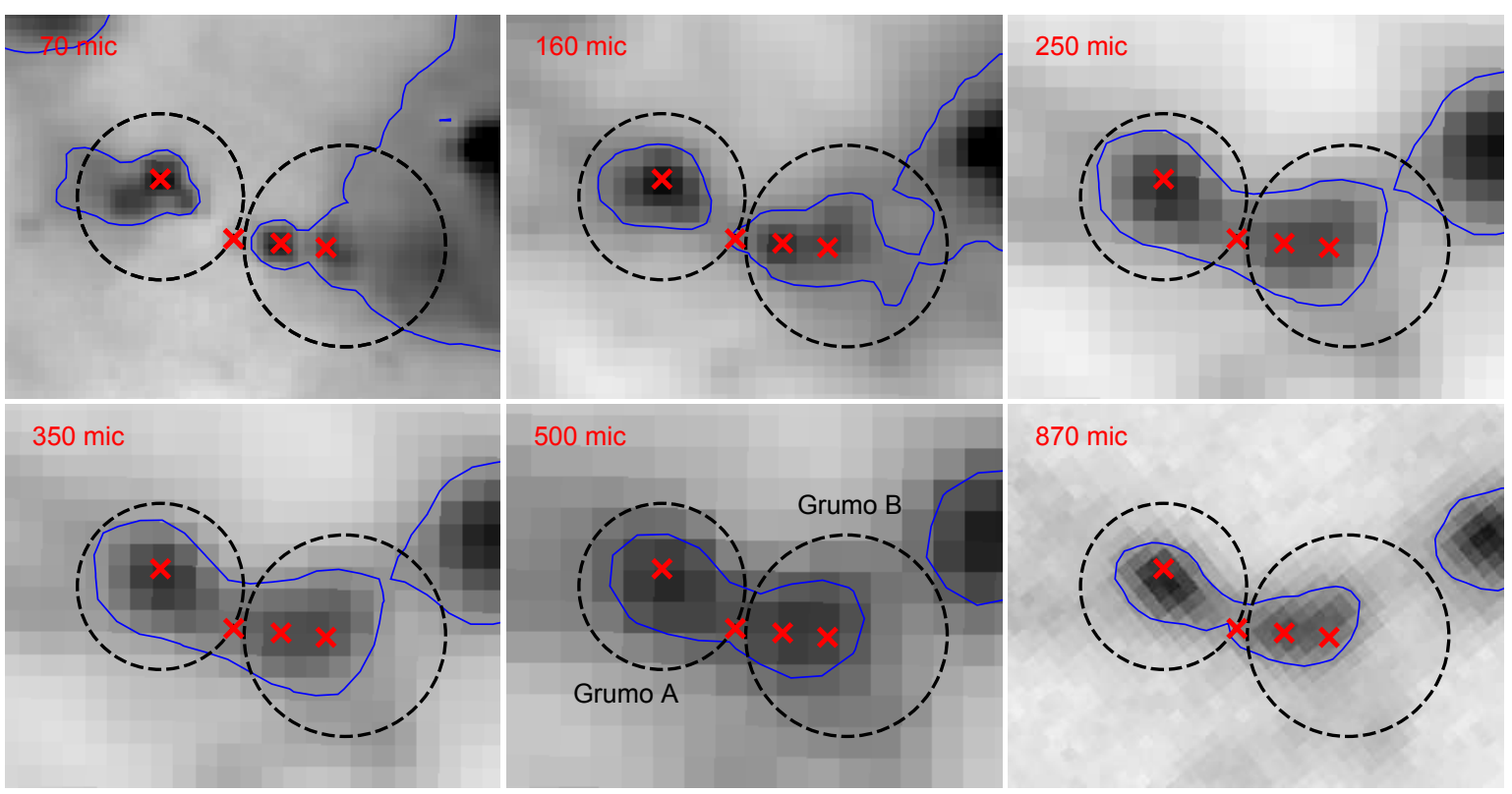

Figura 6.7: Emisión de la IRDC SDC 341.232-0.26 a diferentes longitudes de onda en el lejano infrarrojo. Los círculos muestran la posición y tamaño de los Grumos A y B definidos a partir del gas molecular y las cruces indican las posiciones de los candidatos a EGO. Para cada una de las figuras los contornos corresponden a 20 veces el rms.

Tabla 6.6: Parámetros y propiedades físicas derivados para los grumos IR.

\begin{tabular}{ccccccccc}
\hline \hline & $\mathrm{S}_{160}$ & $\mathrm{~S}_{250}$ & $\mathrm{~S}_{350}$ & $\mathrm{~S}_{500}$ & $\mathrm{~S}_{870}$ & $\mathrm{~T}_{d}$ & $M_{\text {dust+gas }}$ & $n_{\mathrm{H}_{2}}$ \\
{$[\mathrm{Jy}]$} & {$[\mathrm{Jy}]$} & {$[\mathrm{Jy}]$} & {$[\mathrm{Jy}]$} & {$[\mathrm{Jy}]$} & {$[\mathrm{K}]$} & {$\left[M_{\odot}\right]$} & {$\left[10^{3} \mathrm{~cm}^{-3}\right]$} \\
\hline Grumo A & $293 \pm 53$ & $281 \pm 21$ & $151 \pm 8$ & $563 \pm 3$ & $5.2 \pm 0.4$ & 13.6 & $1250 \pm 260$ & $1.3 \pm 0.4$ \\
Grumo B & $421 \pm 53$ & $376 \pm 21$ & $195 \pm 8$ & $74 \pm 3$ & $6.3 \pm 0.6$ & 13.5 & $1540 \pm 330$ & $1.1 \pm 0.3$ \\
\hline \hline
\end{tabular}

fórmula:

$$
f_{(T)}=\frac{S_{\nu}(\nu=160 \mu m)}{S_{\nu}(\nu=250 \mu m)}=\frac{B_{\nu}\left(160 \mu m, \mathrm{~T}_{\mathrm{d}}\right)}{B_{\nu}\left(250 \mu m, \mathrm{~T}_{\mathrm{d}}\right)}\left(\frac{160}{250}\right)^{\beta_{\mathrm{d}}}
$$

donde $B_{\nu}\left(\nu, \mathrm{T}_{\mathrm{d}}\right)$ es la función de Planck para un cuerpo negro y $\beta_{\mathrm{d}}$ corresponde al índice espectral de la emisión térmica del polvo. Se asumió un valor típico $\beta_{\mathrm{d}}=2$. Los resultados se muestran en la Figura 6.9. Los contornos de $8 \mu \mathrm{m}$ son los mismos que se muestran en la Figura 6.1.

Las temperaturas se encuentran en el rango entre $\sim 12$ y $\sim 17 \mathrm{~K}$ para la mayor parte de la IRDC, lo que está de acuerdo con lo obtenido de los ajustes y con lo reportado por Peretto \& Fuller (2009) para otras IRDCs.

Se estimó la masa total (gas + polvo) de los grumos a partir de la ecuación (ver ecuación 2.48)

$$
M_{\text {dust }+ \text { gas }}=\frac{S_{870} d^{2} R_{\mathrm{d}}}{B_{870}\left(T_{d}\right) \kappa_{870}}
$$

donde se adoptó un cociente gas/polvo $R_{\mathrm{d}}=137$ (Draine et al., 2007) y una temperatura media de $15 \mathrm{~K}$. La densidad ambiental se puede calcular como (ver ecuación 2.39) 

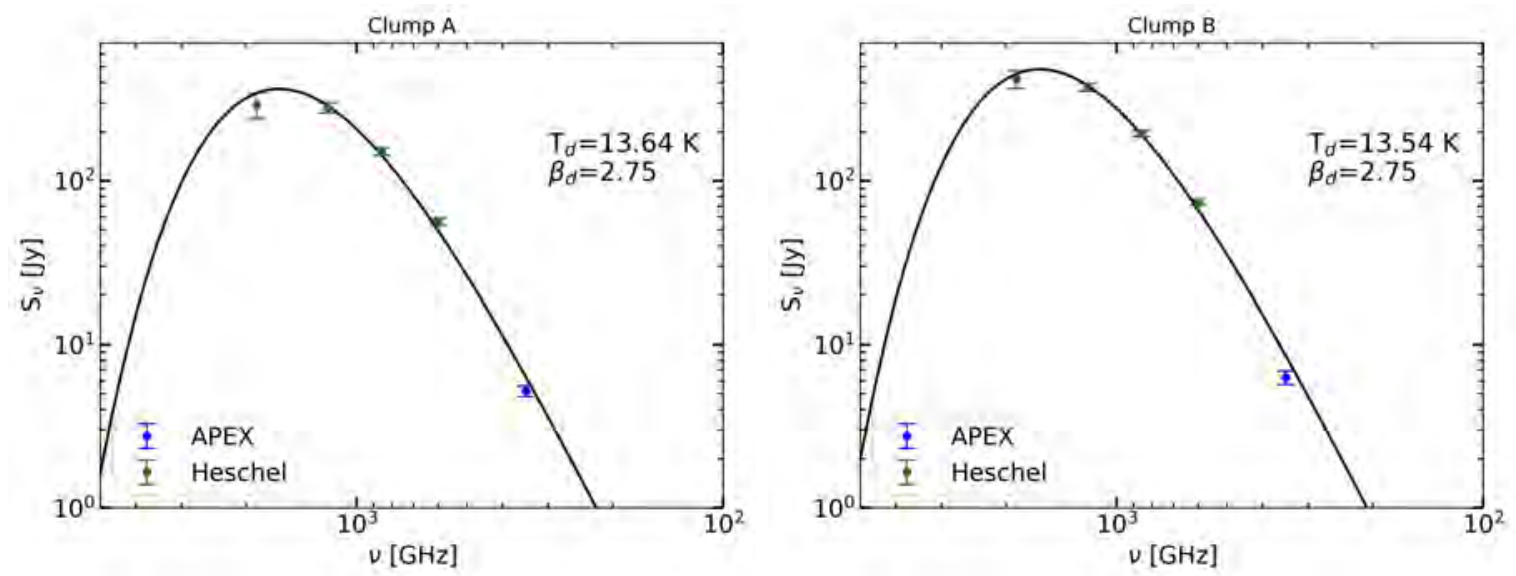

Figura 6.8: Distribuciones espectrales de energía (SEDs) calculadas para el Grumo A (izquierda) y el Grumo B (derecha) a partir de los flujos a 160, 250, 350 y $500 \mu$ m de Herschel y a $870 \mu \mathrm{m}$ de LABOCA.

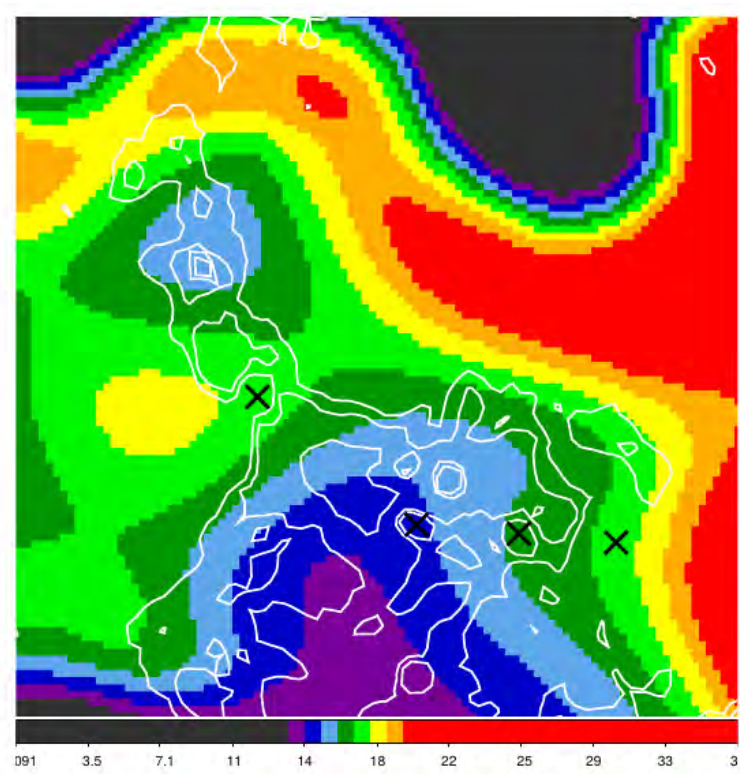

Figura 6.9: Temperatura del polvo obtenida a partir del cociente entre la emisión a 160 y 250 $\mu \mathrm{m}$, superpuesta a los contornos de $8 \mu \mathrm{m}$. Las cruces indican las posiciones de los candidatos a EGO. 
Tabla 6.7: Parámetros físicos obtenidos a partir del ajustes de la SED para los candidatos a YSOs.

\begin{tabular}{lccccc}
\hline \hline & $\begin{array}{c}\text { Edad } \\
10^{4}\end{array}$ & $\begin{array}{c}M_{\text {estelar }} \\
\mathrm{M}_{\odot}\end{array}$ & $\begin{array}{c}M_{\text {env }} \\
\mathrm{M}_{\odot}\end{array}$ & $\begin{array}{c}M_{\text {acr }} \\
10^{-4} \mathrm{M}_{\odot} \mathrm{yr}^{-1}\end{array}$ & $\begin{array}{c}L \\
10^{2} L_{\odot}\end{array}$ \\
\hline EGO 1 & 0.13 & 2.1 & 0.7 & 0.1 & 1.3 \\
EGO 2 & 545 & 3.9 & $8 \times 10^{-6}$ & 0 & 1.9 \\
EGO 3 & 15.7 & 4.1 & 7.1 & 1.4 & 0.8 \\
EGO 4 & 4.1 & 8.6 & 0.09 & 12.5 & 14.4 \\
\hline \hline
\end{tabular}

$$
n_{H_{2}}=\frac{M_{\text {dust }+ \text { gas }}}{\frac{4}{3} \pi R^{3} \mu_{H_{2}} m_{H}}
$$

donde $\mathrm{R}$ es el radio del grumo, $\mu_{H_{2}}=2.76$ es el peso molecular medio (considerando $25 \%$ de He) y $m_{H}$ es la masa atómica del Hidrógeno.

Los resultados están listados en la Tabla 6.6. Las incertezas en los valores de la masa y la densidad ambiental son de aproximadamente del $20 \%$ y del $40 \%$, respectivamente, y se deben principalmente a los errores en la distancia.

Nótese que las masas y densidades de los grumos obtenidas a partir del continuo del polvo y de las líneas moleculares son coincidentes dentro de los errores.

\subsection{Análisis y discusión}

\section{Candidatos a EGO}

Hasta el momento se han determinado parámetros de dos grumos (Grumo A y Grumo B) que fueron identificados en primer lugar a partir de la emisión del gas molecular y luego en su contraparte en el lejano IR. Sin embargo, como se mencionó en la sección anterior, al analizar las imágenes a 70 y $160 \mu \mathrm{m}$, que poseen mejor resolución angular, es posible detectar subestructura en esos grumos, que se corresponde con los candidatos a EGO.

Con el objetivo de estudiar la naturaleza de los candidatos a EGO presentes en la región de la IRDC se graficaron las distribuciones espectrales de energía (Spectral Energy Distributions, SEDs) de cada uno de los EGOs utilizando el algoritmo desarrollado por Robitaille et al. (2007), que puede ayudar a definir el estado evolutivo de una fuente. Esta herramienta ajusta modelos de transferencia de radiación a datos observacionales de acuerdo con un algoritmo de minimización de $\chi^{2}$. Para esto se utilizaron datos disponibles de Spitzer-IRAC y MIPSGAL a $24 \mu \mathrm{m}$, que poseen resolución suficiente como para identificar las fuentes puntuales dentro de los grumos.

Para EGO 2 EGO 3 y EGO 4 se usaron datos de Spitzer-GLIMPSE a 3.6, 4.5, 5.8 y $8 \mu \mathrm{m}$ y MIPSGAL a $24 \mu \mathrm{m}$, que se indican con puntos con barras de error en la Figura 6.10. Para EGO 1 se incluyeron además datos en las bandas J, H y K de 2MASS, indicados con triángulos como límites superiores en el panel superior izquierdo de la Figura 6.10. Las SEDs ajustadas para EGO 1, EGO 3 y EGO 4 son consistentes con objetos estelares jóvenes, mientras que el ajuste de EGO 2 no es muy bueno. En la Tabla 6.7 se muestran los principales parámetros obtenidos para las SEDs.

En las columnas 2 y 3 se muestran la edad y masa $M_{\text {estelar }}$ de la fuente central; en la columna 4 la masa de la envoltura $M_{e n v}$; en la columna 5 , la tasa de acreción $\dot{M}_{a c r}$; en la columna 6, la luminosidad total. Para realizar estos ajustes se adoptó una distancia $d=$ $3.6 \pm 0.4 \mathrm{kpc}$ y una extinción visual de 2-4 mag, típica del medio interestelar. 

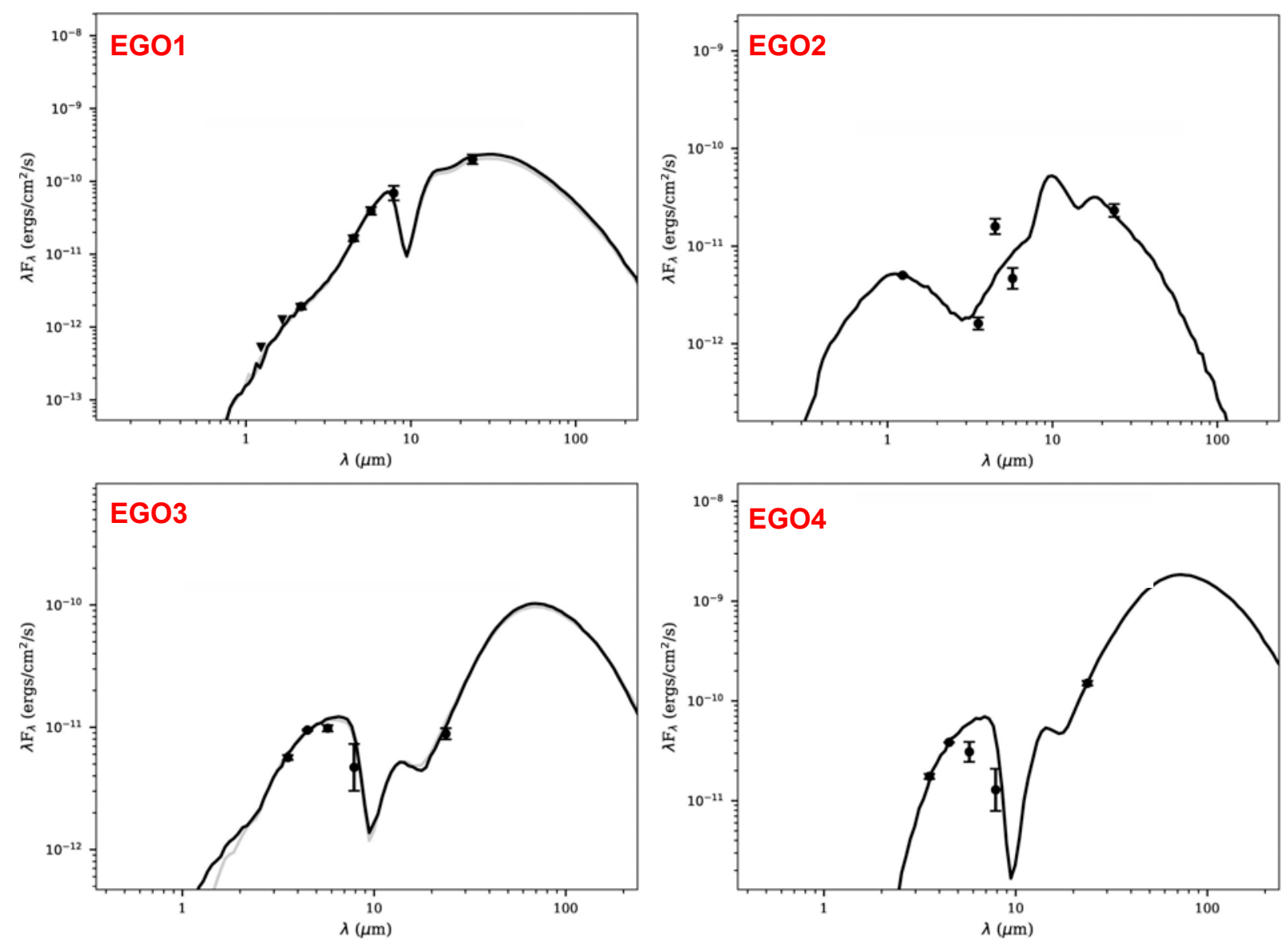

Figura 6.10: Distribución espectral de energía de EGO 1 (arriba izquierda), EGO 2 (arriba derecha), EGO 3 (abajo izquierda) y EGO 4 (abajo derecha). 


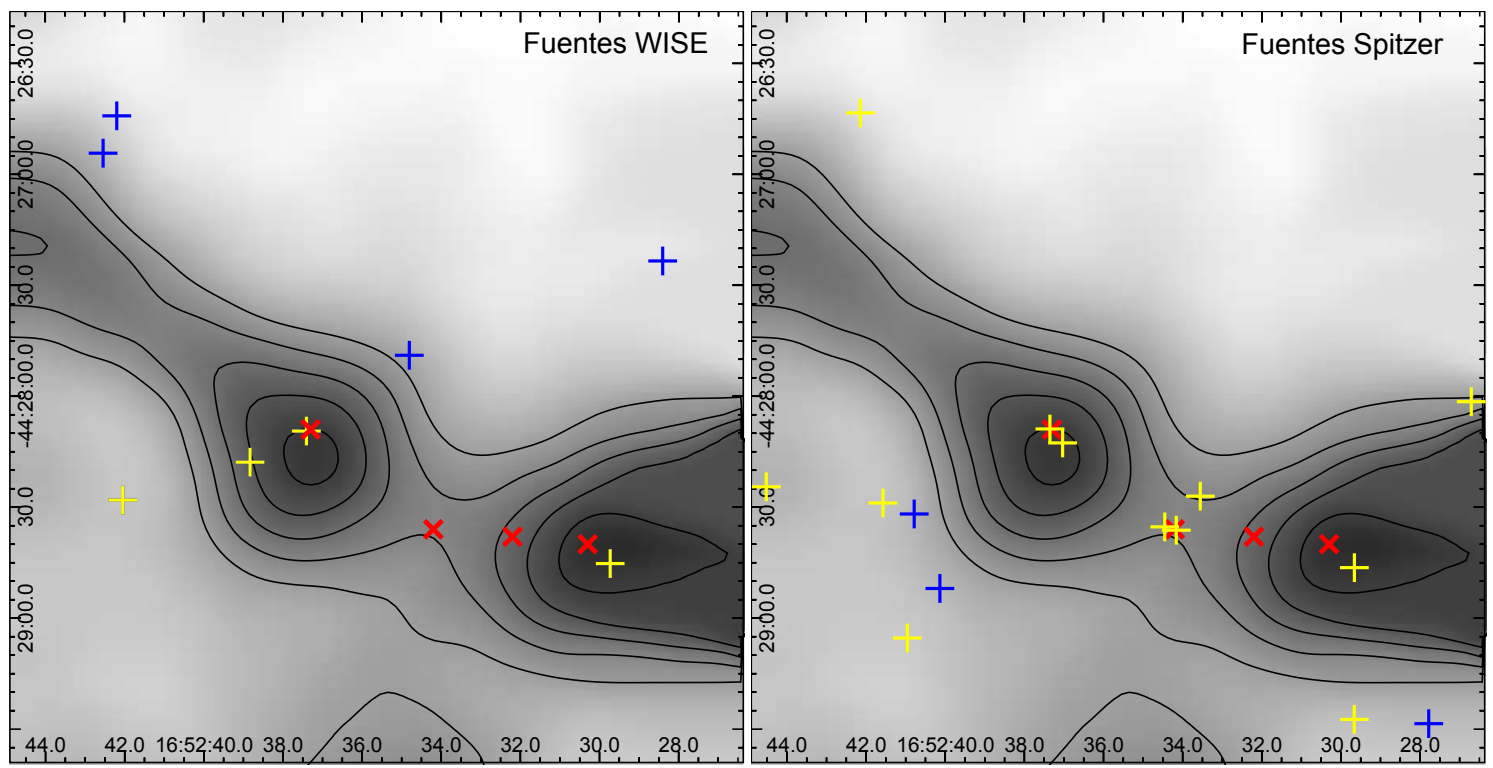

Figura 6.11: Candidatos a YSOs identificados en la región. En ambos paneles se muestran con cruces rojas las posiciones de los EGOs. En el panel de la izquierda se muestran las fuentes WISE clasificadas como candidatos a YSO clase I (amarillo) y clase II (azul). En el panel de la derecha se muestran las fuentes Spitzer clasificadas como candidatos a YSO clase I (amarillo) y clase II (azul).

Siguendo a Robitaille et al. (2007) se puede obtener una estimación del estado evolutivo de las fuentes basado en el cociente de la tasa de acreción y la masa de la fuente central. Para EGO 1, EGO 3 y EGO 4 el cociente $\dot{M}_{a c r} / M_{\text {estelar }}>10^{-6}$, indicando que se trata de YSOs en estadío 0/I, por lo que se trataría de objetos muy jóvenes, mientras que EGO 2 sería un objeto más evolucionado. Esta clasificación en estadíos no está basada en los índices espectrales de las SEDs como la clasificación en clases (ver Sección 1.4.1), sino en base a las propiedades físicas de las fuentes (e. g. masas del disco, tasa de acreción de la envolvente, etc).

Las masas de las envolturas y las edades de EGO 1, EGO 3 y EGO 4 sugieren que están aún inmersas en sus envolturas. De acuerdo con el ajuste, EGO 4 sería el objeto más masivo y EGO 2 el más evolucionado.

Los resultados mostrados en la Tabla 6.7 deben ser tomados con cautela debido a las limitaciones de la herramienta de ajuste que fueron mencionadas en la Sección 2.5 (Robitaille, 2008; Deharveng et al., 2012; Offner et al., 2012).

Para corroborar que, a pesar de las limitaciones del ajuste descriptas, los EGOs son objetos estelares jóvenes se llevó a cabo una búsqueda de candidatos a YSO en varios catálogos infrarrojos, tales como WISE, Spitzer y MSX, según los criterios descriptos en la Sección 1.4.5. Las fuentes WISE y Spitzer candidatos a YSO de clase I y clase II encontrados se muestran en la Figura 6.11. Allí se puede ver que hay varias fuentes clasificadas como clase I sobre los grumos, que coinciden con los EGOs 1, 3 y 4.

Como ya se mencionó en la Sección 1.4.8, los EGOs suelen estar asociados con objetos estelares jóvenes masivos (MYSOs) en regiones de formación estelar. Sin embargo no se han encontrado este tipo de fuentes asociados a los EGOs en esta región.

Por otro lado la emisión a $24 \mu \mathrm{m}$, detectada hacia las cuatro fuentes, sugiere la existencia de gas tibio y protoestrellas embebidas (Jackson et al., 2008). 

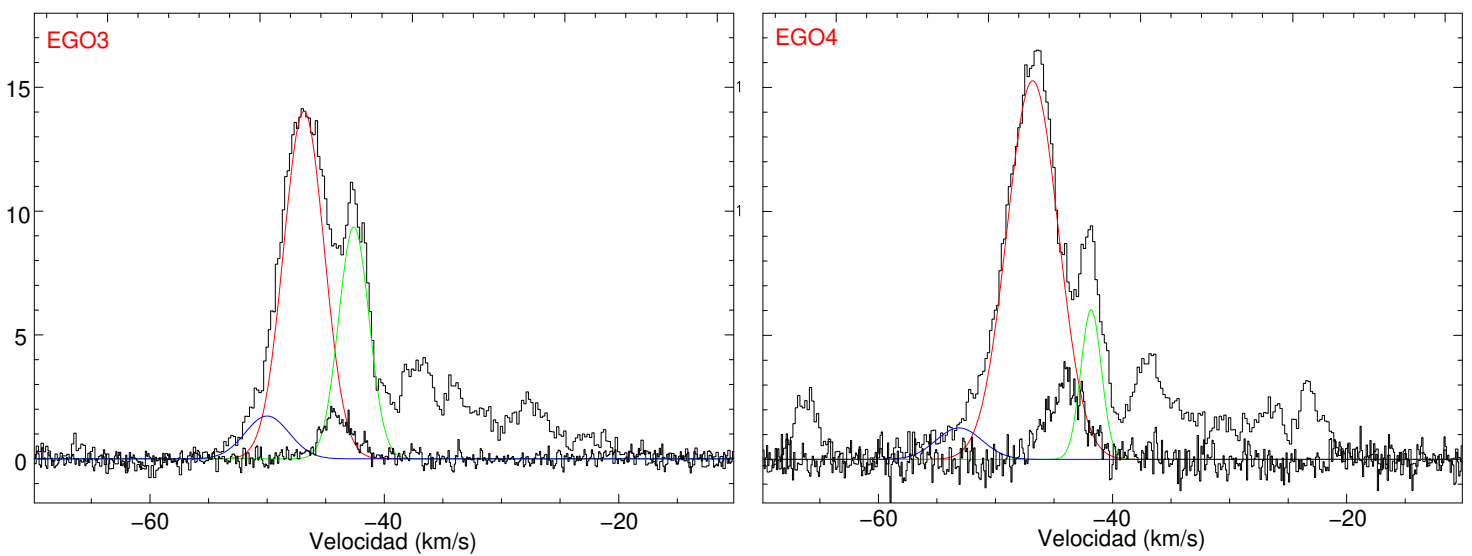

Figura 6.12: Perfiles de ${ }^{12} \mathrm{CO}(2-1)$ y $\mathrm{C}^{18} \mathrm{O}(2-1)$ en la dirección de EGO 3 (izquierda) y EGO 4 (derecha). Se muestran con líneas roja, verde y azul los ajustes gaussianos a las componentes de ${ }^{12} \mathrm{CO}(2-1)$. Las gaussianas indicadas con líneas azules corresponderían a la emisión de posibles alas azules de outflows.

\subsubsection{Evidencias de infalls $\mathbf{y}$ outflows}

Las estrellas en formación suelen mostrar evidencias de infalls y outflows. Una forma de visualizarlos es comparar una línea ópticamente gruesa como la del ${ }^{12} \mathrm{CO}$ con una ópticamente delgada como la del $\mathrm{C}^{18} \mathrm{O}$ en dirección a los candidatos a EGO, tal como se muestra en la Figura 6.12 para EGO 3 y EGO 4. La línea de emisión de ${ }^{12} \mathrm{CO}(2-1)$ presenta una estructura de doble pico con la componente azul más intensa que la roja, y un mínimo entre ambos picos (dip de absorción) cuya velocidad coincide con la velocidad sistémica de la nube molecular, mientras que la línea de $\mathrm{C}^{18} \mathrm{O}(2-1)$ presenta un pico simple que coincide en velocidad con el dip de absorción de la línea ópticamente gruesa. Esta característica es típica de movimientos de infall (para más detalles ver Sección 1.4.7).

De acuerdo con Chen et al. (2010), aunque el outflow y la rotación también podrían producir un perfil corrido hacia el azul a lo largo de una línea de visión particular, el movimiento de infall es el único proceso que produciría consistentemente la asimetría del perfil azul. Esta emisión desplazada hacia el azul puede ser producida debido al gas caliente altamente excitado situado en el lado más alejado del centro de contracción. La emisión de este gas sufre menos extinción que la emisión del material que se ve desplazada hacia el rojo, dado que la temperatura de excitación de las moléculas aumenta hacia el centro de la región (Zhou, 1992). Los espectros hacia EGO 3 y EGO 4 cumplen estas características, sugiriendo la presencia de movimientos infall.

Siguiendo a Mardones et al. (1997), se utilizó el parámetro $\delta \mathrm{v}$ (la diferencia de velocidad entre los picos de la línea ópticamente gruesa y las líneas ópticamente delgadas) para cuantificar la asimetría azul. El parámetro se define como $\delta \mathrm{v}=\left(\mathrm{v}_{\text {thick }}-\mathrm{v}_{\text {thin }}\right) / \Delta \mathrm{v}_{\text {thin }}$, siendo $\mathrm{v}_{\text {thick }} \mathrm{y} \mathrm{v}_{\text {thin }}$ las velocidades de las líneas ópticamente gruesas y delgadas, respectivamente, y $\Delta \mathrm{v}_{\text {thin }}$ el ancho de la línea ópticamente delgada. Valores de $\delta \mathrm{v}<-0.25$ indican que el gas molecular está cayendo sobre el grumo. Se encontró, tanto para el Grumo A como para el Grumo B, $\delta \mathrm{v} \sim-0.06$, lo que apoya la hipótesis de infall. Otro factor que refuerza esta hipótesis son los valores de los cocientes $M_{V I R} / M\left(H_{2}\right)$ que se muestran en la Tabla 6.4. Tal como se describió en la Sección 2.2.3 valores de este cociente menores que 1 indican que la nube no tiene suficiente energía cinética como para frenar el colapso gravitacional. En este caso los valores de $M_{V I R} / M\left(H_{2}\right)$ son mayores o menores que uno según qué modelo de perfil 


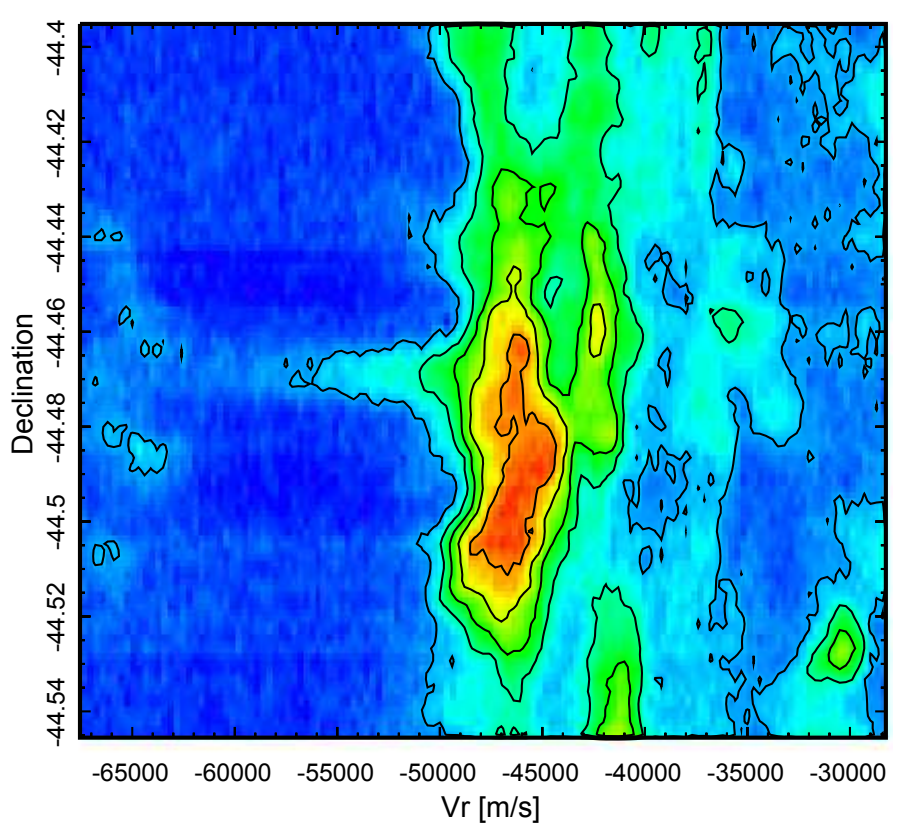

Figura 6.13: Diagrama posición-velocidad hacia EGO 4 (velocidad vs. declinación para un valor fijo de ascensión recta $=16: 52: 35)$. La escala de colores indica temperatura $\mathrm{T}_{m b}$.

de densidad de la nube se considere, por lo que no se puede descartar que haya colapso. Sin embargo, como ya se mencionó en el Capítulo 4, si se tiene en cuenta la estructura jerárquica de las nubes moleculares se puede suponer que los grumos aumentan su densidad hacia el interior, por lo que se debería tomar un valor de $k=126$ lo que da como resultado $M_{\text {vir }} / M\left(H_{2}\right)$ $=0.82$ y 0.73 para los Grumos A y B, respectivamente, lo que indica que estarían colapsando.

Por otro lado, para EGO 3 y EGO 4 se pueden distinguir posibles alas azules desde $\sim-55$ $\mathrm{a}-45 \mathrm{~km} \mathrm{~s}^{-1}$ y desde $\sim-60 \mathrm{a}-50 \mathrm{~km} \mathrm{~s}^{-1}$, respectivamente (representadas por las gaussianas indicadas con línea azul en la Figura 6.12, centradas en -50.5 y $-53 \mathrm{~km} \mathrm{~s}^{-1}$ ) mientras que el rango de velocidad de la línea de $\mathrm{C}^{18} \mathrm{O}(2-1)$ es $\sim-47 \mathrm{a}-40 \mathrm{~km} \mathrm{~s}^{-1}$. Las alas azules fueron definidas a partir del ajuste de componentes gaussianas a los espectros de ${ }^{12} \mathrm{CO}(2-1)$ mostrados en la Figura 6.12 que se encuentran a velocidades más negativas que la emisión de $\mathrm{C}^{18} \mathrm{O}(2-1)$. Las alas rojas no se logran distinguir fácilmente, sin embargo se detectan múltiples componentes en la emisión de ${ }^{12} \mathrm{CO}(2-1)$.

La firma típica de los outflows se puede ver en el diagrama posición-velocidad (ver Sección 1.4.6). Como se muestra en la Figura 6.13 para EGO 4, la extensión de la emisión hacia velocidades de alrededor de $-55 \mathrm{~km} \mathrm{~s}^{-1}$ se corresponde con las alas azules mostradas en la Figura 6.12, indicando que habría un outflow.

\subsection{Conclusiones}

A partir del análisis del gas molecular y el polvo asociado a la IRDC SDC 341.232-0.268 se determinó la presencia de dos grumos moleculares (Grumo A y Grumo B) sobre los que se encuentran proyectados cuatro EGOs y varias fuentes IR de los catálogos WISE y Spitzer clasificadas como candidatos a YSOs clase I.

Se encontró que Grumo A y Grumo B se detectan tanto en CO como en trazadores de alta densidad tales como $\mathrm{HCO}^{+}(1-0), \mathrm{HNC}(1-0)$ y $_{2} \mathrm{H}^{+}(1-0)$, y en el lejano IR $(\lambda>160 \mu \mathrm{m})$. En longitudes de onda del IR medio $(24$ y $70 \mu \mathrm{m})$ para las que la resolución angular es mejor, es 
posible distinguir subestructura en los grumos.

A partir del gas molecular se determinaron masas de $1220 \pm 250$ y $1020 \pm 220 \mathrm{M}_{\odot}$ y densidades ambientales de $(1.3 \pm 0.4) \times 10^{4}$ y $(0.5 \pm 0.2) \times 10^{4} \mathrm{~cm}^{-3}$ para los Grumos A y B, respectivamente. Las densidades ambientales calculadas a partir de $\mathrm{N}_{2} \mathrm{H}^{+}(1-0)$ son mayores $\left(\sim 1.7 \times 10^{5}\right)$ y están de acuerdo con la densidad crítica de esta molécula, que estaría trazando la zona más densa de los grumos. Las masas de gas+polvo y las densidades ambientales calculadas a partir de las imágenes en el IR lejano coinciden, dento de los errores, con las calculadas a partir del gas molecular.

Del ajuste de distribuciones espectrales de energía para los grumos se encontró que la temperatura de polvo de los mismos es de $13.5 \mathrm{~K}$, valor que está dentro del rango de $\mathrm{T}_{d}$ determinado a partir del mapa de temperatura de polvo. Los parámetros calculados coinciden con los determinados por Bergin \& Tafalla (2007) para grumos.

Los valores de masas de virial, la estructura de doble pico asimétrico y la diferencia de velocidad entre los máximos de una línea ópticamente delgada y una ópticamente gruesa sugieren que los grumos estarían colapsando. Además las alas ensanchadas de los perfiles en dirección a EGO 3 y EGO 4 y el diagrama posición-velocidad hacia EGO 4 indican que estos podrían tener outflows. 


\section{Capítulo 7}

\section{Complejo de burbujas infrarrojas S108-S109-S110-S111}

\subsection{Antecedentes de la región}

El complejo de burbujas S108-S111 catalogado por Churchwell et al. (2006) está formado por la burbuja IR S108, que es la de mayor tamaño y más débil a $8 \mu \mathrm{m}$, y por las burbujas S109, S110 y S111 que forman una 'burbuja tripolar'. Estas se pueden ver en la Figura 7.1 donde se muestra en verde la emisión a $8 \mu \mathrm{m}$, en azul a $5.8 \mu \mathrm{m}$ y en rojo a $4.5 \mu \mathrm{m}$. Todas ellas se ven completamente cerradas en $8 \mu \mathrm{m}$. S111 podría estar asociada a un cúmulo estelar ubicado en su interior y detectado por Mercer et al. (2005). Según estos autores el cúmulo está centrado en la posición RA, Dec.(J2000) $=(14: 45: 10,-59: 50: 24)$, tiene un radio de 0!7, y posee 65 estrellas que fueron identificadas a partir del catálogo 2MASS. De las restantes burbujas no hay información, hasta el presente, de objetos estelares asociados.

Churchwell et al. (2006) determinaron la posición de las burbujas y definieron su tamaño a partir de elipses indicando los semiejes menores y mayores tanto internos como externos. Estas elipses se muestran en la Figura 7.2, superpuestas a la emisión de Spitzer-IRAC mostrada en la Figura 7.1, y sus parámetros se detallan en la Tabla 7.1. En la Figura 7.2 también se puede ver que hacia el sureste de la burbuja S109 hay un segundo arco no definido por Churchwell et al. (2006). Algo similar ocurre al suroeste de la burbuja S111 con al menos dos arcos (indicados con línea discontinua roja). Esto podría sugerir que el tamaño real de las burbujas no sería el definido por estos autores.

Hacia esta región se encuentra la fuente IRAS 14416-5937 (RA, Dec.(J2000)=14:45:28.3, -59:49:59) casi en el centro del complejo (ver Figura 7.2). Esta fuente tiene cocientes de flujos $25 \mu \mathrm{m} / 12 \mu \mathrm{m}>3.7$ y $60 \mu \mathrm{m} / 12 \mu \mathrm{m}>19.3$, lo que la transforma en una candidata a sitio de formación de estrellas OB (Churchwell \& Wood, 1989). Las características espectrales de las

\begin{tabular}{lcccccc}
\hline \hline Nombre & $l$ (grados) & $b$ (grados) & $\mathrm{R}_{\text {in }}\left({ }^{\prime}\right)$ & $\mathrm{r}_{\text {in }}\left({ }^{\prime}\right)$ & $\mathrm{R}_{\text {out }}\left({ }^{\prime}\right)$ & $\mathrm{r}_{\text {out }}\left({ }^{\prime}\right)$ \\
\hline S108 & 316.866 & 0.030 & 2.84 & 2.59 & 4.04 & 3.68 \\
S109 & 316.812 & -0.101 & 1.51 & 0.96 & 2.32 & 1.47 \\
S110 & 316.809 & -0.031 & 1.41 & 1.09 & 1.82 & 1.41 \\
S111 & 316.772 & -0.068 & 1.37 & 0.98 & 1.83 & 1.31 \\
\hline \hline
\end{tabular}

Tabla 7.1: Posición y tamaño de las burbujas definidos por Churchwell et al. (2006). Se lista latitud y longitud galáctica y los radios internos mayor y menor $\left(\mathrm{R}_{i n} \mathrm{y} \mathrm{r}_{i n}\right)$ y externos mayor $\mathrm{y}$ menor $\left(\mathrm{R}_{\text {out }} \mathrm{y} \mathrm{r}_{\text {out }}\right)$. 


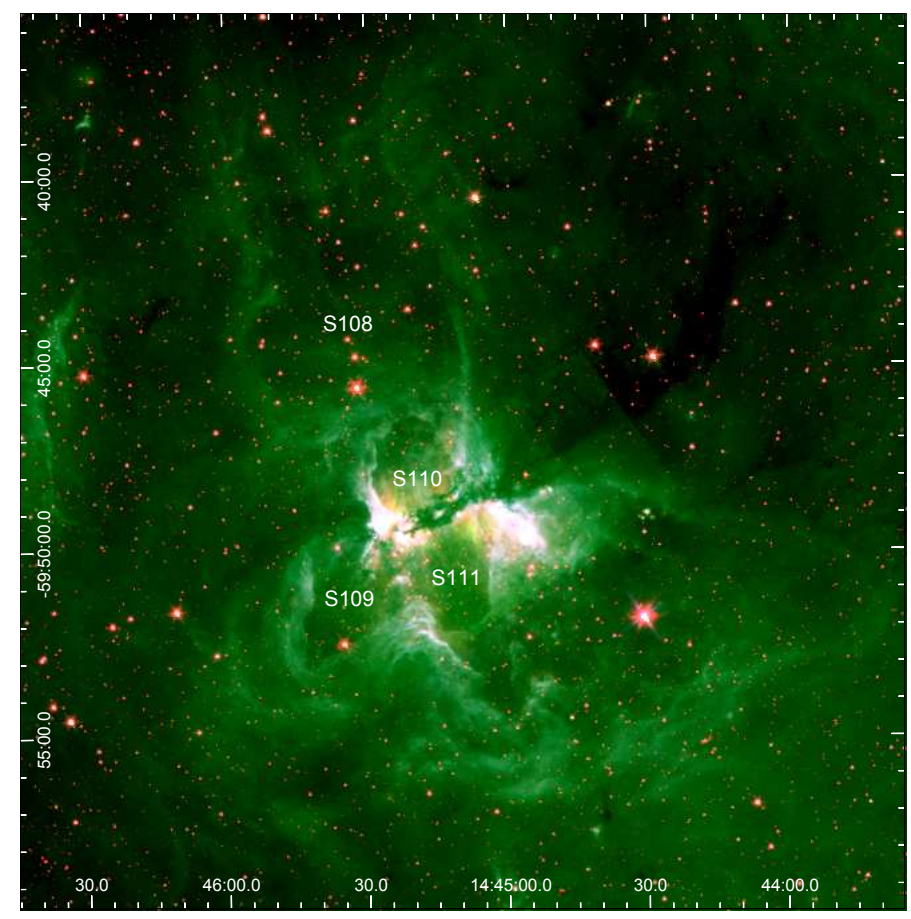

Figura 7.1: Complejo de burbujas S108-S111. Se muestra en verde la emisión a $8 \mu \mathrm{m}$, en azul $5.8 \mu \mathrm{m}$ y en rojo $4.5 \mu \mathrm{m}$.

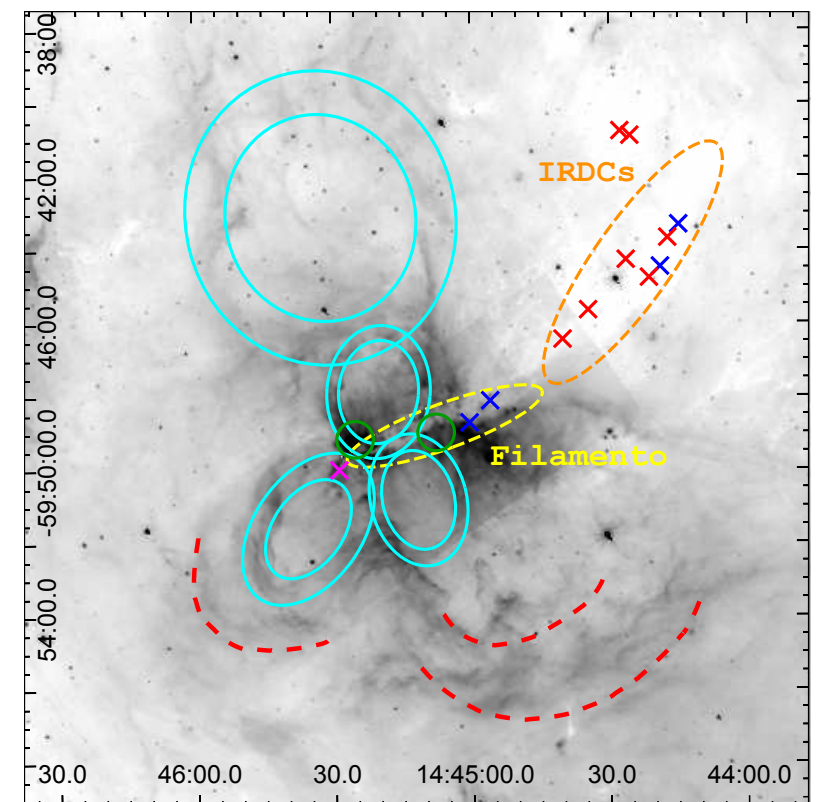

Figura 7.2: Sobre la imagen de $8 \mu \mathrm{m}$ se muestran con elipses color celeste los tamaños de las burbujas definidas por Churchwell et al. (2006). Las líneas discontinuas rojas muestran la presencia de arcos en la emisión de $8 \mu \mathrm{m}$ que estarían centrados en las burbujas S109 y S111. La cruz magenta es la posición de la fuente IRAS 14416-5937. Los círculos verdes corresponden a las fuentes IRAS 14416-5937-A y IRAS 14416-5937-B definidas por Vig et al. (2007). Las cruces azules marcan las posiciones de las IRDCs observadas por Vasyunina et al. (2011). Las cruces rojas corresponden a IRDCs catalogadas por Simon et al. (2006). Finalmente la elipse de línea discontinua de color amarillo muestra el 'Filamento' y la naranja la 'IRDC' tal como fueron definidas en esta tesis. 
fuentes IRAS entre 12 y $100 \mu \mathrm{m}$ fueron estudiadas por Wood \& Churchwell (1989) quienes propusieron que sus colores en el lejano IR podían ser usados para identificar regiones de formación estelar y la catalogaron como UCHII. La posición de esta fuente IRAS coincide también con las posiciones de un máser de OH (Caswell \& Haynes, 1987) que tiene su máximo a una velocidad de $-45.8 \mathrm{~km} \mathrm{~s}^{-1}$, un máser de $\mathrm{H}_{2} \mathrm{O}$ 316.8-00.1 (Caswell et al., 1989) y un máser de metanol $\left(\mathrm{CH}_{3} \mathrm{OH}\right)$ (Walsh et al., 1998).

En las longitudes de onda de radio, donde el gas molecular y el polvo circundante son transparentes, las regiones UCHII aparecen en observaciones de disco simple como fuentes de continuo brillantes y no resueltas. Frecuentemente, y tal como ocurre en el caso de la fuente IRAS 14416-5937, la emisión de continuo de radio está estrechamente asociada con los máseres de $\mathrm{OH}$ y $\mathrm{H}_{2} \mathrm{O}$, una de las primeras indicaciones de que las regiones UCHII están relacionadas con choques y otros movimientos dinámicos en regiones de formación estelar.

La fuente IRAS 14416-5937 ha sido anteriormente estudiada por Bronfman et al. (1996) a partir de datos obtenidos con el telescopio SEST (Swedish-ESO Submillimiter Telescope) que consisten en un apuntamiento centrado en la fuente IRAS a la frecuencia de la línea de $\mathrm{CS}(2-1)(97.981 \mathrm{GHz})$.

Además de CS, se ha observado hacia la región emisión de líneas de $\mathrm{NH}_{3}$ (Vilas-Boas \& Abraham, 2000), CI (Huang et al., 1999), CO (Whiteoak et al., 1982; White \& Phillips, 1983) y $\mathrm{H}_{2} \mathrm{O}$ (Gardner \& Whiteoak, 1984). La emisión se detecta a una velocidad de $-38.3 \mathrm{~km} \mathrm{~s}^{-1}$ lo que, según el modelo de rotación circular galáctica, se corresponde con una distancia cercana de $2.8 \mathrm{kpc}$. Considerando una dispersión en velocidad de $6 \mathrm{~km} \mathrm{~s}^{-1}$, la error en la distancia es del $18 \%(2.8 \pm 0.5 \mathrm{kpc})$.

Vig et al. (2007) estudiaron la región de interacción de las burbujas a partir de imágenes en el lejano infrarrojo a 150 y $210 \mu \mathrm{m}$ tomadas con un fotómetro infrarrojo de dos bandas a bordo de un globo aerostático (TIFR balloon facility) con una resolución angular de $\sim 1^{\prime}$. A partir de esas observaciones identificaron dos fuentes, una de ellas en la zona de contacto de las burbujas S109, S110 y S111 (IRAS 14416-5937-A, RA, Dec.(J2000)=14:45:25.4,-59:49:11) y otra al noroeste de la burbuja S111 (IRAS 14416-5937-B,RA, Dec.(J2000)=14:45:07.8,59:49:0) (ver Figura 7.2). La Figura 7.3 muestra la emisión en 150 y $210 \mu \mathrm{m}$, tomada de Vig et al. (2007). Estos autores publicaron mapas de temperaturas de polvo T(150/210) y de profundidad óptica a $200 \mu \mathrm{m}, \tau_{200}$. A partir de estos determinaron temperaturas de 27 y $24 \mathrm{~K}$ y masas de polvo de 31 y $36 \mathrm{M}_{\odot}$ para IRAS 14416-5937-A y IRAS 14416-5937B, respectivamente. Encontraron también que el máximo de profundidad óptica es 0.06 y se encuentra cerca de IRAS 14416-5937-B. Con el fin de obtener parámetros físicos de la región aplicaron un modelo de transferencia radiativa a las fuentes IRAS14416-5937-A y IRAS 14416-5937-B. Las masas obtenidas del modelo son de 25 y $49 \mathrm{M}_{\odot}$, lo que está de acuerdo con las masas obtenidas a partir de las temperaturas de polvo por esos autores. Del modelo encontraron que la profundidad óptica a $100 \mu \mathrm{m}$ es de 0.02 . La diferencia con lo obtenido a partir del mapa de $\tau_{200}$ podría deberse a inhomogeneidades en el medio o a la presencia de granos de polvo con diferentes propiedades que las que se usaron para el modelo.

El complejo está también asociado a dos nubes oscuras infrarrojas (IRDCs). La zona de las IRDCs fue previamente estudiada por Vasyunina et al. (2011) utilizando las moléculas $\mathrm{CH}_{3} \mathrm{C}_{2} \mathrm{H}, \mathrm{H}_{13} \mathrm{CN}, \mathrm{H}_{13} \mathrm{CO}^{+}, \mathrm{SiO}, \mathrm{C}_{2} \mathrm{H}, \mathrm{HNCO}, \mathrm{HCN}, \mathrm{HCO}^{+}, \mathrm{HNC}, \mathrm{HC}_{3} \mathrm{~N}, \mathrm{CH}_{3} \mathrm{CN}$, ${ }^{13} \mathrm{CS}$ y $\mathrm{N}_{2} \mathrm{H}^{+}$(observaciones de un solo punto). La Figura 7.4, tomada de Vasyunina et al. (2011), muestra la emisión en el IR obtenida con Spitzer-GLIMPSE de las dos regiones correspondientes a IRDC316.72 y IRDC316.76, respectivamente. Cada IRDC fue observada en dos posiciones indicadas como IRDC316.72-1, IRDC316.72-2, IRDC316.76-1 y IRDC316.762. Estas posiciones y el tamaño del haz son mostrados con círculos en la figura. La Figura 7.5, también tomada de este trabajo, muestra los resultados de las observaciones para cada una 

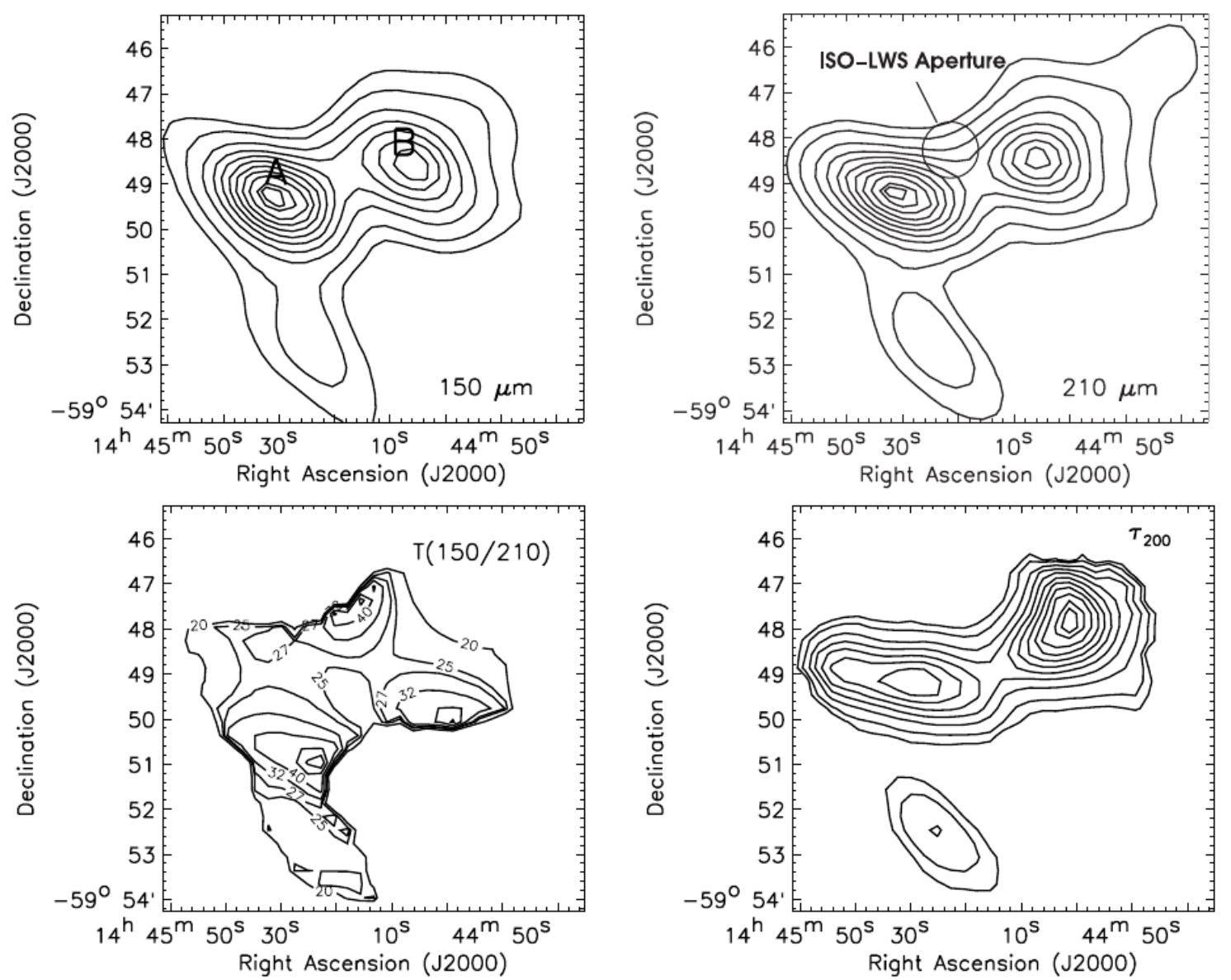

Figura 7.3: Arriba: Mapas de intensidad de la región alrededor de IRAS 14416-5937 a 150 (izquierda) y $210 \mu \mathrm{m}$ (derecha). Se indican las fuentes A y B. Abajo: Mapas de temperatura de polvo T(150/210) (izquierda) y profundidad óptica a $200 \mu \mathrm{m}$ (derecha). Los contornos de $\tau_{200}$ representan el $5,10,20,30,40,50,60,70,80$ y $90 \%$ del valor máximo de 0.06. Imágenes tomadas de Vig et al. (2007). 

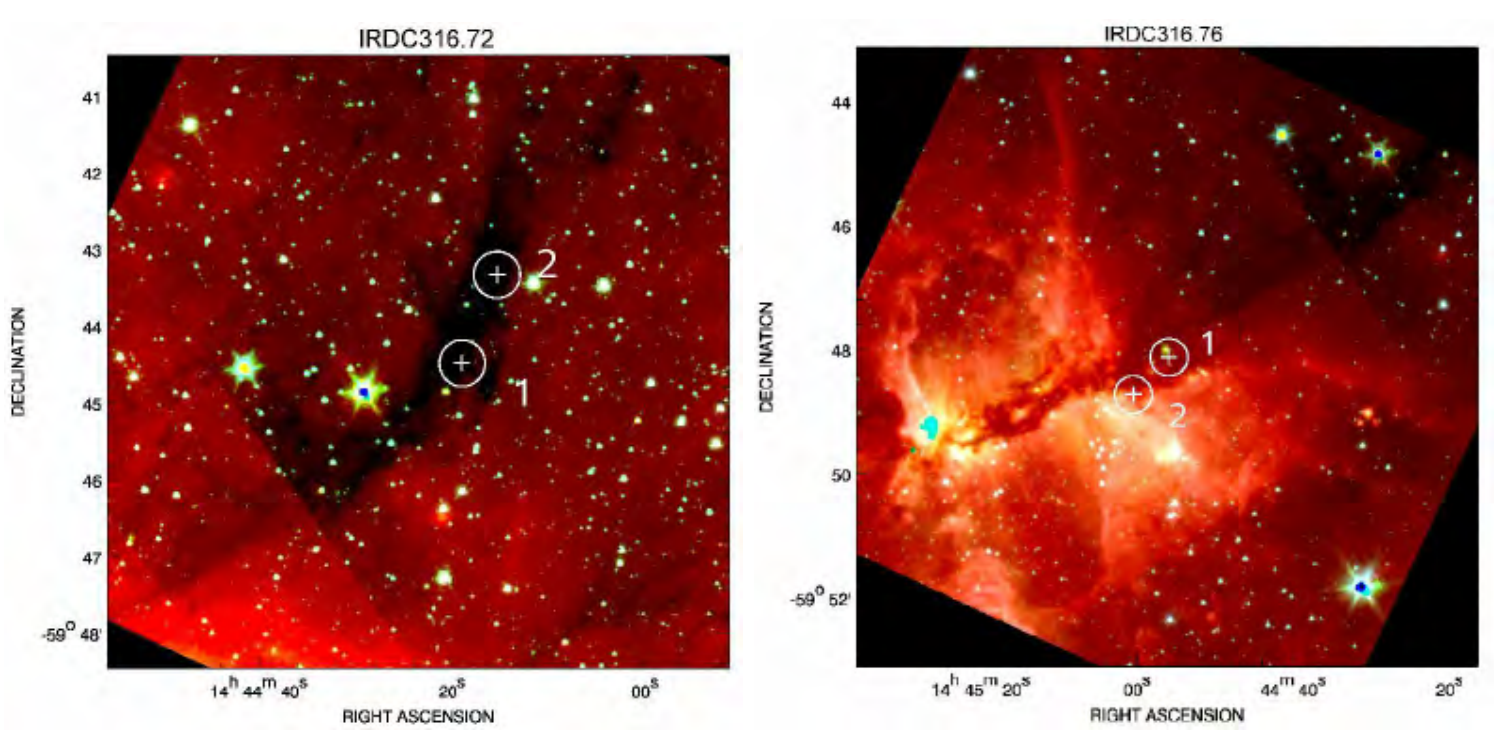

Figura 7.4: Imágenes IR de IRDC316.72 (izquierda) y IRDC316.76 (derecha). Se muestra en azul la emisión a 3.6, en verde a 4.5 y en rojo a $8.0 \mu \mathrm{m}$. Los círculos indican las posiciones y el haz con que fueron observadas las línea moleculares por Vasyunina et al. (2011).

Tabla 7.2: Parámetros de las IRDCs estudiadas por Vasyunina et al. (2011).

\begin{tabular}{lccccc}
\hline \hline Nombre & RA, Dec.(J2000) & $\begin{array}{c}\text { Distancia } \\
{[\mathrm{kpc}]}\end{array}$ & $\begin{array}{c}\mathrm{T}_{\text {exc }}(1) \\
{[\mathrm{K}]}\end{array}$ & $\begin{array}{c}\mathrm{N}\left(\mathrm{H}_{2}\right) \\
{\left[\times 10^{22} \mathrm{~cm}^{-2}\right]}\end{array}$ & Categoría $^{(2)}$ \\
\hline IRDC316.72-1 & $14: 44: 19.000-59: 44: 29.00$ & 2.7 & 26.1 & 1.2 & $\mathrm{M}$ \\
IRDC316.72-2 & $14: 44: 15.400-59: 43: 20.00$ & 2.7 & 24.3 & 1.3 & $\mathrm{Q}$ \\
IRDC316.76-1 & $14: 44: 56.000-59: 48: 08.00$ & 2.7 & 22.6 & 4.1 & $\mathrm{~A}$ \\
IRDC316.76-2 & $14: 45: 00.500-59: 48: 44.00$ & 2.8 & 23.2 & 4.8 & $\mathrm{~A}$ \\
\hline \hline
\end{tabular}

(1): Temperaturas derivadas a partir de transiciones del amoníaco observadas con el radiotelescopio de $64 \mathrm{~m}$ Parkes. (2): 'A' indica grumos activos, 'M' intermedios y 'Q' inactivos tal como se describió en la Sección

$$
\text { 1.4.10. }
$$

de las posiciones observadas en cada IRDC. Los espectros muestran emisión a velocidades de entre -35 y $-45 \mathrm{~km} \mathrm{~s}^{-1}$ (excepto $\mathrm{HCN} \mathrm{y} \mathrm{N}_{2} \mathrm{H}^{+}$cuya extensión en velocidad es mayor debido a la presencia de componentes hiperfinas), compatible con una distancia cinemática de $2.8 \pm 0.5$ kpc. La coincidencia entre la velocidad del gas en las IRDCs y la velocidad para el CS hacia la fuente IRAS sugiere que estos pertenecen al mismo complejo.

Ragan et al. (2012) determinaron que IRDC 316.72-1 aloja un pequeño cúmulo de fuentes infrarrojas embebidas y posee una masa de entre 400 y $950 \mathrm{M}_{\odot}$ derivada de la extinción en $8 \mu \mathrm{m}$ (Linz et al., 2007) y de la emisión a $1.2 \mathrm{~mm}$.

\subsection{Observaciones y base de datos}

El objetivo de este Capítulo es describir el medio interestelar asociado al complejo de burbujas IR y a las IRDCs, estudiar sus propiedades físicas, la cinemática del gas y determinar los posibles procesos de formación estelar que allí tienen lugar.

El estudio de la distribución del gas molecular de la región se llevó a cabo a partir de datos de líneas moleculares obtenidos con los telescopios NANTEN $\left({ }^{12} \mathrm{CO}(1-0)\right)$, APEX $\left({ }^{12} \mathrm{CO}(3-2),{ }^{13} \mathrm{CO}(3-2), \mathrm{C}^{18} \mathrm{O}(3-2),{ }^{12} \mathrm{CO}(2-1) ;{ }^{13} \mathrm{CO}(2-1), \mathrm{C}^{18} \mathrm{O}(2-1)\right)$ y los pertenencien- 

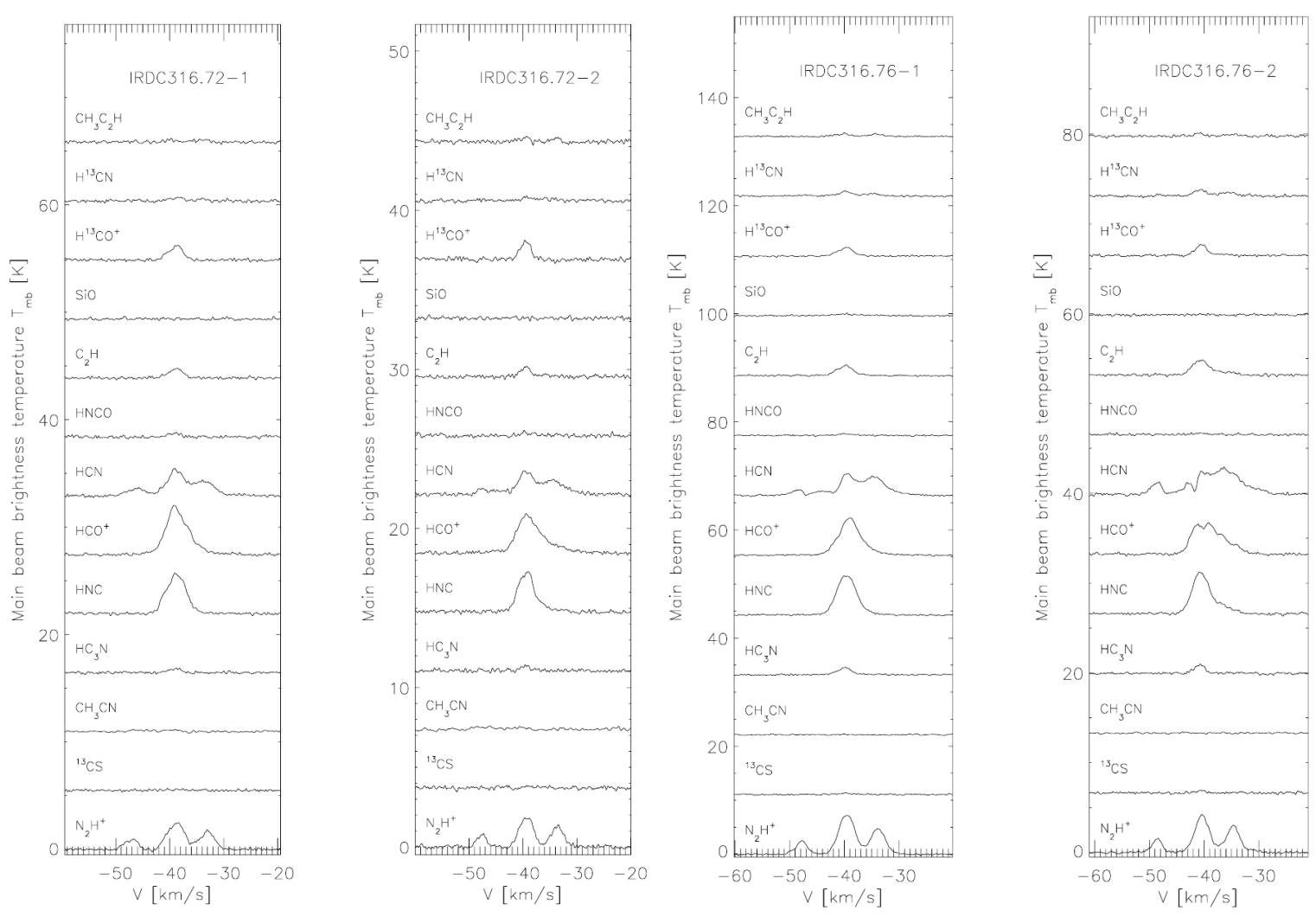

Figura 7.5: Líneas observadas hacia IRDC316.72 (izquierda) y IRDC316.76 (derecha).

tes al relevamiento MALT90 $\left(\mathrm{N}_{2} \mathrm{H}^{+}(1-0), \mathrm{HC}_{3} \mathrm{~N}(10-9), \mathrm{HNC}(1-0), \mathrm{HCO}^{+}(1-0), \mathrm{HCN}(1-0)\right.$, $\left.\mathrm{C}_{2} \mathrm{H}(1-0), \mathrm{HN}^{13} \mathrm{C}(1-0), \mathrm{H}^{13} \mathrm{CO}^{+}(1-0)\right)$ en el que se observaron regiones de $3^{\prime} \times 3^{\prime}$ asociadas a fuentes ATLASGAL, localizadas principalmente en la zona de interacción de la burbuja tripolar y hacia las IRDCs.

Los datos de ${ }^{12} \mathrm{CO}(1-0)$ obtenidos con el telescopio NANTEN tienen una resolución angular de 2 ! 6 , una resolución en velocidad de $1 \mathrm{~km} \mathrm{~s}^{-1}$ y un ruido $r m s$ de $0.16 \mathrm{~K}$.

Con APEX fueron observadas las líneas moleculares ${ }^{12} \mathrm{CO}(2-1),{ }^{13} \mathrm{CO}(2-1)$ y $\mathrm{C}^{18} \mathrm{O}(2-1)$ $\left(\right.$ HPBW $\sim 30^{\prime \prime}$ ) trazando el gas molecular asociado a la burbuja tripolar y a las IRDCs (regiones indicadas por recuadros de línea continua en la Figura 7.6). Por razones instrumentales, y dado que la región que cubre la burbuja tripolar es muy grande, se observaron separadamente dos regiones adyacentes. Los cubos de datos que resultaron de estas observaciones (recuadros 1 y 2 de la Figura 7.6) fueron unidos utilizando el paquete de procesamiento de datos MADR del Dominion Radio Astrophysical Observatory (DRAO). La región de interacción de las burbujas también fue observada en ${ }^{12} \mathrm{CO}(3-2),{ }^{13} \mathrm{CO}(3-2)$ y $\mathrm{C}^{18} \mathrm{O}(3-2)$ (recuadro de línea discontinua en la Figura 7.6), con una resolución de $\sim 20^{\prime \prime}$.

Los datos de ${ }^{12} \mathrm{CO}(2-1) ;{ }^{13} \mathrm{CO}(2-1)$ y $\mathrm{C}^{18} \mathrm{O}(2-1)$ fueron obtenidos con el receptor APEX1 que opera en la banda de 213 a $275 \mathrm{GHz}$ y los de ${ }^{12} \mathrm{CO}(3-2),{ }^{13} \mathrm{CO}(3-2)$ y $\mathrm{C}^{18} \mathrm{O}(3-2)$ con APEX-2 que opera entre 267 y $378 \mathrm{GHz}$ (SHeFI; Vassilev et al., 2008). Todos fueron observados en el modo OTF usando el espectrómetro XFFTS2 que posee un ancho de banda de $2.5 \mathrm{GHz}$ dividido en 32768 canales. Los parámetros observacionales se muestran en la Tabla 7.3. Dado que la diferencia en frecuencia de las líneas de ${ }^{13} \mathrm{CO}$ y $\mathrm{C}^{18} \mathrm{O}$ es menor que el ancho de banda del instrumento, estas pudieron ser observadas simultáneamente centrando la observación en una frecuencia de $219.817837 \mathrm{GHz}$ para las transiciones (2-1) y de 329.959261 $\mathrm{GHz}$ para las transiciones (3-2). Como puede verse en la Figura 7.6, S108 no fue observada 


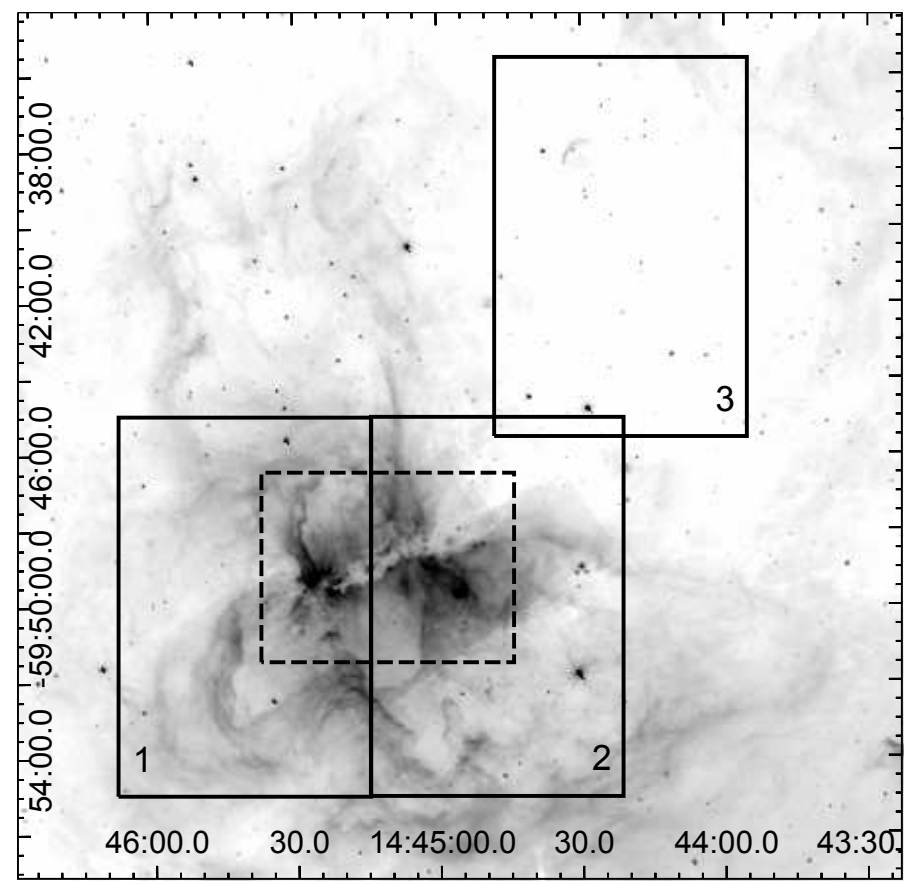

Figura 7.6: Los cuadrados muestran las regiones observadas con APEX sobre la imagen de emisión en $8 \mu \mathrm{m}$.

Tabla 7.3: Parámetros observacionales de las líneas moleculares.

\begin{tabular}{lcccc}
\hline \hline Línea & $\begin{array}{c}\nu_{0} \\
{[\mathrm{GHz}]}\end{array}$ & $\begin{array}{c}\text { HPBW } \\
{[\operatorname{arcsec}]}\end{array}$ & $\begin{array}{c}\Delta \mathrm{v}_{\text {res }} \\
{\left[\mathrm{km} \mathrm{s}^{-1}\right]}\end{array}$ & $\begin{array}{c}r m s \\
{[\mathrm{~K}]}\end{array}$ \\
\hline${ }^{12} \mathrm{CO}(3-2)$ & 345.796 & 19.2 & 0.2 & 0.6 \\
${ }^{13} \mathrm{CO}(3-2)$ & 330.588 & 20.0 & 0.2 & 0.5 \\
$\mathrm{C}^{18} \mathrm{O}(3-2)$ & 329.331 & 20.1 & 0.2 & 0.6 \\
${ }^{12} \mathrm{CO}(2-1)$ & 230.539 & 28.7 & 0.2 & 0.5 \\
${ }^{13} \mathrm{CO}(2-1)$ & 220.399 & 30.0 & 0.2 & 0.5 \\
$\mathrm{C}^{18} \mathrm{O}(2-1)$ & 219.560 & 30.2 & 0.2 & 0.6 \\
\hline \hline
\end{tabular}

con el telescopio APEX y por lo tanto no será incluida en la mayor parte de este capítulo.

El estudio se ha complementado con datos disponibles, tales como imágenes infrarrojas a 3.6, 4.5, 5.8 y $8 \mu \mathrm{m}$ de Spitzer-GLIMPSE, a $24 \mu \mathrm{m}$ de Spitzer-MIPSGAL, en el lejano IR a 70 y $160 \mu \mathrm{m}$ de Herschel-PACS y a 250, 350 y $500 \mu \mathrm{m}$ de Herschel-SPIRE, y en continuo de radio a $843 \mathrm{MHz}$ de SUMSS y a $4.85 \mathrm{GHz}$ del relevamiento GB6/PMN.

La presencia de burbujas infrarrojas y sus zonas de interacción, IRDCs, máseres, fuentes IR y candidatas a regiones HiI ultracompactas, convierten a esta región en un escenario particularmente interesante para el estudio de formación estelar.

\subsection{Análisis de imágenes en el infrarrojo y el continuo de radio}

Para estudiar el polvo interestelar asociado al complejo de burbujas se ha analizado la emisión infrarroja a partir de imágenes obtenidas por los satélites Spitzer, Herschel y pertenecientes al relevamiento ATLASGAL. Estas imágenes se muestran en las Figuras 7.1 y 7.7 


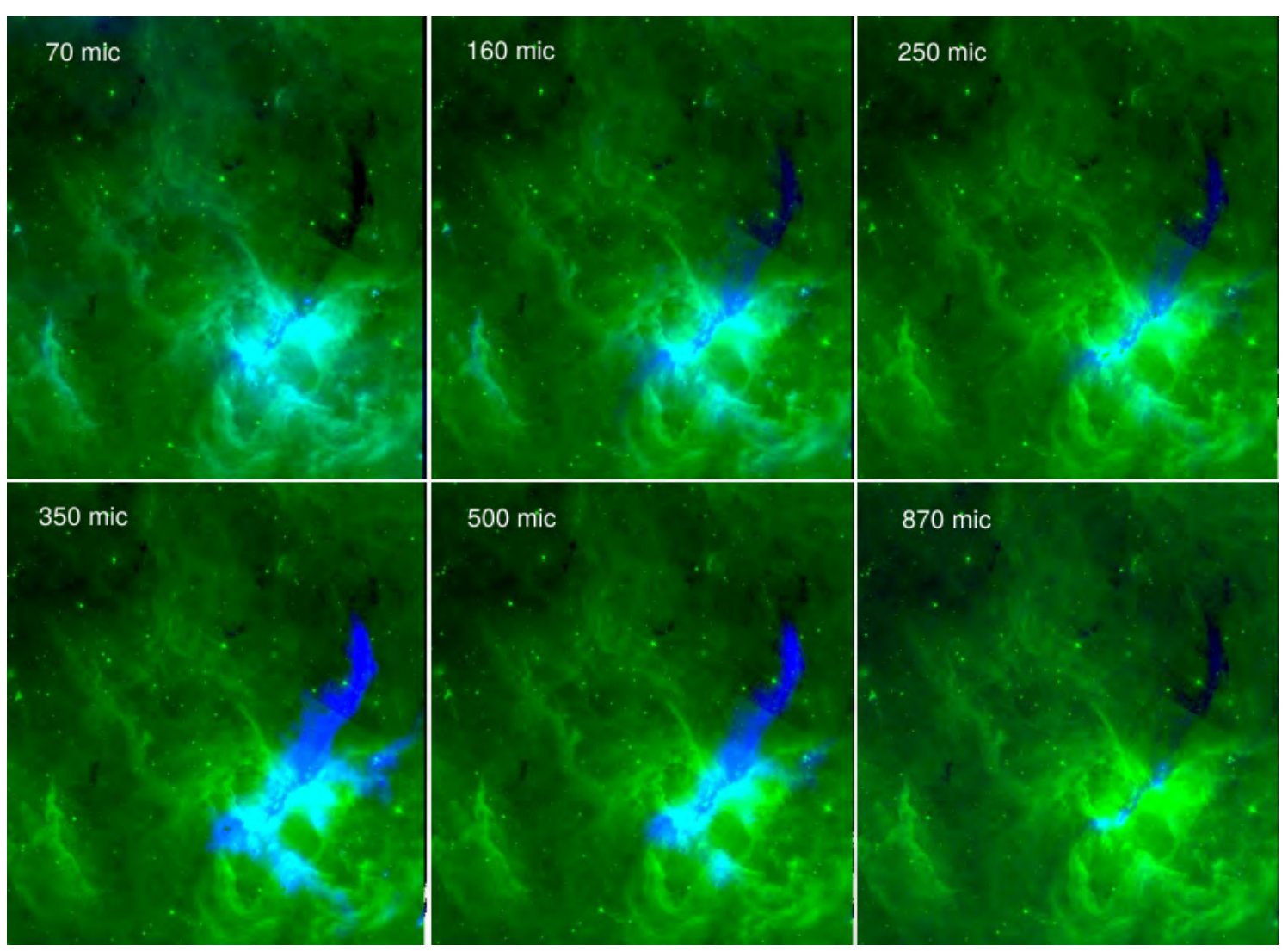

Figura 7.7: En verde se ve la emisión en $8 \mu \mathrm{m}$ de Spitzer-IRAC y en azul la emisión en el IR-lejano de Herschel y LABOCA. Resolución angular: $70 \mu \mathrm{m}=5^{\prime \prime}, 160 \mu \mathrm{m}=13^{\prime \prime}, 250 \mu \mathrm{m}=18^{\prime \prime}$, $350 \mu \mathrm{m}=25^{\prime \prime}, 500 \mu \mathrm{m}=36^{\prime \prime}, 870 \mu \mathrm{m}=19^{\prime \prime}$

y abarcan un amplio rango de longitudes de onda desde los $4.5 \mu \mathrm{m}$ (Spitzer-IRAC) hasta los $870 \mu \mathrm{m}$ (ATLASGAL), lo cual permite identificar polvo a diferentes temperaturas. Como se mencionó anteriormente la emisión en $4.5 \mu \mathrm{m}$ es apropiada para identificar regiones donde se producen choques y es típica de objetos estelares jóvenes masivos. La emisión a 5.8 y 8 $\mu \mathrm{m}$ de Spitzer-GLIMPSE delinea las regiones de fotodisociación (Photodissociation Regions, PDRs) que son las zonas de interacción entre el gas ionizado y el gas neutro.

Un cuidadoso examen de la imagen a $8 \mu \mathrm{m}$ revela una compleja red de arcos y filamentos en toda la región, incluyendo la presencia de estructuras similares a las llamadas 'trompas de elefante', por ejemplo en RA, Dec. $(\mathrm{J} 2000)=(14: 44: 55,-59: 52: 36)$. Esta imagen muestra cómo la emisión a 5.8 y $8 \mu \mathrm{m}$ delinea las burbujas identificadas por Churchwell et al. (2006) como S108, S109, S110 y S111, mientras que emisión extendida a $4.5 \mu \mathrm{m}$ coincide con las fuentes identificadas como IRAS 14416-5937-A y IRAS 14416-5937-B por Vig et al. (2007).

Cada panel de la Figura 7.7 muestra en color verde la emisión a $8 \mu \mathrm{m}$ de IRAC-GLIMPSE, y en azul la emisión a 70, 160, 250, 350 y $500 \mu \mathrm{m}$ de Herschel-PACS y -SPIRE y a $870 \mu \mathrm{m}$ de ATLASGAL.

La emisión a $70 \mu \mathrm{m}$ muestra la región de interacción de las burbujas y rodea parcialmente a S111. Presenta dos máximos, uno de ellos en el punto de contacto de las tres burbujas y otro al noroeste de la burbuja S111, que coinciden con las fuentes IRAS 14416-5937-A y IRAS 14416-5937-B definidas por Vig et al. (2007) a partir de emisión a 150 y $210 \mu \mathrm{m}$.

A $160 \mu \mathrm{m}$ se detectan las mismas zonas que en $70 \mu \mathrm{m}$ y se comienza a identificar emisión sobre las IRDCs (que no emiten en $8 \mu \mathrm{m}$ ). En este caso el máximo también se encuentra en 


\subsection{ANÁLISIS DE IMÁGENES EN EL INFRARROJO Y EL CONTINUO DE RADIO}

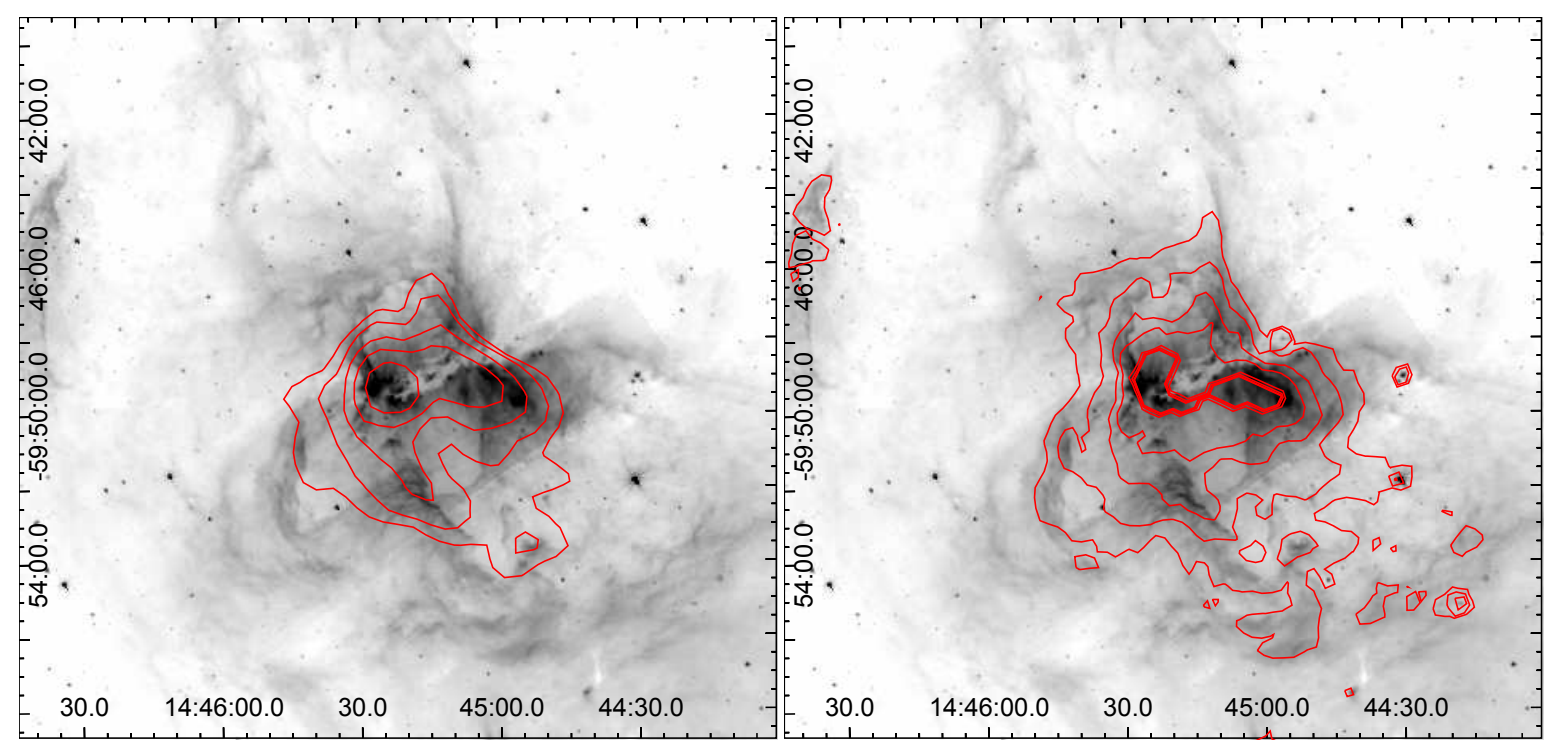

Figura 7.8: Izquierda: continuo de radio (SUMSS) a $843 \mathrm{MHz}$. Contornos: 0.1, 0.2, 0.5, 1 y 2 Jy/beam. Resolución angular: $43^{\prime \prime} \times 50^{\prime \prime}$. Derecha: $24 \mu \mathrm{m}$ de Spitzer-MIPS. Contornos: 100, 200, 300, 500, 1000, $1500 \mathrm{MJy} / \mathrm{sr}$. Resolución angular: 6". La imagen de fondo en ambos paneles muestra en azul, verde y rojo la emisión a 5.8, 8 y 4,5 $\mu \mathrm{m}$, respectivamente.

el punto de contacto de las tres burbujas. Las imágenes a 160 y $70 \mu \mathrm{m}$ de Herschel muestran regiones de polvo tibio sugiriendo la presencia de fuentes excitatrices en el complejo.

La emisión en 250, 350 y $500 \mu \mathrm{m}$ coincide parcialmente con el borde de la burbuja S111 y con las IRDCs, y los máximos de emisión también se encuentran en la zona de interacción de las burbujas. Se identifica con gran claridad un filamento en forma de arco que nace en la fuente IRAS 14416-5937-A, pasa por la zona de interacción de las burbujas y se extiende hasta las IRDCs. Al igual que a 250, 350 y $500 \mu \mathrm{m}$, la emisión a $870 \mu \mathrm{m}$ delinea el filamento que atraviesa la zona de interacción de las burbujas y las IRDCs. Estas longitudes de onda más largas ( $870 \mu \mathrm{m}$ de ATLASGAL y 500, 350 y $250 \mu \mathrm{m}$ de Herschel) muestran las zonas de polvo más frío del complejo, como por ejemplo las IRDCs.

A pesar de que el filamento observado en el lejano IR pasa por la zona de interacción y por las IRDCs, de aquí en más se referirá solamente a la zona de interacción como 'el filamento', y a la parte del mismo que atraviesa las IRDCs se la llamará 'la IRDC', tal como se muestra en la Figura 7.2.

El panel derecho de la Figura 7.8 muestra en contornos la emisión correspondiente a 24 $\mu \mathrm{m}$ de Spitzer-MIPS. La misma coincide con el interior de las burbujas S109, S110 y S111, y tiene su máximo sobre la zona de interacción de las mismas coincidiendo con la emisión a $70 \mu \mathrm{m}$ de Herschel. A su vez los máximos de emisión a 24 y $70 \mu \mathrm{m}$ coinciden con la emisión a $4.5 \mu \mathrm{m}$ de Spitzer-GLIMPSE lo que indica la posible presencia de objetos estelares jóvenes masivos. Del mismo modo, y tal como se puede ver en el panel izquierdo de la Figura 7.8, la emisión en continuo de radio a $843 \mathrm{MHz}$ (SUMSS) muestra gas ionizado en el interior de las burbujas, coincidiendo el máximo de emisión con la posición de la fuente IRAS 14416-5937A. Es notable además, la emisión en $843 \mathrm{MHz}$ de la región que separa las burbujas S109 y S111, indicando la existencia de gas ionizado en la zona. El origen de esta emisión es muy probablemente térmico, debido a que coincide espacialmente con emisión de PAH en $8 \mu \mathrm{m}$ y emisión en $24 \mu \mathrm{m}$ originada por polvo tibio y líneas nebulares.

En resumen, del análisis de las imágenes infrarrojas surge que se está en presencia de una región compleja que posee zonas densas con polvo interestelar frío trazadas por las 
longitudes de onda más largas que evidencian la presencia de IRDCs en la que se podrían estar desencadenando procesos de formación estelar. Las longitudes de onda entre 24 y 160 $\mu \mathrm{m}$, que muestran polvo tibio, evidencian la existencia de fuentes excitatrices, corroborada por la identificación de gas ionizado, lo que indica que las estructuras son regiones HiI (en consonancia con los resultados de Deharveng et al., 2010). Es también el objetivo de este capítulo identificar tales fuentes y estudiar su relación con las burbujas.

\subsection{Análisis de datos de líneas moleculares}

\subsubsection{Distribución global del gas molecular}

\section{Datos de baja resolución angular (NANTEN)}

Los datos de NANTEN con baja resolución permitieron estudiar la distribución a gran escala del gas molecular en la región. Se encontró gas molecular coincidente con las burbujas en el rango de velocidades entre -46 y $-28 \mathrm{~km} \mathrm{~s}^{-1}$, con su máximo de emisión a una velocidad de $-40 \mathrm{~km} \mathrm{~s}^{-1}$. En la Figura 7.9 se muestra la emisión de ${ }^{12} \mathrm{CO}(1-0)$ promediada en este rango de velocidades. Claramente la emisión más intensa coincide con la región de interacción de las burbujas S109-S110-S111 (RA, Dec.(J2000)= 14:45:24.2, -59:49:47.2) y se extiende a lo largo del filamento, hacia el noroeste en dirección a la IRDC y hacia el sur entre las burbujas S109 y S111. A pesar de la baja resolución angular de los datos de NANTEN, se puede decir que esta distribución coincide con la que se observa en el lejando IR. La coincidencia morfológica entre la emisión de ${ }^{12} \mathrm{CO}(1-0)$ y la emisión en las distintas bandas de IR indica que el gas molecular pertenece a la región y se encuentra interactuando con la misma. La emisión molecular mostrada en la Figura 7.9 tiene un $r m s=0.04 \mathrm{~K}$, resultado del promediado en el rango $-46 \mathrm{a}-28 \mathrm{~km} \mathrm{~s}^{-1}$, con una velocidad promedio de $-40 \mathrm{~km} \mathrm{~s}^{-1}{ }^{(1)}$.

La resolución angular de estos datos (2!6) es insuficiente para analizar con detalle el gas asociado a las burbujas y a la IRDC, pero permite llevar a cabo un estudio de la distribución a gran escala del gas molecular. Teniendo datos de una línea molecular solamente se puede realizar una estimación de la densidad columnar de la región a partir de la ecuación

$$
N_{H_{2}}=X I_{C O}
$$

donde

$$
I_{C O}=\int_{\mathrm{v}_{1}}^{\mathrm{v}_{2}} T_{b} d \mathrm{v}
$$

$X$ es constante para toda la Galaxia y $\left(\mathrm{v}_{1}, \mathrm{v}_{2}\right)$ corresponden al rango de velocidades en que se detecta la emisión.

Utilizando esta ecuación y considerando la emisión de CO integrada entre -46 y -28 $\mathrm{km} \mathrm{s}^{-1}$ se obtuvo un total de $18400 \pm 6600 \mathrm{M}_{\odot}$ para el complejo (se asumió un valor de $X=1.9 \times 10^{21} \mathrm{~cm}^{-2} /\left(\mathrm{K} \mathrm{km} \mathrm{s}^{-1}\right)$ obtenido de Strong \& Mattox, 1996), lo que corresponde a una densidad ambiental original de $270 \pm 145 \mathrm{~cm}^{-3}$.

\footnotetext{
${ }^{(1)} \mathrm{Si}$ se tiene un cierto número $\mathrm{N}$ de muestras independientes, sujetas a errores al azar, y se promedian, el ruido del promedio será menor que el de cada una de las muestras, y estará dado por $\sigma_{\text {prom }}=\sigma / \operatorname{sqrt}(N)$, por lo que el $r m s$ de la imagen promediada es de $0.04 \mathrm{~K}$.
} 


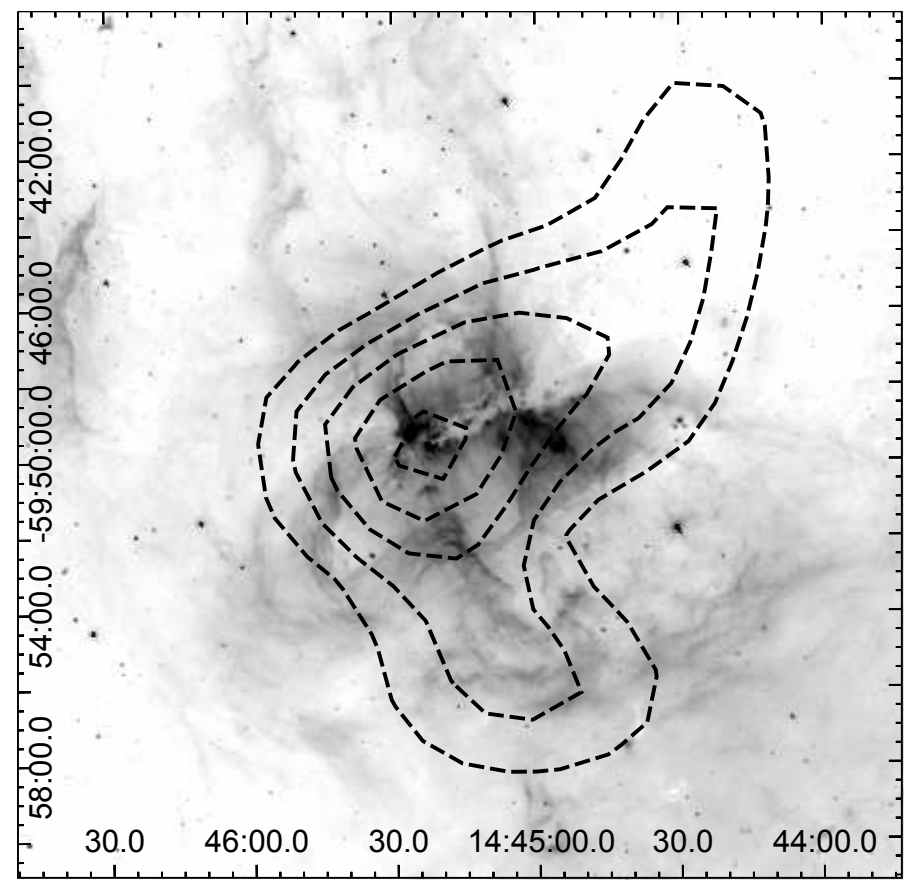

Figura 7.9: Contornos correspondientes a la emisión de ${ }^{12} \mathrm{CO}(1-2)$ promediada entre -46 y $-28 \mathrm{~km} \mathrm{~s}^{-1}$ obtenida con NANTEN $(5,6,7,8$ y $9 \mathrm{~K})$ superpuestos a la imagen de $8 \mu \mathrm{m}$ de Spitzer-IRAC.

\section{Datos de alta $^{(2)}$ resolución angular (APEX)}

Para poder estudiar las condiciones físicas y analizar la dinámica y cinemática de la región se necesitan datos con mejor resolución que los de NANTEN. Es por esto que se llevaron a cabo observaciones de gas molecular utilizando el telescopio APEX. Los datos de APEX tienen una resolución angular de $\sim 30^{\prime \prime}$ (transiciones $\mathrm{J}=2 \rightarrow 1$ ) y de $\sim 20^{\prime \prime}($ transiciones $\mathrm{J}=3 \rightarrow 2$ ) Las últimas son necesarias para caracterizar la región de interacción.

Los objetivos a alcanzar con estas observaciones son los siguientes:

- Identificar el gas molecular ligado al complejo de burbujas IR y la presencia de cáscaras.

- Confirmar que la IRDC pertenece al mismo complejo

- Caracterizar la región de interacción de las burbujas

- Determinar las condiciones físicas de las diferentes regiones (burbujas e IRDCs)

- Identificar grumos de gas denso y estudiar su correlación con la posición de YSOs

- Identificar outflows moleculares ligados a los YSOs

En las siguientes secciones se analizarán separadamente las distintas partes de complejo.

\footnotetext{
${ }^{(2)}$ Los datos con 'alta' resolución angular en Radioastromomía son los que se obtienen con interferómetros, sin embargo como en esta tesis no se usan este tipo de datos, se llamará 'alta' resolución angular a la de un instrumento como APEX por su comparación con la de uno como NANTEN.
} 

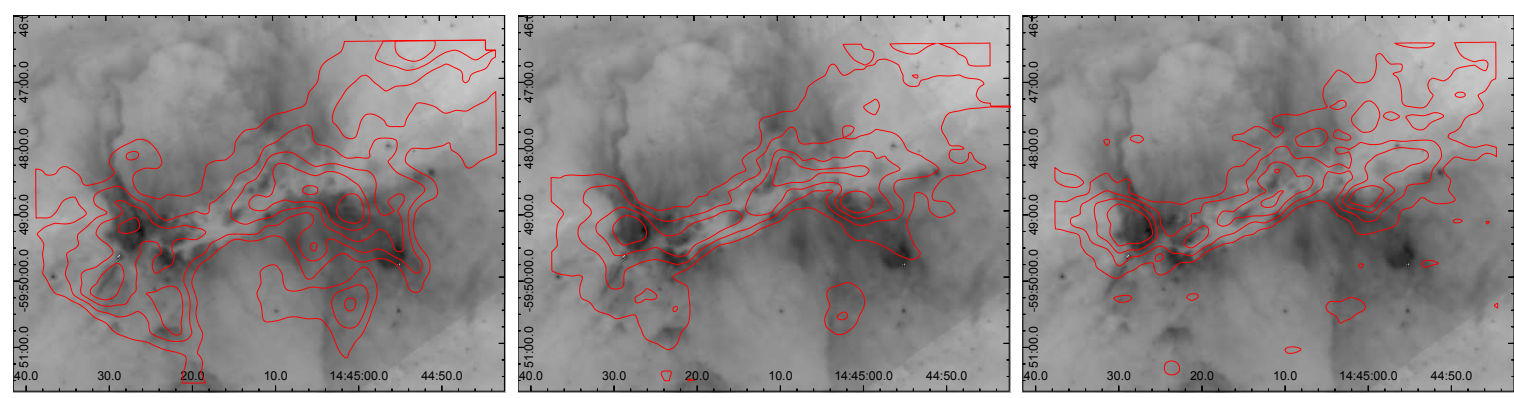

Figura 7.10: Contornos de la emisión promedio $\left(\mathrm{T}_{m b}\right)$ de ${ }^{12} \mathrm{CO}(3-2)$ (izquierda), ${ }^{13} \mathrm{CO}(3-2)$ (centro) y $\mathrm{C}^{18} \mathrm{O}(3-2)$ (derecha) sobre el filamento, superpuestos a la imagen de $8 \mu \mathrm{m}$. Los contornos de ${ }^{12} \mathrm{CO}(3-2)$ corresponden a $5(80 \mathrm{rms}), 6,7,8$, y $9 \mathrm{~K}$, los de ${ }^{13} \mathrm{CO}(3-2)$ a $2(25$ $r m s), 3,4$ y $5 \mathrm{~K}$, y los de $\mathrm{C}^{18} \mathrm{O}(3-2)$ a $0.5(9 \mathrm{rms}), 1,1.5$ y $2 \mathrm{~K}$.

\subsubsection{El filamento en la zona de interacción de las burbujas}

Como se explicó en la Sección 7.3, en la zona de interacción de las burbujas se ve una estructura con forma filamentaria. La misma se caracteriza por la ausencia de emisión en 8 $\mu \mathrm{m}$, se inicia en el punto de contacto de las tres burbujas y continúa por la separación entre S110 y S111 (ver Figura 7.2). Como se puede ver en la Figura 7.8, este filamento coincide con el máximo de la emisión en $24 \mu \mathrm{m}$ y en continuo de radio, revelando la presencia de fuentes excitatrices en la región. Como se mostró en la Figura 7.7 el filamento es delineado por el polvo frío que se evidencia a través de la emisión en el IR lejano. Esta estructura se continúa con la IRDC que se describirá en la Sección 7.4.5.

Los relevamientos del plano galáctico realizados con telescopios infrarrojos han demostrado que las estructuras filamentarias parecen estar omnipresentes en todo el medio interestelar (e.g. Molinari et al., 2016). Recientemente, el análisis cinemático de la emisión de líneas moleculares ha mostrado la existencia de estructuras que se cree que son la representación en velocidades de las estructuras filamentarias detectadas en el IR, y aparecen tanto en las regiones de formación de estrellas de baja y gran masa (e.g. Hacar et al., 2013; Hacar et al., 2016; Henshaw et al., 2017).

En cuanto a la presencia de gas molecular a partir de los datos de NANTEN se puede observar que, tal como se ve en la Figura 7.9, el filamento coincide con el máximo de la emisión de ${ }^{12} \mathrm{CO}(1-2)$.

En la Figura 7.10 se muestran los contornos correspondientes a la emisión promedio de ${ }^{12} \mathrm{CO}(3-2),{ }^{13} \mathrm{CO}(3-2)$ y $\mathrm{C}^{18} \mathrm{O}(3-2)$, observados con APEX, superpuestos a la imagen de 8 $\mu \mathrm{m}$. Para cada molécula los promedios se hicieron sobre todo el rango de velocidades en el que se encuentra emisión. Estos rangos, junto con los valores de rms medidos, se encuentran listados en la Tabla 7.4. Se observa que la emisión para cada una de las moléculas está muy por encina del rms medido, por lo que se puede asegurar que la emisión es muy intensa. Se observa que para todos los casos el filamento es delineado por el gas molecular y contiene gran cantidad de gas.

\subsubsection{Burbuja tripolar}

En lo que sigue se analizará la distribución de la emisión de los diferentes isótopos de CO.

La Figura 7.11 muestra en contornos la emisión correspondiente a ${ }^{12} \mathrm{CO}(2-1)$ en diferentes rangos de velocidades radiales superpuesta a la imagen en $8 \mu \mathrm{m}$. Cada uno de los seis paneles muestra una imagen promediada en un rango de $2 \mathrm{~km} \mathrm{~s}^{-1}$ (10 canales). El rango de velocidad total mostrado es entre $-43 \mathrm{y}-31.2 \mathrm{~km} \mathrm{~s}^{-1}$. En el panel superior derecho de esta Figura $(-39$ 
Tabla 7.4: Parámetros de las imágenes promedio de ${ }^{12} \mathrm{CO}(3-2),{ }^{13} \mathrm{CO}(3-2)$ y $\mathrm{C}^{18} \mathrm{O}(3-2)$ sobre el filamento (Figura 7.10).

\begin{tabular}{cccccc}
\hline \hline Molécula & $\begin{array}{c}\text { Rango de velocidad } \\
{\left[\mathrm{km} \mathrm{s}^{-1}\right]}\end{array}$ & $\begin{array}{c}\Delta \mathrm{v} \\
{\left[\mathrm{km} \mathrm{s}^{-1}\right]}\end{array}$ & Nro canales & $\begin{array}{c}r m s \text { canal } \\
{[\mathrm{K}]}\end{array}$ & $\begin{array}{c}r m s \text { promedio } \\
{[\mathrm{K}]}\end{array}$ \\
\hline${ }^{12} \mathrm{CO}(3-2)$ & {$[-45,-30]$} & 15.0 & 76 & 0.55 & 0.06 \\
${ }^{13} \mathrm{CO}(3-2)$ & {$[-45,-30]$} & 15.0 & 76 & 0.47 & 0.05 \\
$\mathrm{C}^{18} \mathrm{O}(3-2)$ & {$[-43,-33]$} & 10.0 & 52 & 0.55 & 0.08 \\
\hline \hline
\end{tabular}

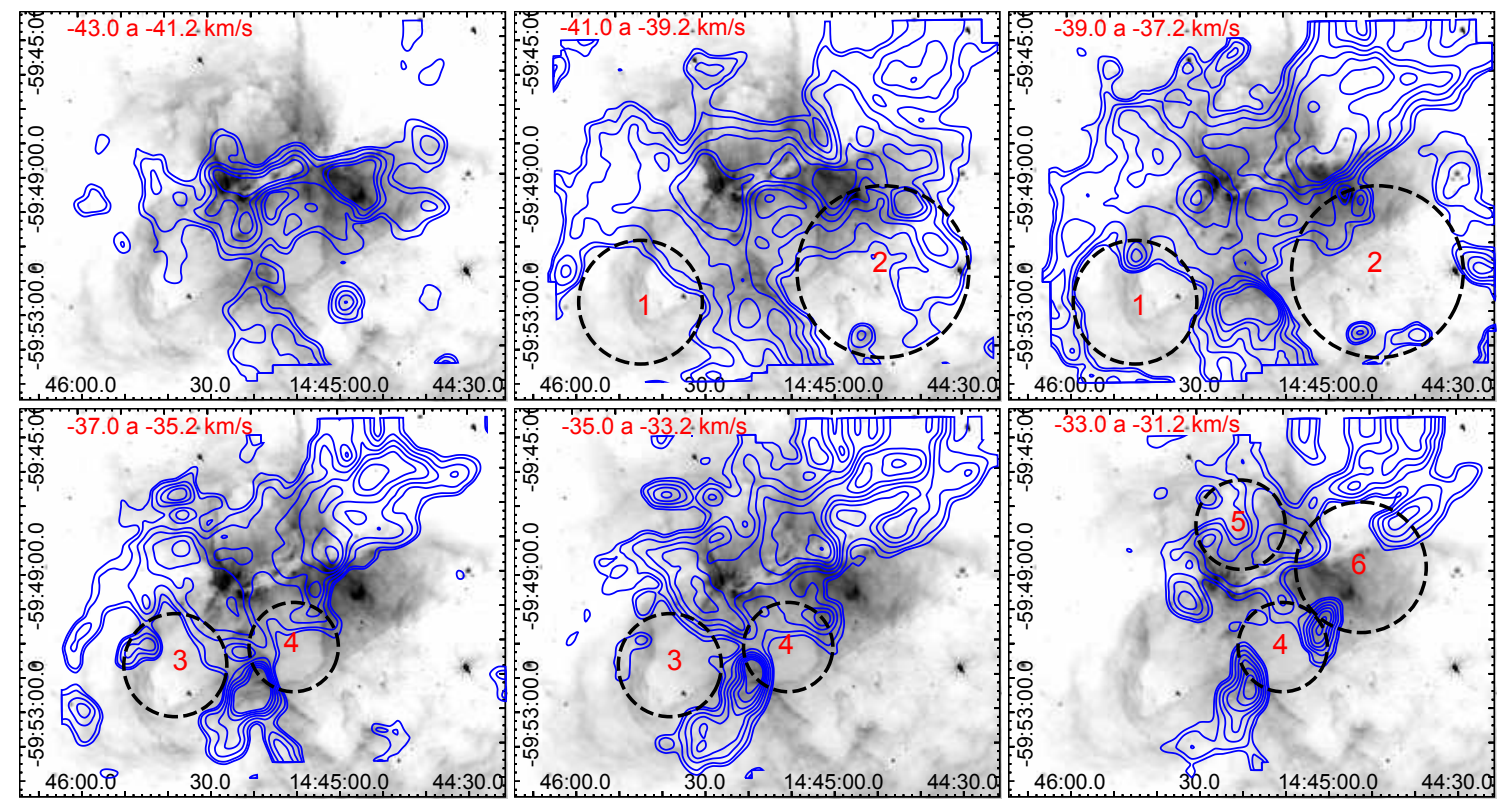

Figura 7.11: Distribución de ${ }^{12} \mathrm{CO}(2-1)$ en el intervalo de velocidades de -43 a $-31.2 \mathrm{~km} \mathrm{~s}^{-1}$ en pasos de $2 \mathrm{~km} \mathrm{~s}^{-1}$. Imagen de fondo: $8 \mu \mathrm{m}$. Contornos: emisión de ${ }^{12} \mathrm{CO}(2-1)$ promediada en los rangos de velocidades indicados en cada panel (10 canales). Los contornos corresponden a 7, 8, 10, 12 y $14 \mathrm{~K}$ en el primer panel, 7, 8, 10, 12, 14, y $16 \mathrm{~K}$ en el segundo panel, 7, 8, $10,12,14,16$ y $18 \mathrm{~K}$ en el tercer y cuarto panel, y $3,4,6,8,10,12,14$ y $16 \mathrm{~K}$ en el quinto y sexto panel. 


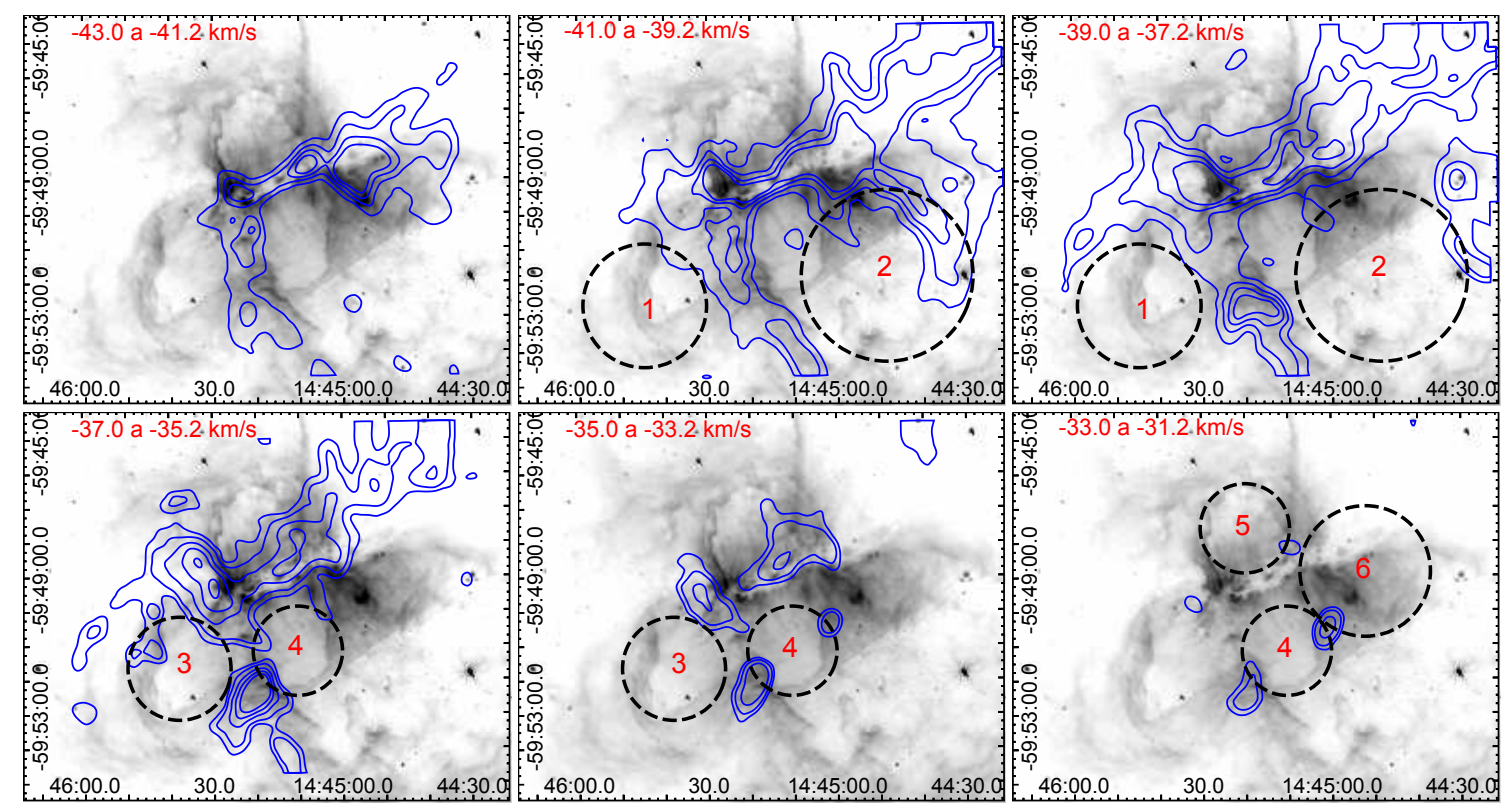

Figura 7.12: Distribución de ${ }^{13} \mathrm{CO}(2-1)$ en el intervalo de velocidades de $-41 \mathrm{a}-29.2 \mathrm{~km} \mathrm{~s}^{-1}$ en pasos de $2 \mathrm{~km} \mathrm{~s}^{-1}$. Imagen de fondo: $8 \mu \mathrm{m}$. Contornos: emisión de ${ }^{13} \mathrm{CO}(2-1)$ promediada en los rangos de velocidades indicados en cada panel. Los contornos correponden a 3, 5,7 y $9 \mathrm{~K}$ en los tres paneles superiores, 2, 3, 5, 7 y $9 \mathrm{~K}$ en el cuarto panel, y 2,3 y $5 \mathrm{~K}$ en los últimos dos paneles.

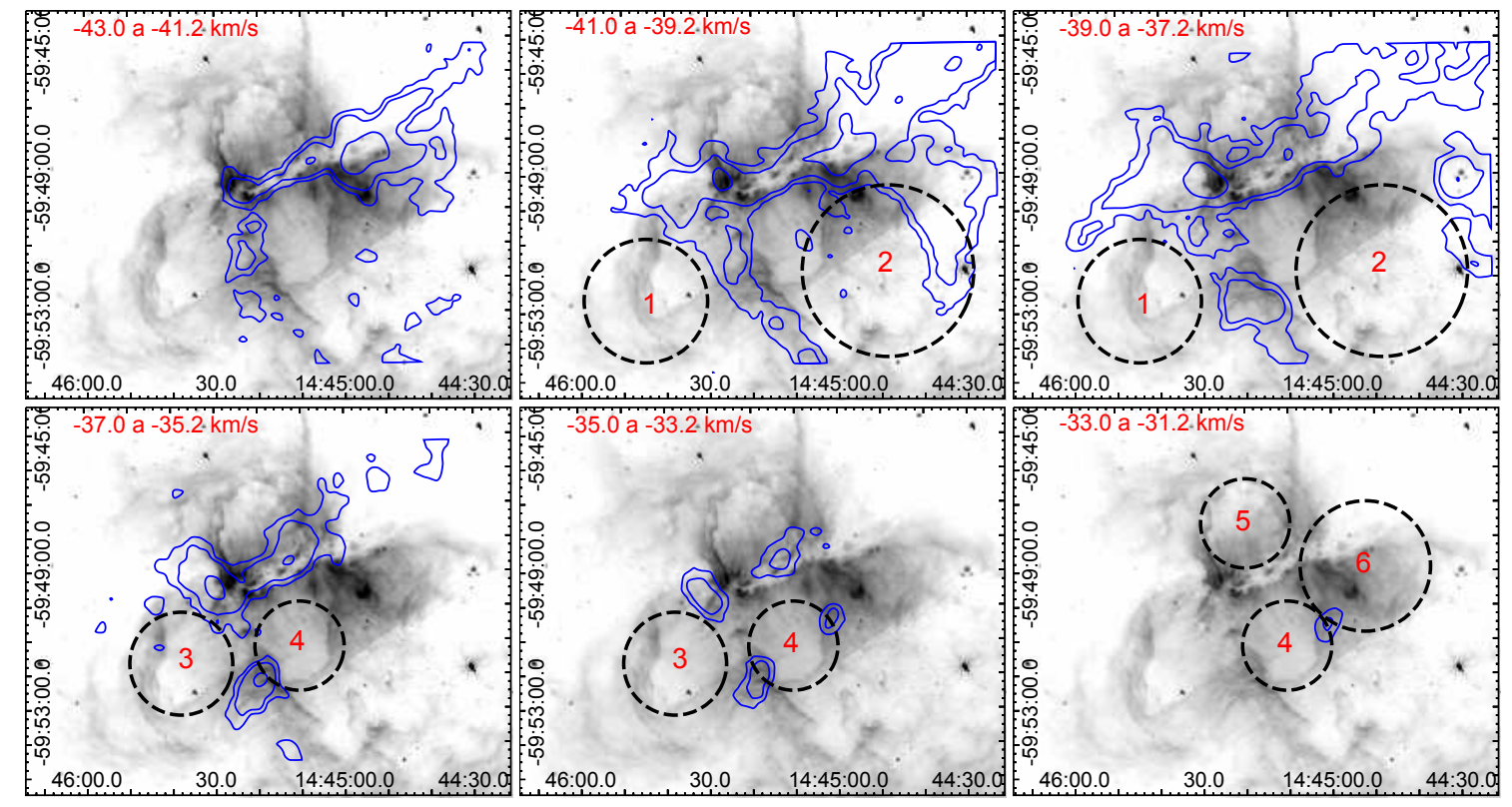

Figura 7.13: Distribución de $\mathrm{C}^{18} \mathrm{O}(2-1)$ en el intervalo de velocidades de -41 a $-29.2 \mathrm{~km} \mathrm{~s}^{-1}$ en pasos de $2 \mathrm{~km} \mathrm{~s}^{-1}$. Imagen de fondo: $8 \mu \mathrm{m}$. Contornos: emisión de $\mathrm{C}^{18} \mathrm{O}(2-1)$ promediada en los rangos de velocidades indicados en cada panel. Los contornos corresponden a $0.5,1 \mathrm{y}$ $3 \mathrm{~K}$. 
a $-37.2 \mathrm{~km} \mathrm{~s}^{-1}$ ) se identifican dos cáscaras de gas molecular indicadas por círculos. Estas cáscaras, identificadas con los números 1 y 2, coinciden con los arcos externos mostrados en la Figura 7.2, y parcialmente con las posiciones y tamaños de las burbujas S109 y S111 definidas por Churchwell et al. (2006). Estas estructuras también se detectan parcialmente entre $-41 \mathrm{y}-39.2 \mathrm{~km} \mathrm{~s}^{-1}$. A velocidades de entre $-37 \mathrm{y}-35.2 \mathrm{~km} \mathrm{~s}^{-1}$ el centro de la cáscara \#1 se desplaza coincidiendo mejor con la burbuja S109 (\#3), los bordes de la cáscara \#2 no se ven definidos, y comienza a identificarse una estructura que rodea el borde superior de la burbuja S111 (\#4). Entre -35 y -33.2 se ve la cáscara rodeando a S109 (\#3), y se detecta la presencia de la cáscara \#4. Finalmente, a velocidades de entre -33 y -31.2 sólo se distingue una parte de la cáscara que rodea S109 (\#3), hacia el oeste en la zona que delimita esta burbuja con S111. Sin embargo a estas velocidades se pueden distinguir cáscaras de gas molecular rodeando a las burbujas S110 (\#5) y S111 (\#4). Además podría sugerirse otra cáscara rodeando parcialmente una región no catalogada como burbuja (\#6). La distribución del gas molecular es claramente muy compleja.

Un análisis similar al anterior puede llevarse a cabo a partir de las Figuras 7.12 y 7.13 que muestran los contornos correspondientes a la emisión de ${ }^{13} \mathrm{CO}(2-1)$ y $\mathrm{C}^{18} \mathrm{O}(2-1)$, respectivamente, promediada en diferentes rangos de velocidades y superpuestos a la emisión de 8 $\mu \mathrm{m}$. Se observa, tal como es de esperar, que la emisión de ${ }^{13} \mathrm{CO}(2-1)$ y $\mathrm{C}^{18} \mathrm{O}(2-1)$ está menos extendida que la de ${ }^{12} \mathrm{CO}(2-1)$ y se concentra mayormente sobre la región del filamento y en el límite entre las burbujas S109 y S111.

En la Figura 7.12 pueden distinguirse algunas escructuras de ${ }^{13} \mathrm{CO}(2-1)$, una de ellas entre -43 y $-37.2 \mathrm{~km} \mathrm{~s}^{-1}$ podría identificarse con la cáscara \#2 definida a partir de la emisión de ${ }^{12} \mathrm{CO}(2-1)$. Entre $-37 \mathrm{y}-35.2 \mathrm{~km} \mathrm{~s}^{-1}$ se detectan dos estructuras parciales que corresponderían a las cáscaras \#3 y \#4. Entre -35 y $-31.2 \mathrm{~km} \mathrm{~s}^{-1}$ solo se detectan grumos sobre los bordes de las cáscaras.

Del mismo modo, en la Figura 7.13 que muestra $\mathrm{C}^{18} \mathrm{O}(2-1)$, se detecta una estructura que rodea parcialmente a la cáscara \#2 entre $-43 \mathrm{y}-37.2 \mathrm{~km} \mathrm{~s}^{-1}$. Entre $-37 \mathrm{y}-31.2 \mathrm{~km} \mathrm{~s}^{-1}$ sólo se observan grumos en los bordes de las cáscaras.

Una vez identificadas las cáscaras, y a fin de determinar el rango de velocidad en que se extiende cada una de ellas, su posición y radio efectivo se llevó a cabo una nueva inspección de los cubos de datos a partir de los que se determinaron los parámetros que se listan en la Tabla 7.5. Además se indica en qué moléculas se puede identificar cada una de las cáscaras y qué cáscaras sólo se observan parcialmente.

La emisión promedio de ${ }^{12} \mathrm{CO}(2-1)$ y ${ }^{13} \mathrm{CO}(2-1)$ en los rangos de velocidad en que se identifica cada una de las cáscaras se muestra en las Figuras 7.14, 7.15, 7.16, 7.17, 7.18 y 7.19 para las cáscaras \#1, \#2, \#3, \#4, \#5 y \#6, respectivamente. A partir de estas se determinó una posición y un radio efectivo para cada una de ellas. Estos rangos de velocidades fueron elegidos a fin de evidenciar la existencia de las cavidades, aunque las cáscaras podrían extenderse un poco más. En efecto se puede observar cómo algunas cáscaras se cierran hacia velocidades mayores o menores que la velocidad central de la cavidad.

La cáscara \#1 es la única que se observa completa en ${ }^{12} \mathrm{CO}(2-1)$ y ${ }^{13} \mathrm{CO}(2-1)$, mientras que la \#2 es la única que se detecta en $\mathrm{C}^{18} \mathrm{O}(2-1)$, aunque se observa incompleta en todas las moléculas. Probablemente esto se deba a que el tamaño de la cáscara excede la región observada. Además en el interior de la cáscara \#2 se detectan dos grumos asociados a estructuras con bordes brillantes en $8 \mu \mathrm{m}$ de tipo 'trompa de elefante'. Estos grumos se muestran en la Figura 7.20 Uno de los grumos se detecta a la misma velocidad que la cáscara (de -37.6 a $-41.8 \mathrm{~km} \mathrm{~s}^{-1}$ ) mientras que el otro se observa a velocidades de entre $-40.6 \mathrm{y}-43.2 \mathrm{~km} \mathrm{~s}^{-1}$, por lo que podría estar ubicado en la pared anterior de la misma.

Por lo tanto se estaría en presencia de una estructura de cáscaras moleculares bien defini- 
Tabla 7.5: Parámetros de las cáscaras definidas a partir de ${ }^{12} \mathrm{CO}(2-1)$.

\begin{tabular}{ccccc}
\hline \hline$\#$ & RA, Dec.(J2000) & $\Delta \mathrm{v}$ & $\mathrm{r}\left({ }^{\prime}\right)$ & Identificadas \\
\hline 1 & $14: 45: 44.869,-59: 52: 39.66$ & {$[-39.6,-37.4]$} & 1.8 & ${ }^{12} \mathrm{CO}(2-1),{ }^{13} \mathrm{CO}(2-1)$ \\
2 & $14: 44: 48.577,-59: 51: 49.94$ & {$[-41.8,-37.6]$} & 2.5 & ${ }^{12} \mathrm{CO}(2-1) \dagger,{ }^{13} \mathrm{CO}(2-1) \dagger, \mathrm{C}^{18} \mathrm{O}(2-1) \dagger$ \\
3 & $14: 45: 37.991,-59: 51: 40.54$ & {$[-36.4,-33.8]$} & 1.5 & ${ }^{12} \mathrm{CO}(2-1),{ }^{13} \mathrm{CO}(2-1) \dagger$ \\
4 & $14: 45: 10.510,-59: 51: 10.85$ & {$[-36.4,-28.2]$} & 1.3 & ${ }^{12} \mathrm{CO}(2-1),{ }^{13} \mathrm{CO}(2-1) \dagger$ \\
5 & $14: 45: 20.020,-59: 47: 36.52$ & {$[-32.4,-26.6]$} & 1.3 & ${ }^{12} \mathrm{CO}(2-1)$ \\
6 & $14: 44: 52.236,-59: 48: 52.57$ & {$[-33.4,-30.8]$} & 1.9 & ${ }^{12} \mathrm{CO}(2-1) \dagger$ \\
\hline \hline
\end{tabular}

†: cáscaras que se detectan incompletas.
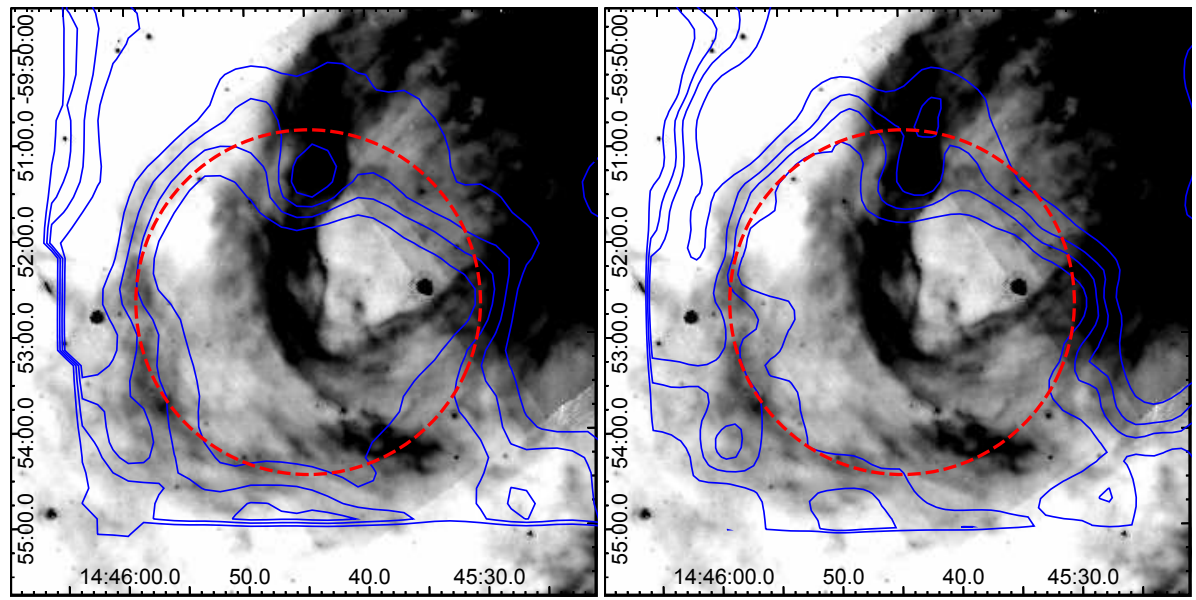

Figura 7.14: Cáscara \#1: Sobre la imagen de $8 \mu \mathrm{m}$ se muestra la emisión promedio de ${ }^{12} \mathrm{CO}(2-1)$ (izquierda; contornos $5,7,9$ y $\left.11 \mathrm{~K}\right)$ y ${ }^{13} \mathrm{CO}(2-1)$ (derecha; contornos 1 , 1.5, 2 y $3 \mathrm{~K}$ ) en el rango de velocidad $(-39.6,-37.4)$ correspondiente a la cáscara molecular \#1. Los círculos muestran la posición y el diámetro efectivo definido para la cáscara.

das en ${ }^{12} \mathrm{CO}(2-1)$ que muestra emisión extendida, y con una contraparte parcial en ${ }^{13} \mathrm{CO}(2-1)$ y $\mathrm{C}^{18} \mathrm{O}(2-1)$ que son moléculas que trazan regiones de mayor densidad, y muestran regiones más densas de las cáscaras. Las cáscaras tienen algunas zonas más densas que otras localizadas sobre el filamento en el límite entre las burbujas S109 y S111 y estarían formadas por varios grumos.

El origen de estas cáscaras moleculares puede buscarse en la expansión de las burbujas IR debida a fotones UV y/o vientos estelares, que perturban el medio ambiente barriendo el gas molecular y creando cáscaras moleculares en las que pueden formarse grumos densos que desencadenen nuevas generaciones de estrellas.

\subsubsection{Identificación de grumos densos}

Puesto que las estrellas se forman por condensación del gas molecular interestelar, es de esperar que las estrellas más jóvenes se encuentren en las regiones más densas de las nubes moleculares. Por ello, para el estudio observacional del proceso de formación estelar, así como del entorno de las estrellas más jóvenes, es interesante disponer de observaciones de moléculas que sólo emitan apreciablemente en condiciones de densidad relativamente alta.

Por esta razón se utilizaron los datos de $\mathrm{C}^{18} \mathrm{O}(2-1)$, que es una molécula menos abundante y por lo tanto mejor trazadora de regiones de alta densidad ambiental, para llevar a cabo una búsqueda de grumos densos en la región con el objetivo de identificar zonas que puedan 


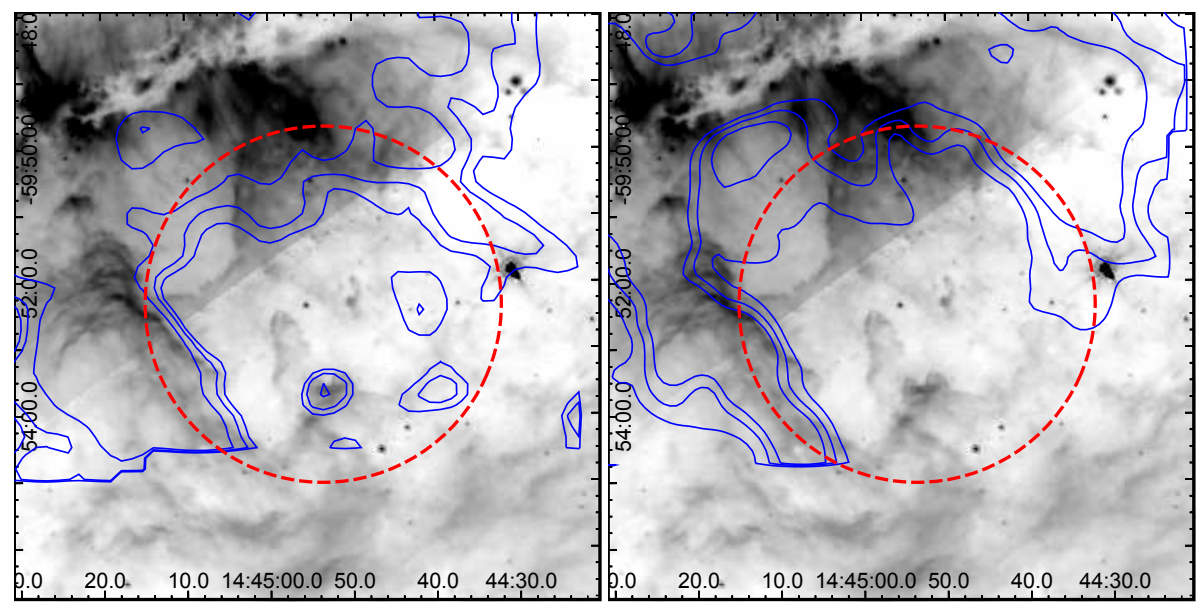

Figura 7.15: Cáscara \#2: Sobre la imagen de $8 \mu \mathrm{m}$ se muestra la emisión promedio de ${ }^{12} \mathrm{CO}(2-1)$ (izquierda; contornos 7, 8 y $10 \mathrm{~K}$ ) y ${ }^{13} \mathrm{CO}(2-1)$ (derecha; contornos 2 , 3 y $4 \mathrm{~K}$ ) en el rango de velocidad $(-41.8,-37.6)$ correspondiente a la cáscara molecular \#2. Los círculos muestran la posición y el diámetro efectivo definido para la cáscara.

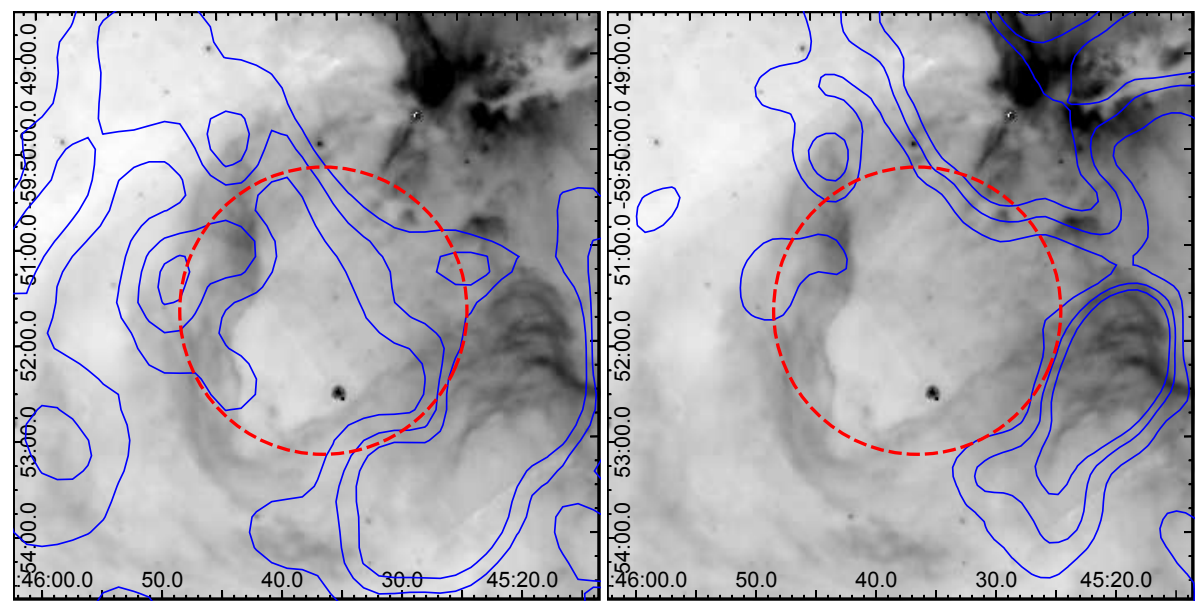

Figura 7.16: Cáscara \#3: Sobre la imagen de $8 \mu \mathrm{m}$ se muestra la emisión promedio de ${ }^{12} \mathrm{CO}(2-1)$ (izquierda; contornos 2 , 4 y $6 \mathrm{~K}$ ) y ${ }^{13} \mathrm{CO}(2-1)$ (derecha; contornos 1 , 2 y $3 \mathrm{~K}$ ) en el rango de velocidad $(-36.4,-33.8)$ correspondiente a la cáscara molecular \#3. Los círculos muestran la posición y el diámetro efectivo definido para la cáscara. 


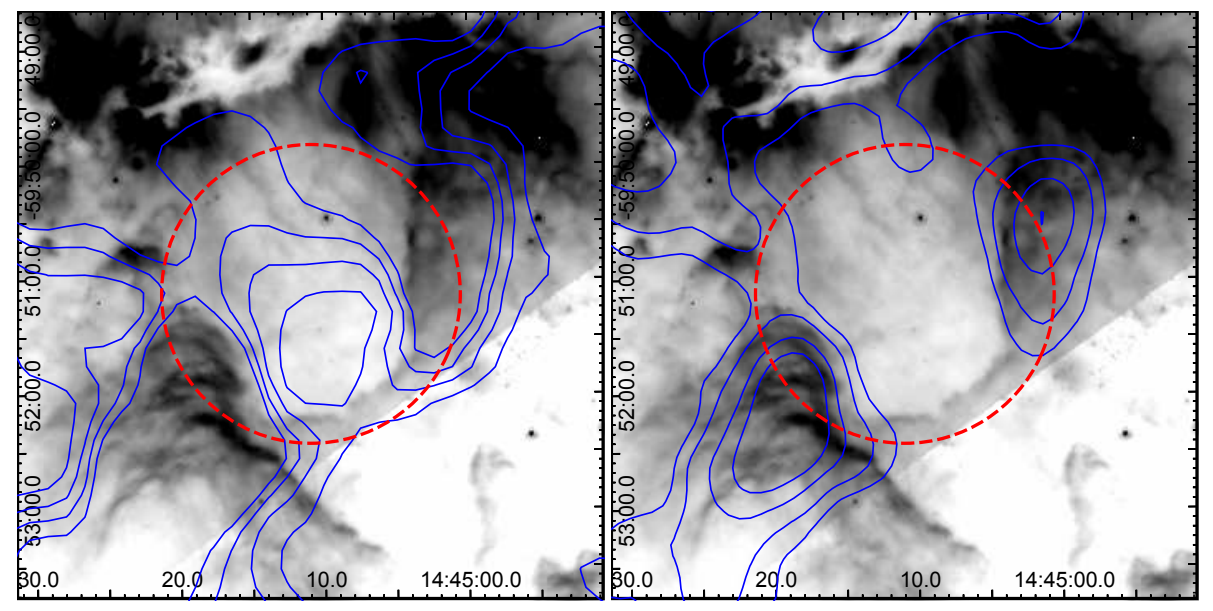

Figura 7.17: Cáscara \#4: Sobre la imagen de $8 \mu \mathrm{m}$ se muestra la emisión promedio de ${ }^{12} \mathrm{CO}(2-1)$ (izquierda; contornos 1.5, 2.5, 3.5 y $4.5 \mathrm{~K}$ ) y ${ }^{13} \mathrm{CO}(2-1)$ (derecha; contornos $0.5,1$ 2 , y $3 \mathrm{~K}$ ) en el rango de velocidad $(-36.4,-28.2)$ correspondiente a la cáscara molecular \#4. Los círculos muestran la posición y el diámetro efectivo definido para la cáscara.
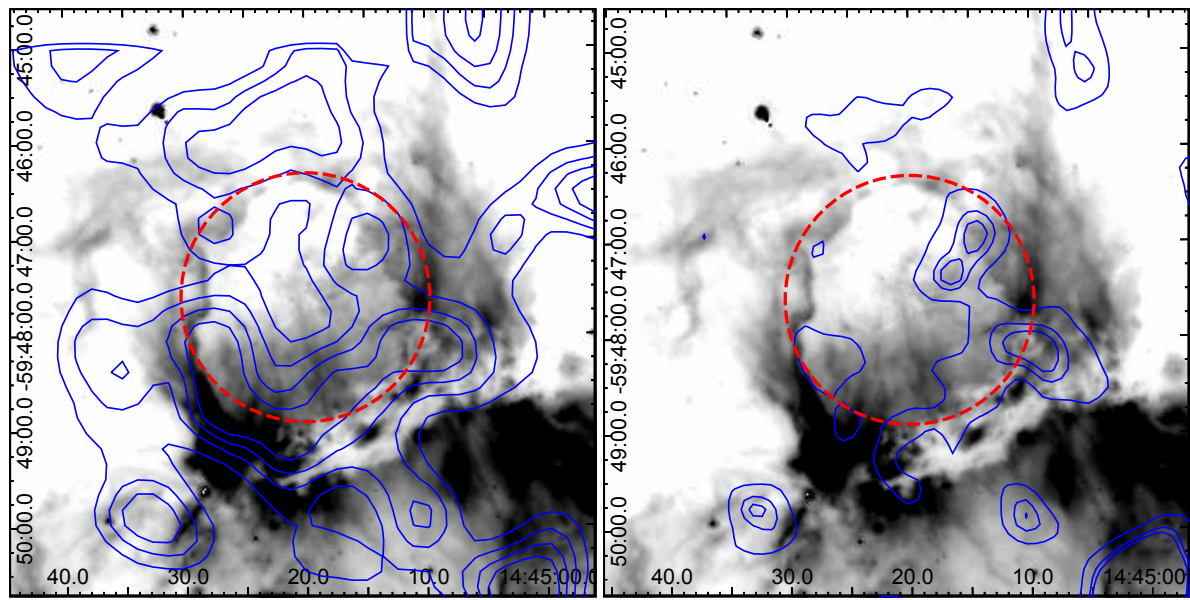

Figura 7.18: Cáscara \#5: Sobre la imagen de $8 \mu \mathrm{m}$ se muestra la emisión promedio de ${ }^{12} \mathrm{CO}(2-1)$ (izquierda; contornos 1.5, 2.5, 3.5 y $\left.4.5 \mathrm{~K}\right)$ y ${ }^{13} \mathrm{CO}(2-1)$ (derecha; contornos 0.1, 0.2 y $0.4 \mathrm{~K})$ en el rango de velocidad $(-32.4,-26.6)$ correspondiente a la cáscara molecular \#5. Los círculos muestran la posición y el diámetro efectivo definido para la cáscara. 


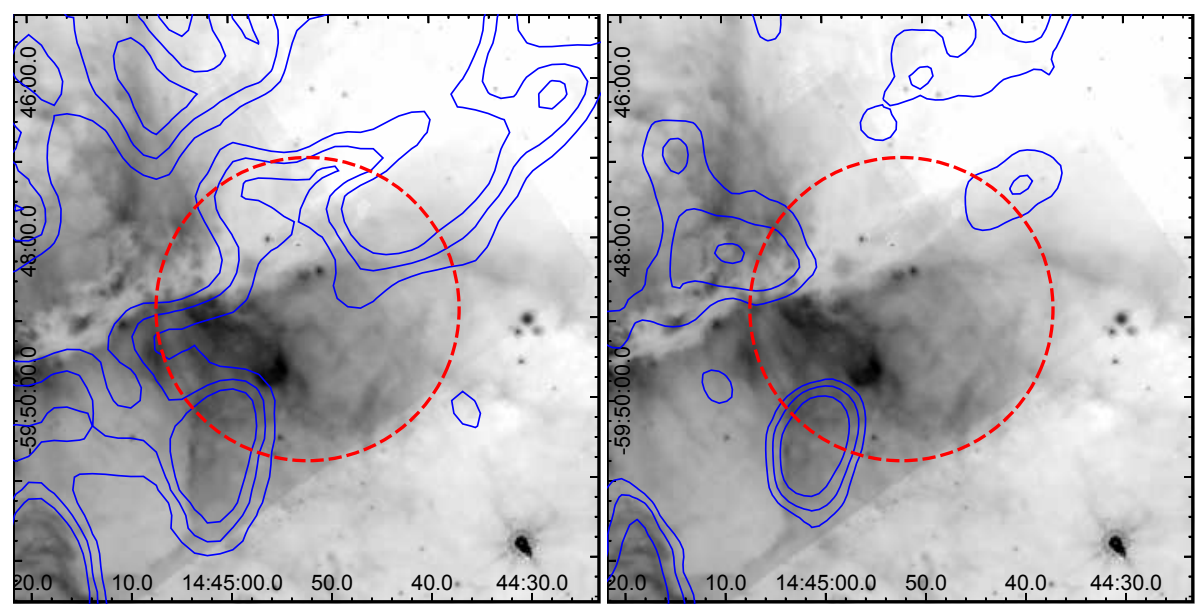

Figura 7.19: Cáscara \#6: Sobre la imagen de $8 \mu \mathrm{m}$ se muestra la emisión promedio de ${ }^{12} \mathrm{CO}(2-1)$ (izquierda; contornos $1.5,3$ y $\left.4 \mathrm{~K}\right)$ y ${ }^{13} \mathrm{CO}(2-1)$ (derecha; contornos 0.5, 1 y $2 \mathrm{~K}$ ) en el rango de velocidad $(-33.4,-30.8)$ correspondiente a la cáscara molecular \#6. Los círculos muestran la posición y el diámetro efectivo definido para la cáscara. La cásacara no coincide con una estructura detectada en el IR.

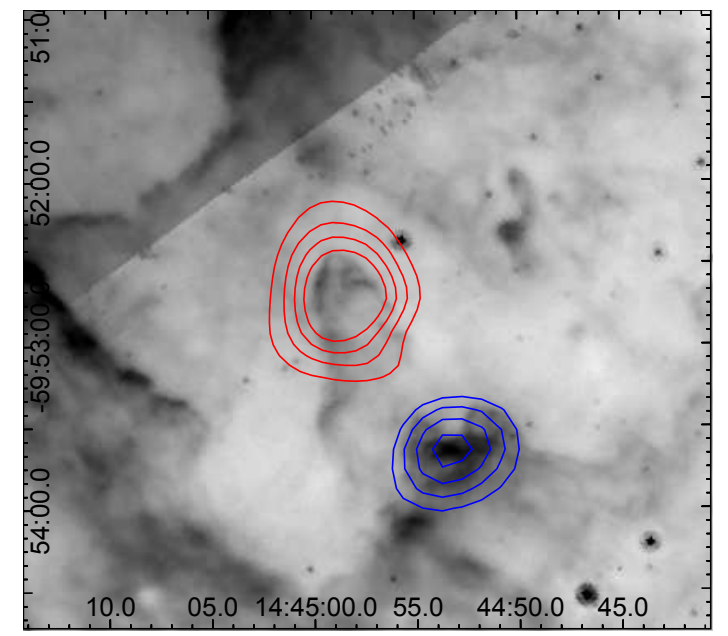

Figura 7.20: Grumos asociados a estructuras de bordes brillantes en el interior de la Cáscara \#2. Los contornos rojos corresponden al promedio entre -40.6 y $-43.2 \mathrm{~km} \mathrm{~s}^{-1}$, y los azules al promedio entre -37.6 a $-41.8 \mathrm{~km} \mathrm{~s}^{-1}$. Para ambos grumos se muestran los contornos de 7 , 8,9 y $10 \mathrm{~K}$. 


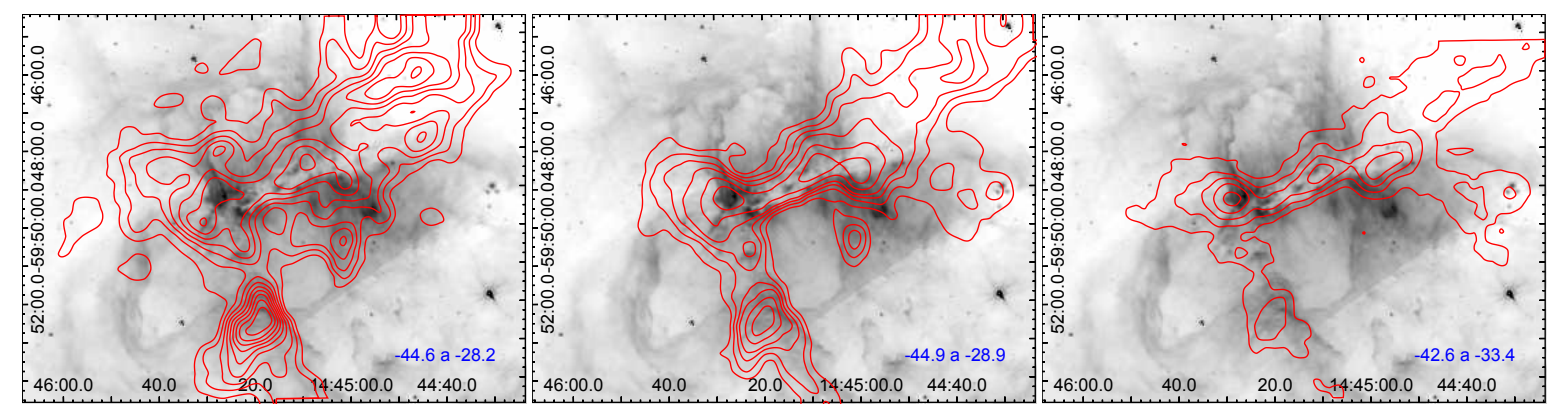

Figura 7.21: Emisión promedio de ${ }^{12} \mathrm{CO}$ (izquierda), ${ }^{13} \mathrm{CO}$ (centro) y $\mathrm{C}^{18} \mathrm{O}$ (derecha) (Tmb) superpuesta a la imagen de $8 \mu \mathrm{m}$. Los contornos corresponden a 5, 6, 7, 8, 9, 10, 11, 12 y 13 $\mathrm{K}$ para el ${ }^{12} \mathrm{CO}, 1.5,2,2.5,3,4$ y $5 \mathrm{~K}$ para el ${ }^{13} \mathrm{CO}$, y $0.5,1,1.5,2$ y $2.5 \mathrm{~K}$ para el $\mathrm{C}^{18} \mathrm{O}$. Los rangos de velocidad en que se hicieron los promedios se indican en cada panel.

Tabla 7.6: Parámetros de las imágenes promedio de ${ }^{12} \mathrm{CO}(2-1),{ }^{13} \mathrm{CO}(2-1)$ y $\mathrm{C}^{18} \mathrm{O}(2-1)$ en la región de las burbujas (Figura 7.21). Se indica el rango en velocidad en que se encuentra la emisión de cada molécula y el número de canales que ocupa, junto con la ruido rms de cada canal individual y el rms que resulta luego de promediar sobre todos los canales indicados canales.

\begin{tabular}{cccccc}
\hline \hline Molécula & $\begin{array}{c}\text { Rango de velocidad } \\
{\left[\mathrm{km} \mathrm{s}^{-1}\right]}\end{array}$ & $\begin{array}{c}\Delta \mathrm{v} \\
{\left[\mathrm{km} \mathrm{s}^{-1}\right]}\end{array}$ & Nro canales & rms canal & rms promedio \\
{$[\mathrm{K}]$} & {$[\mathrm{K}]$} \\
\hline${ }^{12} \mathrm{CO}(2-1)$ & {$[-44.6,-28.2]$} & 16.4 & 81 & 0.25 & 0.028 \\
${ }^{13} \mathrm{CO}(2-1)$ & {$[-44.9,-28.9]$} & 16.0 & 80 & 0.24 & 0.027 \\
$\mathrm{C}^{18} \mathrm{O}(2-1)$ & {$[-42.6,-33.4]$} & 9.2 & 47 & 0.27 & 0.039 \\
\hline \hline
\end{tabular}

relacionarse con la presencia de fuentes IR y posible formación estelar.

Para la identificación de grumos se procedió de la siguiente manera: para cada una de las moléculas se realizó una inspección visual de los cubos de datos, determinándose el rango de velocidades en el que se encontraba emisión, se hizo una imagen promedio de esta emisión y se la analizó en conjunto con el cubo de datos. Se encontró que el rango de velocidad es de -44.6 a $-28.2 \mathrm{~km} \mathrm{~s}^{-1}$ para ${ }^{12} \mathrm{CO}(2-1),-44.9$ a $-28.9 \mathrm{~km} \mathrm{~s}^{-1}$ para ${ }^{13} \mathrm{CO}(2-1)$, y -42.6 a $-33.4 \mathrm{~km} \mathrm{~s}^{-1}$ para $\mathrm{C}^{18} \mathrm{O}(2-1)$. La emisión promedio de estas moléculas en estos rangos de velocidades se muestra en la Figura 7.21 y los parámetros en la Tabla 7.6.

La identificación de los grumos se hizo considerando la emisión en $\mathrm{C}^{18} \mathrm{O}(2-1)$ y luego se buscó su contraparte en los otros isótopos. Esto garantiza que los grumos sean densos y que pueden llegar a formar estrellas. En estos grumos se podrá calcular la masa, determinar si tienen YSOs proyectados y evaluar si podrían estar colapsando.

La Figura 7.22 muestra la emisión de $\mathrm{C}^{18} \mathrm{O}(2-1)$ en seis rangos de velocidades diferentes ${ }^{(3)}$, desde -43 hasta $-31.2 \mathrm{~km} \mathrm{~s}^{-1}$, donde cada panel es un promedio de 10 canales, que corresponden a $2 \mathrm{~km} \mathrm{~s}^{-1}$. Los círculos azules indican los grumos detectados, y han sido numerados desde el 1 al 8 considerando primero los que se encuentran sobre el filamento. No todos los grumos se detectan a las mismas velocidades. El rango total de velocidad en que se observa emisión es de $9.2 \mathrm{~km} \mathrm{~s}^{-1}$, mientras que los grumos se extienden a lo largo de diferentes rangos de velocidades que van desde 1.6 hasta $8.8 \mathrm{~km} \mathrm{~s}^{-1}$.

Las Figuras 7.23 y 7.24 son equivalentes a la Figura 7.22 pero mostrando los contornos

\footnotetext{
${ }^{(3)}$ Las imágenes que se muestran en las Figuras $7.22,7.23$ y 7.24 son las mismas que se muestran en las Figuras $7.11,7.12$ y 7.13 pero con diferentes valores de contornos, de modo de destacar los grumos y no las cáscaras.
} 
Tabla 7.7: Parámetros observacionales de los grumos definidos a partir de la emisión de $\mathrm{C}^{18} \mathrm{O}(2-1)$.

\begin{tabular}{|c|c|c|c|c|c|c|c|c|}
\hline \multirow[t]{2}{*}{$\#$} & \multicolumn{2}{|c|}{$\begin{array}{c}\mathrm{T}_{\operatorname{mb}}^{\max } \\
{[\mathrm{K}]}\end{array}$} & \multicolumn{2}{|c|}{$\begin{array}{c}\Delta \mathrm{v} \\
{\left[\mathrm{km} \mathrm{s}^{-1}\right]}\end{array}$} & \multirow[t]{2}{*}{$\begin{array}{c}\text { Posición } \\
{[\text { RA, Dec.(J2000)] }}\end{array}$} & \multirow[t]{2}{*}{$\begin{array}{l}\text { Rango } \\
{\left[\mathrm{km} \mathrm{s}^{-1}\right]}\end{array}$} & \multirow[t]{2}{*}{$\begin{array}{c}\text { Radio } \\
{\left[{ }^{\prime \prime}\right]}\end{array}$} & \multirow[t]{2}{*}{ Cáscara } \\
\hline & ${ }^{13} \mathrm{CO}$ & $\mathrm{C}^{18} \mathrm{O}$ & ${ }^{13} \mathrm{CO}$ & $\mathrm{C}^{18} \mathrm{O}$ & & & & \\
\hline 1 & 12.0 & 3.6 & 5.0 & 4.3 & $14: 45: 27.738,-59: 49: 11.38$ & {$[-42.6,-34.2]$} & 48.5 & 5 \\
\hline 2 & 9.5 & 2.2 & 1 & 4. & $14: 45: 10.122,-59: 48: 40$ & {$[-43.0,-$} & 41.1 & $5-6$ \\
\hline 3 & 13.0 & 4.0 & 1 & 3.1 & $14: 44: 55.653,-59: 4$ & {$[-42.8$, } & 57.8 & - \\
\hline 4 & 8.0 & 2.4 & 4.0 & 2.8 & 14:44:44.370, $-59:$ & {$[-42.0$,} & 35.4 & 6 \\
\hline 5 & 8.6 & 2.9 & 4.2 & 2.8 & $14: 44: 32.659,-59:$ & {$[-41.6,--$} & 35.1 & - \\
\hline 6 & 9.2 & 2.5 & 4.4 & 2.2 & $14: 45: 21.169,-59: 52: 18$ & {$[-38.8,-34.0]$} & 40.5 & $3-4$ \\
\hline 7 & 3.6 & 1.1 & 3.4 & 1.9 & $14: 45: 01.494,-59:$ & {$[-34.2,-32.2]$} & 34.9 & $2-4$ \\
\hline 8 & 7.4 & 2.0 & 4.0 & 3.0 & $14: 44: 32.523,-59: 49: 14.79$ & {$[-39.0,-37.4]$} & 42.0 & $2-6$ \\
\hline
\end{tabular}

de ${ }^{13} \mathrm{CO}(2-1)$ y ${ }^{12} \mathrm{CO}(2-1)$, respectivamente. Los círculos mostrados en estas figuras son los mismos que los mostrados en la Figura 7.22.

Para definir los grumos se han buscado regiones en donde se encuentren máximos locales en la emisión, por lo tanto los contornos mostrados en las Figuras 7.22, 7.23 y 7.24 se han elegido de modo que evidencien la presencia de estas regiones, y no significa que no se encuentre emisión por fuera de estos niveles. De hecho la emisión de las tres moléculas estudiadas es muy intensa en toda la región observada. Por lo tanto en todos los grumos detectados en $\mathrm{C}^{18} \mathrm{O}(2-1)$ se ve emisión en ${ }^{13} \mathrm{CO}(2-1)$ y ${ }^{12} \mathrm{CO}(2-1)$, aunque al ser esta más extendida a veces no se ve en forma de grumo.

Sólo los grumos \#1, \#2 \#6 y \#7 son detectados en los tres isótopos, mientras que los grumos \#3, \#4, \#5 y \#8 se detectan en $\mathrm{C}^{18} \mathrm{O}(2-1)$ y ${ }^{13} \mathrm{CO}(2-1)$. El grumo \#3, que es uno de los más intensos, no se detecta en ${ }^{12} \mathrm{CO}(2-1)$ pero se observa un máximo en la emisión en una posición cercana a la de este grumo (a unos $50^{\prime \prime}$ de distancia del centro del grumo).

Para cuantificar los parámetros de los grumos se hicieron espectros promedio sobre el área de cada uno de ellos. En la Tabla 7.7 se detallan la temperatura de brillo máxima para ${ }^{13} \mathrm{CO}(2-1)$ y $\mathrm{C}^{18} \mathrm{O}(2-1)$ para cada grumo y el ancho a potencia mitad medido. También se listan la posición, el rango en que se detecta la emisión, el radio equivalente estimado para cada grumo y la cáscara con que se encuantran asociados.

Para determinar el rango de velocidad de cada grumo se midió la temperatura máxima que alcanza y se consideraron parte del grumo aquellos canales en que la temperatura no decae más de un $70 \%$ respecto al máximo.

Para calcular el tamaño de cada uno de los grumos se hizo una imagen promedio de la emisión de $\mathrm{C}^{18} \mathrm{O}(2-1)$ en el rango de velocidades en que se ve cada uno de los grumos. Luego se identificó el contorno cerrado de menor nivel. Esta metodología no resultó apropiada para los grumos que se encuentran sobre el filamento, ya que al estar muy cerca un grumo del otro y al encontrarse embebidos en emisión extendida los contornos cerrados de menor nivel tienen mayor temperatura de brillo y se pierde gran parte de la emisión del grumo. Por esta razón para estos grumos se utilizó otro criterio y se consideró como parte del mismo aquella emisión que estuviera por encima de 30 veces el valor de $r m s$. Finalmente se midió la cantidad de pixeles en la región definida para cada grumo, y considerando el tamaño de los pixeles de la imagen $(1$ !'432 × 1"!432 ) se calculó un valor para el tamaño de cada uno de los grumos. Hay que tener en cuenta que los grumos tienen diversas formas por lo que el radio del grumo solamente sirve para tener una idea del tamaño del mismo. Este valor es igual al radio que tendría un grumo esférico de igual área proyectada y se denomina radio efectivo. 


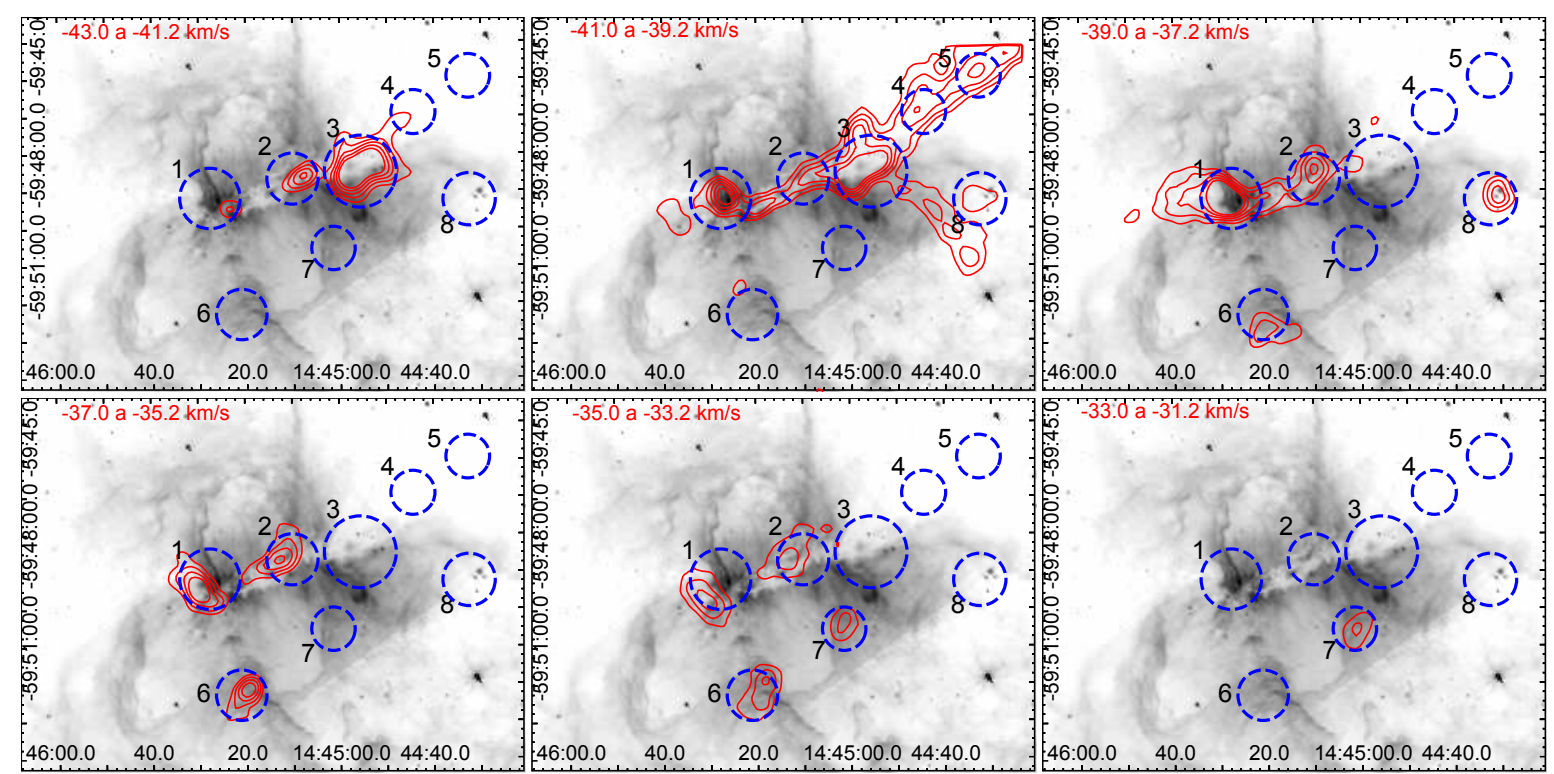

Figura 7.22: Emisión de $\mathrm{C}^{18} \mathrm{O}$ en el rango de velocidades desde -43.0 hasta $-31.2 \mathrm{~km} \mathrm{~s}^{-1}$ indicando los grumos identificados. Cada panel es un promedio de 10 canales $\left(2 \mathrm{~km} \mathrm{~s}^{-1}\right)$. Contornos: paneles 1,2 y $3: 1.5$ a $3.5 \mathrm{~K}$ cada $0.5 \mathrm{~K}$; panel $4: 1.5$ a $3 \mathrm{~K}$ cada $0.5 \mathrm{~K}$; panel 5: $0.5,1$ y $1.5 \mathrm{~K}$; panel 6: 0.5 y $1 \mathrm{~K}$.

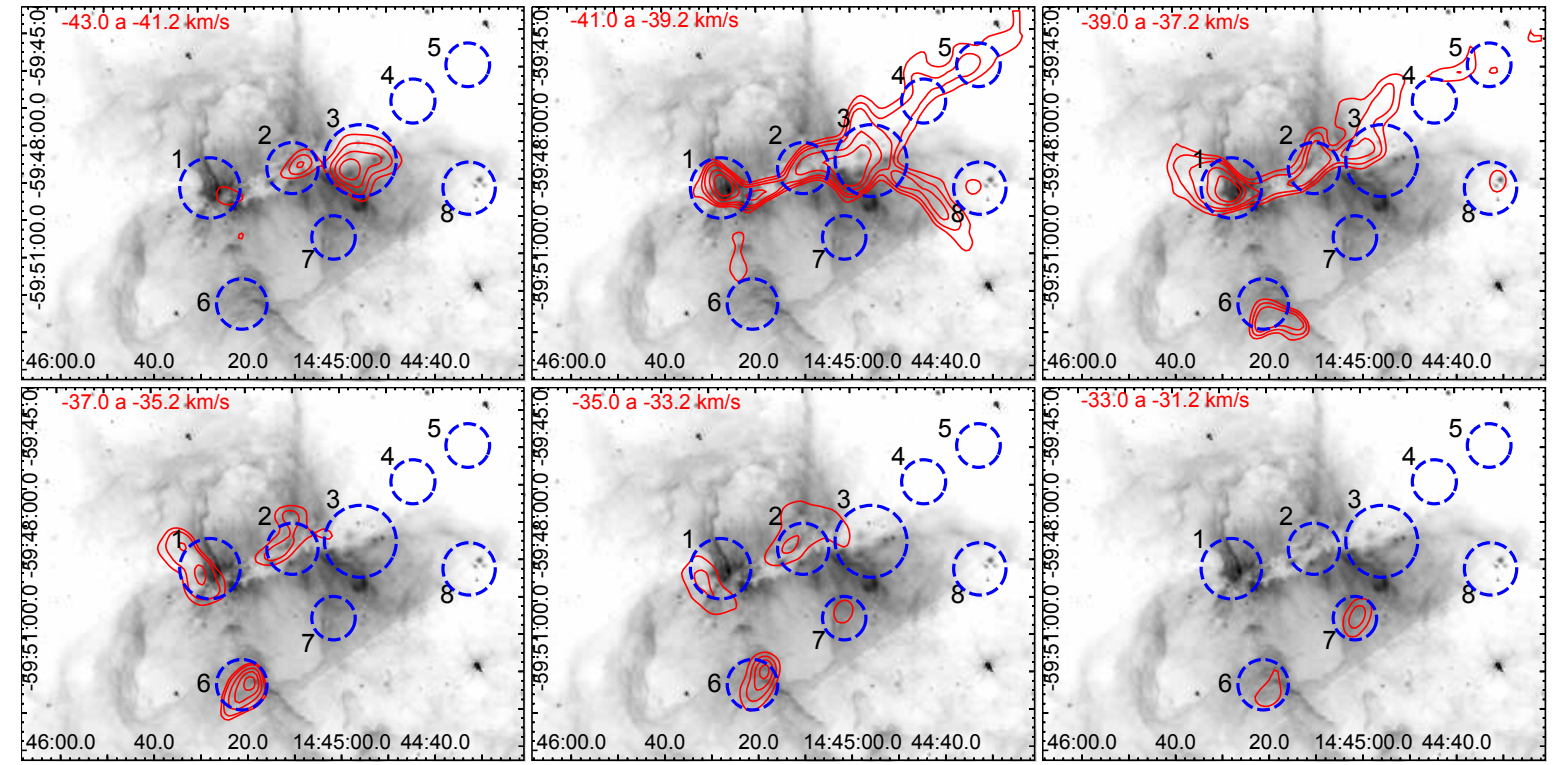

Figura 7.23: Emisión de ${ }^{13} \mathrm{CO}$ en el rango de velocidades desde -43.0 hasta $-31.2 \mathrm{~km} \mathrm{~s}^{-1}$ indicando los grumos identificados en $\mathrm{C}^{18} \mathrm{O}(2-1)$. Cada panel es un promedio de 10 canales $\left(2 \mathrm{~km} \mathrm{~s}^{-1}\right)$. Contornos: panel 1: 7, 9, 11 y $13 \mathrm{~K}$; paneles 2 y $3: 7,8,9,11,13 \mathrm{~K}$; panel 4: 6 , 7, 9, 11 y $13 \mathrm{~K}$; panel 5: 3, 5, 7, y $9 \mathrm{~K}$; panel $6: 3$ y $5 \mathrm{~K}$. 


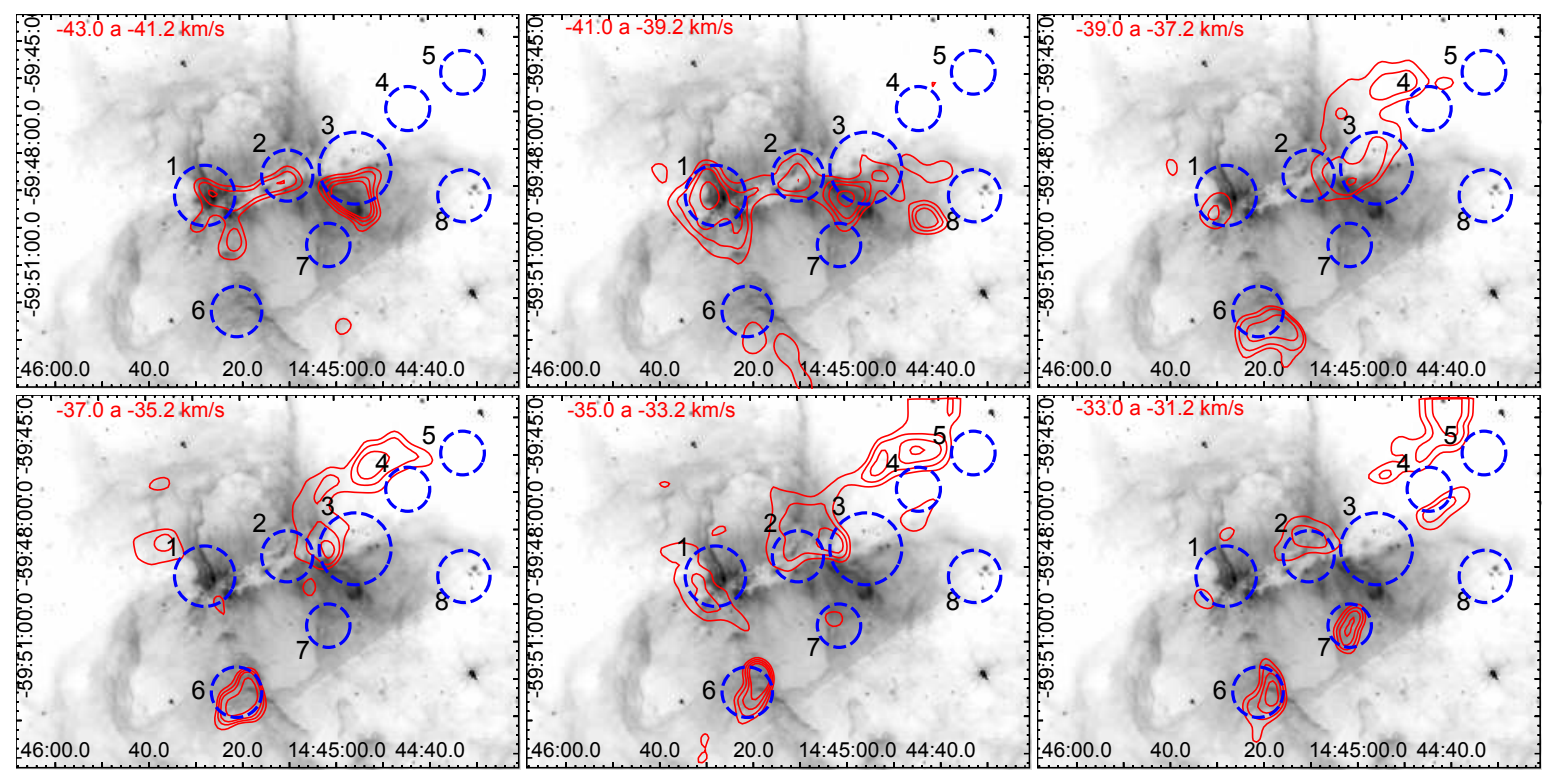

Figura 7.24: Emisión de ${ }^{12} \mathrm{CO}$ en el rango de velocidades desde -43.0 hasta $-31.2 \mathrm{~km} \mathrm{~s}^{-1}$ indicando los grumos identificados en $\mathrm{C}^{18} \mathrm{O}(2-1)$. Cada panel es un promedio de 10 canales $\left(2 \mathrm{~km} \mathrm{~s}^{-1}\right)$. Contornos: panel 1: 12, 14, 16, y $18 \mathrm{~K}$; panel 2: 14, 16, 18 y $22 \mathrm{~K}$; panel 3: 16, 18 y $22 \mathrm{~K}$; panel 4: 14, 16, 18 y $22 \mathrm{~K}$; panel 5: 10, 12, 14 y $16 \mathrm{~K}$; panel 6: 8, 10,12 y $14 \mathrm{~K}$.

\subsubsection{IRDCs}

El análisis del gas molecular asociado a la IRDC se llevó a cabo de modo similar a la región de las burbujas, a partir de los cubos de datos y de imágenes promedio de cada uno de los isótopos en diferentes rangos de velocidades. En las Figuras 7.25, 7.26 y 7.27 se muestra en contornos la emisión de $\mathrm{C}^{18} \mathrm{O}(2-1),{ }^{13} \mathrm{CO}(2-1)$ y ${ }^{12} \mathrm{CO}(2-1)$, respectivamente. En cada panel de estas figuras se muestra la emisión promedio en diferentes rangos de velocidades radiales superpuesta a la imagen en $8 \mu \mathrm{m}$. Cada uno de los seis paneles de cada figura muestra una imagen promediada en un rango de $2 \mathrm{~km} \mathrm{~s}^{-1}$ (10 canales) sobre un rango de velocidad total entre $-43 \mathrm{y}-31.2 \mathrm{~km} \mathrm{~s}^{-1}$ (estas velocidades coinciden con las mostradas en las figuras 7.11, 7.12 y 7.13 para las burbujas).

La Figura 7.25 revela que la emisión más intensa de $\mathrm{C}^{18} \mathrm{O}(2-1)$ se observa entre -41.0 y $-37.2 \mathrm{~km} \mathrm{~s}^{-1}$ delineando la zona de mayor extinción en $8 \mu \mathrm{m}$, que, tal como se evidencia en la Figura 7.7, coincide con emisión en el IR lejano $(\lambda>160 \mu \mathrm{m})$. Esto es similar a lo que ocurre en otras IRDCs, como por ejemplo en la IRDC G34.43+0.24 (Sanhueza et al., 2010) donde el gas molecular tiene la misma distribución que el polvo frío (emisión en $1.2 \mathrm{~mm}$ ). Esto es coherente con el hecho de que el $\mathrm{C}^{18} \mathrm{O}(2-1)$ es una molécula que generalmente traza regiones relativamente frías y densas de las nubes moleculares, y su emisión no es muy extendida. En efecto, la emisión fuera de este rango de velocidad se encuentra apenas por encima del nivel mínimo detectable de emisión $(3 r m s=0.25 \mathrm{~K})$.

En el mismo rango de velocidad en que se encuentra la emisión de $\mathrm{C}^{18} \mathrm{O}(2-1)$ también se observa la máxima emisión de ${ }^{13} \mathrm{CO}(2-1)$, tal como se muestra en la Figura 7.26. Su máximo coincide con la IRDC, que también se observa entre -43.0 y $-41.2 \mathrm{~km} \mathrm{~s}^{-1}$, pero es más extendida. Entre -37.0 y $-31.2 \mathrm{~km} \mathrm{~s}^{-1}$ la emisión de ${ }^{13} \mathrm{CO}(2-1)$ no coincide con la IRDC, sino que traza un filamento que se encuentra cerca de RA(J2000)=14:44:40, y se extiende aproximadamente desde Dec(J2000) $=-59: 39: 20$ hasta Dec $(\mathrm{J} 2000)=-59: 44: 52.00$. Entre -37.0 y $-35.2 \mathrm{~km} \mathrm{~s}^{-1}$ es posible distinguir una cavidad que queda delimitada por la IRDC y el 


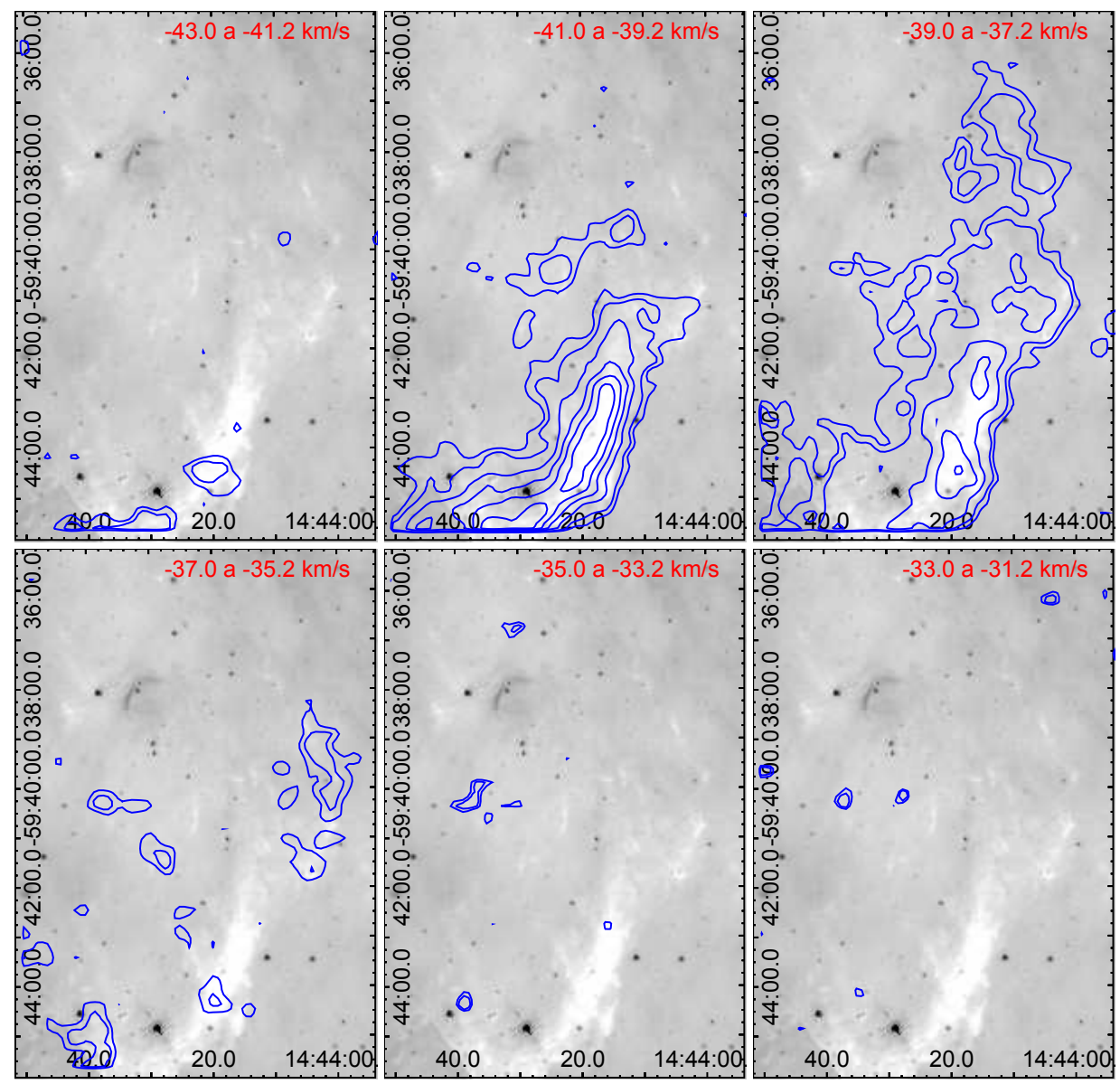

Figura 7.25: Distribución de $\mathrm{C}^{18} \mathrm{O}(2-1)$ en el intervalo de velocidades de -43 a $-31.2 \mathrm{~km} \mathrm{~s}^{-1}$ en pasos de $2 \mathrm{~km} \mathrm{~s}^{-1}$. Imagen de fondo: $8 \mu \mathrm{m}$. Contornos: emisión de $\mathrm{C}^{18} \mathrm{O}(2-1)$ promediada en los rangos de velocidades indicados en cada panel (10 canales). Los contornos corresponden a 0.25 y $0.4 \mathrm{~K}$ en el primer y cuarto panel, $0.3,0.5,1,1.5,2$, y $2.5 \mathrm{~K}$ en el segundo y tercer panel, y 0.25 y $0.3 \mathrm{~K}$ en el quinto y sexto panel.

filamento mencionado.

Finalmente en la Figura 7.27 se puede ver que la emisión de ${ }^{12} \mathrm{CO}(2-1)$ tiene una distribución diferente a la de $\mathrm{C}^{18} \mathrm{O}(2-1)$ y ${ }^{13} \mathrm{CO}(2-1)$, ya que no muestra máximos sobre la IRDC. Esta emisión, mucho más extendida que las anteriores, revela varios máximos en la región, y muestra el filamento delineado por ${ }^{13} \mathrm{CO}(2-1)$.

\subsubsection{Regiones observadas con MALT90}

Para identificar los grumos densos también se pueden utilizar datos del relevamiento MALT90, que incluye observaciones de moléculas trazadoras de alta densidad, tales como $\mathrm{HCO}^{+}(1-0)$ y $\mathrm{HNC}(1-0)$. Se encontraron en la zona 4 regiones observadas por MALT90 asociadas a emisión intensa de ALTASGAL. Las moléculas detectadas en estas regiones son $\mathrm{H}^{13} \mathrm{CO}^{+}(1-0), \mathrm{HN}^{13} \mathrm{C}(1-0), \mathrm{C}_{2} \mathrm{H}(1-0), \mathrm{HCN}(1-0), \mathrm{HCO}^{+}(1-0), \mathrm{HNC}(1-0), \mathrm{HC}_{3} \mathrm{~N}(10-9)$ y $\mathrm{N}_{2} \mathrm{H}^{+}(1-0)$. Sus frecuencias y su utilidad como trazador se encuentran detallados en la Tabla 2.2.

Como se vio en la Sección 7.3, la emisión de ALTASGAL delinea una región en forma de arco sobre la que se encuentran tres de las regiones observadas por MALT90 (ver Figura 


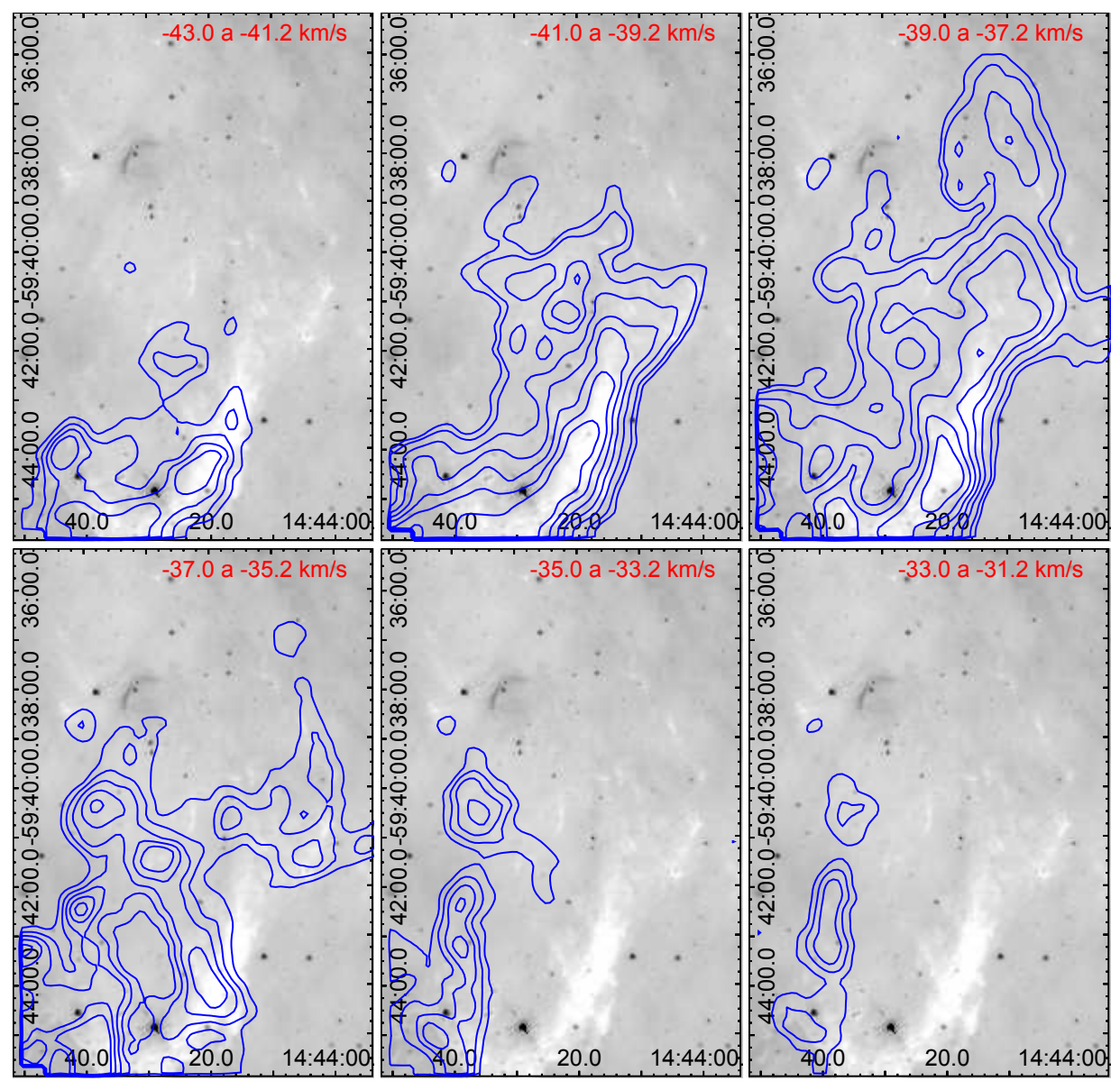

Figura 7.26: Distribución de ${ }^{13} \mathrm{CO}(2-1)$ en el intervalo de velocidades de -43 a $-31.2 \mathrm{~km} \mathrm{~s}^{-1}$ en pasos de $2 \mathrm{~km} \mathrm{~s}^{-1}$. Imagen de fondo: $8 \mu \mathrm{m}$. Contornos: emisión de ${ }^{13} \mathrm{CO}(2-1)$ promediada en los rangos de velocidades indicados en cada panel (10 canales). Los contornos corresponden a 1, 1.5, 2 y $2.5 \mathrm{~K}$ en el primer panel, 1.5, 2, 3, 4, 5, 6 y $7 \mathrm{~K}$ en el segundo y tercer panel, 1 , $1.5,2,2.5$ y $3 \mathrm{~K}$ en el cuarto panel, $1,1.5,2$ y $2.5 \mathrm{~K}$ en el quinto panel, y $1,1.5$ y $2 \mathrm{~K}$ en el sexto panel. 


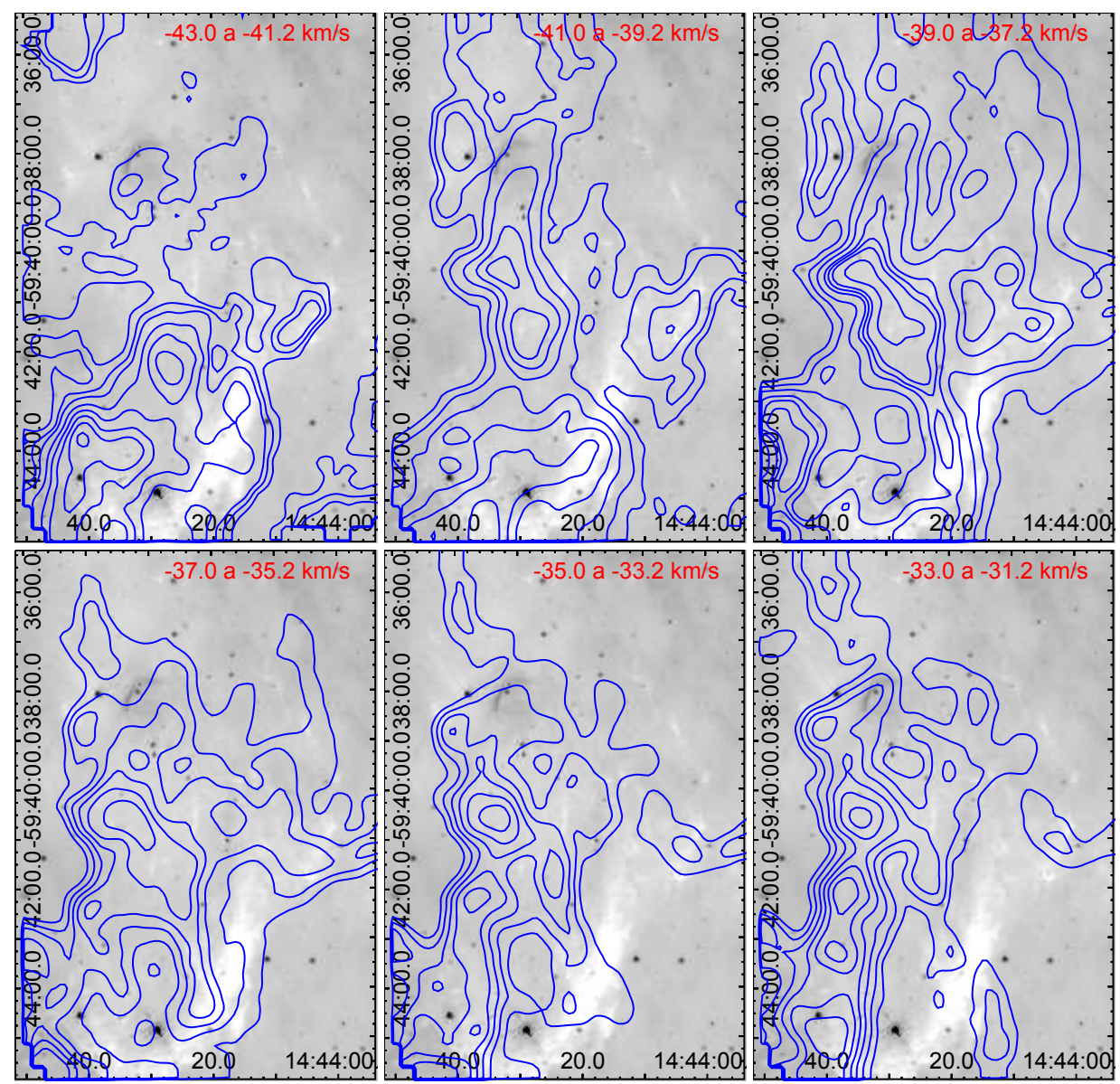

Figura 7.27: Distribución de ${ }^{12} \mathrm{CO}(2-1)$ en el intervalo de velocidades de -43 a $-31.2 \mathrm{~km} \mathrm{~s}^{-1}$ en pasos de $2 \mathrm{~km} \mathrm{~s}^{-1}$. Imagen de fondo: $8 \mu \mathrm{m}$. Contornos: emisión de ${ }^{12} \mathrm{CO}(2-1)$ promediada en los rangos de velocidades indicados en cada panel (10 canales). Los contornos corresponden a $2,2.5,3,4,5,6,7$ y $8 \mathrm{~K}$ en el primer panel, 3, 4, 6, 8, 10 y $12 \mathrm{~K}$ en el segundo panel, 4, 6, $8,10,11,12$ y $14 \mathrm{~K}$ en el tercer panel, $4,6,8,10,12$ y $14 \mathrm{~K}$ en el cuarto y quinto panel, y $2.5,4,6,8,10$ y $12 \mathrm{~K}$ en el quinto y sexto panel. 


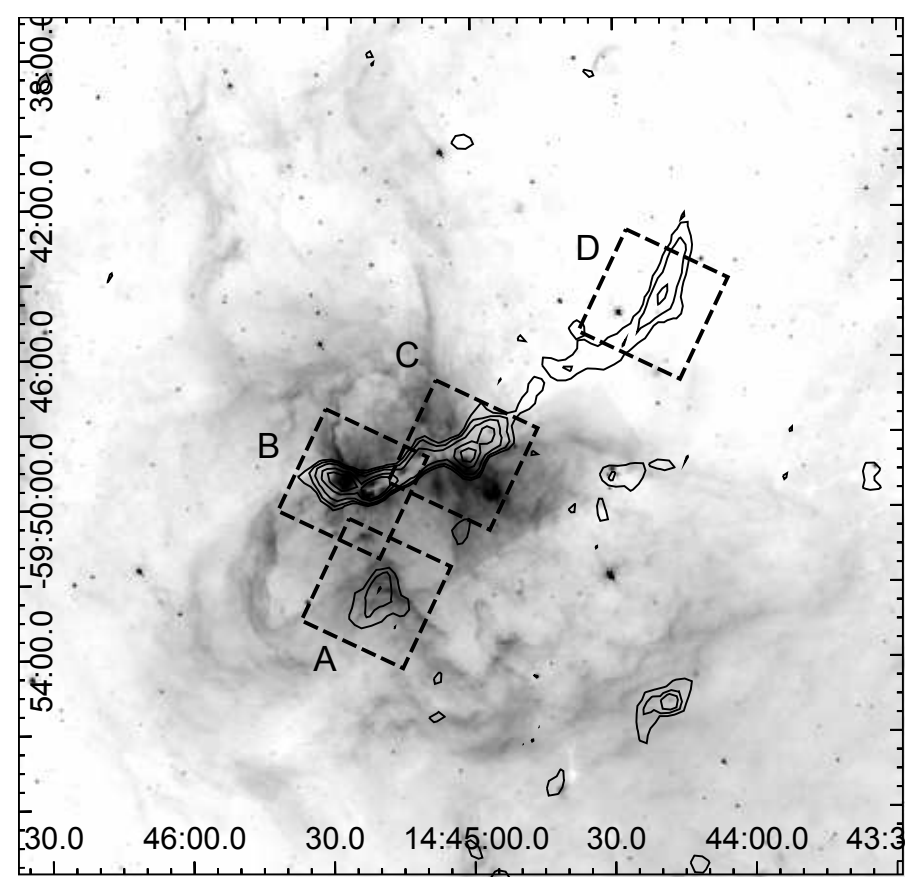

Figura 7.28: Regiones observadas por MALT90 sobre la imagen de emisión en $8 \mu \mathrm{m}$ junto con los contronos de ATLASGAL (0.2, 0.5, 1, 2 y $3 \mathrm{Jy} /$ beam). Los recuadros muestran las regiones observadas sobre el filamento (B y C), sobre la IRDC (D) y en el límite entre las burbujas S 109 y S 111 (A).

\subsection{8).}

Una de ellas (D) centrada en RA, Dec.(J2000) $=(14: 44: 21.584,-59: 44: 37.29)$ coincide con las IRDCs catalogadas por Simon et al. (2006). Allí se encontró emisión de HCN, HNC, $\mathrm{HCO}^{+}$ y $\mathrm{N}_{2} \mathrm{H}^{+}$. Todas estas moléculas son trazadoras de alta densidad. Sus densidades críticas varían entre $10^{4}$ y $10^{5} \mathrm{~cm}^{-3}$, lo que es razonable para IRDCs que suelen tener densidades por encima de $10^{4} \mathrm{~cm}^{-3}$.

Otras dos regiones (B y C) se encuentran sobre el filamento entre las burbujas S110 y S111 (RA, Dec.(J2000) = 14:45:02.098, -59:48:36.97 y RA, Dec.(J2000) = 14:45:25.515, 59:49:24.29). Éste no está catalogado como IRDC (aunque comparte algunas características) y podría ser material comprimido por la expansión de las burbujas. Esta región es químicamente más compleja, ya que allí además de emisión en líneas de $\mathrm{HCN}, \mathrm{HNC}, \mathrm{HCO}^{+} \mathrm{y} \mathrm{N}_{2} \mathrm{H}^{+}$como en la zona de la IRDC, se encontró emisión en $\mathrm{C}_{2} \mathrm{H}, \mathrm{HC}_{3} \mathrm{~N}$ y los isótopos $\mathrm{H}^{13} \mathrm{CO}^{+}$y $\mathrm{HN}^{13} \mathrm{C}$.

Finalmente en la zona entre las burbujas S109 y S111 (A), centrada en RA, Dec.(J2000)= 14:45:20.729, -59:52:17.41, se encontraron las mismas moléculas que en la zona de la IRDC y se suma $\mathrm{C}_{2} \mathrm{H}$ que es un trazador de regiones de fotodisociación.

Se llevó a cabo una búsqueda de emisión de moléculas trazadoras de alta densidad observadas en MALT90 asociadas a los grumos definidos a partir de $\mathrm{C}^{18} \mathrm{O}(2-1)$ en la región de las burbujas (regiones 'A','B' y 'C'). Dado que las regiones observadas por MALT90 cubren un área del cielo menor que las observadas con APEX sólo se pudo analizar la presencia de estas moléculas en los grumos 1, 2, 3 y 6 . El Grumo 1 se encuentra en la región 'B', los Grumos 2 y 3 en la región 'C' y el Grumo 6 en la 'A'. En la Tabla 7.8 se indica cuáles fueron las moléculas detectadas asociadas a cada grumo. Además la velocidad a la que se encuentra la emisión de estas moléculas coincide con los rangos de velocidades de $\mathrm{C}^{18} \mathrm{O}(2-1)$ mostrados en la columna 7 de la Tabla 7.7 por lo que se puede asegurar que se trata de la misma región.

Tal como se puede ver en la Tabla 2.2 del Capítulo 2 todas las moléculas detectadas 
Tabla 7.8: Moléculas trazadoras de alta densidad asociadas a los grumos 1, 2, 3 y 6 definidos a partir de $\mathrm{C}^{18} \mathrm{O}(2-1)$. El símbolo ' $\checkmark$ ' significa que se detecta el emisión y ' $\mathrm{X}$ ' que no se detecta.

\begin{tabular}{lcccc}
\hline \hline & Grumo 1 & Grumo 2 & Grumo 3 & Grumo 6 \\
$\mathrm{N}_{2} \mathrm{H}^{+}(1-0)$ & $\checkmark$ & $\checkmark$ & $\checkmark$ & $\checkmark$ \\
$\mathrm{HC}_{3} \mathrm{~N}(10-9)$ & $\checkmark$ & $\times$ & $\checkmark$ & $\times$ \\
$\mathrm{HNC}(1-0)$ & $\checkmark$ & $\checkmark$ & $\checkmark$ & $\checkmark$ \\
$\mathrm{HCO}^{+}(1-0)$ & $\checkmark$ & $\checkmark$ & $\checkmark$ & $\checkmark$ \\
$\mathrm{HCN}(1-0)$ & $\checkmark$ & $\checkmark$ & $\checkmark$ & $\checkmark$ \\
$\mathrm{C}_{2} \mathrm{H}(1-0)$ & $\checkmark$ & $\checkmark$ & $\checkmark$ & $\checkmark$ \\
$\mathrm{HN}^{13} \mathrm{C}(1-0)$ & $\checkmark$ & $\checkmark$ & $\checkmark$ & $\times$ \\
$\mathrm{H}^{13} \mathrm{CO}^{+}(1-0)$ & $\checkmark$ & $\checkmark$ & $\checkmark$ & $\times$ \\
\hline \hline
\end{tabular}

en esta región poseen densidades críticas de entre $2 \times 10^{5}$ y $5 \times 10^{5}$, excepto $\mathrm{HCN}(1-0)$ cuya densidad crítica es $3 \times 10^{6}$. En todos los grumos se detecta emisión de esta molécula, por lo que se puede asegurar que las densidades de los mismos están por encima de este valor, o sea que son muy densos. En la Figura 7.29 se muestran los perfiles promedio de $\mathrm{C}^{18} \mathrm{O}(2-1)$ y $\mathrm{HCO}^{+}(1-0)$ en dirección a los Grumos 1, 2, 3 y 6 . De todas las moléculas observadas por MALT90 en esta región $\mathrm{HCO}^{+}(1-0)$ es la más intensa.

La Figura 7.30 muestra mapas de momento cero de las moléculas detectadas en las regiones 'A', 'B' y 'C'. En estos se hace evidente la presencia de los Grumos 1, 3 y 6. En dirección al Grumo 2 se observa emisión de todas las moléculas pero no en forma de grumo, sino en forma de filamento uniendo los Grumos 1 y 3 . Además, la figura revela la presencia de un grumo no detectado en $\mathrm{C}^{18} \mathrm{O}(2-1)$, que se encuentra entre los Grumos 1 y 2.

La diferencia en posición entre los grumos detectados a partir de $\mathrm{C}^{18} \mathrm{O}(2-1)$ y de datos de MALT90 puede deberse a que los mapas de momento cero de MALT90 son promedios en todo el rango de velocidad en el que se encuentra emisión mientras que los grumos detectados en $\mathrm{C}^{18} \mathrm{O}(2-1)$ se identificaron en imágenes promedio en rangos de velocidades menores $(2$ $\left.\mathrm{km} \mathrm{s}^{-1}\right)$.

En la Figura 7.31 se muestran los mapas de intensidad integrada de $\mathrm{N}_{2} \mathrm{H}^{+}(1-0), \mathrm{HNC}(1-0)$, $\mathrm{HCO}^{+}(1-0)$ y $\mathrm{HCN}(1-0)$ en la región ' $\mathrm{D}$ '. Todas ellas delinean el filamento oscuro en $8 \mu \mathrm{m}$, y su emisión coincide espacialmente con la observada en $\mathrm{C}^{18} \mathrm{O}(2-1)$ entre -41.0 y $-37.2 \mathrm{~km} \mathrm{~s}^{-1}$ (ver Figura 7.25).

\subsection{Parámetros del gas molecular}

\subsubsection{Calculados a partir de CO}

\section{Cáscaras moleculares}

Para poder determinar los parámetros de las cáscaras moleculares asociadas a las burbujas definidas en la Sección 7.4.3 es necesario precisar un criterio que permita decidir qué pertenece y qué no pertenece a cada cáscara. El criterio utilizado fue el siguiente: Se consideró que las paredes internas de las cáscaras están determinadas por el contorno cerrado de menor nivel en las imágenes promedio. Para definir el borde exterior se determinó que los grumos que se detectaran alrededor de las cavidades sean considerados parte de la cáscara, excepto cuando un mismo grumo aparece asociado a más de un cáscara, caso en el que se divide pasando por su máximo. Esto se fundamenta en el hecho de que las cáscaras se consideran resultado de la expansión de las burbujas IR, y es el modo usual de definir cáscaras en radioastronomía. 

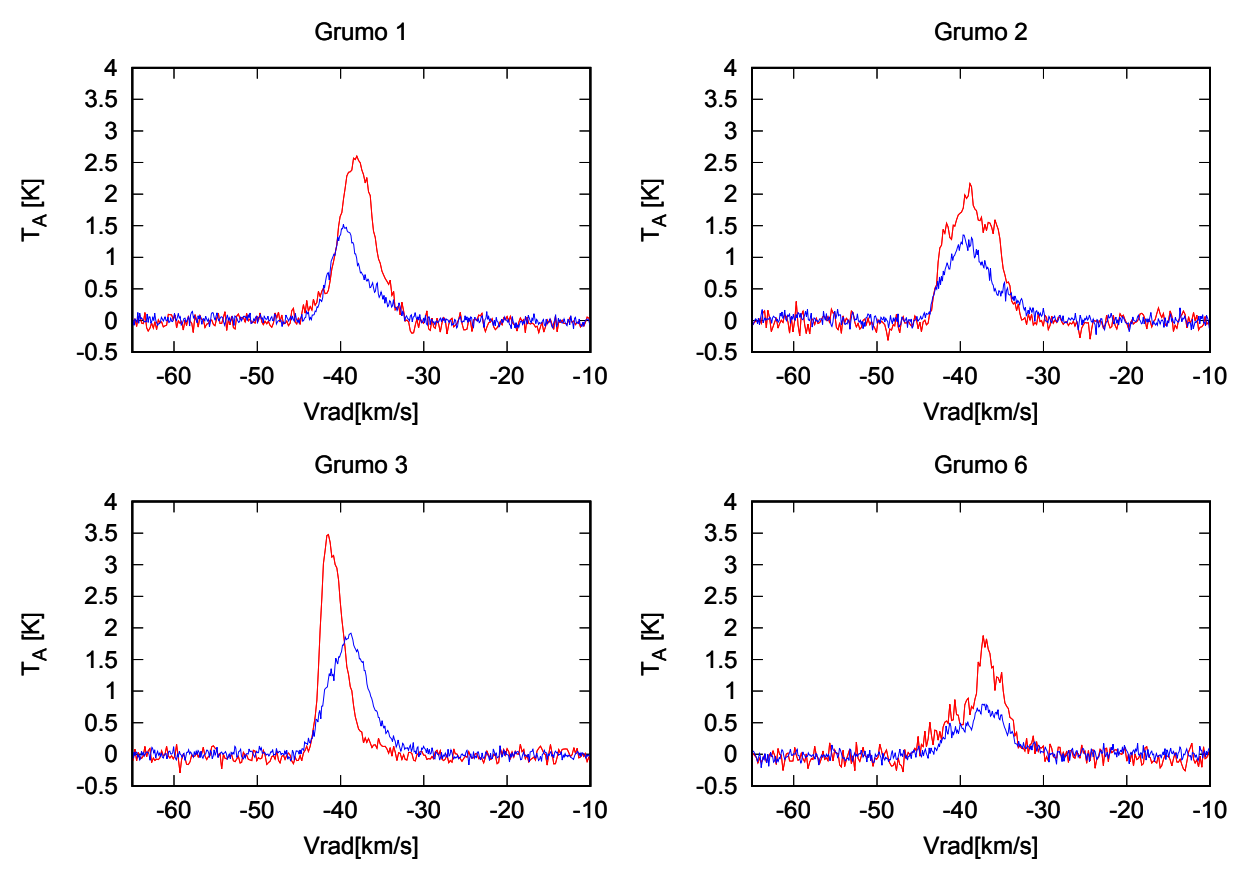

Figura 7.29: Perfiles promedio de $\mathrm{C}^{18} \mathrm{O}(2-1)$ (rojo) y $\mathrm{HCO}^{+}(1-0)$ (azul) para los Grumos 1 , 2,3 y 6 . En el eje de las ordenadas se muestra temperatura de antena $\left(\mathrm{T}_{A}\right)$. Para obtener $\mathrm{T}_{m b}$ se debe multiplicar por 0.436 . 
○े

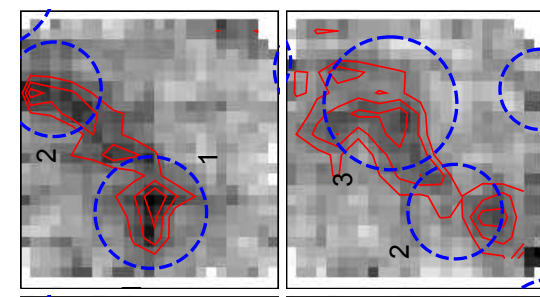

主

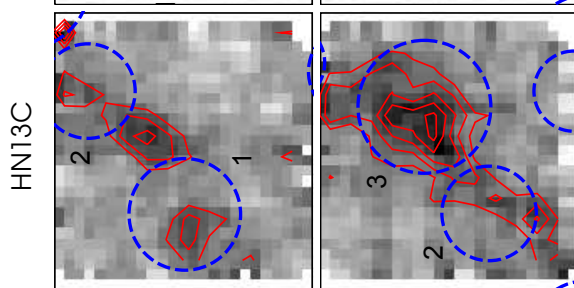

$\stackrel{I}{\mathcal{I}}$

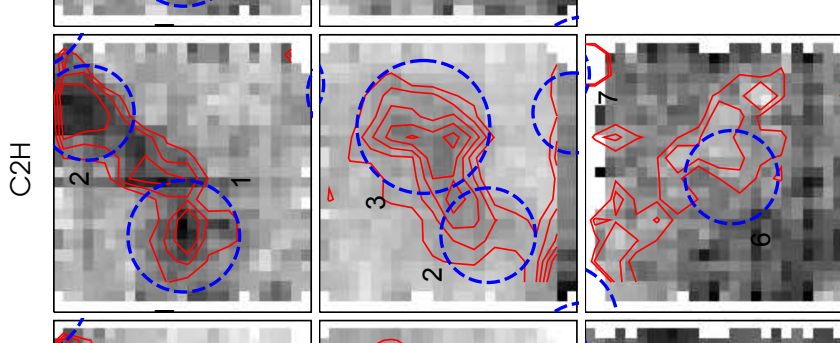

บ

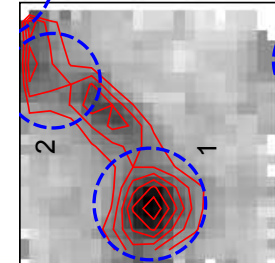

○ั

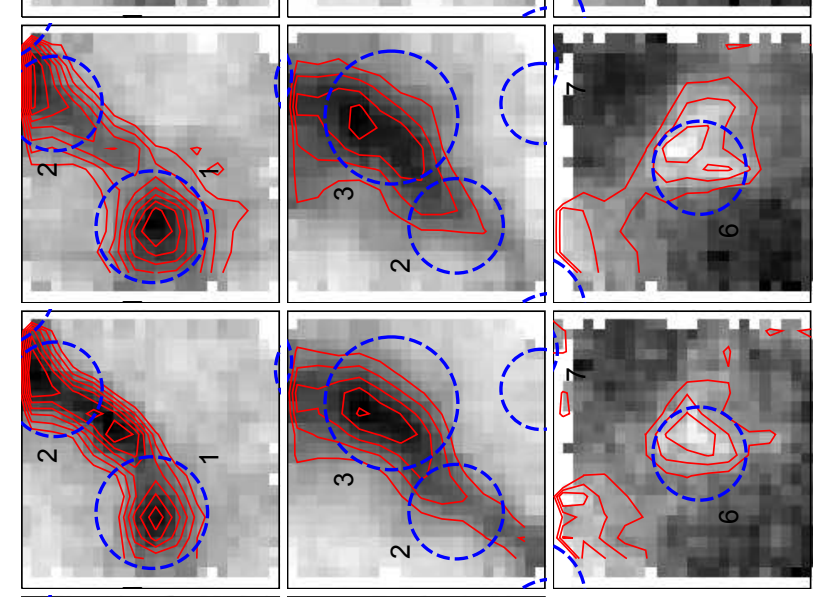

žm

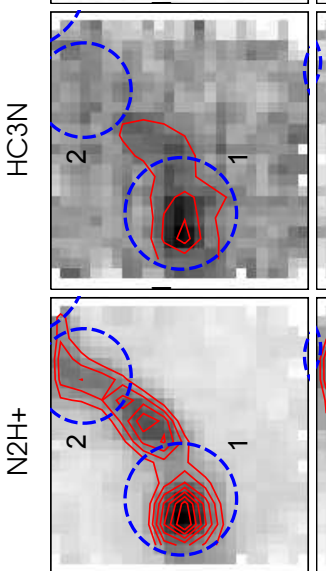

$\infty$

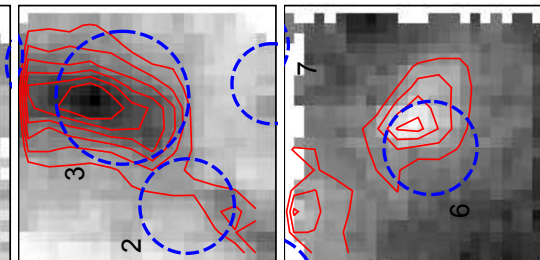

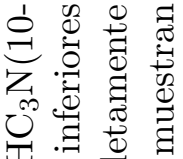

I 0 o

仓ิ

+ 苛

$\begin{array}{cccc}1 & 0 & 0 & 0 \\ & 0 & 0 & 0 \\ 0 & 0 & 0 & 0 \\ 0 & 0 & 0\end{array}$

ช

ปี

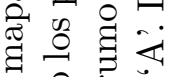

की 융

శี

कृ छ

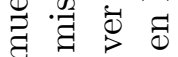

क

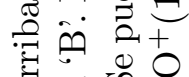

सं थ

ஓं

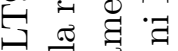

岁包.

- 0 ల

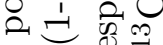

कै + 근

胥

记 $\rightarrow$

艺河

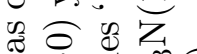

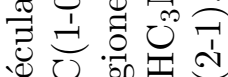

ల.

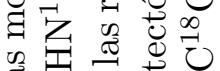

零

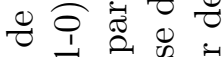

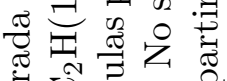

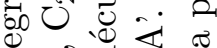

馬

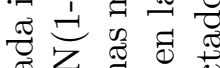

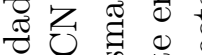

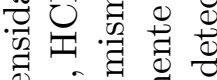

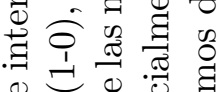

记

กิ $\bigcirc$ के

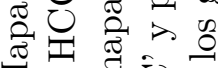

尝当已

$\ddot{0} \hat{0}_{1} 0$

ค.

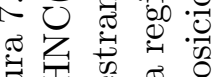

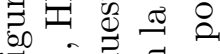

的 

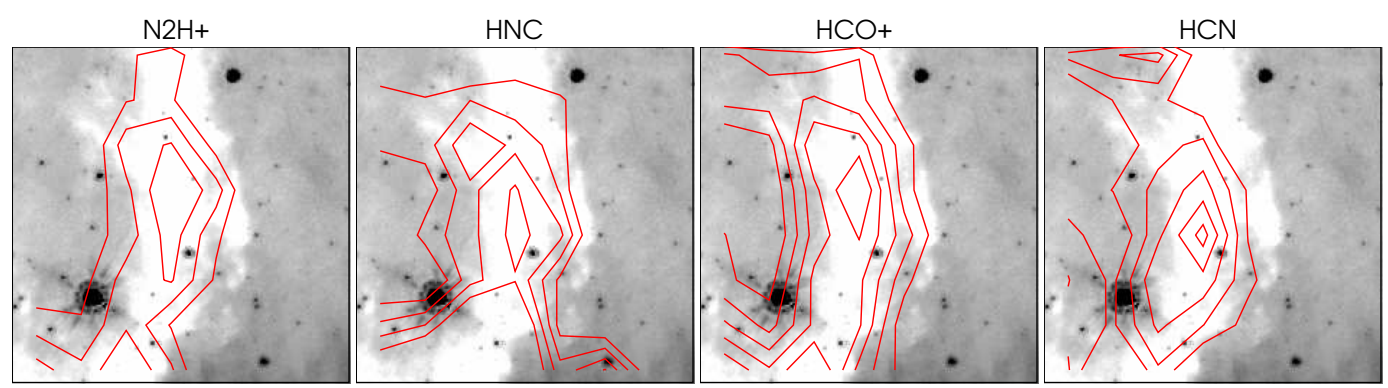

Figura 7.31: Mapas de intensidad integrada de las moléculas detectadas por MALT90 hacia la IRDC.

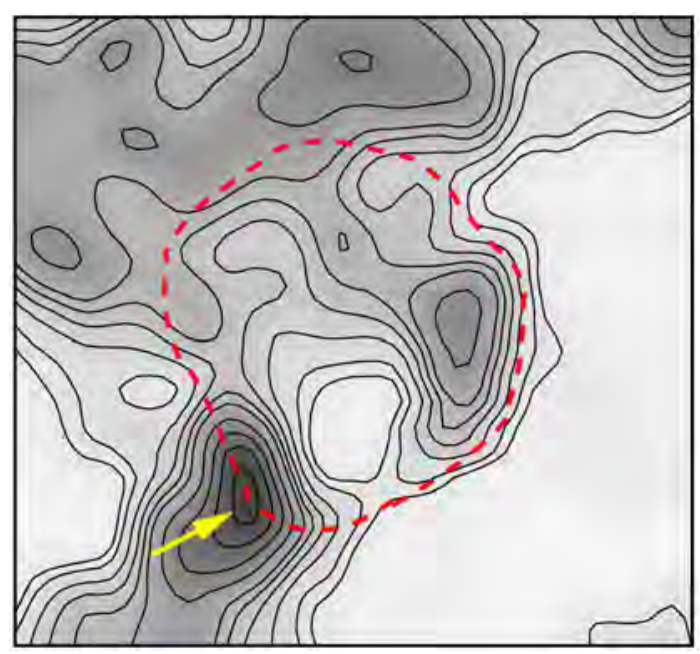

Figura 7.32: Cáscara molecular \#4. Con línea discontinua roja se muestra el borde externo de la cáscara. La flecha amarilla indica el grumo que la cáscara \#4 comparte con otras cáscaras.

En la Figura 7.32 se muestra a modo de ejemplo cómo fue definida el área de la cáscara \#4. El borde exterior de la cáscara (mostrado con línea discontinua roja) fue elegido de modo que los grumos que rodean la cavidad quedaran incluidos dentro de la cáscara, excepto el grumo indicado con la flecha amarilla, del que sólo se tomó parte ya que también se encuentra en la cáscara \#3 (cuya velocidad coincide en parte con la de la cáscara \#4). Así se definieron un radio efectivo interior y uno exterior para cada cáscara. Estos se listan en las columnas 2 y 3 de la Tabla 7.9. En la cuarta columna se listan los radios efectivos medios de las cáscaras.

Dado que, como se mencionó en la Sección 7.4.3, las cáscaras sólo se observan cerradas en ${ }^{12} \mathrm{CO}(2-1)$ (únicamente la cáscara \#1 se detecta cerrada tanto en ${ }^{12} \mathrm{CO}(2-1)$ como en ${ }^{13} \mathrm{CO}(2-1)$ ), se utizó la Ecuación 2.35 para calcular las densidades columnares de $\mathrm{H}_{2}$ y poder determinar las masas. La densidad ambiental fue estimada considerando la masa contenida dentro de un un anillo con radio interior igual al radio efectivo $\mathrm{R}_{\text {eff }}^{\text {int }} \mathrm{y}$ radio exterior igual al radio efectivo $\mathrm{R}_{\text {eff }}^{e x t} \mathrm{y}$ distribuyéndola en un círculo de radio igual a $\mathrm{R}_{\text {eff }}^{\text {med }}$. Las masas moleculares y densidades ambientales de $\mathrm{H}_{2}$ calculadas se muestran en las columnas 5 y 6 de la Tabla 7.9. A los efectos de determinar parámetros se ha considerado una distancia de $2.8 \pm 0.5 \mathrm{kpc}$.

Los valores encontrados para las masas varían entre 800 y $2700 \mathrm{M}_{\odot}$, y las densidades de $\mathrm{H}_{2}$ entre 600 y $10200 \mathrm{~cm}^{-3}$. Los errores en masa y densidad volumétrica corresponden a un $18 \%$ de error en distancia. Estos valores están de acuerdo con los encontrados para otras cáscaras moleculares que se encuentran rodeando burbujas IR (e.g. Yan et al., 2016). 


\begin{tabular}{lccccc}
\hline \hline & $\begin{array}{c}\mathrm{R}_{\text {eff }}^{\text {int }} \\
(\mathrm{pc})\end{array}$ & $\begin{array}{c}\mathrm{R}_{\text {eff }}^{\text {ext }} \\
(\mathrm{pc})\end{array}$ & $\begin{array}{c}\mathrm{R}_{\text {eff }}^{\text {med }} \\
(\mathrm{pc})\end{array}$ & $\begin{array}{c}\text { Masa } \\
\left(\mathrm{M}_{\odot}\right)\end{array}$ & $\begin{array}{c}n_{\mathrm{H}_{2}} \\
\left(\mathrm{~cm}^{-3}\right)\end{array}$ \\
\hline Cáscara 1 & 0.86 & 2.2 & 1.5 & $1700 \pm 600$ & $600 \pm 300$ \\
Cáscara 2 & 1.00 & 2.1 & 1.6 & $2700 \pm 100$ & $1050 \pm 600$ \\
Cáscara 3 & 0.60 & 1.5 & 1.1 & $900 \pm 320$ & $1000 \pm 550$ \\
Cáscara 4 & 0.25 & 1.3 & 0.8 & $800 \pm 280$ & $1200 \pm 700$ \\
Cáscara 5 & 0.50 & 1.1 & 0.8 & $1100 \pm 400$ & $1000 \pm 600$ \\
Cáscara 6 & 0.41 & 1.1 & 0.8 & $900 \pm 320$ & $800 \pm 500$ \\
\hline \hline
\end{tabular}

Tabla 7.9: Parámetros de las cáscaras moleculares definidas en la Sección 7.4.3.

\section{Grumos densos}

A partir de los parámetros observacionales medidos para los grumos definidos en la Sección 7.4.4 se calcularon valores para la profundidad óptica, la temperatura de excitación, la densidad columnar de $\mathrm{H}_{2}$, la masa molecular, el radio y la densidad ambiental de $\mathrm{H}_{2}$ de cada grumo. Estos parámetros, que se estimaron adoptando condiciones de LTE, están listados en la Tabla 7.10.

Dado que los grumos detectados en $\mathrm{C}^{18} \mathrm{O}(2-1)$ se encuentran también en ${ }^{13} \mathrm{CO}(2-1)$ (mientras que en ${ }^{12} \mathrm{CO}(2-1)$ la emisión es extendida), el cálculo de los parámetros se llevó a cabo utilizando los dos primeros. Para determinar las profundidades ópticas $\tau_{13}, \tau_{18}$ y la densidad columnar de ${ }^{13} \mathrm{CO}(2-1)$ se utilizaron las ecuaciones $6.1,6.2,6.3$ y 6.4 y se calculó la temperatura de excitación a partir de la ecuación $2.19 \sin$ hacer la aproximación $\tau \gg 1$. Para ello se consideraron los valores de $\mathrm{T}_{m b}$ y $\Delta \mathrm{v}$ para ${ }^{13} \mathrm{CO}(2-1)$ y $\mathrm{C}^{18} \mathrm{O}(2-1)$ listados en las colunmas 2 , 3,4 y 5 de la Tabla 7.7. Los resultados se muestran en las columnas 2, 3 y 5 de la Tabla 7.10. De los valores de las profundidades ópticas queda claro que el ${ }^{13} \mathrm{CO}$ es ópticamente grueso y el $\mathrm{C}^{18} \mathrm{O}$ es ópticamente delgado.

Una vez obtenida la densidad columnar de ${ }^{13} \mathrm{CO}(2-1)$ se obtiene la del Hidrógeno molecular a partir de la relación de abundancias $\left[\mathrm{H}_{2}\right]\left[{ }^{13} \mathrm{CO}\right]=7.7 \times 10^{5}$ (Wilson \& Rood, 1994). La masa molecular, densidad volumétrica y masa del virial de cada grumo se calcularon usando las ecuaciones $2.38,2.39$ y 2.40 y los resultados se hallan listados en las columnas 7,9 y 10 de la Tabla 7.10. El rango de valores tabulados para las masas del virial depende de cual sea el valor que se tome para la constante $k$ en la ecuación 2.40, que depende del modelo de perfil de densidad asumido.

En la columna 11 de las Tabla 7.10 se muestran los valores de los cocientes $\mathrm{M}_{v i r} / \mathrm{M}_{H_{2}}$. Para los Grumos 1, 3, 5 y 7 este cociente toma valores menores que la unidad para cualquier perfil de densidad que se considere, lo que indica que los grumos podrían estar colapsando, mientras que para el grumo 8 el cociente es menor que la unidad sólo si se considera que la densidad aumenta hacia el centro del grumo. Los valores mayores que la unidad obtenidos para los Grumos 2 y 6 indican que estos no podrían colapsar.

Los valores derivados de masas, radios y densidades son consistentes con los mostrados en la Tabla 1.2 del Capítulo 1, determinados por Bergin \& Tafalla (2007) para otros grumos, y por Yan et al. (2016).

\section{Filamento}

Los parámetros del gas molecular asociado al filamento se estimaron a partir de las observaciones de $\mathrm{C}^{18} \mathrm{O}(3-2)$, asumiendo una temperatura de excitación $\mathrm{T}_{\text {exc }}=17 \mathrm{~K}$ (promedio de las $\mathrm{T}_{\text {exc }}$ calculadas para los grumos que se encuentran sobre el filamento) ${ }^{(4)}$. Se definió

\footnotetext{
${ }^{(4)} \mathrm{Si}$ se asume LTE es posible admitir que la temperatura de excitación es la misma para las transiciones
} 
Tabla 7.10: Parámetros de los grumos listados en la Tabla 7.7 derivados a partir del gas molecular.

\begin{tabular}{|c|c|c|c|c|c|c|c|c|c|c|}
\hline$\#$ & $\tau_{13}$ & $\tau_{18}$ & $\begin{array}{l}\mathrm{T}_{\text {exc }} \\
{[\mathrm{K}]}\end{array}$ & $\begin{array}{c}\mathrm{N}_{13} \mathrm{CO} \\
{\left[10^{16} \mathrm{~cm}^{-2}\right]}\end{array}$ & $\begin{array}{c}\mathrm{N}_{H_{2}} \\
{\left[10^{22} \mathrm{~cm}^{-2}\right]}\end{array}$ & $\begin{array}{l}\mathrm{M}_{H_{2}} \\
\mathrm{M}_{\odot}\end{array}$ & $\begin{array}{c}\text { Radio } \\
{[\mathrm{pc}]}\end{array}$ & $\begin{array}{c}\mathrm{n}_{H_{2}} \\
{\left[10^{4} \mathrm{~cm}^{-3}\right]}\end{array}$ & $\begin{array}{l}\mathrm{M}_{v i r} \\
{\left[\mathrm{M}_{\odot}\right]}\end{array}$ & $\mathrm{M}_{\text {vir }} / \mathrm{M}_{H_{2}}$ \\
\hline 1 & 2.4 & 0.4 & 18 & 7.8 & 4.4 & $1900 \pm 680$ & 0.85 & $1.1 \pm 0.6$ & $700-1180$ & $0.37-0.62$ \\
\hline 2 & 1.5 & 0.2 & 17 & 4.8 & 2.8 & $840 \pm 300$ & 0.72 & $0.8 \pm 0.4$ & $960-1600$ & $1.14-1.90$ \\
\hline 3 & 2.5 & 0.4 & 19 & 6.9 & 3.5 & $2400 \pm 860$ & 1.00 & $0.8 \pm 0.4$ & $1080-1700$ & $0.45-0.70$ \\
\hline 4 & 2.4 & 0.5 & 14 & 5.4 & 2.5 & $700 \pm 250$ & 0.62 & $1.0 \pm 0.5$ & $230-390$ & $0.32-0.56$ \\
\hline 5 & 2.9 & 0.6 & 14 & 6.9 & 2.9 & $890 \pm 320$ & 0.61 & $1.3 \pm 0.7$ & $500-840$ & $0.56-0.94$ \\
\hline 6 & 2.0 & 0.5 & 15 & 5.3 & 1.8 & $930 \pm 330$ & 0.72 & $0.9 \pm 0.5$ & $1530-2500$ & $1.65-2.90$ \\
\hline 7 & 2.5 & 0.6 & 8 & 5.1 & 1.8 & $630 \pm 220$ & 0.60 & $0.9 \pm 0.5$ & $190-320$ & $0.30-0.51$ \\
\hline 8 & 2.0 & 0.4 & 13 & 4.5 & 2.3 & $830 \pm 300$ & 0.73 & $0.7 \pm 0.4$ & $560-940$ & $0.67-1.13$ \\
\hline
\end{tabular}

el tamaño del filamento a partir de $\mathrm{C}^{18} \mathrm{O}(3-2)$ que es la molécula que mejor lo traza. Para esto se hizo un promedio de la emisión de $\mathrm{C}^{18} \mathrm{O}(3-2)$ en el rango de velocidades desde -42.6 hasta $-35.2 \mathrm{~km} \mathrm{~s}^{-1}$, y se consideró parte del filamento a aquella emisión que se encuentra por encima de $1 \mathrm{~K}$. La profundidad óptica y densidad columnar de $\mathrm{C}^{18} \mathrm{O}(2-1)$, la masa de $\mathrm{H}_{2}$ y la densidad ambiental fueron calculados a partir de las ecuaciones 2.22, 2.33, 2.37 y 2.39 utilizando los parámetros observacionales medidos del espectro promedio de $\mathrm{C}^{18} \mathrm{O}(2-1)$ en la región.

Así se estimó un valor de $\tau_{18}=0.1$, una densidad colunmar de $\mathrm{H}_{2}$ de $\mathrm{N}_{H_{2}}=2.2 \times 10^{22} \mathrm{~cm}^{-2}$ (considerando una abundancia $\left[\mathrm{H}_{2} / \mathrm{C}^{18} \mathrm{O}\right]=5.8 \times 10^{6}$, Sanhueza et al., 2010), una masa de $7100 \pm 2600 \mathrm{M}_{\odot}$ y una densidad ambiental de $(1.7 \pm 0.9) \times 10^{3} \mathrm{~cm}^{-3}$.

\section{IRDC}

Los parámetros del gas asociado a la IRDC se calcularon a partir de las observaciones de ${ }^{13} \mathrm{CO}(2-1)$ y $\mathrm{C}^{18} \mathrm{O}(2-1)$, considerando la primera ópticamente gruesa y la segunda ópticamente delgada. A diferencia de como se hizo para el filamento fue necesario utilizar dos isótopos ya que no se cuenta con un valor para la temperatura de excitación. Se definió el tamaño de la IRDC a partir de una imagen promedio de la emisión de $\mathrm{C}^{18} \mathrm{O}(2-1)$ en el rango de velocidades desde -40.8 hasta $-38.0 \mathrm{~km} \mathrm{~s}^{-1}$, y se consideró parte de la IRDC a aquella emisión que se encuentra por encima de $0.5 \mathrm{~K}$. Así mismo se definió la IRDC en ${ }^{13} \mathrm{CO}(2-1)$ en un rango de velocidades desde -41.5 hasta $-37.1 \mathrm{~km} \mathrm{~s}^{-1}$ (un poco más extendido que en $\mathrm{C}^{18} \mathrm{O}(2-1)$ ).

Se utilizaron las ecuaciones 6.1, 6.2, 6.3, 6.4 y 2.19 para calcular las profundidades ópticas $\tau_{13}, \tau_{18}$, la densidad columnar de ${ }^{13} \mathrm{CO}(2-1)$ y la temperatura de excitación.

Así se obtuvieron profundidades ópticas $\tau_{13}=2.5$ y $\tau_{18}=0.8$ (corroborando que ${ }^{13} \mathrm{CO}(2-1)$ es óticamente gruesa), una temperatura de excitación de $12 \mathrm{~K}$ y una masa de $\mathrm{H}_{2}$ de $5400 \pm 2000$ $\mathrm{M}_{\odot}$, lo que corresponde a una densidad ambiental de $(3.4 \pm 1.8) \times 10^{4} \mathrm{~cm}^{-3}$.

\section{Región completa}

Para estimar los parámetros de la región completa se hicieron espectros promedio de toda la emisión de ${ }^{12} \mathrm{CO}(2-1)$ y de ${ }^{13} \mathrm{CO}(2-1)$ en la región de las burbujas por un lado y en la región de la IRDC por el otro. A partir de estos espectros se obtuvo que la masa total de la región, sumando ambos campos observados, es de $23300 \pm 8400 \mathrm{M}_{\odot}$. Estos valores coinciden, dentro de los errores, con los calculados a partir de datos de NANTEN.

$(2-1)$ y $(3-2)$. 
Tabla 7.11: Parámetros de los ajustes gaussianos a las componentes de la emisión de $\mathrm{N}_{2} \mathrm{H}^{+}(1-0)$ para los Grumos 1 y 2.

\begin{tabular}{lccccc}
\hline \hline Grumo & & $\begin{array}{c}\text { Area } \\
{\left[\mathrm{K} \mathrm{km} \mathrm{s}^{-1}\right]}\end{array}$ & $\begin{array}{c}\text { Velocidad } \\
{\left[\mathrm{km} \mathrm{s}^{-1}\right]}\end{array}$ & $\begin{array}{c}\Delta \mathrm{v} \\
{\left[\mathrm{km} \mathrm{s}^{-1}\right]}\end{array}$ & $\begin{array}{c}\mathrm{T}_{\max } \\
{[\mathrm{K}]}\end{array}$ \\
\hline 1 & Grupo 1 & 1.05 & -46.43 & 3.48 & 0.28 \\
& Grupo 2 & 4.18 & -38.28 & 3.90 & 1.01 \\
& Grupo 3 & 2.72 & -32.53 & 4.54 & 0.56 \\
\hline 3 & Grupo 1 & 1.68 & -47.76 & 3.14 & 0.51 \\
& Grupo 2 & 6.08 & -39.43 & 3.52 & 1.62 \\
& Grupo 3 & 3.58 & -33.74 & 3.29 & 1.02 \\
\hline \hline
\end{tabular}

\subsubsection{Calculados a partir de trazadores de alta densidad}

Con el objetivo de conocer los propiedades del gas molecular más denso se estimaron sus parámetros a partir de la emisión de $\mathrm{N}_{2} \mathrm{H}^{+}(1-0)$. Como ya se describió en la Sección 2.2.4 $\mathrm{N}_{2} \mathrm{H}^{+}(1-0)$ posee siete componentes hiperfinas, seis de ellas agrupadas en dos grupos de tres líneas cada uno, más una componente aislada. Tal como se hizo en la Capítulo 6 es posible estimar los parámetros del gas denso a partir de los ajustes de gaussianas a estos grupos de componentes hiperfinas y aplicando las ecuaciones 2.41, 2.42 y 2.43.

Sólo ha sido posible calcular los parámetros para los Grumos 1 y 3 . Esto se debe a que el Grumo 2 no se detecta como tal en $\mathrm{N}_{2} \mathrm{H}^{+}(1-0)$, y no es posible hacer un ajuste de componentes gaussianas al espectro del Grumo6, porque a pesar de que en la imagen promedio mostrada en la Figura 7.30 se detecta emisión, ésta es demasiado débil como para lograr un buen ajuste. Los parámetros de los ajustes gaussianos de las componentes de la emisión de $\mathrm{N}_{2} \mathrm{H}^{+}(1-0)$ se muestran en la Tabla 7.11.

Para los Grumos 1 y 3 se obtuvieron temperaturas de excitación de 5.5 y $6.2 \mathrm{~K}$ y densidades columnares de $\mathrm{N}_{2} \mathrm{H}^{+}$de $1.97 \times 10^{14}$ y $2.51 \times 10^{14} \mathrm{~cm}^{-2}$, respectivamente. Considerando que la abundancia en nubes moleculares oscuras es $\left[\mathrm{N}_{2} \mathrm{H}^{+}\right] /\left[\mathrm{H}_{2}\right]=5 \times 10^{-10}$ (Ohishi et al., 1992), resultaron densidades columnares de $\mathrm{H}_{2}$ de $3.9 \times 10^{23}$ y $5 \times 10^{23} \mathrm{~cm}^{-2}$ y densidades ambientales de $1.4 \times 10^{5}$ y $1.6 \times 10^{5} \mathrm{~cm}^{-3}$ para los Grumos 1 y 3 . Estas densidades ambientales son consistentes con la densidad crítica de $\mathrm{N}_{2} \mathrm{H}^{+}(1-0)$ listada en la Tabla 2.2.

\subsection{Parámetros calculados a partir de la emisión en continuo de radio y del polvo}

Como ya se mencionó, la emisión extendida en el continuo de radio y en $24 \mu \mathrm{m}$ indica la presencia de fuentes excitatrices asociadas a las burbujas IR, por lo que estas serían regiones HII.

Como se describió en la Sección 2.4 del Capítulo 2 es posible obtener un valor para la densidad electrónica y la masa de gas ionizado de una región Hi a partir de la emisión en el continuo de radio. En la Sección 7.3 se analizó la emisión a $843 \mathrm{MHz}$ y se corroboró su presencia en el interior de las burbujas. Sin embargo esta emisión no es apropiada para determinar los parámetros de la región, ya que a bajas frecuencias $(\nu<1 \mathrm{GHz})$ la emisión en continuo de radio de regiones His puede ser ópticamente gruesa.

En cambio, a fin de estimar un valor para la densidad electrónica y la masa del gas ionizado en el interior de las burbujas se utilizaron datos del relevamiento GB6/PMN (Green Bank 6-cm Radio Source Catalog, Gregory et al. 1996; Parkes-MIT-NRAO, Wright et al. 1994) a $4850 \mathrm{MHz}$, con una resolución angular de 3!5. La distribución de esta emisión en la 


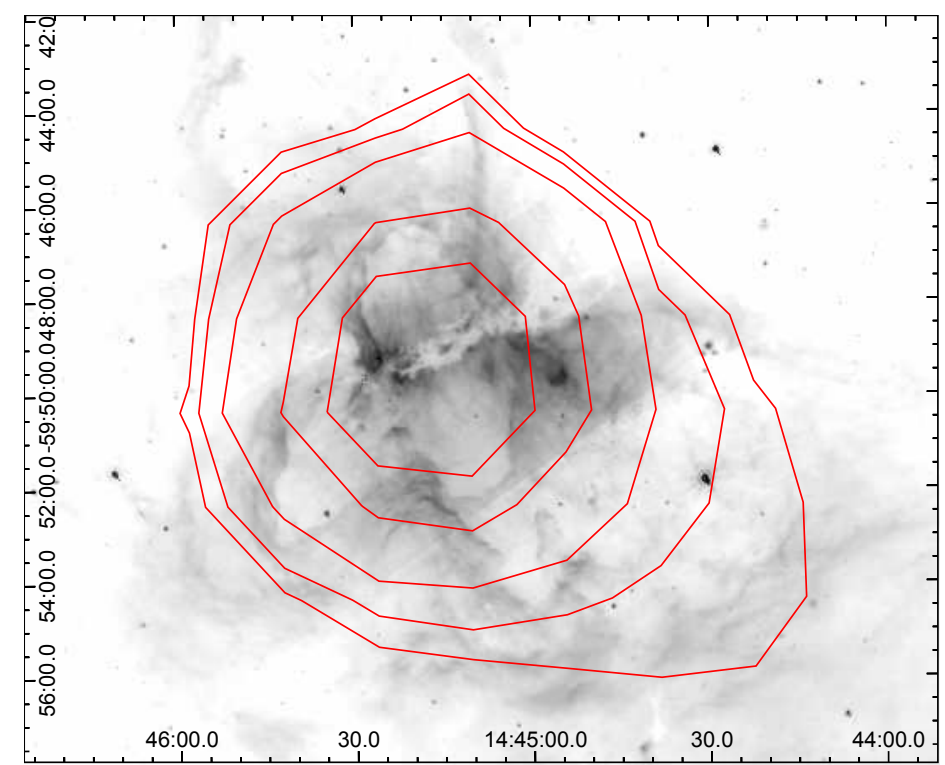

Figura 7.33: Emisión en continuo de radio a $4.86 \mathrm{GHz}$ superpuesta a la imagen de $8 \mu \mathrm{m}$. Datos de 1 relevamiento GB6/PMN.

región se muestra en la Figura 7.33. A pesar de que la resolución angular es peor que la de los datos de SUMSS la frecuencia es apropiada para realizar los cálculos.

Considerando un modelo de región Hit con perfil gaussiano y aplicando las ecuaciones 2.50 y 2.51 se obtuvo una densidad electrónica de $250 \mathrm{~cm}^{-3}$ y una masa de gas ionizado de $1720 \mathrm{M}_{\odot}$. Estos mismos cálculos utilizando los datos de SUMSS arrojan valores de $95 \mathrm{~cm}^{-3}$ y $660 \mathrm{M}_{\odot}$. Estos valores resultan muy pequeños dado que a una frecuencia de $843 \mathrm{MHz}$ la región es ópticamente gruesa y no se detecta toda la emisión originada en ella, sino sólo las zonas más externas.

También es posible estimar un valor para la masa del polvo a partir de la Ecuación 2.48. Para esto es necesario conocer la temperatura del polvo $\mathrm{T}_{d}$. En panel inferior izquierdo de la Figura 7.3 se muestra un mapa de temperatura de polvo calculado a partir del cociente de los flujos en 150 y $210 \mu$ m extraído del trabajo de Vig et al. (2007). Este mapa muestra que las temperaturas de polvo de la región asociada a la fuente IRAS 14416-5937, que corresponden al filamento, toman valores de entre 20 y $40 \mathrm{~K}$.

Asumiendo una temperatura de polvo media de $25 \mathrm{~K}$ para el filamento se estimó a partir de la Ecuación 2.48 una masa de polvo $\mathrm{M}_{d}=50 \mathrm{M}_{\odot}$. Aceptando que la relación gas/polvo es $\mathrm{R}_{d}=137$ (Draine et al., 2007), se obtiene una masa $\mathrm{M}_{\text {gas }}=6850 \mathrm{M}_{\odot}$ para el filamento. Este valor es un poco menor que el calculado a partir de $\mathrm{CO}$, pero está dentro de los errores.

Dado que sólo se conoce la temperatura de polvo para el filamento, ésta masa es la única obtenida por este método.

\subsection{Cúmulos y formación estelar en el complejo}

La existencia de formación estelar activa en la región se analizó a partir de la búsqueda de fuentes candidatas a objetos estelares jóvenes entre las fuentes infrarrojas de los catálogos Spitzer-IRAC, WISE, 2MASS y MSX. Además se llevó a cabo un búsqueda de cúmulos infrarrojos que pudieran estar excitando la región. 


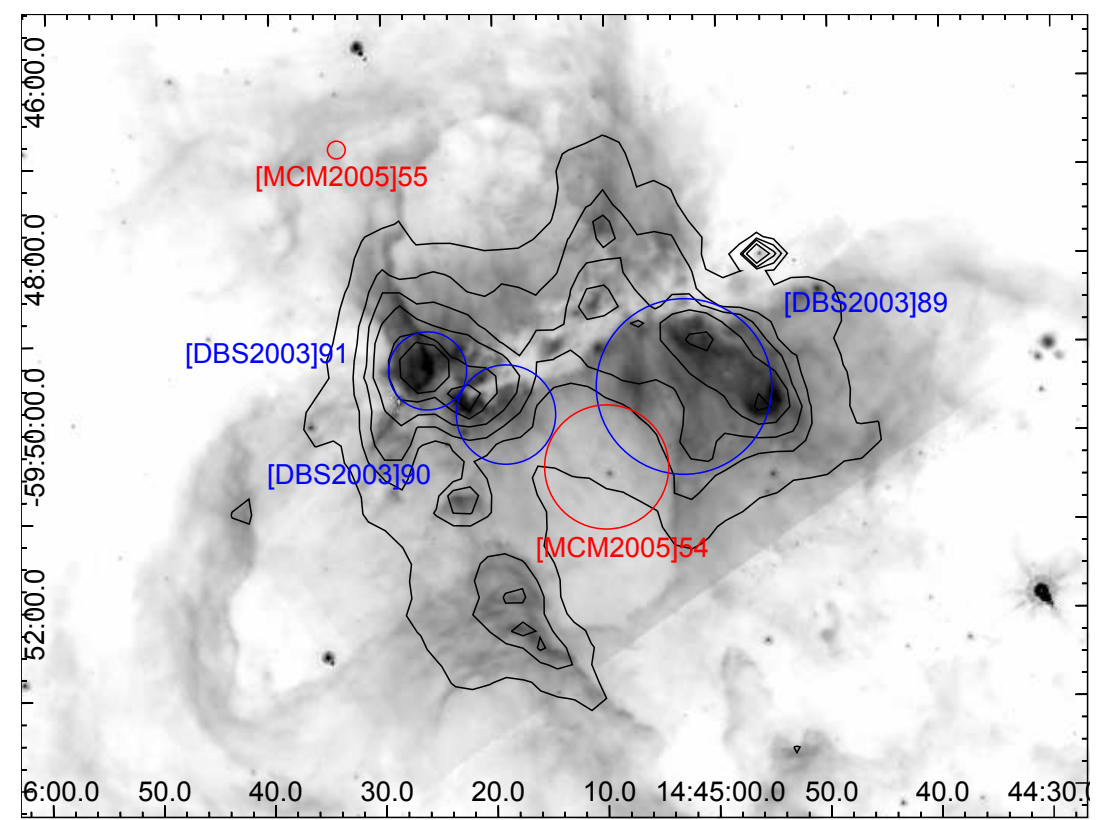

Figura 7.34: Cúmulos identificados en la región de las burbujas. Se muestran la posicion y diámetro de los cúmulos identificados por Mercer et al. (2005) (rojo) y por Dutra et al. (2003) (azul) sobre la imagen de $8 \mu \mathrm{m}$. Los contornos corresponden a la emisión en $70 \mu \mathrm{m}$ de Herschel $(1,2,3,5,10$, y $15 \mathrm{Jy} /$ pixel).

\subsubsection{Cúmulos infrarrojos}

Mercer et al. (2005) identificaron cúmulos infrarrojos realizando una búsqueda sistemática en la región interior de la Galaxia a partir de datos de Spitzer-GLIMPSE, mientras que Dutra et al. (2003) llevaron a cabo su búsqueda a partir de datos de 2MASS en el sector $230^{\circ}<\mathrm{l}$ $<350^{\circ}$. En la Figura 7.34 se muestran las posiciones de cinco grupos de estrellas identificados en la región de las burbujas.

Uno de ellos, [MCM2005b] 54, ya fue mencionado en la Sección 7.1 como posible fuente excitatriz de la burbuja S111. Este, clasificado por Mercer et al. (2005) como asociación estelar, está vinculado a emisión extendida en el infrarrojo medio y se observa en las imágenes de 2MASS. Existe además otra asociación estelar identificada por estos autores, [MCM2005b] 55, que tiene un radio de 0 ' 1 , posee 11 estrellas y también está asociado a emisión extendida en en MIR. Este se encuentra al noreste de la burbuja S109.

Como se muestra en la Figura 7.34 los tres cúmulos identificados por Dutra et al. (2003) se encuentran ubicados sobre máximos de emisión a $70 \mu \mathrm{m}$, que indica polvo tibio. Estos cúmulos IR embebidos podrían ser los responsables de excitar y calentar la región. [DBS2003]91 y [DBS2003]89, clasificados por Dutra et al. (2003) como cúmulos infrarrojos, coinciden con las fuentes IRAS 14416-5937-A y IRAS 14416-5937-B, respectivamente, mientras que [DBS2003] 90 es clasificado como grupo estelar. Estos últimos son sistemas menos densos que los cúmulos pero su relación con la nube sugiere que son sistemas físicamente ligados.

\subsubsection{Búsqueda de candidatos a YSO}

En lo que refiere a las fuentes IR, Vig et al. (2007) analizaron previamente la presencia de las mismas en la región asociada a la fuente IRAS 14416-5937 a partir de los catálogos 2MASS y Spitzer-GLIMPSE identificando candidatos a YSOs. En la Figura 7.35 se muestra la 


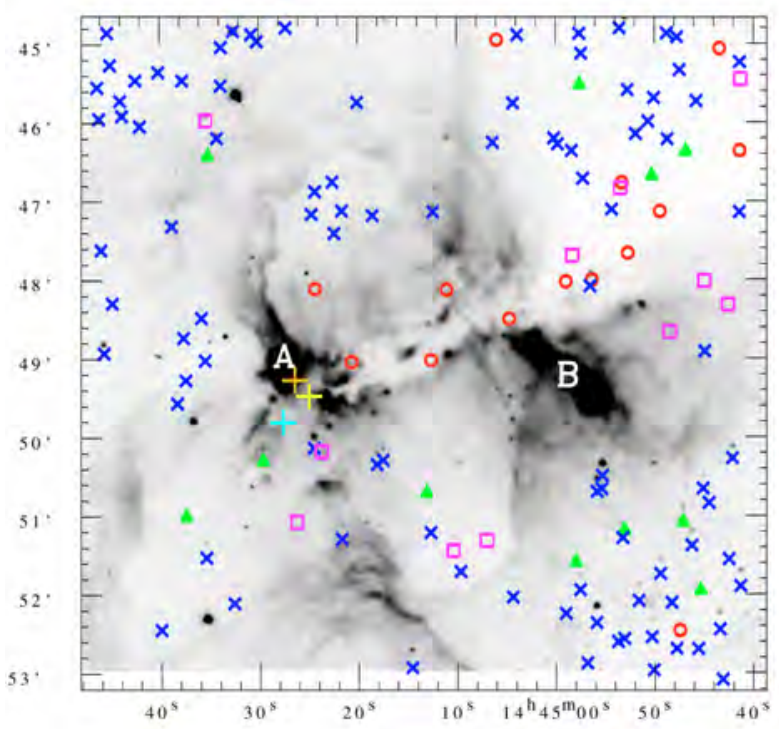

Figura 7.35: Imagen tomada de Vig et al. (2007). Fuentes Spitzer-GLIMPSE superpuestas a la imagen de $5.8 \mu \mathrm{m}$ de la región asociada a IRAS 14416-5937. Los círculos, cuadrados, triángulos y cruces corresponden a fuentes IR clasificadas como candidatos a Clase I, Clase I/II, Clase II y otras fuentes, respectivamente. Máseres de $\mathrm{OH}, \mathrm{H}_{2} \mathrm{O}$ y $\mathrm{CH}_{3} \mathrm{OH}$ se indican con signos + celeste, amarillo y naranja, respectivamente.

distribución espacial de las fuentes Spitzer-GLIMPSE identificadas por dichos autores, junto con las posiciones de los tres máseres de $\mathrm{OH}, \mathrm{H}_{2} \mathrm{O}$ y $\mathrm{CH}_{3} \mathrm{OH}$ encontrados en la región (ver Sección 7.1).

Para completar el análisis realizado por ellos se clasificaron en esta tesis las fuentes WISE y MSX y se repitió la búsqueda de fuentes Spitzer-GLIMPSE y 2MASS en la región completa, incluyendo los bordes de las burbujas y la zona de la IRDC.

A continuación se analizará la distribución de las fuentes IR identificadas a partir de cada uno de los catálogos, tanto sobre la región de las burbujas (que incluye al filamento) como sobre la IRDC.

Las posiciones y flujos de las fuentes IR detectadas en la región se listan en la Tablas 7.14, 7.15, 7.16, 7.17, 7.18, 7.19 y 7.20 en el Anexo de este capítulo.

\subsubsection{Clasificación de fuentes Spitzer}

La Figura 7.36 muestra la ubicación de las fuentes Spitzer catalogadas como candidatos a YSOs en el diagrama color-color separando IRDCs y burbujas. Usando el criterio de Whitney et al. (2004) se identificaron las fuentes candidatos a YSOs, que son las que cumplen [3.6]$[4.5]>0.2,[5.8]-[8]>0.2$ y [4.5]-[5.8] $>0.35$. Una vez identificadas, y siguiendo el criterio de Allen et al. (2004), el estado evolutivo de las mismas se estableció de acuerdo a su posición en los diagramas color-color: las que se encuentran dentro del rectángulo rojo (cruces celestes) son clasificados como clase II y las que quedan por fuera son clasificadas como clase I (cruces violetas). Las fuentes que no cumplen con el criterio de Whitney et al. (2004) se clasifican como clase III (cuadrados amarillos).

\section{Región de las burbujas}

En el panel superior izquierdo de la Figura 7.37 se puede ver que hay un gran número de fuentes Spitzer sobre el filamento, en su mayoría clase I, que son las más jóvenes. Se 

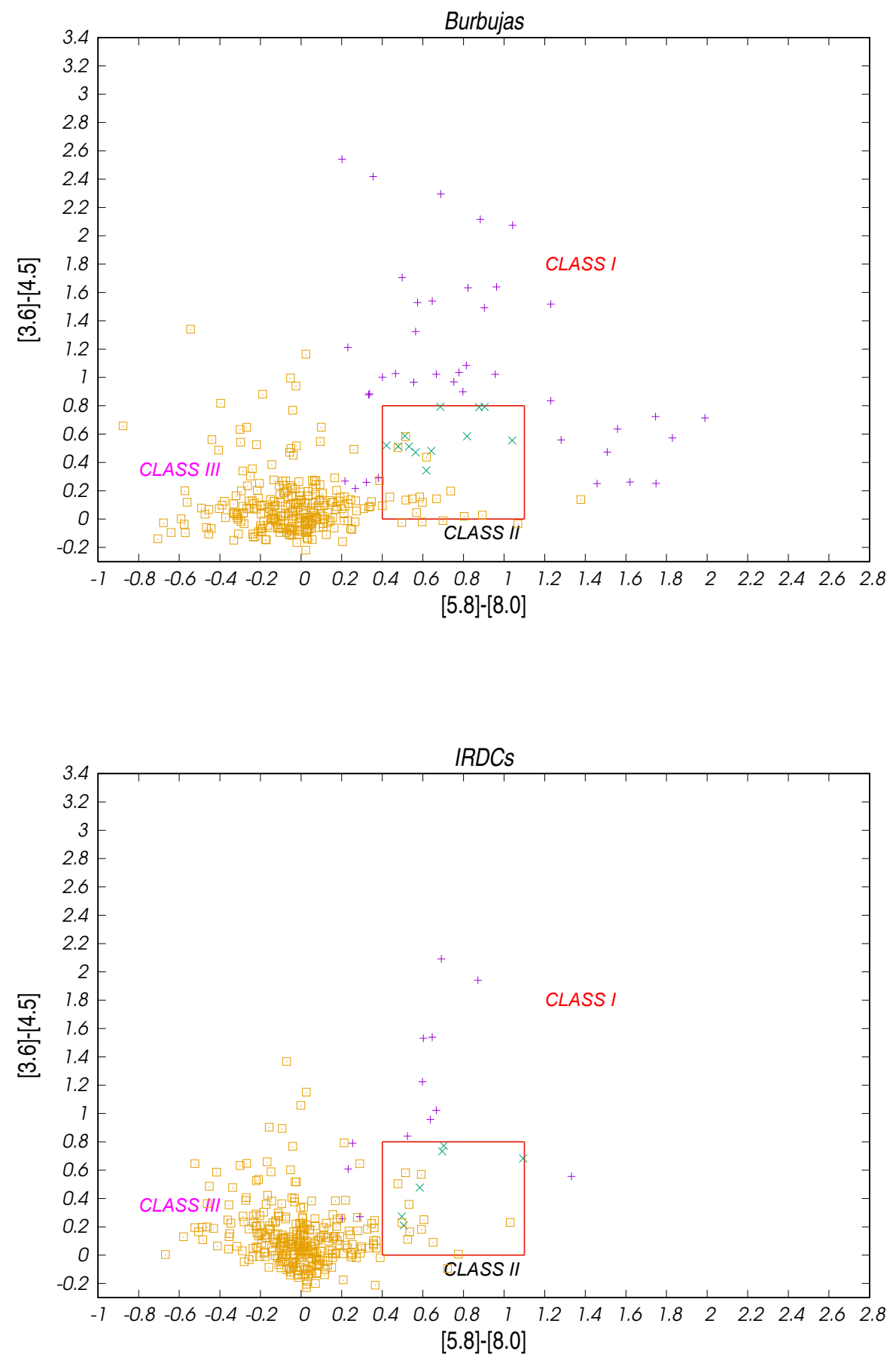

Figura 7.36: Diagrama color-color para las fuentes Spitzer identificadas como candidatos a YSOs sobre la región de las burbujas (arriba) y sobre la IRDC (abajo). 

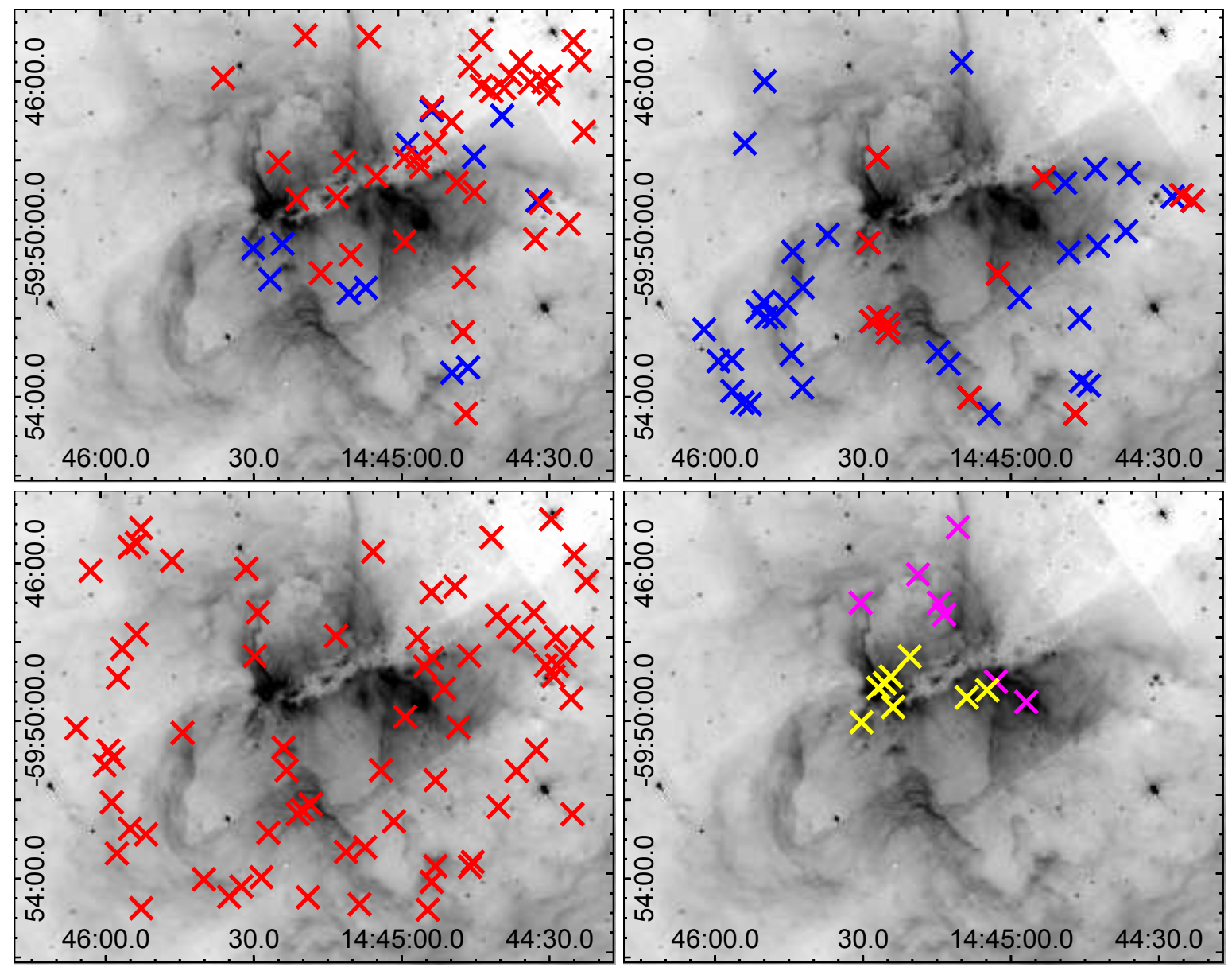

Figura 7.37: Candidatos a YSO identificados en la región de las burbujas. En los paneles superiores se muestran a la izquierda las fuentes del catálogo Spitzer (clase I:rojo, clase II: azul), y a la derecha las fuentes WISE (clase I:rojo, clase II: azul). En los paneles inferiores se muestran a la izquierda las fuentes 2MASS (fuentes con exceso IR) y a la derecha las fuentes MSX identificadas como MYSOs (amarillo) y regiones HII compactas (magenta). 

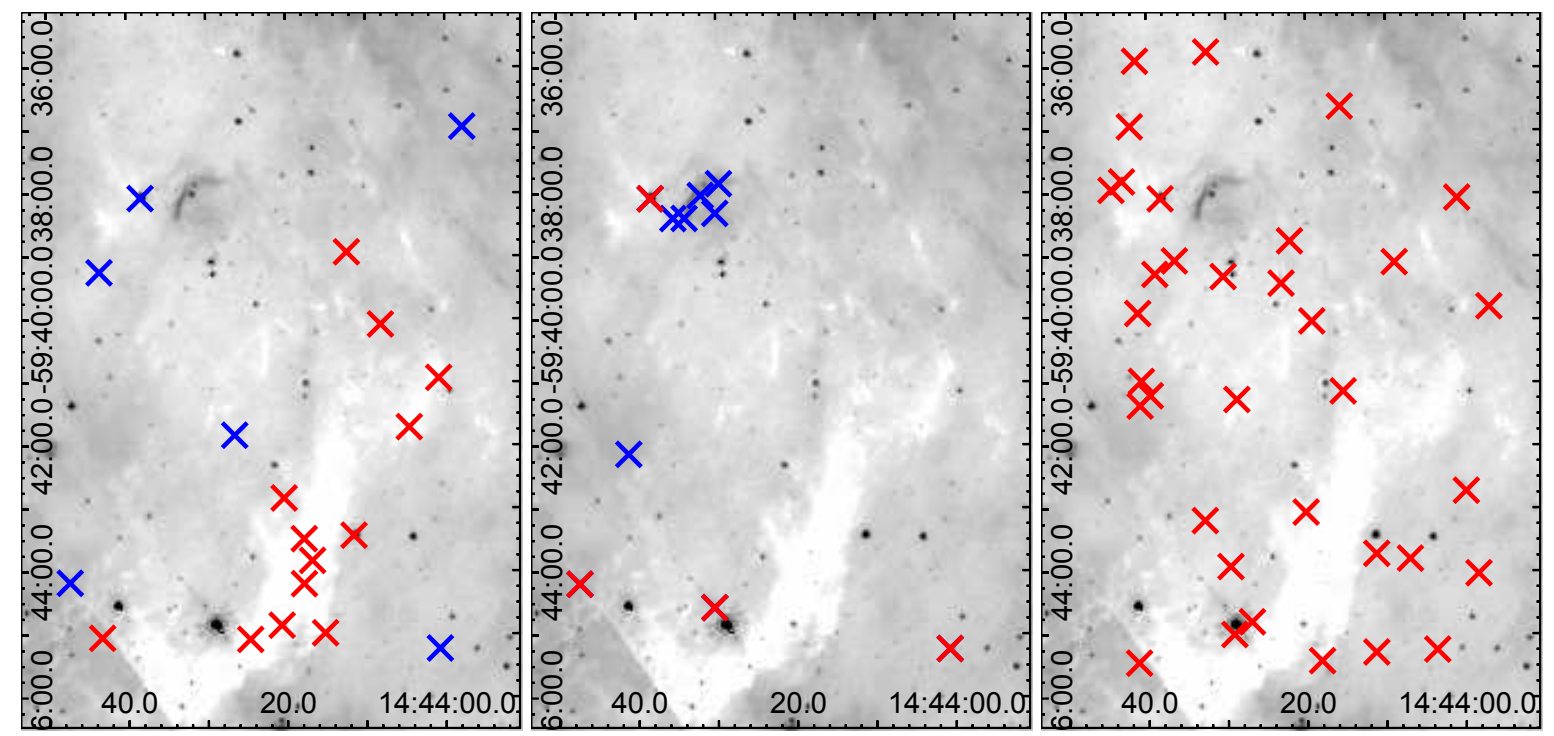

Figura 7.38: Candidatos a YSO identificados en la región de la IRDC. Se muestran a la izquierda las fuentes del catálogo Spitzer (clase I:rojo, clase II: azul), en el centro las fuentes WISE (clase I:rojo, clase II: azul) y a la derecha las fuentes con exceso IR de 2MASS.

ven dos fuentes clasificadas como clase II sobre la fuente IRAS 14416-5937-A de Vig et al. (2007). No se observan fuentes sobre los bordes de las burbujas, excepto al oeste de S111. No se encontraron fuentes IR en el interior de S109 y S110, pero se identificaron 4 YSOS proyectados en el interior de la burbuja S111, posiblemente asociados al cúmulo IR [MCM2005b]54.

\section{Región de la IRDC}

En el panel izquierdo de la Figura 7.38 se ve que las fuentes Spitzer clasificadas como clase I se localizan sobre el arco oscuro en $8 \mu \mathrm{m}$, delineando la IRDC. Las fuentes clase II no parecen estar asociadas a ninguna estuctura que se observe en $8 \mu \mathrm{m}$.

\subsubsection{Clasificación de fuentes WISE}

En cuanto a las fuentes pertenecientes al relevamiento Wide-field Infrared Survey Explorer (WISE), cuyas bandas están centradas en 3.4, 4.6, 12, y $22 \mu \mathrm{m}$ (Wright et al., 2010), se utilizó para clasificarlas el criterio de Koenig et al. (2012) descripto en la Seccion 1.4.5.

\section{Región de las burbujas}

El panel superior derecho de la Figura 7.37 muestra que las fuentes WISE, en su mayoría clasificadas como clase II, están proyectadas sobre los bordes de las burbujas, e incluso sobre los arcos externos detectados en $8 \mu \mathrm{m}$ (ver Figura 7.2). Un grupo de fuentes clase I se detecta sobre la separación de las burbujas S109 y S111. No se detectaron fuentes WISE candidatos a YSO sobre el filamento.

\section{Región de la IRDC}

El panel central de la Figura 7.38 muestra que no se detectan fuentes WISE sobre la IRDC. Sin embargo, se pueden observar 5 fuentes clase II asociadas a una estructura en forma de bowshock detectada en $8 \mu \mathrm{m}$. 


\subsubsection{Clasificación de fuentes 2MASS}

Para la identificación de fuentes 2MASS se consideraron sólo aquellas que poseen excelente calidad fotométrica en las tres bandas (AAA), y fueron clasificadas según el criterio descripto en la sección 1.4.5 considerando para cada fuente los valores del parámetro $q=(\mathrm{J}-\mathrm{H})-$ $1.83 \times(\mathrm{H}-\mathrm{Ks})$. En la Figura 7.39 se muestran los diagramas CC para las fuentes 2MASS detectadas en las regiones de las burbujas y de la IRDC. Los asteriscos color violeta muestran la posición en el diagrama de las estrellas de secuencia principal sin enrojecimiento. Las líneas azul y verde muestran el enrojecimiento de estrellas de tipo espectral O6V y M0III, respectivamente. Los símbolos + indican estrellas de secuencia enrojecidas y las cruces naranja fuentes con exceso IR.

\section{Región de las burbujas}

Las fuentes 2MASS identificadas en la región de las burbujas se muestran en el panel inferior izquierdo de la Figura 7.37. Las fuentes se encuentran distribuidas en toda la región y muchas de ellas aparecen coincidentes con la emisión a $8 \mu \mathrm{m}$. Sin ambargo, también se detectan fuentes que no parecen estar asociadas al complejo. No se encuentran fuentes en el interior de las burbujas ni sobre el filamento.

\section{Región de la IRDC}

En el panel derecho de la Figura 7.38 se muestran las fuentes 2MASS en la región de la IRDC. Estas se encuentran esparcidas por toda la región y no se detecta correlación entre las fuentes 2MASS y la IRDC.

\subsubsection{Clasificación de fuentes MSX}

Finalmente, las fuentes MSX presentes en la región fueron clasificadas según el criterio de Lumsden et al. (2002) descripto en la Sección 1.4.5. Se encontraron fuentes clasificadas como candidatos a MYSOs (con cocientes de flujos F21/F8>2 y F14/F12>1) y como regiones HiI compactas (con F21/F8>2 y F14/F12<1). Como se muestra en el panel inferior derecho de la Figura 7.37, todos los MYSOs están ubicadas sobre el filamento excepto uno que se detecta en el interior de la burbujas S110, mientras que las regiones HiI compactas se localizan o bien en el borde de S110, o bien sobre la fuente IRAS 14416-5937-B de Vig et al. (2007), indicando formación estelar reciente.

\subsubsection{Correlación de fuentes IR y gas molecular}

Lo descripto en las secciones anteriores revela que los objetos clase I, que son los más jóvenes, están proyectados mayormente sobre el filamento y la IRDC. La posición de las fuentes coincide con la emisión en el infrarrojo lejano, y por lo tanto con las zonas más frías de la región. Se observa la presencia de objetos más evolucionados sobre las cáscaras. Además se encuentran objetos estelares jóvenes masivos (MYSOs) sobre el filamento. Esto podría sugerir que la formación de las cáscaras fue anterior a la formación del filamento.

Con el objetivo de estudiar el ambiente en el que las fuentes IR se encuentran inmersas y su interacción con el mismo, se analizó la correlación de los grumos de gas molecular con la posición de las fuentes. En la Figura 7.40 se muestran los contornos de la emisión promedio de $\mathrm{C}^{18} \mathrm{O}(2-1)$ (que se utilizó para definir los grumos) superpuesta sobre la imagen de $8 \mu \mathrm{m}$. Se muestran las posiciones y tamaños de los grumos definidos en la Sección 7.4.4 junto con las fuentes Spitzer, WISE y MSX detectadas.

El Grumo 1, uno de los más masivos, tiene asociados 4 MYSOs. Los valores calculados de las masas de virial para este grumo indican que estaría contrayéndose, por lo que es posible que las fuentes IR asociadas sean objetos que se están formando en el grumo. Este 

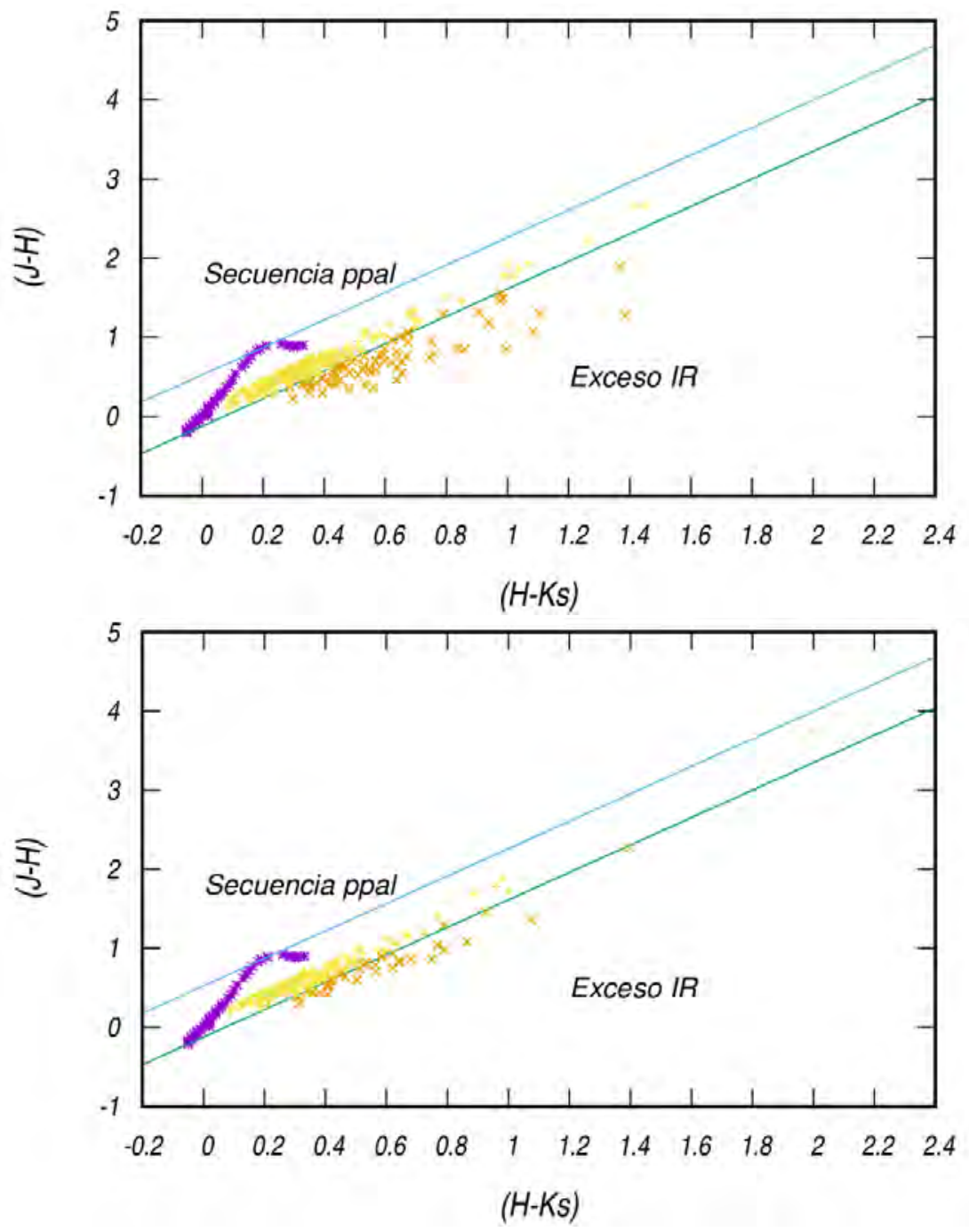

Figura 7.39: Diagramas color-color de las fuentes 2MASS detectadas en la región de las burbujas (arriba) y de la IRDC (abajo). 
grumo coincide con las fuente IRAS 14416-5937-A de Vig et al. (2007) y con el cúmulo IR [DBS2003]91 identificado por Dutra et al. (2003) (ver Figura 7.41).

El Grumo 2 contiene tres fuentes WISE clase I, pero que no se encuentran sobre el máximo de la emisión promedio del grumo. Los valores de masa de virial calculados para este grumo son mayores que los de masa en LTE, por lo que no estaría colapsando. Así, los candidatos a YSO podrían no estar asociados al grumo. Del mismo modo, los YSOs proyectados en los bordes del Grumo 6 son jóvenes, pero sus valores de masa de virial indicarían que no está colapsando.

Coincidentes con los Grumos 3 (que es el de mayor masa), 5 y 7 se detectaron varios candidatos a YSO clase I. Los valores del cociente $M_{V I R} / M_{L T E}<1$ sugieren que estos están colapsando. El Grumo 4 en cambio, a pesar de poseer valores de $M_{V I R} / M_{L T E}<1$ no tiene candidatos a YSO proyectados.

Finalmente, el Grumo 8 contiene YSOs clase I y clase II, aunque sus valores de $M_{V I R} / M_{L T E}$ toman valores mayores o menores que la unidad según el modelo de perfil de densidad del grumo que se considere. Si, como ya se hizo en capítulos anteriores, se supone una estructura jerárquica de nubes moleculares y se supone que el grumo aumenta su densidad hacia el interior, resulta $M_{V I R} / M_{L T E}<1$ y el grumo se estaría contrayendo.

A fin de analizar la presencia de YSOs sobre las cáscaras moleculares, en la Figura 7.42 se muestran los contornos de la emisión promedio de ${ }^{12} \mathrm{CO}(2-1)$ en el rango de velocidad de cada cáscara, junto con las fuentes IR detectadas. En el panel superior izquierdo se puede ver que hay fuentes WISE clasificadas como clase II en el borde este de la cáscara \#1. Las fuentes que se observan en el interior de esta cáscara estarían sobre el borde de la cáscara \#3, tal como se observa en el panel superior izquierdo de la misma figura. También se encuentra un grupo de fuentes clase I en el borde oeste de ambas cáscaras. En el interior de la cáscara \#3 (que coincide con S109) no se detectan fuentes IR.

En el caso de la cáscara \#2 se han identificado varias fuentes clase I y clase II sobre los bordes y algunas en el interior. No se encontraron fuentes IR candidatas a YSO sobre las estructuras de bordes brillantes descriptas en la Sección 7.4.3.

La cáscara \#4 (que coincide con S111) presenta varias fuentes clase I en los bordes y dos fuentes clase II en su interior. Dada la superposición espacial entre las cáscaras \#2 y \#4 es probable que las fuentes encontradas en el interior de la cáscara \#4 se encuentren en los bordes de la \#2. Por otro lado, dos de las fuentes ubicadas sobre la cáscara podrían pertenecer al cúmulo IR [MCM2005b]54.

La cáscara \#5 (que coincide con S110) muestra varias fuentes clasificadas como clase I, MYSOs y regiones Hit compactas ubicadas sobre grumos de gas molecular, y ninguna en su interior.

Finalmente la cáscara \#6 muestra gran cantidad de fuentes en su interior. Esta coincide espacialmente con el filamento, aunque se observa en otro rango de velocidades, por lo que estas fuentes podrían no estar relacionadas con la cáscara sino con el filamento.

En el caso de la región de la IRDC, y tal como se puede apreciar en la Figura 7.43, se encuentran varios candidatos a YSO clase I proyectados sobre la emisión de $\mathrm{C}^{18} \mathrm{O}(2-1)$, que delinea la IRDC, mientras que los YSOs clase II no parecen estar asociados a la misma. 


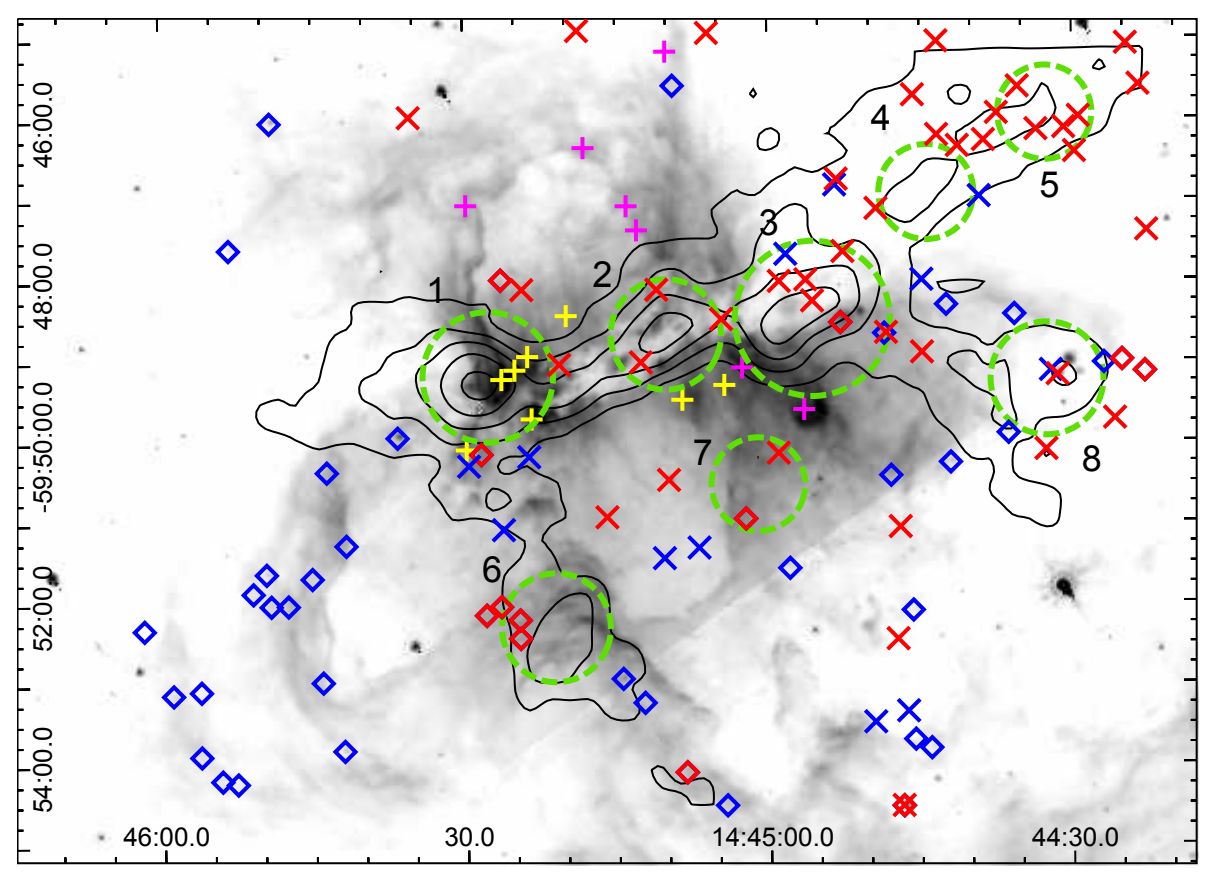

Figura 7.40: Se muestra la emisión promedio de $\mathrm{C}^{18} \mathrm{O}(2-1)$ (contornos: 0.5, 1, 1.5, 2 y $2.5 \mathrm{~K}$ ) junto con la posición y tamaño de los grumos y los candidatos a YSOs superpuestos. Diamantes: WISE. Cruces (X): Spitzer. Rojo: clase I. Azul: clase II. Signos (+): MSX. Amarillo: MYSOs. Magenta: Regiones CHir.

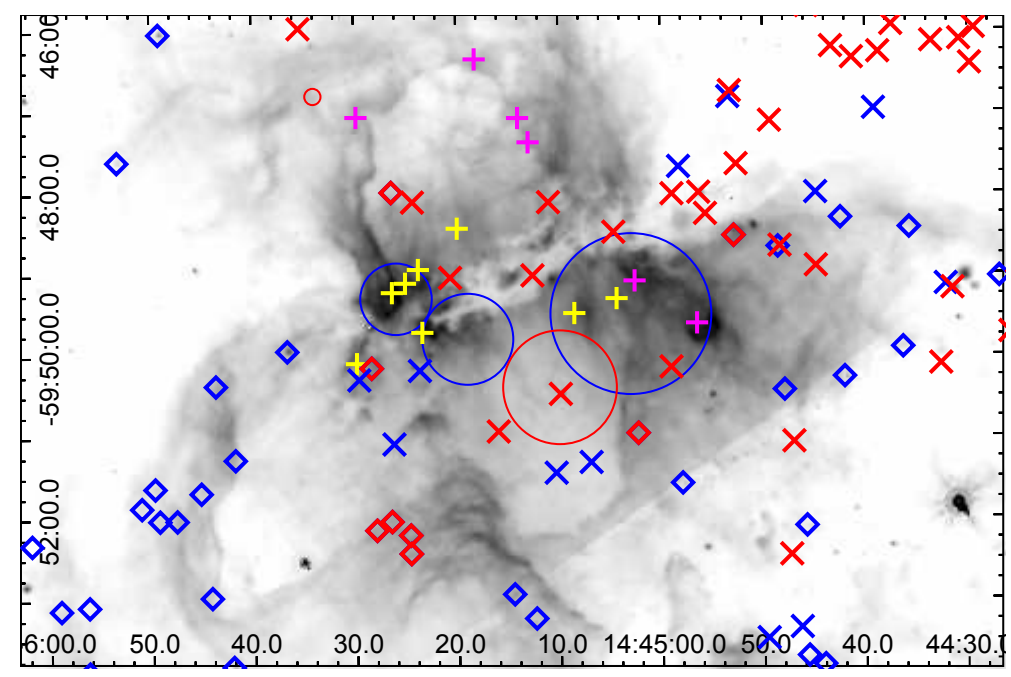

Figura 7.41: Se muestran con círculos las posiciones y tamaños de los cúmulos encontrados por Mercer et al. (2005) (rojo) y Dutra et al. (2003) (azul) junto con los candidatos a YSOs superpuestos. Diamantes: WISE. Cruces (X): Spitzer. Rojo: clase I. Azul: clase II. Signos (+): MSX. Amarillo: MYSOs. Magenta: Regiones CHII. 


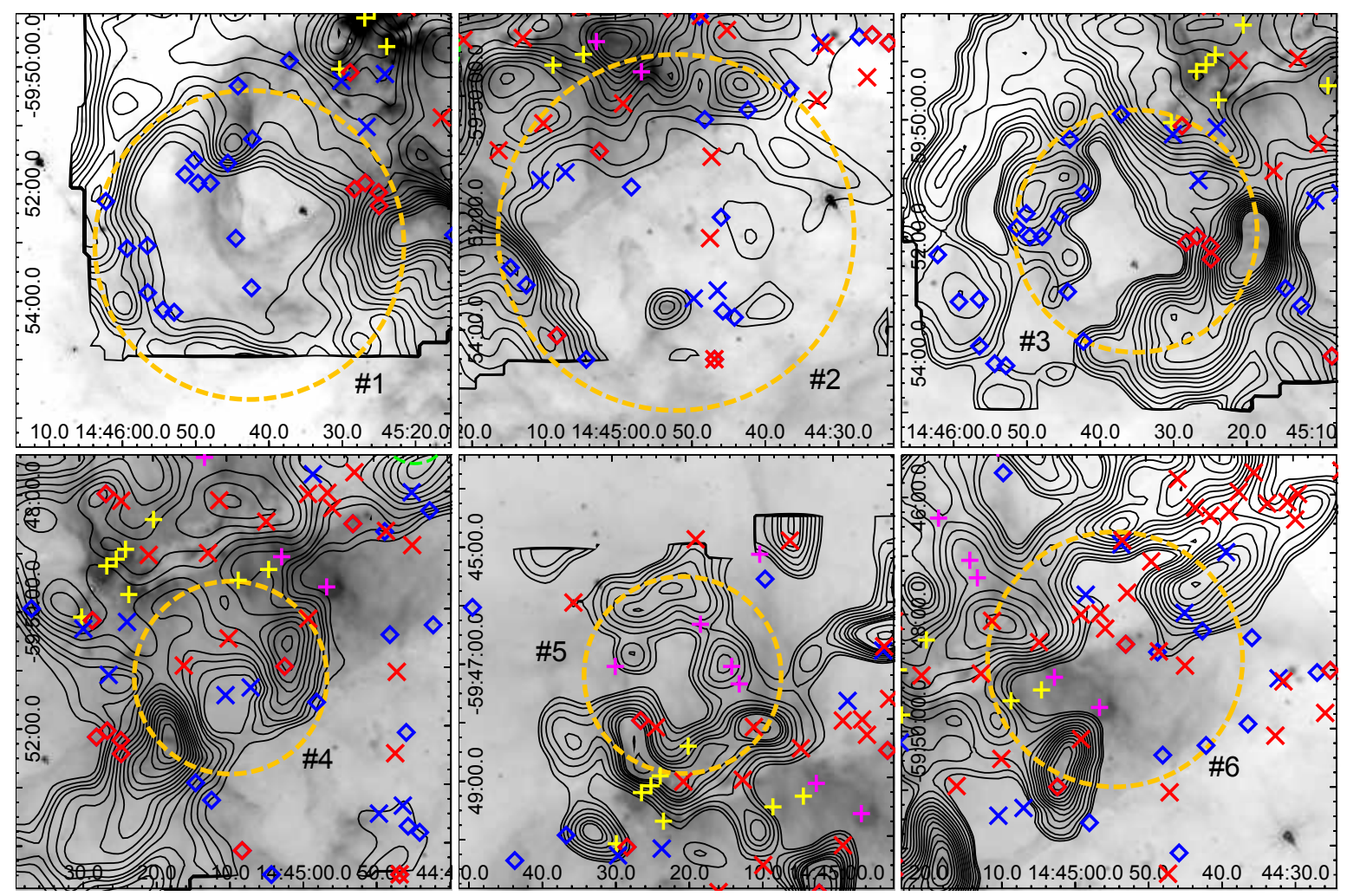

Figura 7.42: Se muestran los contornos de la emisión promedio de ${ }^{12} \mathrm{CO}(2-1)$ para cada una de las cáscaras junto con la posición de los candidatos a YSOs sobre la imagen de $8 \mu \mathrm{m}$. Diamantes: WISE. Cruces (X): Spitzer. Rojo: clase I. Azul: clase II. Signos (+): MSX. Amarillo: MYSOs. Magenta: Regiones CHiı. Los círculos de color naranja muestran el diámetro de las burbujas. El número de cada burbuja se indica en cada panel. 


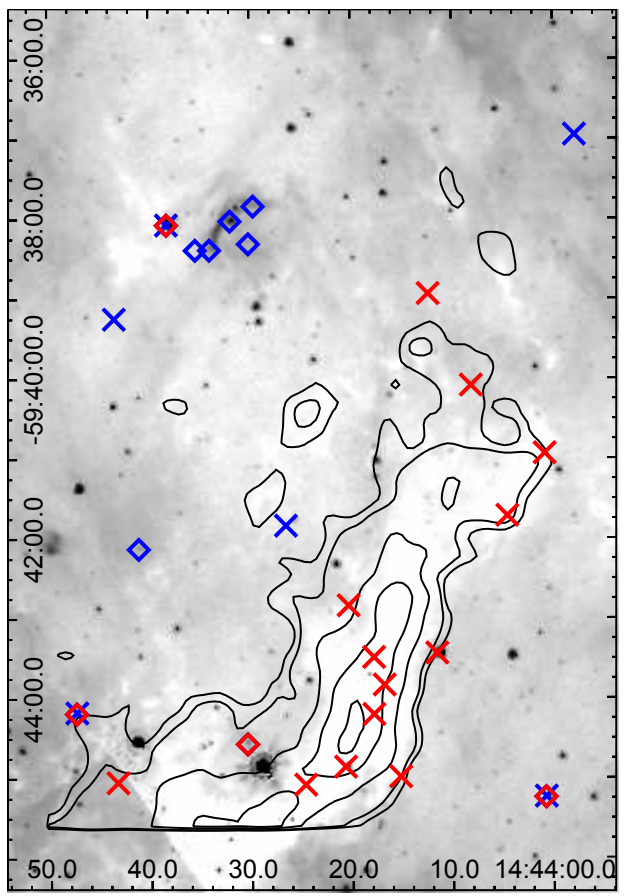

Figura 7.43: Se muestra la emisión promedio de $\mathrm{C}^{18} \mathrm{O}(2-1)$ hacia la IRDC (contornos: 0.35 (3 $r m s), 0.51,1,5$ y $2 \mathrm{~K}$ ) junto con la posición de los candidatos a YSOs. Diamantes: WISE. Cruces (X): Spitzer. Rojo: clase I. Azul: clase II.

\subsection{Discusión}

\subsubsection{Escenario propuesto}

La evidencia mostrada a lo largo de este capítulo (distribución del gas ionizado, polvo tibio, gas molecular y PDR) indica que las burbujas son regiones HiI rodeadas por envolturas de gas molecular (las cáscaras) resultado de la expansión (Deharveng et al., 2010).

Se propone un escenario para esta región en el que las cáscaras \#1 y \#3 formarían parte de una misma estructura observada a diferentes velocidades. Esta hipótesis se apoya en el hecho de que los rangos de velocidad en los que se encuentran ambas cáscaras son adyacentes, y en que coinciden parcialmente en el espacio. Una situación similar se plantea para las cáscaras \#2 y \#4. El panel izquierdo de la Figura 7.44 muestra una representación esquemática de la situación planteada, donde los círculos de línea contínua (que corresponden a las cáscaras \#1 y \#2) están más cerca del observador, y los de línea discontinua (cáscaras \#3 y \#4) están más lejos. Esto se basa en el hecho de que se espera que velocidades más negativas estén más cerca del observador que velocidades de recesión más positivas (tal como se espera para una cáscara en expansión).

Esto sería consistente con el hecho de que la cáscara \#3 tiene mayor densidad molecular y menor tamaño que la \#1. Así, la cáscara \#1 se habría expandido a mayor velocidad que la \#3 al encontrar un medio ambiente menos denso. Una situación similar podría plantearse para las cáscaras \#2 y \#4, ya que, aunque la diferencia en densidad es poca y está dentro de los errores, el tamaño de la \#2 es mucho mayor.

Para acompañar este análisis, en el panel derecho de la Figura 7.44 se muestra un esquema que representa el rango de velocidad en el que se detecta cada cáscara. Allí se puede ver que las cáscaras \#1 y \#2 coinciden mayormente en velocidad y que son las que se encuentran más cercanas al observador, mientras que las cáscaras \#3 y \#4, cuyas velocidades también coinci- 

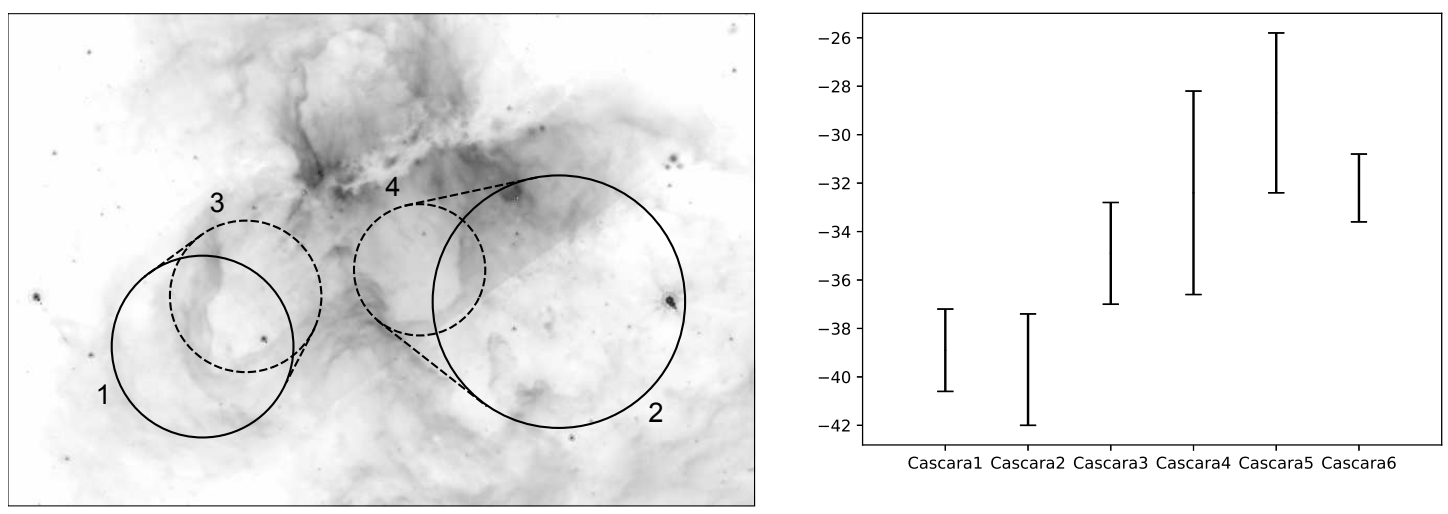

Figura 7.44: Izquierda: Representación esquemática de las regiones Hiı 1+3 y 2+4. Derecha: Rango de velocidad en que se detectan las cáscaras moleculares.

den en parte, se encuentran algo más alajadas, comenzando a detectarse aproximadamente a la velocidad en que dejan de observarse la \#1 y la \#2. Allí se puede apreciar también que la cáscara \#5 se encuentra aún más lejos del observador que las cuatro primeras. Por lo tanto es factible pensar en un escenario en el que las cáscaras forman un único sistema con una cierta inclinación respecto del plano del cielo. Sin embargo, la cáscara \#6, que no tiene una burbujas detectable en el MIR asociada, no sería consistente con en el sistema planteado.

Con respecto al filamento, la existencia de YSOs más jóvenes que los que se identifican sobre las cáscaras, apoya la idea de que esta región de interacción es resultado de la expansión de las regiones HII, ya que los YSOs clase I se podrían haber formado más recientemente que los clase II.

\subsubsection{Estrellas excitatrices y edad de las regiones HII}

Conociendo la densidad electrónica (calculada en la Sección 7.6) es posible determinar qué tipo de estrella sería necesaria para generar una cierta región HII. El número de fotones necesarios para generar una región Hiı con una densidad electrónica $n_{e} \mathrm{y}$ un radio $\mathrm{R}$ está dado por la ecuación (Cichowolski et al., 2009)

$$
\left(\frac{N_{L}}{10^{48} \mathrm{~s}^{-1}}\right)=3.51 \times 10^{-5}\left(\frac{n_{e}^{2}}{\mathrm{~cm}^{-3}}\right)\left(\frac{R^{3}}{\mathrm{pc}}\right)
$$

Considerando $n_{e}=250 \mathrm{~cm}^{-3}$ (ver Sección 7.6) y $R=2.17 \mathrm{pc}$ (correspondiente al ancho a potencia mitad de la emisión en continuo de radio medido en la Figura 7.33) se obtuvo un valor de $N_{L}=22.4 \times 10^{48} \mathrm{~s}^{-1}$. Teniendo en cuenta que la mitad de los fotones estelares son absorbidos por el polvo (Inoue, 2001) el número de fotones total emitido necesario sería de $44.8 \times 10^{48} \mathrm{~s}^{-1}$. El logaritmo decimal de este número es 49.65 , por lo que, según los valores listados en la tercer columna de la Tabla 1.4, se necesita más de una estrella de tipo espectral O3V para obtener tal número de fotones ionizantes.

Considerando que se han encontrado 6 cáscaras en la región, esta cantidad de fotones podría corresponderse con el de 6 estrellas masivas de tipo espectral O7.5V, que serían capaces de ionizar el gas y así generar las regiones. Sin embargo esta suposición no es válida ya que los tamaños de las regiones y las densidades ambientales originales no son iguales.

Para cada cáscara se calcularon los valores del radio de Strömgren inicial, $R_{S_{0}}$, y del tiempo dinámico, $t_{d y n}$, para distintas estrellas excitatrices, a partir de las ecuaciones $1.2 \mathrm{y}$ 
CAPÍTULO 7. COMPLEJO S108-S109-S110-S111

\begin{tabular}{|c|c|c|c|c|c|c|c|c|c|c|c|c|}
\hline \multirow[b]{2}{*}{ TE } & \multicolumn{2}{|c|}{$\overline{\mathrm{C} 1}$} & \multicolumn{2}{|r|}{$\overline{\mathrm{C} 2}$} & \multicolumn{2}{|c|}{$\overline{\mathrm{C} 3}$} & \multicolumn{2}{|r|}{$\overline{\mathrm{C} 4}$} & \multicolumn{2}{|r|}{$\overline{\mathrm{C} 5}$} & \multicolumn{2}{|r|}{ C6 } \\
\hline & $\begin{array}{l}\mathrm{R}_{S_{0}} \\
\text { (pc) }\end{array}$ & $\begin{array}{c}\mathrm{t}_{d y n} \\
\left(10^{5} \mathrm{yr}\right)\end{array}$ & $\begin{array}{l}\mathrm{R}_{S_{0}} \\
(\mathrm{pc})\end{array}$ & $\begin{array}{c}\mathrm{t}_{d y n} \\
\left(10^{5} \mathrm{yr}\right)\end{array}$ & $\begin{array}{l}\mathrm{R}_{S_{0}} \\
\text { (pc) }\end{array}$ & $\begin{array}{c}\mathrm{t}_{d y n} \\
\left(10^{5} \mathrm{yr}\right)\end{array}$ & $\begin{array}{l}\mathrm{R}_{S_{0}} \\
(\mathrm{pc})\end{array}$ & $\begin{array}{c}\mathrm{t}_{d y n} \\
\left(10^{5} \mathrm{yr}\right)\end{array}$ & $\begin{array}{l}\mathrm{R}_{S_{0}} \\
\text { (pc) }\end{array}$ & $\begin{array}{c}\mathrm{t}_{d y n} \\
\left(10^{5} \mathrm{yr}\right)\end{array}$ & $\begin{array}{l}\mathrm{R}_{S_{0}} \\
(\mathrm{pc})\end{array}$ & $\begin{array}{c}\mathrm{t}_{d y n} \\
\left(10^{5} \mathrm{yr}\right)\end{array}$ \\
\hline O3V & 1.4 & 2.79 & 0.9 & 2.98 & 0.2 & 2.04 & - & - & - & - & - & - \\
\hline $\mathrm{O} 4 \mathrm{~V}$ & 1.3 & 2.78 & 0.8 & 3.00 & 0.2 & 2.03 & - & - & - & - & - & - \\
\hline $\mathrm{O} 5 \mathrm{~V}$ & 1.1 & 2.77 & 0.7 & 3.07 & 0.2 & 2.03 & 0.7 & 1.48 & 0.7 & 1.49 & - & - \\
\hline $\mathrm{O} 7 \mathrm{~V}$ & 1.0 & 2.78 & 0.6 & 3.18 & 0.1 & 2.04 & 0.6 & 1.47 & 0.6 & 1.48 & 0.7 & 1.49 \\
\hline $07.5 \mathrm{~V}$ & 0.8 & 2.86 & 0.5 & 3.39 & 0.1 & 2.11 & 0.5 & 1.49 & 0.5 & 1.47 & 0.6 & 1.47 \\
\hline $\mathrm{O} 8 \mathrm{~V}$ & 0.7 & 2.94 & 0.4 & 3.57 & 0.1 & 2.17 & 0.4 & 1.51 & 0.4 & 1.49 & 0.5 & 1.47 \\
\hline $\mathrm{O} 9 \mathrm{~V}$ & 0.4 & 3.37 & 0.3 & 4.35 & 0.06 & 2.50 & 0.2 & 1.69 & 0.3 & 1.63 & 0.3 & 1.57 \\
\hline B0V & 0.3 & 3.98 & 0.2 & 5.33 & 0.04 & 2.96 & 0.2 & 1.96 & 0.2 & 1.87 & 0.2 & 1.76 \\
\hline B0.5V & 0.2 & 4.64 & 0.1 & 6.38 & 0.03 & 3.46 & 0.1 & 2.27 & 0.1 & 2.14 & 0.2 & 1.99 \\
\hline B1V & 0.1 & 5.85 & 0.1 & 8.22 & 0.02 & 4.36 & 0.1 & 2.82 & 0.1 & 2.63 & 0.1 & 2.43 \\
\hline $\mathrm{B} 1.5 \mathrm{~V}$ & 0.1 & 7.35 & 0.1 & 10.5 & 0.01 & 5.50 & 0.06 & 3.52 & 0.06 & 3.27 & 0.07 & 2.99 \\
\hline
\end{tabular}

Tabla 7.12: Radios de Strömgren iniciales $\mathrm{R}_{0} \mathrm{y}$ tiempos dinámicos de expansión $\mathrm{t}_{\text {dyn }}$ para las regiones HiI asociadas a las cáscaras, según el tipo espectral de la estrella ionizante.

1.14 considerando los flujos de fotones de la Tabla 1.4 y tomando como densidades ambientales para cada cáscara las que resultan de distribuir la masa de la cáscara en el área de cada región. Estos resultados se muestran en Tabla 7.12. Allí se puede ver que los tiempos dinámicos para todas las cáscaras son del orden de $10^{5}$ años (excepto en el caso de estrellas excitatrices de tipo espectral B1.5V para la cáscara \#2). Estos resultados muestran que las regiones HiI asociadas a las cáscaras son muy jóvenes.

Analizando los valores encontrados para los tiempos dinámicos resulta que todas las cáscaras coinciden en un valor de $t_{d y n} \sim 2.9 \times 10^{5}$ años, para algún tipo espectral. Si se supone que estas 6 regiones Hit se formaron en el mismo momento y su edad es $\sim 3 \times 10^{5}$ años, es posible determinar un tipo espectral para la estrella excitatriz de cada región. Así se adjudicó una estrella de tipo espectral O8V para la cáscara \#1, O3V para la \#2, B0V para la \#3, B1V para las cáscaras \#4 y \#5 y B1.5V para la cáscara \#6. Sumando el número de fotones ionizantes de estas seis estrellas se obtiene $N_{L}\left(10^{49}\right)=7.0 \mathrm{~s}^{-1}$. Este número es algo mayor que el obtenido a partir de la emisión de continuo de radio. En el caso que las cáscaras \#1 y \#3 formen parte de una única región HII, una estrella O9V podría ser responsable de la ionización. Análogamente si \#2 y \#4 formaran una única estructura sería suficiente con una estrella O7V para ionizar la región. En este caso el número total de fotones en la región sería de $2.2 \times 10^{49}$, algo menor que el obtenido a partir de la emisión de continuo de radio.

Haciendo estas consideraciones es posible proponer un escenario en el que las regiones HiI asociadas a las cáscaras se hubieran formado simultáneamente, y formaran, como ya se sugirió antes, un único sistema.

\subsection{3. ¿Formación estelar inducida?}

Dado que se han encontrado una gran cantidad de objetos estelares jóvenes sobre las cáscaras moleculares se quiere determinar si es posible que el mecanismo collect and collapse pueda estar actuando en la región.

Para determinar si este mecanismo es el responsable de la formación de nuevas estrellas sobre las envolturas densas de las cáscaras moleculares se aplicó el modelo analítico de Whitworth et al. (1994). Para regiones Hiı, el modelo predice la edad de la región Hiı en la que se produce la fragmentación, $t_{\text {frag }}$, el tamaño de la región HiI en ese momento, $R_{\text {frag }}$, la masa de los fragmentos, $M_{\text {frag }}$ y su separación a lo largo de la capa comprimida, $r_{\text {frag }}$.

Los parámetros requeridos para derivar estas cantidades son el flujo de fotones UV de la estrella excitatriz, $N_{L y}$, la densidad ambiental inicial del medio circundante en el cual la 


\begin{tabular}{lccccc}
\hline \hline Cáscara & $\begin{array}{c}t_{\text {frag }} \\
\left(10^{6} \mathrm{yr}\right)\end{array}$ & $\begin{array}{c}R_{\text {frag }} \\
(\mathrm{pc})\end{array}$ & $\begin{array}{c}N_{\text {frag }} \\
\left(10^{21} \mathrm{~cm}^{-2}\right)\end{array}$ & $\begin{array}{c}M_{\text {frag }} \\
\left(10^{3} \mathrm{M}_{\odot}\right)\end{array}$ & $\begin{array}{c}2 r \\
(\mathrm{pc})\end{array}$ \\
\hline 1 & 5.5 & 8.1 & 5.1 & 1.7 & 7.0 \\
2 & 2.7 & 8.5 & 1.1 & 1.2 & 4.6 \\
3 & 1.2 & 2.2 & 2.3 & 1.4 & 5.6 \\
4 & 4.7 & 4.4 & 5.9 & 2.1 & 8.4 \\
5 & 5.1 & 4.9 & 5.5 & 2.1 & 8.5 \\
6 & 3.5 & 8.6 & 8.0 & 1.3 & 5.3 \\
$1+3$ & 1.7 & 3.2 & 1.6 & 1.4 & 5.6 \\
$2+4$ & 2.6 & 8.4 & 1.1 & 1.2 & 4.6 \\
\hline \hline
\end{tabular}

Tabla 7.13: Para cada cáscara se lista el tiempo de fragmentación, el radio de fragmentación, la densidad columnar de los fragmentos, la masa de los fragmentos y la distancia entre los fragmentos que resultan de aplicar el modelo de Whitworth et al. (1994) a las cáscaras.

región HiI está evolucionando, $n_{0}$, y la velocidad del sonido isotérmico en el gas impactado, $a_{0}$.

Se realizaron los cálculos para cada una de las cáscaras considerando el número de fotones ionizantes de las estrellas excitatrices asignadas para cada cáscara en la Sección 7.8.2, la densidad ambiental listada en la columna 6 de la Tabla 7.9, y una velocidad del sonido en el medio de $0.6 \mathrm{~km} \mathrm{~s}^{-1}$. Los resultados se muestran en la Tabla 7.13.

Para las 6 regiones resulta que la escala de tiempo de fragmentación es mucho mayor que la edad dinámica asumida $\left(\sim 3.0 \times 10^{5}\right.$ años $)$, y los radios de fragmentación son mayores que los medidos (ver Tabla 7.9). Por lo tanto, con los parámetros adoptados, las regiones HiI estudiadas serían demasiado jóvenes y no sería posible que esté actuando el mecanismo collect and collapse, que es un proceso relativamente lento para la formación de estrellas en la envoltura molecular de regiones HiI. También se consideró que las cáscaras \#1 y \#,3 al igual que \#2 y \#4, conformaran únicamente dos regiones His, teniendo en cuenta los radios mayores estimados de la Figura 7.44 y una densidad media de $5000 \mathrm{~cm}^{-3}$ para $1+3$ y 1220 $\mathrm{cm}^{-3}$ para $2+4$. Considerando los tiempos y radios de fragmentación así obtenidos, tampoco es posible que el proceso collect and collapse haya podido actuar, mientras que para regiones HiI más grandes y más evolucionadas el mecanismo funciona (e.g. Zavagno et al., 2005). También podría ser evaluado el mecanismo RDI, aunque (excepto en la cáscara \#2) no se observan evidencias de este proceso tales como las estructuras de bordes brillantes y glóbulos cometarios.

Sin embargo la presencia de fuentes IR sobre las grumos densos presentes en las cáscaras que rodean las regiones His sugiere que habría formación estelar. Existen varias razones por las que las edades que se han determinado para estas regiones Hir podrían no coincidir con las derivadas asumiendo un escenario de formación estelar inducida:

- La región es demasiado compleja, y el mecanismo collect and collapse se aplica a regiones Hir aisladas y no considera interación entre las mismas.

- Como ya se mencionó en la Sección 1.4.3, Dale et al. (2015) establecieron que es posible que ocurra la formación de estrellas aún en ausencia de estos mecanismos de formación estelar secuencial.

- Es posible también que las regiones Hir no se hubieran formado simultáneamente. Esta suposición permitió asignar un tipo espectral a la estrella excitatriz de cada región, y 


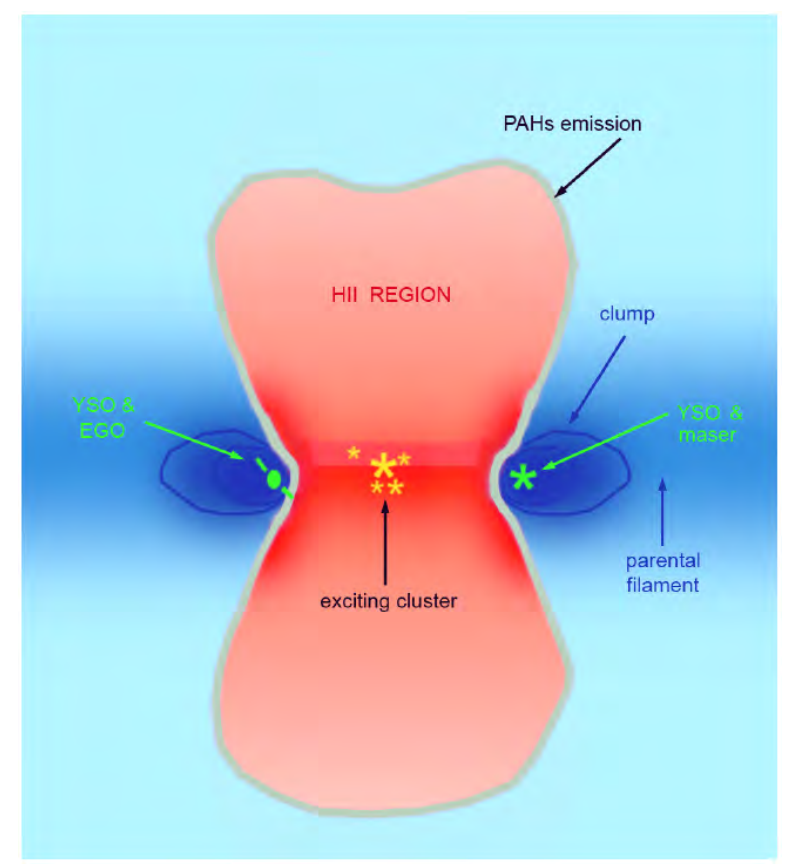

Figura 7.45: Modelo de región Hiı bipolar. Imagen tomada de Samal et al. (2018).

a partir de ello una edad, que podría no ser la real si las cáscaras se hubieran formado en diferentes momentos

- Otra explicación sería que las masas de las cáscaras estén subestimadas, dado que han sido calculadas a partir de la emisión de una sola molécula, lo que también acarrea errores en la estimción de las edades.

Existe además otro escenario muy diferente planteado por Samal et al. (2018), quienes proponen que se trata de una región Hir bipolar. Estos autores presentaron en un trabajo previo (Deharveng et al., 2015) un modelo de región His bipolar en el que las estrellas excitarices se encuentran ubicadas sobre un filamento denso, tal como se muestra en la Figura 7.45 .

Ellos identifican a S110 como el lóbulo norte de la burbuja bipolar, que es perpendicular al filameneto, y a S109 y S111 como el lóbulo sur, cuya morfología resulta muy compleja debido posiblemente a la presencia de estructuras densas e inhomogeneidades que distorsionan el frente de ionización. Sin embargo las fuentes excitatrices de esta región no se han podido identificar con claridad, hecho que Samal et al. (2018) atribuyen a los altos niveles de absorción de la región.

La busqueda de las fuentes excitatrices de la región es clave para poder decidir cuál de los modelos propuestos ajusta a la región.

\subsection{Conclusiones}

Del análisis de la distribución del gas ionizado, polvo tibio, polvo frío y gas molecular en el complejo de burbujas IR S108-S111 se puede concluir:

(1) Se han dentificado 6 cáscaras moleculares, tres de ellas asociadas a las burbujas S109, S110 y S111 y otras dos asociadas a estructuras con forma de arco detectables a partir de la emisión en $8 \mu \mathrm{m}$. La emisión del gas ionizado visto en el continuo de radio y del polvo tibio 
indica que las burbujas IR son regiones Hir. La masas de gas ionizado es de $1720 \mathrm{M}_{\odot}$ con una densidad volumétrica de $250 \mathrm{~cm}^{-3}$.

(2) A partir del estudio de las velocidades de las cáscaras moleculares se propone que cinco de ellas forman parte de un único sistema complejo con una cierta inclinación respecto del plano del cielo.

(3) Se han detectado grumos densos masivos asociados al complejo. Varios de estos grumos poseen cocientes $M_{V I R} / M_{L T E}<1$, indicando que estarían colapsando.

(4) El complejo es una región de formación estelar activa, en la que se localizan una gran cantidad de fuentes IR con características de objetos estelares jóvenes, identificados a partir de diversos catálogos. Se han encontrado muchas fuentes IR sobre las cáscaras, grumos e IRDC. Las fuentes IR detectadas podrían ser nuevas estrellas formándose dentro de los grumos. Varios YSOs coinciden con cuatro cúmulos IR identificados por Dutra et al. (2003) y Mercer et al. (2005).

(5) Se han calculado las masas de las diversas componentes del complejo a través de diferentes métodos. La masa total del complejo calculada a partir de datos de ${ }^{12} \mathrm{CO}(1-0)$ de NANTEN usando el factor $X$ es de $18400 \pm 6600 \mathrm{M}_{\odot}$, mientras que el valor de la masa total calculada a partir de datos de ${ }^{12} \mathrm{CO}(2-1)$ y ${ }^{13} \mathrm{CO}(2-1)$ de APEX suponiendo LTE es de $23300 \pm 8400$ $\mathrm{M}_{\odot}$, compatible con el valor obtenido usando datos de NANTEN. La masa calculada para las distintas componentes del complejo (filamento+cáscaras+IRDC) es de $20600 \pm 7500 \mathrm{M}_{\odot}$ (las masas de los grumos no se sumaron ya que estas ya están incluidas, o bien en las cáscaras moleculares, o bien en el filamento). Todos los valores calculados para la masa total de la región coinciden dentro de los errores. Las masas moleculares y densidades voluméricas para las cáscaras están en el rango 800-2700 M $\odot$ y 600-10200 $\mathrm{cm}^{-3}$. Para los grumos masivos, las masas corresponden a $630-2400 \mathrm{M}_{\odot}$ y las densidades a $(0.7-1.3) \times 10^{4} \mathrm{~cm}^{-3}$.

(6) Considerando que las regiones estudiadas son regiones Hir se determinaron las posibles edades y estrellas excitatrices necesarias para ionizar el gas y mantener la ionización.

(7) Se ha determinado que la región es demasiado joven para que haya actuado el mecanismo de formación estelar collect and collapse tanto considerando individualmente las regiones HII como teniendo en cuenta que las cáscaras \#1 y \#3, por un lado, y \#2 y \#4 por el otro, sean parte de una misma estructura.

(8) El escenario plantedao en esta tesis difiere del propuesto por Samal et al. (2018) quienes proponen que el sistema es una región Hir bipolar. La determinaión de las estrellas excitatrices de la región resulta clave para determinar qué modelo es el más probable. 


\section{Anexo}

Esta sección incluye las tablas con las fuentes IR identificadas como se detalló en las Secciones 7.7.3, 7.7.4, 7.7.5 y 7.7.6, para la región de las burbujas y para la de la IRDC.

Tabla 7.14: Fuentes del catálogo Spitzer candidatos a YSO proyectados sobre las burbujas.

\begin{tabular}{|c|c|c|c|c|c|c|c|}
\hline \multirow[t]{2}{*}{ Nombre } & \multirow{2}{*}{$\begin{array}{c}\alpha \\
\text { [grados] }\end{array}$} & \multirow{2}{*}{$\begin{array}{c}\delta \\
\text { [grados] }\end{array}$} & \multicolumn{4}{|c|}{ Flujo[mag] } & \multirow[t]{2}{*}{ Clase } \\
\hline & & & [3.6] & {$[4.5]^{2}$} & {$[5.8]$} & {$[8.0]$} & \\
\hline G316.7143-00.0709 & $14: 44: 47.3479$ & $-59: 52: 27.9012$ & 13.524000 & 12.007000 & 11.364 & 10.134 & I \\
\hline G316.7029-00.0224 & $14: 44: 32.6196$ & $-59: 50: 6.9792$ & 13.254000 & 11.549000 & 10.903 & 10.405 & I \\
\hline G316.7235-00.0497 & $14: 44: 47.0611$ & $-59: 51: 4.4388$ & 9.496000 & 9.236000 & 8.686 & 8.364 & I \\
\hline G316.8026+00.0261 & $14: 45: 5.93736$ & $-59: 44: 56.3712$ & 11.975000 & 11.724000 & 11.281 & 9.823 & I \\
\hline G316.7430-00.0144 & 14:44:48.3938 & $-59: 48: 39.8304$ & 11.222000 & 10.337000 & 9.545 & 9.208 & I \\
\hline G316.7676-00.0141 & 14:44:58.939 & $-59: 48: 1.3212$ & 14.277000 & 11.859000 & 10.693 & 10.338 & I \\
\hline G316.7596-00.0149 & $14: 44: 55.673$ & $-59: 48: 16.0344$ & 12.805000 & 11.969000 & 11.043 & 9.814 & I \\
\hline G316.7557+00.0079 & $14: 44: 49.3493$ & $-59: 47: 7.5156$ & 12.982000 & 12.016000 & 11.344 & 10.789 & I \\
\hline G316.7582-00.0030 & 14:44:52.6399 & $-59: 47: 39.4296$ & 14.625000 & 12.509000 & 11.128 & 10.245 & I \\
\hline G316.7627-00.0115 & $14: 44: 56.3198$ & $-59: 48: 0.3168$ & 10.955000 & 8.881000 & 7.722 & 6.68 & I \\
\hline G316.8152-00.0380 & $14: 45: 24.353$ & $-59: 48: 6.696$ & 12.021000 & 10.936000 & 9.879 & 9.065 & I \\
\hline G316.7751-00.0263 & $14: 45: 4.66488$ & $-59: 48: 29.4372$ & 14.048000 & 13.412000 & 10.625 & 9.066 & I \\
\hline G316.7898-00.0263 & $14: 45: 11.0352$ & $-59: 48: 7.2072$ & 13.475000 & 12.916000 & 10.03 & 8.749 & I \\
\hline G316.7657+00.0099 & $14: 44: 53.2517$ & $-59: 46: 45.5124$ & 12.733000 & 11.094000 & 10.642 & 9.679 & 1 \\
\hline G316.7865-00.0413 & $14: 45: 12.6139$ & $-59: 49: 1.2108$ & 14.652000 & 13.938000 & 10.647 & & 1 \\
\hline G316.8016-00.0488 & $14: 45: 20.6904$ & & 12.369000 & 11.645001 & 8.922 & & 1 \\
\hline G316.7346-00.0150 & 14:44:44 & $-59: 48:$ & 12.996000 & 12.735000 & 11.197 & 9.577 & I \\
\hline G316.7528-00.0464 & $14: 44: 59.0578$ & $-59: 50: 9.1932$ & 11.429000 & 10.854999 & 10.257 & 28 & 1 \\
\hline G316.7794-00.0733 & $14: 45: 16.0406$ & $-59: 50: 56.2848$ & 12.789000 & 11.465000 & 10.777 & 10.213 & 1 \\
\hline G316.7711-00.0610 & $14: 45: 9.92448$ & $-59: 50: 28.878$ & 11.595000 & 11.343000 & 8.598 & 6. & 1 \\
\hline G316.8272+00.0153 & 14:45:18.7294 & $-59: 44: 54.1824$ & 11.471000 & 11.180000 & 10.819 & 10.439 & I \\
\hline G316.8513-00.0155 & $14: 45: 35.3969$ & $-59: 45: 57.9024$ & 11.158000 & 10.259000 & 9.489 & 8.692 & 1 \\
\hline G316.7072-00.0074 & $14: 44: 31.4777$ & $-59: 49: 11.3844$ & 11.290000 & 10.817000 & 8.165 & 6.657 & 1 \\
\hline G316.6926-00.0107 & $14: 44: 25.8247$ & $-59: 49: 43.9608$ & 12.393000 & 12.177000 & 11.325 & 11.058 & 1 \\
\hline G316.7588+00.0445 & $14: 44: 43.3301$ & $-59: 45: 3.1176$ & 13.290000 & 11.751000 & 10.824 & 10.178 & I \\
\hline G316.7172+00.0539 & $14: 44: 23.4485$ & $-59: 45: 35.514$ & 12.433000 & 11.406000 & 10.8 & 10.335 & I \\
\hline G316.7231+00.0606 & $14: 44: 24.6682$ & $-59: 45: 4.9752$ & 12.233000 & 11.210000 & 10.597 & 9.931 & I \\
\hline G316.7325+00.0366 & 14:44:33.5285 & $-59: 46: 8.904$ & 10.666000 & 9.697000 & 9.129 & 8.377 & I \\
\hline G316.7256+00.0428 & 14:44:29.347 & $-59: 45: 59.202$ & 15.172000 & 12.631000 & 11.869 & 11.667 & 1 \\
\hline G316.7233+00.0358 & $14: 44: 29.7415$ & $-59: 46: 25.3812$ & 13.639000 & 12.761000 & 11.634 & 11.301 & I \\
\hline G316.7274+00.0394 & $14: 4$ & 6 & 93000 & 1000 & 33 & 77 & I \\
\hline G316.7586+00.0324 & 14 & $-59: 4$ & 11.80 & 11.535000 & 11.108 & 10. & I \\
\hline G316.7458+00.0267 & $14: 4$ & $-59: 4$ & 13.6 & 12.395001 & 11.937 & 11. & I \\
\hline G316.7506+00.0270 & 14:44:43.3248 & $-59: 46: 12.7524$ & 14.213000 & 12.721000 & 12.107 & 11.204 & I \\
\hline G316.7397+00.0430 & 14:44:35.346 & $-59: 45: 37.008$ & 14.646000 & 12.351000 & 11.36 & 10.672 & I \\
\hline G316.7414+00.0301 & 14:44:38.7122 & $-59: 46: 16.7844$ & 12.967000 & 11.438000 & 11.016 & 10.442 & I \\
\hline G316.7413+00.0363 & $14: 44: 37.4218$ & $-59: 45: 56.646$ & 12.807000 & 11.806000 & 11.3 & 10.899 & I \\
\hline G316.7030+00.0273 & $14: 44: 22.656$ & $-59: 47: 23.9892$ & 11.526000 & 10.491000 & 9.876 & 9.098 & I \\
\hline G316.6988-00.1018 & $14: 44: 46.8586$ & $-59: 54: 32.0688$ & 9.270000 & 7.637000 & 6.428 & 5.606 & I \\
\hline G316.7978-00.0846 & $14: 45: 26.279$ & $-59: 51: 5.202$ & 11.729000 & 11.247000 & 10.046 & 9.404 & II \\
\hline G316.8098-00.0757 & $14: 45: 29.6681$ & $-59: 50: 17.6748$ & 9.475000 & 9.132000 & 8.426 & 7.809 & II \\
\hline G316.7409-00.0014 & $14: 44: 44.8702$ & $-59: 48: 0.4716$ & 11.624000 & 11.104000 & 10.672 & 10.251 & II \\
\hline G316.7687-00.0084 & $14: 44: 58.278$ & $-59: 47: 41.19$ & 12.882000 & 12.090000 & 11.576 & 10.672 & II \\
\hline G316.7655+00.0087 & $14: 44: 53.4122$ & $-59: 46: 49.8576$ & 10.316000 & 9.845000 & 9.36 & 8.796 & II \\
\hline G316.7993-00.0687 & $14: 45: 23.717$ & $-59: 50: 11.0652$ & 12.041000 & 11.457000 & 10.366 & 9.548 & II \\
\hline G316.7595-00.0710 & $14: 45: 6.96096$ & $-59: 51: 19.35$ & 12.123000 & 11.535000 & 11.163 & 10.652 & II \\
\hline G316.7651-00.0760 & $14: 45: 10.3954$ & $-59: 51: 27.054$ & 11.087000 & 10.574000 & 10.08 & 9.602 & 11 \\
\hline G316.7088-00.0072 & $14: 44: 32.1386$ & $-59: 49: 8.1876$ & 11.226000 & 10.433000 & 9.607 & 8.921 & II \\
\hline G316.7374+00.0191 & 14:44:39.161 & $-59: 46: 58.5012$ & 13.312000 & 12.800000 & 12.032 & 11.501 & II \\
\hline G316.7061-00.0836 & $14: 44: 46.3582$ & $-59: 53: 21.6276$ & 13.104999 & 12.315000 & 11.95 & 11.073 & II \\
\hline G316.7113-00.0885 & $14: 44: 49.6174$ & $-59: 53: 29.6772$ & 11.635000 & 11.079000 & 10.353 & 9.313 & II \\
\hline
\end{tabular}


Tabla 7.15: Fuentes del catálogo Spitzer candidatos a YSO proyectados sobre la IRDC.

\begin{tabular}{lccccccc}
\hline \hline Nombre & $\begin{array}{c}\alpha \\
\text { [grados] }\end{array}$ & $\begin{array}{c}\delta \\
\text { [grados] }\end{array}$ & {$[3.6]$} & {$[4.5]$} & {$[5.8]$} & {$[8.0]$} & \\
\hline G316.7588+00.0445 & $14: 44: 43.3301$ & $-59: 45: 3.1176$ & 13.290000 & 11.751000 & 10.824 & 10.178 & I \\
G316.7058+00.0704 & $14: 44: 15.2256$ & $-59: 44: 58.9992$ & 11.595000 & 10.804000 & 10.26 & 10.006 & I \\
G316.7163+00.0799 & $14: 44: 17.8608$ & $-59: 44: 12.12$ & 13.723000 & 11.631000 & 10.746 & 10.055 & I \\
G316.7171+00.0674 & $14: 44: 20.6986$ & $-59: 44: 51.576$ & 10.314000 & 9.090000 & 8.345 & 7.747 & I \\
G316.7231+00.0606 & $14: 44: 24.6682$ & $-59: 45: 4.9752$ & 12.233000 & 11.210000 & 10.597 & 9.931 & I \\
G316.7428+00.1643 & $14: 44: 12.3898$ & $-59: 38: 56.2524$ & 12.870000 & 12.030000 & 11.179 & 10.655 & I \\
G316.7213+00.0907 & $14: 44: 17.862$ & $-59: 43: 29.2944$ & 12.946000 & 11.988000 & 11.262999 & 10.626 & I \\
G316.7169+00.0864 & $14: 44: 16.8206$ & $-59: 43: 50.0088$ & 14.473000 & 12.942000 & 12.073 & 11.471 & I \\
G316.7306+00.0983 & $14: 44: 20.3686$ & $-59: 42: 50.436$ & 11.966000 & 11.709000 & 11.239 & 11.036 & I \\
G316.7097+00.0970 & $14: 44: 11.5848$ & $-59: 43: 26.1588$ & 6.971000 & 6.362000 & 5.924 & 5.692 & I \\
G316.7067+00.1443 & $14: 44: 0.84744$ & $-59: 40: 56.208$ & 12.110000 & 11.838000 & 11.305 & 11.016 & I \\
G316.7266+00.1507 & $14: 44: 8.1492$ & $-59: 40: 5.1276$ & 12.664000 & 12.108000 & 11.281 & 9.949 & I \\
G316.7084+00.1292 & $14: 44: 4.57464$ & $-59: 41: 43.0044$ & 13.654000 & 11.714000 & 10.723 & 9.852 & I \\
G316.6765+00.0794 & $14: 44: 0.76488$ & $-59: 45: 13.9824$ & 10.704000 & 9.932000 & 8.992 & 8.289 & II \\
G316.8000+00.1320 & $14: 44: 43.4995$ & $-59: 39: 15.0156$ & 13.360000 & 12.883000 & 12.182 & 11.597 & II \\
G316.7983+00.1544 & $14: 44: 38.2769$ & $-59: 38: 4.5492$ & 7.911000 & 7.228000 & 6.406 & 5.312 & II \\
G316.7289+00.2073 & $14: 43: 57.8338$ & $-59: 36: 56.9628$ & 12.006000 & 11.796000 & 11.427 & 10.921 & II \\
G316.7727+00.0542 & $14: 44: 47.3738$ & $-59: 44: 10.482$ & 11.959000 & 11.226000 & 10.335 & 9.64 & II \\
G316.7494+00.1079 & $14: 44: 26.5457$ & $-59: 41: 50.4096$ & 13.005000 & 12.732000 & 12.224 & 11.728 & II \\
\hline \hline
\end{tabular}


Tabla 7.16: Fuentes del catálogo WISE candidatos a YSO proyectados sobre las burbujas.

\begin{tabular}{|c|c|c|c|c|c|c|c|}
\hline \multirow[t]{2}{*}{ Nombre } & \multirow{2}{*}{$\begin{array}{c}\alpha \\
\text { [grados] }\end{array}$} & \multirow{2}{*}{$\begin{array}{c}\delta \\
\text { [grados] }\end{array}$} & \multicolumn{4}{|c|}{ "Flujo[mag] } & \multirow[t]{2}{*}{ Clase } \\
\hline & & & {$[3.4]$} & {$[4.6]$} & {$[12.0]$} & {$[22.0]$} & \\
\hline J144524.67-595226.2 & $14: 45: 24.6735$ & $-59: 52: 26.2153$ & 11.628 & 10.522 & 4.931 & 0.841 & $\mathrm{I}$ \\
\hline J144422.85-594908.8 & $14: 44: 22.8515$ & $-59: 49: 8.86404$ & 12.761 & 11.584 & 6.536 & 4.630 & I \\
\hline J144508.30-595406.5 & $14: 45: 8.30141$ & $-59: 54: 6.57972$ & 12.096 & 11.071 & 5.781 & 3.245 & I \\
\hline J144524.71-595212.5 & $14: 45: 24.7121$ & $-59: 52: 12.5594$ & 11.366 & 10.364 & 4.676 & 2.039 & I \\
\hline J144502.32-595057.9 & $14: 45: 2.32627$ & $-59: 50: 57.9952$ & 10.806 & 9.669 & 2.019 & -2.161 & I \\
\hline J144528.02-595208.8 & $14: 45: 28.0205$ & $-59: 52: 8.83056$ & 11.366 & 10.270 & 5.053 & 0.865 & I \\
\hline J144526.54-595202.4 & $14: 45: 26.5424$ & $-59: 52: 2.47368$ & 11.652 & 10.509 & 5.907 & 0.847 & I \\
\hline J144446.84-595432.2 & $14: 44: 46.8462$ & $-59: 54: 32.274$ & 10.583 & 7.692 & 5.688 & 2.916 & I \\
\hline J144528.43-595009.1 & $14: 45: 28.4373$ & $-59: 50: 9.14964$ & 6.309 & 5.292 & -0.525 & -3.586 & $\mathrm{I}$ \\
\hline J144425.10-594900.3 & $14: 44: 25.107$ & $-59: 49: 0.3234$ & 12.904 & 11.794 & 6.723 & 4.131 & I \\
\hline J144452.89-594832.2 & 14:44:52.8911 & $-59: 48: 32.2621$ & 9.343 & 8.332 & 2.004 & -1.587 & I \\
\hline J144526.42-594759.4 & $14: 45: 26.4239$ & $-59: 47: 59.474$ & 11.771 & 10.624 & 2.463 & 1.853 & I \\
\hline J144444.08-595349.2 & $14: 44: 44.0841$ & $-59: 53: 49.2$ & 11.945 & 11.299 & 6.822 & 2.958 & II \\
\hline J144551.08-595151.8 & $14: 45: 51.0825$ & $-59: 51: 51.8702$ & 12.345 & 11.823 & 7.322 & 3.670 & II \\
\hline J144547.61-595201.1 & $14: 45: 47.6182$ & $-59: 52: 1.19712$ & 10.246 & 9.636 & 5.159 & 2.566 & II \\
\hline J144544.23-595258.1 & $14: 45: 44.2377$ & $-59: 52: 58.1012$ & 11.675 & 11.241 & 6.748 & 7.426 & II \\
\hline J144524.67-595226.2 & $14: 45: 24.6735$ & $-59: 52: 26.2153$ & 11.628 & 10.522 & 4.931 & 0.841 & II \\
\hline J144514.56-595256.6 & $14: 45: 14.562$ & $-59: 52: 56.6566$ & 8.878 & 8.243 & 5.156 & 1.076 & II \\
\hline J144422.85-594908.8 & $14: 44: 22.8515$ & $-59: 49: 8.86404$ & 12.761 & 11.584 & 6.536 & 4.630 & II \\
\hline J144445.66-595342.7 & $14: 44: 45.6644$ & $-59: 53: 42.7502$ & 11.835 & 11.441 & 6.702 & 2.402 & II \\
\hline J144556.32-595352.8 & $14: 45: 56.3276$ & $-59: 53: 52.8544$ & 11.769 & 11.147 & 6.956 & 5.540 & II \\
\hline J144541.85-595116.4 & $14: 45: 41.8518$ & $-59: 51: 16.4772$ & 11.865 & 11.289 & 6.596 & 2.308 & II \\
\hline J144543.72-595021.8 & $14: 45: 43.7269$ & $-59: 50: 21.8742$ & 10.215 & 9.824 & 5.008 & 1.096 & II \\
\hline J144536.69-594956.4 & $14: 45: 36.6966$ & $-59: 49: 56.4326$ & 7.078 & 6.319 & 4.959 & 2.012 & II \\
\hline J144549.31-595201.2 & $14: 45: 49.311$ & $-59: 52: 1.24608$ & 11.069 & 10.607 & 5.978 & 2.171 & II \\
\hline J144556.29-59 & $14: 45: 56.2977$ & $-59: 53: 4.69068$ & 11.260 & 10.908 & 7.936 & 4.746 & II \\
\hline J144508.30-595406.5 & $14: 45: 8.30141$ & $-59: 54: 6.57972$ & 12.096 & 11.071 & 5.781 & 3.245 & II \\
\hline J144524.71-595212.5 & $14: 45: 24.7121$ & $-59: 52: 12.5594$ & 11.366 & 10.364 & 4.676 & 2.039 & II \\
\hline J144601.88-595218.8 & $14: 46: 1.88494$ & $-59: 52: 18.827$ & 11.160 & 10.738 & 8.221 & 5.953 & II \\
\hline J144502.32-595057.9 & $14: 45: 2.32627$ & $-59: 50: 57.9952$ & 10.806 & 9.669 & 2.019 & -2.161 & II \\
\hline J144458.00-595134.9 & $14: 44: 58.0034$ & $-59: 51: 34.9582$ & 10.593 & 10.230 & 5.514 & 0.759 & II \\
\hline J144528.02-595208.8 & $14: 45: 28.0205$ & $-59: 52: 8.83056$ & 11.366 & 10.270 & 5.053 & 0.865 & II \\
\hline J144436.33-594954.8 & $14: 44: 36.3352$ & $-59: 49: 54.8101$ & 11.582 & 10.662 & 6.607 & 4.335 & II \\
\hline J144526.54-595202.4 & $14: 45: 26.5424$ & $-59: 52: 2.47368$ & 11.652 & 10.509 & 5.907 & 0.847 & II \\
\hline J144554.28-595411.0 & $14: 45: 54.2803$ & $-59: 54: 11.0326$ & 12.320 & 11.830 & 7.138 & 6.822 & II \\
\hline J144552.74-595413.2 & $14: 45: 52.7496$ & $-59: 54: 13.284$ & 12.430 & 11.979 & 7.406 & 8.186 & II \\
\hline J144549.75-595137.4 & $14: 45: 49.7524$ & $-59: 51: 37.4465$ & 9.284 & 8.993 & 6.946 & 2.893 & II \\
\hline J144447.96-595026.2 & $14: 44: 47.9611$ & $-59: 50: 26.218$ & 9.718 & 9.306 & 4.527 & 0.618 & II \\
\hline J144442.06-595016.5 & $14: 44: 42.0601$ & $-59: 50: 16.5685$ & 9.435 & 8.480 & 6.938 & 2.204 & II \\
\hline J144446.84-595432.2 & $14: 44: 46.8462$ & $-59: 54: 32.274$ & 10.583 & 7.692 & 5.688 & 2.916 & II \\
\hline J144504.33-595431.3 & $14: 45: 4.33752$ & $-59: 54: 31.396$ & 11.070 & 10.546 & 5.809 & 2.619 & II \\
\hline J144528.43-595009.1 & $14: 45: 28.4373$ & $-59: 50: 9.14964$ & 6.309 & 5.292 & -0.525 & -3.586 & II \\
\hline J144445.82-595206.6 & $14: 44: 45.8259$ & $-59: 52: 6.62232$ & 10.251 & 9.936 & 6.672 & 2.033 & II \\
\hline J144425.10-594900.3 & $14: 44: 25.107$ & $-59: 49: 0.3234$ & 12.904 & 11.794 & 6.723 & 4.131 & II \\
\hline J144442.44-594819.2 & $14: 44: 42.4494$ & $-59: 48: 19.2305$ & 11.065 & 10.574 & 5.963 & 4.052 & II \\
\hline J144426.88-594902.4 & $14: 44: 26.8898$ & $-59: 49: 2.49096$ & 11.810 & 10.952 & 6.599 & 3.238 & II \\
\hline J144452.89-594832.2 & $14: 44: 52.8911$ & $-59: 48: 32.2621$ & 9.343 & 8.332 & 2.004 & -1.587 & II \\
\hline J144448.57-594840.4 & $14: 44: 48.5731$ & $-59: 48: 40.4453$ & 10.056 & 9.168 & 4.788 & 0.857 & II \\
\hline J144553.25-594736.1 & $14: 45: 53.2539$ & $-59: 47: 36.1522$ & 9.331 & 8.954 & 6.733 & 2.873 & II \\
\hline J144526.42-594759.4 & $14: 45: 26.4239$ & $-59: 47: 59.474$ & 11.771 & 10.624 & 2.463 & 1.853 & II \\
\hline J144435.72-594826.3 & $14: 44: 35.7204$ & $-59: 48: 26.3898$ & 10.920 & 10.500 & 5.863 & 4.140 & II \\
\hline J144559.05-595307.1 & $14: 45: 59.0578$ & $-59: 53: 7.17936$ & 11.099 & 10.677 & 6.658 & 4.605 & II \\
\hline J144545.22-595140.9 & $14: 45: 45.229$ & $-59: 51: 40.9219$ & 11.643 & 10.982 & 6.111 & 3.468 & II \\
\hline J144512.42-595314.6 & $14: 45: 12.4202$ & $-59: 53: 14.6324$ & 10.598 & 10.139 & 5.389 & 0.884 & II \\
\hline J144542.16-595349.0 & $14: 45: 42.1609$ & $-59: 53: 49.0906$ & 9.003 & 8.556 & 7.312 & 2.671 & II \\
\hline J144549.11-594601.7 & $14: 45: 49.1125$ & $-59: 46: 1.77528$ & 8.131 & 7.777 & 5.773 & 3.619 & II \\
\hline J144509.35-594535.5 & $14: 45: 9.35347$ & $-59: 45: 35.5424$ & 10.759 & 10.233 & 5.340 & 3.180 & II \\
\hline
\end{tabular}


Tabla 7.17: Fuentes del catálogo WISE candidatos a YSO proyectados sobre la IRDC.

\begin{tabular}{|c|c|c|c|c|c|c|c|}
\hline \multirow[t]{2}{*}{ Nombre } & \multirow{2}{*}{$\begin{array}{c}\alpha \\
\text { [grados] }\end{array}$} & \multirow{2}{*}{$\begin{array}{c}\delta \\
\text { [grados] }\end{array}$} & \multicolumn{4}{|c|}{ Flujo[mag] } & \multirow[t]{2}{*}{ Clase } \\
\hline & & & {$[3.4]$} & {$[4.6]$} & {$[12.0]$} & {$[22.0]$} & \\
\hline J144400.78-594514.4 & $14: 44: 0.788112$ & $-59: 45: 14.4608$ & 11.472 & 10.143 & 7.812 & 4.909 & $\mathrm{I}$ \\
\hline J144430.43-594434.4 & $14: 44: 30.4331$ & $-59: 44: 34.498$ & 10.202 & 8.920 & 6.473 & 2.950 & $\mathrm{I}$ \\
\hline J144438.27-593804.6 & $14: 44: 38.274$ & $-59: 38: 4.6986$ & 7.999 & 6.976 & 3.952 & 1.896 & I \\
\hline J144447.40-594411.3 & $14: 44: 47.409$ & $-59: 44: 11.3791$ & 12.027 & 11.008 & 8.021 & 5.326 & I \\
\hline J144441.16-594207.9 & $14: 44: 41.1614$ & $-59: 42: 7.98912$ & 10.833 & 10.530 & 7.805 & 5.430 & II \\
\hline J144400.78-594514.4 & $14: 44: 0.788112$ & $-59: 45: 14.4608$ & 11.472 & 10.143 & 7.812 & 4.909 & II \\
\hline J144430.43-594434.4 & 14:44:30.4331 & $-59: 44: 34.498$ & 10.202 & 8.920 & 6.473 & 2.950 & II \\
\hline J144435.43-593823.6 & $14: 44: 35.4324$ & $-59: 38: 23.6818$ & 12.013 & 11.592 & 8.396 & 5.356 & II \\
\hline J144438.27-593804.6 & $14: 44: 38.274$ & $-59: 38: 4.6986$ & 7.999 & 6.976 & 3.952 & 1.896 & II \\
\hline J144431.97-593801.9 & $14: 44: 31.9767$ & $-59: 38: 1.90644$ & 7.780 & 7.451 & 6.310 & 4.117 & II \\
\hline J144434.02-593823.6 & $14: 44: 34.0237$ & $-59: 38: 23.6227$ & 11.904 & 11.046 & 6.964 & 4.610 & II \\
\hline J144430.16-593818.9 & 14:44:30.1677 & $-59: 38: 18.9938$ & 12.313 & 11.628 & 6.966 & 3.148 & II \\
\hline J144447.40-594411.3 & 14:44:47.409 & $-59: 44: 11.3791$ & 12.027 & 11.008 & 8.021 & 5.326 & II \\
\hline J144429.68-593750.7 & $14: 44: 29.6827$ & $-59: 37: 50.7396$ & 11.503 & 11.185 & 7.364 & 5.515 & II \\
\hline
\end{tabular}

Tabla 7.18: Fuentes del catálogo 2MASS candidatos a YSO proyectados sobre las burbujas.

\begin{tabular}{|c|c|c|c|c|c|c|c|c|c|}
\hline \multirow{2}{*}{$\begin{array}{c}\alpha \\
\text { (grados) }\end{array}$} & \multirow{2}{*}{$\begin{array}{c}\delta \\
\text { (grados) }\end{array}$} & \multicolumn{3}{|c|}{ Flujo[mag] } & \multirow{2}{*}{$\begin{array}{c}\alpha \\
\text { (grados) }\end{array}$} & \multirow{2}{*}{$\begin{array}{c}\delta \\
\text { (grados) }\end{array}$} & \multicolumn{3}{|c|}{ Flujo[mag] } \\
\hline & & {$[\mathrm{J}]$} & {$[\mathrm{H}]$} & {$[\mathrm{Ks}]$} & & & {$[\mathrm{J}]$} & {$[\mathrm{H}]$} & [Ks] \\
\hline $14: 44: 25.1918$ & $-59: 52: 29.19$ & 13.956 & 13.537 & 13.160 & $14: 45: 59.795$ & $-59: 51: 8.838$ & 15.866 & 14.371 & 13.385 \\
\hline $14: 44: 52.8576$ & $-59: 51: 36.1944$ & 14.343 & 13.933 & 13.518 & 14:45:58.9702 & $-59: 50: 46.2876$ & 15.628 & 14.781 & 13.922 \\
\hline 14:44:32.3623 & $-59: 50: 51.0828$ & 15.245 & 14.840 & 14.306 & $14: 45: 58.0061$ & $-59: 50: 57.3864$ & 15.280 & 14.643 & 14.189 \\
\hline 14:44:40.1443 & $-59: 52: 17.598$ & 15.237 & 14.758 & 14.367 & $14: 46: 2.20032$ & $-59: 46: 12.3852$ & 14.299 & 14.072 & 13.775 \\
\hline $14: 44: 36.4354$ & $-59: 51: 22.7376$ & 15.030 & 14.660 & 14.097 & $14: 45: 56.8843$ & $-59: 48: 55.9872$ & 15.358 & 14.702 & 14.244 \\
\hline $14: 45: 57.5124$ & $-59: 53: 23.3664$ & 14.594 & 14.009 & 13.440 & $14: 45: 53.0518$ & $-59: 47: 49.6428$ & 15.271 & 14.748 & \\
\hline 14:45:54.8042 & $-59: 52: 45.8328$ & 15.873 & 15.093 & 14.501 & $14: 45: 55.9716$ & $-59: 48: 11.8476$ & 15.144 & 14.715 & 14.294 \\
\hline 14:45: & $-59: 52: 54.8292$ & 13.548 & 12.920 & 12.463 & 4:44:30.361 & $-59: 48: 42.5268$ & 14.569 & 14.122 & 13.763 \\
\hline $14: 45: 7.22664$ & $-59: 53: 17.5308$ & 14.852 & 14.359 & 13.968 & $14: 44: 28$. & $-59: 48: 42.444$ & 15.568 & 14.803 & 14.134 \\
\hline 14:45:26.7991 & $-59: 52: 53.9364$ & 15.555 & 14.614 & 13.941 & $14: 44: 2$ & $-59: 48: 58$ & 15.400 & 14.963 & 14.620 \\
\hline 14:45:18.1661 & $-59: 52: 11.2548$ & 15.731 & 14.933 & 14.174 & $14: 44: 2$ & $-59: 49: 32$ & 15.744 & 15.006 & 14.260 \\
\hline $14: 45: 19.879$ & $-59: 52: 19.8228$ & 14.755 & 14.142 & 13.698 & $14: 44: 2$ & $-59: 45: 54$ & 15.045 & 14.644 & 14.315 \\
\hline $14: 45: 20.8404$ & $-59: 52: 26.2524$ & 14.178 & 13.813 & 13.527 & $14: 44: 29$ & $-59: 45: 0.0$ & 14.531 & 13.828 & 13.261 \\
\hline 14:45:22.9726 & $-59: 51: 19.2492$ & 14.816 & 14.222 & 13.697 & & $-59: 47: 43$ & 14.102 & 13.540 & 12.988 \\
\hline $14: 45$ & $-59: 45: 47.5$ & 13.508 & 13.140 & 12.785 & & $-59: 4$ & 15.432 & & 14.176 \\
\hline $14: 44: 45.9034$ & $-59: 48: 27.3132$ & 14.963 & 14.616 & 14.134 & 14:44: & $-59: 46: 41$ & 15.387 & 760 & 14.291 \\
\hline $14: 44: 54.823$ & $-59: 48: 43.0344$ & 15.881 & 14.814 & 13.730 & & $-59: 47: 21$ & 14.808 & & 13.910 \\
\hline 14:44:53.412 & $-59: 46: 50.1672$ & 15.200 & 13.309 & 11.941 & 14: & $-59: 47: 26.1312$ & 14.315 & 13.783 & 13.351 \\
\hline $14: 44: 56.233$ & $-59: 47: 59.3376$ & 15.121 & 14.168 & 13.418 & $14: 44: 41.2303$ & $-59: 45: 26.874$ & 15.532 & 14.239 & 13.450 \\
\hline 14:44:53.347 & $-59: 48: 29.9772$ & 13.766 & 13.472 & 13.078 & $14: 44: 28.3608$ & $-59: 47: 59.7912$ & 15.321 & 13.769 & 12.791 \\
\hline 14:45:29.1931 & $-59: 48: 24.894$ & 14.982 & 14.303 & 13.681 & $14: 44: 26.4658$ & $-59: 48: 28.2888$ & 14.659 & 13.479 & 12.539 \\
\hline $14: 45: 12.7483$ & $-59: 47: 55.1616$ & 14.314 & 13.584 & 13.082 & $14: 44: 22.098$ & $-59: 46: 34.6188$ & 14.976 & 14.214 & 13.682 \\
\hline $14: 45: 23.5694$ & $-59: 50: 44.916$ & 13.948 & 13.640 & 13.290 & 14:44 & $-59: 47: 59.586$ & 15.506 & 14.732 & 14.153 \\
\hline $14: 44: 48.2153$ & $-59: 50: 15.5976$ & 13.558 & 13.100 & 12.463 & $14: 44: 53.0177$ & $-59: 53: 47.2884$ & 15.151 & 13.830 & 12.924 \\
\hline $14: 44: 51.0574$ & $-59: 49: 16.6404$ & 15.300 & 14.453 & 13.458 & 14:44:45.457 & $-59: 53: 41.1108$ & 14.749 & 14.201 & 13.547 \\
\hline 14:45:1.34136 & $-59: 52: 38.46$ & 15.251 & 14.748 & 14.323 & $14: 44: 53.7998$ & $-59: 54: 11.0736$ & 15.859 & 14.808 & 14.132 \\
\hline 14:45:3.89568 & $-59: 51: 20.2932$ & 15.459 & 14.930 & 14.419 & $14: 44: 46.0061$ & $-59: 53: 48.498$ & 15.213 & 14.661 & 14.178 \\
\hline $14: 44: 58.9133$ & $-59: 49: 59.3076$ & 15.560 & 14.868 & 14.214 & 14:45:39.935 & $-59: 54: 4.2084$ & 15.934 & 14.922 & 14.273 \\
\hline $14: 45: 28.5065$ & $-59: 47: 18.5244$ & 15.438 & 14.470 & 13.847 & $14: 45: 52.7018$ & $-59: 54: 47.1924$ & 15.016 & 14.582 & 14.232 \\
\hline $14: 45: 45.726$ & $-59: 45: 58.284$ & 14.007 & 13.566 & 13.224 & $14: 45: 11.0981$ & $-59: 53: 24.4104$ & 15.720 & 14.424 & 13.318 \\
\hline 14:45:51.9142 & $-59: 45: 8.1288$ & 14.343 & 13.715 & 13.274 & $14: 45: 18.8863$ & $-59: 54: 33.0336$ & 15.633 & 14.941 & 14.450 \\
\hline $14: 45: 54.2726$ & $-59: 45: 37.1736$ & 15.849 & 15.038 & 14.402 & $14: 45: 28.3015$ & $-59: 54: 1.8864$ & 15.779 & 15.075 & 14.479 \\
\hline $14: 45: 52.7978$ & $-59: 45: 30.762$ & 15.329 & 14.925 & 14.487 & 14:45:34.872 & $-59: 54: 31.1508$ & 16.018 & 14.953 & 14.280 \\
\hline $14: 45: 30.7428$ & $-59: 46: 11.6436$ & 15.282 & 14.682 & 14.136 & $14: 45: 32.3594$ & $-59: 54: 15.5916$ & 14.892 & 14.034 & 13.436 \\
\hline $14: 45: 58.4287$ & $-59: 52: 5.4192$ & 15.620 & 14.757 & 13.921 & $14: 44: 54.6154$ & $-59: 54: 53.3592$ & 15.468 & 14.000 & 13.026 \\
\hline $14: 45: 43.9577$ & $-59: 50: 20.76$ & 15.247 & 13.967 & 12.581 & $14: 45: 8.43216$ & $-59: 54: 44.2944$ & 13.776 & 13.365 & 13.036 \\
\hline $14: 46: 5.40312$ & $-59: 50: 11.9436$ & 15.307 & 14.665 & 14.133 & & & & & \\
\hline
\end{tabular}


Tabla 7.19: Fuentes del catálogo 2MASS candidatos a YSO proyectados sobre la IRDC.

\begin{tabular}{ccccc}
\hline \hline$\alpha$ & $\delta$ & \multicolumn{3}{c}{ Flujo[mag] } \\
(grados) & (grados) & {$[\mathrm{J}]$} & {$[\mathrm{H}]$} & {$[\mathrm{Ks}]$} \\
\hline $14: 44: 18.1572$ & $-59: 45: 25.4736$ & 14.552 & 13.906 & 13.442 \\
$14: 44: 26.9731$ & $-59: 44: 48.12$ & 14.382 & 13.517 & 12.768 \\
$14: 44: 29.1953$ & $-59: 45: 0.0684$ & 14.531 & 13.828 & 13.261 \\
$14: 44: 41.2303$ & $-59: 45: 26.874$ & 15.532 & 14.239 & 13.450 \\
$14: 44: 11.3107$ & $-59: 45: 17.4276$ & 15.225 & 14.556 & 14.107 \\
$14: 44: 3.62616$ & $-59: 45: 14.9292$ & 15.706 & 14.968 & 14.458 \\
$14: 44: 32.4638$ & $-59: 35: 45.1644$ & 14.593 & 14.194 & 13.891 \\
$14: 44: 36.5417$ & $-59: 39: 3.9744$ & 15.515 & 14.615 & 14.033 \\
$14: 44: 39.0065$ & $-59: 39: 16.704$ & 15.233 & 14.812 & 14.406 \\
$14: 44: 44.453$ & $-59: 37: 56.4096$ & 14.794 & 14.356 & 13.973 \\
$14: 44: 38.2855$ & $-59: 38: 4.7436$ & 13.309 & 11.034 & 9.635 \\
$14: 44: 43.1208$ & $-59: 37: 48.4284$ & 15.602 & 14.670 & 14.048 \\
$14: 44: 41.399$ & $-59: 35: 53.5668$ & 15.373 & 14.844 & 14.440 \\
$14: 44: 42.101$ & $-59: 36: 56.0124$ & 14.423 & 13.668 & 13.049 \\
$14: 44: 41.1914$ & $-59: 39: 53.8236$ & 15.321 & 14.703 & 14.281 \\
$14: 44: 15.7241$ & $-59: 36: 37.2672$ & 13.803 & 13.212 & 12.794 \\
$14: 44: 30.4279$ & $-59: 39: 19.1484$ & 15.022 & 13.658 & 12.585 \\
$14: 44: 22.0634$ & $-59: 38: 45.4488$ & 15.351 & 14.273 & 13.409 \\
$14: 44: 23.1482$ & $-59: 39: 25.7004$ & 14.036 & 13.591 & 13.231 \\
$14: 44: 8.91408$ & $-59: 39: 5.76$ & 15.657 & 14.859 & 14.331 \\
$14: 44: 1.02192$ & $-59: 38: 4.4988$ & 14.359 & 13.831 & 13.423 \\
$14: 43: 58.3956$ & $-59: 44: 2.3496$ & 15.953 & 14.498 & 13.572 \\
$14: 43: 59.9482$ & $-59: 42: 43.6752$ & 14.310 & 13.471 & 12.827 \\
$14: 44: 40.7801$ & $-59: 40: 58.5768$ & 15.907 & 14.869 & 14.101 \\
$14: 44: 39.6682$ & $-59: 41: 12.2676$ & 14.977 & 14.675 & 14.360 \\
$14: 44: 40.9615$ & $-59: 41: 22.002$ & 15.999 & 15.016 & 14.226 \\
$14: 44: 29.6402$ & $-59: 43: 55.3728$ & 15.801 & 14.932 & 14.251 \\
$14: 44: 20.1367$ & $-59: 43: 3.8604$ & 15.834 & 15.012 & 14.369 \\
$14: 44: 32.8402$ & $-59: 43: 11.4564$ & 15.670 & 14.774 & 14.187 \\
$14: 44: 11.2678$ & $-59: 43: 43.4928$ & 14.723 & 14.344 & 14.032 \\
$14: 44: 7.02528$ & $-59: 43: 48.162$ & 15.533 & 14.931 & 14.429 \\
$14: 44: 19.3039$ & $-59: 40: 1.83$ & 14.992 & 14.537 & 14.192 \\
$14: 44: 28.7765$ & $-59: 41: 16.3176$ & 15.006 & 14.218 & 13.655 \\
$14: 43: 57.0276$ & $-59: 39: 48.1248$ & 15.019 & 14.491 & 14.079 \\
$14: 44: 15.4066$ & $-59: 41: 8.5992$ & 15.205 & 14.382 & 13.849 \\
\hline \hline & & & & \\
\hline
\end{tabular}


Tabla 7.20: Fuentes del catálogo MSX candidatos a YSO proyectados sobre las burbujas.

\begin{tabular}{lcccccc}
\hline \hline Nombre & RA, Dec.(J2000) & \multicolumn{4}{c}{ Flujo[Jy] } & \multirow{2}{*}{ Clasificacion } \\
& & A & C & D & E & \\
\hline G316.8095-00.0536 & $14: 45: 25.104-59: 49: 6.6$ & 1.2772 & 23.996 & 43.568 & 81.459 & MYSO \\
G316.8116-00.0727 & $14: 45: 29.88-59: 50: 5.64$ & 1.8387 & 4.8829 & 6.2521 & 17.619 & MYSO \\
G316.8112-00.0566 & $14: 45: 26.4-59: 49: 13.44$ & 14.052 & 35.741 & 41.554 & 188.47 & MYSO \\
G316.8021-00.0614 & $14: 45: 23.448-59: 49: 42.96$ & 9.5385 & 29.909 & 50.372 & 187.31 & MYSO \\
G316.7754-00.0447 & $14: 45: 8.544-59: 49: 29.28$ & 8.6539 & 41.694 & 64.159 & 115.98 & MYSO \\
G316.8083-00.0500 & $14: 45: 23.832-59: 48: 56.52$ & 3.9263 & 28.795 & 48.241 & 106.27 & MYSO \\
G316.7688-00.0383 & $14: 45: 4.392-59: 49: 18.48$ & 5.5006 & 24.959 & 35.744 & 63.077 & MYSO \\
G316.8046-00.0390 & $14: 45: 19.992-59: 48: 26.28$ & 1.3804 & 3.7312 & 4.3808 & 9.1089 & MYSO \\
G316.7670-00.0335 & $14: 45: 2.616-59: 49: 5.52$ & 5.5026 & 9.8214 & 9.3190 & 30.838 & CHII \\
G316.8159-00.0059 & $14: 45: 18.192-59: 46: 21.36$ & 0.4204 & 0.7614 & -0.3938 & 1.3669 & CHII \\
G316.8028-00.0131 & $14: 45: 13.992-59: 47: 4.92$ & 0.8744 & 2.1129 & 1.4829 & 4.8735 & CHII \\
G316.8088+00.0191 & $14: 45: 10.056-59: 45: 10.08$ & 0.4652 & 0.7615 & -0.6139 & 2.6867 & CHII \\
G316.7517-00.0359 & $14: 44: 56.52-59: 49: 36.84$ & 11.973 & 14.564 & 9.0564 & 42.494 & CHII \\
G316.8329-00.0270 & $14: 45: 29.808-59: 47: 3.84$ & 0.7398 & 1.0588 & 0.6356 & 2.7320 & CHII \\
G316.7987-00.0169 & $14: 45: 12.984-59: 47: 22.92$ & 0.2881 & 1.4696 & 0.4453 & 2.2014 & CHII \\
\hline \hline
\end{tabular}




\section{Capítulo 8}

\section{Conclusiones generales}

A lo largo de esta tesis se han estudiado diferentes regiones de formación estelar que no están realacionadas entre sí. Cada una de ellas posee características propias, y sin embargo todas ellas son sitos de formación estelar activa: la cáscara molecular asociada a una región HII, una IRDC y un complejo de burbujas infrarrojas. Todas las regiones fueron estudiadas a partir de observaciones de gas molecular, que se complementaron con datos públicos de imágenes y fuentes puntuales infrarrojas, líneas moleculares de trazadores de alta densidad e imágenes en continuo de radio.

Los principales resultados para cada una de estas regiones son:

- Gum31: La cáscara densa de gas molecular que rodea a la región Hir Gum 31 presenta gran cantidad de fuentes IR clasificadas como objetos estelares jóvenes, por lo que es factible pensar que éste sea un sitio de formación estelar activa. Para investigar esta posibilidad se estudiaron tres regiones asociadas a YSOs sobre la envoltura molecular de Gum 31: IRAS 10351-5816, IRAS 10349-5824 y 2MASS J10365763-5844052. Las tres regiones presentaron a su vez distintos escenarios.

En la región asociada a IRAS 10351-5816 se encuentra una fuente WISE clase I proyectada sobre un grumo denso de gas molecular y polvo que estaría colapsando y formando una nueva estrella. En cambio, en la región de IRAS 10349-5824 se identifican dos fuentes WISE clase II sobre una cavidad rodeada por tres grumos de gas molecular, por lo que se propuso que se trata de una región más evolucionada que la anterior, en la que las estrellas en formación ya han generado una pequeña cavidad en el gas que las rodea. Se estudió también la posibilidad de que esta fuente tenga outflows. Para esto se complementaron las observaciones de gas molecular con datos de líneas de $\mathrm{H}_{2}$ en el infrarrojo cercano observadas con el telescopio Gemini, que revelaron fuentes de continuo con líneas de emisión $\operatorname{Br} \gamma$ en el interior de la cavidad y fuentes extendidas con líneas de diferentes transiciones rotovibracionales de $\mathrm{H}_{2}$ sobre los grumos que la rodean. Los resultados sugieren que la fuente central podría tener un outflow. Finalmente en la región de 2MASS J10365763-5844052 solamente se encontraron dos grumos de gas molecular que no poseen fuentes IR asociadas aunque se determinó que uno de ellos podría estar colapsando.

- SDC 341.232-0.268: Un escenario diferente muestra la IRDC SDC 341.232-0.268. Sobre ella se han detectado cuatro EGOs, que sugieren la presencia de gas chocado. Se encontaron dos grumos de gas molecular asociados a la IRDC que podrían estar colapsando, y se identificaron YSOs clase I proyectados sobre ellos. La hipótesis del colapso es apoyada por los perfiles asimétricos de ${ }^{12} \mathrm{CO}$. Estos grumos también se detectan en el IR lejano indicando la presencia de polvo frío asociado a la IRDC. 
- S108-S111: La última región estudiada es el complejo de burbujas IR S108-S111 y es la que presenta mayor variedad de elementos: allí se encuentra una compleja distribución de burbujas IR, arcos e IRDCs detectados a partir de la emisión de $8 \mu \mathrm{m}$.

Las burbujas IR y arcos están rodeadas de envolturas de gas molecular y presentan emisión en continuo de radio y de polvo tibio en su interior por lo que se estableció que se trata de regiones HiI.

Sobre el filamento en la región de interacción de las burbujas se observa emisión infrarroja en un amplio rango de longitudes de onda, desde $24 \mu \mathrm{m}$, indicando la presencia de polvo tibio y fuentes excitatrices, hasta $870 \mu \mathrm{m}$, mostrando el inicio de una estructura en forma de arco, que no se detecta en $8 \mu \mathrm{m}$, y se continua con la IRDC. También se han identificado grumos densos de gas molecular a partir de $\mathrm{C}^{18} \mathrm{O}(2-1)$ y trazadores de alta densidad.

Se encontró en la región una gran cantidad de candidatos a objetos estelares jóvenes clasificados como clase I (mayormente sobre el filamento, los grumos densos y la IRDC), clase II (principalmete sobre las cáscaras que rodean las burbujas), MYSOs y regiones HiI compactas (sobre la zona de interacción de las burbujas). Tembién se identificaron sobre el filamento cuatro cúmulos IR catalogados.

El hecho de que los objetos clase I estén ubicados preferentemente sobre el filamento y no sobre las cáscaras asociadas a las regiones Hiı, indica que este es más joven que dichas regiones, compatible con que sea el resultado de la interacción de las cáscaras originado en la expansión del gas ionizado.

Se estudió la posibilidad de que estuviera actuando el mecanismo collect and collapse como disparador de la formación de estrellas pero se encontró que la región es demasiado joven como para que este proceso ocurra.

Se plantea como trabajo a futuro en esta región el análisis de los cúmulos IR y un estudio para identificar las estrellas excitatrices del complejo.

En general se ha observado que las IRDCs presentan candidatos a YSOs menos evolucionados y están asociadas a polvo frío, por lo que se trataría de regiónes muy jóvenes. Gum 31 es una región HiI clásica con una densidad original menor, es más grande y más evolucionada que las asociadas a las burbujas. En las ragiones estuiadas sobre cáscara molecular que rodea a Gum 31 se ha encontrado un candidato a YSO clase I, que está asociado a polvo frío al igual que las IRDCs, y uno clase II que es una objeto más evolucionado que ya habría comenzado a formar su propia cavidad. Solo se encontraron candidatos a objetos estelares jóvenes masivos (MYSOs) sobre el filamento asociado a las burbujas, que parece ser la región de formación estelar más activa dentro de las estudiadas. 


\section{Apéndice}

En el presente apéndice se incluyen datos que pueden resultar de ayuda al momento de leer esta tesis.

Tabla 8.1: Factores de conversión y constantes

\begin{tabular}{|c|c|c|}
\hline \multicolumn{3}{|l|}{ Longitud } \\
\hline Micrón & $\mu \mathrm{m}$ & $10^{-6} \mathrm{~m}$ \\
\hline Unidad Astronómica & UA & $1.4960 \times 10^{13} \mathrm{~cm}$ \\
\hline Parsec & $\mathrm{pc}$ & $3.0857 \times 10^{18} \mathrm{~cm}=206265 \mathrm{AU}$ \\
\hline \multicolumn{3}{|l|}{ Masa } \\
\hline Masa del protón & $\mathrm{m}_{p}$ & $1.6726 \times 10^{-24} \mathrm{~g}$ \\
\hline Masa del electrón & $\mathrm{m}_{e}$ & $9.1094 \times 10^{-28} \mathrm{~g}$ \\
\hline Masa del Hidrógeno & $\mathrm{m}_{H}$ & $1.6733 \times 10^{-24} \mathrm{~g}=8.4134 \times 10^{-58} \mathrm{M}_{\odot}$ \\
\hline Masa solar & $\mathrm{M}_{\odot}$ & $1.9891 \times 10^{33} \mathrm{~g}$ \\
\hline \multicolumn{3}{|l|}{ Tiempo } \\
\hline Año & 1 año & $3.1557 \times 10^{7} \mathrm{seg}$ \\
\hline \multicolumn{3}{|c|}{ Carga, energía, densidad de flujo } \\
\hline Carga del electrón & $\mathrm{e}$ & $4.8032 \times 10^{-10} \mathrm{esu}$ \\
\hline Electrón Volt & $1 \mathrm{eV}$ & $1.6022 \times 10^{-12} \mathrm{erg}$ \\
\hline Luminosidad solar & $1 \mathrm{~L}_{\odot}$ & $3.8268 \times 10^{33} \mathrm{erg} \mathrm{s}_{-1}$ \\
\hline Jansky & $1 \mathrm{Jy}$ & $10^{-23} \mathrm{erg} \mathrm{s}^{-1} \mathrm{~cm}^{-2} \mathrm{~Hz}^{-1}=10^{-26} \mathrm{~W} \mathrm{~m}^{-2} \mathrm{~Hz}^{-1}$ \\
\hline \multicolumn{3}{|l|}{ Constantes universales } \\
\hline Velocidad de la luz & $c$ & $2.99792458 \times 10^{10} \mathrm{~cm} \mathrm{~s}^{-1}$ \\
\hline Constante gravitacional & $G$ & $6.6726 \times 10^{8}$ dyn $\mathrm{cm}^{-2} \mathrm{~g}^{2}$ \\
\hline Constante de Plank & $h$ & $6.6261 \times 10^{-27} \mathrm{erg} \mathrm{s}=4.1357 \times 10^{-15} \mathrm{eV} \mathrm{s}$ \\
\hline Constante de Boltzmann & $k$ & $1.3807 \times 10^{-16} \mathrm{erg} \mathrm{K}^{-1}=8.6174 \times 10^{-5} \mathrm{eV} \mathrm{K}^{-1}$ \\
\hline
\end{tabular}


Tabla 8.2: Resolución angular de los datos de catálogos utilizados en esta tesis

\begin{tabular}{ll}
\hline \hline WISE & \\
3.4 & $6^{\prime \prime} 1$ \\
4.6 & $6^{\prime \prime} 4$ \\
12 & $6^{\prime \prime} 5$ \\
22 & $12^{\prime \prime}$ \\
\hline MSX & \\
8.3 & $6^{\prime \prime}$ \\
12 & $6^{\prime \prime}$ \\
14 & $6^{\prime \prime}$ \\
21 & $6^{\prime \prime}$ \\
\hline Spitzer IRAC & \\
3.6 & $1^{\prime \prime} 2$ \\
4.5 & $1^{\prime \prime 2}$ \\
5.8 & $1^{\prime \prime} 2$ \\
8 & $1^{\prime \prime 2}$ \\
\hline Spitzer MIPS & \\
24 & $6^{\prime \prime}$ \\
\hline Herschel PACS & \\
70 & $5^{\prime \prime}$ \\
160 & $13^{\prime \prime}$ \\
\hline Herschel SPIRE & \\
250 & $18^{\prime \prime}$ \\
350 & $25^{\prime \prime}$ \\
500 & $36^{\prime \prime}$ \\
\hline \hline
\end{tabular}

\section{Índice de acrónimos}

A continuación se listan los acrónimos utilizados en esta tesis. Todos ellos provinen de siglas en inglés y no se traducen al español.

EGO: Extended Green Object

FIR: Far-Infrared

HPBW: Half-Power Beam-Width

IR: Infrared

IRDC: Infrared Dark Cloud

MASER: Microwave Amplification by Stimulated Emission of Radiation

MYSO: Massive Stellar Object

NIR: Near-Infrared

PAH: Polycyclic Aromatic Hydrocarbons

PDR: Photodissociation Region

UV: Ultraviolet

YSO: Young Stellar Object 
Figura 8.1: Escalas para la conversión de frecuencia en longitud de onda $(\lambda=c / \nu)$, de frecuencia en temperatura $(\mathrm{T}=h \nu / k)$ y de frecuencia en energía $(\mathrm{E}=h \nu)$.

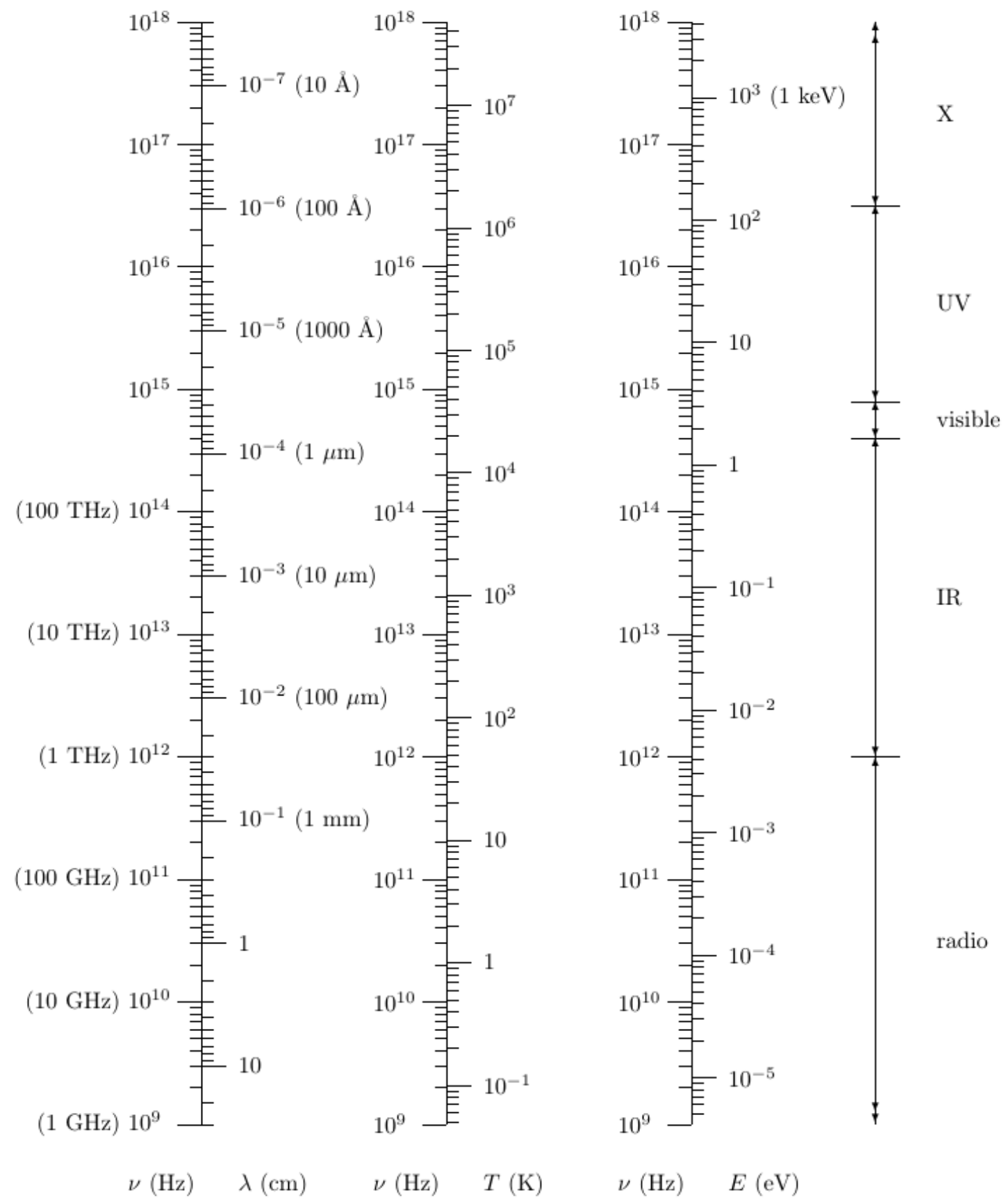




\section{Índice de figuras}

1.1. Distribución de la emisión de Hidrógeno atómico neutro en la Via Láctea . . 6

1.2. Estructura jerrarquica de nubes moleculares . . . . . . . . . . . . . 7

1.3. Estuctura básica y espectro de emisión de PAHs . . . . . . . . . . . . . 9

1.4. Moléculas interestelares . . . . . . . . . . . . . . . . . . . . . . 10

1.5. Espectro de la nube moleclar $\operatorname{Sgr} \mathrm{B} 2(\mathrm{~N}) \ldots \ldots \ldots \ldots$

1.6. Región Hir RCW $120 \ldots \ldots$. . . . . . . . . . . . . . . . . 12

1.7. Esquema de la estructura de una región Hir en expansión . . . . . . . . . . . 15

1.8. Esquema de la estructura de una región de fotodisociación . . . . . . . . . . 16

1.9. Burbuja infrarroja de polvo N $49 \ldots \ldots \ldots \ldots \ldots$. . . . . . . . . 17

1.10. Clasificación de YSOs según su exceso infrarrojo . . . . . . . . . . . . 20

1.11. Región Hit RCW 79 y glóbulos cometarios en Vela-Puppis . . . . . . . . . . . 24

1.12. Esquema de la formación estelar inducida por colisión de nubes moleculares . $\quad 25$

1.13. Diagrama color-color JHK para YSOs de baja masa en el complejo de nubes oscuras Taurus-Auriga . . . . . . . . . . . . . . . . . 27

1.14. Diagrama color-color de fuentes Spitzer . . . . . . . . . . . . . . 28

1.15. Imagen del outflow molecular HH47 ejectado de una protoestrella y esquema de un outflow molecular . . . . . . . . . . . . . . . . . . . . 30

1.16. Espectro de ${ }^{12} \mathrm{CO}(2-1)$ y diagrama posición-velocidad de un outflow . . . . . 31

1.17. Esquema de la formación de líneas moleculares en una envoltura en caída . . 32

1.18. Nebulosa de la Serpiente observada por Spitzer y Herschel . . . . . . . . . . . 34

2.1. Representación de niveles vibracionales y rotacionales en una molécula diatómica 38

2.2. Estructura hiperfina de la molécula $\mathrm{HNC}(1-0) \ldots \ldots \ldots$. . . . . . . . 48

2.3. Estructura hiperfina de $\mathrm{N}_{2} \mathrm{H}^{+} \ldots \ldots \ldots \ldots \ldots$. . . . . . . . . . 49

2.4. Mapa de temperatura de polvo de la región Hir Gum 31 . . . . . . . . . . 51

3.1. Patrón de antena . . . . . . . . . . . . . . . . . . . . 58

3.2. Telescopio APEX . . . . . . . . . . . . . . . . . . . 62

3.3. Esquema de un cubo de datos . . . . . . . . . . . . . . . . . . . 64

4.1. Región Hir Gum 31 . . . . . . . . . . . . . . . . . . . . . . . . 70

4.2. Gas molecular y candidatos a YSOs en Gum $31 \ldots$. . . . . . . . . . . 71

4.3. Emisión a $870 \mu \mathrm{m}$ en Gum 31 . . . . . . . . . . . . . . . . . 73

4.4. Regiones observadas en Gum 31. . . . . . . . . . . . . . . . . . 75

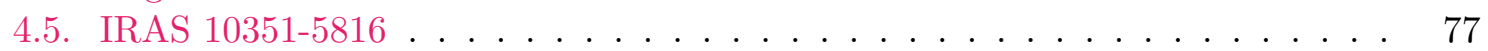

4.6. Emisión IR asociada a IRAS 10351-5816 . . . . . . . . . . . . . . . 77

4.7. Perfiles promedio de ${ }^{12} \mathrm{CO}(3-2)$ y $\mathrm{C}^{18} \mathrm{O}(3-2)$ hacia IRAS $10351-5816 \ldots \ldots$. . . 78

4.8. Gas molecular y emisión a 4.5 y $8 \mu \mathrm{m}$ asociada a IRAS 10349-5824 . . . . . . 82

4.9. Gas molecular en las alas azul y roja asociadas a IRAS 10349-5824 . . . . . . 83

4.10. Emisión de ${ }^{12} \mathrm{CO}$ en la región de la fuente IRAS 10349-5824 . . . . . . . . . . 84 
4.11. Emisión IR asociada a IRAS $10349-5824 \ldots \ldots$. . . . . . . . . . 86

4.12. Perfiles promedio para los grumos en la región asociada a IRAS 10349-5824 . 86

4.13. Emisión de ${ }^{12} \mathrm{CO}$ en la región de la fuente 2MASS J10365763-5844052 . . . . 88

4.14. Emisión de ${ }^{13} \mathrm{CO}$ en la región de la fuente 2MASS J10365763-5844052 . . . . 89

4.15. Emisión de $\mathrm{C}^{18} \mathrm{O}$ en la región de la fuente 2MASS J10365763-5844052 . . . . 90

4.16. Región observada en torno a la fuente 2MASS J10365763-5844052 . . . . . . . 91

4.17. Emisión IR asociada a 2MASS J10365763-5844052 . . . . . . . . . . . . . 91

4.18. Perfiles promedio de los grumos asociados a 2MASS J10365763-5844052 . 92

5.1. Fuentes WISE clase II y fuentes identificadas por Gemini sobre imagen óptica 96

5.2. Distribución de fuentes conspicuas a lo largo de la ranura observada con Gemini 97

5.3. Espectro extraidos de las fuentes extendidas ES1, ES2, ES3 y ES4 . . . . . . 98

5.4. Espectro extraidos de las fuentes de continuo CS1, CS2, CS3 y CS4 . . . . . 98

5.5. Esquema de la superficie de trabajo de un bowshock . . . . . . . . . . . . . . . 101

5.6. Comparación de cocientes de líneas de ES1 y ES2 con modelos . . . . . . . . 105

5.7. Comparación de cocientes de líneas de ES3 y ES4 con modelos . . . . . . . 106

6.1. IRDC SDC $341.232-0.268 \ldots \ldots \ldots \ldots \ldots \ldots$

6.2. Mapas de temperatura de brillo de ${ }^{13} \mathrm{CO}(2-1)$ hacia SDC 341.232-0.268 . . . . 111

6.3. Emisión promedio de ${ }^{13} \mathrm{CO}(2-1)$ hacia SDC 341.232-0.268 . . . . . . . . . . . 112

6.4. Espectros promedio de ${ }^{12} \mathrm{CO}(2-1),{ }^{13} \mathrm{CO}(2-1)$ and $\mathrm{C}^{18} \mathrm{O}(2-1)$ hacia los Grumos

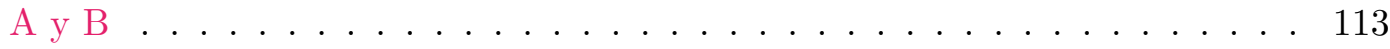

6.5. Perfiles de $\mathrm{HCO}^{+}(1-0), \mathrm{HNC}(1-0)$ y $\mathrm{N}_{2} \mathrm{H}^{+}(1-0)$ hacia los Grumos A y B . . . 116

6.6. Mapas de $\mathrm{HCO}^{+}(1-0), \mathrm{HNC}(1-0)$ y $\mathrm{N}_{2} \mathrm{H}^{+}(1-0)$ hacia SDC 341.232-0.268 . . . 116

6.7. Emisión de la IRDC SDC 341.232-0.26 en el lejano infrarrojo . . . . . . . . . 118

6.8. SEDs calculadas para los Grumos A y B . . . . . . . . . . . . . . . . 119

6.9. Temperatura del polvo hacia SDC 341.232-0.268 . . . . . . . . . . . . . . 119

6.10. Distribución espectral de energía de los EGOs . . . . . . . . . . . . . . 121

6.11. Candidatos a YSOs identificados en la región . . . . . . . . . . . . . . 122

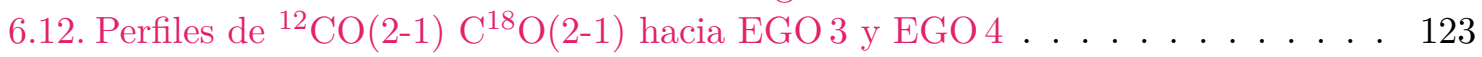

6.13. Diagrama posición-velocidad hacia EGO $4 \ldots \ldots$. . . . . . . . 124

7.1. Complejo de burbujas S108-S111 . . . . . . . . . . . . . . . 128

7.2. Antecedentes de complejo de burbujas . . . . . . . . . . . . . . . 128

7.3. Imágenes tomadas de Vig et al. (2007) . . . . . . . . . . . . . . . . . 130

7.4. Imágenes tomadas de Vasyunina et al. (2011) . . . . . . . . . . . . . . . . 131

7.5. Líneas observadas hacia IRDC316.72 y IRDC316.76 . . . . . . . . . . . . . 132

7.6. Regiones observadas con APEX . . . . . . . . . . . . . . . 133

7.7. Emisión en el lejano IR asociada al complejo S108.S111 . . . . . . . . . . 134

7.8. Emisión de continuo de radio y $24 \mu \mathrm{m}$ asociada al complejo S108-S111 . . . . 135

7.9. Emisión de CO asociado al complejo S108-S111 observada por NANTEN . . 137

7.10. Emisión promedio de ${ }^{12} \mathrm{CO}(3-2),{ }^{13} \mathrm{CO}(3-2)$ y $\mathrm{C}^{18} \mathrm{O}(3-2)$ sobre el filamento . . 138

7.11. Distribución de ${ }^{12} \mathrm{CO}(2-1)$ asociada al complejo S108-S111 . . . . . . . . . . . 139

7.12. Distribución de ${ }^{13} \mathrm{CO}(2-1)$ asociada al complejo S108-S111 . . . . . . . . . . . 140

7.13. Distribución de $\mathrm{C}^{18} \mathrm{O}(2-1)$ asociada al complejo S108-S111 . . . . . . . . . . 140

7.14. Cáscara \#1 . . . . . . . . . . . . . . . . . . . . . . . . . . . 142

7.15. Cáscara \#2 . . . . . . . . . . . . . . . . . . . . . . 143

7.16. Cáscara \#3 . . . . . . . . . . . . . . . . . . . . . . . . 143

7.17. Cáscara \#4 . . . . . . . . . . . . . . . . . . . . . . . . . 144

7.18. Cáscara \#5 . . . . . . . . . . . . . . . . . . . . . . 144 
7.19. Cáscara \#6 . . . . . . . . . . . . . . . . . . . . . . . . . . . . . 145

7.20. Grumos asociados a estructuras de bordes brillantes en la Cáscara \#2. . . . . 145

7.21. Emisión promedio de ${ }^{12} \mathrm{CO},{ }^{13} \mathrm{COy} \mathrm{C}^{18} \mathrm{O}$ hacia las burbujas . . . . . . . . . 146

7.22. Emisión de $\mathrm{C}^{18}$ Ohacia las burbujas a diferentes velocidades. . . . . . . . . . . 148

7.23. Emisión de ${ }^{13} \mathrm{CO}$ hacia las burbujas a diferentes velocidades. . . . . . . . . . 148

7.24. Emisión de ${ }^{12} \mathrm{CO}$ hacia las burbujas a diferentes velocidades. . . . . . . . . 149

7.25. Distribución de $\mathrm{C}^{18} \mathrm{O}(2-1)$ hacia la IRDC . . . . . . . . . . . . . . 150

7.26. Distribución de ${ }^{13} \mathrm{CO}(2-1)$ hacia la IRDC . . . . . . . . . . . . . . . 151

7.27. Distribución de ${ }^{12} \mathrm{CO}(2-1)$ hacia la IRDC . . . . . . . . . . . . . . . 152

7.28. Regiones observadas por MALT90 . . . . . . . . . . . . . . . 153

7.29. Perfiles promedio de $\mathrm{C}^{18} \mathrm{O}(2-1)$ y $\mathrm{HCO}^{+}(1-0)$ de los Grumos $1,2,3$ y 6 . . . 155

7.30. Intensidad integrada de las moléculas detectadas por MALT90 hacia las burbujas 156

7.31. Intensidad integrada de las moléculas detectadas por MALT90 hacia la IRDC 157

7.32. Ejemplo cáscara molecular . . . . . . . . . . . . . . . . . . . 157

7.33. Emisión en continuo de radio a $4.86 \mathrm{GHz} \ldots \ldots$. . . . . . . . . . . 161

7.34. Cúmulos identificados en la región de las burbujas S109-S111 . . . . . . . . . 162

7.35. Fuentes Spitzer-GLIMPSE en región asociada a IRAS 14416-5937 . . . . . . . 163

7.36. Diagrama color-color para fuentes Spitzer candidatos a YSOs . . . . . . . . . 164

7.37. Candidatos a YSO identificados en la región de las burbujas . . . . . . . . . 165

7.38. Candidatos a YSO identificados en la región de la IRDC . . . . . . . . . . 166

7.39. Diagrama color-color para fuentes 2MASS candidatos a YSOs . . . . . . . . 168

7.40. Grumos y candidatos a YSOs sobre emisión promedio de $\mathrm{C}^{18} \mathrm{O}(2-1) \quad \ldots . . .170$

7.41. Cúmulos infrarrojos y candidatos a YSOs detectados sobre el filamento . . . . 170

7.42. Candidatos a YSO en las burbujas sobre emisión promedio de $\mathrm{C}^{18} \mathrm{O}(2-1)$. . 171

7.43. Candidatos a YSO en IRDC sobre emisión promedio de $\mathrm{C}^{18} \mathrm{O}(2-1)$. . . . . . 172

7.44. Representación esquemática de las regiones His $1+3$ y $2+4$, y velocidad en que se detectan las cáscaras . . . . . . . . . . . . . . . . . . 173

7.45. Modelo de región His bipolar. Imagen tomada de Samal et al. (2018) . . . . . 176

8.1. Escalas para la conversión . . . . . . . . . . . . . . . . . . . . . . 189 


\section{Índice de tablas}

1.1. Características de las fases gaseosas del MIE . . . . . . . . . . . . 5

1.2. Propiedades de nubes oscuras, grumos y núcleos . . . . . . . . . . 6

1.3. Propiedades físicas de las nubes moleculares. . . . . . . . . . . . . . 7

1.4. Radios de Strömgren para estrellas de disferente tipo espectral . . . . . . . . 13

2.1. Valores típicos de energía, frecuencia, longitud de onda y temperatura para diferentes tipos de transiciones moleculares . . . . . . . . . . . . . 38

2.2. Frecuencia de transiciones moleculares y densidades críticas . . . . . . . . . 41

2.3. Abundancias moleculares respecto al $\mathrm{H}_{2} \ldots \ldots \ldots \ldots$. . . . . . . . 45

2.4. Factores de conversión de los modelos para calcular la masa de gas ionizado y densidad electrónica de una región Hiı . . . . . . . . . . . . . . . . 52

4.1. Candidatos a YSOs en Gum 31 . . . . . . . . . . . . . . . . . . . 74

4.2. Datos moleculares obtenidos usando APEX en Gum 31. . . . . . . . . . . 74

4.3. Fuentes WISE catalogadas como YSOs detectadas en Gum 31 . . . . . . . 76

4.4. Parámetros de los ajustes gaussianos de la emisión molecular hacia IRAS 10351-

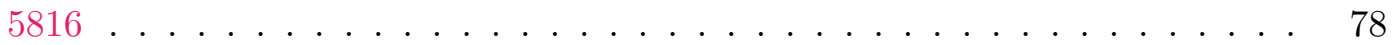

4.5. Parámetros calculados para los grumos en las regiones de las fuentes IRAS 103515816, IRAS 10349-5824, y 2MASS J10365763-5844052 en base a CO . . . . . . 79

4.6. Parámetros derivados para el outflow asociado a IRAS 10349-5824. . . . . . . 80

4.7. Parámetros de los ajustes gaussianos de los perfiles de ${ }^{12} \mathrm{CO}(2-1)$ en dirección a los lóbulos asociados a IRAS $10349-5824 \ldots \ldots$. . . . . . . . . 81

4.8. Parámetros de los ajustes gaussianos a la emisión de gas molecular asociado a

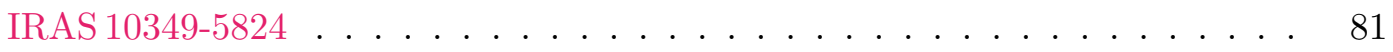

4.9. Parámetros de los ajustes gaussianos a la emisión de gas molecular asociada a 2MASS J10365763-5844052． . . . . . . . . . . . . . . . . 88

5.1. Tabla 1 de Wolfire \& Konigl (1991) . . . . . . . . . . . . . . . . . . . 100

5.2. Cocientes calculados para las fuentes extendidas. . . . . . . . . . . . 103

5.3. Desvío de los cocientes observados respecto a los modelos de Wolfire \& Konigl (1991) . . . . . . . . . . . . . . . . . . . 104

6.1. Emisión máser de metanol a $95 \mathrm{GHz}$ hacia SDC 341.232-0.268 . . . . . . . . . 109

6.2. Parámetros de las líneas observadas hacia SDC 341.232-0.268 . . . . . . . . . 109

6.3. Posición, radio y velocidad de los Grumos A y B definidos a partir de la emisión de ${ }^{13} \mathrm{CO}(2-1) \ldots \ldots \ldots \ldots \ldots \ldots \ldots$

6.4. Parámetros de los grumos moleculares hacia SDC 341.232-0.268 . . . . . . . . 114

6.5. Parámetros de los ajustes de los perfiles extraidos de MALT90 hacia SDC 341.232-

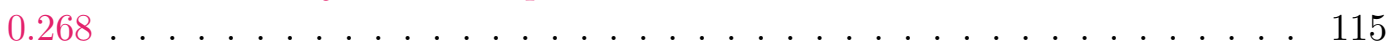


6.6. Parámetros y propiedades físicas derivados para los grumos IR hacia SDC 341.232-

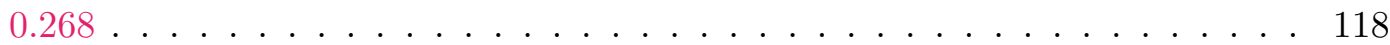

6.7. Parámetros físicos obtenidos a partir del ajustes de la SED para los candidatos

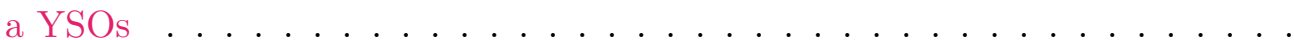

7.1. Posición y tamaño de las burbujas S108, S109, S110 y S111 definidos por Churchwell et al. (2006) . . . . . . . . . . . . . . . . . . . 127

7.2. Parámetros de las IRDCs estudiadas por Vasyunina et al. (2011) . . . . . . . 131

7.3. Parámetros observacionales de las líneas observadas . . . . . . . . . . . 133

7.4. Parámetros de las imágenes promedio de ${ }^{12} \mathrm{CO}(3-2),{ }^{13} \mathrm{CO}(3-2)$ y $\mathrm{C}^{18} \mathrm{O}(3-2)$ sobre el filamento . . . . . . . . . . . . . . . . . . . 139

7.5. Parámetros de las cáscaras definidas a partir de ${ }^{12} \mathrm{CO}(2-1)$. . . . . . . . . . . 142

7.6. Parámetros de las imágenes promedio de ${ }^{12} \mathrm{CO}(2-1),{ }^{13} \mathrm{CO}(2-1)$ y $\mathrm{C}^{18} \mathrm{O}(2-1)$ en la región de las burbujas . . . . . . . . . . . . . . . . . . . . . . 146

7.7. Parámetros observacionales de los grumos definidos hacia las burbujas a partir

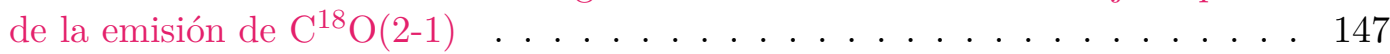

7.8. Moléculas trazadoras de alta densidad asociadas a los grumos 1, 2, 3 y 6 . . 154

7.9. Parámetros de las cáscaras moleculares . . . . . . . . . . . . . . . 158

7.10. Parámetros de los grumos listados en la Tabla 7.7 derivados a partir del gas molecular . . . . . . . . . . . . . . . . . . . . 159

7.11. Parámetros de los ajustes gaussianos a las componentes de la emisión de $\mathrm{N}_{2} \mathrm{H}^{+}(1-0)$ para los Grumos 1 y $2 \ldots \ldots \ldots$. . . . . . . . . . 160

7.12. Radios de Strömgren y tiempos dinámicos para las cáscaras . . . . . . . . . . 174

7.13. Parámetros derivados del modelo de Whitworth et al. (1994) . . . . . . . . . 175

7.14. Candidatos a YSO Spitzer proyectados sobre las burbujas . . . . . . . . . . 178

7.15. Candidatos a YSO Spitzer proyectados sobre la IRDC . . . . . . . . . . . . 179

7.16. Candidatos a YSO WISE proyectados sobre las burbujas . . . . . . . . . . . 180

7.17. Candidatos a YSO WISE proyectados sobre la IRDC . . . . . . . . . . . . 181

7.18. Candidatos a YSO 2MASS proyectados sobre las burbujas . . . . . . . . . . 181

7.19. Candidatos a YSO 2MASS proyectados sobre la IRDC . . . . . . . . . . . 182

7.20. Candidatos a YSO MSX proyectados sobre las burbujas . . . . . . . . . . 183

8.1. Factores de conversión y constantes . . . . . . . . . . . . . . . . 187

8.2. Resolución angular de los datos de catálogos utilizados en esta tesis . . . . . . 188 


\section{Bibliografía}

Allen, L. E., Calvet, N., D'Alessio, P., et al. 2004, ApJS, 154, 363

Allen, T. L., Goddard, J. D., \& Schaefer, III, H. F. 1980, J. Chem. Phys., 73, 3255

Anathpindika, S. V. 2010, MNRAS, 405, 1431

Anderson, L. D., Deharveng, L., Zavagno, A., et al. 2015, ApJ, 800, 101

Aso, Y., Ohashi, N., Saigo, K., et al. 2015, ApJ, 812, 27

Azimlu, M., Fich, M., \& McCoey, C. 2009, AJ, 137, 4897

Battersby, C., Bally, J., Jackson, J. M., et al. 2010, ApJ, 721, 222

Bechtel, H. A., Steeves, A. H., \& Field, R. W. 2006, ApJ, 649, L53

Beck, T. L., Bary, J. S., \& McGregor, P. J. 2010, ApJ, 722, 1360

Benjamin, R. A., Churchwell, E., Babler, B. L., et al. 2003, PASP, 115, 953

Bergin, E. A., Ciardi, D. R., Lada, C. J., Alves, J., \& Lada, E. A. 2001, ApJ, 557, 209

Bergin, E. A. \& Tafalla, M. 2007, ARA\&A, 45, 339

Bertoldi, F. 1989, ApJ, 346, 735

Bertoldi, F. \& McKee, C. F. 1990, ApJ, 354, 529

Beuther, H., Schilke, P., Sridharan, T. K., et al. 2002, A\&A, 383, 892

Beuther, H. \& Sridharan, T. K. 2007, ApJ, 668, 348

Blitz, L. \& Williams, J. P. 1999, ArXiv Astrophysics e-prints

Blondin, J. M., Konigl, A., \& Fryxell, B. A. 1989, ApJ, 337, L37

Boger, G. I. \& Sternberg, A. 2005, ApJ, 632, 302

Bolatto, A. D., Wolfire, M., \& Leroy, A. K. 2013, ARA\&A, 51, 207

Bonnell, I. A. 2007, in Astronomical Society of the Pacific Conference Series, Vol. 367, Massive Stars in Interactive Binaries, ed. N. St.-Louis \& A. F. J. Moffat, 303

Bonnell, I. A., Bate, M. R., \& Zinnecker, H. 1998, MNRAS, 298, 93

Brand, J. \& Blitz, L. 1993, A\&A, 275, 67

Bronfman, L., Nyman, L.-A., \& May, J. 1996, A\&AS, 115, 81 
Brugel, E. W., Shull, J. M., \& Seab, C. G. 1982, ApJ, 262, L35

Cappa, C., Niemela, V. S., Amorín, R., \& Vasquez, J. 2008, A\&A, 477, 173

Cappa, C. E., Duronea, N., Firpo, V., et al. 2016, A\&A, 585, A30

Cappa, C. E., Duronea, N. U., Vasquez, J., et al. 2017, Rev. Mexicana Astron. Astrofis., 53, 79

Carey, S., Noriega-Crespo, A., Price, S., et al. 2005, MIPSGAL : A 24 and 70 Micron Survey of the Inner Galactic Disk with MIPS, Spitzer Proposal

Carraro, G., Patat, F., \& Baumgardt, H. 2001, A\&A, 371, 107

Caselli, P., Myers, P. C., \& Thaddeus, P. 1995, ApJ, 455, L77

Caselli, P., Walmsley, C. M., Tafalla, M., Dore, L., \& Myers, P. C. 1999, ApJ, 523, L165

Caswell, J. L., Batchelor, R. A., Forster, J. R., \& Wellington, K. J. 1989, Australian Journal of Physics, 42, 331

Caswell, J. L. \& Haynes, R. F. 1987, Australian Journal of Physics, 40, 215

Cesarsky, D., Lequeux, J., Abergel, A., et al. 1996, A\&A, 315, L305

Chambers, E. T., Jackson, J. M., Rathborne, J. M., \& Simon, R. 2009, ApJS, 181, 360

Chen, X., Ellingsen, S. P., Shen, Z.-Q., Titmarsh, A., \& Gan, C.-G. 2011, ApJS, 196, 9

Chen, X., Gan, C.-G., Ellingsen, S. P., et al. 2013, ApJS, 206, 9

Chen, X., Shen, Z.-Q., Li, J.-J., Xu, Y., \& He, J.-H. 2010, ApJ, 710, 150

Churchwell, E., Povich, M. S., Allen, D., et al. 2006, ApJ, 649, 759

Churchwell, E., Watson, D. F., Povich, M. S., et al. 2007, ApJ, 670, 428

Churchwell, E. \& Wood, D. O. S. 1989, in Lecture Notes in Physics, Berlin Springer Verlag, Vol. 331, The Physics and Chemistry of Interstellar Molecular Clouds - mm and Sub-mm Observations in Astrophysics, ed. G. Winnewisser \& J. T. Armstrong, 87-92

Cichowolski, S., Romero, G. A., Ortega, M. E., Cappa, C. E., \& Vasquez, J. 2009, MNRAS, 394,900

Comerón, F., Schneider, N., \& Russeil, D. 2005, A\&A, 433, 955

Contreras, Y., Schuller, F., Urquhart, J. S., et al. 2013, A\&A, 549, A45

Cragg, D. M., Sobolev, A. M., Ellingsen, S. P., et al. 2001, MNRAS, 323, 939

Cutri, R. M., Skrutskie, M. F., van Dyk, S., et al. 2003, 2MASS All Sky Catalog of point sources.

Cyganowski, C. J., Brogan, C. L., Hunter, T. R., \& Churchwell, E. 2009, ApJ, 702, 1615

Cyganowski, C. J., Whitney, B. A., Holden, E., et al. 2008, AJ, 136, 2391

Dale, J. E., Haworth, T. J., \& Bressert, E. 2015, MNRAS, 450, 1199 
Deharveng, L., Schuller, F., Anderson, L. D., et al. 2010, A\&A, 523, A6

Deharveng, L., Zavagno, A., Anderson, L. D., et al. 2012, A\&A, 546, A74

Deharveng, L., Zavagno, A., \& Caplan, J. 2005, A\&A, 433, 565

Deharveng, L., Zavagno, A., Salas, L., et al. 2003, A\&A, 399, 1135

Deharveng, L., Zavagno, A., Samal, M. R., et al. 2015, A\&A, 582, A1

Dopita, M. A., Binette, L., \& Schwartz, R. D. 1982, ApJ, 261, 183

Draine, B. T., Dale, D. A., Bendo, G., et al. 2007, ApJ, 663, 866

Draine, B. T., Roberge, W. G., \& Dalgarno, A. 1983, ApJ, 264, 485

Dubner, G. \& Giacani, E. 2015, A\&A Rev., 23, 3

Duronea, N. U., Vasquez, J., Gómez, L., et al. 2015, A\&A, 582, A2

Dutra, C. M., Bica, E., Soares, J., \& Barbuy, B. 2003, A\&A, 400, 533

Dyson, J. E. \& Williams, D. A. 1997, The physics of the interstellar medium

Egan, M. P., Price, S. D., Shipman, R. F., et al. 1999, in Astronomical Society of the Pacific Conference Series, Vol. 177, Astrophysics with Infrared Surveys: A Prelude to SIRTF, ed. M. D. Bicay, R. M. Cutri, \& B. F. Madore, 404

Egan, M. P., Shipman, R. F., Price, S. D., et al. 1998, ApJ, 494, L199

Elmegreen, B. G. \& Lada, C. J. 1977, ApJ, 214, 725

Estalella, R. \& Anglada Pons, G. 2008, Introducción a la física del medio interestelar

Evans, II, N. J. 1999, ARA\&A, 37, 311

Foster, J. B., Jackson, J. M., Barnes, P. J., et al. 2011, ApJS, 197, 25

Foster, J. B., Rathborne, J. M., Sanhueza, P., et al. 2013, PASA, 30, e038

Freyer, T., Hensler, G., \& Yorke, H. W. 2003, ApJ, 594, 888

Freyer, T., Hensler, G., \& Yorke, H. W. 2006, ApJ, 638, 262

Fuente, A. 2001, in ESA Special Publication, Vol. 460, The Promise of the Herschel Space Observatory, ed. G. L. Pilbratt, J. Cernicharo, A. M. Heras, T. Prusti, \& R. Harris, 177

Fuente, A., Martin-Pintado, J., Cernicharo, J., \& Bachiller, R. 1993, A\&A, 276, 473

Fukui, Y., Ohama, A., Kohno, M., et al. 2017, ArXiv e-prints

Fuller, G. A., Williams, S. J., \& Sridharan, T. K. 2005, A\&A, 442, 949

Furukawa, N., Dawson, J. R., Ohama, A., et al. 2009, ApJ, 696, L115

Gardner, F. F. \& Whiteoak, J. B. 1984, MNRAS, 210, 23

Giannini, T., Lorenzetti, D., De Luca, M., et al. 2013, ApJ, 767, 147 
Gregory, P. C., Scott, W. K., Douglas, K., \& Condon, J. J. 1996, ApJS, 103, 427

Griffin, M. J., Abergel, A., Abreu, A., et al. 2010, A\&A, 518, L3

Habe, A. \& Ohta, K. 1992, PASJ, 44, 203

Hacar, A., Kainulainen, J., Tafalla, M., Beuther, H., \& Alves, J. 2016, A\&A, 587, A97

Hacar, A., Tafalla, M., Kauffmann, J., \& Kovács, A. 2013, A\&A, 554, A55

Hanson, M. M., Howarth, I. D., \& Conti, P. S. 1997, ApJ, 489, 698

Haro, G. 1952, ApJ, 115, 572

Hartigan, P. 1989, ApJ, 339, 987

Henshaw, J. D., Jiménez-Serra, I., Longmore, S. N., et al. 2017, MNRAS, 464, L31

Herbig, G. H. 1951, ApJ, 113, 697

Herbst, E. 1978, ApJ, 222, 508

Herbst, E. \& Klemperer, W. 1973, ApJ, 185, 505

Herbst, E., Terzieva, R., \& Talbi, D. 2000, MNRAS, 311, 869

Hildebrand, R. H. 1983, QJRAS, 24, 267

Huang, M., Bania, T. M., Bolatto, A., et al. 1999, ApJ, 517, 282

Inoue, A. K. 2001, AJ, 122, 1788

Jackson, J. M., Finn, S. C., Rathborne, J. M., Chambers, E. T., \& Simon, R. 2008, ApJ, 680, 349

Jackson, J. M., Rathborne, J. M., Foster, J. B., et al. 2013, PASA, 30, e057

Junkes, N., Fuerst, E., \& Reich, W. 1992, A\&A, 261, 289

Kellermann, K. I. \& Verschuur, G. L. 1988, Galactic and extragalactic radio astronomy (2nd edition)

Kessler, M. F., Steinz, J. A., Anderegg, M. E., et al. 1996, A\&A, 315, L27

Koenig, X. P., Leisawitz, D. T., Benford, D. J., et al. 2012, ApJ, 744, 130

Koornneef, J. 1983, A\&A, 128, 84

Kutner, M. L. \& Ulich, B. L. 1981, ApJ, 250, 341

Lada, C. J. 1987, in IAU Symposium, Vol. 115, Star Forming Regions, ed. M. Peimbert \& J. Jugaku, 1-17

Lada, C. J. \& Adams, F. C. 1992, ApJ, 393, 278

Lada, C. J. \& Kylafis, N. D., eds. 1991, NATO Advanced Science Institutes (ASI) Series C, Vol. 342, The physics of star formation and early stellar evolution

Langer, W. D. \& Penzias, A. A. 1990, ApJ, 357, 477 
Larson, R. B. 1969, MNRAS, 145, 271

Lefloch, B. \& Lazareff, B. 1994, A\&A, 289, 559

Linz, H., Klein, R., Looney, L., et al. 2007, in IAU Symposium, Vol. 237, Triggered Star Formation in a Turbulent ISM, ed. B. G. Elmegreen \& J. Palous, 440-440

Lumsden, S. L., Hoare, M. G., Oudmaijer, R. D., \& Richards, D. 2002, MNRAS, 336, 621

MacLaren, I., Richardson, K. M., \& Wolfendale, A. W. 1988, ApJ, 333, 821

Mardones, D., Myers, P. C., Tafalla, M., et al. 1997, ApJ, 489, 719

Mercer, E. P., Clemens, D. P., Meade, M. R., et al. 2005, ApJ, 635, 560

Mezger, P. G. \& Henderson, A. P. 1967, ApJ, 147, 471

Miettinen, O. 2012, A\&A, 542, A101

Miettinen, O. 2014, A\&A, 562, A3

Minniti, D., Lucas, P. W., Emerson, J. P., et al. 2010, New A, 15, 433

Molinari, S., Schisano, E., Elia, D., et al. 2016, A\&A, 591, A149

Molinari, S., Swinyard, B., Bally, J., et al. 2010, PASP, 122, 314

Mul, P. M. \& McGowan, J. W. 1980, ApJ, 237, 749

Najita, J., Carr, J. S., \& Tokunaga, A. T. 1996, ApJ, 456, 292

Offner, S. S. R., Robitaille, T. P., Hansen, C. E., McKee, C. F., \& Klein, R. I. 2012, ApJ, 753,98

Ohama, A., Dawson, J. R., Furukawa, N., et al. 2010, ApJ, 709, 975

Ohishi, M., Irvine, W. M., \& Kaifu, N. 1992, in IAU Symposium, Vol. 150, Astrochemistry of Cosmic Phenomena, ed. P. D. Singh, 171

Ohlendorf, H., Preibisch, T., Gaczkowski, B., et al. 2013, A\&A, 552, A14

Oka, T., Onodera, Y., Nagai, M., et al. 2012, ApJS, 201, 14

Ossenkopf, V. \& Henning, T. 1994, A\&A, 291, 943

Ott, S. 2010, in Astronomical Society of the Pacific Conference Series, Vol. 434, Astronomical Data Analysis Software and Systems XIX, ed. Y. Mizumoto, K.-I. Morita, \& M. Ohishi, 139

Pearson, P. K. \& Schaefer, III, H. F. 1974, ApJ, 192, 33

Perault, M., Omont, A., Simon, G., et al. 1996, A\&A, 315, L165

Peretto, N. \& Fuller, G. A. 2009, A\&A, 505, 405

Poglitsch, A., Waelkens, C., Geis, N., et al. 2010, A\&A, 518, L2

Preibisch, T., Roccatagliata, V., Gaczkowski, B., \& Ratzka, T. 2012, A\&A, 541, A132 
Purcell, C. R., Longmore, S. N., Burton, M. G., et al. 2009, MNRAS, 394, 323

Ragan, S., Henning, T., Krause, O., et al. 2012, A\&A, 547, A49

Rathborne, J. M., Jackson, J. M., \& Simon, R. 2006, ApJ, 641, 389

Rathborne, J. M., Jackson, J. M., Zhang, Q., \& Simon, R. 2008, ApJ, 689, 1141

Rathborne, J. M., Simon, R., \& Jackson, J. M. 2007, ApJ, 662, 1082

Rawlings, J. M. C., Redman, M. P., Keto, E., \& Williams, D. A. 2004, MNRAS, 351, 1054

Rawlings, J. M. C., Taylor, S. D., \& Williams, D. A. 2000, MNRAS, 313, 461

Reipurth, B. \& Bally, J. 2001, ARA\&A, 39, 403

Rimmer, P. B., Herbst, E., Morata, O., \& Roueff, E. 2012, A\&A, 537, A7

Robitaille, T. P. 2008, in Astronomical Society of the Pacific Conference Series, Vol. 387, Massive Star Formation: Observations Confront Theory, ed. H. Beuther, H. Linz, \& T. Henning, 290

Robitaille, T. P. 2017, A\&A, 600, A11

Robitaille, T. P., Whitney, B. A., Indebetouw, R., \& Wood, K. 2007, ApJS, 169, 328

Robitaille, T. P., Whitney, B. A., Indebetouw, R., Wood, K., \& Denzmore, P. 2006, ApJS, 167,256

Rohlfs, K. \& Wilson, T. L. 2004, Tools of radio astronomy

Salpeter, E. E. 1955, ApJ, 121, 161

Samal, M. R., Deharveng, L., Zavagno, A., et al. 2018, A\&A, 617, A67

Sandford, II, M. T., Whitaker, R. W., \& Klein, R. I. 1982, ApJ, 260, 183

Sanhueza, P., Garay, G., Bronfman, L., et al. 2010, ApJ, 715, 18

Sanhueza, P., Jackson, J. M., Foster, J. B., et al. 2012, ApJ, 756, 60

Schilke, P., Walmsley, C. M., Pineau Des Forets, G., et al. 1992, A\&A, 256, 595

Schuller, F., Menten, K. M., Contreras, Y., et al. 2009, A\&A, 504, 415

Shu, F. H., Lizano, S., \& Adams, F. C. 1987, in IAU Symposium, Vol. 115, Star Forming Regions, ed. M. Peimbert \& J. Jugaku, 417-433

Shull, J. M. \& Hollenbach, D. J. 1978, ApJ, 220, 525

Simon, R., Jackson, J. M., Rathborne, J. M., \& Chambers, E. T. 2006, ApJ, 639, 227

Simpson, R. J., Povich, M. S., Kendrew, S., et al. 2012, MNRAS, 424, 2442

Smith, L. J., Norris, R. P. F., \& Crowther, P. A. 2002, MNRAS, 337, 1309

Snell, R. L. 1987, in IAU Symposium, Vol. 115, Star Forming Regions, ed. M. Peimbert \& J. Jugaku, 213-236 
Snell, R. L., Loren, R. B., \& Plambeck, R. L. 1980, ApJ, 239, L17

Stahler, S. \& Palla, F. 2004, The Formation of the Stars.

Stahler, S. W., Palla, F., \& Ho, P. T. P. 2000, Protostars and Planets IV, 327

Strömgren, B. 1939, ApJ, 89, 526

Strong, A. W. \& Mattox, J. R. 1996, A\&A, 308, L21

Sugitani, K., Fukui, Y., \& Ogura, K. 1991, ApJS, 77, 59

Tafalla, M. 2011, in Highlights of Spanish Astrophysics VI, ed. M. R. Zapatero Osorio, J. Gorgas, J. Maiz Apellaniz, J. R. Pardo, \& A. Gil de Paz, 442-453

Talbi, D., Ellinger, Y., \& Herbst, E. 1996, A\&A, 314, 688

Tan, J. C. \& McKee, C. F. 2002, in Astronomical Society of the Pacific Conference Series, Vol. 267, Hot Star Workshop III: The Earliest Phases of Massive Star Birth, ed. P. Crowther, 267

Tasker, E. J. \& Tan, J. C. 2009, ApJ, 700, 358

Tielens, A. G. G. M. \& Hollenbach, D. 1985, ApJ, 291, 722

Torrelles, J. M., Patel, N., Gómez, J. F., Anglada, G., \& Uscanga, L. 2005, Ap\&SS, 295, 53

Turner, B. E., Herbst, E., \& Terzieva, R. 2000, ApJS, 126, 427

Vassilev, V., Meledin, D., Lapkin, I., et al. 2008, A\&A, 490, 1157

Vasyunina, T., Linz, H., Henning, T., et al. 2011, A\&A, 527, A88

Vazzano, M. M., Cappa, C. E., Vasquez, J., Rubio, M., \& Romero, G. A. 2014, A\&A, 570, A109

Verschuur, G. 2007, The Invisible Universe

Vig, S., Ghosh, S. K., Ojha, D. K., \& Verma, R. P. 2007, A\&A, 463, 175

Vilas-Boas, J. W. S. \& Abraham, Z. 2000, A\&A, 355, 1115

Walborn, N. R. 1982, AJ, 87, 1300

Walsh, A. J., Burton, M. G., Hyland, A. R., \& Robinson, G. 1998, MNRAS, 301, 640

Wang, Y., Zhang, Q., Rathborne, J. M., Jackson, J., \& Wu, Y. 2006, ApJ, 651, L125

Watson, C., Povich, M. S., Churchwell, E. B., et al. 2008, ApJ, 681, 1341

Watt, G. D., White, G. J., Millar, T. J., \& van Ardenne, A. 1988, A\&A, 195, 257

Weaver, R., McCray, R., Castor, J., Shapiro, P., \& Moore, R. 1977, ApJ, 218, 377

White, G. J. \& Phillips, J. P. 1983, MNRAS, 202, 255

Whiteoak, J. B., Otrupcek, R. E., \& Rennie, C. J. 1982, Proceedings of the Astronomical Society of Australia, 4, 434 
Whitney, B. A., Indebetouw, R., Babler, B. L., et al. 2004, ApJS, 154, 315

Whitney, B. A., Wood, K., Bjorkman, J. E., \& Cohen, M. 2003a, ApJ, 598, 1079

Whitney, B. A., Wood, K., Bjorkman, J. E., \& Wolff, M. J. 2003b, ApJ, 591, 1049

Whitworth, A. P., Bhattal, A. S., Chapman, S. J., Disney, M. J., \& Turner, J. A. 1994, MNRAS, 268, 291

Wilson, T. L. \& Rood, R. 1994, ARA\&A, 32, 191

Wolfire, M. G. \& Cassinelli, J. P. 1987, ApJ, 319, 850

Wolfire, M. G. \& Konigl, A. 1991, ApJ, 383, 205

Wood, D. O. S. \& Churchwell, E. 1989, ApJS, 69, 831

Wright, A. E., Griffith, M. R., Burke, B. F., \& Ekers, R. D. 1994, ApJS, 91, 111

Wright, E. L., Eisenhardt, P. R. M., Mainzer, A. K., et al. 2010, AJ, 140, 1868

Yamaguchi, N., Mizuno, N., Moriguchi, Y., et al. 1999, PASJ, 51, 765

Yan, Q.-z., Xu, Y., Zhang, B., et al. 2016, AJ, 152, 117

Yorke, H. W. \& Sonnhalter, C. 2002, ApJ, 569, 846

Zavagno, A., Deharveng, L., Brand, J., et al. 2005, in IAU Symposium, Vol. 227, Massive Star Birth: A Crossroads of Astrophysics, ed. R. Cesaroni, M. Felli, E. Churchwell, \& M. Walmsley, 346-351

Zhou, S. 1992, ApJ, 394, 204

Zinnecker, H. \& Yorke, H. W. 2007, ARA\&A, 45, 481

Ziurys, L. M., Adande, G. R., Edwards, J. L., et al. 2015, Origins of Life and Evolution of the Biosphere, 45, 275 